\title{
Die kulturelle Konstruktion von Verwandtschaft unter den Bedingungen der Reproduktionstechnologien in Deutschland
}

\author{
Dissertation \\ zur Erlangung des sozialwissenschaftlichen Doktorgrades \\ der Sozialwissenschaftlichen Fakultät der \\ Universität Göttingen
}

vorgelegt

von

IRIS SCHRÖDER

aus

Salzgitter

Göttingen 2003 
1. Gutachterin: Prof. Dr. Brigitta Hauser-Schäublin.

2. Gutachterin: Prof. Dr. Heidi Rosenbaum.

Tag der mündlichen Prüfung: 01.Oktober 2002 
Für meine Eltern

und meine Tochter Stella Theresa 


\section{Inhalt}

Vorwort 1

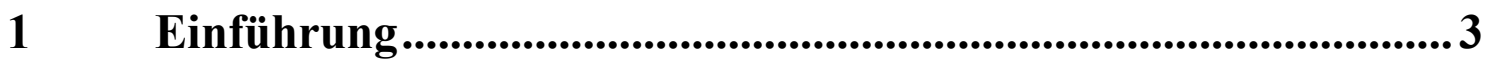

2 Theoretische Überlegungen und die ,Schärfung des Blicks' .... 16

2.1 Die Konstruktion von Verwandtschaft in der Ethnologie..........................16

2.1.1 Verwandtschaftssysteme als Repräsentationen ,natürlicher“ Beziehungen .....16

2.1.2 Die Suche nach einem Vater ..................................................................... 18

2.1.3 „A Critique in the Study of Kinship“: Die Dekonstruktion eines Modells.......21

2.2 Das euro-amerikanische Verwandtschaftskonzept: Die Betonung der körperlichen Prozesse ........................................................................................24

2.2.1 Die Entwicklung von Blutsverwandtschaft als dominantes

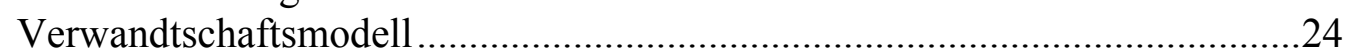

2.2.2 Das biomedizinische Konzept von Zeugung...............................................28

2.2.3 Die soziale Konstruktion der ,natürlichen Fakten“ ........................................30

2.3 Körper, Leib und Selbst ............................................................................31

2.3.1 Die Entwicklung des Körpers als Objekt oder Die soziale Entbettung..............32

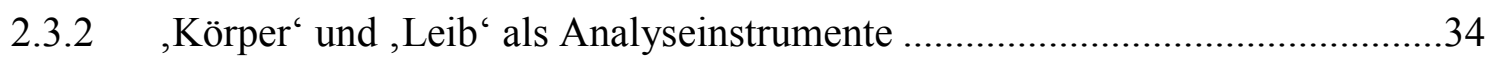

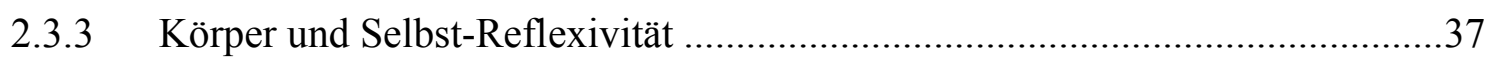

$3 \quad$ Methodisches Vorgehen..................................................................41

3.1 Die ethnologische Forschung in Deutschland ...........................................41

3.2 Datenerhebung und Auswertung ..............................................................44

4 Die Konstruktion von Verwandtschaft im bundesdeutschen rechtlichen Diskurs..............................................55

4.1 Rechtliche Definitionen und Zuordnungen von Personen.............................54

4.1.1 Verwandtschaft, Schwägerschaft und Familie ...............................................54

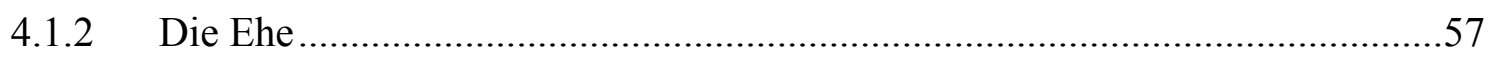

4.1.3 Verwandtschaft - Kindschaft - Elternschaft...............................................59

4.2 Rechtswirkungen gesetzlicher Zugehörigkeiten..........................................64

4.3 Staatlich legitimierte Wege und Möglichkeiten der ,assistierten“ Familiengründung .....................................................................................668

4.3.1 Die Reproduktionstechnologien in Deutschland .........................................69

4.3.1.1 Der gesellschaftliche Rahmen: Das Embryonenschutzgesetz, das Sozialgesetzbuch, die „Richtlinien zur assistierten Befruchtung“ und die Kassenleistungen..... 
4.3.1.2 Die Behandlungsmethoden männlicher und weiblicher „Sterilität““ .75

4.3.1.3 Eine kurze Betrachtung der Konsequenzen der heterologen Insemination bezüglich der Zuordnungen, Rechte und Pflichten der beteiligten Personen

4.3.2 Adoptionen und Pflegschaften: Ein Blick auf die rechtliche Situation

5 „Verwandtschaft ${ }^{66}$ und das ,eigene“6 Kind: Der persönliche Diskurs.

5.1 Die Konstruktion von Zugehörigkeit und Angehörigkeit im Kontext der Reproduktionsmedizin ................................................................................92

5.1.1 Deszendenz und Affinalität .................................................................... 92

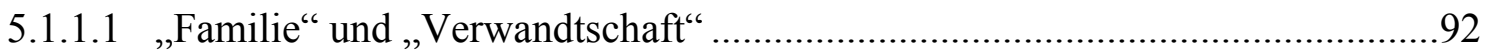

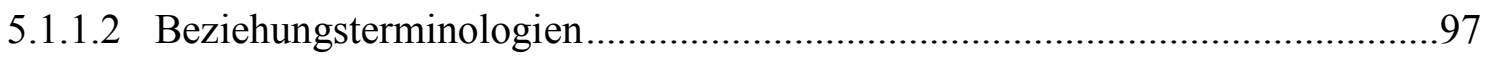

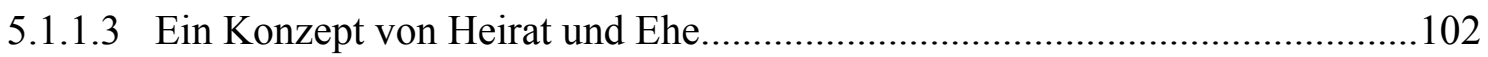

5.1.1.4 Die Beendigung einer Ehe und die Auswirkungen auf Vorstellungen von Verwandtschaft.

5.1.2 Die zeitliche Dimension von Zugehörigkeit: Die gemeinsame

Vergangenheit und ihre Bedeutung für die Vorstellung von

Verwandtschaft.

5.1.3 Die sozialen Konsequenzen von „Verwandtschaft“ “....................................114

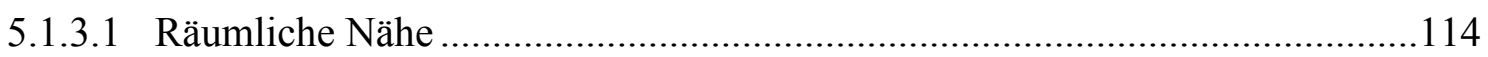

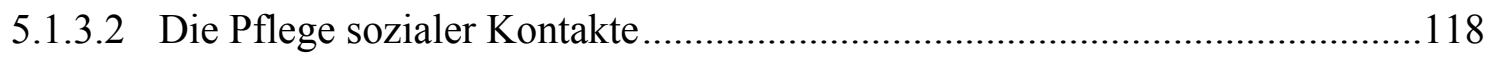

5.1.3.3 Ökonomische und moralische Unterstützungen und Verpflichtungen ............125

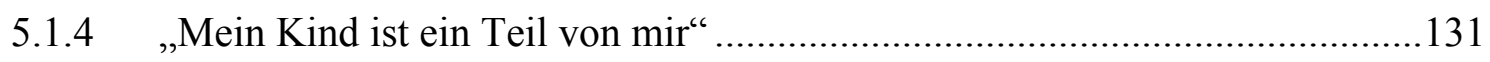

5.1.4.1 Physische und psychische Ähnlichkeiten.....................................................131

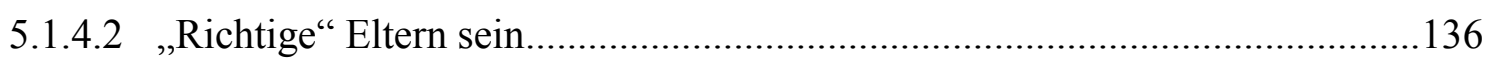

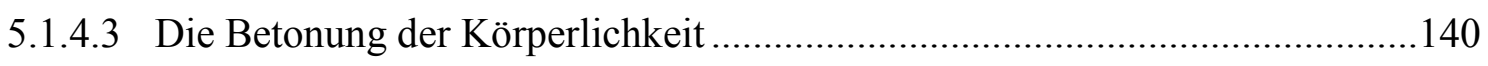

5.2 Ein Blick auf die Konstruktion von Zugehörigkeit und Angehörigkeit im Kontext von Adoptionen und Pflegschaften

5.2.1 Soziale Interaktionen und der Aspekt der gemeinsamen Lokalität als Basis enger emotionaler Bindungen ........................................................143

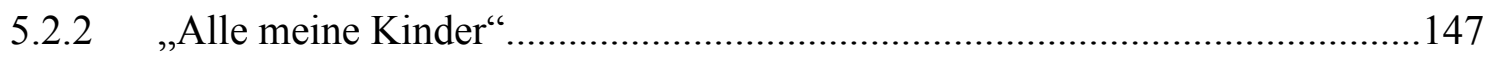

5.2.2.1 „Sie ist so sehr meine eigene Tochter, so von ihrem Wesen her.“...................148

5.2.2.2 „Eltern wird man aus durchwachten Nächten, durch Wickeln und Freude

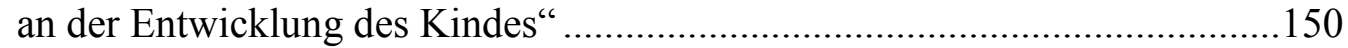

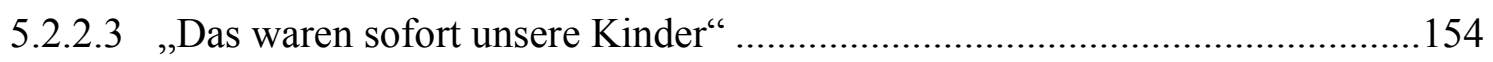

6 „Zeugung 6 im Schnittpunkt verschiedener Diskurse............. 160

6.1 Die innermedizinische Perspektive ...........................................................162

6.1.1 „Man muss sich auch mal von dem Mythos Zeugung verabschieden

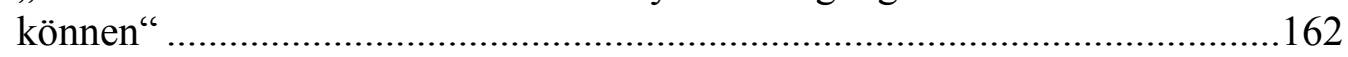

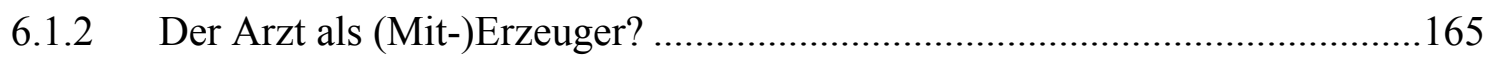

6.2 Die Sichtweise der Paare...........................................................................174 


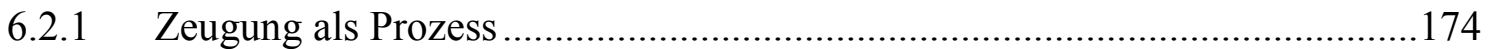

6.2.2 Die Gleichsetzung von Zeugung und Befruchtung .....................................179

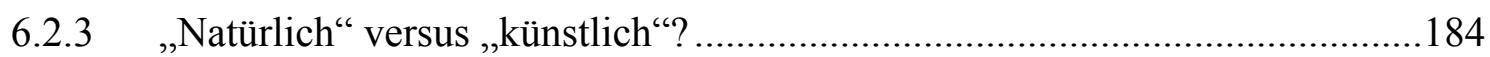

6.3 Fallbeispiel: Zeugung mit ,,Spendersperma“ ${ }^{6}$.............................................191

7 „Verwandtschaft ${ }^{66}$ und Reproduktionstechnologien in Deutschland aus ethnologischer Perspektive.

7.1 Die soziale Konstruktion von Blutsverwandtschaft und ihre spezifischen Konsequenzen in verschiedenen Kontexten vor dem Hintergrund der reproduktiven Technologien..........................................200

7.2 Das ,eigene“ Kind im Schnittpunkt von Körper, Selbst und Person.......213

7.3 Die Konstruktion der Reproduktion .......................................................219

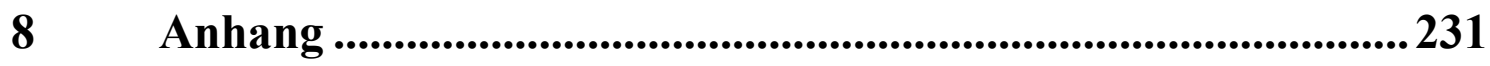

Anhang 1: Fragebogen Reproduktionsmedizin................................................232

Anhang 2: Leitfadeninterview Reproduktionsmedizin .......................................234

Anhang 3: Leitfadeninterview Adoptiv- und Pflegeeltern ................................237

Anhang 4: Soziobiografische Angaben zu den zitierten Personen......................239

Anhang 5: Glossar medizinischer Fachausdrücke ..............................................246

Anhang 6: Abkürzungsverzeichnis...............................................................248

$9 \quad$ Literatur ...................................................................................... 249 


\section{Vorwort}

Diese Arbeit ist entstanden aus dem Forschungsprojekt „Verwandtschaft und Humantechnologien in Deutschland“", das von 1996 bis 2001 am Institut für Ethnologie der Universität Göttingen durchgeführt wurde. Dieses Forschungsvorhaben stand unter der Leitung von Professorin Brigitta Hauser-Schäublin und wurde von der Deutschen Forschungsgemeinschaft finanziert. Als wissenschaftliche Mitarbeiter und Mitarbeiterinnen waren daran mit unterschiedlichen inhaltlichen Forschungsschwerpunkten Dr. med Lutz Freudenberg (bis 1998), Dr. Vera Kalitzkus, Dr. Imme Petersen und ich beteiligt. Die Resultate publizierten wir gemeinsam in dem Buch „Der geteilte Leib. Die kulturelle Dimension von Organtransplantation und Reproduktionsmedizin in Deutschland (Hauser-Schäublin et al. 2001 im Campus Verlag, Frankfurt am Main). Die Dissertationen von Vera Kalitzkus (2001) und Imme Petersen (2002) bieten weiterhin vertiefte Einblicke in spezielle Einzelaspekte.

Meine Dissertation basiert auf von mir in dem Forschungsprojekt erhobenen Datenmaterial und führt es unter spezifischen Fragestellungen weiter. Ich möchte an dieser Stelle den Ärzten und Ärztinnen, die mich an ihrem beruflichen Alltag teilhaben ließen und mir viele Fragen beantwortet haben, danken. Ohne die Offenheit der Patienten und Patientinnen und der Teilnehmerinnen und Teilnehmer der Selbsthilfegruppe für ,ungewollte Kinderlosigkeit“ hätte diese Arbeit jedoch nicht geschrieben werden können. Durch Erzählungen über ihre persönlichen Hintergründe, ihre Gefühle und Erlebnisse sowie die konkreten Erfahrungen ermöglichten sie mir einen tieferen Einblick in die verschiedenen Bereiche der Anwendung und des Umgangs mit den Reproduktionstechnologien. Ihnen allen danke ich für ihr Vertrauen.

Dieser Dank gebührt auch den Adoptiv - und Pflegeeltern, die mich in ihre privaten Räumlichkeiten einluden und sich viel Zeit nahmen für die Gespräche. Sie gaben mir dadurch die Chance ihre Einstellungen, Sorgen und Freuden besser zu verstehen.

Die Bearbeitung und Auswertung meines umfangreichen Datenmaterials ist durch den Einsatz der wissenschaftlichen Hilfskraft Annika Schmitt, die die Transkriptionen der Tonbänder geduldig und genau durchführte, wesentlich erleichtert worden. Die Diskussionen und der Austausch mit meinen Kolleginnen Vera Kalitzkus und Imme Petersen gaben mir immer wieder neue Anregungen und ermöglichten mir, meine Fragestellungen und die Herangehensweise an mein Thema kritisch zu überdenken. Ganz besonders hat mir die Unterstützung und der fachliche Rat von Brigitta Hauser-Schäublin geholfen. Ich danke ihnen allen sehr für die langjährige gute Zusammenarbeit. 
Die intensive Beschäftigung mit einer spezifischen Thematik beinhaltet immer auch die Gefahr einer gewissen „Betriebsblindheit“. Die Zeit und die Mühe, die Dr. Michael Dickhardt und Susanne Kessel in das Lesen und Korrigieren meiner Texte investiert haben, haben wesentlich dazu beigetragen, mich wieder „sehend“ zu machen.

Auf dem Weg durch den Dschungel der Formatierungen hat mich Markus Göbel begleitet und unterstützt. Seine fachliche Kompetenz, aber auch seine Ruhe und Geduld haben in der nervenaufreibenden Endphase dieser Arbeit sehr zu ihrer Vollendung beigetragen.

Ohne das kontinuierliche Zutrauen und den Beistand meiner Eltern hätte ich diese Arbeit so nie schreiben können. Ihnen und meiner Tochter Stella, die mir immer wieder gezeigt hat, dass es wichtigere Dinge im Leben gibt als eine Dissertation, möchte ich diese Arbeit widmen. 


\section{$1 \quad$ Einführung}

„The most significant development in the study of kinship has been the growing awareness of the cultural specificity of what were previously taken for granted to be the natural facts on which all kinship systems were presumed to be built.“

(Holy 1996:165)

Mit der Durchsetzung der Reproduktionstechnologien ${ }^{1}$ zu Beginn der 1980er Jahre zur Behandlung von „Sterilität“2 konnte Fortpflanzung aus der intimen sexuellen Beziehung eines Paares herausgelöst und die Erzeugung von Kindern mit Hilfe medizinischtechnischer Methoden bewirkt werden. Im Zentrum der Anwendung der reproduktiven Technologien steht dabei die Vorstellung von leiblicher Verwandtschaft, die auf der Annahme basiert, dass „,natürliche“ Verwandtschaft auf geteilten Körpersubstanzen aufbaut. Auf der Grundlage der Überlegung, Verwandtschaft im Sinne einer kulturell konstruierten Form von Verbundenheit zu begreifen, als ein System kulturellen Wissens, durch welches die soziale Praxis wahrgenommen wird, das jedoch Konzepte von Sexualität und geschlechtlicher Fortpflanzung berührt, ${ }^{3}$ haben sich verwandtschaftsethnologische Untersuchungen auch den Verwandtschaftsvorstellungen der westlichen Gesellschaften zugewandt. So haben beispielsweise Schneider (1980 [1968]) für Amerika, Strathern (1992a) und Edwards (2000) für England die Bedeutsamkeit von Blutsverbindungen als konstituierend für besondere Formen sozialer Beziehungen, die als Verwandtschaft konzipiert werden, nachgewiesen und die so wichtige Kongruenz biologischer und sozialer Verwandtschaft dargelegt. Sie konnten zeigen, dass „,natürliche“

\footnotetext{
Wobei hier nach Stanworth (1987:10f) empfängnisfördernde Technologien betrachtet werden. Stanworth unterscheidet vier Gruppen von Technologien, die in die menschliche Fortpflanzung eingreifen. Die ersten beiden umfassen medizinische Eingriffe, die die Geburtenkontrolle und den Umgang mit Schwangerschaft und Geburt regeln. Die dritte Kategorie, Technologien der pränatalen Vorsorge, und die vierte, empfängnisfördernde Technologien, sind jedoch erst in diesem Jahrhundert entwickelt worden und charakteristisch für euro-amerikanische Gesellschaften.

2 Ich verwende in meiner Arbeit doppelte Anführungszeichen bei bestimmten Begriffen, die ich aus dem gesellschaftspolitischen Diskurs übernehme. Ich möchte sie dadurch als kulturelle Selbstverständlichkeiten sichtbar machen, die ich hier aber nicht detailliert reflektiere. Mit einfachen Anführungszeichen kennzeichne ich die offiziell verwendeten Begriffe und distanziere mich damit von ihnen. So handelt es sich bei dem Begriff der „Sterilität“ auf den ersten Blick um eine wissenschaftliche Deskription, die sich auf körperliche ,Störungen ' bezieht. Er wird in der medizinischen Praxis verwendet, wenn nach ein bis zwei Jahren mit regelmäßigem ungeschützten Geschlechtsverkehr keine Schwangerschaft eingetreten ist. Unter „Sterilität“ werden primär die körperlichen ,Störungen“ zusammengefasst, die eine Empfängnis und Schwangerschaft verhindern. „Infertilität“ bezeichnet die Situation, dass es zwar zu einer Empfängnis kommt, der Embryo allerdings nicht bis zur Geburt ausgetragen werden kann. „Männliche Infertilität“ bezieht sich auf die Zeugungsunfähigkeit des Mannes. In der Andrologie bezieht sich die Bezeichnung „Sterilität“ auf beide Partner (Ulrich et al. 1988; Pschyrembel 1993:1461). Ich werde in meiner Arbeit den Begriff der „Sterilität“" sowohl für Männer als auch für Frauen verwenden.

3 Auch wenn diese Konzepte unterschiedlich ausgeprägt sind und unterschiedlich betont werden (vgl. HauserSchäublin 1998:55).
} 
Verwandtschaft auch sozial über Körper und Körpersubstanzen definiert wird. Ihren Untersuchungen zufolge vereint Verwandtschaft in den euro-amerikanischen Gesellschaften ${ }^{4}$ zwei Dimensionen in sich: eine biologische, d.h. der Verwandtschaftsgrad zwischen Personen wird bestimmt vom Anteil der Körpersubstanzen, die sie miteinander teilen, und eine soziale, was bedeutet, dass eine Person als verwandt gilt, wenn ihr Verwandtschaftsgrad sozial anerkannt ist. Diesem Konzept liegt die Trennung von ,Natur' und Kultur zugrunde, wobei die biologische Verwandtschaft der ,Natur' zugeordnet wird (Strathern 1992b:15). Zeugung und Fortpflanzung gelten als ,natürliche“ Phänomene und bilden die Voraussetzung für Verwandtschaftsvorstellungen, die der Kultur zugeordnet werden. Nach Strathern stellt Verwandtschaft das Bindeglied zwischen dem Bereich der ,Natur' und dem der Kultur dar:

„Human kinship is regarded as a fact of society rooted in facts of nature. Persons we recognise as kin divide into those related by blood and those related by marriage, that is, the outcome of or in prospect of procreation. However, the process of procreation as such is seen as belonging not to the domain of society but to the domain of nature. Kinship thus connects the two domains." (Strathern 1992b:16-17; Hervorhebung im Original)

Da die Konstruktion von „,natürlicher“ Verwandtschaft in euro-amerikanischen Gesellschaften eng mit Körper und Körperlichkeit verbunden ist, rücken auch die neuen Reproduktionstechnologien immer mehr in den Mittelpunkt zahlreicher Studien, da sie die Möglichkeit eröffnen, den Geschlechtsverkehr, den Schneider (1980 [1968]:40) als zentrales Symbol des amerikanischen Verwandtschaftskonzeptes bei der Schaffung der kulturellen Einheit der Kernfamilie bezeichnet hat, abzuschaffen und durch die außerkörperliche Zusammenführung von Ei- und Samenzellen zu ersetzen. Fortpflanzung wird somit zunehmend zu einem öffentlichen Ereignis, an deren Durchführung außer dem Paar zahlreiche weitere Personen wie beispielsweise Ärzte und Ärztinnen, medizinische Mitarbeiter und Mitarbeiterinnen ${ }^{5}$ beteiligt sein müssen (vgl. Franklin 1993b:545, 1995:336).

Ethnologische Untersuchungen, die sich mit Verwandtschaft und „assistierter“ Fortpflanzung im Kontext der Reproduktionstechnologien beschäftigt haben, sind bisher hauptsächlich in den USA und England durchgeführt wurden. So erörtern die Autorinnen unter anderem die Frage, ob die neuen Methoden technologischer Interventionen in die Fortpflanzung, und damit in den Bereich der ,Natur', ,traditionelle‘ Vorstellungen

\footnotetext{
4 Zwar sind die Ergebnisse der Untersuchungen über europäische Verwandtschaftsvorstellungen nicht ohne weiteres auf den amerikanischen Kontext übertragbar und vice versa. Da das Konzept der Blutsverwandtschaft jedoch in beiden Kontexten eine zentrale Rolle spielt, werde ich diesen zusammenfassenden Begriff auch in meiner Arbeit verwenden (vgl. z.B. Franklin 1995:336, Strathern 1995:348).

5 Auf die zusätzliche Angabe der grammatikalisch weiblichen Form verzichte ich im Folgenden. So ich es nicht ausdrücklich erwähne, handelt es sich immer um Männer und Frauen.
} 
von Zeugung und der Schaffung neuen Lebens verändert haben: Führen die Schöpfung von Leben im Auftrag anderer Personen, die multiple Partizipation an der Zeugung sowie Körperbeziehungen ohne soziale Beziehungen zu einer Veränderung von Konzepten über Elternschaft, Verwandtschaft sowie über menschliche Ursprünge (vgl. z.B. Edwards et al. 1993; Franklin 1993b, 1995, 1997; Stacey 1992b)? Im Zusammenhang mit der Zeugung menschlichen Lebens stellen sich somit Fragen nach der Bedeutung von Verwandtschaft und deren Interpretation sowie nach dem Einfluss der medizinischtechnischen Möglichkeiten auf die kulturelle Konstruktion von Verwandtschaft:

„With the assistance to conception comes also assisted origins, assisted relations, assisted genealogy, and assisted futures. The meaning of such assistance is not merely additive: it is transformative. One does not only derive new relations, but new ways of understanding relatedness, new implications of relatedness, new joys of relatedness, and new fears about bringing the dangers of relatedness, or of bringing new relatedness into being." (Franklin 1993a:128-129)

Die Reproduktionstechnologien schaffen durch die ,assistierte“ Fortpflanzung nicht nur neue Individuen, womit sie in den Bereich der ,Natur ${ }^{6}$ eingreifen, sondern lassen gleichzeitig auch neue soziale interpersonelle Beziehungen entstehen. Marilyn Strathern spricht deshalb nicht nur von ,assisted procreation“ sondern von ,assisted kinship“ (1992b:14), weil Verwandtschaft zwar als ein soziales Phänomen betrachtet wird, das jedoch auf Tatsachen der ,Natur' aufbaut. Wie - neben anderen Autorinnen - Stanworth (1987) und Strathern (1992b) aufgezeigt haben, konnten die Reproduktionstechnologien zu einer Auftrennung von ehemals als Einheit wahrgenommenen Konzepten von Mutterschaft, Vaterschaft und Elternschaft überhaupt führen. ${ }^{6}$ Umgekehrt wirken ,traditionelle‘ Vorstellungen von Verwandtschaft und deren Konstituierung auf den Umgang mit Reproduktionstechnologien. So haben beispielsweise Untersuchungen von Leihmutterschaften in den USA gezeigt (Dolgin 1995, Ragoné 1994, Tong 1995), nicht nur wie und inwieweit solche durch den Einfluss der Medizin möglich gewordenen Formen der Zeugung von Kindern immer noch von ,traditionellen' Vorstellungen von Verwandtschaft geprägt sind, sondern auch wie Leihmutterschaft dementsprechend

\footnotetext{
6 Vor der Einführung der Reproduktionstechnologien wurden in euro-amerikanischen Gesellschaften zwei Dimensionen von Elternschaft anerkannt: die ,natürliche“ (biologische) und die soziale Elternschaft, d.h. die Eltern, die ein Kind aufziehen. Mit der Etablierung der Technologien entstanden nun drei Dimensionen von Elternschaft: die ,natürliche“ Elternschaft, d.h. die Eltern, die ihre Kinder ohne medizinische Hilfe zeugen und zur Welt bringen, die biologische und die soziale Elternschaft (vgl. Strathern 1992b:19ff). Gespaltene soziale Elternschaft ist kein neues gesellschaftliches Phänomen (Hoffmann-Riem 1989:389), doch hat sich erst durch die Reproduktionstechnologien die gewollte Aufspaltung biologisch-sozialer Elternschaft etabliert. Sie eröffnete weiterhin die Möglichkeit, auch biologische Elternschaft zu fragmentieren, in dem beispielsweise ein befruchtetes Ei aus einer außerkörperlichen Befruchtung nicht in die Gebärmutter der „Spenderin“ zurückgebracht werden musste, sondern einen anderen Frau eingepflanzt werden konnte. So konnte das gesellschaftlich neue Phänomen der sogenannten gespaltenen Mutterschaft entstehen.
} 
reinterpretiert wird; Die Aspekte, die den ,traditionellen“ Vorstellungen der Zeugung von ,eigenen“ Kindern widersprechen, werden weitgehend ausgeblendet.

Die Reproduktionstechnologien haben somit nicht nur für „ungewollt kinderlose“7 ${ }^{\text {(7) }}$ Pare neue Möglichkeiten eröffnet, um Kinder zu bekommen, in dem sie bei der Fortpflanzung assistieren, sondern sie haben auch zu der Schaffung von Verwandtschaft geführt, in denen vertraute Annahmen über die biologischen Verbindungen aufgegeben werden müssen. Kulturelle dominante Vorstellungen, die die menschliche Reproduktion in den Bereich der ,Natur' eingeordnet und verwandtschaftliche Beziehungen mit Bezug auf die biologische Fortpflanzung definiert haben, können dadurch in Frage gestellt werden. So stellt Strathern (1993b:140) fest: ,[...] the NRT [New Reproductive Technologies] themselves provide a new context in which to think about human affairs. [...] They have the potential to make us think again about what we take for granted, what we look for in family life, how we regard the relationship between parents and children" (vgl. auch Edwards 2000:34). So beschäftigen sich verwandtschaftsethnologische Untersuchungen über Reproduktionstechnologien z.B. auch mit Prozessen der ,Normalisierung und deren widersprüchlichen Dimensionen, wobei ein Schwerpunkt der Betrachtungen auf den sich verändernden Bedeutungen der „natürlichen Tatsachen“ liegt. So rückten insbesondere ins Zentrum der Untersuchungen von Franklin (1997) die Erfahrungen im Umgang mit der ,achieved conception“. Sie kann zeigen, dass der Prozess der ,Normalisierung' auch eine ,Naturalisierung' und Routinisierung beinhaltet und sich im Kontext fortschreitender technologischer Assistenz neue Dimensionen der ,natural facts of life“" ablesen lassen.

Die Ergebnisse der ethnologischen Untersuchungen über amerikanische und englische Verwandtschaftsvorstellungen lassen sich, ohne vorweg greifen zu wollen, in wesentlichen Aspekten auf Deutschland übertragen. Die Vorstellung, dass „natürliche“ Verwandtschaft über den Körper, d.h. die biogenetische Verschmelzung von Körpersubstanzen, konstruiert wird, führt weiterhin zu der Frage nach der Konstituierung von Körper. Der Körper steht als Gegenstand vielfältiger Diskurse ${ }^{8}$ im Mittelpunkt der Körperethnologie (anthropology of the body). Dabei geht es u.a. um ,the making of the modern body“, die Entwicklung der modernen Anatomie, Biologie und Medizin unter be-

\footnotetext{
7 Die Bezeichnung „ungewollt kinderlos“ übernehme ich in meiner Arbeit, da sie auch als Eigenbezeichnung von den von mir interviewten Personen im Kontext der Reproduktionsmedizin verwendet wird. Dieser Begriff impliziert jedoch auch gleichzeitig einen ,Mangel` (vgl. Lang 1992:10) und rekurriert auf ein Leiden, das durch die Abwesenheit eines Kindes entsteht. „Ungewollte Kinderlosigkeit“ wird besonders im gesellschaftspolitischen Diskurs verwendet zur Beschreibung eines Zustandes, der als abweichend vom ,Normalen` gilt und dadurch per se eine Behandlungsnotwendigkeit einbezieht. Des weiteren wird dieser Zustand primär als ein Symptom von „Sterilität“ angesehen, dadurch medikalisiert und dem Zuständigkeitsbereich der Biomedizin zugeordnet, auch wenn nur teilweise organische Erkrankungen deren Ursache sind.

8 Im Sinne Foucaults (1974) verstehe ich Diskurse als Macht- und Handlungsfelder, die an bestimmte Gruppen und Akteure gebunden sind bzw. von diesen kontrolliert werden (vgl. auch Hauser-Schäublin et al. 2001:254).
} 
sonderer Berücksichtigung der ,Erfindung‘ bzw. ,Festlegung“ von geschlechtsspezifischen Körpern (sex) aufgrund biologischer „Fakten“, wie sie beispielsweise in den Studien von Laqueur und Gallagher (1987) oder Duden (1991) behandelt werden. So geht die Körperhistorikerin und -theoretikerin Barbara Duden davon aus, dass es sich bei dem Körper nicht um einen ahistorischen biologischen Stoff handelt, der je nach Epoche lediglich unterschiedlich interpretiert und repräsentiert wird. Ihrer Ansicht nach wird der Körper überhaupt erst durch und in historischen und kulturell spezifischen Konzepten konstituiert. Der Körper gilt demnach nicht als naturwissenschaftliche Größe, sondern als Bedeutungsträger für kulturelle und gesellschaftliche Vorstellungen, die wiederum kulturspezifisch und historisch gewachsen sind: „The body itself is a cultural creation. [...] Even when we focus on the physicality of the body as flesh and bone, we still perceive it in cultural terms“" (Sault 1994:14).

Auch der Soziologe Giddens (1991) beschäftigt sich mit den Bedingungen, unter denen der Körper als Bedeutungsträger fungiert. Er arbeitet diese Bedingungen für die Spätmoderne ${ }^{9}$ heraus, die insbesondere geprägt ist durch die institutionalisierte Reflexivität, d.h., dass neue Erkenntnisse verwendet werden, um Aspekte des gesellschaftlichen Lebens kontinuierlich zu bearbeiten und zu modifizieren (vgl. auch Beck 1986:26). Das entscheidende Merkmal der Reflexivität der Spätmoderne ist die Institutionalisierung im Rahmen einer besonderen Form der Rationalität und die Anerkennung dieser Reflexivität als Wert an sich. Diese Reflexivität betrifft auch das Individuum und damit Konzepte von Körper und Selbst, da, wie Giddens postuliert, in der Spätmoderne über den individuellen Körper das Selbst dargestellt wird, das sich im ,reflexiven Projekt des Selbst“" ständig bestätigt oder erneuert (1991:5). Da auch der Körper keine ,natürliche‘, d.h. unveränderliche, Gegebenheit mehr ist im Sinne eines passiven Objektes, unterliegen demnach Körper und Selbst einem kontinuierlichen Prozess der Bearbeitung bzw. Anpassung (Giddens 1991:218).

In der westlichen Gesellschaft der Spätmoderne steht dem Diskurs des Körpers als aufteilbare, ergänzbare sowie reparierbare Größe, derjenige vom , geschlossenen“ kontrollierbaren Körper des Subjekts gegenüber. Die Betrachtung des Körpers als Ansammlung von Einzelteilen, losgelöst vom Selbst, macht es möglich, sich von bestimmten Organen, Körperteilen und -zellen trennen zu können, ohne dass das Selbst davon betroffen sein muss (vgl. Sault 1994:300-301). Dieses biomedizinische Körperbild produziert auch eine entsprechende Praxis, d.h. nur auf Grundlage dieses Konzeptes ist die Durchführung beispielsweise der Reproduktionstechnologien überhaupt möglich. Ande-

\footnotetext{
9 Die Spätmoderne ist nach Giddens (1991:21) geprägt von der Entkoppelung von Zeit und Raum in Bezug auf soziale Beziehungen, von Entbettungsmechanismen und Expertensystemen, die er zusammen als abstrakte Systeme bezeichnet, vor allem aber durch die institutionalisierte Reflexivität.
} 
rerseits wird eine enge Verbindung zwischen Körper, Selbst und Identität angenommen und es steht im Vordergrund, die physische Geschlossenheit des Körpers zu bewahren und zu kontrollieren, da nur so die persönliche Identität sichergestellt ist. Diese Vorstellung muss mit Einschränkungen kein Gegensatz sein, sondern ist eine Folge der Lösung des Selbst vom Körper. Wen man das Selbst und den Körper für relativ unabhängig voneinander hält, kann an dem Körper ,gearbeitet' werden, um ihn zu einem entsprechenden Ausdruck des Selbst zu machen bzw. beides einander anzugleichen. Der Körper ist demnach eng verbunden mit der Erfahrung des Selbst und dem Erleben sozialer Beziehungen, in denen das Individuum zur Person ${ }^{10}$ wird. Die Vorstellung des individuellen Selbst als sicher im eigenen Körper verankert, wird jedoch durch das Symptom der ,ungewollten Kinderlosigkeit“, dessen Ursache im medizinischen Diskurs in der körperlichen „Sterilität“" lokalisiert wird, in Frage gestellt. Durch das Gefühl der biografischen Dissonanz, die der Zustand der „ungewollten Kinderlosigkeit“ mit sich bringt, gerät die Selbst-Identität ${ }^{11}$ in eine Krise. Dieser empfundene Bruch hängt auch direkt mit den gesellschaftlichen Erwartungen an die geschlechtliche und soziale Rolle von Mann und Frau zusammen. Die Reproduktionsmedizin arbeitet somit an der Schnittstelle zwischen Körper, Selbst und Person.

Die vorliegende Arbeit versteht sich im dargelegten Forschungskontext als ein Beitrag $\mathrm{zu}$ den Vorstellungen von Zugehörigkeit und Angehörigkeit (ideas of relatedness) unter den Bedingungen der Reproduktionstechnologien in Deutschland, da eine Beschäftigung mit „Verwandtschaft“12 und den neuen reproduktiven Technologien im Rahmen der Ethnologie in Deutschland Neuland darstellt. Auf dem Hintergrund kulturvergleichender Studien zu Verwandtschaft und Zeugung, die aufzeigen, warum sich Menschen

\footnotetext{
10 In der Ethnologie führte Marcel Mauss (1978 [1938]) das Konzept von Person als eine kulturelle Kategorie ein, das gesellschaftlich geformt wird. Als kulturelles Konzept ist die Idee von Person in kulturelle Bedeutungssysteme eingebunden und variiert somit interkulturell. Grace Harris griff die Kategorien von Mauss bezüglich Selbst und Person auf und schlug vor, Personsein als eine Art Status zu betrachten, der denjenigen Gesellschaftsmitgliedern zugeteilt wird, die bestimmte Qualifikationen für eine angemessene Teilnahme am sozialen Leben besitzen. Das Konzept von Person entsteht somit im sozialen Kontext und sozialen Handeln: „Dealing with a concept of person entails conceptualizing the human or other being as an agent, the author of action purposively directed towards a goal. By 'human person' I mean a human being publicly considered as an agent. In this sense, to be a person means to have a certain standing (not 'status') in a social order, as agent-in-society“ (Harris G. 1989:602). Das Konzept von Person impliziert gleichzeitig die Vorstellung von Selbst und Selbstwahrnehmung in Vergangenheit und Zukunft, da soziales Handeln immer auch durch wahrgenommene Ziele, gelebte Erfahrungen, beurteilte Situationen und getroffene Wahl motiviert ist. Einen ausführlichen Überblick über Konzepte von Selbst und Person im ethnologischen Diskurs bietet Csordas (1994).

11 Die sichere ,Verankerung' des Selbst im Körper steht in enger Verbindung mit der kontinuierlichen Eigenwahrnehmung und Anerkennung durch das soziokulturelle Umfeld. Selbst-Identität ist nach Giddens (1991:218) das Gefühl eines ungebrochenen Verlaufs der eigenen Lebensgeschichte.

12 Untersuchungen zur Konstituierung von Verwandtschaft finden sich im deutschsprachigen Raum nur wenige (Fehlmann-von der Mühll 1978, Fenner 1984). Die meisten soziologischen Studien (König 1974, Weber-Kellermann 1974) haben die Frage nach der Konstruktion von Verwandtschaft allerdings ausgeklammert und sich primär auf Interaktionen und die Funktion von Verwandtschaft sowie Formen der Familie konzentriert, „Verwandtschaft“ aber letztlich als gegeben hingenommen (z.B. Lüschen 1988, Rosenbaum 1982, Wagner/Schütze 1998).
} 
in bestimmten Kulturen ,verwandt" fühlen und die die emischen Kriterien bestimmter Kategorisierungen sowie deren Auswirkungen auf das soziale Handeln herausarbeiten, lassen sich die kulturellen Selbstverständlichkeiten deutlich machen und hinterfragen. Diese Studien legen dar, dass kulturelle Vorstellungen von Verwandtschaft verschiedene Dimensionen beinhalten können. „Verwandtschaft“ in euro-amerikanischen Gesellschaften konstituiert sich primär über den Austausch von Körpersubstanzen basierend auf einem einmaligen ,Zeugungsakt ${ }^{`}$ und gilt damit als eindeutig und festgelegt. Die Fortpflanzungsgemeinschaft von Mann und Frau stellt jedoch nicht die einzige Voraussetzung für die Schaffung von Deszendenz dar. Vorstellungen von Zeugung können auch das Einwirken übernatürlicher Kräfte beinhalten. Auch kann die Konstituierung und das Aufrechterhalten von als Verwandtschaft konzipierten Beziehungen durch einen kontinuierlichen Austausch von Substanzen (wie z.B. Blut und Nahrungsmittel durch Ko-Konsumption) ${ }^{13}$ zwischen verschiedenen Personen stattfinden und dadurch immer wieder neu geschaffen werden (vgl. Carsten 1995, 2000b; Hauser-Schäublin 1989, 1991, 1995).

Die Ethnologie mit ihren Methoden (vgl. Kap. 3) bietet somit die Möglichkeit, die spezifischen Charakteristika der kulturellen Konstruktionen und Vorstellungen von Verwandtschaft, Körper und Selbst in verschiedenen kontext- und gruppenspezifischen Diskursen der eigenen Gesellschaft herauszuarbeiten. Diskurse dürfen sinnvollerweise nicht losgelöst von Handlungen und Entscheidungen betrachtet werden. So sollen die Prozesse untersucht werden, die dazu führen, dass Menschen schließlich bestimmte Entscheidungen treffen, ebenso wie die daraus ableitbaren Handlungen und deren Konsequenzen. Mein theoretischer Ansatz unterscheidet sich von den bisherigen euroamerikanischen Studien durch die von mir gewählte konzeptionelle Unterscheidung in ,Körper‘ und ,Leib“ ${ }^{\text {14 }}$. Da der Körper in der euro-amerikanischen spätmodernen Gesellschaft immer auch verkörpertes Selbst ist, besitzt er in seiner verleiblichten Form eine besondere Bedeutung für die Selbst-Identität (vgl. Kap. 2.3). Die analytische Trennung von Körper und Leib ermöglicht das Herausarbeiten der Spannungsfelder zwischen dem Körper als Objekt reproduktionsmedizinischer Behandlungen und dem subjektiven Leib-

\footnotetext{
13 So zeigt Carsten (1995) am Beispiel der Malayen auf Pulau Langkawi, dass die Tatsache der Ko-Konsumption als die Grundlage von „Verwandtschaft“ gilt. Zum einen ist von Bedeutung, als Kind zusammen gestillt worden zu sein, da man über das gemeinsame Milchtrinken das gleiche Blut erhält, das im Körper zirkuliert. Zum anderen ist das gemeinsame Essen von gekochtem Reis ein weiterer zentraler Aspekt, da Blut und Fleisch des Menschen aus gekochtem Reis entstehen. Im Körper einer Mutter transformiert Reis zu Muttermilch und zu Blut. Das Kind erbt das Blut der Mutter und wird von ihr gestillt, was ein doppeltes Band an Zugehörigkeit schafft. Über den Prozess des Kochens von Reis auf dem Herd des Hauses für den Mann und die Kinder wird auch der Ehemann ,blutsverwandt', was hier keine Vorstellung von Inzest beinhaltet. Muttermilch und Blut gelten als Inbegriffe des Lebens und Verwandtseins.

14 Diese konzeptionelle Unterscheidung von ,Körper` und ,Leib` bildete bereits die Grundlage für die Analyse der empirischen Daten im Rahmen des Forschungsprojektes „Humantechnologien und Verwandtschaft in Deutschland“ (siehe Hauser-Schäublin et al. 2001).
} 
empfinden, in denen sich die Menschen befinden, die direkt von den Technologien betroffen sind oder diese anwenden.

Für meine Arbeit ergeben sich aus dem Dargelegten folgende Fragen: Die deutschen Gesetze sind Teil der gesamtgesellschaftlichen Rahmenbedingungen, die den Umgang mit den Reproduktionstechnologien sowie Adoptionen und Pflegschaften mitbestimmen. Sie beinhalten bestimmte Einstellungen zu Wertbegriffen, die Einfluss auf Entscheidungen und Handlungen haben. So werde ich analysieren, wie Verwandtschaft im rechtlichen Diskurs in Deutschland konstruiert wird, und welche Konsequenzen diese Definitionen und Regelungen für das soziale Handeln haben. Welche Mittel und Wege ,assistierter“ Familiengründungen bietet der deutsche Staat an, und welche Möglichkeiten wählen Menschen in verschiedenen Situationen und Kontexten aus diesem Spektrum aus? In der Untersuchung des persönlichen Verwandtschaftsdiskurses sollen die unterschiedlichen Kriterien von Verwandtschaft und ihre Konstituenten in verschiedenen sozialen Kontexten analysiert werden. Weiterhin wird es um das Herausarbeiten der Zeugungsvorstellungen und -definitionen gehen und um die Frage, inwieweit diese mit „natürlicher“ Verwandtschaft zusammenhängen. Welche Rolle spielt der materielle Körper bei der Konstituierung von ,natürlicher“ Verwandtschaft? Werden die Reproduktionstechnologien reflexiv angewendet, um über Eingriffe am Körper das Selbst zu verändern und damit den eigenen Lebensvorstellungen entsprechend anzupassen, d.h. Mutter und Vater zu werden und eine Familie zu gründen, und als solche auch im sozialen gesellschaftlichen Handeln Bestätigung zu erfahren? Greifen die neuen reproduktiven Technologien in dominante Modelle von Verwandtschaft ein, werden neue Formen und Definitionen von Verwandtschaft (direkt oder indirekt) geschaffen, und in welchem Verhältnis stehen diese zu ,traditionellen“ Vorstellungen von Verwandtschaft? Lassen sich darüber hinaus Einflüsse auf verschiedene Diskurse, wie sie in Deutschland über Verwandtschaft und Körper geführt werden, feststellen, die wiederum handlungsweisend für die konkrete soziale Praxis sind?

Die von mir in meiner Arbeit dargestellten kontext- und gruppenspezifischen Diskurse sind somit Ergebnisse der direkten oder indirekten Auseinandersetzung mit den neuen Fortpflanzungstechnologien in Deutschland bezüglich Vorstellungen und Umgang mit Verwandtschaft, Körper, Selbst und Person. Zum einen geht es primär um die Menschen, die mit Hilfe der Reproduktionsmedizin ein Kind bekommen wollen, aber auch um die Ärzte, die diese medizinischen Verfahren anwenden. Zum anderen werfe ich einen Blick auf Adoptionen und Pflegschaften im Sinne einer Vergleiches. ${ }^{15}$ Im Kontext der Reproduktionsmedizin erfolgt die Entscheidung für eine Form der ,assistierten“

15 ,Neue' Formen von Verwandtschaft und Familie, wie sie im Zusammenhang mit Schwulen- und Lesbengemeinschaften zu erörtern wären, werden hier ausgeklammert (vgl. dazu beispielsweise Weston 1991, Hayden 1995). 
Familie, die auf geteilter biogenetischer Körpersubstanz basiert, Adoptiv- und Pflegefamilien müssen jedoch ohne die Konstituente des Körpers auskommen. Der Blick auf eine Vergleichsgruppe ermöglicht das Herausarbeiten der Multivokalität, d.h. auch von konträren Ansichten und Bedeutungen je nach Handlungskontexten, und das Untersuchen der Konstituierung von Verwandtschaft im Spannungsfeld von Körper, SelbstIdentität, Körper als Objekt und subjektivem Leibempfinden sowie sozialer Verwandtschaft.

Da Deutschland durch das Embryonenschutzgesetz (ESchG) ${ }^{16}$ von 1991 Regeln hinsichtlich der Forschung und Anwendung der reproduktiven Technologien verabschiedet hat, die Verfahren wie „Eizellen- und Embryonenspende“ und die dadurch mögliche gespaltene Mutterschaft, In-vitro-Fertilisation (IVF) und Mikroinjektionen (ICSI) ${ }^{17}$ bei gleichgeschlechtlichen sowie unverheirateten Paaren und Behandlungen nach der Menopause nicht gestatten, fallen diese Verfahren nicht in meinen Untersuchungsbereich. $\mathrm{Zu}$ der durch die heterologe Insemination ${ }^{18}$ möglichen Aufspaltung in biologische und soziale Vaterschaft äußert sich das Embryonenschutzgesetz nicht und schließt diese somit nicht aus (siehe Kap. 4.3.1.1). Wenn allerdings die finanziellen Voraussetzungen bei „ungewollt kinderlosen“ Paaren gegeben sind, bestehen durchaus die Möglichkeiten, Behandlungen auch grenzüberschreitend in Anspruch zu nehmen. Aus diesem Grund bearbeite ich mein empirisches Datenmaterial zwar vor dem Hintergrund der skizzierten Forschungsergebnisse anderer Ethnologen aus dem euro-amerikanischen Kontext zu dieser Thematik, deren Ergebnisse ich jedoch aufgrund der gesellschaftlichen Rahmenbedingungen in Deutschland nicht in allen Aspekten überprüfen kann.

Die vorliegende Dissertation bietet eine Sichtweise auf die Konstruktion von Verwandtschaft in Deutschland, und zwar unter den Bedingungen der Reproduktionstechnologien in historisch und sozial eingegrenzten Situationen, die jedoch nicht losgelöst vom gesamtgesellschaftlichen Kontext gelesen werden kann. Aufgrund des Fehlens ethnologischer Arbeiten dieser Art in Deutschland will sie somit dazu beitragen, diese Forschungslücke aufzuarbeiten.

\section{Aufbau der Arbeit}

Meine Dissertation ist eingebunden in das DFG-Forschungsprojekt „Verwandtschaft und Humantechnologien in Deutschland“, das unter der Leitung von Prof. B. Hauser-

\footnotetext{
16 Ein Abkürzungsverzeichnis findet sich im Anhang.

17 Ein Glossar der medizinischen Fachausdrücke findet sich im Anhang.

18 Die heterologe Insemination ist die Befruchtung der Eizelle mit „Spendersperma“. Eine ausführliche Darstellung der einzelnen reproduktionsmedizinischen Techniken erfolgt in Kapitel 4.3.1.2.
} 
Schäublin am Institut für Ethnologie der Universität Göttingen von 1996 bis 2001 durchgeführt wurde. In diesem Projekt war ich als wissenschaftliche Mitarbeiterin tätig und beschäftigte mich schwerpunktmäßig mit dem Bereich der Reproduktionstechnologien. Vera Kalitzkus bearbeitete den Teilaspekt der Organtransplantation aus Sicht der direkt Betroffenen, und Imme Petersen konzentrierte sich auf die bundesdeutschen Parlamentsdebatten im Zusammenhang mit dem Embryonenschutzgesetz und dem Transplantationsgesetz. Die Resultate veröffentlichten wir in dem Buch „Der geteilte Leib. Die kulturelle Dimension von Reproduktionsmedizin und Organtransplantation in Deutschland“" (Hauser-Schäublin et al. 2001). Für vertiefte Einblicke in spezielle Einzelaspekte waren jedoch detaillierte Einzelstudien nötig (Kalitzkus 2001, Petersen 2002). Das von mir erhobene empirische Datenmaterial habe ich bereits in die Projektpublikation eingebracht sowie interpretiert und werde dies unter den spezifischen Fragestellungen meiner Dissertation hier weiterführen. ${ }^{19}$

Meine Arbeit wird wie folgt aufgebaut sein: Da es mir um die kulturspezifischen Vorstellungen von Verwandtschaft geht, möchte ich im zweiten Kapitel zunächst theoretische Vorüberlegungen anstellen sowie die ,Schärfung des Blicks' auf meine Thematik erreichen. Anhand eines kurzen Überblicks über die Wissenschaftsgeschichte werde ich die Konstruktion von Verwandtschaft in der Ethnologie aufzeigen, in der deutlich wird, wie die Brille der Forschenden seit Morgan (1871) durch das Konzept vom Körper als Medium für Vorstellungen von Zugehörigkeit und Angehörigkeit (relatedness) gefärbt war, und welche Auswirkungen dies auf die weitere verwandtschaftsethnologische Forschung hatte. Erst mit Schneider (1984), der Blutsverwandtschaft als kulturelle Konstruktion, die eine Möglichkeit darstellt soziale Beziehungen zwischen Individuen und Gruppen zu etablieren, als ethnozentristisch dekonstruierte, veränderte sich die Perspektive und die Frage nach der Konstituierung von sozialen Beziehungen in den untersuchten Gesellschaften trat in den Mittelpunkt (Kap. 2.1).

In zweiten Teil von Kapitel zwei stelle ich die Konstruktion und Entwicklung von Blutsverwandtschaft als dominantes Modell in Europa dar und arbeite auf der Grundlage verwandtschaftsethnologischer Untersuchungen die spezifischen Charakteristika des euro-amerikanischen Verwandtschaftskonzeptes heraus.

In meiner Arbeit werde ich die konzeptionelle Trennung von ,Körper` und ,Leib` anwenden (Kap. 2.3). Die Idee von Verwandtschaft ist in Deutschland eng mit Körper und Körperlichkeit verbunden und somit von der Anwendung der reproduktiven Technologien direkt betroffen. Ich werde zunächst das biomedizinische Körpermodell darstellen,

19 An den entsprechenden Stellen werde ich explizit darauf hinweisen, welche Zitate oder Textausschnitte bereits von mir in der Projektpublikation veröffentlicht wurden. 
das die (reproduktions)medizinische Praxis dominiert, soweit es für das Verständnis der Thematik meiner Arbeit relevant ist, und mich dann der Definition, der Herleitung und dem Gebrauch der Begriffe ,Körper' und ,Leib` zuwenden. In der westlichen spätmodernen Gesellschaft (vgl. Giddens 1991:1) wird im biomedizinischen Körperbild der Körper als Objekt und damit als teilbar, formbar, reparierbar und abtrennbar vom subjektiv erfahrbaren Leib konzipiert. Andererseits wird er aber auch als geschlossene Einheit betrachtet, die nicht nur eine physische Größe, sondern auch verkörpertes Selbst ist, dessen Wahrnehmung über den Leib verläuft. Der enge Zusammenhang von Körper, Leib und Selbst bildet somit einen theoretischen Hintergrund bei der Analyse meines Datenmaterials und ermöglicht die Beschäftigung mit der Frage nach dem Spannungsfeld zwischen Körper als Objekt von Behandlungen und der subjektiven Leiberfahrung der Personen, die direkt oder indirekt mit der Reproduktionsmedizin zu tun haben.

Im dritten Kapitel lege ich meinen empirischen Zugang an das Thema dar, da eine ethnologische Forschung in der eigenen Gesellschaft spezifische Vor- und Nachteile im Vergleich zu Studien in ,fremden' Gesellschaften hat. Im Anschluss daran beschreibe ich die von mir angewandten Methoden der Datenerhebung und Auswertung.

In Kapitel vier beschäftige ich mich mit dem rechtlichen Diskurs von Verwandtschaft als öffentliche Diskursform anhand der bundesdeutschen Gesetzgebung, der die rechtlichen Definitionen von Zugehörigkeit und Angehörigkeit als auch die Rechtsfolgen, die sich aus dieser Zuordnung ergeben, umfasst (Kap. 4.1 und 4.2). Ein besonderer Schwerpunkt liegt dabei auf der gesetzlich geregelten Eltern-Kind-Beziehung im Kindschaftsreformgesetz von 1998, aus dem sich konstitutive Elemente von Mutterschaft und Vaterschaft in Deutschland herausarbeiten lassen. Bei genauerer Betrachtung zeigt sich, dass nur bestimmte Formen von Verbundenheit als Verwandtschaft konzipiert werden und ihren Eingang ins Recht gefunden haben. Die gesellschaftlichen Rahmenbedingungen und die Gesetzgebungen, die zu entsprechenden Regelungen bezüglich ,,assistierter" Familiengründungen in Deutschland geführt haben, werden in Kapitel 4.3 dargelegt, wozu sowohl die Anwendung der Reproduktionstechnologien als auch die Möglichkeit von Adoptionen und Pflegschaften gehören. Hier gebe ich detaillierte Informationen zu beiden Bereichen, die notwendig sind, um den Kontext der Erfahrungen meiner Gesprächspartner zu verstehen. Dieses Kapitel umfasst die medizinischen Behandlungsmethoden von „Sterilität“ ebenso wie eine kurze historische Einordnung und eine Beschreibung der gegenwärtigen rechtlichen Situation von Adoptionen und Pflegschaften. Auf der Grundlage der in Kapitel 4.1 beschriebenen legal anerkannten Eltern-KindBeziehungen möchte ich hier in einem Exkurs verdeutlichen, welche zivilrechtlichen Folgen die Anwendung der heterologen Insemination auf die Statusdefinitionen und -zuordnungen der an der Erzeugung des Kindes beteiligten Personen haben können, insbesondere da hier, fremde` Zeugungssubstanzen beteiligt sind. Im weiteren Verlauf 
meiner Arbeit werde ich dann der Frage nachgehen, inwieweit diese Aspekte in der konkreten reproduktionsmedizinischen Praxis thematisiert werden.

Im fünften Kapitel wende ich mich konkret meinem empirischen Material zu. Der Schwerpunkt liegt auf dem persönlichen Diskurs über Verwandtschaft der befragten Personen im Kontext von Reproduktionsmedizin (5.1) sowie Adoptionen und Pflegschaften (5.2). Auf der Grundlage des im vierten Kapitel beschriebenen rechtlichen Diskurses gehe ich der Frage nach, ob und inwieweit sich dieser in der persönlichen Konstituierung von enger Verbundenheit, die als Verwandtschaft konzipiert wird, wiederfinden lässt, welche Kategorien von Verwandten unterschieden werden sowie deren Konstituenten. Durch den Vergleich zwischen der Konstruktion von Verwandtschaft sowohl im Kontext der Reproduktionsmedizin, die den Schwerpunkt dieses Kapitels bildet, als auch von Adoptionen und Pflegschaften, ist es möglich, situations- und kontextspezifische Definitionen und Vorstellungen von Verwandtschaft in Deutschland zu analysieren. Bei beiden befragten Gruppen geht es um die angestrebte oder schon vollzogene Transformation eines Paares zu einem legal anerkannten Elternpaar und damit um die Schaffung neuer sozialer Beziehungen. Erstere streben die Erzeugung eines „eigenen“ leiblichen Kindes und damit sowohl die biologische als auch die soziale Elternschaft an, letztere sind ausschließlich in sozialer Hinsicht mit dem Kind verbunden und übernehmen ,nur' die soziale Elternschaft. Ich arbeite hier die verschiedenen Positionen und Argumentationsstränge hinsichtlich der Konzepte vom „eigenen“ Kind heraus vor dem Hintergrund, dass der Körper eine symbolische Funktion bei der Konstruktion von Verwandtschaft übernimmt und den Ansatzpunkt bildet, um das Selbst zu konstruieren.

Im sechsten Kapitel beschäftige ich mich in Anlehnung an die Projektpublikation (Hauser-Schäublin et al. 2001:97-111) intensiver und ausführlicher mit den verschiedenen Diskursen über Zeugung im Kontext der Reproduktionstechnologien. Zum einen geht es um die Perspektive der Ärzteschaft, sowohl kraft ihres Amtes als auch als Privatperson, zum anderen um die Perspektive der Paare, die die medizinischen Behandlungsmöglichkeiten nutzen. Auf der theoretischen Grundlage der analytischen Trennung von Körper und Leib lassen sich hier sehr deutlich die Widersprüchlichkeiten und Brüche seitens der betroffenen Paare, aber auch der Ärzteschaft im Umgang mit , traditionellen“ Konzepten von Verwandtschaft, Zeugung und Fortpflanzung unter den Bedingungen der Reproduktionstechnologien herausarbeiten. Die Durchführung und Anwendung der Behandlungen machen eine Konzentration auf den Körper als teilbares und reparierbares Objekt notwendig und setzen eine prinzipielle Loslösbarkeit von Ei- und Samenzellen vom Körper und sozialen Beziehungen voraus. Das kulturell dominante Konzept von ,echter" Verwandtschaft, die sich über geteilte Körpersubstanz konstituiert, verortet jedoch den Blutsverwandten auch symbolisch im individuellen Leib. Zur Überwindung dieses Dilemmas werden von allen Beteiligten verschiedenste Mechanismen aktiviert, 
die ich hier darstellen und diskutieren werde. Die Konstituierung von Verwandtschaft über Körper und Körperlichkeit mit Hilfe der reproduktiven Technologien ist in jedem Fall unabdingbar, zumindest über ein Elternteil wie im Fall der Zeugung mit „Spendersperma $^{\text {“20 }}$. Dieser Anteil der Verbindung wird entsprechend sozial betont, wohingegen gleichzeitig der ,fremde“ Anteil eines „Spenders“ sozial ausgeblendet wird. Die beabsichtigte Anonymität zielt auf ein Unsichtbarmachen und Verhindern des Entstehens sozialer Beziehungen hin und ist ein Aspekt des Umgangs mit Körpersubstanzen in Deutschland.

Im siebten Kapitel fasse ich noch einmal die wichtigsten Ergebnisse meiner Untersuchung zusammen und interpretiere sie auf der Grundlage meines theoretischen Werkzeuges.

20 Den Begriff des „Spenders“, ebenso wie „Samenspende“ und „Eizellspende“, verwende ich im Folgenden mit doppelten Anführungszeichen, da es sich nicht um wissenschaftlich-beschreibende Termini handelt. Das Wort „Spenden“ impliziert bereits eine soziale Interpretation und suggeriert eine Gabe, die als uneigennütziger Akt mit sozialer Anerkennung verstanden werden will. Dies trifft jedoch gerade im Fall der „Samenspende“ in Deutschland nicht zu, da die „Spender“ in der Regel alle eine finanzielle Entschädigung erhalten. Für eine kritische Auseinandersetzung mit dem Begriff der "Spende“ in Deutschland aus ethnologischer Perspektive verweise ich auf HauserSchäublin et al. (2001:226ff). 


\section{Theoretische Überlegungen und die, Schärfung des Blicks ${ }^{6}$}

\subsection{Die Konstruktion von Verwandtschaft in der Ethnologie}

\subsubsection{Verwandtschaftssysteme als Repräsentationen „natürlicher“ Beziehungen}

Die Verwandtschaftsethnologie bildet eines der Kerngebiete der Ethnologie und ist zudem eines der ältesten kontinuierlich bearbeiteten Gebiete des Faches. Lewis Henry Morgan (1818-1881), der als Begründer der Verwandtschaftsethnologie gilt, begann im 19. Jahrhundert, Verwandtschaftssysteme zu beschreiben und zu klassifizieren. ${ }^{21}$ Verwandtschaftsterminologien und ihre Klassifikationsweise waren für ihn der Schlüssel zum Verständnis der zugrundeliegenden Verwandtschaftssysteme. Anhand seines gesammelten Materials veröffentlichte er 1871 sein Werk „Systems of Consanguinity and Affinity of the Human Family“, in dem er als erster systematisch die verschiedenen Verwandtschaftsterminologien aufführte und zur gleichen Zeit eine Methode etablierte, die Eingang fand in die ethnologische Forschung. Alle Ethnologen, egal welcher Gesellschaft sie sich zuwandten, versuchten zunächst das Vokabular der familiären Beziehungen herauszufinden (Zonabend 1996:15). Morgan unterschied zwischen konsanguinen und affinen Beziehungen und teilte die Termini der konsanguinen Verwandtschaftssysteme in deskriptive und klassifikatorische ein. Deskriptive Systeme reflektierten für ihn die „natürlichen“ Beziehungen, d.h. die biologische Verwandtschaft, zwischen den Familienmitgliedern.

„As a system it is based upon a true and logical appreciation of the natural outflow of the streams of blood, of the distinctiveness and perpetual divergence of these several streams, and of the difference in degree numerically, and by line of descent, of the relationship of each and every person to the central Ego. It is therefore a natural system, founded upon the nature of descents and may be supposed to have been of spontaneous growth.“ (Morgan 1970 [1871]:468f)

Mit Hilfe primärer Termini wie beispielsweise Vater/Mutter, Bruder/Schwester, Ehemann/Ehefrau, Sohn/Tochter wurden die direkten Verwandten begrifflich von den übrigen getrennt (Morgan 1970 [1871]:142, 468), und jeder Terminus wurde einer bestimmten genealogischen Position zugewiesen. Jede Beziehung konnte somit nach

21 Siehe dazu auch Trautmann (1987). 
Morgan begrifflich klassifiziert werden. Die Zusammengehörigkeit der linear verbundenen Menschen wurde noch durch die Unterscheidung zwischen linearen und kollateralen Verwandten in den Verwandtschaftsbezeichnungen betont und der ,Wert ${ }^{6}$ der Verwandtschaft nahm mit der Entfernung von Ego ab (Morgan 1970 [1871]:13, 142f). In deskriptiven Systemen folgten die Termini ,the streams of the blood and it is in accordance with the nature of descents" (1868:438f). Für Morgan basierte das deskriptive Verwandtschaftssystem auf der Ehe und damit der sexuellen Beziehung zwischen Mann und Frau, die die Grundlage bildete für die Institution der Familie: Die konsanguine Familie stellte für ihn den Beginn aller menschlichen sozialen Organisation dar. ${ }^{22}$

Klassifikatorische Systeme bezeichneten hingegen Kategorien von Verwandten. So konnte der gleiche Terminus sowohl für den Vater als auch den Vaterbruder verwendet werden, der diese somit vom Mutterbruder unterschied. Andere wiederum bezeichneten die Mutter und ihre Schwestern mit dem gleichen Begriff und unterschieden diese von den Schwestern des Vaters. ${ }^{23}$ Nach Morgan fußten diese Verwandtschaftssysteme auf dem Organisationsprinzip des „Stammes“ im Gegensatz zu den deskriptiven, die auf dem Organisationsprinzip der Familie und ihren Mitgliedern basierten. Erstere teilten die Mitglieder in Gruppen ein und bezeichneten diese mit bestimmten Termini, wobei Kategorien von Verwandten bedeutender waren als Individuen. ${ }^{24}$

Morgans Einteilungen reflektierten die europäischen Vorstellungen von Familie und Verwandtschaft, die von „,natürlichen“ Zusammengehörigkeiten ausgingen sowie von der Vorstellung einer Mutter, die das Kind zur Welt bringt und eines Vaters, der das Kind zeugt. Die biologische und soziale Vaterschaft wurde als ,natürliche“ Einheit betrachtet und eine Differenzierung zwischen genitor und pater kam in Morgans Modell nicht vor. Morgan führte die Blutsverwandtschaft als zentrale Kategorie in die Ethnologie ein, indem er Verwandtschaft über den Prozess der biologischen Reproduktion definierte. „Echte“ Verwandtschaft galt somit an den Körper gebunden, was über viele Jahre die ethnologische Verwandtschaftsforschung prägte.

\footnotetext{
22 Morgan formulierte ein Entwicklungsschema der Institution Familie, das mit der monogamen Familie als höchster Entwicklungsstufe endete (siehe Morgan 1970 [1871]:480ff) und begründete damit die erste wissenschaftliche Reihenfolge der Entwicklungsformen der Familie. Mit der monogamen Familie ging die Terminologie nach Morgan von dem klassifikatorischen in den deskriptiven Typ über (Morgan 1970 [1871]:492f).

23 Spätere Ethnologen haben nachgewiesen, dass die Unterteilung in deskriptive und klassifikatorische Systeme nicht aufrecht erhalten werden kann, da letztlich alle klassifikatorisch sind. Termini wie beispielsweise „Schwester“ oder „Bruder“ sind nicht deskriptiv, weil diese Begriffe die Geburtsfolge der Geschwister unberücksichtigt lässt. In vielen Gesellschaften spielt dieser Umstand jedoch eine wesentlich bedeutendere Rolle bezüglich sozialer Konsequenzen und Pflichten als das Geschlecht (Hauser-Schäublin 1991:309).

24 Für die klassifikatorischen Systeme suchte Morgan eine Erklärung auf der Grundlage anderer Ehe- und Familienformen wie der Gruppenehe zwischen leiblichen und kollateralen Brüdern und Schwestern, der Polygamie und der Polyandrie. Die verschiedenen Stufen der Familie basierten jeweils auf einer entsprechenden Eheform (Morgan 1910 [1877]:47, 435).
} 
„Die Vorstellung von Blut als dem Medium, das ,richtige' Verwandte miteinander verbindet, wurde zu einem wissenschaftlichen Begriff erhoben, obwohl er letztlich ein Wertbegriff ist und per se keine andere Definition von Banden zwischen als eng verwandt geltenden Menschen zulässt.“ (Hauser-Schäublin 1998:61; Hervorhebung im Original)

\subsubsection{Die Suche nach einem Vater}

$\mathrm{Zu}$ Beginn des 20. Jahrhunderts rückten Diskussionen über den Zusammenhang von Zeugung und Fortpflanzung und damit zusammenhängend die Frage nach Elternschaft, insbesondere Vaterschaft, in den Mittelpunkt des Interesses der Verwandtschaftsethnologie. ${ }^{25}$ Diese Debatten konnten nur geführt werden, da von der Idee biologischer Verwandtschaft ausgegangen wurde, die einen Vater notwendig machte, und unter der Voraussetzung, Verwandtschaft allein im Sinne von Blutsverwandtschaft aufzufassen. Die Vorstellung von Vaterschaft als Grundlage jeder genealogisch organisierten Gruppe führte zu einer besonderen Sichtweise von Verwandtschaft und Zeugungsvorstellungen in anderen Gesellschaften.

„Denn nur, wenn man davon ausgeht, dass Vaterschaft im Ursprung biologische Vaterschaft sein muss und die Vorstellung von Zeugung eines Menschen nur Zeugung im Sinne des Verschmelzens biogenetischer Substanzen bedeuten kann, machen solche Debatten Sinn." (Hauser-Schäublin 1998:62f; Hervorhebung im Original)

Für Malinowski (1963 [1930]) galt die Kernfamilie (Eltern und Kind) als ein universelles Merkmal jeder Gesellschaft, die er als ,procreative institution of mankind“ (1963 [1930]:57) bezeichnete. Er sah die Familie als biologische und kulturelle Einheit und für ihn galt die Ehe als notwendige Voraussetzung für das Hervorbringen von legitimen Kindern (legitimate sexuality). Mit dieser Auffassung schloss er sich ganz Morgan an, der die konsanguine Familie als Ursprung jeder Sozialorganisation ansah (siehe Kap. 2.1.1).

Das Modell der Nuklearfamilie wurde selbstverständlich von Europa auf andere Kulturen übertragen, was zur Folge hatte, dass die Suche nach Vaterschaft eine herausragende Rolle spielte. Ausgehend von Malinowskis Beobachtungen bei den Trobriandern, deren Zeugungsvorstellungen den traditionellen euro-amerikanischen Vorstellungen von der Kontinuität zwischen sexuellen Beziehungen, Schwangerschaft und menschlicher Reproduktion entgegenstanden, entspann sich die Diskussion um die „Unkenntnis der Vaterschaft“ (ignorantia paternitatis). In der Debatte ging es um die Frage, ob diese Kultu-

25 Eine ausführliche Darstellung über Vorstellungen von und den Umgang mit Zeugung im ethnologischen Wissenschaftsdiskurs gibt Franklin (1997:17-73). 
ren den Zusammenhang von Sexualität und Zeugung nicht kannten oder ihn sprachlich nur nicht ausdrückten. Malinowski war der Auffassung, dass der Geschlechtsverkehr für die Trobriander als rein mechanischer Akt galt, der den Weg für das spirituelle Kind öffnen sollte, und nicht als die Ursache einer Schwangerschaft anerkannt wurde. Malinowski schloss daraus, dass diese ignorantia paternitatis durch einen Mangel an wissenschaftlicher Kenntnis entstanden war: „Sexual intercourse has nothing to do with conception“ (1929:164). Den Männern wurde seiner Ansicht nach zwar ein physischer Beitrag an der Entwicklung des Kindes zugesprochen, der jedoch nicht gleichzusetzen war mit Vaterschaft im biologischen Sinne. Im Unterschied zu Morgans Modell, der von der ,natürlichen“ Einheit biologischer und sozialer Vaterschaft ausging, formulierte Malinowski hier ein Konzept von sozialer Vaterschaft, indem er den Ehemann der Mutter in der Kultur der Trobriander als „Vater“ bezeichnete, sich aber dennoch mit der Frage beschäftigte: „Are the natives really entirely ignorant of physiological fatherhood?“ (1929:153).

Leach kritisierte in seinem Artikel „Virgin Birth“ (1967) den Eurozentrismus Malinowskis und bezeichnete das von ihm beschriebene Phänomen als ,,ignorance of physiological paternity“ (1967:94). Seiner Ansicht nach würden alle Kulturen den Zusammenhang zwischen Sexualität und Zeugung kennen, diesem jedoch unterschiedlich große Bedeutung beimessen bzw. religiöse oder mythische Erklärungen etablieren. Für die unterschiedlichen Erklärungsebenen zeigte er einen Widerspruch in der eigenen Kultur bezüglich Zeugungsvorstellungen auf. Hierfür zog er das Christentum heran, das die unbefleckte Empfängnis, die Jungfrauengeburt (virgin birth), propagierte, obwohl alle wüssten, dass ein Mann zur Zeugung eines Kindes nötig sei. Dieses diente seiner Auffassung nach dazu, das Dogma des göttlichen Kindes zu betonen und nicht die Abwesenheit der körperlichen Vaterschaft abzuleugnen.

Spiro (1968) versuchte den Sachverhalt mit einem funktionalistischen Ansatz zu erklären. In seinem Artikel „Virgin Birth, Parthenogenesis and Physiological Paternity: An Essay in Cultural Interpretation“ bestätigte er Malinowskis Einschätzung von der Unkenntnis der physiologischen Vaterschaft, betrachtete diese Unkenntnis aber auf zwei verschiedenen Ebenen. Einerseits ging es ihm um die Abwesenheit von Wissen, andererseits um die Ablehnung von bestimmtem Wissen (1968:256). Er argumentierte nicht von einem biologischen oder kulturellen Standpunkt aus, sondern zog die Psychologie Sigmund Freuds heran, mit deren Hilfe er die Vorstellung der Trobriander als eine Funktion erklärte, den Ödipus-Konflikt symbolisch zu lösen. Dies war seiner Auffassung nach der Versuch, eine kognitive und symbolische Antwort zu liefern, um zu leugnen, dass der Vater eines Mannes, obwohl er mit der Mutter verheiratet ist, sein genitor wäre. Der Sohn bestreitet damit, dass er in einem Geschlechtsakt seiner Eltern gezeugt wurde. Spiro griff auch die Interpretation von Leach hinsichtlich des Glaubens an die 
unbefleckte Empfängnis des Christentums an, indem er darstellte, dass es nicht darum ging, Jesus einen genitor abzusprechen, sondern darum, dass er keinen menschlichen genitor hatte. Dieses von Leach gewählte Beispiel erklärte jedoch nicht die ignorantia paternitatis erklären, sondern setzte im Gegenteil ein kulturelles Wissen über physiologische Vaterschaft voraus (vgl. Spiro 1968:250).

Hastrup schaltete sich 1978 in die Diskussion ein und vertrat die Ansicht, dass es in Malinowskis Untersuchung über die Zeugungsvorstellungen der Trobriander nicht um die Kenntnis bzw. Unkenntnis der Vaterschaft ging, sondern um Kategorien, denen in einer Kultur bestimmte Bedeutungen zuerkannt werden und die mit anderen Kategorien in Beziehung gesetzt wurden.

Auch Delaney kritisierte 1986 in ihrem Artikel „The Meaning of Paternity and the Virgin Birth Debate“, dass die physiologische Vaterschaft keine universale Kategorie darstellte, wie es immer wieder in der virgin birth-Kontroverse angenommen wurde. Vaterschaft wäre ihrer Ansicht nach weder eine physische Einheit noch eine natürliche Tatsache. Ein kulturelles Verständnis von Elternschaft „has to do with the symbols, meanings and beliefs by which life is thought to come into being. It provides a view of what life is, how and by what or whom it comes into being and for what purpose, what the person is (both male and female), how persons are related to each other, the non-human world and the cosmos“ (1986:506). Delaney zufolge existierte Elternschaft als ein Konzept innerhalb eines Systems von kulturellen Vorstellungen, das nicht unabhängig von seinem Kontext betrachtet werden könnte, ohne dessen Bedeutung $\mathrm{zu}$ verzerren (1986:495). Auch Vaterschaft bildete demnach eine eigene kulturelle Kategorie, die im jeweiligen kulturellen Kontext betrachtet und interpretiert werden und in Beziehung zu anderen kulturellen Konzepten gesetzt werden müsste, insbesondere zu Mutterschaft. Vaterschaft wäre nicht mit der Anerkennung der physiologischen Verbindung des Vaters zum Kind gleichzusetzen wie bei der Verbindung zwischen Mutter und Kind, die aufgrund der Schwangerschaft und Geburt als maßgebliche Beziehung galt. Delaney argumentierte auch dahingehend, dass nicht die Unkenntnis der Trobriander über die Vaterschaft den strittigen Punkt darstellte, sondern die Unwissenheit der Ethnologen, die ihre eigene Kultur nicht genau kannten. Sie führte zwei Gründe an: Zum einen hätten sie den christlichen Hintergrund für den Symbolismus der jungfräulichen Geburt mit seinen monogenetischen Vorstellungen von Schöpfung und Zeugung nicht angesprochen, zum anderen hätten sie die Tatsache ignoriert, dass die „natürlichen Fakten“ der 


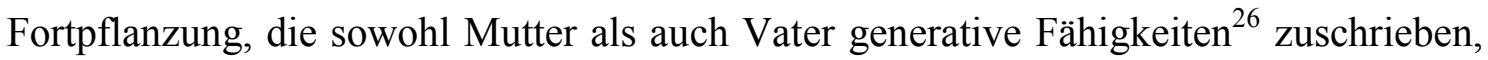
neueren Datums wären. ${ }^{27}$

Die virgin birth-Kontroverse um die Frage nach Vaterschaft innerhalb der Ethnologie veranschaulicht die dringende Suche nach einem Vater in nicht-westlichen Zeugungsvorstellungen. Dabei wurde das Fehlen der physiologischen Rolle des Vaters nicht als das Fehlen einer Beziehung ausgelegt, sondern nur als das Fehlen von Wissen. Ohne die Prämisse, dass Verwandtschaftssysteme mit Biologie zu tun haben und somit ein physiologischer Vater vorhanden sein muss, wäre diese Diskussion nicht führbar gewesen. Die Eindeutigkeit von Mutterschaft wurde jedoch nie in Frage gestellt. ${ }^{28}$

\subsection{3 „A Critique in the Study of Kinship“: Die Dekonstruktion eines Modells}

1984 veröffentlichte der Kulturanthropologe David Schneider sein Buch „A Critique in the Study of Kinship“, in dem er kritisch bisherige Verwandtschaftsstudien in der Ethnologie betrachtete. Dieses Werk führte zu einer Um- bzw. Neuorientierung in der verwandtschaftsethnologischen Forschung. Schneider hinterfragte, welches Vorverständnis und welche Einflüsse den bisherigen Verwandtschaftsstudien zugrunde lagen. Er war der Ansicht, dass diese Studien keine realen Tatsachen wiedergäben, sondern Realitäten konstruierten (1984:4f).

Euro-amerikanische Verwandtschaftsvorstellungen gingen zurück auf Maine (1861), McLennon (1865) und Morgan (1871), die Blutsverwandtschaft als zentrale Kategorie einführten und Verwandtschaft über den Prozess der biologischen Reproduktion definierten. Schneider zeigte auf, dass die Grundannahme „blood is thicker than water“ (1984:165) euro-amerikanischer Ethnologen in der Vorstellung begründet lag, dass Verwandtschaft aus ,Blutsbanden“ bestände, die unauflösbar und gleichzeitig stärker seien als alle anderen menschlichen Beziehungen, da sie biologisch und nicht kulturell determiniert seien. Als Beispiel zog Schneider Malinowskis Forderungen nach einer Unterscheidung zwischen Blutsverwandten und anderen Formen von Beziehungen wie

\footnotetext{
26 Siehe auch Kap. 2.2.2.

27 Vgl. dazu Hauser-Schäublin (1989) und Hiatt (1990), die die Zeugungsvorstellungen der Trobriander interpretieren.

28 Siehe dazu auch Barnes (1973), der der Frage nachgegangen ist, ob Vaterschaft kulturell variabler sei als Mutterschaft. Er rekurrierte in seiner Analyse auf das aristotelische Zeugungsmodell, das den Samen des Mannes als alleinigen genitor definierte (siehe auch Kap. 2.2.2). Dieses besäße allerdings seiner Meinung nach aus ethnologischer Perspektive keine universale Gültigkeit. Er war der Ansicht, dass die Gründe für die Vorstellung „ein Kind - ein genitor" im kulturellen Kontext gesucht werden müssten. Dennoch sah Barnes den Unterschied zwischen Vater- und Mutterschaft darin, dass Mutterschaft aufgrund der körperlichen Vorgänge von Schwangerschaft und Geburt sichtbar und somit kulturell festgeschriebener als Vaterschaft sei. Daraus zog er den Schluss, dass Vaterschaft (genitor) im Gegensatz zu „,natürlicher“ Mutterschaft (genetrix) einen sozialen Status darstelle. Diese Prämisse der ,Natürlichkeit', die Mutterschaft damit seit Malinowski zugewiesen wurde, hat besonders von Seiten feministisch orientierter Ethnologinnen immer wieder zu Kritik geführt (vgl. Collier/Rosaldo/Yanagisako 1982, Moore 1988).
} 
beispielsweise die nach Malinowskis Ansicht selten praktizierte Adoption ${ }^{29}$ heran, auf deren Grundlage sich in der Verwandtschaftsethnologie eine Unterscheidung in „,true“ or ,fictive“ or ,putative“ or ,classificatory“ kin“ etablierte (Schneider 1984:172).

Schneider war weiterhin der Überzeugung, dass aus dieser Grundprämisse die ethnologische Doktrin der genealogischen Einheit der Menschheit (genealogical unity of mankind) abgeleitet wurde. Dieser Stammbaum der Menschheit als Doktrin basierte auf den drei Annahmen, dass erstens Verwandtschaft ein universales Phänomen sei und somit alle Kulturen eine Theorie menschlicher Reproduktion besäßen bzw. ähnliche Vorstellungen von biologischer Verwandtschaft hätten, zweitens dass diese genealogisch definierten Kategorien transkulturell vergleichbar seien, ungeachtet ihres kulturellen Kontextes $^{30}$, und drittens dass anders gelagerte Vorstellungen von Zugehörigkeit und Angehörigkeit, etwa Aussagen über qualitativ verschiedene Zeugungsbeiträge wie zum Beispiel Blut oder Knochen eines Menschen, keinen Einfluss auf die ethnologische Interpretation von Genealogien hätten. ${ }^{31}$

In seinem Buch kritisierte Schneider, dass euro-amerikanische Volksmodelle in die verwandtschaftsethnologischen Untersuchungen und somit in die Beschreibung und Analyse von außereuropäischen Kulturen hineingetragen wurden, was seiner Ansicht nach dazu führte, dass Ethnologen in der Annahme, dass Verwandtschaft als universales Phänomen in allen Kulturen prinzipiell wesensgleich sei, Verwandtschaftssysteme interkulturell miteinander verglichen. Abweichende Klassifikationen und Zeugungsvorstellungen, die nicht den „,biologischen Tatsachen“ entsprachen, wurden in der Ethnologie zunächst mit dem fehlenden Wissen einer Gesellschaft erklärt (siehe dazu auch Morgan 1970 [1871]:476ff; Malinowski 1929:153). ${ }^{32}$ Schneider bezweifelte eine kulturelle Vergleichbarkeit von Verwandtschaftssystemen und erkannte Blutsverwandtschaft nur als ein mögliches Erklärungsmodell für soziale Beziehungen. Es müsste darum gehen, kulturelle Konstrukte zunächst emisch zu verstehen und erst in einem weiteren

\footnotetext{
29 Malinowski war der Auffassung, dass Blutsbeziehungen zwar auch kulturell abgebildet werden könnten, „but statistically speaking, the biological ties are almost invariably merely reinforced, redetermined, and remoulded by the cultural ones“ (1963 [1930]:62).

30 Eine Mutter nimmt somit beispielsweise eine bestimmte Position innerhalb eines genealogisch aufgebauten Verwandtschaftsdiagramms ein, was interkulturell verglichen werden kann. Bedeutungen und Inhalte dieser Position stehen dabei nicht im Vordergrund.

31 So scheinen Genealogien gleichwertige und -artige Beiträge der beiden Eltern bei der Entstehung des Kindes zu zeigen. Qualitativ unterschiedliche Beiträge werden dabei vernachlässigt und sind nicht zu erkennen. Somit lassen Genealogien keine Aussage über die Beschaffenheit der genealogischen Beziehung zu.

32 Auch Holy (1996:154f) ist der Ansicht, dass allein die Tatsache, dass Männer überall Kinder zeugen und Frauen diese gebären würden, nicht bedeute, dass jede Kultur den genealogischen Beziehungen dieselben Bedeutungen und Wertigkeiten zuschreibe wie die westliche Kultur. Selbst wenn eine Kultur die Gemeinsamkeit von Blut zwischen einigen Menschen anerkenne, müsse es sich nicht um ein „Blut ist dicker als Wasser“-Postulat handeln: „In studying kinship as a system of genealogical relations, anthropologists were studying something that may be simply the product of their methodology rather than what may be the culturally specific constructions of relatedness in the societies studied“" (Holy 1996:155).
} 
Schritt eine etische Interpretation zu vollziehen. Dabei sei es für den Forscher unerlässlich, sich über den ,ethnocentric bias“ klar zu werden, ,[...] simply because our , explanatory“ ideas are in their nature ethnocentristic“" (Schneider 1992:630).

Schneiders Veröffentlichung hat dazu geführt, dass Verwandtschaft in der Ethnologie nicht mehr als ein universales Phänomen betrachtet wurde, dass auf der Anerkennung von genealogischen Verbindungen basiert, sondern dass die Forschung sich zunehmend darauf konzentriert hat zu erkennen, wie Vorstellungen über Beziehungen und Verbundenheit (ideas of relatedness) innerhalb einer Kultur definiert werden.

„To adopt a wider definition of kinship than a system of relations arising from procreation is grounded in the realisation that we cannot treat alternative concepts of kinship as differing cultural elaborations of universal natural or biological facts as this precludes the possibility that different societies might conceptualise differently what makes people related to each other." (Holy 1996: 166)

Die zentrale Frage, die sich für Carsten bezüglich Verwandtschaftsstudien stellt, ist folgende:

„How do the people we study define and construct their notions of relatedness and what value and meaning do they give them? It seems to me that we would do better to use the term ,kinship " to characterize the relatedness that people act and feel. In this way we may arrive at a new and more flexible study of kinship in anthropology.“ (1995:236)

Verwandtschaft wird somit definiert als kulturelle Praxis, die mit biogenetischer Verwandtschaft, mit Stammbäumen und naturwissenschaftlich definierten Vererbungsgesetzen a priori nichts zu tun hat. Die wesentliche Veränderung innerhalb der Verwandtschaftsethnologie ist die Tatsache, dass rezente Studien sich auf die emischen Konzeptualisierungen und Konstruktionen von sozialen Beziehungen konzentrieren. Da Zeugungsvorstellungen nach Hauser-Schäublin (1995:73ff) „die Sprache von Verwandtschaft sprechen“ lassen sich durch deren Untersuchung Rückschlüsse auf die Konstruktion von Verwandtschaft anderer Kulturen ziehen, wie auch Franklin im Folgenden herausstellt:

„Conception stories not only tell us where we come from - they tell us who we are, how we are related to others, what our kin obligations are, and how we are situated in patterns of inheritance and descent. They are central to the acquisition of a specific cultural identity, to definitions of sexual difference, personhood, parenthood and procreation." (Franklin 1992: 80f)

Aufgrund dieser Überlegungen ist es möglich und sinnvoll, sich im Rahmen der Verwandtschaftsethnologie und deren Erkenntnissen über die Konstituierung spezifischer Formen von Verbundenheiten in nicht-westlichen Kulturen auch den unterschiedlichen 
Diskursen über die Konstruktion von Verwandtschaft und Zeugungsvorstellungen in euro-amerikanischen Gesellschaften und in einem weiteren Schritt im Verlauf der Arbeit auch dem deutschen Kontext zuzuwenden.

\subsection{Das euro-amerikanische Verwandtschaftskonzept: Die Betonung der körperlichen Prozesse}

Die Überblicksdarstellung über die verwandtschaftsethnologische Wissenschaftsgeschichte hat deutlich gemacht, dass die Betonung des Körpers und der biologischen Reproduktion als zentrale Kriterien für die Konstituierung von „echter“ Verwandtschaft durch westliche Ethnologen Auswirkungen auf Studien von Verwandtschaftskonzepten nicht-westlicher Gesellschaften hatten. Erst seit Schneider (1984) veränderte sich der Blick und die emischen Konstruktionen von enger Verbundenheit rückten in den Mittelpunkt ethnologischer Studien. Im folgenden Kapitel werde ich mich nun Untersuchungen zuwenden, die sich mit dem euro-amerikanischen Konzept von Verwandtschaft beschäftigt haben und aufzeigen, dass Blutsverwandtschaft als kulturelle Konstruktion eine Möglichkeit darstellt, soziale Beziehungen zwischen Individuen und Gruppen zu etablieren.

\subsubsection{Die Entwicklung von Blutsverwandtschaft als dominantes Verwandtschaftsmodell}

Die historische Entwicklung des Modells von Blutsverwandtschaft in Europa und dessen christliche Ursprünge hat der englische Sozialanthropologe Jack Goody (1989 [1983]) anhand der Veränderungen des europäischen Erscheinungsbildes von Verwandtschaft im Verlauf der Jahrhunderte nachgezeichnet. In seinem Buch „Die Entwicklung von Ehe und Familie in Europa“ beschreibt Goody den Einfluss der christlichen Kirche in der Aufstellung von Heirats- und Erbschaftsbestimmungen, insbesondere durch die Einführung von Eheverboten, und in der Entwicklung zur Anerkennung von Blutsverwandtschaft als ,echter“ Verwandtschaft. Die Idee der Blutsverwandtschaft in europäischen Gesellschaften ist nach Goody christlichen Ursprungs. In der Bibelfassung von König Jakob heißt es: „Keiner unter euch soll sich irgendwelchen Blutsverwandten nahen, um mit ihnen geschlechtlichen Umgang zu haben“ (Vers 6 des 3. Levitikus 18, zitiert nach Goody 1989 [1983]:62). Goody zeigt auch auf, dass bereits in vorchristlicher Zeit das germanische Verwandtschaftssystem, das auf der Geschwistergruppe basierte, über das gemeinsame Blut konstruiert wurde. Die Grade des germanischen Systems unterschieden sich von dem römischen Verwandtschaftssystem. Die römische Zählweise berechnete die Zahl von Generationsschritten von Ego aus (auf- und absteigend). So galt Ego mit den Eltern im ersten Grad verwandt, mit den Geschwistern im 
zweiten Grad usw. Ab dem siebten Grad galt man nach römischen Recht nicht mehr als verwandt. Bei der germanischen Zählweise bildete die Geschwistergruppe den Ausgangspunkt. Mit ihnen war Ego im ersten Grad verwandt. Daraus ergibt sich, dass Ego beispielsweise nach germanischem Recht mit seiner Cousine im zweiten Grad und nach römischer Zählweise mit ihr im vierten Grad verwandt war (Goody 1989 [1983]:151153). Im römischen Verwandtschaftssystem wurde demnach die (vertikale) Linearität, im germanischen System die Kollateralität als Hauptprinzip einer horizontalen Achse betont.

Im 11. Jahrhundert vereinheitlichte die Kirche jedoch die beiden verwendeten unterschiedlichen Berechnungsmethoden. Als Zählweise der Verwandtschaftsgrade wurde nur noch die germanische (und nicht mehr die römische) anerkannt. Dies hatte weitreichende Konsequenzen für Heirats- und Erbschaftsangelegenheiten, da die Heiratsverbote nun auf den siebten germanischen Grad ausgedehnt wurden, der im römischen System dem 13. oder 14. Grad entsprochen hatte. Diese neue Berechnungsweise erweiterte den Kreis derjenigen, die aufgrund der kirchlichen Bestimmungen als Blutsverwandte galten und nicht mehr geheiratet werden durften. Damit waren auch erhebliche soziale Folgen verbunden. Mit der Einführung der exogamen Eheregelungen durch die Kirche wurde eine nennenswerte Akkumulation von Besitz innerhalb einer Verwandtengruppe stark erschwert, da innerhalb dieser Gruppe kein Erbe weitergegeben werden durfte. ${ }^{33}$ Auch nachdem die Verbote 1215 auf den vierten Verwandtschaftsgrad reduziert wurden, waren viele Ehen auf einen kirchlichen Dispens von den Eheverboten angewiesen, die für die Kirchen eine erhebliche Geldquelle darstellten.

Neben der Trennung zwischen Bluts- und Affinalverwandten hatte die Kirche in Europa auch Einfluss auf eine weitere Verwandtschaftsgruppe, die „fiktive“ oder „spirituelle“ Verwandtschaft wie Adoption und Patenschaften. Mittels Adoption und Patenschaften, die nach Goody (1989 [1983]:211-212) auch vorchristlichen Ursprungs waren, konnte zwischen Blutsverwandten sowie zwischen Nicht-Verwandten eine „fiktive“ Verwandtschaft hergestellt werden, wodurch die Möglichkeit bestand, Familienbande zu intensivieren oder zu erweitern (siehe auch Jussen 1991). Patenschaften, und damit die Trennung zwischen ,natürlicher“ und „fiktiver“"Verwandtschaft, begannen gegen Ende des vierten Jahrhunderts, nachdem von der Erwachsenentaufe zur Kindtaufe übergegangen wurde. Damit fand ein Wandel von elterlicher zu nicht-elterlicher Patenschaft statt, indem die Paten Zeugen der geistigen Geburt eines Kindes bei der Taufe wurden und die

33 Siehe dazu auch Hauser-Schäublin (1998:57; Hervorhebung im Original): „Endogame Heiratsregelungen und beziehungen sind - so wissen wir aus dem ethnologischen Studium außereuropäischer Gesellschaften - das Mittel, um Gruppenidentität und -solidarität, vor allem jedoch Besitz und/oder Status von Klassen und Kasten, aufrecht zu erhalten und zu stärken. Indem es nun plötzlich nicht mehr erlaubt war, Menschen aus einer näheren sozialen Umgebung zu heiraten und man gezwungen wurde, Partnerinnen von anderen Verwandtschaftsverbänden zu heiraten, hatte dies zweifellos, zumindest längerfristig, eine Schwächung des eigenen Verbandes zur Folge“. 
geistige Elternschaft übernahmen. Auch die Adoption wurde schon in vorchristlicher Zeit praktiziert, insbesondere wenn ein Mann keinen leiblichen Erben hatte. Das Bestreben der Kirche war es jedoch, die Praktiken der Adoption zu verbieten, da bei fehlenden Erben der Besitz einer Verwandtschaftsgruppe der Kirche zufiel. Ab dem fünften Jahrhundert setzte die Kirche durch, dass adoptierte Kinder nicht mehr erbberechtigt waren (Goody 1989 [1983]:113). Mit Hilfe der Verurteilung von Adoption, der Wiederheirat von Witwen und sämtlicher Ehen mit Affinalverwandten und „fiktiven“ Verwandten konnte die Kirche somit zum einen „fiktive“ Erben ausschließen, zum anderen bot sie den Witwen, für die eine Wiederheirat ausgeschlossen war, eine Versorgung in eigens gegründeten Witwenorden. Bei deren Ableben fiel zumeist der Kirche deren Besitz zu. Gerade im Mittelalter war die Bedeutung dieser Zuwendungen erheblich. Die Folge dieser kirchlichen Bestimmungen war eine immense Vermehrung des kirchlichen Besitzes und eine gleichzeitige Verarmung von Verwandtschaftsverbänden.

Aus den obigen Ausführungen lässt sich erkennen, dass sich unter dem Einfluss der Kirche ein Verwandtschaftssystem entwickelte, in dem eine Hierarchisierung zwischen Bluts- und Adoptionsverwandtschaft stattfand und als die „wahren“ Verwandten nur noch die Blutsverwandten galten, die im Rahmen der Ehe als einzig legitime Institution durch einen körperlichen Akt gezeugt wurden. Nicht mehr die Kollateralität, sondern einzig die Linearität von Verwandtschaft und Deszendenz wurde nunmehr betont. Gleichzeitig wurde ein Ego als zentraler Referenzpunkt eingeführt, von dem aus der Grad der Blutsverwandtschaft berechnet wurde.

„Die Bemühungen der Kirche zielten hingegen darauf ab, die auf der Geschwistergruppe basierende Berechnungsweise durch eine Methode zu ersetzen, die auf der Einheit des Fleisches (unitas carnis) beruhte und durch den Akt der Eheschließung (oder copula) eingesetzt wurde; ein System also, das auf dem verheirateten Paar und seinen Kindern fußte. Eine derartige Berechnungsmethode war darauf ausgerichtet, die Bedeutung der Nachkommen innerhalb der direkten Linie besonders hervorzuheben und Verwandte der Seitenlinien auszuschließen.“ (Goody 1989 [1983]:158; Hervorhebungen im Original)

Das amerikanische Verwandtschaftskonzept wurde zum ersten Mal von David Schneider (1980 [1968]) in ethnologischer Perspektive analysiert, das er als ein kulturelles System mit einer spezifischen symbolischen Logik betrachtet. In seinem Werk „American Kinship. A Cultural Account“ zeigt er auf, dass die Idee des gemeinsamen Blutes eine dominante Rolle in den amerikanischen Vorstellungen von Verwandtschaft ${ }^{34}$ spielt, $^{2}$

34 Schneider hat jedoch nur Daten innerhalb der urbanen, weißen Mittelschicht gesammelt (Schneider 1980 [1968]:121), was ihm immer wieder Kritik einbrachte. Kritisch äußert sich u.a. auch Scheffler (1976) zum Umgang mit den Aussagen der Informanten, die Schneider seiner Ansicht nach zu wörtlich genommen habe. Er akzeptiert dessen Unterteilung von Verwandten in „relatives by blood“ und ,relatives in law“ anhand des empirischen Materials 
und dass die Einheit von biologischer und sozialer Verwandtschaft im Mittelpunkt steht. Blutsverwandte (relatives by blood) teilen in der Regel eine „natürliche“ Substanz und eine soziale Beziehung miteinander. Affinalverwandte (relatives by marriage) (Schneider 1980 [1968]:25ff) teilen nur die soziale Beziehung miteinander, woraus sich ergibt, dass biologisch und sozial unterschiedliche Kriterien darstellen, um verwandtschaftliche Beziehungen zu charakterisieren. Die Ehe stellt einen rechtlichen Vertrag aufgrund einer Willensentscheidung des einzelnen dar und kann durch Scheidung wieder gelöst werden. „Natürliche“ Verbindungen entstehen durch Zeugung und werden dadurch unauflöslich (vgl. Schneider 1980 [1968]:23ff). Blutsverwandte sind aufgrund der geteilten biogenetischen Substanz, dem gemeinsamen Blut, die „wahren“ Verwandten, von denen man sich nicht wie von Affinalverwandten trennen kann, da das Gesetz der ,natürlichen Ordnung“" erstere miteinander verbindet.

„Blood is a matter of birth, birth is a matter of sexual intercourse. Sexual intercourse is an act which is undertaken and does not just happen. Yet as an act, it is natural. Its outcome is conception, which is followed by birth, and these are natural, too.“ (Schneider 1980 [1968]:38)

Nach Schneider gewinnt der Geschlechtsverkehr eine zentrale Bedeutung, da er Ausdruck der sozialen Beziehung zwischen den Eheleuten ist und gleichzeitig die biologische Verbindung zwischen Eltern, Kindern sowie Geschwistern herstellt.

„The distinctive features which define the members of the family and differentiate them from each other and which at the same time define the family as a unit and distinguish it from all other cultural units are those which are contained in the symbol sexual intercourse. Father is the genitor, mother the genetrix of the child which is their offspring. Husband and wife are in sexual relationship and theirs is the only legitimate and proper sexual relationship. Husband and wife are lovers and the child is the product of their love as well as the object of their love; it is in this sense that there are two kinds of love which define family relationships, one conjugal and the other cognatic, and it is in this sense that love is a synonym for sexual intercourse." (Schneider 1980 [1968]:43)

Somit entsteht über den rechtlichen Vertrag der Ehe die kulturelle Einheit der Kernfamilie aus dem Ehepaar mit seinen Nachkommen, die durch eine gemeinsame Körperlichkeit miteinander verbunden sind; Zum einen über den Geschlechtsverkehr, der Ausdruck der sexuellen Beziehung des Ehepaares ist, zum anderen wird durch den Akt der Zeugung die biologische Identität zwischen Eltern und Kind hergestellt. Durch diese

nicht, sondern unterscheidet selbst zwischen Individuen, die als verwandt gelten, d.h. Blutsverwandten, und ,relatives of other kinds", die nicht als Verwandte angesehen werden wie Pflegemutter, Priester (Vater). Scheffler misst den „real relatives“ eine sehr viel größere Bedeutung bei. Als Grundlage erkennt er die biologische (über gemeinsames Blut verbundene) Familie an. Verwandtschaftliche Beziehungen, die darüber hinaus gehen, sind für ihn ,relatives by extension“. 
geteilte Körperlichkeit (shared biogenetic substance) entsteht zwischen den Familienmitgliedern ein emotionales Zusammengehörigkeitgefühl (diffuse enduring solidarity), was Schneider mit Liebe gleichsetzt (1980 [1968]:50). ${ }^{35}$

Im Zentrum sowohl des europäischen als auch des amerikanischen Verwandtschaftskonzeptes steht die Vorstellung von Blutsverwandtschaft als „echte“ Verwandtschaft, die untrennbar mit dem Körper verbunden ist. Zwischen Blutsverwandten besteht eine physische Verbindung über eine ,natürliche“ Substanz, die diejenigen teilen, die als miteinander verwandt gelten, und die untrennbar verbunden ist mit der Vorstellung der Schaffung von Blutsverwandten durch Geschlechtsverkehr und Zeugung im Rahmen der Ehe. Das Idiom der ,Natur' ist entscheidend in der Vorstellung, dass eine geteilte Körperlichkeit auch in sozialer Hinsicht unauflöslich miteinander verbindet: „The family is formed according to the laws of nature and it lives by rules which are regarded by Americans as self-evidently natural“ (Schneider 1980 [1968]:34). ${ }^{36}$ Die Idee der ,Natürlichkeit‘ dieser Verbindungen führt auch zu einer Trennung zwischen „echter“ und „künstlicher“ bzw. „fiktiver“ Verwandtschaft, wozu Adoptionen und Patenschaften gezählt werden. Dieser Unterscheidung liegt die Vorstellung zugrunde, dass „fiktive“ Verwandtschaft der echten nur nachgebildet sein kann, wodurch sie eine andere kulturelle Bewertung erfährt.

\subsubsection{Das biomedizinische Konzept von Zeugung}

Es ist deutlich geworden, dass im Zentrum euro-amerikanischer Vorstellungen von „natürlicher“ Verwandtschaft die Idee von Blutsverwandtschaft steht, die jedoch im Zeitalter gentechnologischer Forschung vom Konzept der gemeinsamen Gene fast abgelöst wurde. Biogenetische Verwandtschaft und die körperlichen Prozesse der Schaffung neuen Lebens stehen im Mittelpunkt dieses Konzeptes. Diese Sichtweise ist ge-

\footnotetext{
35 Gerade die „natürliche“ Verwandtschaft gilt auf eine besondere Weise mit Gefühlen - Emotionen im weitesten Sinne - belegt, was für den europäischen Raum vor allem Medick und Sabean (1986) nachgewiesen haben. So kann vermutet werden, dass zumindest streckenweise die für amerikanische (weiße Mittelklasse) Verhältnisse herausgearbeiteten Vorstellungen zu Verwandtschaft auch für Mitteleuropa zutreffen (vgl. Goody 1989 [1983]).

36 Franklin (1997:54) hat allerdings aufgezeigt, dass Schneider den Begriff der ,Natur` für drei unterschiedliche Aspekte verwendet und zwischen diesen nicht differenziert: Zum einen setzt er ,Natur' gleich mit ,Biologie“ in Bezug auf ,shared biogenetic substance“, zum anderen verwendet er ,Natur' zur Beschreibung dessen, ,what animals do" ebenso wie für die menschliche ,Natur" in „man is a special part of nature“. Franklin demonstriert die Widersprüche in Schneiders Analyse zwischen ,Natur' als ein klares symbolisches Idiom im amerikanischen Verwandtschaftskonzept und ,Natur' oder ,Biologie“ als Bereich wissenschaftlicher Fakten. „On the one hand, Schneider was arguing that there is no such thing as a biological fact per se in American kinship systems - there are only cultural interpretations of them. On the other hand, he was also arguing that there are 'natural facts' within science which are true and which are separate from the cultural constructions of them" (Franklin 1997:55; Hervorhebung im Original). Dennoch war gerade das Hervorheben der Rolle von ,Natur' und ,Biologie" im amerikanischen Volksmodell von Verwandtschaft und in der ethnologischen Analyse von Verwandtschaft (vgl. Schneider 1984) ebenso wie der Beginn, die Verbindungen zwischen diesen beiden Bereichen aufzuzeigen, das Besondere an Schneiders Werken für das weitere wissenschaftliche Vorgehen (siehe dazu auch Strathern 1992a:194, 1995:222).
} 
prägt von den Naturwissenschaften der Biologie, insbesondere der Genetik, die den Körper als komplexen, aus Zellen bestehenden Organismus definieren. ${ }^{37}$ REFIn jeder Körperzelle befindet sich die gesamte und gleiche genetische Information eines Organismus, der sich aus 50.000 bis 100.000 Untereinheiten, den Genen, zusammensetzt (vgl. Keller/Günther/Kaiser 1992:8). Bei der Frau reifen nach Beginn der Geschlechtsreife, die durch den Beginn der ersten Regelblutung definiert ist, ungefähr alle vier Wochen mehrere Eizellen heran, von denen meist nur eine einzige voll zur Ausreifung kommt. Nach dem Eisprung etwa in der Mitte des Zyklus kann die Eizelle von befruchtungsfähigen Samenfäden im Eileiter befruchtet werden. In der neu entstandenen Zelle vereinigen sich die genetischen Informationen von Mann und Frau und es entsteht ein neues Individuum. Für die euro-amerikanischen Zeugungsvorstellungen ist die von dem Wissen der Naturwissenschaften abgeleitete kausale Beziehung zwischen Geschlechtsverkehr und Schwangerschaft entscheidend. ${ }^{39}$ Durch den Geschlechtsakt können Ei- und Samenzellen aufeinander treffen und miteinander verschmelzen, woraus neues menschliches Leben entsteht. Impliziert in dieser biogenetischen Zeugungsvorstellung ist die Überzeugung, dass ein Kind je zur Hälfte die Erbinformation von Mutter und Vater in sich trägt und dass deren Rekombination die genetische Ausstattung des Embryos darstellt.

Durch die Forschung und Anwendung im Zusammenhang mit Reproduktions- und Gentechnologien wird dieses Zeugungsmodell jedoch immer differenzierter. Die Loslösung der Sexualität von der Fortpflanzung und die Möglichkeit der Zusammenführung von Zeugungssubstanzen außerhalb des Körpers im Labor ebenso wie die Visualisierung der einzelnen Stadien führen dazu, dass die genetischen Prozesse zunehmend als die „natürlichen Fakten“ anerkannt werden und immer mehr ins Zentrum des Zeugungsmodells rücken. ${ }^{40}$ Dies lässt sich auch daran verdeutlichen, dass der Zeugungszeitpunkt als Schaffung eines neuen Individuums auf das Stadium der Verschmelzung der beiden elterlichen Keimzellen festgelegt wird. Die genetisch definierte Konzeption,

\footnotetext{
${ }^{37}$ Betrachtet man jedoch die Überlieferungen der verschiedenen Zeugungsvorstellungen im Verlauf der Jahrtausende wird deutlich, dass jede Zeit ihre ,Wahrheit‘ über die Anatomie des menschlichen Körpers und die Fortpflanzungsvorgänge besaß (vgl. Laqueur 1986; Franklin 1992:76).

38 Eine detaillierte Beschreibung über die Zeugungs- und Vererbungslehren der Antike und ihr Nachwirken findet sich bei Lesky (1951).

39 Siehe dazu auch Strathern (2001:364): „Die Beziehung des Geschlechtsaktes zur Zeugung ist daher nicht einfach eine technische. Er dient der Reproduktion der Elternschaft als dem erwarteten Ergebnis einer Vereinigung, bei der die Partner durch Gender voneinander unterschieden sind. Von allen anderen abgesehen spielt er daher eine konzeptuell signifikante Rolle bei der Fortpflanzung.“

40 So führte Hiatt die Unterscheidung in „real“ and „pseudo“ Zeugungsvorstellungen in die Ethnologie ein (1971:80). Als „reale“ Zeugungsvorstellungen wurden diejenigen klassifiziert, die wie das euro-amerikanische Modell biogenetisch „wahre“ Tatsachen widerspiegelten. Die trobriandrische Zeugungstheorie (siehe auch Kap. 2.1.2) galt danach als ein Beispiel für eine ,pseudo“ Zeugung. Mit der Dekonstruktion des Modells von Blutsverwandtschaft (vgl. Schneider 1984) ist jedoch auch das biogenetische Zeugungsmodell als eine kulturelle Konstruktion entschlüsselt worden.
} 
und nicht wie früher die physische Verschmelzung, gewinnt damit eine hohe Bedeutung als ,Zeugungsakt‘ (vgl. Franklin 1995:331).

\subsubsection{Die soziale Konstruktion der „natürlichen Fakten““}

Durch die zunehmende Reduzierung der „,natürlichen Fakten“ auf genetische Prozesse wird die biologische Fundierung der Kernfamilie als soziale Abbildung ,natürlicher Tatsachen“ noch verstärkt. Strathern postuliert, dass den ,natürlichen Fakten“ Ausdruck verliehen wird durch die Vorstellung der biologischen, emotionalen und sozialen Einheit der Kernfamilie bestehend aus Eltern und Kindern: „Biology is rooted in an order of reality to which social arrangements must attend, not the other way round" (Strathern 1992b:26). Nach Strathern ist die Vorstellung im euro-amerikanischen Verwandtschaftskonzept dominant, dass soziale Anordnungen und Ausgestaltungen die ,natürlichen Fakten" nur abbilden und dementsprechend diesen nachgeordnet werden. Somit werden diese sozialen Ausgestaltungen zu den sozialen Konstruktionen der als „,natürlich“ konzipierten Fakten. In der Kernfamilie manifestiert sich die Verbindung zwischen ,Natur' und Kultur von Verwandtschaft: Das Ehepaar ist durch die Heirat miteinander affinalverwandt, und die Kinder sind durch den Akt der Zeugung mit Eltern und Geschwistern blutsverwandt. Da diese körperlichen Prozesse zum Bereich der ,Natur gezählt werden, erfolgt auch eine Anerkennung der ,Natürlichkeit‘ der Beziehungen zwischen Familienmitgliedern. Diese Vorstellung hat Auswirkungen auf die Bedeutung, die der Eltern-Kind-Beziehung zuerkannt wird. Das Konzept von Blutsverwandtschaft als Basis von „echter“ Verwandtschaft impliziert die Vorstellung, dass „,natürliche“ Eltern die biologischen Eltern sind, und die soziale Elternschaft dieser nachgeordnet ist:

„Kinship, who one is related to, is established in the popular American view by the prenatal act of conception. By the time one is born, in fact at the moment of conception, it is established who one's, real' kin are. The use of such terms as ,real mother' and ,real father' attests to the importance in this ideology of the presence or absence of what is understood as an actual physiological connection, as does the cultural fascination with genealogy. (Over fifty do-it-yourself genealogy books are currently in print in the United States. The recent phenomenon of adopted children searching for their ,real' parents is additional testimony to the power of the notion that ,real' kinship is determined by birth.) Kinship, according to this ideology, is a matter of shared blood (or genes) by which one is eternally and immutably related.“" (Meigs 1989:36)

Die Eltern-Kind-Beziehung gilt im euro-amerikanischen Verwandtschaftskonzept als eine ausschließliche Verbindung, die für jeden Menschen eine besondere Bedeutung besitzt. Diese beruht auf der Überzeugung, dass jeder über die reproduktive Verbindung die individuelle biologische Identität erhält (Strathern 1992b:24ff, siehe auch Schneider 
1980 [1968]:39f), unabhängig davon, ob das Kind seine Eltern kennt oder nicht. Da diese „natürlichen Fakten“ des Lebens sich dem menschlichen Zugriff entziehen, spielt es keine Rolle, ob die Beziehungen, die sich über genetische Substanzen konstituieren, aktiviert werden oder nicht, „for Euro-Americans there is no getting around that ties exist with those persons whose genetic substances are combined at the child's conception. This is taken as a fact of life“" (Strathern 1993a:14). Dies führt im euroamerikanischen Verwandtschaftskonzept zum einen zu der Vorstellung, dass jeder biologische Eltern hat, und dass zum anderen die „facts of life“ eine rationale Begründung für die ,Natürlichkeit‘ von Familien- und Verwandtschaftsbeziehungen abgeben. ${ }^{41}$

\subsection{Körper, Leib und Selbst}

Ethnologische Untersuchungen haben sich mit kulturspezifischen Sichtweisen von Konzepten über Verwandtschaft, Körper, Selbst und Person beschäftigt. Vor diesem Hintergrund sollen im Folgenden die euro-amerikanischen bzw. deutschen Vorstellungen dargelegt werden. Zunächst werde ich auf die kulturhistorischen Voraussetzungen und die kulturelle Bedingtheit von ,Körper` eingehen, wie er die heutige medizinische Praxis, und damit auch die Reproduktionstechnologien, die die Schaffung von körperlicher Verwandtschaft propagiert, in weiten Teilen beherrscht. ${ }^{42}$ Als nächster Schritt erfolgt die Beschäftigung mit der konzeptionellen Trennung von ,Körper' und ,Leib‘, wie ich sie für die Analyse meines empirischen Materials verwende. Mit diesen Begrifflichkeiten werden verschiedene Aspekte der leiblich-körperlichen Einheit bezeichnet: Körper als objektivierbares und materiell Konkretes wie er auch Gegenstand von medizinischen Behandlungen ist, Leib als subjektives Wahrnehmungs- und Empfindungsorgan. In einem weiteren Schritt zeige ich den Zusammenhang von Körper, Leib und Selbst auf, da der Körper als individuell verkörpertes Selbst einen zentralen Wert der westlichen Gesellschaft der Spätmoderne darstellt (vgl. Giddens 1991) und in seiner leiblichen Dimension konstitutiv für das Selbst ist.

\footnotetext{
41 Auch bei diesen als gegeben angenommenen „,natürlichen Fakten“ handelt es sich um soziale Konstruktionen, wie u.a. Franklin (1990) gezeigt hat. Auf diesen Aspekt werde ich im weiteren Verlauf dieser Arbeit noch näher eingehen.

42 Es ist mir bewusst, dass die unter 2.3.1 dargestellten kulturhistorischen Entwicklungen des biomedizinischen Körpermodells nur einen Ausschnitt darstellen und keine umfassende Begründung des gegenwärtigen Körperbildes bieten. Detaillierter kann hier jedoch nicht darauf eingegangen werden.

43 Diese konzeptionelle Unterscheidung von ,Körper` und ,Leib` bildete bereits die Grundlage für die Analyse der empirischen Daten im Rahmen des Forschungsprojektes „Humantechnologien und Verwandtschaft in Deutschland“ (siehe Hauser-Schäublin et al. 2001).
} 


\subsubsection{Die Entwicklung des Körpers als Objekt oder Die soziale Entbettung}

Die Grundlage für die moderne Medizin basiert auf der Anatomie Galens, welche das erste große wissenschaftliche medizinische Konzept des Abendlandes, das fast 2000 Jahre Gültigkeit besaß, die Humoralpathologie, ablöste. ${ }^{44}$ In der Mitte des 16. Jahrhunderts rückte der menschliche Körper ins Licht der Öffentlichkeit: Andreas Vesalius (1514-1564), ein flämischer Arzt, sezierte Leichen, um von der Erfahrung über tote Körper Kenntnisse über das Funktionieren des Körpers und Einblicke in Krankheiten zu gewinnen. Er enthüllte damit die Anatomie Galens, dessen Sektionen nicht der Erforschung dienten, sondern lediglich der Bestätigung des auf philosophischer Grundlage Erkannten, als er 1543 seine Fabrica de humani corporis herausbrachte (Winau 1982:289). Das Sektieren und Anschauen wurde nun als die Basis aller Aussagen über den Körper proklamiert, der in einem radikalen Empirismus zu Quelle und Prüfstein allen anatomischen Wissens wurde.

Damit wurde die Anatomie als neue Wissenschaft begründet, die für Jahrhunderte zur Grundlagenwissenschaft der westlichen Medizin wurde. Die neue anatomische Betrachtungsweise des Körpers führte auch zu der Erkenntnis, dass die Körper von Männern und Frauen in ihrem biologischen Bau unterschiedlich waren. Die Fortpflanzung, die dem weiblichen Körper zugeordnet wurde, stellte das entscheidende Kriterium für die Festlegung der Geschlechter dar. ${ }^{45}$ Vorher erklärte man sich, nach dem Modell Galens, die morphologische Differenz dadurch, dass bei Männern die Genitalien nach außen gestülpt waren, bei Frauen jedoch aufgrund ihrer mangelnden vitalen Hitze innen lagen (Laqueur 1990:25ff); Körperlich gab es zwischen Männern und Frauen keine Opposition, sondern es handelte sich um graduelle Differenzierungen eines einzigen Körpers.

Mit dem Sezieren toter Menschen fiel auch die Auftrennung in einen körperlichmateriellen Körper und einen geistigen Denk- und Erkenntnisbereich zusammen. Die Trennung von Körper und Geist geht auf René Descartes zurück (1596-1650), der bei-

\footnotetext{
44 Das Konzept der Humoralpathologie (Säftelehre) wurde im 2. Jahrhundert n. Chr. von dem griechischen Arzt Galen formuliert. Den vier Elementen Luft, Wasser, Feuer und Erde, die als konstituierend für die gesamte Welt angesehen wurden, entsprachen im Menschen die vier Körpersäfte Blut, Schleim, Galle und schwarze Galle. Weiterhin gab es noch die Kategorien wie die vier Lebensalter, die vier Jahreszeiten, die vier Temperamente oder die vier Qualitäten. Wenn die Säfte des Menschen gut gemischt waren, galt er als gesund (Eukrasie). Wenn die Säfte jedoch aus dem Gleichgewicht gerieten, wurde der Mensch demzufolge krank (Dyskrasie). Außerdem herrschte die Ansicht vor, dass die Seele des Menschen durch die Substanz des Körpers beeinflusst wurde. Je nach Mischung der dem Körper eigenen vier Qualitäten Wärme, Kälte, Trockenheit, Feuchtigkeit veränderte sich auch der Charakter der Seele. Die Seele konnte folglich durch rechte Ernährung und Lebensweise (Diätetik) beeinflusst werden (Hauke 1937).

45 Im 19. Jahrhundert etablierte sich die Gynäkologie als eine weibliche ,Sonderanthropologie‘ und spielte bei der Geschlechterkonstruktion eine entscheidende Rolle. Die biologisch determinierten Geschlechter von ,Mann " und ,Frau' wurden entdeckt und davon auch eine gesellschaftliche Aufgabenverteilung zwischen den Geschlechtern abgeleitet (vgl. Lindemann 1994:4). Durch die „,natürlichen“ Prozesse von Schwangerschaft und Geburt wurde die Frau dem Bereich der ,Natur` zugeordnet (siehe dazu auch Petersen 2000:49ff).
} 
des in ein hierarchisches Verhältnis zueinander setzte: Den Geist über den Körper. Er begründete den von der philosophischen Souveränität der Vernunft überzeugten modernen Rationalismus (Schmidt 1982:116). Es ging darum, Wissen um die ,Natur' nicht mehr an den antiken Autoritäten festzumachen, sondern in der Übereinstimmung mit der ,Natur' selbst. Die ,Natur' wurde folglich ihre eigene Autorität und war zugleich Erkenntnis- und Zergliederungsobjekt (Eckart 1998:209).

Descartes entwarf in seiner Theorie eine Neubewertung der ,Natur' mit dem Menschen als „maitre et possesseur de la nature“ (zitiert nach Eckart 1998:209). Sein Glauben an die Vernunft drückte er mit dem Zitat ,ich denke, also bin ich“ (cogito, ergo sum) aus. Der Mensch wurde in dieser rationalen Idee als gespaltene (dualistische) Gestalt betrachtet: Er war zum einen „denkende Substanz“ (res cogitans) mit einem Bewusstsein und zum anderen Körpersubstanz, wobei als das Merkmal des Körpers die Ausdehnung im Raum galt (res extensa). Alle Eigenschaften des Körpers müssen demnach als Modifikationen derselben gedeutet werden und alle physikalischen Vorgänge als Lageveränderungen oder als Bewegungen (König 1989:52). Diese Theorie wurde bekannt als die „kartesianische Lebensmechanik““46. Eine Ausnahme gab es jedoch: Die Epiphyse oder Zirbeldrüse (glandula pinealis) galt als der Sitz der wahrnehmenden und denkenden Seele (anima rationalis) (Eckart 1989:198; Winau 1983:217f). Von besonderer Bedeutung für die westliche Medizin war die aus der kartesianischen Trennung von Geist und Körper hervorgegangene Dichotomie von Subjekt und Objekt; Der Körper wurde säkularisiert und damit zum Objekt (vgl. Pfleiderer 1995:169). ${ }^{47}$

Der Körper wurde allerdings immer weiter auf seine Einzelteile und das Funktionieren derselben reduziert (Winau 1983:224). Es herrschte die Meinung vor, dass sich Veränderungen des Körpers in kleineren Einheiten abspielen müssten. Diese Einheiten waren die in Organen vorkommenden Gewebe. Störungen in der Funktion eines Gewebes müssten zwangsläufig zu fehlerhafter Funktion eines Organs führen: „Krank wird nicht mehr der Körper; krank wird nur noch ein Organ, vielleicht nur noch ein Gewebe in einem Organ. Mit der Lokalisierung der Krankheit geht in gleichem Maße die Einheit des Körpers verloren“(Winau 1983:221).

Der nächste Schritt war die von Rudolf Virchow (1821-1902) im 19. Jahrhundert entwickelte Zellularpathologie. Virchow wies nach, dass nicht Organe oder Gewebe, sondern letztlich immer die einzelnen Zellen Träger krankhafter Veränderungen waren

\footnotetext{
46 Die beiden Hauptwerke von Descartes, in denen er die physikalischen Grundzüge dieser Theorie darlegt, heißen „Principia philosophiae“ (Amsterdam 1644) und „De homine“ (Leiden 1662) (Eckart 1998:198; Winau 1983:217).

47 Diese Zweiteilung und die unterschiedliche Bewertung der beiden Teile hatte weitreichenden Einfluss auf das Bild des Menschen in den westlichen Kulturen und prägt dieses auch gegenwärtig, wie im weiteren Verlauf noch gezeigt wird (siehe auch Hauser-Schäublin et al. 2001:80f).
} 
(Winau 1982:294). Er postulierte drei Grundregeln: Erstens, dass allein die Zelllehre den „einzig möglichen Ausgangspunkt aller biologischen Doktrinen“ bilden könnte, zweitens, dass die Zelle die kleinste aller vitalen Einheiten wäre, und drittens, dass jeder Zelle eine andere vorausgegangen sein müsste (omnis cellula a cellula) (Eckart 1998:272f). Damit wurde zum ersten Mal dem Konzept der Humoralpathologie (Säftelehre) ein ebenso umfassendes Konzept der Solidarpathologie gegenübergestellt, die in den festen Bestandteilen des Körpers die Ursachen für eine Krankheit suchte. Diese bildet bis heute die Basis des medizinischen Denkens, zumindest der Schulmedizin. Nach Winau hatte das Körperkonzept des Mediziners fatale Folgen: „Nicht der gesamte Körper interessiert den Arzt, er existiert für ihn nicht mehr, alles muss lokalisiert werden, alles hat seinen spezifischen Sitz“ (1982:294). Der Körper als Ganzes ist der iatrotechnischen Medizin ${ }^{48}$ aus dem Blick geraten und hat sich aufgelöst in Organe, Organteile und Funktionen. Der damals begonnene Prozess wurde durch die damit zusammenhängende Spezialisierung der Ärzte noch intensiviert (Winau 1982:295).

\subsection{2 ,Körper' und ,Leib' als Analyseinstrumente}

Da der Schwerpunkt meiner Arbeit auf dem individuell erlebten und empfundenen Körper $^{49}$ liegt, verwende ich die Unterscheidung zwischen Körper und Leib. Dabei gehe ich vom objektivierbaren abtrennbaren Körper und vom Leib als subjektivem Wahrnehmungs- und Empfindungsorgan aus. Ich bin mir bewusst, dass beide Aspekte letztlich nicht klar voneinander zu trennen sind, da der Körper auch immer eine leibliche Dimension enthält. Die analytische Trennung von Körper und Leib soll jedoch dazu dienen, die Widersprüche, die sich aus dem Körper als medizinischem Behandlungsobjekt mit seiner ganz besonderen Geschichte und dem Leib als individueller Befindlichkeit und als Organ der Welterfahrung schlechthin ergeben, sichtbar zu machen und erklären zu können. Die Reproduktionstechnologien arbeitet genau in dem Spannungsfeld zwischen

\footnotetext{
48 Das Wort „Iatro“ stammt aus dem griechischen. „Iatrik“ ist die Heilkunst bzw. ärztliche Kunst. Ivan Illich hat als Medizinkritiker den Begriff der „Iatrogenesis“ geprägt. Es ist zusammengesetzt aus den griechischen Vokabeln für „Arzt“ (iatros) und für Ursprung (genesis). Zu den iatrogenen Leiden gehören, laut Illich (1975:20), nur solche Krankheiten, die nicht ausgebrochen wären, wenn eine entsprechende, fachlich gebotene Behandlung vorgenommen worden wäre. Rothschuh (1978:417-419; zitiert nach Winau 1982:296) verwendete den Begriff vom ,iatrotechnischen Körper“, der heutzutage weite Teile der modernen Medizin prägt, und definierte folgende Grundsätze dieses Körperkonzeptes: 1. Der Organismus ist nicht von besonderen vitalen Kräften gelenkt, es gibt keine Lebenskraft, 2. Alle Lebensvorgänge sind chemische oder physikalische Prozesse, 3. Der Zusammenhang aller Funktionsglieder ist streng naturgesetzlich determiniert, 4. Die Kenntnis der Bedingungen, unter denen Organe arbeiten, erlaubt, Lebensprozesse in beliebiger Weise zu beeinflussen, 5. Krankheit ist eine Störung physikalischer oder chemischer Prozesse, die sich in morphologischen Veränderungen der Organe niederschlägt. Sie sind mit adäquaten Mitteln objektiv nachweisbar, 6. Die Therapie verfolgt das Ziel, unmittelbar und gezielt in die gestörte Funktion einzugreifen. Dies kann der Arzt am besten, wenn er über lückenlose Kenntnisse zur Reparatur verfügt.

49 In der Körperethnologie wurde dafür der Begriff embodiment eingeführt. Er bezeichnet die Fokussierung auf den individuell gelebten Körper. Der Körper gilt dabei jedoch nicht nur als Objekt, das in seiner Beziehung zur Kultur untersuchbar ist, sondern als Subjekt seiner Kultur (Csordas 1994:5).
} 
dem aufteilbaren Körper als Objekt medizinischer Diskurse und Praktiken einerseits und dem geschlossenen und individuell kontrollierten Körper als verleiblichte Integrität und persönliche Identität andererseits. Nur auf der Grundlage der Vorstellung eines trenn- und aufteilbaren Körpers, der die Loslösung von Zeugungssubstanzen und ihre außerkörperliche Zusammenführung ermöglicht, können die reproduktiven Technologien angewendet werden. Gleichzeitig ist das Ziel dieser Behandlungen die Konstituierung von Leiblichkeit im Form von leiblichen Nachkommen.

Die deutsche Sprache ermöglicht durch die Differenzierung der Begriffe ,Körper` und ,Leib‘ dem doppelten Aspekt von Körper Ausdruck zu verleihen. Die Wörter ,Leben“ und ,Leib“ sind etymologisch über das mittelhochdeutsche lip verwandt, das eng verwandt ist mit dem niederländischen lijt und dem englischen life. ${ }^{50}$ Der Leib ist demnach mit Leben synonym, d.h. er ist ein lebendiger, beseelter Körper. Körper geht auf das lateinische corpus zurück. Der heutige Begriff des Körpers bezieht sich nicht mehr nur den menschlichen Leib, sondern wird in verschiedenen Dimensionen verwendet: ,Körper“ bezeichnet „ein stoffliches und räumliches Gebilde“ oder auch eine „Stoffmasse“ (vgl. Hauser-Schäublin et al. 2001:133). ,Leib“ wird im allgemeinen nur dem Lebendigen zugesprochen. Illich (1975:216) konstatiert dazu:

"Der Körper als klinisch verstandener Organismus ist etwas anderes als der Leib. Noch Ende des 18. Jahrhunderts machen zeitgenössische Wörterbücher einen deutlichen Unterschied: ,Körper', das ist etwas Totes, Dingliches, Tierisches (Leichname, Hölzer, Hunde haben ,Körper'), Leib ist, was Mensch ist. Leib und Leben gehören sinnmäßig zusammen, aber was ,Leib“ im Laufe der Wortgeschichte jemals meinte, wird oft erst verständlich, wenn er dem Blut oder der Seele oder dem Herzen gegenübergestellt wird.“

In der deutschen Philosophie hat der Leib eine lange Tradition (vgl. Waldenfels 2000:15). Husserl, der als Begründer der Phänomenologie gilt (1859-1938), beschreibt den Leib als das Wahrnehmungsorgan des erfahrenden Subjektes (1993:144). Der Leib ist seiner Ansicht nach Träger von „Empfindnissen“ (1993:149), durch die sich Welt konstituiert, und damit auch das Orientierungszentrum des Ichs (1993:152f). Diesem Leib kann sich das Ich nicht entziehen. Jede Erfahrung ist somit eine sinnliche leibliche Wahrnehmung: „Der Leib hat nun für sein Ich die einzigartige Auszeichnung, dass er den Nullpunkt aller Orientierungen in sich trägt“ (1993:158). Husserl spricht auch von „Leibkörper“, was auf einen Zusammenhang von Leiblichkeit und Körperlichkeit hinweist. Der Leib konstituiert sich somit auf doppelte Weise, er ist sowohl physische Materie, die eine bestimmte Beschaffenheit aufweist als auch Wahrnehmungsorgan. Auch der Philosoph Waldenfels (2000:254) erwähnt die Doppelheit des Leibes: Einmal tritt er

50 Die folgenden Ausführungen basieren auf dem etymologischen Wörterbuch der deutschen Sprache von Adolf (1937) und dem Duden Herkunftswörterbuch (1997). 
als fungierender Leib auf, d.h. er ist beteiligt an der Konstituierung von Welt und somit Wahrnehmungsorgan, und einmal als Körperding, was bedeutet, er ist ein Ding in der Welt wie jedes andere und kann ge- und vermessen werden. Beide Aspekte sind jedoch nicht klar voneinander zu trennen, sondern ineinander verschränkt. Dabei kommt es zu einer Selbstverdoppelung des Leibes in Leib und Körper (Waldenfels 2000:265).

Auf der Grundlage von Husserls Phänomenologie beschäftigte sich auch Merleau-Ponty (1908-1961) mit dem Verhältnis zwischen Ich und Leib. So schreibt er: ,[...] mein Leib steht nicht vor mir, sondern ich bin in meinem Leib, oder vielmehr ich bin mein Leib [...]“ (1966 [1945]:180). Das reflektierende Subjekt ist ein verleiblichtes Wesen, das von der Welt nicht getrennt ist und kann sich nur seiner bewusst sein auf der Basis der Leiblichkeit: Für Merleau-Ponty gibt es demnach keine Wahrnehmung jenseits der Leiblichkeit. Er hat damit indirekt Körper von Leib abgegrenzt, denn der Leib gilt ihm als Gesichtspunkt zur Welt (siehe auch Hauser-Schäublin et al. 2001:135). MerleauPonty fasst zusammen: „Und auf diese Weise ist der Leib Ausdruck der gesamten Existenz, nicht als deren äußere Begleiterscheinung, sondern weil sie sich in ihm realisiert“" (1966 [1945]:198). Auch der deutsche Philosoph Schmitz entwickelte eine Leibtheorie, die eine explizite Unterscheidung zwischen Körper und Leib trifft. Er versteht unter ,Leib‘ dasjenige, was innerlich gespürt wird, das affektive Betroffensein, während ,Körper‘ das ist, was durch die Sinne äußerlich wahrgenommen wird. Leiblichkeit bedeutet für Schmitz ein Empfinden, nicht ein Wahrnehmen, was impliziert, dass es auch nicht zu behandeln ist (vgl. Waldenfels 2000:112).

Die konzeptionelle Unterscheidung zwischen Körper und Leib, so wie sie implizit von Merleau-Ponty und explizit von Schmitz vorgenommen wurde, ermöglicht es bei der Analyse des Umgangs und des Erlebens von Reproduktionstechnologien zu untersuchen, in welchem Verhältnis der Körper zum Leib steht. Auch die von der Leibphilosophie beeinflussten Körper-Theoretikerinnen (vgl. z.B. Duden 1991; Lindemann 1993, 1994, 1996; List 1993) unterscheiden zwischen dem Körper als Bild und damit als äußerlich, objektiviert und instrumentalisiert und dem Leib als lebendigen, sinnlich erfahrenden, ganzheitlichen Körper. Die alltägliche Leibeserfahrung der Subjekte wird hier einem biologischen, sexualwissenschaftlichen und medizinischen Wissen über den Körper entgegengehalten. Lindemann (1993, 1996:166) beschäftigt sich zum einen mit den sozial relevanten Wissenskonzepten vom Körper, und zum anderen damit, wie der Körper von Individuen gespürt wird. ${ }^{51}$ Körpergefühle, -erfahrungen und -wahrnehmungen

\footnotetext{
51 Den engen Zusammenhang von Körperkonstruktion und Leiberfahrung und die historische Wandlung des leiblichen Empfindens, zeigt Duden (1991) am Beispiel des schwangeren Frauenleibes auf. Sie vergleicht das Erleben der Leibesfrucht unter der Haut mit der Besichtigung des durch Ultraschallaufnahmen veräußerten Fötus auf dem Bildschirm und kann damit kulturkritisch zeigen, wie die subjektive Erfahrungsvielfalt, das intuitive und empirische Wissen und die Sinnlichkeit als Erkenntnismittel zunehmend ausgeschlossen werden.
} 
sind ihrer Ansicht jedoch immer schon gesellschaftlich vermittelt. Sie sind eingebettet in historisch gewordene, kulturell vermittelte soziale Lebenswelten. ${ }^{52}$ Bei diesen Ansätzen gilt der empfindende, spürende, wahrnehmende Leib als individueller Zugang zur Welt und als entscheidende Quelle aller Erkenntnisse (siehe auch Merleau-Ponty 1966 [1945]). Die Leiblichkeit - unser embodiment - ist unsere existentielle menschliche Daseinsform, an die sich die Erfahrung von Raum und Zeit bindet.

\subsubsection{Körper und Selbst-Reflexivität}

Im biomedizinischen Körpermodell der westlichen Gesellschaft geht man seit Descartes von einer Trennung des Menschen in Körper und Geist/Seele ${ }^{53}$ aus, wobei der Geist letztlich als über den Körper erhaben gilt. ${ }^{54}$ Kennzeichnend für dieses kulturspezifische Körpermodell ist die Vorstellung vom Körper als einem Objekt mit einer materiellen Realität, die physisch wahrnehmbar ist. Aus der Vorstellung vom Körper als einer Sammlung von Einzelteilen, die hinzugefügt oder entfernt werden können, ergibt sich auch eine Betrachtung des Körpers als losgelöst von seinem Selbst (vgl. Sault 1994). Dieses Konzept impliziert die Möglichkeit, sich von Teilen seines Körpers trennen zu können, ohne dass das Selbst betroffen ist. In der westlichen (Schul-)Medizin „,body and self are understood as distinct and separable entities; illness resides in either the body or the mind. Social relations are seen as partitioned, segmented, and situational - generally discontinuous with health or sickness“ (Scheper-Hughes/Lock 1987:21). Körper und Selbst gelten dabei als unterscheidbare und trennbare Entitäten. Ich verstehe den Begriff des ,Selbst' nach Scheper-Hughes/Lock (1987:14) im Sinne von Eigenwahrnehmung eines individuellen Körper-Selbst (der auch eng mit ,Identität‘ zusammenhängt), der analytisch zu unterscheiden ist von dem der ,Person“. Als Person wird die kulturelle Konzeption eines Menschen hinsichtlich seiner gesellschaftlichen und sozialen Identität bezeichnet (Scheper-Hughes/Lock 1987:14). Für meine Untersuchung greife ich diese analytische Trennung zwischen Selbst und Person auf und begreife Körper in seiner verleiblichten Form als das Bindeglied zwischen beiden. ${ }^{55}$ Beide Aspekte eines Individuums sind veränderliche Konstituierungen, die durch Diskurse und Praktiken entstehen

\footnotetext{
52 Der Begriff der Lebenswelt geht zurück auf Husserl und bezeichnet die Welt, wie sie sich in der alltäglichen Erfahrung dem handelnden Menschen darbietet. In dieser Lebenswelt existiert das Individuum, sie ist ihm vertraut und bildet den Hintergrund aller Tätigkeiten.

53 Im Zusammenhang mit dem kartesischen Dualismus werden Seele und Geist häufig begrifflich nicht streng getrennt, sondern synonym verwendet. Auch Descartes hat beide Kategorien oftmals vermischt, wobei es sein Hauptanliegen war, wie Andrew Strathern zeigt, die absolute Trennung zwischen dem materiellem Körper und der Seele dazulegen (1996:41-42, 139).

54 Siehe dazu Kapitel 2.3.1.

55 Diese Meinung wird jedoch nicht allgemeinhin geteilt. So unterscheidet beispielsweise Grace Harris (1989) zwischen Person und Selbst, wohingegen oftmals in der Literatur auch eine Gleichsetzung von Selbst, Person und Individuum erfolgt (Morris 1994:1).
} 
und im sozialen Kontext verhandelbar sind. Die gegenseitige Abhängigkeit von Person und Selbst im westlichen Kontext verdichtet sich auch in der Idee von Individualität, die in der ethnographischen Literatur als das entscheidende Charakteristikum des westlichen Selbst (und damit auch der Person) dargestellt wird.

„Western conception of the person as a bounded, unique, more or less integrated motivational and cognitive universe, a dynamic centre of awareness, emotion, judgement, and action organized into a distinctive whole and set contrastively both against other such wholes and against its social and natural background, is, however incorrigible it may seem to us, a rather peculiar idea within the context of the world's cultures." (Geertz 1983:290)

Ethnologische Studien, wie beispielsweise die von Becker über „Body, Self and the Society. The View from Fiji“ (1995) zeigen auf, dass nicht-westliche Konzepte des Selbst nicht individuell, sondern eher ,fundamentally interpersonal“, kollektiv gesellschaftlich und an sozialer Interaktion orientiert sind (Becker 1995:4-5). Nach ScheperHughes/Lock (1987:21) gilt in vielen nicht-westlichen Kulturen der Körper als integrierter Aspekt des Selbst und der sozialen Beziehungen.

Die Vorstellung der Trennung von Körper und Selbst stellt jedoch einen zentralen Wert der westlichen Gesellschaft in Frage, das verkörperte Selbst. So existiert die Idee, dass der Körper nach Idealvorstellungen geformt werden kann zum Beispiel durch Techniken wie Ernährungsvorschriften, Bodybuilding, Schönheitspflege oder Medikamente, deren Ziel es ist, den eigenen Körper und seine Geschlossenheit zu bewahren. Diese Vorstellung muss nicht unbedingt einen Gegensatz zur oben genannten Lösung des Selbst vom Körper darstellen, sondern kann auch dessen Folge sein. Wenn man das Selbst und den Körper für relativ unabhängig voneinander hält, kann an dem Körper ,gearbeitet' werden, um ihn zu einem entsprechenden Ausdruck des Selbst zu machen bzw. beide einander anzugleichen. Manipulationen am Körper sind möglich, da die westliche Gesellschaft von einer Einzigartigkeit des Menschen ausgeht und die Individualität eine hohe Bewertung erfährt. Nach Giddens gilt der Körper in der Spätmoderne als symbolische Repräsentation des Selbst; Körper und Selbst sind untrennbar verbunden. Das Selbst ist somit verkörpert (embodied) (Giddens 1991:56). Es empfindet sich im und durch den körperlichen Leib und ist von diesem nicht zu trennen. ${ }^{56}$ Dieser Einschätzung liegt auch das Konzept eines autonomen individuellen Selbst zugrunde. Das

\footnotetext{
56 Giddens trifft in seiner Arbeit in englischer Sprache keine Unterscheidung zwischen ,Körper‘ und ,Leib“. Auf der Ebene der Begrifflichkeit liegt die Schwierigkeit darin, dass die englische Sprache nicht wie das Deutsche die verschiedenen Termini bereitstellt. Die unterschiedlichen Bedeutungsspektren existieren dennoch. So spricht Giddens vom Körper (body) und vom verkörperten Selbst (embodied self). Mit ,Körper' bezieht er sich primär auf den Körper im biomedizinischen Sinn, der formbar ist, und an dem als Behandlungsobjekt gearbeitet werden kann. Er greift jedoch auf Merleau-Ponty zurück, wonach unsere Fähigkeit, in der Welt als kompetenter sozialer Akteur zu handeln, in unserer Leiblichkeit begründet ist. So enthält für Giddens der Körper immer auch die Dimension des Leibes als Organ der subjektiven Wahrnehmung und Empfindung.
} 
Selbst ist jedoch nicht ein festgelegter gegebener Zustand, sondern ein reflexiver Prozess, d.h. es muss vom Individuum immer neu bestätigt und reflexiv an neue Bedingungen angepasst werden (Giddens 1991:75). Dies geschieht durch ein ständiges Angleichen zwischen dem soziokulturellen Umfeld und dem Selbst und eine Anpassung bzw. Veränderung desselben. Die leibliche Integrität, d.h., wenn das Selbst sicher im Körper verankert ist, steht deshalb in enger Verbindung mit der Person, der regelmäßigen Anerkennung durch andere im sozialen Handeln. Beide sind verkörpert, in dem wie andere meinen Körper sehen, und so wie ich mich fühle. Gerade weil die beiden Aspekte eines Individuums, das (subjektive) Selbst und die Person, wie sie im Erleben sozialer Beziehungen entsteht, verkörpert sind, bildet die routinisierte Kontrolle über den Körper ein Mittel, um die eigene Selbst-Identität aufrecht zu erhalten (vgl. Hauser-Schäublin et al. 2001:258). Unter Selbst-Identität versteht Giddens den reflexiven Prozess zwischen innen und außen, der sich über die Kontinuität der eigenen Biografie konstituiert.

„In the post-traditional order of modernity, and against the backdrop of new forms of mediated experience, self-identity becomes a reflexive organised endeavour. The reflexive project of the self, which consists in the sustaining of coherent, yet continuously revised, biographical narratives, takes place in the context of multiple choice as filtered through abstract systems. (Giddens 1991:5)

Diese Selbst-Identität hängt eng mit dem Gefühl von biografischer Kontinuität zusammen. Ein ungebrochener Verlauf der eigenen Lebensgeschichte vermittelt somit ein Gefühl von innerer Authentizität. Diskontinuitäten, also Brüche oder Veränderungen, müssen integriert werden, da ansonsten die eigene Biografie nicht weiter entwickelt werden kann und die Selbst-Identität in eine existentielle Krise gerät (Giddens 1991:79). Damit verbunden ist sowohl die Vorstellung von der eigenen Handlungsfähigkeit (agency) und damit zusammenhängend der Anspruch auf eigene Kontrolle (Giddens 1991:61f). In einer, wie Giddens es nennt, post-traditionalen Umgebung der Spätmoderne sind weder körperliches Aussehen noch Verhalten etwas Festgelegtes, so dass ,the body participates in a very direct way in the principle that the self has to be constructed“" (1991:100). Ebenso wie das Selbst gilt auch der Körper nicht mehr als festgelegte Einheit, sondern ist verwoben in die Reflexivität der Spätmoderne (1991:218). Nach Giddens (1991:20) ist diese Reflexivität ein wichtiges Merkmal der spätmodernen Gesellschaft, die sich - v.a. durch schnelle technische und medizinische Fortschritte laufend verändert und deshalb extreme Anforderungen ans Individuum stellt (siehe auch Hauser-Schäublin et al. 2001:257). Durch den Einfluss der Moderne wurde der Körper emanzipiert, womit er die Voraussetzung für seine reflexive Neustrukturierung bietet. Der Körper ,is part of an action system rather than merely a passive object“ (Giddens 1991:77), was bedeutet, dass an ihm gearbeitet werden kann. Körper und Selbst werden 
somit zu einer Stätte von Interaktionen, Verwendung und Wiederverwendung, die reflexiv organisierte Prozesse und Expertenwissen verbinden.

Die hier dargelegten analytischen Konzepte erlauben mir, die unterschiedlichen Diskurse über Verwandtschaft, Körper, Leib, Selbst und Person der Menschen aufzuzeigen, die direkt oder indirekt von den Reproduktionstechnologien tangiert werden. Besonders im Zusammenhang mit den Vorstellungen der Schaffung von Verwandten und Verwandtschaft und den Konzepten von einem ,eigenen“ Kind bietet dieser theoretische Hintergrund die Möglichkeit zu untersuchen, welche Mittel und Wege die von mir interviewten Personen aufgrund ihrer „,ungewollten Kinderlosigkeit“ zur Überwindung dieses Zustandes aus dem Spektrum der staatlich legitimierten Formen zur ,assistierten“ Familiengründung auswählen. So lässt sich unter anderem der Frage nachgehen, ob Reproduktionstechnologien reflexiv verwendet werden, d.h. um über Eingriffe am Körper das Selbst und damit die Biografie der Selbst-Identität zu verändern und somit den eigenen Lebensvorstellungen von einer Kleinfamilie zu entsprechen. Das Ziel der Reproduktionsmedizin ist die Überwindung des Zustandes der „ungewollten Kinderlosigkeit““ und die Schaffung von Leiblichkeit (das leibliche Kind) durch die ,Reparatur' des Körpers. Dieses kulturspezifische biomedizinische Körperkonzept, dessen Entwicklung in Kapitel 2.3.1 beschrieben wurde, bildet den Erfahrungshintergrund sowohl für die Paare, die sich in reproduktionsmedizinischer Behandlung befinden, als auch für die Ärzteschaft. Die analytische Trennung zwischen Körper und Leib ermöglicht die Untersuchung des Erlebens und Empfindens der Paare im Spannungsfeld von medizinisch definiertem, objektivierbaren und abtrennbaren Körper und ihrer individuell erlebten subjektiven Leiberfahrung ebenso wie die Analyse der Perspektive der Ärzte kraft ihres Amtes im Umgang mit Körpern und ihrer individuellen Leiblichkeit. 


\section{Methodisches Vorgehen}

\subsection{Die ethnologische Forschung in Deutschland}

In meiner Untersuchung versuche ich, ein zentrales Thema der Ethnologie, die Verwandtschaftsethnologie, mit den von ihr erarbeiteten Erkenntnissen auf eine komplexe Gesellschaft anzuwenden. Auf dem Hintergrund kulturvergleichender Studien zu Zeugung und Verwandtschaft wende ich den für ethnologische Arbeiten typischen holistischen Ansatz an mit einer Kombination verschiedenster Untersuchungsmethoden wie beispielsweise die teilnehmende Beobachtung, ausführliche qualitative Interviews sowie Erhebungen mittels Fragebögen. Empirische Daten werden im Rahmen einer ethnologischen Forschung in besonderem Maße durch die Feldforschung gewonnen, d.h. es geht um zeitintensive, langdauernde oder auch in Abständen im gleichen Gebiet fortgesetzte Forschungen zur Erfassung qualitativ dichter Daten (vgl. Girtler 2001; Jensen 1995). In meiner konkreten Forschung zur Erhebung des empirischen Material habe ich die Methode der teilnehmenden Beobachtung eingesetzt, da sie mir einen intensiven Einblick in die Erfahrungswelt der von ,ungewollter Kinderlosigkeit“" Betroffenen im Kontext der Reproduktionsmedizin ermöglichte. Sie war außerdem sinnvoll, um vor allem gruppenspezifisches Handeln (wie z.B. Beratungen und Behandlungen, Treffen von Selbsthilfegruppen u.ä.) zu dokumentieren, da verbale Antworten auf von mir als Wissenschaftlerin gestellte Fragen nur einen Teil von effektiven Einstellungen und Meinungen darstellten, die zum Treffen von Entscheidungen und konkreten Handlungen führten. Die teilnehmende Beobachtung machte es möglich, dass durch sie verbale Äußerungen mir gegenüber ergänzt, zum Teil auch korrigiert werden konnten.

Die Feldforschungsaufenthalte in den verschiedenen reproduktionsmedizinischen Zen$\operatorname{tren}^{57}$, die mit einer Ausnahme (s.u.) zwischen zwei und drei Monaten dauerten, bildeten einen Schwerpunkt meiner empirischen Forschung. Sie ermöglichten mir das Kennenlernen der verschiedenen medizinischen Techniken, der täglichen Abläufe und der Organisationsstrukturen der Kliniken. Durch meine Anwesenheit bei den verschiedenen Untersuchungen und Behandlungen sowie eigene Beobachtungen entstand eine ,Vertrauensbasis“ zu den „ungewollt kinderlosen“ Paaren, die anschließende ausführliche qualitative Interviews zu einem großen Teil erst ermöglichten. Häufig teilten mir die Paare explizit mit, dass sie nie mit mir gesprochen hätten, wenn wir uns nicht so oft

57 Da im Kontext von Adoptionen und Pflegschaften keine stationäre Feldforschung mit teilnehmender Beobachtung möglich war, gehe ich hier nur auf die konkrete Forschungssituation in den reproduktionsmedizinischen Kliniken ein. Die empirische Datenerhebung bei Adoptiv- und Pflegeeltern beschreibe ich in Kapitel 3.2. 
begegnet wären. Sie empfanden zum einen meine (erworbene) Kenntnis der verschiedenen medizinischen Methoden und der Eingriffe als hilfreich, da sie somit im Gespräch nicht zu langen Erklärungen ansetzen mussten, ebenso wie sie es begrüßten, das ich durch meinen Aufenthalt ihre „Kinderwunsch-Geschichte“ schon ein bisschen kannte. Die mehrmonatige Feldforschung hatte den Vorteil, dass sich die Paare Zeit nehmen konnten, um in Ruhe zu überlegen, ob sie sich zu einem Gespräch bereit erklären möchten. Die einen stimmten schon bei den ersten Treffen spontan zu, andere brauchten für diese Entscheidung länger. Die Aussagen der Paare bestätigten die Vorteile einer ethnologischen Untersuchung mit einer kontinuierlichen teilnehmenden Beobachtung. Sie betonten das Gefühl, ernst genommen zu werden, da sich jemand so viel Zeit nehmen würde, um sich mit ihnen zu beschäftigen. Dieses positive Gefühl wirkte sich besonders auf die Länge und inhaltliche Ausführlichkeit der Gespräche aus. Zunächst fiel es den Interviewpartnern oftmals schwer, über ,etwas Alltägliches und Selbstverständliches“ wie Familie, Verwandtschaft, Ehe, Elternschaft oder Kinder zu sprechen mit der Begründung, sie hätten noch nie darüber nachgedacht oder könnten sich auch nicht vorstellen, worum es mir in meiner Untersuchung konkret ginge. Die gemeinsame Sprache $^{58}$ von Forscherin und ,Beforschten“ erleichterte den Zugang zu den kulturellen Selbstverständlichkeiten und Wertvorstellungen, da in den Gesprächen deutlich wurde, dass „über Familienangelegenheiten reden“ bedeutete, sich einem intimen Bereich zu nähern, der sowohl das persönliche Geschlechts- und Gefühlsleben als auch die gesellschaftliche Moral berührte. Im Verlauf der Interviews kamen fast alle befragten Personen ins Reden und waren dann der Meinung, das es sich doch um ein Thema handele, dass für alle von Bedeutung sei. Das offene Gespräch über den ,unerfüllten Kinderwunsch $^{\text {“59 }}$, die persönlichen und medizinischen Hintergründe, Gefühle und Erlebnisse sowie die konkreten Erfahrungen mit der Reproduktionsmedizin bereiteten zunächst mehr Schwierigkeiten, wobei dennoch sowohl Frauen als auch Männer, die sich zu einem Interview mit mir bereit erklärten, auch nach anfänglichen Hemmungen sehr offen über ihre Empfindungen und Erfahrungen redeten.

\footnotetext{
58 Nicht für alle befragten Paare war das Deutsche die Muttersprache, wurde jedoch von allen fließend verwendet.

59 Zur kulturellen Konstruktion des „Kinderwunsches“ siehe auch ausführlicher Beck-Gernsheim (1988), HauserSchäublin et al. (2001), Petersen (2000) und Stein/Sproll (1995). Im Zusammenhang mit der ,ungewollten Kinderlosigkeit“ ist auch der „unerfüllte Kinderwunsch“ ein von den Betroffenen selbst verwendeter Begriff. Er wird jedoch ebenso wie „ungewollte Kinderlosigkeit“ besonders im öffentlichen Diskurs zur Beschreibung eines Zustandes, der als abweichend vom ,Normalen' gilt, eingesetzt und impliziert dadurch per se eine Behandlungsnotwendigkeit. Der Begriff des „unerfüllten Kinderwunsches“ wird dabei semantisch wie der Name einer Krankheit benutzt und nimmt meiner Ansicht nach noch expliziter Bezug auf ein ,Leiden“ der Betroffenen, mit dem - wie auch Petersen (2000:73f) gezeigt hat - die Anwendung der Reproduktionsmedizin legitimiert wird. Die Betonung der Reproduktionsmedizin als „Hilfe für Paare mit unerfülltem Kinderwunsch“ und der Verwendung des Begriffes „Kinderwunsch-Therapie“ zeigt auch die Tendenz, nicht die körperlichen Eingriffe, sondern das Kind als Motivation und Ziel der Behandlungen in den Mittelpunkt zu rücken.
} 
$\mathrm{Zu}$ welchen Personen ich jedoch letztlich Zugang bekam, und was mir in den Interviews erzählt (oder eben auch nicht erzählt) wurde, war abhängig von dem Bild der Paare von mir als Forschende generell im Rahmen der Klinik und weiterhin von ihrem persönlichen Eindruck im Verlauf der Interviews. So stellten sie mir mehrfach sehr direkte Fragen u.a. zu meiner Person und zu meinen Lebensumständen (,Sind Sie eigentlich verheiratet?“, „Haben Sie selbst Kinder?“, „Wollen Sie Kinder?“). Es wurde deutlich, wie sehr meine Positionierung bestimmte Blickwinkel eröffnete und andere verschloss. Mein akademischer Grad, durch den ich vielen Paaren bildungsmäßig überlegen war, und meine ,Nähe‘ zur Ärzteschaft, die ich in ihrer täglichen Routine in den verschiedensten Situationen teilnehmend begleitete, führte zunächst zu einem hierarchischen Ungleichgewicht. Einige Paare äußerten sich erst in den Interviews offen zu dem Gefühl der Beklommenheit, der Unsicherheit und des Beobachtet-werdens, das sie durch meine Gegenwart bei den ärztlichen Gesprächen und Untersuchungen empfunden hatten. Dennoch waren viele Personen bereit, sich von mir interviewen zu lassen, da wir uns aufgrund der längerandauernden Feldforschungsaufenthalte des öfteren trafen und sich, wie oben schon erwähnt, eine gewisse ,Vertrautheit' einstellte. In den Gesprächen wurde die hierarchische Asymmetrie oft dadurch wieder ausgeglichen, dass zum einen die Befragten die ,Experten' insbesondere bezüglich der reproduktionsmedizinische Behandlungen waren, und zum anderen viele Interviews in deren privaten Räumlichkeiten stattfanden, in denen sie sich sicherer bewegten und mit denen sie vertrauter waren als ich (siehe dazu auch 3.2).

Was sich bei meiner ethnologischen Forschung in Deutschland als schwierig erwies, war die Optik des fremden Blicks auf die ,eigene“ Gesellschaft zu richten. „Der Blick von draußen“ (Hauser-Schäublin 1997:16), das Wissen über Konzepte von Verwandtschaft in ,fremden' Kulturen, ermöglichte es zwar, die ,eigenen' Vorstellungen mit der verfremdeten ethnologischen Brille zu beobachten. Dennoch hat sich gezeigt, dass aufgrund von Distanzierungsschwierigkeiten die Problematik gerade im Prozess des Erkennens des ,Eigenen“ lag, dem das Abwägen und Gegenüberstellen mit dem ,Fremden` folgen musste (vgl. Hauser-Schäublin 1995:7). Die ,Fremdheit‘ war offensichtlicher während der teilnehmenden Beobachtung in den reproduktionsmedizinischen Kliniken. Mir waren durch eigene regelmäßige Besuche bei einer Gynäkologin zwar die technischen Gerätschaften und bestimmte Untersuchungen bekannt, den Bereich der Reproduktionsmedizin kannte ich jedoch nicht. Aufgrund meiner Ausbildung besaß ich keine speziellen (fortpflanzungs)medizinischen Kenntnisse und Fertigkeiten, ebenso wenig wie ich die medizinische Fachsprache beherrschte. Es kristallisierte sich heraus, dass die spezifische Schwierigkeit der Ärzte darin lag, mit einer Kulturwissenschaftlerin und ihren spezifischen Forschungsmethoden umzugehen. Es wurde schnell deutlich, dass ich seitens der Ärzteschaft im naturwissenschaftlich-medizinischen Bereich als ,Exotin“ galt „mit ganz interessanten Themen“, die zum Teil zwar geduldet, jedoch in 
der direkten Feldforschung oftmals mit Skepsis betrachtet wurde. Den Ärzten fiel es aufgrund ihrer naturwissenschaftlichen Ausbildung schwer, insbesondere die Methode der teilnehmenden Beobachtung zu verstehen, da ich damit direkt in ihren Berufsalltag eindrang, sie begleitete und bei der Ausübung ihres Berufes beobachtete, Fragen stellte und speziell auch auf die Interaktionen mit den Paaren achtete. Dies war für die meisten ungewohnt und führte in einigen Fällen auch zur direkten Ablehnung meiner Forschung. In allen vier von mir besuchten Kliniken wurde mir jedoch prinzipiell ermöglicht, die reproduktionsmedizinisch tätigen Ärzte zu begleiten, wenn auch die individuelle Kooperationsbereitschaft und Offenheit unterschiedlich ausgeprägt war.

Ich möchte gern an dieser Stelle betonen, dass weder während meiner konkreten Feldforschungsphase noch in späteren oder wiederholten Gesprächen seitens der betroffenen Paare mir gegenüber geäußert wurde (oder ich den Eindruck hatte), dass ich durch meine Fragen erst Probleme ausgelöst habe, die eigentlich gar nicht vorhanden gewesen waren. „Wenn man in der Scheiße wühlt, fängt sie auch an zu stinken“ war die Meinung einiger Ärzte, mit denen ich vorab sprach, und die meiner Untersuchung ablehnend gegenüber standen. Diese Ansicht habe ich zum damaligen Zeitpunkt nicht geteilt und teile sie vor dem Hintergrund meiner Erfahrungen mit meinen Gesprächspartnern auch immer noch nicht.

\subsection{Datenerhebung und Auswertung}

\section{Datenerhebungen im Kontext der Reproduktionsmedizin}

Wie schon in Kapitel 3.1 deutlich wurde, bildete den Schwerpunkt meiner empirischen Datenerhebung die Methode der teilnehmenden Beobachtung, die für ethnologische Feldforschungen zentral ist. Um eine Forschung in einer Praxis oder Klinik für Reproduktionsmedizin durchführen zu können, nahm ich zunächst schriftlichen und telefonischen Kontakt auf, um mein Vorhaben zu erklären. Ich bat um Unterstützung, musste allerdings feststellen, dass die Resonanz nur zum Teil positiv war. Einige der von mir angefragten Kliniken antworteten erst gar nicht, andere standen einer ethnologischen Untersuchung mit ihren spezifischen Methoden prinzipiell skeptisch gegenüber. Nur von den Zentren, in denen ich letztendlich meine stationäre Feldforschung durchführte, bekam ich eine positive Rückmeldung. Nach Besuchen vor Ort, in denen ich mir selbst einen ersten Eindruck verschaffen konnte, ob eine Forschung durchführbar wäre, und bei denen ich den Ärzten und medizinischen Mitarbeitern mein Vorhaben ausführlich vorstellte, entschied ich mich, die vier Kliniken für Reproduktionsmedizin (s.u.) in unterschiedlichen Bundesländern aufzusuchen. Diese Entscheidung war einerseits davon geprägt, möglichen kulturellen Unterschieden innerhalb Deutschlands Rechnung zu 
tragen, da sich auch die landesärztlichen Bestimmungen voneinander unterschieden, was einen direkten Einfluss auf den Umgang mit der Reproduktionsmedizin hatte: So war in Bayern die reproduktionsmedizinische Behandlung an einen Trauschein gebunden, wohingegen es in Hessen und Niedersachsen ohne größere Schwierigkeiten möglich war, mit einer Genehmigung der Landesärztekammer auch unverheiratete Paare zu behandeln. Andererseits wollte ich der Frage nachgehen, ob sich an unterschiedlichen Kliniken auch unterschiedliche Einstellungen und Handlungen widerspiegeln, worauf ich im Folgenden näher eingehe.

Zunächst hospitierte ich 1997 einige Tage in der Frauenklinik des UniversitätsKlinikums in Göttingen (Niedersachsen), einer staatlich finanzierten Klinik mit einer „Abteilung für IVF (In-vitro-Fertilisation)“. ${ }^{60}$ Das Einzugsgebiet des Klinikums umfasste in erster Linie Südniedersachsen und Nordhessen sowie die angrenzenden neuen Bundesländer. Die Durchführung der reproduktionsmedizinischen Behandlungen erfolgte ambulant, d.h. die Paare bzw. die Frauen kamen mehrere Male zur Zykluskontrolle und Ultraschalluntersuchungen in die Routinesprechstunden und konnten am Tag eines medizinischen Eingriffs wie Eizellenpunktion und Embryonentransfer (siehe dazu Kap. 4.3.1.2) bei problemlosem Verlauf die Klinik wieder verlassen. Die medizinischen Maßnahmen zur Behandlung von ,ungewollt kinderlosen“ Paare wurden nur im Rahmen der Ehe und ausschließlich mit Körpersubstanzen der Ehepartner durchgeführt.

Bei dem „IVF-Zentrum“ in Wiesbaden (Hessen), wo ich 1997 fast drei Monate und 1998 einen Monat verbrachte, handelte es sich um eine staatlich finanzierte Tagesklinik, die sich ausschließlich auf die Behandlung ,ungewollt kinderloser“ Paare konzentrierte und in der alle Eingriffe ambulant durchgeführt wurden. Die meisten Paare des Zentrums kamen aus Hessen oder den angrenzenden Bundesländern, jedoch reisten auch vereinzelt Paare aus Norddeutschland oder der Schweiz an. Weitere Paare waren türkischer, bosnischer, iranischer oder irakischer, zum Teil auch amerikanischer Herkunft. In Wiesbaden wurden nach Genehmigung der hessischen Landesärztekammer auch unverheiratete Paare behandelt. Diese Genehmigungen mussten vom jeweiligen Paar beantragt und dem Arzt vorgelegt werden. Die medizinischen Therapien wurden in diesen Fällen jedoch nicht von den Krankenkassen bezahlt, die in ihren Richtlinien festgelegt haben, dass eine reproduktionsmedizinische Behandlung nur im Rahmen einer Ehe finanziert wird (siehe Kap. 4.3.1.1). Weiterhin besaß das Zentrum eine Spermabank, so dass die Befruchtung mit „Spendersperma“ (heterologe Insemination) hier möglich war.

60 Die zeitliche Begrenzung dieses Aufenthaltes war nicht meine Entscheidung, sondern beruhte auf der Entscheidung der dort tätigen Ärzte, die eine längere Forschung aus verschiedenen Gründen letztlich ablehnten (vgl. dazu auch Kap. 3.1). Bei der folgenden Beschreibung meiner Untersuchungseinheiten werde ich die Göttinger Universitätsklinik daher nur kurz erwähnen. 
Einen weiteren Feldforschungsaufenthalt führte ich 1997 für zweieinhalb Monate an einer Frauenklinik in München (Bayern) durch, eine staatlich finanzierte Klinik, in der die Reproduktionsmedizin nur einen Bereich neben gynäkologischen Vorsorge- und Routineuntersuchungen, operativen Eingriffen, der Schwangerschaftsbetreuung u.a.m. bildete. Da dort jedoch schon seit zwölf Jahren reproduktionsmedizinische Maßnahmen durchgeführt wurden, waren die Klinik und deren Ärzte in ganz Deutschland und auch im angrenzenden Ausland bekannt. Obwohl die meisten der dort behandelten Paare aus dem süddeutschen Raum kamen, reisten auch aus Österreich und der Schweiz Betroffene an. Auch hier befanden sich Paare ausländischer Herkunft (Türkei, Iran, Irak) in Behandlung. In der reproduktionsmedizinischen Abteilung fanden täglich Routinesprechstunden statt, und parallel dazu wurden Eizellpunktionen und Embryotransfers im Operationsbereich durchgeführt, die auch mit stationären Aufenthalten von mindestens einer Nacht verbunden waren. Die reproduktionsmedizinischen Therapien erfolgten nur im Rahmen der Ehe, und die Klinik führte keine heterologen Inseminationen durch.

Die letzte stationäre Feldforschung fand in der Deutschen Klinik für Fortpflanzungsmedizin (DKF) in Bad Münder (Niedersachsen) 1997 für zwei Monate statt, bei der es sich um die größte deutsche private Klinik handelte. Die Paare kamen aus der ganzen Bundesrepublik, die Behandlung erfolgte ambulant und musste privat bezahlt werden. Für die Dauer der zehn- bis vierzehntägigen Behandlung wohnten die Paare oft in den Appartements der Klinik oder in Ferienwohnungen am Ort. Im Unterschied zu den anderen von mir besuchten Zentren wurde die Deutsche Klinik häufig in den Medien erwähnt, da der Vorsitzende des Direktoriums eine medienfreundliche Einstellung besaß und daher zahlreiche Interviews gab. Da die hier durchgeführten medizinischen Behandlungen nicht von den Krankenkassen bezahlt wurden, waren regelmäßig in verschiedenen regionalen und überregionalen Zeitungen Werbeanzeigen zu finden, und es gab ausführliches Informationsmaterial, das auf Anfrage kostenlos verschickt wurde. Propagiert wurde hier besonders eine „ganzheitliche Medizin“, d.h. „,das Paar als Paar ernst zu nehmen und sowohl die persönliche Paarsituation als auch Umweltbedingungen und Ernährung bei der Behandlung ungewollter Kinderlosigkeit zu berücksichtigen“. Ein Kriterium für die Entscheidung, dort eine längere Feldforschung durchzuführen, war, dass die hier anzutreffenden Paare sich bewusst für privatärztliche Behandlungen entschieden, was mit einem hohen finanziellen Eigenaufwand verbunden war. ${ }^{61}$ Auch in Bad Münder wurden alle in Deutschland zulässigen reproduktionsmedizinischen Behandlungsmethoden angewendet.

611999 wurde die Deutsche Klinik für Fortpflanzungsmedizin (DKF) neu strukturiert. Der bisherige Bereich der Privatklinik wurde aufgelöst und gehört nun, bis auf eine privatärztliche Praxisgemeinschaft, zum kassenärztlich zugelassenen IVF-Zentrum der Deutschen Klinik. 
Während meiner Feldforschungsaufenthalte nahm ich, mit Ausnahme der Universitätsklinik in Göttingen, an den täglichen Routinesprechstunden teil, in der Ultraschalluntersuchungen und Zykluskontrollen durchgeführt wurden, war bei homologen und heterologen Inseminationen anwesend, bei Eingriffen wie Bauchspiegelungen (Laparoskopie), röntgenologischen Untersuchungen der Eileiter und der Gebärmutter (Hysterosalpingographie), Hodenbiopsien ebenso wie bei den Eileiterpunktionen und Embryonentranfers. Ich konnte den Biologen und medizinischen Mitarbeitern bei der Spermaaufbereitung, bei der In-vitro-Fertilisation (IVF) sowie bei der Mikroinjektion (ICSI) über die Schulter schauen. Weiterhin hatte ich die Gelegenheit, an den „KinderwunschSprechstunden“" zwischen Ärzten und Paaren teilzunehmen, die ein Gespräch zur Abklärung der (medizinischen) Vorgeschichte, eine erste Diagnose und Beratung umfasste. Dies war mir auch in Göttingen an zwei Tagen möglich. Während oder nach diesen verschiedenen Situationen machte ich mir Notizen und verfasste ausführliche Protokolle, in denen ich Angaben zum Ort und zu den Anwesenden machte sowie den Ablauf, die angesprochenen Themen und die Länge der Gespräche festhielt. Während der gesamten Feldforschung ergaben sich 50 Gesprächsprotokolle von Erst- und Zweitgesprächen zwischen Ärzten und Paaren. Meine täglichen Beobachtungen in den verschiedenen Bereichen der Kliniken habe ich systematisiert und in Tagesprotokollen festgehalten.

Die Methode der Erhebung mittels Fragebögen wurde an jedem der angeführten Untersuchungsstandorte angewendet (siehe Anhang). ${ }^{62}$ Entweder verteilte ich die Fragebögen selbst oder bat die Ärzte um Verteilung oder Versendung. Da die Resonanz auf die Fragebögen und deren Rücklauf jedoch weniger positiv war als erwartet, dienten sie letztlich zur Unterstützung der von mir persönlich gesammelten Daten oder als Ergänzung der ausführlichen Gespräche. Es hat sich gezeigt, dass fast alle der zurückgesandten Fragebögen von mir verteilt worden waren. Dies entspricht auch meiner Erfahrung während der gesamten Feldforschungen, in der die meisten Interviews dadurch zustande kamen, dass die Paare mich durch einen kontinuierlichen Aufenthalt kannten und ich mein Forschungsvorhaben persönlich erklären konnte.

Für die Durchführung der Interviews entschied ich mich, die Methode eines halbstandardisierten Interviews mit einem thematischen Leitfaden und verschiedenen Fragenkomplexen auszuwählen, die ich mit den Betroffenen, zum Teil auch mit einigen Ärzten, führte (siehe Anhang). ${ }^{63}$ Dieser Befragungsform liegt kein absolut gültiger und aus-

\footnotetext{
62 Zwischen den einzelnen empirischen Phasen im laufenden Jahr wurden die Fragebögen aufgrund der gewonnenen Erfahrungen überarbeitet und gegebenenfalls ergänzt.

63 Ich sprach fast alle Ärzte, in deren Sprechstunden und Untersuchungen ich eine teilnehmende Beobachtung durchführte, darauf an, ob sie auch zu einem Interview mit mir bereit wären. Aus den verschiedensten Gründen lehnten jedoch einige ab. Es ist mir wichtig hier zu erwähnen, dass nicht ich die Auswahl der ärztlichen Interviewpartner traf, sondern dass die Entscheidung für oder gegen ein Interview mit mir allein auf einer persönlichen Entscheidung der Ärzte beruhte.
} 
schließlicher Fragenkatalog zugrunde, sondern vielmehr ein mehr oder weniger flexibel aufgebautes und anzuwendendes Frageschema (vgl. Atteslander 1975:91). Als Interviewerin konnte ich unverstandene Fragen erklären und Nachfragen stellen, um die Antworten zu präzisieren. Diese Methode ermöglichte es, nötigenfalls - der jeweiligen Situation entsprechend - Fragen umzuformulieren oder sie zu einem früheren oder späteren Gesprächszeitpunkt zu stellen. Ein weiteres Merkmal eines halbstandardisierten Interviews stellt nach Atteslander die Formulierung der Frage in alltäglichen, vertrauten Worten dar. Während der Interviews versuchte ich weiterhin offen zu sein für Themenkomplexe, die von Seiten der Betroffenen formuliert wurden, da nicht alle meine Fragen für die jeweiligen Interviewpartner relevant waren.

Insgesamt führte ich 45 Interviews von ein bis zwei Stunden Dauer durch. 28 Interviewpartner waren Paare, 17 Frauen. Nach jedem Interview, das ich auf Tonband aufnahm, erstellte ich ein Protokoll, in dem ich u.a. festhielt, wo das Interview stattfand, wer anwesend war, ob es Störungen gab, wie lange es dauerte, und welche Themen besprochen wurden. Darüber hinaus machte ich Notizen zu non-verbalen Elementen, zur Atmosphäre, zur Interaktion zwischen den Interviewpartnern und mir und über meine persönlichen Gefühle vor, während und nach dem Interview.

Bei den 16 im Jahr 1997 im IVF-Zentrum in Wiesbaden aufgenommenen Interviews handelte es sich mit zwei Ausnahmen um Gespräche mit verheirateten Personen. Sie wurden alle in deren privaten Räumlichkeiten geführt und dauerten ca. ein bis zwei Stunden. Dieser Umstand wirkte sich positiv auf die Gespräche aus, da die Befragten nicht unter Zeitdruck standen und sich zum anderen auf einem ,sicheren Terrain' befanden. Die Gesprächsatmosphäre war symmetrischer als in den Räumen der Klinik, da ich zwar die Forschende und Fragende und meine Gesprächspartner die Beforschten und Befragten waren, gleichzeitig aber kam ich als fremder Gast in das den Paaren vertraute Heim, in dem ich mich nicht auskannte, und war in dieser Atmosphäre nicht mehr so eng mit der Klinik und der Behandlung verknüpft (vgl. Kap. 3.1). Im Januar 1998 verbrachte ich ein weiteres Mal einen Monat in Wiesbaden. Nach einer ersten Übersicht meiner 1997 gewonnenen empirischen Daten wollte ich eine zweite kurze Phase zur Vorstellung und Diskussion erster Ergebnisse und teilweiser Vertiefung des Materials durchführen. Ich konnte vier Zweitinterviews mit Frauen führen, die nach einer Behandlung ein Kind bekommen hatten, zwei davon mit Hilfe der Methode der heterologen Insemination, eine durch die Mikroinjektion (ICSI) und eine nach vielen erfolglosen IVF-Behandlungen (In-vitro-Fertilisation). Mit ihnen konnte ich nochmals über verschiedene Aspekte sprechen und diese vertiefen.

Die 14 Interviews in München wurden bis auf vier in einem Sprechzimmer der Klinik geführt, da sie mir zumeist durch die Ärzte nach der „Kinderwunsch-Sprechstunde“ vermittelt wurden; Alle Personen waren verheiratet. Da die Befragten nach dem Ge- 
spräch mit den Ärzten oft nicht mehr viel Zeit hatten, dauerten diese Gespräche ungefähr 45 bis 60 Minuten. Auch wirkte sich die Tatsache, dass wir uns in einem Raum der Klinik befanden - mit großem Schreibtisch und gynäkologischem Stuhl - zum Teil auf das Verhalten der Befragten aus. Die Distanz zwischen mir als derjenigen, die Fragen stellte (wie kurz zuvor der Arzt) und den Paaren als denjenigen, die Auskunft gaben, war deutlich auffälliger als bei den Gesprächen in privater Umgebung, in der die Paare entspannter waren, was sich auch auf die Länge, Ausführlichkeit und inhaltliche Tiefe der Interviews auswirkte (s.o.).

In Bad Münder führte ich 12 Interviews von ein- bis zweistündiger Dauer durch. Außer einer Person waren alle verheiratet. Die Interviews wurden mit zwei Ausnahmen in den Räumen der Klinik geführt, wobei es sich um ein Bibliothekszimmer mit kleinem Tisch und Sesseln handelte und nicht um ein Sprechzimmer mit medizinischen Gerätschaften. Die meisten befragten Paare hatten für die Behandlung einen längeren Aufenthalt über mehrere Tage vor Ort eingeplant, was sich positiv auf die Gespräche auswirkte, da sie Zeit hatten und meine Fragen ausführlich beantworteten. Mit den anderen Gesprächspartnern verabredete ich zusätzliche Termine in privater Umgebung.

Des weiteren konnte ich insgesamt zehn Interviews mit Ärzten und medizinischen Mitarbeitern in den Räumen der verschiedenen Kliniken von ungefähr einstündiger Dauer führen. ${ }^{64}$ Einerseits wurden sie als Funktionsträger und Repräsentanten ihres Berufsstandes befragt, andererseits standen ihre individuellen lebensweltlichen Orientierungen und Einstellungen im Mittelpunkt. Zusätzlich zu den aufgezeichneten Interviews ergaben sich zwischen den Untersuchungen immer wieder kürzere oder längere Gespräche zu bestimmten Aspekten der Behandlung oder einfach zur Beantwortung meiner Fragen.

Einen Teil meiner empirischen Daten erhob ich durch teilnehmende Beobachtung bei einer lokalen Selbsthilfegruppe für ,ungewollte Kinderlosigkeit“. In Deutschland existiert seit 1995 der Bundesverband der Selbsthilfegruppen für Fragen ,ungewollter Kinderlosigkeit“ „Wunschkind e.V.“ mit Sitz in Berlin, der u.a. den Erfahrungsaustausch der regionalen und lokalen Selbsthilfegruppen koordinieren soll, Ansprechpartner für Medien, Politik und Verbände sein will und in der Öffentlichkeit für Aufklärung und Auseinandersetzung mit dem Thema „ungewollte Kinderlosigkeit“" sorgen möchte. Die Selbsthilfegruppen auf regionaler und lokaler Ebene sind zwar überwiegend an den Bundesverband „Wunschkind e.V.“ angegliedert, im Mittelpunkt ihrer Gruppenarbeit steht allerdings die Situation der Einzelnen. Der Schwerpunkt liegt hier auf gegenseitigem Austausch von Erfahrungen, Weitergabe von Informationen und gegenseitiger

64 Neun dieser Interviews sind auf Tonband aufgezeichnet; über ein Gespräch fertigte ich nachträglich Notizen an. 
Unterstützung. Mein Entschluss, eine teilnehmende Beobachtung in einer lokalen Selbsthilfegruppe durchzuführen, war geprägt von der Überlegung, dass es sich bei den Teilnehmenden um ,ungewollt Kinderlose“ handelte, die in Gesprächen mit anderen Betroffenen Hilfe bei der Entscheidungsfindung für oder gegen die Inanspruchnahme einer reproduktionsmedizinischen Behandlung suchten, die sich in Behandlungen befanden, die eine Behandlung aus persönlichen Gründen abgebrochen hatten oder ,erfolglos' abbrechen mussten. Ich erhoffte mir somit ein breiteres Spektrum von Betroffenen, die sich in einem anderen Kontext als in den Kliniken mit ihrer Situation auseinander setzten und die einen aktiven Umgang mit ihrem ,Zustand' anstrebten. Des weiteren erschien es mir sinnvoll, nicht nur direkt vor oder in den reproduktionsmedizinischen Behandlungen auf die Paare zu treffen, da ich in den Kliniken oftmals mit der ärztlichen Seite und damit dem medizinischen Diskurs über ,ungewollte Kinderlosigkeit“" identifiziert wurde. Von der Teilnahme an einer Selbsthilfegruppe versprach ich mir einen anderen und tieferen Einblick in die persönlichen Erfahrungen und Schwierigkeiten, die die Situation der ,ungewollten Kinderlosigkeit“" mit sich brachte.

Aufgrund eines Zeitungsberichtes über eine „Selbsthilfegruppe für ungewollte Kinderlosigkeit" kontaktierte ich die dort angegebene Ansprechpartnerin mit der Bitte um eine Teilnahme an den regelmäßig stattfindenden Treffen. Im Jahr 1997 und bis Mitte 1998 konnte ich einmal monatlich an den Begegnungen teilnehmen und einen guten Kontakt für Einzelgespräche aufbauen. In der Regel erstellte ich im nachhinein ein ausführliches Protokoll über die angesprochenen Themen, die Stimmung, über die Interaktion zwischen den Teilnehmenden sowie über meine ganz persönlichen Gefühle. Aus diesen Treffen ergaben sich zusätzlich drei Interviews von zwei- bis dreistündiger Länge mit zwei Paaren und einer Frau in privaten Räumen. Der Umstand, dass ich meine Interviewpartner schon über mehrere Monate regelmäßig getroffen hatte, wirkte sich sehr positiv auf die Gespräche aus.

Meine Gesprächspartner aus dem Kontext der Reproduktionsmedizin waren zwischen 26 und 42 Jahre alt. Aus den gewählten Feldforschungsorten ergab sich, dass die Interviews in erster Linie mit Personen durchgeführt wurden, die sich vor oder in einer reproduktionsmedizinischen Behandlung befanden, mindestens eine ,erfolgreiche ${ }^{6} \mathrm{Be}-$ handlung hinter sich hatten, die zu der Geburt eines Kindes geführt hat, oder die sich aus verschiedenen Gründen zu einer Behandlungspause entschlossen hatten mit dem Wunsch, diese in absehbarer Zeit wieder fortzusetzen. Es stellte sich heraus, dass dies auch für die Teilnehmenden der Selbsthilfegruppe zutraf. Paare, die die Behandlungen aus unterschiedlichsten Gründen abgebrochen hatten oder abbrechen mussten, befanden sich entgegen meiner Erwartungen nicht in der Selbsthilfegruppe, so dass ich mit ihnen keine Gespräche führen konnte. 
Obwohl sich auch zahlreiche ausländische Paare in reproduktionsmedizinischer Behandlung befanden, bei deren Erstgesprächen mit den Ärzten oder Untersuchungen ich anwesend war und teilnehmend beobachten konnte, handelte es sich bei allen Personen, die sich zu ausführlichen Interviews mit mir bereit erklärten oder meine Fragebögen beantworteten, um Deutsche. Dieser Umstand hing vermutlich in erster Linie mit sprachlichen Verständigungsschwierigkeiten zusammen, da viele ausländische Paare (und insbesondere die Frauen) die deutsche Sprache nur sehr schlecht beherrschten, zum anderen schien es spezifische Hemmungen bezüglich der Thematik meiner Studie zu geben, die jedoch nicht direkt geäußert wurden/konnten und über die daher nur spekuliert werden kann. Ich möchte an dieser Stelle ausdrücklich auf die mir bewusste Schwierigkeit hinweisen, dass sich Paare ausländischer Herkunft in reproduktionsmedizinischen Behandlungen befinden, aus genannten Gründen jedoch in meiner Studie persönlich nicht zu Wort kommen.

\section{Datenerhebungen im Kontext von Adoptionen und Pflegschaften}

Ich hatte mich entschlossen, als Vergleichsgruppe für meine Studie im Bereich der Reproduktionsmedizin meinen Blick auf Adoptiv- und Pflegeeltern zu richten, die ein Kind/Kinder adoptiert oder zur Pflege angenommen hatten. Hier ging es mir um eine genauere Betrachtung der Vorstellungen von sozialen Beziehungen, die sich nicht über geteilte Körpersubstanz konstituierten. Aus diesem Grund nahm ich Kontakt zur Adoptions- und Pflegekindstelle eines Jugendamtes auf, da die Jugendämter die staatlichen Anlaufstellen für Adoptionsbewerber und Pflegeeltern in Deutschland darstellen. Nach Gesprächen mit den zuständigen Mitarbeitern wollten diese meine Forschung gern unterstützen. Eine teilnehmende Beobachtung scheiterte jedoch daran, dass dafür eine offizielle Genehmigung des Dienststellenleiters notwendig war, die ich jedoch ohne Angabe von Gründen nicht erhielt. Die Mitarbeiter des Jugendamtes erklärten sich jedoch bereit, Briefe an Adoptionsbewerber, Adoptiv- und Pflegeeltern zu verteilen, in denen ich mein Forschungsvorhaben beschrieb und um persönliche Gespräche bat. Leider bekam ich keine Rückmeldung von angeschriebenen Paaren, auch nicht auf nochmaliges Nachfragen seitens des Jugendamtes. So entschloss ich mich im November und Dezember 1997, Aufrufe in drei regionalen Tageszeitungen zu veröffentlichen mit einer Beschreibung meines Forschungsschwerpunktes und der Bitte an Adoptiv- und Pflegeeltern, sich mit mir in Verbindung zu setzen. Daraufhin meldeten sich insgesamt 15 Paare und sieben Frauen, mit denen ich von Februar bis April 1998 ausführliche Interviews führen konnte.

Da im Kontext von Adoptionen und Pflegschaften keine teilnehmende Beobachtung möglich war, erhob ich meine Daten mit Hilfe halb-standardisierter Interviews. Meinen thematischen Leitfaden modifizierte ich zu diesem Zweck, da ich vorab keine Informa- 
tionen über die spezifischen individuellen Erfahrungen und Erlebnisse hatte, wie das im Kontext der Reproduktionsmedizin durch die längerandauernde Begleitung der Paare in der Feldforschung zum Teil der Fall war (siehe Kap. 3.1). Der Bereich über Definitionen und Vorstellungen von Verwandtschaft blieb identisch, den Bereich über Zeugung und Elternschaft leitete ich jedoch mit einer offenen erzählgenerierenden Frage ein: „Mich interessiert ihre persönliche Geschichte von dem Zeitpunkt an, als sie sich gemeinsam oder jeder für sich entschlossen haben, dass sie ein Kind/Kinder haben oder mit einem Kind/Kindern zusammen leben möchten, bis zum heutigen Tag. Erzählen Sie mir doch bitte, welche Wege sie gegangen sind, welche Entscheidungen sie getroffen haben und warum?“" (siehe Anhang). Mit dieser Frage wollte ich den Teil des Gesprächs zwar thematisch vorstrukturieren, allerdings ging es mir auch darum, die Befragten ihre Geschichte nach ihren subjektiven Schwerpunkten gestalten zu lassen. Es stellte sich heraus, dass ein großer Teil der Interviewten auch eigene Erfahrungen mit den Reproduktionstechnologien gemacht hatte, was dazu führte, dass ich im Sinne eines Vergleichs auf bestimmte Themenkomplexe in der Nachfragephase näher einging. Die 22 auf Tonband aufgezeichneten Interviews mit Adoptiv- und Pflegeeltern fanden alle in privaten Räumlichkeiten statt und hatten eine Dauer von ein bis drei Stunden. Auch nach diesen Interviews erstellte ich zusätzlich ausführliche Protokolle (siehe oben).

\section{Datenauswertung}

Nach Abschluss der empirischen Datenerhebung erfolgte eine detaillierte Transkription der Interviews. Für die qualitative Auswertung und Interpretation der Interviews waren verschiedene Methoden notwendig, die von dem Computerprogramm AQUAD ${ }^{65}$ unterstützt wurden. Bei der Inhaltsanalyse lehnte ich mich unter anderem an das analytische Ablaufmodell von Mayring (2000) an. Diese Analyse richtete sich sowohl auf das Thema als auch darauf, „durch den Text Aussagen über den emotionalen, kognitiven und Handlungshintergrund der Kommunikatoren zu machen“ (Mayring 2000:47). Zunächst wurden die von mir aufgenommenen und transkribierten Interviews nach einem allgemeinen Themenkatalog wie beispielsweise Familie, Verwandtschaft, Ehe, Kind(er), „Kinderwunsch“, Zeugung durchgesehen und einzelne Gesprächsabschnitte den jeweiligen Problembereichen zugeordnet. Aus den Themenbereichen extrahierte ich dann ein System von Kategorien, wobei zwei Arten von Kategorien zu unterscheiden waren: die Kategorien von ,außen' (die von mir festgelegten) und von ,innen“ (sich aus den Interviews ergebende). Durch den Interviewleitfaden wurden bestimmte Kategorien von , außen' bereits vorgegeben. Die Kategorien von ,innen' wurden mit Hilfe von AQUAD

65 Aquad Five for Windows, Version 5.51. Software Package for the Analysis of Qualitative Data, Günther L. Huber, Universität Tübingen, 1999. Vertrieb über Verlag Ingeborg Huber, Schwangau. 
schrittweise erarbeitet und in einem Kategoriensystem mit Haupt- und Unterkategorien (vgl. Mayring 1993) zusammengestellt, wie z.B. eigenes Kind, Körper, Leiblichkeit, Normalität, natürlich, künstlich. Die Entwicklung der Kategorien und die entsprechende Zuordnung sind als meine eigene Interpretationsleistung anzusehen und finden sich in den Kapiteln fünf und sechs meiner Arbeit.

Da ich im Sinne einer Multivokalität die von mir interviewten Personen selbst zu Wort kommen lassen möchte, habe ich in der Darstellung meines empirischen Materials sehr viele Zitate verwendet. Ich sehe die zitierten Aussagen meiner Gesprächspartner als gleichwertige Beiträge an und habe sie daher, trotz einer wortgenauen Transkription, hier der Schriftsprache angeglichen, sprachlich geglättet sowie grammatikalisch vervollständigt. Gekennzeichnet habe ich sie durch geringeren Zeilenabstand. Auslassungen ebenso wie von mir vorgenommene Ergänzungen zum besseren Verständnis sind mit [...] gekennzeichnet, Redepausen mit drei Punkten. Bei längeren Interviewausschnitten, in denen ich Nachfragen stellte, habe ich meine Redebeiträge mit den Anfangsbuchstaben des Vor- und Zunamens gekennzeichnet (I.S.). So bin ich auch bei den Aussagen meiner Gesprächspartner verfahren, deren Namen jedoch zum Schutz ihrer Anonymität verändert wurden. Das angegebene Alter bezieht sich auf den Zeitpunkt der Interviews. Nähere soziobiografische Angaben finden sich im Anhang. 


\section{Die Konstruktion von Verwandtschaft im bundesdeutschen rechtlichen Diskurs}

Nach den theoretischen Vorüberlegungen, die Einbindung meiner Arbeit in den ethnologischen Kontext und die Darstellung der methodischen Herangehensweise, werde ich mich im folgenden Kapitel der Konstituierung von Verwandtschaft als einer spezifischen Formen von Zugehörigkeit und Angehörigkeit (relatedness) in der bundesdeutschen Gesetzgebung zuwenden, d.h. legal zugeschriebenen und anerkannten Zugehörigkeiten und Angehörigkeiten. So werde ich Definitionen von Verwandtschaft betrachten ebenso wie die verschiedenen Kategorien von Verwandtschaft, deren Konstituenten und Bedeutungen. Gesetze betrachte ich als eine öffentliche Diskursform, die auch Anweisungen für das konkrete soziale Handeln geben. Gesetzgebungen und die Diskussionen, die zu den entsprechenden Regelungen geführt haben, unterscheiden sich von einem Staat zum anderen, nicht zuletzt aufgrund unterschiedlicher kultureller Traditionen, und beinhalten bestimmte Einstellungen zu Werten und Vorstellungen. Als Teil der gesamtgesellschaftlichen Rahmenbedingungen bestimmen sie auch den Umgang mit Möglichkeiten von ,assistierten“ Familiengründungen wie den Reproduktionstechnologien sowie mit Adoptionen und Pflegschaften und gehören zu der Erfahrungswelt der von „ungewollter Kinderlosigkeit“ betroffenen Personen. Dabei sind sie nicht statisch, sondern veränderbar und spielen darüber hinaus eine zentrale Rolle bei der Etablierung einer bestimmten gesellschaftlichen Vorstellung von ,ungewollter Kinderlosigkeit“" und der Schaffung einer Akzeptanz ihrer Behandlung.

\subsection{Rechtliche Definitionen und Zuordnungen von Personen}

\subsubsection{Verwandtschaft, Schwägerschaft und Familie}

Die wichtigsten Rechtsvorschriften, die Verwandtschaft nach dem Gesetz begründen und die sich aus ihr ergebenden Rechtsfolgen, sind im deutschen Grundgesetz (GG) und dem Bürgerlichen Gesetzbuch (BGB) festgelegt. Das Familienrecht des Bürgerlichen Gesetzbuches $(\mathrm{BGB})^{66}$ definiert exakt, wer mit wem als verwandt gilt und welche Rechte und Pflichten sich mit diesem Status verknüpfen (siehe Kap. 4.2). Unter Verwandtschaft wird hier Bluts- oder leibliche Verwandtschaft (Kognation) verstanden. Nach dem Bürgerlichen Gesetzbuch $§ 1589$ wird Verwandtschaft wie folgt definiert:

66 Lucke (1998:62) weist darauf hin, dass sich im Bürgerlichen Gesetzbuch zwar ein Ehe-, Familien- und Scheidungsrecht findet, jedoch kein eigenes Verwandtschaftsrecht. 
„Personen, deren eine von der anderen abstammt, sind in gerader Linie, Personen, die nicht in gerader Linie verwandt sind, aber von der selben dritten Person abstammen, sind in der Seitenlinie verwandt. Der Grad der Verwandtschaft bestimmt sich nach der Zahl der sie vermittelnden Geburten.“

Dementsprechend gelten Großeltern, Eltern und Kinder als in gerader Linie verwandt, Geschwister in der Seitenlinie. Eltern und Kinder werden in gerader Linie als Verwandte ersten Grades, Großeltern und Enkel als Verwandte zweiten Grades definiert. In der Seitenlinie ist Ego mit seinen Geschwistern im zweiten Grad verwandt, mit Geschwisterkindern im dritten Grad. Geschwisterkinder untereinander sind im vierten Verwandtschaftsgrad miteinander verwandt.

Des weiteren regelt ein spezieller Paragraph die „Verwandtschaft durch Adoption“, wobei es sich hierbei um Verwandtschaft im Rechtssinn handelt; Durch die Annahme als Kind wird ein Kindschaftsverhältnis durch Rechtsakt „künstlich“ begründet. Die Gesetzgebung unterscheidet zwischen der Adoption Minderjähriger und Volljähriger. Nach $\S 1754$ I 1 BGB besitzt ein minderjähriges adoptiertes Kind die rechtliche Stellung eines ehelichen Kindes der Ehegatten. Zu seiner bisherigen genetischen Verwandtschaft erlischt das Verwandtschaftsverhältnis mit der Adoption (§ 1755 I 1 BGB). Ein adoptierter Volljähriger hingegen wird nur mit den Adoptiveltern verwandt, nicht aber mit deren Verwandten (§ 1770 I BGB). Darüber hinaus bleibt in diesem Fall die genetische auch als rechtliche Verwandtschaft erhalten (§ 1770 II BGB).

Von Verwandtschaft unterschieden wird die Schwägerschaft. In $\S 1590$ I BGB heißt es: „Die Verwandten eines Ehegatten sind mit dem anderen Ehegatten verschwägert $[\ldots]^{\prime c}$. Der Grad richtet sich dabei nach dem Verwandtschaftsgrad, der die Schwägerschaft vermittelt. Laut Absatz II § 1590 BGB dauert die Schwägerschaft auch nach Auflösung der sie vermittelnden Ehe an. Dies gilt jedoch nur für die schon in der Ehe begründeten Schwägerschaften; Neue Schwägerschaften können nach Auflösung der Ehe nicht mehr begründet werden. Ebenso wie die Verwandtschaft gliedert sich die Schwägerschaft in Verschwägerte in gerader Linie, z.B. Stiefeltern und Stiefkinder, Schwiegereltern und Schwiegerkinder, sowie in Verschwägerte in der Seitenlinie. Als stiefverwandt werden das nicht blutsverwandte Kind des Ehepartners, der nicht blutsverwandte Ehepartner eines Elternteils und die mit ihm genealogisch verbundenen Personen bezeichnet (vgl. Fenner 1984:51). Die stiefverwandte Verbindung ist demnach gekennzeichnet durch die Heirat zwischen Ego und dem Elternteil eines mit Ego nicht blutsverwandten Kindes. Ehegatten sind im Sinne des Bürgerlichen Gesetzbuches weder verwandt noch verschwägert. Ihr Verhältnis zueinander ist besonders geregelt.

Im Gegensatz zum BGB liefert das Strafgesetzbuch (StGB) eine genaue Definition des Begriffes ,Angehörige‘. Nach $\S 111$ I StGB gelten folgende Personen als Angehörige: 
„a) Verwandte und Verschwägerte gerader Linie, der Ehegatte, der Verlobte, Geschwister, Ehegatten der Geschwister, Geschwister der Ehegatten, und zwar auch dann, wenn die Beziehung durch eine nichteheliche Geburt vermittelt wird, wenn die Ehe, welche die Beziehung begründet hat, nicht mehr besteht, oder wenn die Verwandtschaft oder Schwägerschaft erloschen ist.

b) Pflegeeltern und Pflegekinder“.

Im übrigen ist der Begriff der Angehörigen in einzelnen Gesetzen verschieden weit gefasst (vgl. z.B. §§ 530, 569 a, 1579 Nr. 2, § 11 I Nr. 1 Strafgesetzbuch).

Das Grundgesetz stellt Ehe und Familie (Art. 6 Abs. 1) unter den besonderen Schutz des Staates. Der deutsche Begriff der ,Familie` wird am Ende des 18. Jahrhunderts aus dem lateinischen familia entlehnt. Dieser Begriff hat wiederum einen Bedeutungswandel erlebt - als Bezeichnung von allem, was zum Haus gehört (Personen, Sachen, unfreies Gesinde) bis hin zur Beschreibung einer personenrechtlichen Gemeinschaft (Segalen 1997; Zonabend 1996). ${ }^{67}$ Historisch wurde ,Familie“ nicht als Interaktionssystem verstanden, sondern mit Haus und Besitz als Sachgegebenheit gleichgesetzt (Lüschen 1989:441f). Bis in das 19. Jahrhundert hinein galten Haus und Hof als Wirtschaftseinheit in Landwirtschaft, Handwerk und Handel. Die Industrialisierung führte zu einer erhöhten Mobilität, aus ,Haus und Hof* wurde für viele die gemietete Wohnung und arbeitsteilige Wirtschaft ersetzte die vorher übliche Universalproduktion. Die Familie im Sinne der oben beschriebenen Hausgemeinschaft konnte nicht mehr untergebracht werden und wurde wirtschaftlich funktionslos. Übrig blieb die Kern- oder Kleinfamilie (Zwei-Generationen- oder Gattenfamilie). ${ }^{68}$

Unter den gegenwärtigen deutschen Rechtsbegriff der Familie fallen Eltern und Kinder; Ehepartner ohne Kinder bilden hingegen keine Familie im Rechtssinn. Andererseits kam es verfassungsrechtlich (Art. $6 \mathrm{GG}$ ) auf Ehelichkeit oder Nichtehelichkeit für die Familienbeziehung auch schon früher nicht an (Lüderitz 1999:23), d.h. auch die nicht

\footnotetext{
67 Auf die Mehrdeutigkeit des Begriffs ,Familie' soll hier nur hingewiesen werden. Auf die Etymologie des Wortes, die unterschiedlichsten Verwendungen und Bedeutungen kann in dieser Arbeit nicht eingegangen werden.

68 Die ältere Familiensoziologie hat aus dieser Entwicklung ein „Kontraktionsgesetz der Familie“ (Durkheim 1921) abgelesen. Jedoch wird in neuerer Zeit eingeräumt, dass jede Gesellschaft ein komplexes Familienbild zeigt (König 1976). Eng verknüpft mit der Entstehung der bürgerlichen Familie war die soziale und rechtliche Ausdifferenzierung von Eltern-Kind-Beziehungen. Die außerhäusliche Produktion wurde zur Domäne des Mannes. Den Frauen wurde die Sorge für Haus, Kinder und Ehemann zugeteilt. Parallel zu einer neuen geschlechtsspezifischen Arbeitsteilung wurde durch Vorstellungen vom ,natürlichen“ Wesen der Frau und ihrer biologischen Fähigkeit zur Mutterschaft ihre soziale Zuständigkeit für die Betreuung und Versorgung der Kinder abgeleitet. Die ,Natur' des Kindes verlangte dementsprechend die Fürsorge der Mutter (Schütze 1991). Zunehmend verband sich mit der Umstrukturierung von Familie auch deren spezifische Gefühlswelt: die Intimisierung und Emotionalisierung von Familienbeziehungen. Näher werde ich in dieser Arbeit nicht auf historische Veränderungen der Zusammensetzung und Funktionen der Familie eingehen. Mit den Wandlungen, die die Institution der Familie durchmachte, haben sich u.a. Volkskundler und Soziologen beschäftigt (vgl. Fehlmann-von der Mühll 1978; König 1976; Lüschen 1970; Mitterauer 1977; Weber-Kellermann 1974). Eine Analyse der gesellschaftlichen Bedingungen von Familie und Verwandtschaft im Feudalismus und Kapitalismus und ihre Determiniertheit durch Wirtschaft und Gesellschaft liefert Rosenbaum (1982).
} 
durch eine Ehe gegründete Gemeinschaft von Eltern und Kindern gilt als Familie. Großeltern werden nicht zur Familie gerechnet, aber der Gesetzgeber ist nicht gehindert, sie wie Mitglieder der engeren Familie zu behandeln (siehe Kap. 4.2). Dagegen zählen zur Familie die Kinder nur eines Ehegatten, die mit dem Stiefelternteil lediglich verschwägert sind, ferner auch Pflegekinder. Im bürgerlichen Recht kommt der Begriff der ,Familie‘ kaum vor. Statt der Familie verwendet das BGB häufiger den traditionellen Begriff der ,Hausgemeinschaft‘ (Lüderitz 1999:23f).

\subsubsection{Die Ehe}

\section{Die Eheschließung}

Die Ehe ist eine rechtlich anerkannte, mit Eheschließungswillen eingegangene, grundsätzlich auf Lebensdauer bestimmte Lebensgemeinschaft zwischen Mann und Frau (§ 1353 Abs. 1 S.1 BGB), welche ökonomische, sexuelle sowie soziale Rechte und Pflichten beinhaltet und durch den unmittelbaren Heiratsvollzug eine öffentliche Bestätigung erfährt. Die Ehe kommt durch eine entsprechende Willenserklärung von Mann und Frau zustande. ${ }^{69}$ Die Voraussetzungen einer wirksamen Eheschließung bestimmen sich nach dem Ehegesetz (EheG). Es handelt sich dabei um die Verschiedenheit des Geschlechts der Ehepartner ${ }^{70}$, der Ehefähigkeit, dem Fehlen von Eheverboten, dem Fehlen von Willensmängeln und dem Einhalten von Formvorschriften. Es ist keine uneingeschränkte Partnerwahl erlaubt, sondern es gibt immer einen Personenkreis, aus dem der Ehepartner nicht stammen darf (Exogamieregeln). So ist gesetzlich festgeschrieben, dass die Ehe monogam ist. Ein gesetzliches Eheverbot gilt heute zwischen Verwandten in gerader Linie, zwischen voll- oder halbbürtigen Geschwistern sowie zwischen Verschwägerten in gerader Linie, wobei im Falle von Schwägerschaft Befreiung vom Eheverbot erteilt werden kann. Die im Bürgerlichen Gesetzbuch und im Ehegesetz geregelte Ehe ist ein privates Rechtsverhältnis. Daher enthält das Gesetz kein Leitbild über die persönliche Ausgestaltung (Lüderitz 1999:34). Die Entscheidung, ob und wann in dieser Form der Gemeinschaft Kinder geboren werden, bleibt einzig den Eheleuten überlassen.

69 Zur historisch-soziologischen Entwicklung der Institution Ehe siehe z.B. Goody (1989 [1983]), Segalen (1986).

70 Seit kurzem können jedoch auch homosexuelle Paare eine eheähnliche Lebensgemeinschaft eingehen. 


\section{Der Ehename}

Das Gesetz weist der Familie eine besondere Bedeutung zu (Art. 6 GG). Wie sich am Beispiel des Namensrechtes zeigt, wird die Zugehörigkeit einer Person zu ihrer Fortpflanzungsfamilie und nicht zu ihrer Abstammungsgruppe betont. Ursprünglich war die Namenszuweisung vom Gesetzgeber viri- und patrinominal geregelt: Die Frau nahm den Namen ihres Ehemannes an und die Kinder bekamen diesen Familiennamen als Geburtsnamen. Seit 1993 gibt es aber eine Neuregelung, der zufolge die Ehegatten einen gemeinsamen Familiennamen (Ehenamen) bestimmen. Der Ehename kann gemäß $\S 1355$ Abs. 2 BGB nur der Geburtsname eines Ehegatten sein (dabei kann es sich auch um einen Namen aus einer Adoption handeln). Durch diese Regelung soll vermieden werden, dass Doppelnamen zu Ehenamen werden. Nach $\S 1355$ Abs. 1 S.3 BGB kann aber auch jeder Ehegatte seinen vor der Ehe geführten Namen weiterführen. Außerdem ist es nach $\S 1355$ Abs. 4 BGB n.F. möglich, dass zwar ein Ehename geführt wird, jedoch derjenige Ehegatte, dessen Name nicht Ehename ist, seinen Namen dem Ehenamen beifügt. Soll ein gemeinsamer Ehename geführt werden, so bedarf es einer Erklärung der Ehegatten.

\section{Die Ehescheidung}

Unter einer Ehescheidung versteht das deutsche Recht die Auflösung der Ehe durch ein gerichtliches Urteil mit Wirkung für die Zukunft aufgrund bestimmter Scheidungsgründe. Dieses Verständnis von der Ehescheidung stellt einen Mittelweg zwischen zwei extremen Positionen dar. Während in der Aufklärungszeit die Ehe als ein Vertrag zweier Individuen definiert wurde, der jederzeit durch einen Aufhebungsvertrag beseitigt werden konnte, hält die katholische Kirche die Ehe grundsätzlich für unauflösbar. Die Ehescheidung als gemeindeutsche Einrichtung beruht auf dem Personenstandsgesetz von 1875, das die Trennung von Tisch und Bett zu echten Scheidungsgründen erklärte. Mit dem 1. EheRG (Ehereformgesetz vom 14.06.1976 - im wesentlichen Teil in Kraft seit 01.07.1977) wurde das Ehescheidungsrecht wieder in das BGB zurückgenommen, aus dem es 1938 herausgelöst wurde. Das bisher geltende Verschuldungsprinzip wurde durch ein reines Zerrüttungsprinzip abgelöst. Danach reicht die objektive Ehezerrüttung als einziger Scheidungsgrund aus, so dass es auf Eheverfehlungen der Eheleute nicht mehr ankommt (Limbach 1989:229f). Die Ehe wird durch ein Gerichtsurteil geschieden $(\S 1564 \mathrm{BGB})^{71}$

71 Die Scheidungsrate in Deutschland betrug im Jahr $200046 \%$ im Vergleich zu den geschlossenen Ehen (IDEASpektrum Nr. 22, 30. Mai 2001; Internet-Quelle: http: //www.idea.de). 


\subsubsection{Verwandtschaft - Kindschaft - Elternschaft}

Das Kindschaftsreformgesetz (KindRG) als Teil des Familienrechts, das am 01. Juli 1998 in Kraft trat, behandelt in erster Linie die Eltern-Kind-Beziehungen (personenstandsrechtliche Zuordnungen, Sorgerecht und Unterhaltsverpflichtungen). ${ }^{72}$ Das Elternrecht ist durch Art. 6 II, III GG als Grundrecht geschützt, womit Eltern gleichzeitig das ,natürliche' Recht und die Pflicht zur Pflege und Erziehung ihrer Kinder haben. Der Staat nimmt jedoch besonderes Interesse an der Erziehung der Kinder. Das Elternrecht wird als ein im Interesse des Kindes pflichtgebundenes Recht verstanden, so dass die Persönlichkeitsrechte des Kindes Inhalt und Schranken des elterlichen Erziehungsrechts mitbestimmen (Lüderitz 1999:237). Das Gesetz gliedert Abstammung von der Unterhaltspflicht (Trennung von Status und Unterhalt). Die bisher geltende Unterscheidung von ehelichen und nicht-ehelichen Kindern wurde nunmehr beseitigt und die Rechtsstellung beider Eltern weitgehend angeglichen.

Die personen- und vermögensrechtliche Zuordnung eines Kindes zu seinen Eltern folgt in Deutschland in erster Linie der ,natürlichen Abstammung“. Dieser Aspekt, der sich auf $\S 1589$ BGB „Verwandtschaft“ bezieht, füllt zahlreiche Seiten und ist darüber hinaus auch als Art. 2 Abs. 1 „Jeder hat das Recht auf Kenntnis seiner genetischen Abstammung“ (Schutz des Persönlichkeitsrechts) im Grundgesetz (GG) verankert. Mit Bezug auf Lüderitz (1999:243) ist Abstammung, und insbesondere Abstammung vom Vater, jedoch weitgehend eine Rechtsregel, da sie nur mit wissenschaftlich aufwendigen Methoden festgestellt werden kann. Dennoch regeln die im Kindschaftsreformgesetz neugefassten $\S \S 1591$ bis 1600 e BGB auf der Basis des durch $§ 1589$ BGB definierten Begriffs von „Verwandtschaft“ die Statusvoraussetzungen, die sich grundsätzlich weiterhin an der Abstammung orientieren bzw. die einen ,erfahrungsmäßigen' Schluss auf „natürliche Abstammung“ zulassen (vgl. Lüderitz 1999:243). Die sogenannten Personenstandsbeteiligten können jedoch durch Rechtsgeschäfte (Anerkennung) oder ihr Unterlassen (Anfechtung) auf die rechtliche Zuordnung des Kindes einwirken, d.h. sich über „tatsächliche Abstammung“ hinwegsetzen.

\footnotetext{
72 Die elterliche Sorge ( $\$ 1626$ BGB) umfasst allgemein die Vertretung des Kindes. Sie teilt sich auf in Personenund Vermögenssorge. Die Personensorge ist wiederum unterteilt in die tatsächliche Personensorge (Pflege, Erziehung, Aufenthaltsbestimmung des Kindes) und die Vertretung in persönlichen Angelegenheiten (Anmeldung in der Schule, Zustimmung zu ärztlichen Behandlungsmaßnahmen) (Oberloskamp 1993:4). Nach $§ 1626$ III gehört das Umgangsrecht für beide Elternteile zu den Grundsätzen der elterlichen Sorge. Es dient dem Wohl des Kindes und umfasst auch den Umgang des Kindes mit anderen Personen, ,zu denen das Kind Bindungen besitzt, wenn ihre Aufrechterhaltung für seine Entwicklung förderlich ist" (beispielsweise Großeltern, Geschwister, Stiefelternteile sowie „Personen, bei denen das Kind längere Zeit in Familienpflege war“) (§1695 BGB).
} 


\section{Abstammung von der Mutter}

Die rechtliche Zuordnung eines Kindes zur Mutter ist seit dem Kindschaftsreformgesetz von 1998 ausdrücklich in $\S 1591$ BGB geregelt: Als „,natürliche“ Mutter gilt diejenige Frau, die das Kind geboren hat. Schon vor dieser gesetzlichen Fixierung galt die gebärende Frau als die Mutter, jedoch existierten keine Rechtsregeln. Kindesvertauschung oder Pflege konnten zwar eine soziale Mutterbeziehung begründen, die möglicherweise besondere rechtliche Regelungen erforderte; sie berührte jedoch Mutterschaft als solche nicht (vgl. Lüderitz 1999:244).

Die Entwicklung der Reproduktionstechnologien macht nun die Aufspaltung der ehemals als Einheit wahrgenommenen „natürlichen“ Mutterschaft in genetische (Herkunft der Eizelle) und biologische (austragende Frau) Mutter möglich. In Deutschland gilt die Zuordnung eines Kindes zu der Frau, die es geboren hat, auch bei gespaltener Mutterschaft. ${ }^{73}$ In diesem Fall setzt der Gesetzgeber den $\S 1589$ BGB außer Kraft, da nicht die genetische Abstammung über Mutterschaft entscheidet. Die rechtliche Zuordnung steht im Einklang mit der Bewertung der Mutter-Kind-Beziehung während der Schwangerschaft in der Diskussion um das Embryonenschutzgesetz und dem gesetzlich geregelten Verbot von gespaltener Mutterschaft. Die genetische Mutter hat keinerlei Rechte, d.h. die Mutterschaft kann weder von der „Ei- oder Embryonenspenderin“ noch vom Kind angefochten werden.

\section{Abstammung vom Vater}

Eine der Mutterschaft vergleichbare evidente „natürliche“ Zuordnung des Kindes zum Vater fehlt. Das Recht verhilft daher dem Kind, seinen Vater zu ,finden', indem es die rechtliche Zuordnung, zumindest vorläufig, an Tatbestände knüpft, die eindeutig erkennbar sind und eine Abstammung wahrscheinlich machen. Dies sind die Ehe mit der Mutter und förmliche Anerkennung ( $\$ 1592$ Nr. 1, 2 BGB n.F.). Auch das reformierte Kindschaftsrecht knüpft eine Vaterschaftsbeziehung in erster Linie an eine sichtbare rechtliche Institution an: Das in einer Ehe geborene Kind stammt vom Ehemann der Mutter ab, solange die Vaterschaft nicht angefochten wird (§ 1592 Nr. 1 BGB n.F., $\S 1593$ BGB a.F.). Vorher ist die Anerkennung durch einen Dritten nicht wirksam (§ 1594 II BGB). Nach Lüderitz (1999:247) begünstigt dieser fast zwangsläufige institutionelle Schutz faktisch das eheliche Kind und lässt es weiterhin als wünschenswert erscheinen, Kinder von Anfang an in formalisierten Elternbeziehungen aufwachsen zu

\footnotetext{
73 In Deutschland wird diese jedoch durch das Embryonenschutzgesetz (ESchG) von 1991 explizit verboten (siehe Kap. 4.3.1.1). Eine gesetzliche Zuordnung zur genetischen Mutter kommt auch schon wegen des Kindes nicht ernsthaft in betracht, da sie praktisch zunächst ,Mutterlosigkeit' bedeutet hätte (vgl. Gaul 1998:116). Ein mutterloses Kind ist jedoch im deutschen Verwandtschaftskonzept nicht denkbar.
} 
lassen (vgl. auch Gaul 1998:64). Dies gilt auch, wenn die Ehe durch Tod aufgelöst wurde und danach innerhalb von 300 Tagen ein Kind geboren wird. Neu hingegen ist, dass das nach der Wiederverheiratung der Frau geborene Kind grundsätzlich dem neuen, nicht dem früheren Ehemann zugerechnet wird, außer wenn ersterer die Vaterschaft erfolgreich anficht; somit ist es wieder Kind des früheren Ehemannes.

Wird das Kind nicht in einer Ehe oder innerhalb von 300 Tagen nach ihrer Auflösung durch Tod geboren, so wird Vater, wer es anerkennt (§ 1592 Nr. 1 BGB). Die Vaterschaft anerkennen heißt sich zum Kind bekennen, gleichgültig aus welchen Motiven (Glaube an Abstammung, Liebe zur Mutter) (vgl. Lüderitz 1999:248). Der die Vaterschaft anerkennende Mann kann eine Abstammung behaupten, auch wenn sie nach seiner Kenntnis ausgeschlossen ist. Die Wirksamkeit der Vaterschaftsanerkennung wird (zunächst) davon nicht berührt. Lüderitz ist der Auffassung, dass es in diesem Zusammenhang nicht zutreffend sei, von einer „Wissenserklärung“ zu sprechen. Vielmehr handele es sich seiner Ansicht nach um eine „Willenserklärung“ und damit um ein Rechtsgeschäft, das den Status des Kindes bestimme und verändere. Die Anerkennung der Vaterschaft ist zwar rechtstechnisch ein einseitiger Akt (des Mannes), begründet jedoch einen Status, der die biologische und soziale Beziehung spiegeln soll (Lüderitz 1999:250). Die Rechtsordnung respektiert eine bestehende Intimbeziehung (MutterKind) dadurch, dass sich ein Mann als Vater nicht ohne weiteres aufdrängen kann. Die Anerkennung ist damit von der Zustimmung der unmittelbar Betroffenen abhängig. Früheres Recht folgte strikt rechtslogischen Strukturen: Da die Anerkennung den Personenstand des Kindes veränderte, war dessen Zustimmung erforderlich. Sie war innerhalb einer Frist von sechs Monaten nach Beurkundung der Anerkennung zu erklären. Erforderlich war in der Regel - da die Anerkennung meist während der Minderjährigkeit des Kindes erfolgte - eine Erklärung durch den gesetzlichen Vertreter; Dieser wiederum war das Jugendamt (vgl. Gaul 1998:74). Eine Zustimmung der Mutter war nicht vorgesehen. Heute ist die Zustimmung der Mutter erforderlich, denn es wird davon ausgegangen, dass sie am ehesten weiß, wer der, wirkliche“ Vater ist. Faktisch wird sie durch die Vaterschaftsanerkennung - jedenfalls zunächst - mehr betroffen als das Kleinkind. Mit ihrer Zustimmung vertritt sie daher ihre eigenen Interessen und die des Kindes.

Der durch die Geburt in der Ehe oder Anerkennung geschaffene legale Status des Kindes kann durch Anfechtung verändert werden. Das Familiengericht entscheidet über die Vaterschaftsfeststellung auf Klage des Mannes gegen das Kind oder auf Klage der Mutter oder des Kindes gegen den Mann (§ 1600c BGB) Diese neue Regelung räumt der Mutter erstmals ein Klagerecht in eigener Person ein. In einem gerichtlichen Feststellungsverfahren wird als Vater vermutet, wer der Mutter während der Empfängniszeit (vom 300. bis zum 181. Tag vor der Geburt) beigewohnt hat (§ 1600d I, II BGB). Diese 
Vermutungsregel gilt nicht, wenn schwerwiegende Zweifel vorliegen. Dann wird zumeist durch medizinische Gutachten die ,wahre' Abstammung durch das Gericht geklärt. Die Anfechtung der legal anerkannten Vaterschaft ist formalisiert: Es ist eine Klage notwendig, das Klagerecht ist befristet, die Klagefrist ist ausdrücklich und abschließend geregelt (binnen einer Frist von zwei Jahren). Diese Regelung soll der Stabilisierung einer grundlegenden Beziehung im Leben des Kindes dienen, auch wenn die Rechtsordnung ihre tatsächliche Ausfüllung nicht garantieren kann. Zugleich soll die Personengruppe, die in der Regel das Kind umgibt (Familie, im früheren Recht: Ehe) vor Außenstörungen geschützt werden. Ist die Klage hiernach zulässig, so führt sie, wenn sie begründet ist, zur gerichtlichen Feststellung, dass der Ehemann oder der bisher Anerkennende nicht der biologische Vater des Kindes ist. Wird die Klage abgewiesen, so bleibt es bei der Zuordnung des Kindes durch institutionelle Verbindung oder autonome Entscheidung: Rechtlich anerkannter Vater bleibt der Ehemann bzw. der Anerkennende. Zur Anfechtung berechtigt sind der vermeintliche Vater (Ehemann der Mutter im Fall des $\S 1592$ Nr.1 BGB, Anerkennender im Fall des $\S 1592$ Nr.2 BGB), die Mutter und das Kind ( $\$ 1600$ BGB). Dem Kind, d.h. bei einem minderjährigen Kind als gesetzliche Vertreterin der Mutter, wird das Recht gewährt, bei berechtigten Zweifeln die Anerkennung der Vaterschaft anzufechten. Abgeleitet wird dieses Recht aus Art. 2 Abs. 1 GG, der jedem Menschen das Recht auf gerichtliche Feststellung seiner ,tatsächlichen Abstammung“ gewährleistet. Dieses Recht basiert auf der Überzeugung, dass die Abstammung für die „Individualitätsfindung und damit für die Persönlichkeitsentwicklung von erheblicher Bedeutung“ ist (Lüderitz 1999:257; vgl. auch SteinHilbers 1994:201).

Aus meinen bisherigen Ausführungen über die gesetzlich fixierte Eltern-KindBeziehung in Deutschland wird deutlich, dass Vaterschaft nicht allein durch die biologische Verbindung zum Kind definiert ist. Biologische Vaterschaft ist zwar mit der Möglichkeit verbunden, sie mit sozialer Vaterschaft zu verknüpfen. Es besteht jedoch keine zwangsläufige Verbindung zwischen der biologischen Vaterschaft und der Anerkennung der formalrechtlichen Vaterschaft. Es wird demnach unterschieden zwischen den konstitutiven Elementen von Mutterschaft und Vaterschaft. Mutter des Kindes ist in Deutschland die Frau, die das Kind austrägt und zur Welt bringt. Damit verbunden ist das Konzept einer unteilbaren Einheit von Mutterschaft (genetische, biologische und soziale Mutter), d.h., dass es letztlich nur eine Mutter geben kann. Die Identität eines Kindes wird demnach nicht nur durch die genetische Identität, die ihm von der genetischen Mutter vermacht wird, sondern auch durch die psycho-soziale Mutter-Kind- 
Bindung während der Schwangerschaft geprägt. ${ }^{74}$ Die als „,natürlich“ und untrennbar konzipierte Verbindung zwischen körperlicher und sozialer Mutterschaft stellt ihr kulturell konstitutives Element dar. Das Konzept von Mutterschaft beinhaltet somit eine physische und soziale Dimension. ${ }^{75}$ Vaterschaft muss hingegen sozial bestätigt werden, um eine legale Anerkennung zu erfahren.

Deutlich wird diese kulturspezifische Sichtweise auch anhand der Arbeiten von Dolgin (1995) und Tong (1995), die sich mit der rechtlichen Problematik von gespaltener Elternschaft in den USA beschäftigt haben. Sie zeigen anhand von strittigen Rechtsfällen (u.a. dem weltweit bekannt gewordenen Fall „Baby M.“) auf, dass „echte“ Mutterschaft , verhandelbar' ist, der Vater jedoch über seinen genetischen Zeugungsbeitrag eindeutig definiert wird. ${ }^{76}$ Die in Kapitel 2.1 dargestellte Konstruktion von Verwandtschaft in der Ethnologie hat gezeigt, dass die Suche nach einem Vater, die in der Diskussion um die ignorantia paternitatis und die sich anschließende virgin-birth-Kontroverse deutlich wurde, der physiologischen Vaterschaft eine bedeutende Rolle zugewiesen hat. Dies war nur möglich auf der Grundlage, Verwandtschaft allein im Sinne von biologischer Verwandtschaft zu begreifen. Das aristotelische Zeugungsmodell „Ein Kind - Ein genitor", das über zwei Jahrtausende als gültig anerkannt wurde, wies dem biologischen Vater den alleinigen kreativen Anteil an der Zeugung zu (Kap. 2.2.2). Erst die Genetik dieses Jahrhunderts hat diese Denkweise widerlegt, in dem sie den Frauen einen

74 Aus diesem Grund wurde beispielsweise in den Parlamentsdebatten um das bundesdeutsche Embryonenschutzgesetz die gespaltene Mutterschaft als gravierender für das Leben des Kindes bezeichnet als gespaltene Vaterschaft. Es wurde davon ausgegangen, dass die genetische Mutter dem Kind nicht nur zum gleichen Anteil wie der genetische Vater dem Kind die genetische Identität verleiht, sondern darüber hinaus durch die biologische und psycho-soziale Beziehung zum Kind während der Schwangerschaft Einfluss auf dessen Persönlichkeitsentwicklung nimmt (vgl. Petersen 2000:105-110). Daher würde das Kind bei Kenntnis, dass durch „Eizellspende“ zwei Frauen (die genetische und die austragende) seine Existenz mitbedingt haben, auf spezielle Probleme bei seiner Identitätsfindung stoßen. Aufgrund der herausragenden Bedeutung der Mutter-Kind-Bindung im Konzept von Mutterschaft, ist auch die bewusste Aufspaltung in biologische und soziale Mutter durch Leihmutterschaft in Deutschland nicht erlaubt (siehe Kap. 4.3.1.1).

75 Diese kulturelle Konstruiertheit von Mutterschaft wird detailliert von Stein/Sproll (1995:5-32) aufgezeigt u.a. anhand der Darstellung der Geschichte der Mütterlichkeit vom 17. bis ins 20. Jahrhundert. Auch die Vorstellung von „natürlicher“ Mutterliebe hat sich entsprechend der gesellschaftlichen Realität gewandelt und wurde inzwischen zur unangetasteten Ideologie: „Es bedurfte jahrhundertelanger Beeinflussung, Manipulation und ideologischer Arbeit, um die sogenannten mütterlichen Eigenschaften und die Mutterliebe zum angeblich natürlichen Bestandteil des weiblichen Wesens werden zu lassen. Nicht die Liebe einer Mutter zu ihrem Kind ist als eigentlicher gefühlsmäßiger Neuerwerb zu bewerten, sondern die dahinter stehenden ,kulturellen Deutungsmuster“" (Stein/Sproll 1995:31). Raymond (1995:81f) betont insbesondere die Einbettung von Mutterschaft in soziale, politische und historische Kontexte und betrachtet sie in erster Linie als Beziehung. Jede Frau, die schwanger wird, tritt ihrer Ansicht nach in eine persönliche und soziale Beziehung mit dem Fötus, der ein Kind werden kann. Sie betont jedoch, dass diese Beziehung nicht immer positiv sei. Schwangerschaft stelle zwar eine Beziehung dar, die ein bedeutsame Bindung zwischen den Betroffenen fördern könne, aber nicht müsse - je nach Situation.

76 Auch in den USA geht es um die Suche nach der „echten“ Mutter, und es wird davon ausgegangen, dass es nur eine Mutter geben kann. Auf der Suche nach der „,echten“ Mutter geht es hier allerdings darum, die Frau zu ermitteln, die möglichst viele Aspekte des amerikanischen Konzeptes von Mutterschaft in sich vereinigen kann, wozu auch die sozialen und wirtschaftlichen Verhältnisse zählen, die am ehesten den Vorstellungen von Familie entsprechen: „To achieve the desired results, the courts that heard Baby M. and Johnson composed the biological facts so that the child was given to the parents with the more middle-class, traditional, home" (Dolgin 1995:63; Hervorhebungen im Original). Zum kulturellen Umgang mit Leihmutterschaften in den USA siehe auch Ragoné (1994, 1998). 
gleichwertigen Zeugungsbeitrag zuerkannte. Anhand der Analyse der bundesdeutschen Gesetzgebung ist deutlich geworden, dass Mutterschaft in Deutschland idealerweise als unteilbare Einheit konzipiert wird. Soziale Mutterschaft ergibt sich allerdings nicht allein als Option aus dem biogenetischen Anteil, sondern gilt als „natürliche“ Konsequenz der körperlichen Verbindung zwischen Mutter und Kind durch Schwangerschaft und Geburt. Zwar gründet sich auch die Ausübung der sozialen Vaterschaft in Deutschland auf den ,natürlichen Fakten“ der Fortpflanzung, dennoch ist deutlich geworden, dass die bundesdeutsche Gesetzgebung eine ,Verhandelbarkeit' der personenstandsrechtlichen Zuordnung eines Kindes durch Anerkennung oder Anfechtung der Vaterschaft zulässt. Trotz der kulturellen Vorstellung von ,natürlicher Abstammung“ als Grundlage der Konstituierung von eindeutiger, unveränderbarer und somit „echter“ Verwandtschaft, kann sich der deutsche Staat jedoch im Fall sozialer Dissonanzen und bei Einigkeit aller Personenstandsbeteiligten über diese hinwegsetzen.

\subsection{Rechtswirkungen gesetzlicher Zugehörigkeiten}

Die juristischen Festlegungen von Verwandtschaft haben direkte Auswirkungen auf das soziale Handeln. Die bundesdeutsche Gesetzgebung regelt in Bezug auf Familie und Verwandtschaft im wesentlichen drei Bereiche: den Schutz, die Versorgung und die Erbfolge. Dabei werden alle rechtlichen Wirkungen, die sich aus den Verwandtschaftsbeziehungen ergeben, durch die Art und den Grad der Verwandtschaftsbeziehung bestimmt. Rechtsfolgen der Verwandtschaft sind in erster Linie Unterhaltspflichten ( $\S 1601$ ff BGB), Erbrechte ( $\S 1924$ ff BGB) und Eheverbote ( $\$ 1307$ BGB). Diese Rechtsfolgen sind nicht nur nach Graden verschieden, sondern gehen auch bei Verwandten in gerader Linie vielfach weiter als bei Verwandten in der Seitenlinie.

Bevor Unterhalt gewährt werden muss, wird überprüft, ob einerseits ein Unterhaltsanspruch berechtigt und andererseits eine Unterhaltsverpflichtung zuzumuten ist (vgl. $\S \S 1602$ und 1603 BGB). $§ 1606$ I BGB schreibt die Reihenfolge der Unterhaltspflichtigen vor: „Die Abkömmlinge sind vor den Verwandten der aufsteigenden Linie unterhaltspflichtig“. Ist eine Person unterhaltsberechtigt, so sind seine Kinder vor seinen Eltern zum Unterhalt verpflichtet. Geschwister sind nicht verpflichtet, sich gegenseitig Unterhalt zu zahlen. Ebenso sind immer die „näheren“ vor den „entfernteren“ Verwandten (z.B. Kinder vor den Enkeln) unterhaltspflichtig (§ 1606 II BGB), vor den Verwandten jedoch zunächst die Ehegatten (§ 1608 BGB). Gibt es mehrere bedürftige Verwandte, so regelt $\S 1609$ BGB die Reihenfolge.

Die $\S \S 1924$ bis 1930 BGB regeln das gesetzliche Erbrecht von Verwandten. Bei der Erbfolge werden nicht nur die Verwandten in gerader Linie, sondern auch die Seitenverwandten berücksichtigt. Grundsätzlich beerben der Ehegatte und die Verwandten den 
Verstorbenen. Dabei erben „nähere“ Verwandte vor den „entfernteren“. Der Verstorbene kann durch ein Testament eine andere als die gesetzliche Erbfolge festlegen. Durch die Pflichtteilregelung werden jedoch die Interessen der nächsten Verwandten gewahrt (§2303 BGB). Für das gesetzliche Erbrecht ist nicht die genetische, sondern ausschließlich die rechtliche Verwandtschaft von Bedeutung, die beide nicht immer übereinstimmen müssen. Dies kommt zum Beispiel bei einer Adoption zum Tragen, oder wenn ein Kind in einer Ehe geboren, jedoch nicht vom Ehemann gezeugt wurde. Dieses Kind ist beiden legal anerkannten Elternteilen gegenüber erbberechtigt.

Durch die Eheschließung wird zwischen den Ehegatten ein personenrechtliches Gemeinschaftsverhältnis gegründet, aus dem sich gewisse Rechte und Pflichten ergeben. Die Rechtswirkungen der Ehe sind die Pflicht zur ehelichen Lebensgemeinschaft (§ 1353 Abs.1 S.2 BGB), Anspruch auf Schutz und Versorgung sowie die Pflicht zur ehelichen Treue, die jedoch keine Rechtspflicht zum Geschlechtsverkehr beinhaltet. Ebenso gibt es keine Verpflichtung der Ehegatten zur Kindererzeugung (vgl. Lüderitz 1999:34). Im Hinblick auf die Pflichtenteilung unter den Eheleuten gilt heute der Grundsatz der freistehenden Rollenverteilung gemäß § 1356 BGB (vor Neufassung des $\S 1356$ BGB durch das EheG vom 01.07.1977 galt das Prinzip der „Hausfrauenehe“). Während des Bestehens der Ehe hat jeder Ehegatte gegen den anderen einen Unterhaltsanspruch gemäß $\S 1360$ S. 1 BGB, der den gesamten Lebensbedarf der Ehegatten und der unterhaltsberechtigten Kinder umfasst.

Verwandtenbeziehungen führen, wie sich anhand der bisherigen Ausführungen feststellen lässt, einerseits zu Rechten, andererseits bringen sie jedoch auch Verpflichtungen mit sich. So werden Verwandte und Verschwägerte im Vormundschaftsrecht besonders berücksichtigt ( $\$ 1779$ II BGB). Als bedeutendes Element ist die Sonderbehandlung von Verwandten aufgrund des Angehörigenstatus zu nennen, die besonders im Strafrecht in Erscheinung tritt. $§ 242$ Strafgesetzbuch (StGB) sieht vor, einen Diebstahl oder eine Unterschlagung unter Angehörigen nur zu verfolgen, wenn dies beantragt wird. Des weiteren genießen Angehörige ein Zeugnisverweigerungsrecht (§§ 52, 55, 63, 72 Strafprozessordnung StPO). Der Beischlaf zwischen Verwandten, d.h. wer mit einem leiblichen Nachkommen, einem leiblichen Vorfahren oder Geschwistern den Beischlaf vollzieht, wird gemäß $§ 173$ I-III StGB strafrechtlich verfolgt. Zwischen Verwandten sowie zwischen Geschwistern und Verschwägerten in gerader Linie dürfen keine Ehen geschlossen werden. Heiraten dürfen jedoch Onkel und Nichte, Cousine und Cousin oder Schwager und Schwägerin. Auch das persönliche Umgangsrecht mit dem Kind ist in $\S 1634$ BGB geregelt. Dieses Umgangsrecht steht ausschließlich den Kindseltern zu, nicht jedoch beispielsweise dessen Großeltern oder Geschwistern. § 1666 BGB sieht allerdings vor, dass Beziehungen des Kindes zu seinen Verwandten aufrecht erhalten werden müssen, es sei denn, es liegen verständliche Gründe für eine Zuwiderhandlung 
vor. Die Entscheidungsgewalt darüber, wer mit dem Kind Umgang haben darf, obliegt also letztendlich den Eltern, wobei der Gesetzgeber aber hier die Interessen des Kindes klar vor die der Verwandten stellt.

Gesamthaft betrachtet lässt sich zusammenfassen, dass Verwandtschaft in Deutschland formalrechtlich nur über Abstammung definiert ist, d.h. Teil dieser konstruierten Form von Verbundenheit ist die geschlechtliche Fortpflanzung. Personen, die unter den ethnologischen Terminus der Affinalverwandtschaft fallen, werden hingegen der Kategorie Schwägerschaft zugeordnet. Eine Ausnahme bildet die Adoption, auf die ich in Kapitel 4.3.2 noch detaillierter eingehen werde. In rechtlicher Hinsicht sind Adoptierte jedoch den konsanguinen Verwandten gleichgestellt. Ehegatten gelten weder als verwandt noch als verschwägert. Eheliche Beziehungen werden durch einen freiwilligen Willensentscheid beider Ehepartner eingegangen und können unter bestimmten Gründen durch ein gerichtliches Urteil wieder aufgelöst werden. Die Ehescheidung betrifft allerdings in rechtlicher Hinsicht nur die Verbindung zwischen den beiden Ehepartnern, nicht aber die durch die Eheschließung entstandenen Schwägerschaften. Sie dauern auch über die Auflösung der Ehe hinaus an (vgl. Lucke 1998:70). Die Ehe ist zwar an eine äußere Form gebunden, deren persönliche Ausgestaltung jedoch den Eheleuten selbst überlassen bleibt. Sie gilt nach dem Bürgerlichen Gesetzbuch (BGB) als ein privates Rechtsverhältnis, das nicht gekoppelt ist an die Fortpflanzung. Der Rechtsbegriff der Familie ist nicht eindeutig, wenn auch Eltern und Kinder definitiv dazugehören. Lüderitz (1999:23) stellt jedoch heraus, dass es verfassungsrechtlich (Art. 6 GG ,Schutz der Familie") nicht auf die Ehelichkeit oder Nichtehelichkeit für die Familienbeziehung ankommt. Eine Familie gilt somit rechtlich nicht ausschließlich als konsanguine Einheit, da auch Stiefkinder, die mit einem Elternteil nur verschwägert sind, sowie Pflegekinder vom Gesetzgeber dazugezählt werden. Wie gezeigt, haben beispielsweise Großeltern, der Ehegatte, der Verlobte, Geschwister, Ehegatten der Geschwister, Geschwister der Ehegatten, Stiefeltern und -kinder sowie Pflegeeltern und -kinder einen rechtlichen Status als Angehörige, der jedoch in einzelnen Gesetzen unterschiedlich weit gefasst ist.

Weiterhin konnte ich zeigen, dass der rechtlich anerkannte Verwandtschaftsstatus mit bestimmten Rechtsfolgen wie Unterhaltspflichten, Eheverbote und Erbrechte verbunden ist. Dabei werden auch die Grade der Verwandtschaft wesentlich. Die Rechtswirkungen sind jedoch nicht nur nach Verwandtschaftsgraden verschieden, sondern gehen beispielsweise auch bei Verwandten in gerader Linie weiter als bei Verwandten in der Seitenlinie. So bestehen Unterhaltsverpflichtungen nur zwischen Verwandten in gerader Linie, nicht aber zwischen Verwandten in der Seitenlinie, nicht einmal zwischen Geschwistern. Verwandte in der Seitenlinie erben erst ab der zweiten Erbordnung, wohingegen Geschwistern kein Pflichtteilsrecht zusteht. Das Verhältnis zwischen Ehegatten 
ist rechtlich verankert und umfasst Schutzwirkungen sowie gegenseitige Verantwortung, was sich zum Beispiel auf allgemeine Hilfs- und Unterhaltsverpflichtungen bezieht.

Den Rechtsbeziehungen zwischen Eltern und Kindern wird eine zentrale Bedeutung beigemessen. Anhand des Kindschaftsreformgesetzes von 1998 (vgl. Kap. 4.1.3) ist zu erkennen, dass „natürliche Abstammung“ den zentralen Aspekt darstellt, der primär die Zuordnung zwischen Eltern und Kind(ern) und damit von Verwandtschaft bestimmt. Die Statusbeziehung eines Kindes zur Mutter ist in Deutschland eindeutig geregelt, indem Schwangerschaft und Geburt als die Kriterien für Mutterschaft festgelegt sind, wohingegen die Kriterien für die Zuordnung zu einem Vater heterogener sind. Die rechtliche Vaterschaftsanerkennung ist zum einen an die Institution der Ehe geknüpft, zum anderen richtet sie sich im Fall der Geburt eines Kindes außerhalb einer Ehe nach den Angaben der Kindsmutter. In den Fällen, in denen eine eindeutige Statuszuordnung des Kindes und somit Zuordnung zu einer bestimmten Verwandtschaft möglich ist, ist für den deutschen Staat die Frage nach der „tatsächlichen“ Abstammung nicht von primärer Relevanz; Ist demnach die strukturelle Einheit einer Kernfamilie gewährleistet, mischt sich der Staat nicht ein. Hier wird das Spannungsverhältnis zwischen der eindeutigen, ausschließlichen und dauerhaften Konzeption der biologisch begründeten Verwandtschaft und das damit einhergehende Ideal der Kongruenz biologischer und sozialer Elternschaft einerseits und den sozialen ,Dissonanzen' andererseits deutlich. Formalrechtlich muss die genetische nicht mit der personenstandsrechtlichen Zuordnung übereinstimmen, d.h. eine Einheit von biogenetischer und sozialer Elternschaft, insbesondere Vaterschaft, ist staatlicherseits für die Zuordnung von Personen nicht maßgeblich. Die Anerkennung der Vaterschaft eines (Ehe-)Mannes kann als eine willentliche Entscheidung bezeichnet werden, deren Motivation unterschiedlicher Art sein kann, die jedoch den Status des Mannes sowie des Kindes definiert. Nach deutschem Recht hat ein Mann keinen Anspruch darauf, die (biologische) Vaterschaft einzuklagen, solange diese entweder vom Ehemann der Mutter anerkannt bzw. nicht angefochten wurde, oder von einem anderen Mann mit Zustimmung der Mutter anerkannt wurde. Es lässt sich somit feststellen, dass es für den deutschen Staat letztlich nicht ausschlaggebend ist, wie sich die Kategorie „Verwandtschaft“ konstituiert, auch wenn diese per definitionem nach $\S 1589$ BGB auf Abstammung beruht. Relevant sind eindeutige personenstandsrechtliche Zuordnungen und damit Zugehörigkeiten und Angehörigkeiten von verwandten Personen, was bestimmte Rechte und Pflichten mit sich bringt, wobei der Unterhaltsverpflichtung zwischen Verwandten in gerader Linie, insbesondere zwischen Eltern und Kindern, eine wesentliche Bedeutung zukommt. Dies führt zu der These, dass etablierte Wertvorstellungen der Einheit einer Kernfamilie - in ideologischer und struktureller 
Hinsicht - mit Hilfe der bundesdeutschen Gesetzgebung verstärkt werden, auch wenn in genetischer Hinsicht dieses Ideal einer ,normalen' Familie nicht zutrifft. ${ }^{77}$

\subsection{Staatlich legitimierte Wege und Möglichkeiten der ,assistierten“ Familiengründung}

Der deutsche Staat bietet verschiedene Wege an, um eine legal anerkannte Familie zu gründen. So besteht für Paare, die durch sexuelle Intimbeziehungen keine Kinder bekommen, unter bestimmten Voraussetzungen die Möglichkeit, medizinisch-technische Maßnahmen zur Erzeugung eines leiblichen Kindes - zumindest über ein Elternteil - in Anspruch zu nehmen. Dadurch kann das Paar zu einem Elternpaar mit Nachkommen und somit zu der kleinsten und intimsten ,natürlichen“ Verwandtschaftseinheit, einer Familie, werden (vgl. Kap. 4.1). Seit der Geburt des ersten Retortenbabys 1978 in England entstanden weltweit immer mehr reproduktionsmedizinische Zentren. In Deutschland kam 1982 das erste Baby zur Welt, das mit Hilfe der Methode der In-vitroFertilisation (IVF) gezeugt wurde. Erst 1991 trat jedoch das bundesdeutsche Embryonenschutzgesetzes (ESchG) in Kraft, das mit strafrechtlichen Regelungen möglichen Missbräuchen der Fortpflanzungstechniken begegnen will. ${ }^{78}$ Die juristischen und gesellschaftlichen Verhältnisse hinken demnach den fortschreitenden medizinischtechnologischen Erkenntnissen hinterher und werden diesen angepasst. Diesen Vorgang bezeichnet Giddens (1995:54) als ,,institutionalisierte Selbst-Reflexivität“ und weist ihn als wesentliches Merkmal der Gesellschaften der Moderne aus (Kap. 2.3.3). Nach und nach entstanden in allen Industrienationen Gesetze, die einerseits die medizinischtechnischen Möglichkeiten begrenzten, diese aber andererseits auch grundsätzlich legalisierten. Im Folgenden werde ich zunächst die spezifischen gesetzlichen Bestimmungen im Zusammenhang mit den Reproduktionstechnologien, die zulässigen Anwendungsmethoden, deren Leistungsvoraussetzungen und Finanzierungen in Deutschland aufzeigen. Weiterhin erfolgt eine detaillierte Beschreibung der einzelnen Maßnahmen zur Behandlung von „Sterilität“.

Ein weiterer staatlich legitimierter Weg zur Familiengründung besteht über die Adoption eines Kindes oder die Übernahme einer Pflegschaft. Diese so entstandenen Familienformen werden in Deutschland legal und/oder sozial anerkannt, basieren jedoch nicht auf geteilter Körpersubstanz und werden somit nicht als „natürliche“ Verwandtschaft

\footnotetext{
77 Dies gilt insbesondere auch für den Fall der Familienbildung mit Hilfe der heterologen Insemination, worauf jedoch in Kapitel fünf und sechs jeweils noch genauer eingegangen wird.

78 Ich werde hier nicht die Gesetzesgenese und die darüber geführten öffentlichen Diskussionen wie beispielsweise zur Kostenübernahme durch die Krankenkassen u.ä. darstellen. Eine ethnologische Analyse der Parlamentsdebatten zum bundesdeutschen Embryonenschutzgesetz bietet Petersen (2000).
} 
konzipiert. ${ }^{79}$ Als eine Methode, den Fortbestand einer Familie bei Kinderlosigkeit zu gewährleisten, kann die Adoption jedoch auf eine lange Tradition zurückblicken. Schon in vorchristlicher Zeit stellte die Adoption eine übliche Methode zur Sicherung der Erbfolge dar (vgl. Kap. 2.2.1). Im christlichen Mittelalter verlor sie allerdings weitgehend ihre Bedeutung und wurde erst im 19., teilweise erst im 20. Jahrhundert, mit der Entwicklung der heutigen Rechtssysteme wieder in fester, von Staat zu Staat unterschiedlicher Form, eingeführt. Stark gewandelt hat sich das Selbstverständnis der Adoption und der mit ihr befassten überwiegend staatlichen Stellen. Im Mittelpunkt des Adoptionsverfahrens steht heute nicht mehr die Familiengründung „ungewollt kinderloser“ Paare, sondern de jure allein das Wohl des schon geborenen Kindes (vgl. Wild 1998: 264-267). Ich werde im folgenden Kapitel auf die rechtliche Lage und die Voraussetzungen für die Bewerbungsverfahren als Adoptiv- und Pflegeeltern eingehen.

\subsubsection{Die Reproduktionstechnologien in Deutschland}

\subsubsection{Der gesellschaftliche Rahmen: Das Embryonenschutzgesetz, das Sozialgesetzbuch, die „Richtlinien zur assistierten Befruchtung“ und die Kassenleistungen}

Neue medizinisch-technische Entwicklungen im Zusammenhang mit den Reproduktionstechnologien führten Ende der 1980er Jahre zu zahlreichen Diskussionen um neue juristische Rahmenbedingungen. ${ }^{80}$ Das Embryonenschutzgesetz (ESchG), das sich mit der zulässigen Anwendung der reproduktiven Technologien und deren Missbrauch beschäftigt, wurde im Oktober 1990 im Bundestag verabschiedet und trat zum 01. Januar 1991 in Kraft. ${ }^{81}$ Zur gleichen Zeit wurde mit der Änderung des Sozialgesetzbuches der finanzielle Rahmen für die Etablierung der In-vitro-Fertilisation (IVF) als einer Leistung der Gesetzlichen Krankenversicherung geschaffen.

Mit der Einführung des Embryonenschutzgesetzes erhielt der (ausgebildete und dafür qualifizierte) Arzt die Verantwortung für die Durchführung reproduktionsmedizinischer

\footnotetext{
79 Siehe Kapitel 4.1 „Verwandtschaft durch Adoption“.

80 Teile dieses Kapitels wurden wörtlich oder sinngemäß von mir schon in Hauser-Schäublin et al. (2001) veröffentlicht.

81 Hervorgehoben soll an dieser Stelle werden, dass es sich um die Auffassung zum Zeitpunkt der Verabschiedung des Embryonenschutzgesetzes handelt. Im Zusammenhang mit den rezenten Diskussionen um die embryonale Stammzellforschung werden Stimmen lauter, die eine Überarbeitung und Anpassung des Gesetzes an aktuelle Forschungsentwicklungen fordern. Gesetze oder Regelungen, die Forschung an Embryonen und embryonale Stammzellforschung unter Auflagen erlauben, existieren bereits in Dänemark, Schweden und Finnland, Großbritannien, Italien und Spanien. In Frankreich, Belgien und Portugal werden Gesetze vorbereitet. In Deutschland wurde am 30.01.2002 beschlossen, den Import menschlicher (,überzähliger') Embryonen aus dem Ausland zu Forschungszwecken zuzulassen. Die Herstellung von Embryonen bleibt zunächst jedoch verboten.
} 
Verfahren (Befruchtung, Embryonentransfer, Kryokonservierung) (§ 9 ESchG). Damit trug er nun auch allein die strafrechtlichen Konsequenzen. Im einzelnen erlaubt das Gesetz die Befruchtung außerhalb des Körpers ausschließlich zum Zweck der Fortpflanzung, um das Entstehen überzähliger Embryonen zu verhindern ( 1 ESchG), es verbietet fremdnützige Experimente mit menschlichen Embryonen und deren extrakorporale Weiterentwicklung ( $§ 2$ ESchG), untersagt die Erzeugung von Klonen und Chimären/Hybriden ( $\S \S 6$ und 7 ESchG), gewährleistet das Recht auf Selbstbestimmung über die eigene Fortpflanzung und will Formen der gespaltenen Mutterschaft durch „Eizellspende“ oder „Ersatzmutterschaft“ verhindern (§ 1 ESchG). Auch Schwangerschaften von Frauen im post-menopausalen Alter, was durch „Eizellspende“ prinzipiell möglich wäre, sind aufgrund des Embryonenschutzgesetzes in Deutschland nicht erlaubt ( $\$ 4$ Abs. 1 Nr. 3 ESchG). Ziel des Gesetzes ist es, die Manipulation menschlichen Lebens zu verhindern. Bereits der Versuch, die Erbinformation menschlicher Keimzellen zu verändern bzw. solche zur Befruchtung zu verwenden, steht unter Strafe ( $\$ 5$ ESchG). ${ }^{82}$ Deutschland hat im Vergleich zum europäischen Ausland die restriktivsten Gesetze aufgestellt. Das Verbot jeglicher fremdnütziger Verwendung von Embryonen, die nicht seiner Erhaltung dienen, schließt eine Forschung an Embryonen aus. Ebenfalls ist keine Forschung erlaubt, die eine Präimplantationsdiagnostik ermöglichen oder verbessern könnte, bei der einem Embryo nach den ersten Zellteilungen eine oder mehrere Zellen entnommen und auf chromosomale oder genetische Defekte untersucht werden. ${ }^{83}$ Darüber hinaus verbietet das Embryonenschutzgesetz die Geschlechtswahl, um zu verhindern, dass im Rahmen der „künstlichen“ Befruchtung über das Geschlecht des künftigen Kindes vorab entschieden wird ( $\$ 3 \mathrm{ESchG)}$.

Nach den Motiven des Embryonenschutzgesetzes beschränkt sich der Schutz des Embryos auf seine Existenz in vitro. Nachdem der Embryo in die Gebärmutter der Frau transferiert worden ist und sich dort eingenistet hat, greift das Strafgesetzbuch $(\S \S 218$ ff StGB) ein, welches den Embryo nur vor der vorsätzlichen Tötung im Mutterleib schützt. Allerdings weist auch der Schutz in vitro einen fragmentarischen Charakter auf, da mit der Gewährleistung des Embryonenschutzes durch das Strafrecht familienrechtliche und medizinrechtliche Auswirkungen nicht geregelt wurden. Das Embryonen-

\footnotetext{
82 Das Embryonenschutzgesetz definiert den Embryo in $\S 8$ als die befruchtete, entwicklungsfähige menschliche Eizelle vom Zeitpunkt der Kernverschmelzung an, sowie auch jede einem Embryo entnommene totipotente (= in ihrer Entwicklung noch nicht festgelegte) Zelle, die sich unter gegebenen Voraussetzungen zu einem Individuum entwickeln kann. In den ersten 24 Stunden nach der Kernverschmelzung gilt die befruchtete menschliche Eizelle als entwicklungsfähig, ausgenommen diejenigen Eizellen, bei denen schon vor Ablauf der ersten 24 Stunden festgestellt wird, dass sie sich nicht über das Eizellstadium hinaus entwickeln. Als Keimbahnzellen werden alle Zellen definiert, die in einer Zell-Linie von der befruchteten Eizelle bis zu den Ei- und Samenzellen des aus ihr hervorgegangenen Menschen führen und alle Eizellen vom Eindringen der Samenzelle an bis zu der mit der Kernverschmelzung abgeschlossenen Befruchtung.

83 In Großbritannien sind beispielsweise Forschungen an Embryonen unter einem Genehmigungsverfahren bis zum 14. Tag möglich, so dass auch die Präimplantationsdiagnostik praktiziert werden kann (Wiesenbart 1990).
} 
schutzgesetz beschränkt sich somit darauf, mit strafrechtlichen Regelungen möglichen Missbräuchen neuer Fortpflanzungstechniken zu begegnen. Kontrovers diskutierte Problemfelder, über die in dem Zeitrahmen der 11. Wahlperiode, in der das Gesetz verabschiedet wurde, keine abschließende Regelung gefunden werden konnte, klammert das Embryonenschutzgesetz dagegen aus. So schweigt es zur vielfachen Forderung, die „künstliche“ Befruchtung nur bei Ehepaaren anzuwenden. Damit bleibt auch nach Inkrafttreten des Embryonenschutzgesetzes die Entscheidung dem behandelnden Arzt überlassen, ob auch andere Personen (insbesondere nicht-eheliche Lebensgemeinschaften) behandelt werden dürfen. ${ }^{84}$ Das Embryonenschutzgesetz verfolgt als ein zentrales Ziel, die gespaltene Mutterschaft schon im Vorfeld zu verhindern („Leihmutterschaften“ werden per Gesetz unter Strafe gestellt), schweigt aber über die gespaltene Vaterschaft, d.h. eine Befruchtung mit „Spendersperma“, und schließt diese somit nicht aus. Es werden nur einige Einschränkungen für die Verwendung von „Spendersamen“ vorgenommen: Die Auswahl nach Geschlechtschromosomen, somit auch bei einer „Samenspende", und jede postmortale künstliche Befruchtung sind verboten. Außerdem wird die Zustimmung des „Samenspenders“ vorausgesetzt, und die heterologe Insemination sowie die In-vitro-Fertilisation dürfen nur von Ärzten ausgeführt werden, die nach der ärztlichen Berufsordnung der Ärztekammer nachweisen müssen, dass die berufsrechtlichen Anforderungen erfüllt sind (vgl. Petersen 2000:66-70). Weitere Folgeprobleme, die sich aus der Verwendung von „Spendersperma“ ergeben, wie z.B. die Gewährleistung der Anonymität des ,Spenders“ oder stattdessen das Recht des Kindes auf Kenntnis seiner genetischen Abstammung sowie Kriterien für die Aufbewahrung, Dokumentation und Kommerzialisierung von „Spendersamen“ (z.B. Regelungen über Samenbanken) finden jedoch im Embryonenschutzgesetz keine Erwähnung. ${ }^{85}$

\section{Das Gesundheitsreformgesetz (GRG) und das Sozialgesetzbuch (SGB)}

Schon 1967 wurde im Rahmen eines Bundessozialgerichtsurteils Kinderlosigkeit juristisch als Krankheit definiert, doch erst 1990 erfolgte ein gesetzliche Entscheidung. Anfang 1987 trat ein Einheitlicher Bewertungsmaßstab in Kraft (EBM), der durch den Bundesausschuss der Ärzte und Krankenkassen abgestimmt worden war. Damit waren Maßnahmen zur „künstlichen“ Befruchtung als eigenständige Gebührenordnungspositionen abrechnungsfähig. Mit der Einführung des Gesundheitsreformgesetzes (GRG)

\footnotetext{
84 Die Berufsordnung für Ärzte, die bereits auf dem 88. Ärztetag 1985 verabschiedet wurde, legt allerdings für alle Mediziner verbindlich fest, die Durchführung der In-vitro-Fertilisation (IVF) sowie den Gameten- und Embryotransfer grundsätzlich auf Ehepaare zu beschränken. Ausnahmen werden nur nach Zustimmung einer Ethikkommission möglich, die von einer Ärztekammer eingerichtet sein muss (siehe Richtlinien zur Durchführung der assistierten Reproduktion 1998:A-3168).

$85 \mathrm{Zu}$ diesem Punkt sind die Gesetzgebungen der Länder bei der Regelung der Reproduktionstechnologien nicht einheitlich (vgl. Franklin 1993a).
} 
Anfang 1989 wurden sie jedoch wieder aus dem Leistungskatalog der Krankenkassen entfernt und bis Mitte 1990 nicht anerkannt. ${ }^{86}$ Mit der Einführung des $\S 27$ a in das 5. Buch des Sozialgesetzbuches (SGB V), das am 01.07.1990 in Kraft trat, wurden die Methoden der „künstlichen“ Befruchtung wieder als Kassenleistung anerkannt. Sie können nach ärztlicher Feststellung dann durchgeführt werden, wenn hinreichende Aussicht auf das Herbeiführen einer Schwangerschaft besteht, die Maßnahmen nicht mehr als viermal ohne Erfolg durchgeführt worden sind, wenn es sich um Ehepaare handelt und ausschließlich deren Keimzellen verwendet werden. Voraussetzung für die Durchführung einer Behandlung ist für die Ehepaare eine Beratung von einem Arzt, der die Behandlung nicht selbst durchführt. ${ }^{87}$ Man könnte also annehmen, dass Paare, die sich einer reproduktionsmedizinischen Behandlung unterziehen bzw. unterzogen haben, alle zu Behandlungsbeginn ,ungewollt kinderlos“ sind, d.h. keine Kinder haben bzw. ohne Kinder leben. Entgegen dieser Vermutung lassen sich jedoch die Paare in zwei Gruppen untergliedern: Zum einen die ,primär Kinderlosen“, wozu ich die Personen zähle, die noch nie ein Kind gezeugt oder geboren haben, zum anderen die „sekundär Kinderlosen“, die bereits ein Kind oder mehrere Kinder durch Geschlechtsverkehr bekommen haben und bei denen sich die Einlösung eines weiteren „Kinderwunsches“ nicht realisieren lässt (entweder mit demselben oder einem neuen Partner). Es lässt sich demnach festhalten, dass die Kategorie der ,ungewollt kinderlosen“ Personen, deren „Sterilität“, und als Folge der Zustand der „ungewollten Kinderlosigkeit“, als Leiden und damit als behandlungswürdig anerkannt wird, sehr heterogen ist. ${ }^{88}$ Auch nach der Geburt eines Kindes, das durch medizinisch-technische Maßnahmen entstanden ist, können Paare weiterhin als „steril“ diagnostiziert und damit als „ungewollt kinderlos“ klassifiziert werden, und die Kosten für die reproduktionsmedizinische Behandlungen für ein zweites Kind von den Krankenkassen übernommen werden (s.o.). Diesen Aspekt möchte ich hier erwähnen zur Hervorhebung der Tatsache, dass es sich bei den Reproduktionstechnologien nicht um Heilmethoden handelt, die die Ursachen der ,Krankheit ${ }^{89}$ beheben,

\footnotetext{
86 Während Private Krankenkassen weiterhin diese Kosten übernahmen, mussten Versicherte der Gesetzlichen Krankenversicherung die Behandlungskosten privat zahlen.

87 Als weitere Änderung des Sozialgesetzbuches wurde der § 121a ergänzt (Genehmigung zur Durchführung „künstlicher“ Befruchtung). Die Genehmigung kann die zuständige Landesbehörde an Vertragsärzte, ermächtigte Ärzte, ermächtigte ärztlich geleitete Einrichtungen oder zugelassene Krankenhäuser erteilen. Voraussetzung dafür ist, dass sie die notwenigen fachlichen und personellen Bedingungen erfüllen.

88 Die öffentliche Repräsentation von „Sterilität“ verknüpft mit Gefühlen von Verzweiflung und Hoffnungslosigkeit ist jedoch ein Merkmal, das alle „ungewollt kinderlosen“ Paare vereint. Dieses Stigma, das der „Sterilität“ anhaftet, beschreibt Pfeffer als direkte Folge ihrer Repräsentation: „Besides their involuntary childlessness there is one characteristic which the infertile are said to share, that of desperation. The word desperation or some such synonym appears so frequently in conjunction with infertility that sometimes it appears that what troubles infertile men and women is not the absence of the child as such but some form of emotional disorder related to their failure" (1987:82). Auf diesen Aspekt gehe ich im weiteren Verlauf dieser Arbeit noch näher ein.

89 Außer gegebenenfalls beim Öffnen eines Eileiterverschlusses, der Entfernung von Myomen, Zysten oder Endometrioseherden, die eine „,natürliche“ Empfängnis erschweren oder verhindern, wie auch eine ,erfolgreiche“ Refertilisierung (siehe dazu Kap. 4.3.1.2).
} 
sondern um technologische Verfahren, die zur Überbrückung eines sozialen Leidenszustandes an der „ungewollten Kinderlosigkeit“ verhelfen können. Dies wird besonders deutlich an der Tatsache, dass sich zahlreiche Paare auch für ein zweites Kind wieder in die „Kinderwunsch-Behandlung“ begeben, d.h. trotz der Geburt eines Kindes sind sie nicht weniger ,kinderwunschkrank'.

\section{Richtlinien zur Durchführung der ,assistierten“ Befruchtung und die Kassenleistungen}

Nach der Einführung der In-vitro-Fertilisation (IVF) Anfang der 1980er Jahre in Deutschland hat die Bundesärztekammer „Richtlinien zur Durchführung von In-vitroFertilisation (IVF) und Embryotransfer (ET) als „Behandlungsmethode der menschlichen Sterilität" erarbeitet. Diese Richtlinien sind durch Beschluss des 88. Deutschen Ärztetages 1985 Bestandteil der Berufsordnung geworden. Sie wurden nach Inkrafttreten des Embryonenschutzgesetzes 1991 novelliert und vom Deutschen Ärztetag als „Richtlinien zur Durchführung des intratubaren Gametentransfers, der In-vitroFertilisation mit Embryotransfer und anderer verwandter Methoden" verabschiedet. Aufgrund von Modifikationen der Methoden und der Entwicklung neuer diagnostischer und therapeutischer Verfahren erfolgte 1998 eine zweite Novellierung dieser Richtlinien (,Richtlinien zur Durchführung der assistierten Reproduktion“). Es werden dort Leistungsvoraussetzungen, Methoden, medizinische Indikationen, der Umfang der Maßnahmen, aber auch die Beratung des Ehepaares formuliert, sowie eine Auflistung der Qualifikationsanforderungen und Genehmigungsvorbehalte vorgelegt, die für die Methoden Voraussetzung sind. Außerdem werden die einzelnen Arbeitsgruppen ${ }^{90}$ explizit aufgefordert, zum Zwecke der Verfahrens- und Qualitätssicherung eine EDV-gestützte Dokumentation entsprechend dem Fragenkatalog des Deutschen IVF-Registers (DIR) zu erstellen. Die Ärztekammer beauftragt dieses Dokumentationszentrum, jährlich einen Bericht über die Arbeit der zugelassenen IVF-Zentren zu erstellen und zu veröffentlichen. $^{91}$

\footnotetext{
90 Von einer Arbeitsgruppe spricht man, da bestimmte fachliche und personelle Voraussetzungen als Zulassungsbedingungen für die Durchführung der In-vitro-Fertilisation mit Embryotransfer erfüllt sein müssen. Qualifiziertes Personal aus sechs Teilbereichen gehören zu einer Arbeitsgruppe: 1. Endokrinologie der Reproduktion, 2. Gynäkologische Sonographie, 3. Operative Gynäkologie, 4. Experimentelle oder angewandte Reproduktionsbiologie mit dem Schwerpunkt der In-vitro-Kultur, 5. Andrologie, 6. Psychosomatische und psychotherapeutische Versorgung.

91 Seit dem Jahr 1982 gibt es in der Bundesrepublik Deutschland eine freiwillige Registrierung von IVF-Zentren. In den Jahren 1986/87 kam es zur Gründung eines jährlich stattfindenden Treffens der deutschen IVF-Gruppen. Mit zunehmenden berufspolitischen und vor allem abrechnungstechnisch schwieriger werdenden Problemen verlangten zahlreiche IVF-Gruppen eine Vertretung, die sich besonders im Bundesinnenministerium für Arbeit und Soziales Gehör verschaffen konnte. Es folgte die Gründung einer Arbeitsgemeinschaft Endokrinologie und Fortpflanzungsmedizin (AGGEF) der Deutschen Gesellschaft für Gynäkologie und Geburtshilfe. Im Mai 1996 wurde eine weitere Interessenvereinigung gegründet, der Bundesverband Reproduktionsmedizinischer Zentren e.V., welcher besonders
} 
Die Richtlinien äußern sich im Gegensatz zum Embryonenschutzgesetz konkreter zu der Verwendung von „Spendersperma“. So kann es kann dann verwendet werden, wenn eine andere Methode der ,assistierten“ Reproduktion im homologen System wegen „Unfruchtbarkeit“ des Mannes nicht möglich ist. Die Verwendung von einem SpermaGemisch verschiedener „Spender“ muss dabei jedoch ausgeschlossen sein, da das allgemeine deutsche Persönlichkeitsrecht das Recht auf Kenntnis der genetischen Abstammung umfasst (vgl. Kap. 4.1). Aus diesem Grund kann der Arzt dem „Samenspender" keine Anonymität zusichern, sondern muss ihn darauf hinweisen, dass er dem Kind gegenüber zur Nennung des „Spendernamens“ verpflichtet ist und sich nicht auf die ärztliche Schweigepflicht berufen kann. Ebenso sollen das Paar und der „Samenspender" darüber aufgeklärt werden, dass die Vaterschaft sowohl vom sozialen Vater als auch von dem Kind selbst angefochten werden kann (Richtlinien zur Durchführung der assistierten Reproduktion 1998:3170f).

Die Kassenleistung ist an den ehelichen Status gebunden und nur die Keimzellen der Ehepartner dürfen verwendet werden. Kein Anspruch auf eine Behandlung besteht in der Regel nach einer Sterilisation, Ausnahmen müssen genehmigt werden. Die Kosten der Behandlungen werden bis zu einem Alter der Frau von 40 Jahren übernommen. Ausnahmen sind nur bei Frauen zulässig, die das 45. Lebensjahr noch nicht überschritten haben, und sofern die Krankenkasse nach gutachterlicher Beurteilung der ,Erfolgsaussichten' eine Genehmigung erteilt hat. ${ }^{92}$ Die Gesetzlichen Krankenkassen zahlten von Mitte 1990 bis Anfang 1999 bei entsprechender medizinischer Indikation bis zu sechs (homologe) Inseminationen, vier IVF-Zyklen oder zwei GIFT-Versuche (intratubarer Gametentransfer). ${ }^{93}$ Seit ca. 1992 hat sich die Behandlung männlicher „Sterilität“ mit Hilfe der Methode der Intrazytoplasmatischen Spermieninjektion (ICSI) durchgesetzt, wodurch die Behandlung mit GIFT rapide abgenommen hat. Die Kassen übernahmen bis zu vier IVF/ICSI-Versuche. In einer Stellungnahme der kassenärztlichen Bundesvereinigung und der Spitzenverbände der Krankenkassen vom 26. November 1998 wurde jedoch festgelegt, dass die Kostenübernahme für ICSI auszuschließen sei. Begründet wurde diese Entscheidung mit ,dem Fehlen fundierter Aussagen, die eine Beurteilung von etwaigen mit der Methode der Intrazytoplasmatischen Spermieninjektion verbundenen Risiken wie erhöhte Fehlbildungsraten und vermehrte Chromosomenanomalien erlauben würden“ (Reproduktionsmedizin 1999:84f). ${ }^{94}$ Die gesamte Kryo-

die berufspolitischen Interessen der in freier Praxis oder privater Klinik tätigen IVF-Spezialisten vertritt. An der Datenerfassung des IVF-Registers nahmen für das Berichtsjahr 1995 insgesamt 65 Gruppen teil (Beier 1997:30).

92 Eine Altersbegrenzung des Mannes besteht nicht.

93 Eine genaue Beschreibung der einzelnen Techniken erfolgt in Kapitel 4.3.1.2.

94 Gegen dieses Urteil wurde sowohl von Seiten der Reproduktionsmediziner als auch von Seiten betroffener Paare scharf protestiert. Seit Herbst 2001 werden die entstehenden Kosten der Behandlungen aufgrund eines Urteils des 
konservierung (das Tiefgefrieren beispielsweise vom Sperma des Ehemannes, von befruchteten Eizellen und Hodengewebe) ebenso wie die finanziellen Aufwendungen für die heterologe Insemination gehören nicht zur Leistungspflicht der Gesetzlichen Krankenkassen. Auch die anfallenden Kosten bei den mikrochirurgischen Eingriffen zur Gewinnung von Samenzellen aus dem Hoden oder Nebenhoden (MESA/TESE) unter Vollnarkose wird nicht übernommen mit der Begründung, dass es sich nicht um kurative (heilende) Maßnahmen handelt.

\subsubsection{Die Behandlungsmethoden männlicher und weiblicher „Sterilität“6}

Im Rahmen des Embryonenschutzgesetzes haben sich verschiedene Behandlungsmethoden männlicher und weiblicher „Sterilität" in der medizinischen Praxis etabliert, deren Kosten wie oben gezeigt mit wenigen Ausnahmen von den Gesetzlichen wie den Privaten Krankenversicherungen bei entsprechender medizinischer Indikation und Aussicht auf das Herbeiführen einer Schwangerschaft übernommen werden. Die medizinische Definition von „Unfruchtbarkeit“ bezieht sich auf die Situation, dass nach ein bis zwei Jahren mit regelmäßigem ungeschützten Geschlechtsverkehr keine Schwangerschaft eingetreten ist. ${ }^{95}$ Dabei fällt auf, dass von einer klaren Unterscheidung zwischen „Fruchtbarkeit“" und „Unfruchtbarkeit“ ausgegangen wird. Die spezifischen Umstände des Paares werden hier nicht berücksichtigt, die jedoch wesentlich zu einer scheinbaren „Unfruchtbarkeit“" beitragen können. So ist es beispielsweise möglich, dass das Paar eine Wochenendbeziehung lebt und entsprechend zeitlich determinierte sexuelle Beziehungen hat, der Eisprung der Frau aber in der Woche stattfindet, oder dass generell wenige sexuelle Kontakte stattfinden bzw. diese selten im Koitus enden. Eine Folge der engen statistischen Definition von „Unfruchtbarkeit“ sind die immer wieder in den Medien erwähnten Prozentzahlen von 10 bis $20 \%$,ungewollt kinderloser“ (Ehe)paare in Deutschland, die jedoch keine Angaben darüber machen, ob es sich um den Prozentsatz aller Kinderlosen in der deutschen Bevölkerung handelt, um den Anteil aller Kinderlosen der „fortpflanzungsfähigen Altersgruppen“ oder um diejenigen Kinderlosen, die eine ärztliche Behandlung gesucht haben. ${ }^{96}$

In Deutschland werden reproduktionsmedizinische Technologien in Kliniken oder Praxen durchgeführt, die sich ausschließlich auf die Fortpflanzungsmedizin spezialisiert haben (privat- und kassenärztliche Einrichtungen), sowie in spezifischen Abteilungen

Bundessozialgerichts (nach der Klage eines Paares) in der Regel wieder weitgehend übernommen. Aufwendungen für eine Behandlung sind im übrigen nach $\S 33$ EstG als außergewöhnliche Belastung steuerlich abzugsfähig.

95 In meiner Feldforschung habe ich es jedoch erlebt, dass Paare schon nach einem halben bis einem Jahr in die „Kinderwunsch-Sprechstunde“ der Kliniken kamen.

96 Wobei sich hier allerdings auch die gesellschaftliche Idealvorstellung, dass zu einem verheirateten Paar Kinder gehören, widerspiegelt. 
größerer Kliniken (beispielsweise Universitätskliniken oder auch staatliche Frauenkliniken). Diese Zentren sind frei zugängig, d.h. nach Voranmeldung kann ein Termin zu einem Erstgespräch mit einem Arzt vereinbart werden. Am Anfang jeglicher Diagnose und auch Therapie steht die Selbstdefinition der Frau/des Paares als „ungewollt kinderlos“. Mit dieser Vorab-Definition wird ein Gynäkologe oder direkt ein reproduktionsmedizinisches Zentrum aufgesucht. Bei jedem Erstgespräch (auch „KinderwunschSprechstunden“ genannt) zwischen dem Arzt und dem „ungewollt kinderlosen“ Paar erfolgt eine ausführliche Erhebung der Vorgeschichte (Anamnese). Diese wird zumeist in Form eines Fragebogens ermittelt. Gefragt wird unter anderem nach Alter, Größe, Gewicht, Operationen im Bauch und Unterleib, Krankheiten, früheren Erkrankungen, Zyklusverlauf, Regelblutung, bisherige Form der Verhütung, vorausgegangenen Schwangerschaften und deren Verlauf, Fehlgeburten, Entzündungen der Eierstöcke, der Scheide oder der Hoden/Nebenhoden, nach der Dauer des „Kinderwunsches sowie Art und Dauer der bisher durchgeführten Diagnostik und gegebenenfalls der Therapien. Auch die Sexualität des Paares, zumeist die Häufigkeit der sexuellen Kontakte, spielt eine wichtige Rolle (Feige et al. 1997:104ff).

Der Mann wird einer urologischen Untersuchung unterzogen, um den andrologischen „Sterilitätsfaktor“ zu bestimmen. Diese sieht neben einer Hormonanalyse eine körperliche Untersuchung der äußeren Genitalien vor sowie die Erstellung eines Spermiogramms, um die Spermien auf Qualität, Quantität und Beweglichkeit zu prüfen. Bei der körperlichen Untersuchung der Frau werden zunächst die äußeren und die inneren Genitalien kontrolliert. Es folgen weitere Untersuchungen zur Abklärung der weiblichen „Sterilität“ wie die Hormonanalyse ${ }^{97}$ anhand abgenommenen Blutes, die Basaltemperaturmessung ${ }^{98}$ und/oder der Postcoital-Test ${ }^{99}$. Sie beziehen sich vor allem auf Störungen der Abläufe im Eierstock (Schirren et al. 1989:73ff). Die Durchgängigkeit der Eileiter sowie Verwachsungen oder gutartige Tumore der Gebärmuttermuskulatur (Myome) können mit Hilfe einer röntgenologischen Untersuchung der Eileiter und der Gebärmutter (Hysterosalpingographie) überprüft werden. Dabei wird die Gebärmutter mit Kontrastmittel gefüllt und dann mit Hilfe von Röntgenaufnahmen festgestellt, ob das Kontrastmittel über die Eileiter in den Bauchraum abfließen kann. Eine weitere Möglichkeit ist die Bauchspiegelung (Laparoskopie) als gleichzeitig diagnostische und the-

\footnotetext{
97 So kann zum Beispiel ein erhöhter Prolaktin-Wert unterschiedliche Zyklusstörungen bewirken (Prolaktin wird auch als Still- oder Stresshormon bezeichnet).

98 Störungen des Zyklus und der Eierstockfunktion können mit Hilfe der Basaltemperaturmessung festgestellt werden.

99 Beim Postcoital-Test wird die Frau nach verordnetem Geschlechtsverkehr zur Untersuchung einbestellt, um das Vorhandensein von lebensfähigen Spermien im oberen weiblichen Geschlechtstrakt nachzuweisen.
} 
rapeutische Maßnahme, bei welcher der Bauchraum betrachtet und kleinere Eingriffe vorgenommen werden können. Hierfür ist eine Vollnarkose notwendig. ${ }^{100}$

Im wesentlichen sind zwei Behandlungsmöglichkeiten der männlichen „Sterilität“ zu nennen: die medikamentös-hormonelle und operative Behandlung. Gaben des männlichen Geschlechtshormons können die Spermienreifung anregen und zu einer Erhöhung der Dichte normal geformter und beweglicher Spermien führen (Winkler 1994:105). Durch chirurgische Eingriffe werden z.B. Krampfadern am Hoden (Varikozelen) entfernt, welche sich negativ auf die Spermienreifung auswirken. Des weiteren können Verschlüsse der Samenleiter operativ behoben werden. Ebenfalls operativ kann eine Refertilisation erfolgen. Hier wird nach einer Sterilisation die Durchlässigkeit der Samenleiter wieder hergestellt (Schirren et al. 1989:169-186). Mit Hilfe des operativen Eingriffs der mikrochirurgischen Spermatozoen-Aspiration aus dem Nebenhoden (MESA) können Spermienvorstufen direkt aus den Nebenhoden gewonnen werden. Während einer Vollnarkose wird der Nebenhoden punktiert und gleichzeitig werden die Punktate auf das Vorhandensein von Samenzellen untersucht. Bei einem positiven Nachweis wird der Eingriff beendet, um in der Zukunft gegebenenfalls neu punktieren zu können. Eine weitere Möglichkeit, Samenzellen mikrochirurgisch zu gewinnen, bietet die testikuläre Spermienextraktion (TESE). Auch dieser Eingriff erfolgt unter Vollnarkose, wobei aus Teilen des Hodengewebes Samenzellen herausgelöst werden.

Die Behandlung der weiblichen „Sterilität“ kann mit Hilfe von Hormontherapien erfolgen, die sowohl eigenständige Behandlungen oder auch Teil der komplexeren medizinischen Therapien sein können. Es werden Hormonpräparate in Form von Tabletten, Spritzen oder einer Pumpe verabreicht, um die Eierstöcke zur Follikelreifung zu stimulieren, Eisprünge auszulösen und die Gebärmutterschleimhaut auf eine Schwangerschaft vorzubereiten. Diese Behandlung wird regelmäßig fachärztlich kontrolliert. Verschlüsse der Eileiter und Verwachsungen können mittels chirurgischer Eingriffe behandelt werden. Dabei wird versucht, die Eileiter wieder durchgängig zu machen und Verwachsungen zu lösen. Teilweise geschieht dies durch eine Bauchspiegelung, teilweise durch mikrochirurgische Operationen. Ebenfalls können Endometrioseherde ${ }^{101}$, Zysten sowie Myome operativ entfernt werden.

\footnotetext{
100 Ich möchte hier explizit darauf hinweisen, wie umfangreich die Untersuchungen am Körper der Frau sind im Vergleich zu denen, die am Körper des Mannes vorgenommen werden zur Abklärung der Ursachen der ,ungewollten Kinderlosigkeit“. Diese Unterschiede werden auch deutlich an den im weiteren Verlauf beschriebenen Behandlungsmethoden, die primär den weiblichen Körper als Behandlungsobjekt in den Mittelpunkt stellen, auch bei der Diagnose ,(eingeschränkte) männliche Zeugungsunfähigkeit“".

101 Endometriose ist eine Erkrankung, bei der sich Teile der Gebärmutterschleimhaut außerhalb der Gebärmutter angesiedelt haben.
} 
Im Folgenden möchte ich nun die medizinischen Therapien darstellen, mit deren Hilfe versucht wird, die weibliche und/oder männliche „Sterilität“ zu überbrücken, um eine Befruchtung und anschließende Schwangerschaft zu erreichen. ${ }^{102}$ Es gibt zwei Varianten der Insemination.

Eine homologe Insemination mit aufbereitetem Sperma wird durchgeführt, wenn beim Ehemann oder Partner Störungen bezüglich Menge, Form und Beweglichkeit der Samenfäden vorliegen, bei einem vorzeitigen Samenerguss, Erektionsstörungen oder immunologischer ${ }^{103}$ „Sterilität“ auf Seiten des Mannes oder der Frau. Die Spermien gelangen dabei direkt in den Gebärmutterhals oder die Gebärmutter (intrazervikal oder intrauterin), was ihre Überlebenschancen steigern kann. Das durch Masturbation gewonnene Sperma des Ehemannes oder Partners wird mit einem Kulturmedium versetzt und zentrifugiert. Danach wird es mittels eines dünnen, weichen Katheters direkt in die Gebärmutter oder den Gebärmutterhals gebracht. Der Zyklus wird gleichzeitig beobachtet, und gegebenenfalls erfolgt eine Stimulation und die Auslösung des Eisprungs.

Unter heterologer Insemination versteht man die Verwendung von „Spendersperma“. Anstelle von Sperma des Ehemannes wird auf kryokonserviertes Sperma eines Spenders zurückgegriffen, das vor der Insemination aufgetaut wird. Mit Hilfe der Kryokonservierung kann das Sperma über Jahre hinweg aufbewahrt werden. Die heterologe Insemination wird zumeist bei „Zeugungsunfähigkeit“ oder bei der Weitergabe von schweren genetischen Erkrankungen des Mannes angewandt. Zur Steigerung der ,Erfolgsaussichten' wird die Frau bei einer Befruchtung mit „Spendersperma“ teilweise ebenfalls hormonell stimuliert.

Unter In-vitro-Fertilisation (IVF) mit Embryotransfer (ET) versteht man die außerkörperliche Befruchtung nach hormoneller Vorbehandlung und Zyklusüberwachung der Frau. Die Indikationen für eine In-vitro-Fertilisation sind überwiegend Eileiterverschluss oder fehlende Eileiter (Tubenfaktor). Zunächst werden die Eierstöcke mit Hilfe hoch dosierter Hormongaben über ein bis zwei Wochen ruhig gestellt (DownRegulation). Anschließend erfolgt eine Stimulierung der Eierstöcke durch tägliche Hormongaben (Tabletten, Spritzen) mit gleichzeitiger Zyklusüberwachung durch Ultra-

\footnotetext{
102 Angaben zu ,Erfolgsquoten “ der einzelnen Methoden werde ich hier nicht machen, da diese in der Regel aufgrund der Aussagen der jeweiligen reproduktionsmedizinischen Zentren erstellt werden. Dabei zählt aus medizinischer Perspektive als ,Erfolg' entweder die erreichte Befruchtung von Eizellen, die Anzahl der Frauen, an denen ,erfolgreich' ein Embryonentransfer vorgenommen wurde oder erzielte Schwangerschaften in einem sehr frühen Stadium. Weder sagen diese Quoten etwas über die Anzahl der geborenen Kinder aus (die baby take home-Rate), noch beziehen sie die aus medizinischen Gründen abgebrochenen Behandlungsversuche (die ,Misserfolge') mit ein (z. B. eine zu geringe oder zu hohe Stimulation, die nicht zur Punktion führt, oder die nicht erfolgte Befruchtung der Eizellen im Reagenzglas).

103 Bestimmte Abwehrmechanismen im Körper können sogenannte Antikörper bilden, die sich an die Samenzellen anlagern und diese in der Beweglichkeit hindern, wodurch eine Befruchtung erschwert bzw. unmöglich wird. Diese Antikörper können sowohl von der Frau als auch vom Mann selbst gebildet werden.
} 
schalluntersuchungen und Hormonbestimmungen in Blut und Urin. Durch die hormonelle Stimulierung kommt es zu einer Reifung mehrerer Follikel. ${ }^{104}$ Der Eisprung wird wiederum mit einer Hormonspritze ausgelöst. Kurz bevor der Eisprung stattfindet, wird eine vaginale, ultrasonografisch gesteuerte Follikelpunktion durchgeführt. Um die Eizellen zu gewinnen, wird ein stabförmiger Ultraschallkopf mit einer Punktionsnadel in der Führungshülse durch die Scheidenwand zum Eierstock eingeführt. Dort werden die Follikel angestochen und die Flüssigkeit mitsamt der Eizelle abgesaugt (Beier 1997:22ff). Die Punktionen werden ohne Narkose, mit Teil- oder Vollnarkose durchgeführt. $^{105}$

Nach der Beurteilung der Follikelflüssigkeit unter dem Mikroskop werden die reifen Eizellen im Inkubator (Brutkasten) gelagert. Das Sperma des Partners wird auf Anzahl und Beweglichkeit überprüft. Dieses im Labor aufbereitete Sperma wird im Inkubator in einer Petrischale mit den Eizellen zusammengebracht. Nach ca. 48 Stunden haben sich die Eizellen - wenn es zur Befruchtung und zur Zellteilung gekommen ist - bis zum Vier- oder Achtzellstadium geteilt und werden in die Gebärmutter transferiert. Die ,überzähligen` befruchteten Eizellen im Vorkernstadium können auf Wunsch des Ehepaares sofort kryokonserviert werden. ${ }^{106}$ Sie werden für einen späteren Transfer aufgetaut, um dann unmittelbar in den Uterus der Frau transferiert zu werden. Für diese Zyklen, die sogenannten Kryozyklen, ist jedoch zusätzlich eine hormonelle Behandlung der Frau einschließlich einer ultrasonografischen Überwachung üblich. Der Embryotransfer (ET) erfolgt in der Regel ohne Narkose durch die Scheide und den Gebärmuttermund, indem ein dünner Plastikkatheter in die Gebärmutter eingeführt wird. In den meisten Fällen ruhen die Frauen zwischen einer halben und einer Stunde, seltener verbleiben sie 24 Stunden in der Klinik. Zur Förderung der Einnistung erfolgt eine hormonelle Unterstützung, die das „Schwangerschaftsmilieu“ der Gebärmutterschleimhaut begünstigen soll. In den folgenden ca. 14 Tagen muss abgewartet werden, ob sich ein oder mehrere Embryonen einnisten und eine Schwangerschaft entsteht.

\footnotetext{
104 Durch hormonelle Behandlungen der Eierstöcke werden sogenannte Superovulationen ausgelöst. Im Vergleich zum Spontanzyklus sind damit angeblich organisatorische Vorteile verbunden, da sich die Zyklusüberwachung vereinfachen und das Auslösen des Eisprungs somit besser determinieren lässt.

105 Zur Organisation der Rahmenbedingungen einer Follikelpunktion und die dabei verwendete unterschiedliche Medikation der einzelnen IVF-Zentren in Deutschland siehe auch Barbian/Berg (1997:64-69).

106 Da die Befruchtung von Eizellen in vitro zum Zweck des Einfrierens in Deutschland nicht erlaubt ist (was im Ausland allerdings zum reproduktionsmedizinischen Alltag gehört), wird das Pronukleus-Stadium, das sogenannte Vorkernstadium, kryokonserviert. Dieser Vorkern gilt noch nicht als Embryo. Es bilden sich aus den Chromosomen (genetisches Material) der Eizelle und der Samenzelle zwei Zellkerne in einer Eizelle. Erst wenn diese beiden Vorkerne miteinander verschmelzen, ist eine vollständige menschliche Körperzelle entstanden (Zygote). Das Pronukleusstadium stellt demnach ein Zwischenstadium dar. Die Eizelle ist bereits befruchtet, aber männliches und weibliches Erbmaterial haben sich noch nicht verbunden. Kryokonservierte Vorkerne werden in flüssigem Stickstoff eingefroren und bei $-196{ }^{\circ} \mathrm{C}$ eingelagert.
} 
Intratubarer Gametentransfer (GIFT): Die Befruchtung erfolgt in vivo, im Körper. Sie verläuft bis zur Eizellentnahme mit der In-vitro-Fertilisation identisch. Dabei werden die gewonnenen reifen Eizellen mit dem aufbereiteten Sperma in den Eileiter eingespült. Für die Methode des intratubaren Gametentransfers muss mindestens ein Eileiter durchgängig sein; sie wird u.a. bei ungeklärter „Sterilität“ beider Partner oder geringer Spermienzahl angewendet. ${ }^{107}$

Intrazytoplasmatische Spermieninjektion (ICSI) ${ }^{108}$ mit Embryotransfer (ET): Diese Therapieform wurde im deutschen IVF-Register erstmals für die Jahre 1994 und 1995 vermerkt. Sie wird angewendet bei der Diagnose „männliche Zeugungsunfähigkeit“", d.h. aufgrund der Spermaquantität, -qualität oder -beweglichkeit ist eine Befruchtung der Eizelle nicht möglich. Das Vorgehen bei der Mikroinjektion ist einschließlich der Punktion mit der Methode der In-vitro-Fertilisation (IVF) identisch. ${ }^{109}$ Danach wird jedoch jeweils ein Spermium mit einer Mikropipette aufgenommen und durch die äußere Eihülle direkt in die Eizelle injiziert. Nach einer erfolgten Befruchtung und Teilung in ein Vier- oder Achtzellstadium erfolgt der Embryotransfer wie oben bei der In-vitro-Fertilisation beschrieben.

Bei den beschriebenen operativen Eingriffen bestehen die gleichen Risiken wie bei anderen chirurgischen Eingriffen auch, d.h. es kann zu Verletzungen innerer Organe der Frau kommen, zu unkontrollierten Blutungen ebenso wie zu Infektionen. Des weiteren besteht ein Anästhesierisiko bei Vollnarkose im Zusammenhang mit Laparoskopien oder Follikelpunktionen. Insbesondere bei den Therapien, die mit einer Stimulationstherapie verbunden sind, gibt es immer das Risiko von Mehrlingsschwangerschaften. Durch die Hormonbehandlungen, mit denen im Zusammenhang mit einer IVFBehandlung sogenannte Superovulationen ausgelöst werden, kann es zu einer Eierstocküberreaktion mit Ausbildung großer Eierstockzysten (Überstimulation) kommen. Über die Nebenwirkungen der Hormonpräparate besteht immer noch Uneinigkeit. Seitens der Ärzteschaft überwiegt die Ansicht, dass fruchtbarkeitssteigernde Medikamente keine kurz- oder langfristigen Konsequenzen nach sich ziehen, Kritiker sprechen jedoch von starken Gewichtsschwankungen, Übelkeit und Schwindelgefühl. Die von mir befragten Frauen waren geteilter Meinung und hatten unterschiedliche Erfahrungen gemacht. Im Beisein der Ärzteschaft äußerten sie sich jedoch in den seltensten Fällen kri-

\footnotetext{
107 Die Therapieverfahren wie der intratubare Gamtentransfer (GIFT), der intratubare Transfer von Eizellen im Vorkernstadium (PROST), der intratubare Zygotentransfer (ZIFT) und der intratubare Embryotransfer (EIFT/TET) haben an Zahl und Bedeutung seit der Einführung der Methoden der Mikroinsemination (ICSI) verloren (Feige et al. 1997:124).

108 Auch Mikroinjektion genannt.

109 Die Mikroinjektion ist ohne vorhergehende IVF-Behandlung somit nicht möglich, d.h. die beschriebenen Eingriffe müssen trotz diagnostizierter männlicher „Sterilität“ am Körper der Frau vorgenommen werden.
} 
tisch. Da diese der Überzeugung waren, dass es keine körperlichen Nebenwirkungen gebe, verschwiegen sie oftmals ihre subjektiven (leiblichen) Empfindungen, von denen sie mir in den persönlichen Interviews berichteten.

\subsubsection{Eine kurze Betrachtung der Konsequenzen der heterologen Insemination bezüglich der Zuordnungen, Rechte und Pflichten der beteiligten Personen}

Aus dem bisher Dargelegten ist deutlich geworden, dass Verwandtschaft im rechtlichen Diskurs in Deutschland als eine besondere Form sozialer Beziehungen dargestellt wird, die sich über eine geteilte biogenetische Körpersubstanz konstituiert und soziale Konsequenzen beinhaltet. Die Reproduktionstechnologien propagieren die Schaffung von leiblichen Kindern eines (Ehe-)Paares und arbeiten somit an der Schnittstelle zwischen Ehe, Familie und Verwandtschaft. Die Anwendung der Maßnahmen zur Behandlung von „Sterilität“, insbesondere bei der Verwendung von Keimzellen Dritter, werfen jedoch auch Fragen auf nach den Auswirkungen der Vorstellungen von Verwandtschaft als materielle, auf Körpersubstanzen basierende, Beziehungen zwischen Menschen und deren statusrechtliche Zuordnungen, Zugehörigkeiten, Rechten und Pflichten.

Wie ich zeigen konnte, geht es in der deutschen Gesetzgebung um eindeutige personenstandsrechtliche Zuordnungen von Personen, wobei „natürlicher“ Verwandtschaft im Verhältnis zu anderen Kategorien von Verwandtschaft eine zentrale Bedeutung beigemessen wird. Bei dem im homologen System gezeugten Kind mittels Insemination, IVF, GIFT oder ICSI ist diese Zuordnung ebenso wie die damit zusammenhängenden gesetzlich festgelegten Rechte und Pflichten eindeutig geregelt, da es genetisch von der Mutter und deren Ehemann abstammt. Anders verhält es sich bei dem heterolog gezeugten Kind, das in einer Ehe geboren wird. Als Mutter gilt immer die Frau, die es geboren hat (siehe $\S 1591$ BGB). In rechtlicher Hinsicht gilt das Kind solange als Kind des Ehemannes der Mutter, bis dessen Vaterschaft erfolgreich angefochten wird. Auch im Fall der heterologen Insemination ist die Anfechtung der Vaterschaft durch den Ehemann möglich, da der Bundesgerichtshof jedem Mann bei berechtigten Zweifeln die Anfechtung der Vaterschaft innerhalb einer festgelegten Klagefrist zubilligt; Dies gilt auch für den Ehemann bei vorheriger Zustimmung zur „Samenspende“. Das gegenwärtige deutsche Recht kennt keine bindenden Personenentscheidungen vor der Geburt. Ein nichteheliches Kind kann somit zwar vor der Geburt anerkannt werden ( $\$ 1594$ BGB), jedoch ist diese Anerkennung aufgrund des geltenden Rechts anfechtbar ( $\$ 1600$ BGB n.F.). Vom Anfechtungsrecht zu trennen ist jedoch die Unterhaltspflicht. Dass das Kind bei Vertragsabschluss noch nicht gezeugt war, ist in diesem Fall kein Hindernis. Die Einwilligung zur Unterhaltsverpflichtung ist nicht ohne weiteres widerrufbar. In diesem Fall wird unterschieden zwischen dem Status als Vater und dem Unterhalt (vgl. Lüderitz 
1999:277). Wenn die Vaterschaft erfolgreich angefochten wurde, ist der Mann folglich nicht mehr gesetzlich verpflichtet, den Unterhalt für das Kind zu zahlen. An die Stelle der gesetzlichen Unterhaltsverpflichtung tritt nun allerdings eine rechtsgeschäftliche Unterhaltsverpflichtung, da der Ehemann der heterologen Insemination seiner Ehefrau zugestimmt hat. Dies hat zur Folge, dass der Unterhaltsanspruch des Kindes auch bei der Anfechtung der Vaterschaft durch den Ehemann bestehen bleibt. Anders stellt sich die Situation jedoch dar, wenn das Kind (oder als dessen gesetzliche Vertreterin die Mutter) die Vaterschaft anficht. Wurde die Vaterschaft durch das Kind erfolgreich angefochten, entfällt der Unterhaltsanspruch des Kindes und damit auch sein Erbrecht. ${ }^{110}$

Ein weiterer Aspekt sind die Rechtsbeziehungen zwischen dem Kind und den an seiner Erzeugung beteiligten Personen wie zum Arzt und zum „Samenspender“. Wenn der Ehemann erfolgreich die Vaterschaft anfechten konnte, hat das Kind einen Anspruch darauf, die gerichtliche Feststellung des „Samenspenders“ zu verlangen. Im gegenwärtigen Recht ist der „Samenspender“ Vater im Sinne von $\S 1592$ Nr 3 BGB n.F., daher als solcher feststellbar und damit unterhaltspflichtig, wenn vom sozialen Vater kein Unterhalt zu erwarten ist (vgl. Lüderitz 1999:278f). Da es eine gesetzliche Verpflichtung für den Arzt zur Dokumentation der Unterlagen über den „Samenspender“ gibt, kann dieser somit ermittelt werden. Nach vorherrschender juristischer Meinung haftet der Arzt auf Schadensersatz, wenn er dieser Pflicht nicht nachkommen kann und der „Samenspender“ nicht ermittelbar ist. Begründet wird diese Auffassung u.a. damit, dass der Arzt das Persönlichkeitsrecht des Kindes verletzt hat.

Dieser kurze Exkurs macht deutlich, dass die Anonymität des ,Spenders“, die sowohl den Paaren als auch den „Spendern“ von Seiten der Ärzteschaft zugesichert wird ${ }^{111}$, und damit verbunden dessen soziale Ausblendung, ein im Extremfall nicht einzuhaltendes Versprechen darstellt. Durch das gesetzliche Verbot der Anonymisierung der Spenderdaten (vgl. Lüderitz 1999:278) soll dem Kind das in der Bundesverfassung festgelegte Recht auf Kenntnis seiner Abstammung zugesichert werden (Art. 2 Abs. 1 GG). Dieses Recht basiert auf der herausragenden Bedeutung, die der genetischen Herkunft für die Identitätsbildung eines Kindes in Deutschland zugesprochen wird. Darin spiegelt sich auch das biogenetische Zeugungsmodell wider, das davon ausgeht, dass durch Fort-

\footnotetext{
110 Vom Anfechtungsrecht zu trennen ist demnach die Unterhaltsverpflichtung. Die Einwilligung des Ehemannes in die Durchführung der heterologen Insemination kann als Vertrag zugunsten des Kindes verstanden werden. Dieser Vertrag ist nicht ohne weiteres widerrufbar, d.h. auch wenn der Ehemann die Vaterschaft erfolgreich angefochten hat, kann ihm zwar der Status als Vater aberkannt werden, die Verpflichtung zur Zahlung des kindlichen Unterhalts besteht jedoch weiterhin. Anders verhielt es sich in einem Fall, in dem die Mutter nach Scheidung der Ehe dem sozialen Vater, der weiterhin Unterhalt zahlte, das Umgangsrecht verweigerte und als gesetzliche Vertreterin des Kindes Anfechtungsklage erhob. Nachdem die Anfechtung anerkannt wurde, stellte der Mann die Zahlungen ein. Der Bundesgerichtshof gab ihm dabei Recht (BGH NJW 1995, 2031).

111 Siehe dazu auch Kapitel 5.1.4 und 6.
} 
pflanzung die genetischen Informationen eines Elternpaares weitergegeben werden, die die Grundlage bilden für die genetische Identität und die Individualität des Kindes (Kap. 2.2.2). Diese „natürlichen“ Verbindungen zwischen den biologischen Eltern und dem Kind gelten als festgelegt und damit unveränderlich. Was jedoch auffällt, ist die Tatsache, dass sich das Recht auf Kenntnis der Abstammung einzig auf die Aszendenz bezieht, da nur das Kind auf gerichtliche Feststellung der genetischen Vaterschaft klagen kann (siehe auch Kap. 4.1.3). Dem „Samenspender“ ist ein Einklagen der genetischen Vaterschaft, also das Recht auf Kenntnis der Deszendenz, verwehrt, solange ein Mann, in der Regel der Ehemann der Mutter, die Vaterschaft anerkennt. Sofern der Ehemann zur Übernahme der sozialen Vaterschaft bereit ist, erfährt - unter dem Gedanken der Priorität von Eheschutz und „Kindeswohl“"112 - die biologische Vater-Kind-Beziehung seitens des Gesetzgebers keine Anerkennung (vgl. Stein-Hilbers 1994:200). In diesem Fall wird die soziale Dimension von Vaterschaft höher gewichtet als die biologische und die strukturelle Einheit einer Kernfamilie aufrecht erhalten. Wie ich schon in Kapitel 4.1.3 herausgestellt habe, mischt sich der Staat nicht in die persönliche Gestaltung der rechtlich als Verwandtschaft anerkannten Beziehungen ein, solange die personenstandsrechtliche Zuordnung eindeutig ist. Es bleibt somit dem persönlichen Ermessen eines Elternpaares überlassen, ob es dem Kind mitteilt, dass es mit Hilfe einer „Samenspende“ entstanden ist und somit mit dem „Spender“ (biogenetisch) verwandt ist. Erfährt das Kind jedoch von der gespaltenen Vaterschaft, kann es sich auf das Recht auf Kenntnis der Abstammung berufen, das es ihm ermöglicht, die rechtlich anerkannte Vaterschaft anzufechten. Bei Feststellung des „Samenspenders“ könnte dieser demnach aufgrund des Primats von biologischer Verwandtschaft ( $\$ 1589$ BGB) in Deutschland den formalrechtlich anerkannten Verwandtschaftsstatus als Vater übernehmen. $\mathrm{Ob}$ damit auch die Übernahme der sozialen Vaterschaft und damit das Ausüben elterlicher Verantwortung verbunden ist, bleibt allerdings Teil der individuellen Handlungsfreiheit und Ausgestaltung verwandtschaftlicher Statusbeziehungen, in die sich der Gesetzgeber nicht einmischt.

\footnotetext{
112 Das BGB verwendet den Begriff des „Kindeswohls“ als orientierenden Begriff zur Ausübung der elterlichen Sorge (§ 1627) und zur Regelung elterlicher Konflikte bei Eingriffen in die elterliche Sorge. Coester (1986) benennt vier Grundfunktionen des Kindeswohlpostulats: Es legitimiert Eingriffe in die Familienautonomie (z.B. bei Kindesmisshandlungen), es dient als Entscheidungsmaßstab bei positiv zu treffenden Maßnahmen (z.B. im Umgangsrecht), es ist Verfahrensrichtlinie bei richterlichen Interventionen, und es bezeichnet einen rechtspolitischen Gestaltungsauftrag an den Gesetzgeber. Longino (1998:49) spricht im Zusammenhang mit dem „Kindeswohl“ von der Entwicklung einer selbstständigen und selbstverantwortlichen Persönlichkeit mit der gleichzeitigen Fähigkeit zur sozialen Koexistenz. Weiterhin fällt seiner Ansicht darunter eine Kontinuität von Beziehungen, die sich in stabilen äußeren Verhältnissen und dauernden Gefühlsbindungen ausdrückt.
} 


\subsubsection{Adoptionen und Pflegschaften: Ein Blick auf die rechtliche Situation}

Adoptionen und Pflegschaften sind Formen der Familienbildung, in denen ein Kind mit Personen in einer Familienkonstellation zusammenlebt, die im allgemeinen nicht blutsverwandt mit ihm sind. Das wesentliche Unterscheidungsmerkmal von Adoptionen und Pflegschaften liegt in der legislativ-normativen Spaltung der Elternschaft, die bei der Adoption vollständig vollzogen wird: Mit der Adoption eines Kindes erlöschen alle verwandtschaftlichen Bindungen, inklusive sämtlicher Rechten und Pflichten, zur Herkunftsfamilie, und das Kind wird rechtlich der Adoptivfamilie zugeordnet. Dieser Statuswechsel betrifft auch alle Deszendenten des Adoptivkindes. Der Wechsel von der Herkunftsfamilie zur Adoptivfamilie ist somit endgültig und nicht umkehrbar. ${ }^{113}$ Bei der Pflegschaft bleibt das Kind grundsätzlich weiterhin Mitglied der Herkunftsfamilie, unabhängig davon, ob die Eltern das Sorge- oder Umgangsrecht ausüben. Zwar kann die Unterbringung des Kindes in der Pflegefamilie auf Dauer angelegt sein (sogenannte Dauerpflegschaften), doch besteht prinzipiell immer die Möglichkeit einer Rückkehr des Kindes in die Herkunftsfamilie.

Wie ich in Kapitel 4.1.3 gezeigt habe, spricht die deutsche Gesetzgebung den biologischen Eltern grundsätzlich die „natürliche“ Fähigkeit zu, „die Erziehung und Betreuung eines minderjährigen Kindes [...] innerhalb einer harmonischen Gemeinschaft" am ehesten zu gewährleisten (Longino 1998:52). Hierzu sollen Kontinuität und Stabilität in äußeren Verhältnissen und sozialen Bindungen gehören, die Emotionalität der ElternKind-Bindung und die Erziehung als zentrale Aufgabe der Eltern. Können die leiblichen Eltern diese Aufgaben nicht oder nur unzureichend erfüllen, wird als Alternative die Unterbringung in einer Adoptiv- oder Pflegefamilie gesehen (Wittland-Mittag 1992:17). Die Kriterien, die von potentiellen Pflegeeltern erfüllt werden sollen, sind ,,angemessene wirtschaftliche und räumliche Verhältnisse, Erziehungstüchtigkeit, gefestigte charakterliche und sittliche Eigenschaften sowie kein zu großer Altersabstand zum Kind“ (Oberloskamp 1993:12). Für Adoptiveltern ergeben sich als Voraussetzungen neben dem angemessenen Alter vor allem die Stabilität der Ehe und die „Erziehungsfähigkeit“ der Bewerber (Oberloskamp 1993:94). ${ }^{114}$

\section{Adoptionen}

Die Adoption wurde im ersten reichseinheitlichen Bürgerlichen Gesetzbuch im Jahre 1900 als „Annahme an Kindes Statt“ gesetzlich festgeschrieben:

\footnotetext{
${ }^{113}$ Dieser Aspekt wird gegenwärtig jedoch diskutiert (vgl. Schmitt 2000).

114 Detailliertere Darstellungen zur Gesetzeslage bieten Oberloskamp (1993) und Schlieffen (1994).
} 
„Die Adoption soll edeldenkenden Familien - insbesondere Adligen - die Möglichkeit der Namenserhaltung bieten, wenn diese keine eigenen Nachkommen haben oder auch einem würdigen Erwachsenen einen Nachfolger und Erben verschaffen, ebenfalls unter dem Gesichtspunkt der Erhaltung des Namens.“" (InfoAdoption 1994:7)

Im Vordergrund standen hier eindeutig die Interessen der Annehmenden. Die bedeutendsten Funktionen der Adoption waren der Namenserhalt und die Schaffung eines Erben. Die Adoptierenden mussten kinderlos und mindestens 50 Jahre alt sein. Das Recht sah nur die Volljährigenadoption vor. Die verwandtschaftlichen Beziehungen blieben zwischen dem Adoptierten und seiner Ursprungsfamilie weiterhin rechtlich bestehen.

Die Funktionsverschiebung bei der Adoption von „einem Mittel zur Gewinnung eines Erben für kinderlose Familien der Oberschicht hin zu einem Mittel der Fürsorge“ (InfoAdoption 1994:14) hatte seinen Anfang bereits in der Weimarer Republik, als sich die staatlichen Fürsorgestellen mit einer großen Zahl von Kriegswaisen konfrontiert sah. Doch erst im Reformgesetz von 1950 wurde das „Wohl des Kindes“ zum zentralen Vermittlungskriterium. Die Adoption diente nun dazu, für das Kind neue Eltern zu finden - nicht umgekehrt. Im Zuge dieser Umorientierung wurde 1961 das Alter der Adoptierenden auf 35 Jahre gesenkt und bereits 1950 Ausnahmen von der Regelung der Kinderlosigkeit zugelassen (InfoAdoption 1994:13). 1973 wurde das Alter weiter auf 25 Jahre gesenkt, doch erst durch das neue Adoptionsgesetz vom 02.07.1976 wurde die Kinderlosigkeit als Annahmevoraussetzung ersatzlos gestrichen. Durch die Einführung des neuen Adoptionsgesetzes wurde der Zustand beendet, dass die Adoptivkinder mit den leiblichen Eltern per Gesetz verwandt blieben. Zuvor bestand lediglich eine persönliche, aber keine rechtliche Verbindung zwischen den Adoptivkindern und den übrigen Familienangehörigen. 1976 wurde die Volladoption eingeführt, durch die das adoptierte Kind in der Adoptivfamilie den Status eines leiblichen Kindes mit allen Rechten und Pflichten hat. ${ }^{115}$ Durch die Adoption erhält das Kind den rechtlichen Status eines leiblichen Kindes der Adoptiveltern. Die Adoption, die früher durch zivilrechtlichen Vertrag zwischen Angenommenen und Annehmenden vereinbart wurde, wobei bestimmte Vertragsbedingungen (zum Beispiel Ausschluss von der Erbfolge) frei verhandelbar waren, wird jetzt durch Gerichtsbeschluss ausgesprochen und hat damit viel stärkere Rechtswirkung. Sie ist faktisch unumkehrbar.

Laut $§ 1641$ I BGB ist die Voraussetzung für die Annahme eines Kindes, dass sie dem „Wohl des Kindes“ dient und zu erwarten ist, dass zwischen ihm und den Annehmen-

115 Dies ist zum Beispiel auch vor allem für die Großeltern (Eltern der Adoptiveltern) von Bedeutung, denn sie sind, falls die Adoptiveltern nicht mehr für den Unterhalt des Kindes aufkommen können, unterhaltspflichtig. 
den ein Eltern-Kind-Verhältnis entsteht. Letzteres soll in einer ,,angemessenen“ Probezeit angestrebt werden ( $§ 1744$ BGB). Diese Zeit umfasst in der Regel ein Jahr und wird als Adoptionspflege bezeichnet (InfoAdoption 1994:23). Adoptieren dürfen nur in der Regel nur heterosexuelle verheiratete Paare oder eine ledige Person. Außerdem darf ein Ehegatte das Kind des anderen Ehegatten annehmen (Stiefkindadoption) (§ 1741 II BGB). Bis zur Kindschaftsrechtsreform 1998 konnten ledige Elternteile ihre eigenen Kinder adoptieren. Die Annahme als Kind bedarf grundsätzlich der Einwilligung des Kindes, wenn es das 14. Lebensjahr vollendet hat, sowie der leiblichen Eltern. Die Eltern dürfen ihre Einwilligung frühestens acht Wochen nach der Geburt geben. Die Einwilligung darf nur gegeben werden, wenn schon Adoptionswillige vorhanden sind, d.h., dass die Einwilligung nicht „blanko“ gegeben werden kann (Oberloskamp 1993:114). Die leiblichen Eltern müssen die Adoptiveltern nicht kennen. Ohne Einwilligung der Adoptiveltern können bei einer Inkognitoadoption die leiblichen Eltern den Aufenthalt des Kindes nicht erfahren. Ebenso bleiben die Herkunftseltern den Adoptiveltern unbekannt. Mit der Einwilligung in die Adoption verwirken die Herkunftseltern ihr Recht auf Umgang mit dem Kind. Die vollständige Identität der Herkunftseltern wird, soweit sie der Adoptionsvermittlungsstelle bekannt ist, jedoch im Annahmevertrag vermerkt. Damit wird dem Kind, das mit 16 Jahren ein Recht auf Einsicht in die Adoptionsakte hat (Schlieffen 1994:52), die spätere Suche nach den leiblichen Eltern ermöglicht.

Bei der am häufigsten praktizierten Form der Inkognitoadoption „erlöschen das Verwandtschaftsverhältnis des Kindes und seiner Abkömmlinge zu den bisherigen Verwandten und die sich aus ihm ergebenden Rechte und Pflichten“ ( $\$ 1755$ BGB). Das Kind erhält den Namen der Adoptivfamilie und sein Vorname darf auf Antrag ebenfalls geändert werden, wenn dies dem „Wohle des Kindes“ dient (§ 1757 BGB). Mittlerweile werden auch Formen von offener Adoption durchgeführt: „Bei der offenen Adoption wissen die abgebenden Eltern, in welcher neuen Familie ihr Kind nun aufwachsen wird“ (Schlieffen 1994:52). Welche Informationen (und soziale Beziehungen) dieses Wissen enthält, ist Aushandlungssache zwischen den beteiligten Personen sowie der Adoptionsvermittlungsstelle. Mindestens umfasst die offene Adoption jedoch die Einbeziehung der Herkunftseltern in die Auswahl der Adoptivfamilie, meist auch ein Zusammentreffen der Elternpaare, wenn das Kind in die neue Familie gegeben wird. Gesetzlich ist die offene Adoption nicht geregelt. So haben Adoptiveltern jederzeit das Recht, Kontakte zu reglementieren, abzubrechen oder erst gar nicht zuzulassen. Auf die Möglichkeit der offenen Adoption hinzuweisen, in welcher Form auch immer, liegt wiederum im Ermessen der zuständigen Adoptionsvermittlungsstelle. 


\section{Pflegschaften}

Der Ausdruck „Pflegekind“ ist zwar neueren Datums (im allgemeinen Sprachgebrauch erst seit etwa hundert Jahren üblich), jedoch haben Pflegschaftsverhältnisse, wenn auch nicht in rechtlich anerkannter Form, im Europa der Neuzeit eine lange Tradition. Ziehund Kostkinder als zusätzliche Familienmitglieder ebenso wie als Arbeitskräfte waren weit verbreitet. Rosenbaum (vgl. 1998:68) zeigt auf, dass für bäuerliche Familien des 17. bis 19. Jahrhunderts erweiterte Familienkonstellationen durch Gesinde, Stiefverwandtschaft und Ziehkinder nichts ungewöhnliches waren. Durch die Produktionsweise der bäuerlichen Haushalte, die eine solch komplexe Verwandtschaftskonfiguration möglich und auch nötig machte, war die Abgrenzung von leiblicher und nicht-leiblicher Verwandtschaft kaum denkbar. Bis in die 60er Jahre des 20. Jahrhunderts wurde die Familienpflege nicht als gleichwertige Alternative zur Heimunterbringung betrachtet. Heute ist sie jedoch fester Bestandteil der staatlichen Maßnahmen zur „Hilfe zur Erziehung".

Gesetzliche Grundlage des Pflegekinderwesens ist das seit Januar 1991 geltende Kinder- und Jugendhilfegesetz (KJHG), welches Teil des Sozialgesetzbuches ist. In ihm sind u.a. das Recht des ,jungen Menschen“ auf „Förderung seiner Entwicklung und Erziehung zu einer eigenverantwortlichen und gemeinschaftsfähigen Persönlichkeit" (§ 1 KJHG), die „Aufgaben der Jugendhilfe“ ( 2 KJHG) und die „Beteiligung von Kindern und Jugendlichen“" an allen sie betreffenden Entscheidungen der öffentlichen Jugendhilfe ( $§ 8 \mathrm{KJHG}$ ) festgelegt. Des weiteren werden die Merkmale der verschiedenen Förderungsangebote ( $§ 22-26 \mathrm{KJHG})$ und Erziehungshilfen ( $\S 27-41 \mathrm{KJHG})$ beschrieben sowie die „Beratung und Unterstützung bei der Ausübung der Personensorge“ (§ $18 \mathrm{KJHG})$, die „Leistungen zum Unterhalt des Kindes oder des Jugendlichen“ (§ $39 \mathrm{KJHG}$ ), die „Herausnahme des Kindes oder des Jugendlichen ohne Zustimmung des Personensorgeberechtigten“ (§ $43 \mathrm{KJHG})$ und die „Pflegeerlaubnis“ (§ $44 \mathrm{KJHG}$ ) geregelt, um nur die für die Familienpflege bedeutendsten Abschnitte zu nennen. ${ }^{116} \mathrm{Im}$ KJHG von 1991 wird als „Pflegeperson“ definiert, ,wer ein Kind oder einen Jugendlichen außerhalb des Elternhauses in seiner Familie regelmäßig betreut oder ihm Unterkunft gewähren will“ (§ $44 \mathrm{KJHG}$ ). Grundsätzlich bleiben die Herkunftseltern Träger des Elternrechts, da das Pflegekind mit allen Rechten und Pflichten Mitglied der Herkunftsfamilie bleibt, sowohl bei sogenannter familienersetzender Familienpflege als auch dann, wenn den Herkunftseltern das Sorgerecht vollständig entzogen wurde.

\footnotetext{
116 Allerdings umfasst das KJHG nur einen Teil der Kinder- und Jugendhilfe. In den anderen Abschnitten des Sozialgesetzbuches, im Grundgesetz, im Bürgerlichen Recht, im Jugendgerichts-, Einkommenssteuer-, Bundeskinder-, Bundessozialhilfegesetz und anderen Gesetzestexten finden sich weitere Bestimmungen zur Kinder- und Jugendhilfe, die ebenfalls das Pflegekindwesen betreffen.
} 
„Handelt es sich um ,Alltagsfragen“ von Pflege, Erziehung und Beaufsichtigung, so sind die Pflegeeltern zuständig. Sind es dagegen Fragen von größerer Tragweite (Grundentscheidungen über Schul- und Berufswahl, gravierende medizinische Fragen), so müssen die leiblichen Eltern gefragt werden. Das Aufenthaltsbestimmungsrecht verbleibt grundsätzlich bei den leiblichen Eltern, jedenfalls soweit die Grundsatzfrage, wo das Kind gepflegt und erzogen wird, betroffen ist.“ (Oberloskamp 1993:52)

In der Regel behält auch der Elternteil, dem die Personensorge nicht zusteht, die Befugnis zum persönlichen Umgang mit dem Kinde (§ 1634 BGB). Neben schriftlichen Kontakten und Telefonaten umfasst dieses Umgangsrecht vor allem das Recht auf Besuchskontakte. Sehr häufig werden Regelungen über Zuständigkeiten und Verteilung der elterlichen Personen- und Vermögenssorge in einem Pflegevertrag festgehalten. Grundsätzlich endet ein Pflegevertrag mit der Volljährigkeit des Kindes. Er ist sowohl von Pflegeeltern- als auch von Herkunftselternseite kündbar. An seiner Ausarbeitung soll Fachpersonal beteiligt sein sowie die personensorgeberechtigten Eltern, das Kind und die Pflegeeltern. Letzteren kann die Pflegeerlaubnis entzogen werden, wenn sie sich nicht an die Regelungen des Vormundschaftsgerichts und des Jugendamtes bzw. des Pflegeplanes halten (Oberloskamp 1993:53). Halten sich die Herkunftseltern nicht an die Regelungen, werden ihre Rechte beschnitten (vgl. Wiemann 1998:40f).

Es gibt verschiedene Formen von Pflegschaften, wobei nur die Tages- und die Vollzeitpflege eindeutig im KJHG geregelt werden. Erstere ist rechtlich der „Förderung von Kindern in Tageseinrichtungen“" wie Kindergärten oder Schulen gleichgestellt ( $\$ 23$ KJHG). Die Vollzeitpflege ist „eine zeitlich befristete Erziehungshilfe oder eine auf Dauer angelegte Lebensform“ ( $\$ 33 \mathrm{KJHG})$. Die Vollzeitpflege kann zum einen eine „familienergänzende“ oder auch eine „familienersetzende“ Lebensform sein. Grundsätzlich soll jedoch die Familienergänzung im Vordergrund stehen und das Ziel die Wiederherstellung der Herkunftsfamilie sein. Die Herkunftseltern bleiben gegenüber ihrem Kind unterhaltspflichtig, was bedeutet, dass sie, wenn sich das Kind in Vollzeitpflege befindet, einen regelmäßigen Unterhalt zahlen müssen. Dieser kann, je nach ihrer finanzieller Situation, teilweise oder ganz vom Jugendamt übernommen werden (Oberloskamp 1993:55). Pflegeeltern erhalten regelmäßige, meist monatliche, Unterhaltsleistungen, genannt „Pflegegeld“. Diese setzen sich aus einer zur Abdeckung des Lebensbedarfs dienenden Betrag und einem als Anerkennung für die Erziehungsleistung gedachten Betrag zusammen. Vollzeitpflegeeltern haben außer dem Anspruch auf Unterhaltsleistungen nach $\S 39 \mathrm{KJHG}$ beispielsweise auch Anspruch auf Kindergeld, Eintragung des Kindes in die Lohnsteuerkarte, Baukindergeld sowie Wohngeld. Erbberechtigt bleiben die Kinder gegenüber ihren leiblichen Eltern, nicht gegenüber den Pflegeeltern. Unter bestimmten Voraussetzungen ist es möglich, dass das Pflegekind den Familiennamen der Pflegefamilie trägt (Oberloskamp 1993:28). 
Da „echte“ Verwandtschaft im rechtlichen Diskurs in Deutschland über Abstammung und damit Körper und Körperlichkeit konstituiert wird, gilt sie im Unterschied zu ,nur“ sozialen Verbindungen (z.B. durch Heirat, Adoptionen, Pflegschaften) als eindeutig festgelegt und unauflöslich. Eine Aushandelbarkeit und damit Modifizierbarkeit wird nur den Beziehungen zuerkannt, die ihren Ursprung nicht in der ,Natur ${ }^{\star}$ haben. Idealerweise besteht Verwandtschaft allerdings aus einer biologischen und sozialen Dimension, wobei das Soziale die Biologie (die damit der ,Natur' gleichgesetzt wird) abbildet bzw. dieser folgt. Dies zeigt sich an der rechtlichen Festlegung der primären Elternverantwortung durch die „,natürlichen“ Eltern auf der Basis des Konzeptes von ,natürlicher Abstammung“. Es impliziert die Vorstellung, dass die biologische Elternschaft die Ausübung der sozialen Elternschaft beinhaltet bzw. nach sich ziehen soll, wodurch jedoch auch die soziale der biologischen Elternschaft nachgeordnet wird: ,Nur' soziale Elternschaft beruht nicht auf „natürlichen“ Fakten, ist durch menschlichen Willensentscheid entstanden und wird als Ersatz für die „,natürliche“ Elternschaft angesehen. Das „,natürliche“ Elternrecht wird nur dann eingeschränkt, und staatliche Eingriffe in die Kernfamilie werden legitimiert, wenn die biologischen Eltern die Aufgabe der Kindesfürsorge und -erziehung nicht oder nur eingeschränkt übernehmen. Diese Feststellungen lassen den Bezug zu dem u.a. von Goody (1989 [1983]), Schneider (1980 [1968]) und Strathern (1992a, 1993b) beschriebenen euro-amerikanischen Verwandtschaftskonzept (Kap. 2.2) herstellen, in dessen Zentrum die Idee der Blutsverwandtschaft steht, die über den Prozess der biologischen Reproduktion geschaffen wird. Ein Paar wird somit nur aufgrund der biologischen Vorgänge zu Eltern, deren soziale Anerkennung den „natürlichen Fakten“ folgt. Auch aus der Analyse des rechtlichen Verwandtschaftsdiskurses in Deutschland hat sich die Vorstellung herauskristallisiert, dass die Einheit von biologischer und sozialer Elternschaft in der Kernfamilie, die in ihrer historischen Tradition auf der Institution der Ehe beruht, als ideale Form des Zusammenlebens gilt, die das „Kindeswohl" garantiert.

Die gesellschaftlichen Rahmenbedingungen in Deutschland schaffen auch die Voraussetzungen für die technologische Absicherung der naturalisierten Form der Kernfamilie, indem sie die Anwendung der Reproduktionstechnologien auf verheiratete Paare oder zumindest heterosexuelle nicht-eheliche Lebensgemeinschaften beschränken (Kap. 4.3.1.1). ${ }^{117}$ Ist eine Einheit aus biologischer und sozialer Elternschaft wie im Fall der heterologen Insemination, Adoption und Pflegschaften nicht gegeben, so ,schützt ${ }^{\star}$ der deutsche Staat zumindest die Einheit der sozialen Form einer Kernfamilie, in dem er

\footnotetext{
117 Ein Leiden unter dem Zustand der „ungewollten Kinderlosigkeit“ wird somit beispielsweise alleinstehenden Frauen und Männern, lesbischen und schwulen Paare aufgrund der dominanten rechtlichen Verwandtschaftsvorstellung in Deutschland abgesprochen. Ihnen ist die Nutzung der Reproduktionsmedizin in Deutschland grundsätzlich verwehrt, da sie nicht die notwendigen Kriterien für ,natürliche“ Elternschaft, die sich idealerweise im Rahmen einer Ehe über den Prozess der biologischen Reproduktion konstituiert, erfüllen.
} 
situations- und motivationsspezifisch der sozialen Dimension von Elternschaft eine höhere Gewichtung beimisst und rechtlich-normativ eingreift (Kap. 4.1.3, 4.3.1.3, 4.3.2). Elternschaft bzw. elterliche Verantwortung kann in diesen Fällen auch von sozialen Eltern übernommen werden, die nicht über den Prozess der biologischen Reproduktion mit dem Kind verbunden sind. Jedoch ist auch die Übernahme der ,nur' sozialen Elternschaft in der Regel auf heterosexuelle Ehepaare beschränkt.

Ich habe in diesem Kapitel die staatlich legitimierten Möglichkeiten von ,assistierten“ Familiengründungen ${ }^{118}$ aufgezeigt, doch soll nicht unerwähnt bleiben, dass sich die Voraussetzungen für deren Inanspruchnahme unterscheiden. So ist es für ,ungewollt kinderlose“ Paare möglich, aufgrund einer körperlichen Diagnose von „Sterilität“ die Methoden, die die Reproduktionsmedizin bietet, in Anspruch zu nehmen und die Behandlungen von den Krankenkassen finanzieren zu lassen. Bei Eigenübernahme der Kosten liegt es im Ermessen des Arztes, Behandlungen auch ohne eindeutige medizinische Indikationen durchzuführen. Auskünfte über ihre persönlichen Motivationen für ein Kind, die Vorstellungen über die Ausgestaltung ihrer (potentiellen) Rollen als Vater oder Mutter und Nachweise über eine gesicherte finanzielle Grundlage für die Gründung einer Familie werden weder verlangt noch erwartet. Im Fall eines Adoptionsbewerbungsverfahrens müssen die ,ungewollt kinderlosen“ Bewerber jedoch auf bürokratischem Wege ihre ,Qualifikation`zur Elternschaft unter Beweis stellen, in dem sie in persönlicher und finanzieller Hinsicht darlegen, warum sie ein Kind adoptieren möchten und wie sie sich das Leben mit dem Adoptivkind vorstellen würden. Es wird vorausgesetzt, dass eine Trauerarbeit über eine mögliche „ungewollte Kinderlosigkeit“ schon geleistet wurde, d.h., dass das potentielle Adoptivkind keinen ,Ersatz' darstellt für ein leibliches Kind. Steht im Fall der Anwendung der Reproduktionsmedizin das Paar und dessen „Kinderwunsch“ im Mittelpunkt, so soll es hier ausschließlich um das „Wohl“ und die Interessen des schon geborenen Kindes gehen. Wie ich gezeigt habe, ist die Möglichkeit der Übernahme einer Pflegeelternschaft, die nicht mit einer legal anerkannten Elternschaft verbunden ist, weniger problematisch. Für die das Pflegekind vermittelnden staatlichen Instanzen zählen in erster Linie die vorhandenen persönlichen Möglichkeiten und räumlichen Gegebenheiten, die dem Kind eine regelmäßige Betreuung und „Hilfe bei der Entwicklung“ gewährleisten. Der zwischen Pflege- und Her-

\footnotetext{
118 Auf die ,assistierte“ Form einer Familiengründung in Form von Stiefelternschaften bin ich hier nicht eingegangen, da diese durch den Akt einer Eheschließung zwischen zwei Personen entstehen. In diesem Fall beruht die Entscheidung zu einer ,Familiengründung' auf einer bewussten persönlichen Entscheidung beider Partner, die die Übernahme der sozialen Elternschaft durch den Stiefelternteil beinhalten kann, aber im Unterschied zu den von mir genannten Beispielen, in denen der Wille entweder nach biologischer und sozialer oder ausschließlicher (kontinuierlicher) sozialer Elternschaft im Mittelpunkt steht, nicht muss (abhängig beispielsweise davon, ob das Kind/die Kinder des Ehepartners mit im Haushalt leben oder nur am Wochenende oder im Urlaub zu Besuch kommen).
} 
kunftseltern geschlossene Pflegevertrag ist darüber hinaus im Unterschied zu der biologischen Elternschaft sowie der Adoptionselternschaft kündbar.

Auf dem Hintergrund der Analyse des bundesdeutschen rechtlichen Verwandtschaftsdiskurses, der das Spannungsverhältnis zwischen biologischer und sozialer Verwandtschaft und die ,Naturalisierung' von sozialer Verwandtschaft, die sich über den Körper und biologische Fortpflanzung konstituiert, gezeigt hat, wende ich mich im Folgenden konkret meinem empirischen Material zu und damit dem persönlichen Diskurs von Verwandtschaft, wie er in verschiedenen sozialen Kontexten geführt wird. Diskurse können nicht losgelöst von Handlungen und Entscheidungen betrachtet werden, so dass es hier auch um die Prozesse gehen soll, die Menschen dazu führen, bestimmte Entscheidungen zu treffen und danach zu handeln. So werde ich der Frage nachgehen, ob in den Kontexten unterschiedliche Diskurse über Verwandtschaft im Spannungsfeld von Körper als Objekt und Leib, Selbst und Person geführt werden, und welche Kriterien bei dem aus den verschiedenen Diskursen resultierenden Entscheidungsprozess, der daraus ableitbaren Handlung und deren Konsequenzen eine Rolle spielen. 


\section{5 „Verwandtschaft" und das ,eigene“" Kind: Der persönliche Diskurs}

\subsection{Die Konstruktion von Zugehörigkeit und Angehörigkeit im Kontext der Reproduktionsmedizin}

\subsubsection{Deszendenz und Affinalität}

\subsubsection{1 „Familie“6 und „Verwandtschaft"}

In der Untersuchung des persönlichen Konzeptes von Verwandtschaft hat sich gezeigt, dass die befragten Personen, sei es vor oder während reproduktionsmedizinischer Behandlungen, als die bedeutendste, emotionalste und identitätsstiftende Form der Zugehörigkeit und Angehörigkeit diejenige definieren, die die eigene Verortung in eine Gemeinschaft, die als „Familie“ definiert wird, betrifft. Anhand der Interviews wird deutlich, dass diese Gemeinschaft von Personen auch als „Blutsverwandtschaft“ bezeichnet wird, ein Begriff, der sowohl die Vorstellung einer körperlichen Verbindung durch eine gemeinsame Körpersubstanz impliziert, die sich nur über den Prozess der Fortpflanzung herstellen lässt, sowie die Naturhaftigkeit dessen, was „echte“" Verwandte verbindet. ${ }^{119}$ Dies sollen einige Zitatstellen exemplarisch veranschaulichen:

„Die Familie ist im engeren Sinn die Blutsverwandtschaft.“

„Die Familie ist eben aus einem Fleisch und Blut gebaut, und man wird in eine Familie hineingeboren. Da gehört man einfach hin.“

„Blutsverwandtschaft, also damit meine ich meine Familie, sagt ja schon aus, dass das gleiche Blut durch unsere Adern fließt. Das schweißt eben zusammen. Da kommt man her, da gehört man hin und das kann man auch nicht ablegen. Außerdem drückt das auch aus, wer eben nicht dazu gehört. Das muss ja nicht gleich was Negatives sein, aber es gibt einfach Personen, mit denen bin ich definitiv nicht blutsverwandt und werde es auch niemals werden.“

\footnotetext{
119 Strathern und Franklin (1993) haben darauf hingewiesen, dass Blut als ein einzigartiges kulturelles Idiom von Zeugungsbeziehungen in Europa gilt, wodurch ein komplexes kulturelles Wissen von Ursprüngen, Deszendenz, Vererbung und Ebenen von Verbundenheit ausgedrückt wird. Dieses Wissen ist sowohl wichtig für die individuelle Identität, ebenso wie es die Basis bildet für einen weiten Bereich sozialer Institutionen und Handlungen. Diese Feststellung trifft auch für meine Untersuchung in Deutschland zu, wie ich im weiteren Verlauf noch zeigen werde, wobei die Idee von Blutsverwandtschaft auch mit biogenetischer Verwandtschaft gleichgesetzt wird (siehe auch Kap. 5.1.4).
} 
„Zur Verwandtschaft gehöre ich. Da muss ich nichts für tun. Besonders schätze ich die Familientreffen, bei denen ich mich total wohl fühle. Da kommt dann die ganze Familie, alle die dazu gehören.“

Anhand dieser Aussagen zeigt sich, dass im persönlichen Diskurs sowohl der Begriff der ,Familie' als auch der, Verwandtschaft' verwendet wird, wenn es um die Zuordnung von nahestehenden Personen geht, wobei es sich um zwei Termini handelt, die in den Gesprächen auch alternativ oder synonym gebraucht wurden. Bei dem Versuch einer näheren inhaltlichen Definition der beiden Begriffe wird jedoch deutlich, dass für die Befragten definitiv ein Unterschied zwischen beiden besteht, zum einen auf der Ebene der formalen Zuordnung bzw. Abgrenzung von Personen, zum anderen auf der Emotions- und Handlungsebene. Auch diesen Aspekt möchte ich anhand von einigen Zitaten verdeutlichen:

„Die Familie ist der engere Kreis, die Verwandtschaft der weitere.“

„Die Familie ist die engere Verwandtschaft.“

„Familie ist für mich ein engerer Begriff. Die Verwandtschaft ein größerer Personenkreis.“

„Die Familie sind diejenigen, die mir am nächsten sind. Zur Familie ist die Bindung größer."

„Familie ist für mich der innerste Zirkel von persönlichen Beziehungen, die verwandtschaftlichen Ursprungs sind, wobei sich das in vielen Generationswechseln oder Ergänzungen auch wandelt.“"

„Die Familie ist die Kernzelle von dem Ganzen. Da findet man erst mal die Geborgenheit. Verwandtschaft, das ist das größere Umfeld um die Familie herum. Das ist wie mit Haus und Ortschaft, im Haus wohnt man, da fühlt man sich wohl, und die Ortschaft außen herum gehört auch dazu. So ähnlich ist es mit der Verwandtschaft.“”

„Verwandtschaft ist das Anhängsel von der Familie.“

Im Fall der interviewten Personen, die die reproduktionsmedizinischen Methoden nutzen, um ein erstes Kind zu bekommen, lässt sich feststellen, dass in den überwiegenden Fällen die Konsanguinen der gleichen und aufsteigenden Generation, also die Eltern („Vater/Mutter") und die Geschwister („Bruder/Schwester“), zur Familie gezählt werden. Bei der Zuordnung von Muttersmutter und -vater sowie Vatersmutter und -vater („Großmutter/-vater“) sind die Aussagen unterschiedlich; In einigen Fällen gelten sie als Teil der Familie, in anderen gehören sie explizit in die Kategorie ,Verwandte'. Auch die angeheirateten Partner der Geschwister (,Schwager/Schwägerin“) und deren weibliche und männliche Kinder (,Nichte/Neffe“) gelten nur zum Teil zur Familie zugehörig, was mehrheitlich abhängig ist von weiteren Kriterien wie dem Wohnort, der Häufigkeit der Kontakte, dem persönlichen Verhältnis und der emotionalen Bindung. Ebensolches gilt für die Affinalen wie die Eltern des Ehepartners/der Ehepartnerin (,Schwiegermutter/- 
vater"), die Geschwister (,Schwager/Schwägerin“) und deren männliche und weibliche Kinder („Neffe/Nichte“).

Der eigene Ehe- oder Lebenspartner wird dann als Teil der Familie genannt, wenn ein gemeinsames Kind vorhanden ist. Entweder wird der als Familie bezeichnete Personenkreis erweitert um den Ehepartner und das Kind, oder es wird unterschieden zwischen der ,eigenen Familie“, ,unserer Familie“, und der ,anderen Familie“: „Das ist auch eine Familie, aber eine andere. Die richtige Familie, also das sind wir jetzt. Wir drei, meine Frau, mein Kind und ich" (Bernhard Baumann, 42 Jahre). Solange in einer Partnerschaft noch kein Kind geboren ist, beziehen sich die Antworten mehrheitlich auf die Art von Familie, die in der Literatur als Herkunfts- oder Orientierungsfamilie bezeichnet wird (vgl. Fehlmann-von der Mühll 1978:31f), da sich die Ehepartner noch nicht selbst als eigenständige Familie definieren.

„Man ist in seiner eigenen Familie aufgewachsen, und wir haben jetzt eine neue Familie gegründet. Und diese Familie besteht dann für mich in erster Linie erst mal aus dem Paar plus dem Nachwuchs, und darüber hinaus gibt es immer wieder Personen, die da hinein kommen. Jeder der Partner hat ja seine eigene Familie mitgebracht. Dann würde ich den Kreis enger ziehen, erst einmal das Paar plus das Kind, das ist die Familie. Und dann pflanzt sich das immer weiter fort, aber die Familie findet ihren Ursprung im Paar plus Kind.“ (Helga Bachmann, 39 Jahre)

Zu der Kategorie ,Verwandte' zählen die Seitenlinien sowohl mütterlicher als auch väterlicherseits, d.h. Egos Mutterbrüder und -schwestern, die Vaterbrüder und schwestern, deren Ehepartner sowie deren männliche und weibliche Kinder (,Tante/Onkel, Cousin/Cousine“). Darüber hinaus werden auch die Mutter- und Vatermutter sowie der Mutter- und Vatersvater („Großmutter/-vater"), deren Geschwister („Großtante und -onkel“), die Eltern (,Schwiegereltern“) von Egos Ehemann oder -frau, die Geschwister sowie deren Partner („Schwager/Schwägerin“) und deren männliche und weibliche Kinder („Nichte/Neffe“) dazu gerechnet. Der Kreis, der als verwandt bezeichnet wird, kann jedoch auch noch wesentlich umfangreicher sein.

Ein Kriterium für die Zuordnung einzelner Personen zur Verwandtschaft ist die Kenntnis ihrer genealogischen Position. Wer genealogisch verortet werden kann, muss allerdings wiederum nicht unbedingt als verwandt bezeichnet werden, wie im weiteren Verlauf noch gezeigt wird. An diesem Punkt sind die gewonnenen Daten sehr heterogen. Innerhalb der Kategorie ,Verwandtschaft' wird deutlich unterschieden in ,nahe“ und „entfernte“ Verwandtschaft, eine Differenzierung, die nach unterschiedlichen Kriterien vollzogen wird. Es kann jedoch festgestellt werden, dass kein klar umrissenes und einheitliches Prinzip zur Unterscheidung zwischen „,nahen“ und „fernen“ Verwandten und zur Begrenzung des Personenkreises, der als Verwandtschaft betrachtet wird, zu erken- 
nen ist. ${ }^{120}$ Insbesondere die „ferne“ Verwandtschaft kann zum einen diejenige sein, die Ego als genealogisch entfernt versteht wie beispielsweise Verwandte aus der Generation der Großeltern oder die Kinder der Cousins und Cousinen, zum anderen kann es sich jedoch auch um genealogisch näherstehende Personen handeln wie Tanten, Onkels, Cousins und Cousinen mütterlicher- oder väterlicherseits, zu denen aus verschiedensten Gründen die emotionale Bindung oder der Kontakt fehlt. Bei wenigen befragten Personen handelt es sich dabei auch um Geschwister, in keinem Fall um Vater oder Mutter. Diese Zuordnung von Personen hängt also sowohl von der genealogischen Nähe oder Distanz ab als auch von dem persönlichen Verhältnis, das oft in direktem Zusammenhang steht mit der Kontakthäufigkeit (siehe dazu auch Kap. 5.1.3.2). ${ }^{121}$

Adoptierte Personen gelten immer als Verwandte, wohingegen die persönliche Zuordnung von Stiefeltern und Stiefgeschwistern zu Egos Verwandtschaft abhängig ist von deren persönlicher Kenntnis sowie dem Verhältnis zueinander. „Verwandt“" wird hier eher in einer Bedeutung verwendet, die eng mit Kontakt und Sympathie zu tun hatte, wie die folgenden Beispiele zeigen:

„Da ist zum Beispiel Michaels Stiefoma. Das ist die Mutter von der zweiten Frau seines Vaters. Die wird jetzt 85. Das ist eine total geile Frau finden wir beide, und zu der haben wir einen ganz engen Bezug und besuchen sie oft. Ich sag immer, das ist meine Wahlomi, weil das so eine tolle Frau ist. Ich bewundere sie sehr. Michael geht das genauso. Mit der fühlen wir uns sehr verwandt, obwohl ich es ja überhaupt nicht bin. Michaels Stiefmutter zum Beispiel würde er bzw. hat er noch nie als verwandt bezeichnet. Mit der kann er einfach nicht so. Es ist nicht so, dass sie sich nicht leiden können, aber das Gefühl stimmt nicht so. Geht mir übrigens auch so." (Ute Wille, 36 Jahre)

„Der eine ist mein Vater und der andere ist mein Stiefvater. Der ist ja auch angeheiratet. Mein Vater ist auch noch wichtig, aber mein Stiefvater ... Das hat nichts mit verwandt zu tun, also schon gar nicht mit blutsverwandt, sondern mit der menschlichen Beziehung zueinander. Von meinem Vater her habe ich Stiefgeschwister, aber irgendwie habe ich mit denen nichts zu tun. Ich kenne die ja auch kaum. Ich sehe meinen Vater, aber die beiden eigentlich nie. Daher würde ich die auch nicht als Verwandte sehen, auch wenn sie es schon irgendwie sind über meinen Vater eben.“ (Jörn Budecker, 36 Jahre)

Viele Interviewpartner berichten auch von Personen, zu denen keine konsanguine oder gesetzliche Verbindung existiert, wie beispielsweise Freunde der Eltern, Lebenspartner einer Tante oder eines Onkels väterlicher- oder mütterlicherseits, die Eltern einer ange-

\footnotetext{
120 Lüschen (1988:153) hat in einer Untersuchung, die in Köln und Bremen durchgeführt wurde, gezeigt, dass beim vierten Verwandtschaftsgrad (Kinder oder Ehepartner von Cousins und Cousinen) für die meisten Befragten Verwandtschaft aufhört. In anderen Fällen werden auch Cousins und Cousinen zweiten Grades noch als verwandt bezeichnet (vgl. dazu auch Fenner 1984:38-43).

121 Dies bestätigt die Aussage von Edwards und Strathern (2000:160) über Vorstellungen von Nähe in einer englischen Kleinstadt: „'Closeness' summons affective ties, the obligations and duties such ties entail, and the warmth and mutual care with which relationships are sustained. 'Closeness' also points to distance, and 'distant relatives' are those with whom interaction is infrequent, with whom obligations are at a minimum, and with whom confidences are unlikely to be shared. From this perspective, close relatives can become distant ones."
} 
heirateten Tante oder die Eltern der Frau von Egos Bruder, die jedoch sowohl in der Kindheit als auch zum Zeitpunkt der Interviews mit Verwandtschaftstermini wie Tante oder Onkel angesprochen werden. ${ }^{122}$ Diese Beziehungen sind geprägt von intensiven, oft langjährigen Kontakten sowie von gegenseitiger Sympathie, werden jedoch nicht als Verwandtschaftsbeziehungen definiert. Nur in zwei Interviews finden sich für diese Arten von Zuordnung auch die Verwendungen von „quasi-verwandt“ oder „halbverwandt".

Im folgenden Beispiel wird der Begriff der, Verwandtschaft' noch in anderen Bedeutungen angewendet als bisher dargestellt. So beschrieb Ilona Ottmers (30) ihre Beziehung zu einer Person namens Silke als „Seelenverwandtschaft“ oder „,verwandt im Geiste“:

„Zu der Silke, da habe ich eine ganz besondere Beziehung. Da stimmt einfach alles oder zumindest sehr viel. Das nenne ich dann Seelenverwandtschaft. Was nützt einem die schönste Blutsverwandtschaft, wenn man nicht auch verwandt im Geiste ist. Und mit so vielen Blutsverwandten verbindet mich eben nichts, was den Geist angeht. Und das bin ich mit der Silke auf jeden Fall. Wir kennen uns schon sehr lange, wissen fast alles voneinander, können uns absolut vertrauen und mögen eben auch die gleichen Dinge. Ja, und das ist eben genau das Besondere an der Verbindung, dieses Gefühl einer besonderen Verwandtschaft.“

Mit diesem Begriff bzw. in dieser Verwendung des Begriffes ,Verwandtschaft‘ wird explizit eine persönliche Form von Verbundenheit der beiden Frauen betont, die sich primär durch Sympathie, Zuneigung sowie gemeinsamen Vorlieben und Abneigungen konstituiert. Er drückt in diesem Fall besondere Qualitäten dieser Verbindung aus, die Frau Peters bei vielen Blutsverwandten nicht findet. Damit grenzt sie sich ausdrücklich gegen die Annahme ab, dass Blutsverwandtschaft a priori positive Beziehungen beinhaltet. Sie verwendet den Begriff der, Verwandtschaft' in diesem Zusammenhang metaphorisch, was darauf hinweist, dass Verwandtschaft als eine besondere Form sozialer Beziehungen konzipiert wird, die idealerweise affektive Bindungen beinhaltet.

Das existierende Verwandtschaftssystem in Deutschland wird gemäß verwandtschaftsethnologischer Kategorisierung als bilateral bezeichnet mit bilinearer Deszendenz ${ }^{123}$,

\footnotetext{
122 Die Verwendung verwandtschaftlicher Terminologien in unterschiedlichen Kontexten wird in Kapitel 5.1.1.2 noch detaillierter dargestellt. Auf die in der Literatur als „fiktive“ oder „,spirituelle“ Verwandtschaft (vgl. Goody 1989 [1983]:211-212) bezeichnete Form der Patenschaft werde ich hier nicht näher eingehen, da sie von meinen Interviewpartnern zwar als eine Kategorie von Personen erwähnt wurde, zu denen es enge Verbindungen geben kann, auf die jedoch in den Interviews - auch auf mein Nachfragen - nicht ausführlicher Bezug genommen wurde. Es liegt mir somit kein ausreichendes Datenmaterial vor, um diese Form sozialer Beziehungen in meiner Arbeit analysieren und diskutieren zu können.

123 Bestimmte Deszendenzbeziehungen statten Individuen mit verschiedenen Pflichten, Rechten und Privilegien im Hinblick auf andere Personen wie auf verschiedene Aspekte des Soziallebens aus. Name, Familienzugehörigkeit, Wohnort, Rang, Besitz und ethnischer wie nationaler Status einer Person können von solchen Zuschreibungen aufgrund von Deszendenz abhängen (Harris, M. 1989:177).
} 
die formal keine Unterscheidung trifft zwischen der Matri- und der Patrilinie in aszendenter wie deszendenter Generationenfolge ${ }^{124}$ und die somit zu unendlichen Verzweigungen führt. Es entstehen Verwandtschaftsgruppen, die auch als kindred bezeichnet werden (Harris, M. 1989:182). Sprechen die Interviewpartner von „Familie“ und meinen damit nicht nur ihre Kernfamilie, bestehend aus Eltern und Kindern, so beziehen sie sich demnach auf ihre kindred. ${ }^{125}$ Hauptmerkmal der kindred in Deutschland ist, dass der Weite und Tiefe bilinearer Deszendenzableitung theoretisch keine Grenzen gesetzt sind.

Die Interviews zeigen, dass ,Familie‘ kein homogener Begriff ist, sondern in verschiedenen Kontexten unterschiedlich verwendet wird. Auf meine Frage „Wer gehört zu Ihrer Familie?" wurden bei Paaren ohne Kinder die Eltern und Geschwister genannt, bei Paaren mit Kindern ebenso der Ehepartner und die Kinder. Die Frage „Haben Sie Familie?" wurde im Gegensatz dazu von ersteren häufig verneint, da dabei nach Meinung der befragten Personen die Frage nach Kindern impliziert war. „Nein, ich habe keine Kinder" war darauf eine häufige Antwort. Daran wird deutlich, dass es sich jeweils um einen unterschiedlichen Bezugsrahmen handelt. Bei ersterer Frage bezieht sich die Antwort auf die gleiche und die aufsteigende Generation (Aszendenz), bei zweiterer auf die Filiation bzw. Deszendenz. ,Familie‘ im Unterschied zu ,Verwandtschaft‘ gilt bei den befragten Personen jedoch eher als ein geschlossenes System, das mit Begriffen bezüglich Stabilität, Gemeinschaft und Kommunikation beschrieben wird. ,Familie“ kann somit auch als ein affektiver Terminus bezeichnet werden, der den emotionalen Aspekt einer Beziehung ausdrückt. ,Verwandtschaft‘ stellt im persönlichen Diskurs ein offeneres System dar, in dem ein Wechsel der zugezählten Personen eher möglich ist als bei ,Familie‘.

\subsubsection{Beziehungsterminologien}

Es ist deutlich geworden, dass Verwandtschaft ein Kriterium der Kategorisierung sozialer Beziehungen darstellt, wobei die Kategorie der Verwandten nicht einheitlich definiert ist, sondern wiederum in verschiedene Kategorien eingeteilt ist, die sich unterschiedlich konstituieren. Dies zeigt sich auch an der Verwendung von Verwandtschaftsbezeichnungen, mit denen man Verwandte anredet oder (anderen gegenüber) bezeichnet. Verwandtschaftsterminologien haben seit der Etablierung der Ethnologie Mitte des

\footnotetext{
124 „Innerhalb ein und derselben Gesellschaft können verschiedene Arten der Deszendenzableitung vorkommen, sofern sich die Abstammungsregeln auf verschiedene Vorstellungs- und Verhaltensbereiche beziehen“ (Harris, M. 1989:181).

125 Daraus folgt, dass Ego und Egos Geschwister einer kindred angehören, die nur auf sie hin definiert werden kann, d.h., dass verschiedene Personen immer unterschiedlichen kindreds angehören.
} 
19. Jahrhunderts eine große Rolle gespielt und sind mit dem Namen Lewis Henry Morgan verbunden (Kap. 2.1.1). Ein Merkmal der Verwandtschaftsterminologie in Deutschland ist die Unterscheidung von Bezeichnungs- und Anredeformen. Erstere entsprechen einem theoretischen Modell, bezeichnen eine soziale Kategorie und geben die sozialen Erwartungen an eine Beziehung an, die mit einem bestimmten Verwandtschaftsterminus verbunden sind, zweitere bieten ein breites individuelles Spektrum und drücken die jeweils individuelle persönliche Beziehung aus (Fehlmann-von der Mühll 1978:12). Die Verwandtschaftsterminologie ist demnach die Gesamtheit und das System aller solcher Termini in einer Kultur und muss jeweils von einem Ego aus betrachtet werden (vgl. Fischer 1990:97ff). Ich werde im Folgenden detaillierter auf die Begriffe eingehen, die sich aus meinem empirischen Material herauskristallisiert haben zur Bezeichnung und zur Anrede von Personen, die Ego als Verwandte versteht, ebenso wie auf die „fiktive“ Verwendung von Verwandtschaftstermini (vgl. Fehlmann-von der Mühll 1978:11). Dabei lege ich ein Hauptaugenmerk auf die Art und Weise der Anrede und die damit zum Ausdruck gebrachte (als positiv oder negativ empfundene) persönliche Beziehung. ${ }^{126}$

Die Bezeichnungsformen setzen sich aus Grundformen sowie abgeleiteten Formen zusammen. Als Grundform gilt beispielsweise der Terminus Mutter, der zu Großmutter, Schwiegermutter oder Stiefmutter modifiziert werden kann (vgl. Schneider 1980 [1968]:22). Im Deutschen kommt auch die Kombination von Verwandtschaftsbezeichnung plus Name in Anrede und Bezug (,Tante Maria“) vor. Weiterhin gibt es Verwandte, die nur mit dem Namen angeredet werden, von denen anderen gegenüber jedoch die Verwandtschaftstermini verwendet werden (,mein Bruder"). Meine Interviews zeigen, dass die zusammengesetzten Formen in der Anrede kaum gebraucht werden. So reden beide Ehegatten ihre Affinalverwandten mit denselben Formen an: Vornamen für Schwiegereltern und Geschwister des Ehepartners, in nur 4 von 35 Fällen werden die jeweiligen Schwiegereltern mit „Mutter/Vater“ bezeichnet. Im Regelfall werden diese Personen geduzt, wobei sich anhand meines empirischen Materials feststellen lässt, dass die direkte Anrede der angeheirateten Verwandten oftmals nach Möglichkeit vermieden wird. Am deutlichsten fällt es bei Egos Schwiegereltern auf, da offensichtlich eine gewisse Zurückhaltung existiert, eine andere Frau als die eigene Mutter mit dem Terminus ,Mutter' oder gar ,Mama' anzureden ebenso wie einen anderen Mann mit dem Terminus ,Vater' oder ,Papa'. Die Zitate aus Interviews mit Elfriede Zimmermann (35) und Sybille Mainer (36) zeigen, dass diese Termini mehrheitlich für die biologischen Eltern,

\footnotetext{
126 Vgl. dazu auch Edwards (2000:232), die konstatiert, dass „kin terms are not sluggish; they do more than fix and locate people in relation to each other, they also connote special types of relationship and are thought to create particular kinds of ambience, with or without (or with more or less) shared substance."
} 
insbesondere für die eigene Mutter, gebraucht werden, da sie eine einmalige, vertraute und emotionale Beziehung zum Ausdruck bringen sollen.

„Meine Mutter ist die Mutti oder die Mama, meine Schwiegermutter wird seit jeher von allen Mutter genannt, was für mich am Anfang sehr komisch war. Es war ja nicht meine Mutter und ich hatte sie dann mit ,Frau' und dem Familiennamen angesprochen zuerst. Das fand sie gar nicht gut und sie sagte dann ,Ich bin die Mutter für alle‘. Und dann habe ich gesagt na gut, dann ist es halt die Mutter. Alle anderen werden mit dem Vornamen angesprochen."(Elfriede Zimmermann)

„Ich könnte zu meinen Schwiegereltern nie Mama und Papa sagen. Weil ich nur einen Vater und eine Mutter habe. Das ist etwas ganz anderes. Da ist man sich ganz anders vertraut. Und die liebe ich eben auch ganz anders und sie mich.“ (Sybille Mainer)

Es zeigt sich auch, dass sich die Situation bei der Geburt eines Kindes auflösen kann, da die Schwiegereltern dann oft mit Oma und Opa bezeichnet und die direkten Anreden vermieden werden. So stellte Helga Bachmann (39) fest:

„Also das war schon irgendwie eine große Erleichterung, als unser Sohn kam. Da konnte ich dann immer über Oma und Opa sprechen, und wenn wir mit ihnen zusammen waren, habe ich es eigentlich immer über meinen Sohn laufen lassen. Ich meine, ich habe einfach vermieden, sie direkt anzusprechen. Mit einigen Ausnahmen klappt das auch ganz gut. Ich weiß nicht, ob sie es merken, aber sie reden jetzt von sich auch fast nur noch von Oma und Opa."

Wie ich schon im vorherigen Kapitel beschrieben habe, wird das deutsche Verwandtschaftssystem in der Verwandtschaftsethnologie als bilateral kategorisiert, was bedeutet, dass sowohl die patri- als auch die matrilinearen Verwandten theoretisch gleichbedeutend sind ${ }^{127}$ (was sich auch in der verwandtschaftsterminologischen Gleichsetzung von Tanten und Onkeln mütterlicherseits und väterlicherseits ausdrückt ebenso wie in der Symmetrie in den Begriffen für Bluts- und Affinalverwandte). ${ }^{128}$ Mein empirisches Material zeigt jedoch deutliche Unterschiede im sozialen Handeln und in den Anredeformen. Die Varietäten sind beispielsweise im Gebrauch der Anredeformen zu finden. Im Fall von Tanten und Onkels mütterlicher- wie väterlicherseits wird die Bezeichnungsform in der Anrede in erster Linie von der jüngeren zur älteren Generation verwendet, was als Zeichen von Respekt gegenüber der Autorität des Alters gesehen wird.

\footnotetext{
127 Das Verwandtschaftssystem, dass den Euro-Amerikanern vertraut ist, wird in der Ethnologie als Eskimo-System bezeichnet (Murdock 1949:223f). Folgendes sind die wichtigsten Merkmale dieses Systems: 1. Keine der Bezeichnungen für Egos Kernverwandte wird außerhalb der Kernfamilie benutzt. 2. Es wird keine terminologische Unterscheidung zwischen Verwandten mütterlicherseits und väterlicherseits gemacht. Das heißt, Kreuz- und Parallelcousins/-cousinen und Kreuz- und Paralleltanten/-onkel werden terminologisch miteinander gleichgesetzt. In diesen Merkmalen spiegelt sich die Tatsache wider, dass Gesellschaften, die die Eskimo-Terminologie verwenden, im allgemeinen keine korporierten Abstammungsgruppen umfassen. So sind für Ego sowohl Mutters- bzw. Vatersschwester „Tante“ und „Onkel“ als auch deren angeheiratete Partner.

128 Vowinckel (1995:84) beschreibt jedoch, dass diese verwandtschaftsterminologische Gleichsetzung bis zur Mitte des 16. Jahrhunderts in dieser Form nicht existierte. Die Geschwister des Vaters wurden ursprünglich als Base und Vetter bezeichnet, die Geschwister der Mutter als Muhme und Oheim.
} 
In vielen Fällen wird die Anrede ,Tante' oder ,Onkel' dann weggelassen, sobald der Neffe oder die Nichte ein gewisses Alter erreicht hat. Wie Elfriede Zimmermann (35) erzählte, fordern entweder die Älteren die Jüngeren direkt dazu auf, „doch nun endlich mal das Tante oder Onkel wegzulassen“, oder die Jüngeren verwenden von sich aus nur noch die Vornamen zur Anrede.

E.Z.: „Also ältere Personen habe ich schon mit Tante und Onkel angesprochen. Früher als Kind die Geschwister meiner Eltern. Das tue ich eigentlich nicht mehr.“

I.S.: „Und wann hat sich das geändert?““

E.Z.: „Als ich dann älter wurde, so 16 oder 17 Jahre. Entweder die haben das direkt gesagt oder man hat das gespürt, dass das nicht mehr erwünscht war. Und bei den jüngeren Personen, da ist das nicht mehr so.“

I.S.: „In welche Generation würden Sie denn die Anrede ,Tante' einordnen?“

E.Z.: „Ich nenne nur noch eine Person Tante, und die ist jetzt so um die 65. Also schon ältere Personen. Die Onkels so um die 50 spreche ich mit Vornamen an, die sind eigentlich alle recht jung geblieben und wollen das so. Da habe ich einfach irgendwann den Vornamen verwendet und das war völlig okay.“

In welcher Beziehung stehen jedoch Verwandtschaftsterminologien und das konkrete interpersonale Verhalten zueinander? Gibt es eine gegenseitige Beeinflussung von theoretischem Modell und aktueller Realität? Wie im Folgenden deutlich wird, müssen beide Bereiche zum gegenseitigen Verständnis bekannt sein, und es existieren Wechselwirkungen zwischen ihnen. Nach Schneider (1980 [1968]) ist das Weglassen der verwandtschaftlichen Anredeformen als Zeichen einer intimen affektiven Beziehung zu deuten, die auf bewusster gegenseitiger Zuneigung beruht und nicht auf einem vorgegebenem Verwandtschaftsgrad. Die Interviews zeigen jedoch, dass die ausschließliche Verwendung des Vornamens einerseits eine nun eher egalitäre Beziehung ausdrücken kann und damit Zeichen eines persönlichen Verhältnisses ist, andererseits wird gerade die Bedeutung der Benutzung einer Bezeichnungsform auch in der direkten Anrede als Kosewort hervorhoben, um somit eine besondere affektive Beziehung ausdrücken. Wie heterogen die Verwendung der Anredeformen sein kann, zeigt auch das folgende Beispiel von Tim Bergmann (38). Hier werden ausschließlich die Vornamen verwendet, um auszudrücken, dass kein persönliches Verhältnis vorhanden ist (vgl. auch Strathern 1992a:20):

„Also meine Onkels und Tanten nenne ich nur mit Vornamen. Und das mache ich ganz bewusst. Da ist das Verhältnis einfach nicht da. Ich muss gestehen, ,Tante' oder ,Onkel ${ }^{6}$ zu sagen wäre mir da einfach zu intim und persönlich. Das Verhältnis zur Putzfrau ist herzlicher als das zu meinem Patenonkel. Das liegt in den Wurzeln, die die Familie schon vor Ewigkeiten entzweit haben."

Aber auch Personen, die von Ego nicht der Kategorie ,Verwandte ${ }^{6}$ zugeordnet werden, werden mit Verwandtschaftstermini bezeichnet. So gibt es Beispiele, dass Nachbarn, zu 
denen guter Kontakt seitens Egos Eltern gepflegt wird, oder Freunde der Eltern in der Kindheit als „Tante“ oder „Onkel“ angeredet wurden oder noch werden. Daran wird deutlich, dass mit diesen Kategorien bestimmte kulturell geprägte Vorstellungen verbunden sind, die nicht dem genauen biologischen Verwandtschaftsgrad entsprechen müssen: Was eine Tante oder ein Onkel ist, und wie sie oder er sich zu verhalten hat, findet auch in der sprachlichen Kategorisierung ihren Ausdruck. Menschen, die biologisch nicht verwandt sind, werden oftmals als „Tante“ bezeichnet, wenn sie eine bestimmte soziale Rolle erfüllen. Wird bei Verwandten, wie oben beschrieben, die Bezeichnungsform als Anrede ab einem gewissen Alter oft weggelassen, kann sie im Fall von „nicht-verwandten“ Personen bestehen bleiben. Robert Toll (39) und Melanie Toll (32) erzählten, dass sie die Verwendung des Vornamens beispielsweise als der Beziehung nicht angemessen empfänden:

R.T.: „Ich kann sie doch jetzt nicht wieder siezen, da ich sie schon so lange kenne. Aber nur mit dem Vornamen anreden geht auch nicht, dazu ist die Beziehung nicht eng oder zumindest nicht formal verwandtschaftlich. Ist schwer auszudrücken. Es ist kein Problem, Verwandte irgendwann nur noch mit dem Vornamen anzureden. Meist passiert das, wenn man sowieso oft was zusammen macht. Aber auch die, die man nicht so oft sieht, sagen bei einem Familienfest plötzlich: ,Nun lass mal gut sein mit Tante und Onkel, sag einfach Brigitte und Dieter'. Aber unsere Nachbarin, die ist jetzt 70 Jahre, die sagt doch so was nicht zu mir. Also was mach ich? Ich bleib einfach weiter Kind und sage Tante, dann gibt's keine Probleme. Meine Frau sagt auch Tante zu ihr, siezt sie aber. Verstehe ich auch, sie kennt die Frau ja auch nicht. Ich duze sie natürlich. Das Problem gibt es übrigens auch ständig auf irgendwelchen Familienfeiern. Da kommen dann unzählige Verwandte von mir, die ich selber ja noch nicht mal unbedingt alle kenne. Manchmal weiß ich noch nicht mal, wie die heißen. Wenn, dann sage ich zu meiner Frau ,Das ist die Tante Ilse, das ist die Cousine zweiten Grades von meiner Mutter'. Meine Frau sagt dann ,Hallo, nett sie kennen zu lernen' oder so was ähnliches. Manchmal sagt dann so eine Tante, Sag ruhig du zu mir und nenne mich auch Tante Ilse'. Oft sagen sie aber auch nichts. Dann weiß meine Frau nicht, was sie machen soll. Am besten gar nicht ansprechen. Aber wenn es nicht anders geht, sagt sie ,sie' und Frau Hermann.“

M.T.: „Das ist echt schwierig in solchen Situationen. Was machst du? Willst nicht unhöflich sein, aber das sind doch fremde Leute. Da kann ich doch nicht einfach ,Tante' sagen. Da komme ich mir blöd vor. Der Vorname wäre aber zu direkt. Ich versuche immer, möglichst drum herum zu reden und vermeide jede persönliche Ansprache. Ich mein, ich habe doch einfach nichts mit denen zu tun, außer dass sie irgendwie mit meinem Mann verwandt sind.“

R.T.: „Allerdings geht es mir bei euren Treffen genauso. Habe immer keine Ahnung, wer das ist und wie ich die nennen soll. Die meisten sind aber gut drauf und bieten von selbst das Du an und den Vornamen. Bei meinen eigenen Leuten ist das alles irgendwie selbstverständlich, bei ihren muss ich immer erst überlegen.“

Das oben zitierte Beispiel zeigt auch die Unsicherheiten auf, die im Zusammenhang mit dem Siezen oder Duzen von Personen verbunden sind. Duzen wird als Ausdruck einer persönlichen Beziehung verstanden. So werden in der Regel die Verwandten der Matri- 
und der Patrilinie selbstverständlich geduzt (,schließlich ist man ja verwandt“), im Fall der Affinalverwandten über eine Eheschließung (hier „Schwiegerverwandtschaft“ genannt) kann es jedoch zu Verlegenheiten kommen. So stellen sich zahlreiche meiner Interviewpartner die Frage, ob Personen, die man vorher noch nie gesehen habe und die man auf Festen und Feiern kennenlerne, nun geduzt werden, da sie mit dem Ehepartner verwandt seien, oder weiterhin gesiezt werden sollen, da sie ,ja eigentlich Fremde sind“. Die „eigene“ Verwandtschaft scheint demzufolge mit einer gewissen Grundvertrautheit verknüpft zu sein, die dazu führt, dass Personen, egal wie genealogisch und altersmäßig entfernt sie von Ego sind, und auch wenn man sie bis zum Erwachsenenalter noch nie gesehen hat, sich in der Regel gegenseitig duzen und es darüber nur in den seltensten Fällen Unsicherheiten gibt. Die von mir Befragten berichten explizit über ihre Schwierigkeiten in dieser Hinsicht mit den Affinalverwandten, die bei „fremden“ Personen so nicht auftauchen. Wenn sich Fremde mit dem Vornamen anreden, drückt das im Erwachsenenalter meistens eine persönliche Beziehung aus, wobei diese unterschiedlich eng sein kann. In diesem Zusammenhang werden auch „Bekannte“, „Freunde“ und „Arbeitskollegen“ erwähnt. Es lässt sich feststellen, dass diese Beziehungen, außer zu den Arbeitskollegen, als freiwillig ausgesuchte Beziehungen definiert werden, bei denen das Duzen und die Anrede des Vornamens entweder vom Zeitpunkt des Kennenlernens an oder erst später von beiden Parteien im gegenseitigen Einverständnis ausgehandelt wird. Eine Ausnahme bilden hier die (sich in hierarchisch gleicher oder ähnlicher Position befindlichen) Arbeitskollegen, bei denen des öfteren der „Zugzwang“ erwähnt wurde, der dazu führte, dass ,,wer dazu gehören will, auch mitduzen muss, sonst ist man schnell außen vor. Das gehört einfach mit dazu. Siezen tun wir natürlich die Abteilungsleiter und die anderen von oben. “ Arbeitskollegen fallen explizit in die Kategorie von Personen, mit denen man zwar im beruflichen Kontext ein gutes Verhältnis haben könne, was jedoch keine Bedeutung für das Privatleben haben müsse (wobei hier Ausnahmen durchaus vorkommen und Kollegen oder Kolleginnen auch gute Freunde sein können).

\subsubsection{Ein Konzept von Heirat und Ehe}

Wie ich in Kapitel 4.1.2 gezeigt habe, definiert das Bürgerliche Gesetzbuch (BGB) die Institution der Ehe als einen sozialen Kontrakt, als rechtlich anerkannte, auf Lebensdauer angelegte Lebensgemeinschaft zwischen Mann und Frau, die durch eine Willenserklärung beider Partner zustande kommt und durch die Heirat eine öffentliche Bestätigung erfährt. Auch in der Ethnologie versteht man unter Heirat den rechtlich-sozialen Akt, durch den die Gemeinschaft von Mann und Frau als Ehegemeinschaft hergestellt 
wird. Oft zählen dazu auch eine kirchliche Zeremonie und ein Fest (Bargatzky 1997:105). ${ }^{129}$ Eine Heirat bedeutet einen Wandel im Status jeder Person (von „ledig“ zu „,verheiratet“/,Ehemann“ und „Ehefrau“) und führt zu neuen sozialen Beziehungen (Affinalbeziehungen). Mit dem Ehegatten gilt man in Deutschland per Gesetz weder als verwandt noch als verschwägert, wohingegen die Verwandten des Ehegatten zwar laut Bürgerlichem Gesetzbuch (BGB) nicht als Verwandtschaft, jedoch als Schwägerschaft anerkannt werden. Mein Datenmaterial zeigt jedoch, dass diese Unterscheidung in der deutschen Alltagssprache nicht gebräuchlich ist.

In Deutschland sowie in den meisten westeuropäischen Gesellschaften wird die Ehe heute als emotionale und freiwillig eingegangene Verbindung anerkannt (vgl. auch Fehlmann-von der Mühll 1978:37). Es existieren zwar bestimmte gesetzliche Regelungen für die Partnerwahl (siehe Kap. 4.2), überdies gilt die Ehe jedoch als eine prinzipiell individuelle Angelegenheit. Die ehelichen Verbindungen führen zu neuen rechtlich anerkannten Zugehörigkeiten (siehe Kap. 4.1 „Schwägerschaft“), jedoch existieren in Deutschland nur wenige gesetzlich festgelegte Regelungen, die das soziale Handeln zwischen diesen Personen bestimmen (Kap. 4.2). Sie beschränken sich auf Unterhaltspflichten gegenüber den direkten Verwandten und dem Ehepartner sowie auf Erbrechte, die sowohl Verwandte in gerader Linie als auch die Seitenverwandten berücksichtigen. Egos affinale Beziehungen sind von diesen Bestimmungen nicht betroffen, d.h. moralische und ökonomische Obligationen können zwischen den Individuen ausgehandelt werden. Die durch die Schließung einer Ehe rechtlich anerkannten Zugehörigkeiten und Angehörigkeiten müssen somit auch nicht zwangsläufig zu aktiven Handlungsbeziehungen zwischen den jeweiligen Verwandtschaftsgruppen der Eheleute führen, die wie in vielen außereuropäischen Gesellschaften - auf dem Prinzip der Reziprozität beruhen (Holy 1996:129).

Der Aspekt der Freiwilligkeit ist in Deutschland eine wesentliche Grundvoraussetzung für die Eheschließung zwischen zwei Personen. Die freiwillige und persönliche Auswahl des Ehepartners nach bestimmten Kriterien haben wesentlichen Einfluss auf die Vorstellungen von einer ehelichen Lebensgemeinschaft. Ausschließlich die emotionale Zuneigung zu einer bestimmten Person wurde in den von mir geführten Interviews als Grund genannt, mit dieser das ganze Leben verbringen zu wollen, wie die folgenden Zitate exemplarisch zeigen:

\footnotetext{
129 In vielen außereuropäischen Gesellschaften gilt die Ehe häufig als ein Kontrakt zwischen den Verwandten des Mannes und denen der Frau, der die gegenseitigen Beziehungen beider Seiten festlegt, die in der Regel über den Tod der Ehepartner hinausgehen. Bestimmungen wie Levirat (der Bruder des verstorbenen Ehemannes oder ein anderer Verwandter heiratet die Witwe) und Sororat (der Witwer heiratet eine Schwester oder andere Verwandte der Frau) sorgen für den Fortbestand dieser Beziehungen. In patrilinearen Gesellschaften gelten beispielsweise die Kinder, die der Bruder des verstorbenen Mannes mit der Witwe zeugt, nicht als seine, sondern als die Kinder seines verstorbenen Bruders, da in dessen Namen der „Brautpreis“ gezahlt wurde (vgl. Holy 1996:128).
} 
„Ich bin eine Romantikerin. Eine Heirat in Weiß war von klein auf die logische Konsequenz, wenn ich den entsprechenden Partner dazu gefunden habe. Die Ehe ist mir sehr wichtig. Sie sollte auf Lebenszeit halten und durch dick und dünn gehen. Eine lebenslange Partnerschaft ohne Rücktrittsrecht. Das ist neben der Familie der Haltpunkt, wo ich mich vollkommen fallen lassen kann, wo ich nichts spielen muss." (Yvonne Menge, 36 Jahre)

„Heiraten tut man ausschließlich aus Liebe zum Anderen. Wirtschaftliche Gründe gibt es doch heute keine mehr. Steuern kann man kaum sparen und Zusammenleben tut man doch auch schon. Es ist eine Bestätigung, dass man sich liebt und das ganze Leben zusammen verbringen will.“ (Wilfried Stiepel, 38 Jahre)

In Kapitel 4.2 konnte ich außerdem zeigen, dass gesetzlich festgelegte Verpflichtungen für die jeweiligen Ehepartner existieren, die das eheliche Miteinander regeln, wozu unter anderem der gegenseitige Anspruch auf Schutz und Versorgung gehört. Dieser Aspekt der Reziprozität sowie das Gefühl der Solidarität finden sich auch in meinen Interviews. Das Gefühl zusammen zu gehören, füreinander da zu sein und das Leben gemeinsam meistern zu wollen, beinhaltet in der Vorstellung auch den Beistand im Falle von Krankheiten oder bei anderen schwerwiegenden Problemen, wie das Ehepaar Britta und Hans Kuschnick (31 und 37 Jahre) erzählte:

B.K.: „Die Ehe ist eine Gemeinschaft, wo der eine für den anderen da ist. Liebe gehört auch mit dazu, aber ich sage immer, je älter man wird, desto weniger bedeutet einem Sex. Es ist die Gemeinschaft, jemanden zu haben, auf den man vertrauen kann, der für einen da ist in allen Situationen. Das ist für mich die Ehe. Man geht ein Leben gemeinsam.“

H.K.: „Das geht mal auf, mal ab, aber man ist füreinander da und hilft sich so gut es geht. Eben auch, wenn man krank ist, dass man sich auf den anderen verlassen kann, dass der einen pflegt oder sich um einen kümmert. Genauso, wie wenn man zusammenlebt, wenn es einem gut geht. Und wir haben gerade auf uns bezogen schon so viel in unserer Ehe durchgemacht durch das Ganze [die „ungewollte Kinderlosigkeit“]. Und das sind ja alles Belastungen, die an einer Ehe reißen. Und das immer alles durchzustehen, da ist es gut, wenn man viel Verständnis füreinander hat. Und den anderen respektieren, das ist das Wichtigste. Wenn man sagt, mit dem möchte ich alt werden. Manchmal in Krisensituationen, wenn man denkt, es geht nicht mehr, hält einen die Ehe auch zusammen. Dass man eben mal geheiratet hat und sich zueinander bekannt hat. Ohne die Ehe wäre es vielleicht bei uns auch schon mal eher auseinandergegangen. Aber irgendwas hat uns immer zurückgehalten. Vielleicht so das Ideal, wenn man verheiratet ist, dann kann man das schaffen, dann sollte man eben für den anderen da sein und sich nicht aus dem Staube machen."

Die Zugehörigkeit und „das Bekennen“ zum Ehepartner kann auch durch einen gemeinsamen Ehenamen demonstriert werden. Das bundesdeutsche Recht bietet seit 1993 die Möglichkeit, dass beide Ehepartner ihren vor der Ehe geführten Namen beibehalten und einen der beiden Namen als sogenannten Familiennamen bestimmen können, was im Fall gemeinsamer Kinder von Belang ist. Die im Kontext der Reproduktionsmedizin geführten Interviews zeigen aber, dass die alte Regelung dominiert, bei der der Ehena- 
me virinominal bestimmt wird (vgl. Fenner 1984). Die befragten Frauen entschieden sich bewusst dazu, den Namen ihrer Ehemänner anzunehmen und empfanden diesen Schritt als bewusstes Bekennen zum Partner, wie hier bei Britta Kuschnick (31) und Marion Glück (33) deutlich wird:

„Ja, das war eine bewusste Entscheidung. In gewissen Sachen bin ich da vielleicht ein bisschen konservativ, und das ist ein Schritt, den man sich bewusst überlegen sollte. Name behalten oder Doppelname ist nicht Wasser, nicht Wein, das ist sich irgendwie nicht bekennen können." (Britta Kuschnick)

„Schon wie wir das Aufgebot bestellt haben, da wird man danach gefragt. Und das war für mich von vorneherein klar, dass ich den Namen meines Mannes annehme. Da hat es auch keine Diskussionen gegeben wegen Doppelnamen und so. Die mag ich sowieso nicht, muss ich ehrlich sagen. Da muss man soviel schreiben, und manche passen auch nicht so zusammen. Vielleicht dass mein Mann ... aber der wollte das auch gar nicht. Und mir war das egal. Es ging ja darum, dass wir heiraten, dass wir eine Familie gründen wollen, und da spielt der Name eigentlich keine Rolle. Wobei es schon ein sich bekennen zueinander ist, auch nach außen hin. Da ist eben klar, dass man zusammen gehört.“ (Marion Glück)

Von 42 interviewten verheirateten Personen im Kontext der Reproduktionsmedizin, trugen nur zwei Frauen ihre jeweiligen Geburtsnamen auch nach der Eheschließung weiter, drei hatten einen Doppelnamen. In keinem Fall nahm der Ehemann den vor der Ehe geführten Namen der Frau an.

Im Zusammenhang mit der Vorstellung von und der Entscheidung für eine Ehe spielt der Aspekt eines gemeinsamen Kindes und damit die Gründung einer Familie für die Paare eine bedeutende Rolle. Wie das folgende Zitat beispielhaft zeigt, empfand Markus Treis (30), dass ein Paar ohne Kind vom sozialen Umfeld nicht als gleichwertige feste Gemeinschaft angesehen wird wie ein Paar mit Kind(ern), definierte jedoch auch persönlich eine Partnerschaft ohne Kind nicht als Familie:

„Zu einer Familie gehören für mich Kinder. Als Paar bleibt man doch immer zu zweit, zwei einzelne Personen. Es wird von anderen nie als so etwas Festes und Verbundenes angesehen, als wenn man Kinder hat. Aber mit Kindern wird man zu einer Familie. Man wird zu einer Einheit, man gehört zusammen. Und dieses Gefühl von Zusammengehörigkeit als Familie gibt nochmal eine andere Geborgenheit als vorher. Die Situation verändert sich, es beginnt irgendwie ein neuer Lebensabschnitt.“

Maike Schmidt (34) betonte den Aspekt des Fortbestandes der Familie, wobei sich aus dem mir vorliegenden Gesamtinterview ergibt, dass hier im Zusammenhang mit dem Fortbestand der Familie sowohl die Herkunftsfamilien der Ehepartner gemeint sind als auch das Paar selbst, das ihrer Ansicht nach in einem Kind weiterlebe:

„Ich möchte einfach, dass unsere Familie fortbesteht, dass es nicht einfach irgendwann zu Ende ist. Beide Zweige, von ihm und von mir, leben ja in so einem Kind weiter. Und wir beide als Familie auch. Außerdem fragt man sich ja doch, was kommt denn dabei heraus, also ich meine, so von einem selbst. Sieht das Kind vielleicht aus wie ich, oder 
wie mein Mann, oder wird es eine Mischung aus uns beiden. Das wünscht man sich doch, dass die Eigenschaften, die man an dem Partner so liebt, dass die sich auch in dem Kind wiederfinden. Ja, und die eigenen natürlich auch. Eben eine Mischung wäre das Optimale. [...] Mit einem eigenen Kind wäre nach einem auch nicht einfach alles vorbei, da kommen vielleicht noch Enkel und so geht die Familie weiter."

Ein gemeinsames leibliches Kind wird von den Befragten als Ausdruck einer besonders engen affektiven Bindung zwischen den Ehepartnern genannt und als ,Produkt ${ }^{6}$ einer Liebesbeziehung. Der „Kinderwunsch“ resultiert aus dieser Liebesbeziehung, wie das Ehepaar Juliana und Tim Bergmann (33 und 38 Jahre) berichtete:

T.B.: „Das ist der Wunsch, ewig zusammen zu leben und zu bleiben. Wenn man das Gefühl hat, das ist der Partner, mit dem man sich ideal ergänzt, sich gegenseitig einfach gut tut. Das Zusammenleben lohnt sich für jeden. Auch der Zusammenhalt und der Kinderwunsch, oder diese Möglichkeit, eine Familie zu gründen.“

J.B.: „Eine Ehe ist, Lust zu haben, mit jemandem zusammen zu leben. Im Augenblick, und ich hoffe, dass es so bleibt, dass man zusammen alt werden kann. Möglichst viel Zeit zusammen verbringen. Und der Kinderwunsch entsteht daraus. Ich habe immer gedacht, irgendwann werde ich mal Kinder haben, aber als mein früherer Freund ganz viele haben wollte, da habe ich die Koffer gepackt. Das war nicht der richtige Mann. Ich denke, dieser Kinderwunsch resultiert aus einer Beziehung. Dass man das Vertrauen hat, dass man das wagen kann. Weil man sich ja schon in eine ziemliche Abhängigkeit begibt."

Weiterhin kristallisiert sich aus den Interviews heraus, dass für die Paare eine ideale Vorstellung von der biologischen und sozialen Einheit der Kernfamilie bestehend aus Eltern und Kind(ern) dominiert. Der individuelle „Kinderwunsch“ ist zwar oftmals schon vor der Heirat vorhanden, jedoch ist die Vorstellung von einem gemeinsamen Kind dem Konzept von Ehe im Kontext der Reproduktionsmedizin inhärent. ${ }^{130}$ Ein Paar definiert sich mit seinen Nachkommen als ,eigene“ Familie, die auch als emotionale Einheit mit engen und intimen Bindungen gilt. Ein gemeinsames Kind wird oft als „Zeichen der Liebe" bezeichnet und als Erweiterung der Partnerschaft angesehen.

Sa.H.: ,Ich wollte schon immer eine Familie mit Kindern. Jemanden, mit dem ich mich ganz tief verbunden fühle. Und dann ein gemeinsames Kind. Also schon eine Erweiterung, eine Bereicherung. Ich könnte mir nicht vorstellen, ein Kind zu kriegen ohne meinen Mann. Für mich ist das eine Erweiterung der Partnerschaft. Ich glaube, mit ihm ein Kind zu haben und von ihm. Das ist es beides. Das ist auch eine Bewusstseinserweiterung, eine Erweiterung der eigenen Person, sich selber in neuen Räumen erleben. Sich selber auch als Paar erleben."

St.H.: „Außerdem weiß man dann, wem man sein Haus und all den Kram vererben kann. Das ist doch ein ganz anderes Gefühl, als wenn man weiß, dass alles, was man

\footnotetext{
130 Siehe dazu auch Nave-Herz (1987:22), die von einer „kindorientierten Ehegründung“ spricht, und die der Auffassung ist, dass erst die emotionale kindorientierte Partnerbeziehung gegenwärtig zur Eheschließung führt.
} 
sich aufgebaut hat, an andere Leute geht.“ (Ehepaar Sabine und Stefan Hiller, 33 und 38 Jahre)

An obigen Aussagen wird deutlich, dass hier Kinder als Teil der Liebesbeziehung der Erwachsenen gelten, aus der der „Kinderwunsch“ entspringt. Kinder zu bekommen mit dem Partner wird auch als Teil eines neuen Lebensabschnittes bezeichnet, der häufig nach beruflicher Qualifikation, langjähriger Arbeit, nach dem Kauf einer Wohnung oder einem Hausbau beginnen soll, wobei letzteres eng verknüpft ist mit der Vorstellung von Vererbung und Weitergabe von Eigentum an Nachkommen. ${ }^{131}$ Anhand meines empirischen Materials zeigt sich allerdings, dass gerade die Frauen ein Konzept von einer Mutter- und Familienrolle internalisiert haben, das oftmals in direktem Widerspruch zu ihrer Erwerbstätigkeit zum Zeitpunkt der Interviews steht, welches aber eine zentrale Bedeutung für ihre Lebensplanung zu haben scheint: Die Eheschließung wird als Voraussetzung für die spätere Geburt von Kindern angesehen, die wiederum eng verknüpft ist mit der Vorstellung einer ganzen oder zumindest teilweisen Berufsunterbrechung in einer neuen Lebensphase als Mutter. Insbesondere bei den Frauen, die sich schon länger in einer reproduktionsmedizinischen Behandlung befinden, lässt sich eine zunehmende Orientierung an einer ,traditionellen' Mutterrolle feststellen. Nur wenige Frauen halten den Willen beispielsweise nach einer Teilzeitbeschäftigung aufrecht, im Gegenteil äußern viele den Wunsch, bei ,erfolgreicher' Therapie ganz auf die Berufstätigkeit zu verzichten. ${ }^{132}$ Es wird jedoch deutlich, dass bei den meisten der interviewten Frauen eine ,traditionelle' Familienorientierung schon zu Beginn der reproduktionsmedizinischen Behandlung vorhanden ist, die durch die Reproduktionstechnologien noch verstärkt wird und eine individuelle Lebensplanung ohne Kind kaum noch zulässt. Kinder bleiben somit individuell und gesellschaftlich ein zentrales Moment im Leben der von mir interviewten Personen, insbesondere der Frauen. Dies widerspricht den Aussagen von Beck-Gernsheim (1988:110, 113), die davon ausgeht, dass Kinder in den letzten Jahren eine immer geringere Bedeutung erhalten, d.h. nicht mehr das vorrangige Lebensziel darstellen. Mein Datenmaterial zeigt jedoch, dass es primär eher um den ,richtigen Zeitpunkt $^{6}$ des Kinder bekommens als neuen Lebensabschnitt mit neuen Erfahrungen, verknüpft mit dem Bedürfnis nach einer bürgerlichen Kleinfamilie geht, in der sich die beiden Verwandtschaftsprinzipien von Deszendenz bzw. Filiation und Affinalität verbinden (vgl. Schütze/Wagner 1998:7). ${ }^{133}$

\footnotetext{
131 Siehe dazu auch den Kontext von Adoptionen und Pflegschaften in Kapitel 5.2.

132 Stein und Sproll sind der Ansicht, dass viele Frauen durch Kinder eine Aufgabe zu finden meinen, die ihnen ein Abwenden von der leistungs- und konkurrenzorientierten Männerwelt ermöglicht. Somit würden Kinder funktionalisiert zur (Neu-)Bestimmung weiblicher Lebensentwürfe entweder außerhalb der Berufswelt oder durch Schaffung neuer Berufsperspektiven, wie z.B. durch Berufswechsel oder Arbeitszeitverkürzung (1995:68). Ich möchte diesen Aspekt hier erwähnen, kann dazu aber aufgrund meines empirischen Materials keine eigenen Aussagen dazu machen.

${ }^{133}$ Siehe dazu auch Kapitel 5.1.4.2.
} 


\subsubsection{Die Beendigung einer Ehe und die Auswirkungen auf Vorstellungen von Verwandtschaft}

In vielen außereuropäischen Gesellschaften werden im Fall einer Heirat bestimmte Gaben von den Verwandten des Mannes an die Seite der Frau gegeben, auch „Brautpreis“ genannt. Sie garantieren den Rechtsanspruch der Verwandtschaftsgruppe des Ehemannes auf die Kinder (vgl. Radcliffe-Brown 1950). Da ein Auseinanderbrechen der Ehe, besonders wenn Kinder geboren sind, für beide Seiten erhebliche Unannehmlichkeiten mit sich bringen würde - etwa die Rückgabe des inzwischen für die Heirat anderer Söhne verplanten „Brautpreises“ - ist das Interesse beider Seiten an der Stabilität der Ehe groß. Eine Ehe ist somit keine Sache, deren Aufrechterhaltung man alleine den Eheleuten überlässt.

Wie ich in der Analyse des rechtlichen Diskurses gezeigt habe, gilt die Ehe in Deutschland ausschließlich als ein sozialer Kontrakt zwischen zwei Personen, der zwar neue formalrechtliche Zugehörigkeiten schafft, womit jedoch keine rechtlich fixierten Handlungsanweisungen verbunden sind. Da es sich bei der Institution der Ehe um eine ausschließlich soziale Verbindung zwischen zwei Personen handelt, die alleinig durch den freien Willensentscheid von Mann und Frau entsteht, so gilt auch ihre Auflösung durch Scheidung als persönliche Angelegenheit der Ehepartner (ermöglicht durch den Staat). Die Fragen nach dem Verbleib der Kinder sollen von den beiden Elternteilen geregelt werden, und nur im Fall einer fehlenden Einigung greift eine staatliche Instanz ein. Dies trifft auch für die güterrechtliche Auseinandersetzung zu. Zur Ehescheidung existieren im BGB zahlreiche Bestimmungen, die erfüllt sein müssen, damit eine Ehe per Gesetz geschieden werden kann (vgl. Kap. 4.1.2). Es wird also deutlich, dass ein Individuum persönlich entscheiden und wählen kann, mit wem es eine eheliche Gemeinschaft eingehen will, ebenso wie durch einen Willensentscheid eine Trennung möglich ist. Auch wenn es, im Unterschied zu zahlreichen außereuropäischen Gesellschaften, keine kulturell determinierten Allianzbeziehungen zwischen den jeweiligen Verwandtengruppen gibt, habe ich schon darauf hingewiesen, dass sowohl eine Eheschließung als auch eine Ehescheidung auf Egos Vorstellungen von Verbundenheit und das soziale Beziehungssystem Einfluss hat, was ich im Folgenden näher erläutern werde.

Durch die Heirat wird Egos theoretisches Beziehungsmodell um die Kategorie ,Schwägerschaft‘ erweitert. Sie beruht auf der gesetzlichen Handlung der Eheschließung, ist jedoch rechtlich von einer Ehescheidung nicht betroffen (vgl. Kap. 4.1.1). Die Interviews ${ }^{134}$ zeigen allerdings, dass sich im konkreten sozialen (verwandtschaftlichen) Han-

\footnotetext{
134 Die Interviews im Kontext der Reproduktionsmedizin sind alle mit Paaren geführt worden, die von ihren Ansichten und Erfahrungen über Scheidungen in ihrer Verwandtschaft sprachen und somit auf eine Trennung als Verwandte reagierten. Es waren keine Personen dabei, die eine Scheidung selbst durchlebt hatten und über ihre persönli-
} 
deln die Situation anders darstellt. Mit einer Ausnahme dominiert die Auffassung, dass mit einer Scheidung die Verwandtschaft zum angeheirateten Ehepartner offiziell beendet sei. Die rechtliche Lage war den von mir Befragten nicht bekannt. Nur in einem Interview bezeichnete Sonja Göbel (37) die geschiedene erste Frau ihres Onkels noch als „,irgendwie verwandt und zur Familie gehörig“, was direkt damit zusammenhängt, dass sie während der Ehezeit ein sehr gutes persönliches Verhältnis zueinander hatten:

„Von meinem Onkel, meiner Mutter ihrem Bruder, die erste Frau, mit der bin ich irgendwie immer noch verwandt. Die zweite Frau, von der er jetzt geschieden ist, mit der hatte ich von vorneherein nicht viel zu tun. Die wollte mir in mein Leben hereinreden und war nur drei Jahre jünger wie ich. Da habe ich dann gesagt, ,in meinen Augen bist du nur die zweite Frau von meinem Onkel' ${ }^{6}$. Ich habe keinen großen Kontakt gepflegt und zu seiner ersten Frau habe ich immer noch ein super Verhältnis. Das ist für mich auch eine Tante gewesen, zwar nicht in dem Sinne, dass ich ,Tante' zu ihr gesagt habe, sondern mehr wie ein Kumpel. Wenn ich irgendwelche Probleme hatte, konnte ich zu ihr gehen. Das war auch bei unserer Hochzeit so, zu der Zeit war sie schon geschieden, und sie kam zur kirchlichen Trauung und wollte dann gehen. Da sagte ich ,warum' und sie sagte ,ich gehöre nicht mehr zur Familie, ich bin ja geschieden von deinem Onkel ${ }^{6}$. Und da habe ich gesagt, du gehörst in meinen Augen noch zur Familie'. Wir sind wirklich gute Freunde, gute Kumpels. Wenn es mir nicht gut geht, ruft sie an oder ich bei ihr. Die zählt auf jeden Fall dazu. Und jetzt von meiner Schwägerin, von meinem Mann seiner ältesten Halbschwester, die lebt in Scheidung. Mit ihrem Mann unterhalte ich mich so, wenn wir uns halt treffen. Der Kontakt ist schon da, zum Geburtstag rufe ich ihn auch mal an, weil er mir schon ein bisschen Leid tut, weil er jetzt so alleine ist. Da kümmere ich mich ein bisschen drum. Um das Gewissen zu beruhigen, sagen wir mal so."

Auch anhand der folgenden Beispiele möchte ich verdeutlichen, dass die Überzeugung, dass das durch die Ehe begründete Verwandtschaftsverhältnis im Fall einer Scheidung aufgelöst wird, den weiteren persönlichen Kontakt zum geschiedenen Ehepartner nicht zwangsläufig beeinträchtigen muss. So war Ute Wille (36) beispielsweise der Ansicht, dass sie zumindest theoretisch den Wunsch nach einer Weiterführung der Beziehung habe, wenn die Beziehung von gegenseitiger Zuneigung geprägt sei:

„Also bei meinem Mann in der Familie, da gibt es nur Scheidungen, bei mir keine einzige. Sein Bruder ist zwei Jahre jünger und auch geschieden. Wir haben eigentlich kein schlechtes Verhältnis zu seiner ersten Frau, aber auch nicht so ein besonders gutes. Wir haben sie mal in der Stadt gesehen und haben beide festgestellt, dass sie sehr gut aussieht. Sie hat mittlerweile auch ein Kind. Aber wir haben eigentlich keinen Kontakt mehr. Aber das ist nichts Bösartiges. Ich hätte auch kein Problem zu Geschiedenen den Kontakt zu halten, wenn ich den Menschen akzeptieren würde. Weil ich finde, das ist die urpersönliche Sache von den Beiden, die sich haben scheiden lassen. Ich würde immer versuchen, im Bekanntenkreis zu den Paaren, die sich getrennt haben, weiterhin gute Kontakte zu pflegen, auch wenn man jetzt nicht mehr verwandt ist. Eigentlich den-

chen Erfahrungen mit Verhaltensweisen von Verwandten (insbesondere Affinalverwandten) und die Auswirkungen auf ihr Beziehungssystem erzählen konnten. 
ke ich, dass bestimmt kein Thema wäre, dass die Geschiedenen auch noch dazugehören, emotional jedenfalls. $\mathrm{Zu}$ Festen kommen die dann sicher nicht mehr. Ist ja dann auch keine Verwandtschaft mehr. Wenn jemand sich scheiden lässt, ist das aber eine Sache zwischen den Zweien und muss nicht in der Familie breitgetreten werden oder nach außen hin. Das geht niemanden was an. Das müssen die unter sich ausmachen.“

Es kristallisiert sich heraus, dass bei den von mir befragten Personen die Meinung dominiert, dass staatlich anerkannte Zugehörigkeiten und Angehörigkeiten im Fall einer Eheschließung bzw. -scheidung zwischen Ehepartnern wechseln, die persönlichen Beziehungen zwischen Affinalverwandten jedoch im Fall von gegenseitiger Zuneigung weitergeführt werden können bzw. sollen. Wie das folgende Zitat von Marion Wieger (34) zeigt, kann die Beziehung zu dem Affinalverwandten nach einer Scheidung besser sein als zur konsanguinen Verwandtschaft. Es wird deutlich, dass hier die Kriterien der emotionalen Verbundenheit aufgrund häufiger sozialer Kontakte verknüpft mit gegenseitigen Hilfeleistungen im Gegensatz zur Kategorie ,Verwandtschaft ${ }^{6}$ besonders hervorgehoben werden. Deutlich wird jedoch auch, dass Frau Wieger die Situation als „komisch“ empfindet, da sie nur mit der Cousine durch das Kriterium der Verwandtschaft verbunden ist und nicht mit dem geschiedenen Mann. Diese Beziehungskonstellation scheint gerade in der speziellen Situation einer Ehescheidung als ungewöhnlich angesehen zu werden, da, wie ich schon zeigen konnte, eine Konstituente von Verwandtschaft auch die Vorstellung von gelebten sozialen Beziehungen beinhaltet. „Komisch“ und vermutlich daher erwähnenswert ist für Frau Wieger an der Situation, dass sie zu ihrer Cousine keinen Kontakt mehr hat, nur zu deren geschiedenem Mann, der doch gar keine Verwandtschaft mehr ist:

„Meine Cousine ist geschieden, aber das ist eine ganz komische Situation. Mit der Cousine habe ich überhaupt nichts zu tun, obwohl wir doch eigentlich verwandt sind. Das war aber schon immer schwierig mit der von meiner Seite aus und von ihrer mit mir auch. Aber mit dem Exmann haben wir sehr viel zu tun, weil der uns auch viel im Haus hilft. Der wohnt hier immer noch im Ort. Der ist super nett und handwerklich sehr geschickt. Wir helfen ihm auch, wenn er was braucht. Zu dem haben mein Mann und ich ein gutes Verhältnis, zu meiner Cousine gar keins. Das ist schon irgendwie merkwürdig. Wenn ich die sehe, dann ist das wie jemand, der sonst im Ort wohnt. Trotzdem kam die zum Familientreffen letztens bei uns, also als meine Mutter ihren 65. Geburtstag gefeiert hat. Da war sie eingeladen, weil sie ja die Tochter von der Schwester meiner Mutter ist, ihr Ex-Mann aber nicht mehr. Das ist bei unseren Feiern genau anders herum.“

Da Frau Wieger zu ihrer Cousine keinen Kontakt mehr pflegt (was auch umgekehrt der Fall zu sein scheint), kommt es bei Festen, die sie oder ihr Mann veranstalten, nicht zu Entscheidungssituationen bezüglich der einzuladenden Personen. Da das persönliche Verhältnis zum Zeitpunkt des Interviews ausschließlich zu dem geschiedenem Mann existiert, wird auch nur dieser von ihr eingeladen. Die Situation ist jedoch eine andere bei sogenannten Familientreffen, bei denen er aufgrund der Scheidung nicht mehr anwesend ist. Die Berechtigung zur Anwesenheit ist in diesem Fall abhängig von dem 
Kriterium der Zugehörigkeit zu einer bestimmten Verwandtschaft, die jedoch im persönlichen Diskurs mit der Scheidung als beendet gilt. ${ }^{135}$

\subsubsection{Die zeitliche Dimension von Zugehörigkeit: Die gemeinsame Vergangenheit und ihre Bedeutung für die Vorstellung von Verwandtschaft}

Verknüpft mit der Vorstellung von enger Verbundenheit, die als Verwandtschaft konzeptualisiert wird, ist auch deren zeitliche Dimension. Am empirischen Material im Kontext der Reproduktionsmedizin lässt sich erkennen, dass eine gemeinsame $\mathrm{Ab}$ stammung auch einen Bezug auf eine gemeinsame Geschichte signalisiert, die damit eine spezifische Konstituente dieser Form von Zugehörigkeit darstellt. In den meisten Interviews wird die konsanguine Verwandtschaft von der Affinalverwandtschaft unterschieden als „Verwandtschaft mit unterschiedlicher Qualität“. Mit dieser Aussage wird Bezug genommen auf eine größere emotionale Nähe, die man zu den Blutsverwandten verspüre. Begründet wird diese damit, dass man die „Blutslinie“ länger und besser kenne und gemeinsame (positive und negative) Erfahrungen gemacht habe. Gemeinsames Blut schafft demnach eine gemeinsame Basis, auf der besondere Beziehungen aufgebaut werden können. Wie die folgenden Beispiele zeigen, spielt die zeitliche Dimension in diesem Zusammenhang eine wesentliche Rolle, da als ein zentraler Grund für die emotionale Nähe angegeben wurde, dass ,man immer zusammengelebt hat und sich dadurch auch irgendwie ähnlich ist“.

„Ich finde, man ist aufgrund dieser Blutsverwandtschaft viel mehr gefordert oder ich fühle mich aufgefordert, bestimmte Dinge in irgendeiner Form zu ertragen, zu übergehen oder zu glätten. Man gehört einfach noch ganz anders zusammen. Bei der Schwiegerverwandtschaft fühle ich mich nicht so stark herangezogen. Also Schwierigkeiten mit meiner Schwägerin oder Schwiegermutter empfinde ich als weniger problematisch und habe nicht ständig das Druckgefühl, ich müsste das in irgendeiner Form ausgleichen. Das ist anders bei meiner Schwester. Wenn ich mit ihr ein Problem habe, fühle ich mich viel mehr aufgefordert, mich damit auseinander zu setzen, daran was zu bewegen. [...] Ich glaube, das hat einen emotionalen Ursprung. Einfach von Kindheit an. Das Gefühl, dass mich jemand großgezogen hat, umhegt hat und gepflegt hat. In allen Situationen hat man versucht mir zu helfen. Also ein Gefühl, das ich meiner angeheirateten Familie gegenüber nicht empfinde, sondern nur der eigenen Verwandtschaft. Was nicht heißt, dass in der Schwiegerfamilie nicht auch Personen vorkommen, die mir eben durch Zufall persönlich liegen und aufgrund der Jahre, die ich mit diesen Personen nun

\footnotetext{
135 Nur in einem Gespräch wurden durch die Scheidung betroffene Kinder erwähnt. Egos Verwandtschaft zu den Kindern blieb dem Interview zufolge bestehen, auch wenn sich der persönliche Kontakt reduziert hat, da die Kinder bei dem angeheirateten (jetzt geschiedenen) Elternteil leben. In allen anderen Fällen gab es keine gemeinsamen Kinder, so dass es in den Gesprächen in erster Linie um die Veränderung der Beziehungen zwischen den erwachsenen Personen ging.
} 
schon verbracht habe, sind diese Personen mir auch sehr nahe. Aber das ist etwas, was ich auch Freundinnen gegenüber empfinden könnte.“ (Barbara Berger, 33 Jahre)

„Man ist einfach miteinander groß geworden. Das verbindet einen unheimlich viel. Weil man was Gemeinsames hat, na ja eben gemeinsames Blut. Und es ist auch so, dass man Eigenschaften hat, die einen verbinden. Also, man ist sich ähnlich auf eine gewisse Art und Weise. Ich hänge einfach an meinem Bruder, ohne dass wir so viele innige Gespräche haben. Wenn irgendetwas ist, dann wird schon noch mehr Sensibilität freigesetzt als bei Freunden. Bei Freunden ist man auch sensibel, aber nie so tiefgründig sensibel wie bei Geschwistern“ (Ute Wille, 36 Jahre)

Die Herkunftsfamilie und die ,eigenen“ Verwandten werden als diejenigen mit der größten persönlichen Bedeutung und dem intensivsten Verhältnis genannt. Die Vorstellung, dass Blutsverwandtschaft Personen, besonders Eltern und Kinder, eng verbindet, ist vorherrschend. Durch das lange, emotional intensive und räumlich nahe $\mathrm{Zu}$ sammenleben in einem Haushalt und den alltäglichen und vertraulichen Umgang miteinander, entwickelt sich zwischen ihnen eine Bindung, die sich in dieser Art zwischen genealogisch entfernteren Verwandten oder Affinalverwandten nicht findet, wie Jan Stephan (39) berichtete:

„In einer Familie, da wird man groß. Da kennt man die Geschwister von klein auf, das ist was ganz anderes. Letztlich verbindet einen ja nun auch das gleiche Blut. Ich kann mich mit meiner Schwester über jedes Thema unterhalten. Da ist ein Vertrauen und eine Basis da. Das wird nicht weiter rausgetragen, das bleibt in der Familie. Mit anderen, das braucht schon eine gewisse Zeit, eine Vertrauensbasis aufzubauen, dass man sich über alles unterhalten kann. [...] In einer Familie da wächst man zusammen, da wird man gemeinsam groß, man kennt sich und das Vertrauen ist da.“

Obwohl Aussagen wie die oben zitierten in den Interviews eindeutig dominieren, gibt es jedoch auch immer wieder kritische Einschätzungen, die die gemeinsame Vergangenheit, die Familienmitglieder oder ,nahe“ Verwandte verbindet, zwar als eine mögliche Grundlage für Beziehungen anerkennen, die jedoch auch oft nicht ausreicht, ,wenn da nicht auch eine Form von Seelenverwandtschaft ist" (siehe auch Kap. 5.1.1.1). Dazu Sandra Töpfer (36):

„Oftmals ist Blutsverwandtschaft ja nicht der Freipass für Liebe. Peters Mutter ist im Grunde nie eine Mutter. Er sagt selbst immer, er würde sich von seiner Mutter scheiden lassen. Er hat mit dieser Frau nichts zu tun. Da stimmt einfach nichts zwischen denen, auch wenn die so lange zusammengelebt haben und natürlich auch viel miteinander erlebt haben."

Die zeitliche Dimension von Zugehörigkeit drückt sich auch in genealogischen Kenntnissen aus. ${ }^{136}$ Gute genealogische Kenntnisse führen oft dazu, dass die eigene Verortung

136 Der Vollständigkeit halber sollen hier auch Besitztümer erwähnt werden, die von einer Generation an die nächste weitergegeben werden und über die sich ein persönlicher Bezug zur Vergangenheit herstellen lassen kann (wobei 
in eine Abstammungslinie eine größere persönliche Bedeutung annimmt. ${ }^{137}$ Die Idee einer gemeinsamen Geschichte verknüpft vergangene mit gegenwärtigen und zukünftigen Generationen. Je umfangreicher das Wissen über die Verwandtschaft ist, desto gröBer ist oft auch das Interesse, zu den noch lebenden Personen Kontakte zu knüpfen. Das Wissen über die Abstammung von einem gemeinsamen Vorfahren (wozu auch eine Übereinstimmung der Familiennamen gehören kann) führt in vielen Fällen zu einem Gefühl von Zugehörigkeit und Gemeinschaft und dazu, dass Ego zu diesen Personen eine gewisse Grundvertrautheit voraussetzt. Dazu gehört auch die Idee, zu bisher unbekannten, weit entfernt lebenden Personen Kontakt aufnehmen zu können bzw. zu wollen, da man mittels Stammbäumen Verwandtschaft nachweisen kann. So erzählte Bettina Schulz (30) über ihre Erfahrungen:

B.S.: „Wir [sie und ihr Mann] haben jetzt auch mal ein bisschen Ahnenforschung betrieben. Und da waren wir vor einem Jahr in Südtirol und haben dann mal die ganzen Hellers [ihr Mädchenname] abgefahren, weil uns das wahnsinnig interessiert hat. Wir haben dann die Leute besucht.“"

I.S.: „Und wie haben Sie das gemacht? Sind Sie dort hingegangen und haben gesagt ,Guten Tag, ich bin mit Ihnen verwandt‘?“

B.S.: „Nee, wir sind da also hin und haben dann erst mal nachgefragt, ob sie das denn auch sind. Dann haben wir uns vorgestellt. Wir haben also wirklich dagestanden und haben gesagt, wir stammen von dem und dem ab. Die meisten fanden das total interessant und bei den meisten waren wir dann zu Kaffee und Kuchen, und dann haben wir Bilder gesehen. Von meiner Oma habe ich noch welche bekommen, die ich noch nie gesehen hatte. Ich glaube, das hat allen gefallen. Die haben ja von uns gar nichts gewusst. Aber irgendwie liegt es wohl doch am gleichen Blut (lacht), man kriegt doch gleich einen Draht zueinander und fühlt sich gar nicht so fremd.“

I.S.: „Hat sich denn daraus auch ein Kontakt ergeben?“

B.S.: „Ja, wir haben bei der einen geschlafen, und dieses Jahr waren wir schon wieder da."

Anhand des dargestellten Materials lassen sich die Analysen von Schneider (1980 [1968]) für Amerika sowie Strathern (1992a) und Edwards (2000) für England, die nachgewiesen haben, dass hinter der Vorstellung von ,natürlicher“ Verwandtschaft die Idee biogenetischer Verwandtschaft steht, auch auf den persönlichen Diskurs im Kontext der Reproduktionsmedizin in Deutschland übertragen. „Natürliche“ Verwandtschaft und die daraus resultierenden Beziehungen werden im Gegensatz zu ,nur` sozialer Ver-

weniger finanzielle Erbschaften gemeint sind als Land, Haus, Möbel oder Schmuck). In den Interviews wurden diese jedoch nur selten erwähnt.

137 Eine persönliche Genealogie ist die Darstellung der genealogischen Kenntnisse einer Person zu einem bestimmten Zeitpunkt. Genealogien werden hier in Anlehnung an Fischer (1990:155ff) verstanden als Repräsentation von Kenntnissen, aber auch von Überzeugungen und Interessen. In diesem Sinne ist jede Genealogie eine subjektive Darstellung, die die Situation widerspiegelt, wie sie durch eine bestimmte Person oder Gruppe zu bestimmten Zeiten wahrgenommen wird. 
wandtschaft als unauflöslich verstanden. Dieses Konzept beinhaltet zudem die Vorstellung, dass Verwandtschaft existent ist, ob man sie kennt oder nicht. Gemäß dieser Definition von Verwandtschaft können beispielsweise verwandtschaftliche Bindungen entdeckt werden, von deren Existenz man bislang keine Ahnung gehabt hat. ${ }^{138}$ Am bisher Dargelegten ist allerdings auch deutlich geworden, dass die befragten Personen zwar immer wieder ,Blutsbande' als Basis für als Verwandtschaft konzipierte Beziehungen hervorheben, jedoch die konstitutiven Elemente emotionaler Bindungen insbesondere gemeinsame Erfahrungen als Interaktions- und Solidargemeinschaft sind. Um diese konkreten Erfahrungen und das soziale Handeln geht es im folgenden Kapitel.

\subsubsection{Die sozialen Konsequenzen von „Verwandtschaft"“}

\subsubsection{Räumliche Nähe}

Ein bedeutendes in den Interviews genanntes Element im Zusammenhang mit den Vorstellungen von Familie oder ,naher“" Verwandtschaft ist eine räumliche Nähe, das Zusammenleben in einem Haus, auf demselben Grundstück, in demselben Ort oder in Nachbarorten. Da meine Interviewpartner allesamt älter als 26 Jahre alt waren, existieren keine rechtlichen Regelungen mehr bezüglich des sozialen Alltags, die für minderjährige Kinder das Zusammenleben mit den Eltern oder einem Elternteil vorsieht. Die Entscheidung, auch als Volljähriger mit Lebens- bzw. Ehepartner im gleichen Ort zu wohnen oder zumindest in relativer Nähe von Eltern und Geschwistern sowie der ,nahen Verwandtschaft" scheint somit eine freiwillige zu sein. Es herrscht jedoch eine hohe geografische Mobilität vor, da viele der befragten Personen (zumeist) berufsbedingt von ihrem Geburtsort oder dem Wohnort der Eltern wegziehen, wobei über die Hälfte jedoch wieder zurückkehrt, da ihnen die räumliche Nähe von Familie sehr viel bedeutet: Aussagen zufolge in erster Linie wegen der intensiven emotionalen Bindungen, aber auch wegen der Gemeinschaft, den gegenseitigen Hilfeleistungen, Solidaritäten und Selbstverständlichkeiten zwischen Mitgliedern der Familie oder „nahen“ Verwandtschaft, worauf ich auch in den folgenden Kapiteln noch ausführlicher eingehen werde. Friedrich Walter (41) berichtete von seinen persönlichen Gründen, wieder an den Wohnort der Eltern zurückzuziehen:

\footnotetext{
138 Eine kritische Reflektion dieser Vorstellung von genealogischen Verbindungen und der ,gemeinsamen Biologie“ gibt Marvin Harris (1989:29) und verweist auf die kulturelle Konstruktion dieser als „wahre“ Verwandtschaft konzipierten Beziehungen: „This is not only because 'true' biological links are mostly unknowable but, more importantly, because the assumed biological relations among the members of any given population are not the result of random mating as not all types of mating are allowed, equally preferred or occur with the same degree of probability. The assumed genealogical relations which are seen as arising out of procreative activities are therefore culturally constructed. They are a socially structured set of relations culturally conceptualised as natural relations.“
} 
„Ich bin berufsbedingt weggezogen und habe diese Zeit auch sehr genossen. In der Großstadt zu leben, viel zu unternehmen, keine oder nur wenig familiäre Verpflichtungen zu haben, einfach nur mal abzuhängen, das war schon klasse. Da haben wir uns auch kennen gelernt [er und seine Frau]. Nach drei Jahren ungefähr war uns aber klar, dass wir zurück wollten. Meine Frau kommt witzigerweise aus einem Nachbarort von mir. Wir haben also beide unsere ganze Familie da wohnen. Tja, und dann haben wir überlegt, dass wir ja auch gern mal Kinder haben wollen und dann am liebsten in der Nähe unserer Familie sein wollen. Klar haben wir hier auch Leute, die wir kennen, also auch Freunde. Aber wenn es drauf ankommt, dann kann man sich schon voll und ganz auf die Familie verlassen, die helfen irgendwie selbstverständlicher, und man macht das ja selber auch. Jedenfalls wohnen wir jetzt wieder ganz in der Nähe von meinen und von ihren Eltern, haben regelmäßig Kontakt, helfen uns, und wenn das Kind dann hoffentlich kommt, hat es eben auch gleich die engste Familie um sich herum. Das war uns einfach total wichtig, dass es diese Gemeinschaft so erleben kann.“

Der Rückzug hängt also auch damit zusammen, dass das Paar eine „eigene“ Familie gründen, also ein Kind bekommen will, und dieser Umstand dazu führt, dass der Wunsch nach familiärer Nähe und dem damit assoziierten Gefühl von Vertrauen und Zusammengehörigkeit wieder eine größere persönliche Bedeutung annimmt. Das ,Familie leben', das in der Lebensphase vom Auszug aus dem Elternhaus, beruflicher Qualifikation, Ausübung des Berufes, Zusammensein mit einem Partner eine geringere Bedeutung hatte, gewinnt wieder an Wert, wenn der Wunsch nach einer „eigenen“ Familie auftaucht. $^{139}$

Die Interviewten, die in ländlicher oder kleinstädtischer Umgebung wohnten, lebten in unmittelbarer Nähe oder im näheren Umkreis zu den Eltern und Geschwistern. Bei gemeinsamer Residenz wohnten mehr Paare im Haus der Eltern des Ehemannes als im elterlichen Haus der Ehefrau. Waren die Geschwister noch jünger (im Durchschnitt bis 20 Jahre alt), lebten auch sie im selben Haus, entweder in der Wohnung der Eltern oder in weiteren Wohnungen des Hauses. In einem konkreten Beispiel wurde davon berichtet, dass die Wohnungen je nach Generation wechselten: Im Erdgeschoss wohnten zur Zeit die Eltern, im ersten Stock das befragte Paar, im oberen Stockwerk jüngere Geschwister. Hier handelte es sich um das Wohnhaus der patrilinearen Aszendenten des Ehemannes, in das die Ehefrau mit eingezogen war (virilokale Residenz). Im Todesfall eines Elternteils des Ehemannes war vorgesehen, dass das Paar in das Erdgeschoss ziehen werde, der jüngere Bruder mit Freundin oder Frau in den ersten Stock und der verbleibende Elternteil in das obere Geschoss. Im Todesfall beider Elternteile werde auf lange Sicht einer der Nachkommen des befragten Paares wieder in das obere Stockwerk einziehen. Dieses Beispiel zeigt auf, dass hier eine gemeinsame Lokalität verschiedener Generationen im Fall von Haus- oder Grundstückseigentum der Patri- oder Matrilinie

\footnotetext{
139 Dies bestätigt auch die Untersuchung von Bien und Marbach, die feststellen, dass $30 \%$ aller Familien im selben Ort wohnen wie mindestens ein Großelternpaar (1991:33).
} 
eines Ehepartners erwünscht ist, entweder wird eine Wohnung im Haus bezogen oder es wird auf dem gleichen Grundstück ein eigenes Haus gebaut. Mein Material zeigt, dass zumeist im Fall von mehreren Geschwistern nicht alle in einem Haus leben, sondern eine hausinterne Wohnung häufig entweder von dem ältesten Sohn oder der ältesten Tochter mit Ehepartner (und gegebenenfalls Kind) bezogen wird oder von demjenigen, der sich beruflich im selben Ort etabliert hat. Diese Regelung hängt sehr oft eng mit Erbschaftsregelungen zusammen. Die Geschwister sind zwar alle gleichberechtigt erbberechtigt (vgl. Kap. 4.2), jedoch geht zumindest das Haus bei gemeinsamer Residenz an denjenigen über, der schon zu Lebzeiten der Eltern dort wohnt. Die anderen Geschwister erben andere Besitztümer im gleichen Wert oder werden ausbezahlt (zu Lebzeiten von den Eltern oder nach deren Ableben vom Haupterben). Näher gehen die Befragten zumeist nicht auf Erbschaftsangelegenheiten ein, machen jedoch deutlich, dass diejenigen, die mit oder auf dem Grundstück der Eltern wohnen, nach deren Tode auch diejenigen sein werden, an die Haus und/oder Grundstück übergehen.

Die Interviews zeigen, dass weitaus häufiger als das Wohnen in demselben Haus die Situation vorkommt, dass die befragten Paare im selben Ort oder in einem Nachbarort wohnen wie entweder die Eltern und Geschwister der Frau oder des Mannes. Eine Regelmäßigkeit in der Wahl des Wohnortes lässt sich nicht erkennen. ${ }^{140}$ Oft ist die Wahl des Wohnortes identisch mit dem Geburtsort oder dem Ort der Sozialisation, sehr häufig allerdings abhängig vom Arbeitsplatz. Letzterer spielt eine sehr große Rolle und führt, wie im obigen Interview schon erwähnt, oft zum Wegzug. In vielen Gesprächen wird dieser Umstand sehr bedauert, da damit eine Reduktion des persönlichen Kontaktes zu der Familie zusammenhängt. Ein Austausch findet dann überwiegend über das Telefon statt, was von den wenigsten als Äquivalent gesehen wird.

Aus den Gesprächen geht hervor, dass die Beziehungen zu den in der Nähe wohnenden Eltern und Geschwistern überwiegend regelmäßig sind, geprägt von persönlichen Kontakten. Eine räumliche Nähe oder gar eine gemeinsame Lokalität stellt den Aussagen zufolge prinzipiell ein wesentliches Kriterium oder gar eine Voraussetzung für regelmäBige Kontakte dar, die dazu führen können, dass das persönliche Verhältnis und die gegenseitigen Bindungen stärker sind als gegebenenfalls bei räumlicher Distanz (vgl. auch Fehlmann-von der Mühll 1978:60). Je größer der Wohnort, desto weiter weg wohnen oftmals die Eltern und Familienmitglieder. Zu ,engeren“ Verwandten wird der persönliche Kontakt meist durch telefonischen ersetzt, zu „weiterer“ Verwandtschaft verliert sich häufig der Kontakt mit der räumlichen Entfernung.

${ }^{140}$ Festgelegte post-maritale Residenzregeln existieren in Deutschland auch nicht. 
Ein weiterer Aspekt wird in den folgenden Beispiele deutlich, die zeigen, dass die Basis für eine affektive Beziehung im Fall der Konsanguinen als ,,in die Wiege gelegt“ betrachtet wird. Sie ist verbunden mit dem Wissen um eine ,natürliche“ Zugehörigkeit, die auch nach längerer Trennung wieder aktivierbar ist, anders als im Fall der Affinalverwandten. Das Verhältnis zu den Affinalverwandten muss sich erst aufbauen und ist sehr stark abhängig und geprägt von gegenseitiger Sympathie. Aus den Interviews lässt sich jedoch auch ablesen, dass ein häufiger und intensiver persönlicher Kontakt, der durch eine gemeinsame Lokalität oder kurze Anlauf- oder Anfahrtswege zueinander entsteht bzw. gefördert wird, ein wesentliches Kriterium für eine enge emotionale Verbundenheit darstellt. So zeigt sich, dass einerseits die „eigene“ Familie von den Interviewpartnern als sehr bedeutend genannt wird, im sozialen Handeln des täglichen Lebens jedoch die Affinalverwandtschaft eine gewichtigere Rolle einnehmen kann, je größer die räumliche Nähe und damit auch verbunden je häufiger die persönlichen Treffen sind. So berichtete Katrina Rudig (33):

„Viele, die ganz viel zählen, sind keine Blutsverwandten. Meine Schwiegereltern zum Beispiel. Ich habe mir das lange überlegt, ob sie zu meiner Familie oder Verwandtschaft dazugehören. Aber sie zählen schon dazu, weil man sich so oft sieht und so dicht beieinander wohnt. Da bleibt auch eine gewisse emotionale Anbindung nicht aus. Wenn die $150 \mathrm{~km}$ weg von hier wohnten, wäre das ganz anders. Ich merke das schon, dass es bei meinen Eltern und meinen Schwestern weniger wird aufgrund der räumlichen Distanz. Das ist dann zwar sofort wieder da, wenn man sich sieht und sie stehen mir gefühlsmäßig ganz nah, weil wir einfach natürlicherweise zusammengehören.“

Den Aspekt des gemeinsam verbrachten Alltags erwähnte auch Marion Wieger (34):

„Meine Schwiegereltern sind mir sehr wichtig. Überhaupt auch Leute, mit denen ich meinen Alltag verbringe. Da gehört mein Schwiegervater eben auch direkt dazu, weil wir zusammen eine Firma haben. Da sehen wir uns jeden Tag, und außerdem haben wir auf dem Grundstück der Schwiegereltern gebaut. Da man sich ständig sieht und auch was vom anderen mitkriegt und sich auch gegenseitig zur Seite steht, wenn man so dicht zusammen wohnt, fühlt man sich schon auch sehr verbunden.“

Wie ich schon erwähnt habe, dominiert in den meisten Fällen bei meinen Interviewpartnern die Vorstellung einer positiven affektiven Beziehung zu Familie oder Verwandtschaft. In Erzählungen aus der konkreten gelebten Verwandtschaftspraxis wird jedoch auch deutlich, dass es zum Teil zahlreiche negative Erfahrungen und Erlebnisse mit Mitgliedern der Familie oder der Verwandtschaft gibt, gerade aufgrund von zu enger räumlicher Nähe, die u.a. Gefühle von Kontrolliert-sein oder Verpflichtet-sein hervorrufen. Dennoch lässt sich konstatieren, dass durch diese Gefühle das obige Konzept prinzipiell nicht in Frage gestellt wird, wie auch Bernhard Baumann (42) erzählte:

„Ich muss Ihnen ehrlich sagen, dass ich bzw. meine Frau und ich schon eine Menge negative Sachen erlebt haben mit unseren Familien, die hier auch im Ort wohnen. Oft denke ich, das ist einfach zu eng, wir müssten hier mal weg. Ständig will jemand etwas von uns oder hängt uns auf der Pelle. Das nervt mich manchmal wahnsinnig an, beson- 
ders an den Wochenenden. Aber das gehört ja auch wieder dazu, wenn man eine Familie ist und füreinander da ist. Da muss man wohl mal in den sauren Apfel beißen. Man würde das ja wohl alles nicht machen, wenn da nicht Gefühle füreinander wären.“

\subsubsection{Die Pflege sozialer Kontakte}

Wie bisher deutlich wurde, hat jedes Individuum in Deutschland theoretisch zahlreiche Verwandte sowohl über die Patri- als auch über die Matrilinie. In der sozialen Praxis kann Ego jedoch nicht mit allen diesen Personen Beziehungen pflegen, d.h. eine Auswahl nach bestimmten Kriterien ist notwendig. Aus den Interviews kristallisiert sich heraus, dass diese Auswahl im konkreten Handeln anhand von verwandtschaftlichen Interaktionen im Zusammenhang mit bestimmten Festlichkeiten von besonderer Relevanz ist, ebenso wie bei der Pflege der sozialen Kontakte im Alltag, was auch schon im vorherigen Kapitel deutlich wurde.

Von wesentlicher Bedeutung für die Auswahl von Verwandten, mit denen Ego interagiert, ist der Einfluss von bestimmten Personen, die verwandtschaftliche Kontakte pflegen, zu Treffen einladen und somit als Verbindungsglied zwischen verschiedenen Verwandten fungieren. Dabei handelt es sich zumeist um Egos Eltern, insbesondere die Mutter (vgl. auch Fenner 1984:128, Fehlmann-von der Mühll 1978:65):

„Ja die Eltern, die sind immer noch der zentrale Anlaufpunkt. Wenn da jetzt Geburtstage sind, dann kommen meine Geschwister und einige Tanten und Onkels auch. Und manchmal auch Cousins oder Cousinen, je nachdem ob sie in der Nähe wohnen. Dann werden sie auch eingeladen. Wir treffen uns da, und da gibt es Kaffee und Kuchen. Die würde ich sonst nicht mehr unbedingt sehen. Jeder hat halt so seinen eigenen Kreis und nur begrenzte Kapazitäten. Aber wenn die alle zu meinen Eltern kommen, na eigentlich ist es ja meine Mutter, die das alles organisiert. Wenn meine Schwester kommt aus der Lüneburger Heide, dann übernachtet die immer dort, und dann ist es selbstverständlich, dass wir dann auch hinfahren. Die kommt aber auch hierher, wenn der Kleine [der Sohn] zum Beispiel Taufe hat.“ (Britta Meyer, 33 Jahre)

Wird Egos Kontakt zu den Eltern seltener oder stirbt die organisierende und vermittelnde Person, vermindert sich häufig auch die Interaktion mit den anderen Verwandten oder hört gänzlich auf. Gerade die Beziehung zu Verwandten dritten und vierten Grades sind abhängig von deren Verhältnis zu den Ego nahestehenden Verwandten. Je größer der Kontakt des vermittelnden Verwandten zu einer Abstammungslinie (Matri- oder Patrilinie), je intensiver auch Egos Verbindung zu diesen Personen; Wenn demnach das Verhältnis zwischen der Mutter und deren Geschwistern gut ist, ist Egos Kontakt zu diesen und deren Kindern in der Regel größer. Dieser Aspekt findet sich auch bei Christoph Ottmers (33):

„Früher war unsere Familie sehr eng zusammen, als die Großmutter noch lebte. Da gab es allsonntäglich Treffen beim Sonntagskuchen. Da kamen dann alle hin und man sah sich regelmäßig, auch die ganzen Tanten, Onkels und die Kinder, also Cousins und 
Cousinen. Da war immer ganz schön was los. Hat sich aber nach dem Tod der Großmutter sehr gelockert. Jetzt gibt es noch mal Treffen zu Weihnachten oder so. Wenn vielleicht meine Mutter mehr Kontakt zu ihren Geschwistern hätte und die mal einladen würde, dann würde ich die wahrscheinlich auch öfter mal sehen. Aber so von mir aus werde ich da jetzt nicht aktiv. Mein Vater geht regelmäßig mit seinem Bruder zum Angeln oder macht irgendwas anderes mit ihm. Dadurch sehe ich den noch relativ oft, da ich auch ab und zu mal mitgehe.“

$\mathrm{Zu}$ bestimmten Anlässen kommen Personen zusammen, die von den Befragten entweder als ,Familie' oder als ,Verwandtschaft ${ }^{6}$ kategorisiert werden. Solche Treffen können eingebettet sein in andere Aktivitäten wie Schützenfeste, Weinfeste o.ä., bei denen man zufällig Verwandte aus dem Ort oder den Nachbarorten trifft, oder es handelt sich um spezielle Familienfeste. Dieser Begriff umfasst in erster Linie Weihnachten und Ostern. Geburtstage werden unterschiedlich kategorisiert, wobei „runde“ Geburtstage im Gegensatz zu „normalen“ Geburtstagen zumeist als Familienfeste gelten.

„Normale“ Geburtstage werden zumeist informell gefeiert und es wird nicht schriftlich dazu eingeladen. Oftmals wird überhaupt nicht eingeladen, sondern bestimmte Personen kommen im Falle räumlicher Nähe einfach vorbei. Dabei handelt es sich häufig um Egos Eltern, Geschwister, deren Partner und Kinder je nach Alter, weiterhin abhängig vom persönlichem Verhältnis um die Schwiegereltern, Schwager oder Schwägerin mit Partnern und Kindern. Es kommt vor, dass der eigene Geburtstag entweder einmal mit den oben aufgezählten Personen und einmal mit Freunden und Bekannten gefeiert wird, da die Eltern und die Großeltern der aufsteigenden Generation angehören und oftmals andere Interessen oder Vorstellungen von Geburtstagsfeiern haben. $\mathrm{Zu}$ diesen Punkten sind die Interviewaussagen jedoch sehr heterogen. So können bei Geburtstagen oben genannte Personen auch entweder gleichzeitig oder zumindest mit zeitlichen Überschneidungen anwesend sein. In diesem Fall kommen häufig die Eltern (und gegebenenfalls die Schwiegereltern, Großeltern, Tanten und Onkels) am Nachmittag zum Kaffee und die Freunde stoßen irgendwann dazu. Freunde kommen oft entweder ohne direkte Einladung vorbei oder werden kurzfristig telefonisch informiert. Eltern und Geschwistern gegenüber (bei Wohnortnähe) wird eine gewisse Verpflichtung zur Einladung empfunden, bei „entfernteren“ Verwandten entscheiden Alter, Sympathie und Kontakt. Als wichtiger Aspekt wurde von Ute Wille (36) - wie auch von vielen anderen - genannt, dass die Gäste zusammenpassen müssten.

„Zum Geburtstag kommt die Familie sowieso, also die Eltern, mein Bruder und Frau mit Kindern und meine eine Tante, die für mich wie eine Schwester ist. Manchmal nervt es mich sogar, weil ich dann lieber Freundinnen eingeladen hätte. Und dann ist die ganze Bude voller Verwandtschaft. Vor allen Dingen, weil die Kinder haben und der Geräuschpegel laut ist. Aber ich kann und will sie natürlich auch nicht ausladen. Und das müsste ich ja tun, weil die immer von alleine kommen, was mich zum einen sehr freut, zum anderen würde ich eben auch ganz gern mal anders feiern. Ich hole das dann eben mit Freundinnen manchmal nach, das passt dann einfach besser. Mein Opa ist schon 
sehr alt, der will in Ruhe seinen Kuchen essen und nicht Sekt trinken, tanzen und rumgackern."

„Runde“ Geburtstage haben einen formelleren Charakter, da sie in größerem Rahmen gefeiert werden. Dies führt dazu, dass die Befragten hinsichtlich der einzuladenden Personen einen größeren Druck empfinden. Anhand des folgenden Interviews mit Lars Jansen (31) wird auch deutlich, dass das Gefühl der Verpflichtung die verwandten Personen betrifft, nicht die Freunde. Diese werden aufgrund der gegenseitigen Sympathie und des guten persönlichen Verhältnisses „freiwillig“ eingeladen.

„Als ich meinen 30. Geburtstag feiern wollte mit einer großen Gartenparty mit Grillen, Tanzen usw. fühlte ich mich plötzlich verpflichtet, mehr Verwandte einzuladen als sonst. Im Normalfall wird akzeptiert, dass man außer der Familie niemanden einlädt, weil das einfach zuviel wird. Man kann ja nicht jedes Jahr 30 Leute im Wohnzimmer unterbringen und mit Kaffee und Kuchen versorgen. Außerdem wird akzeptiert, dass man auch Bedarf hat, Freunde einzuladen. Aber wenn etwas Größeres anliegt und dann auch noch im Garten, wo viel Platz ist, ja dann wollen alle kommen. Na gut, vielleicht nicht diejenigen, die $200 \mathrm{~km}$ entfernt wohnen, aber alles, was im Umkreis von 50 Kilometern wohnt, und das sind schon so einige. Da gibt es noch zwei Schwestern von meiner Mutter, die noch nicht 60 Jahre alt sind und gerne feiern. Ob die Männer mitkommen, weiß ich nicht. Eingeladen sind sie natürlich, kann ja nicht sagen, die sollen nicht kommen. Dann habe ich da auch noch Cousins mit Frauen, aber ohne Kinder. Die sehe ich vielleicht zweimal im Jahr irgendwo, aber bei dem 30. Geburtstag von dem einen war ich letztes Jahr auch. Also werden die auch eingeladen. Na ja, ich zähle die jetzt nicht alle auf, aber da kommen mindestens schon mal 20 Leute von der Familie und Verwandtschaft dazu. Außer natürlich meine Großeltern, die sind einfach zu alt für eine Gartenparty. Die können nur am Nachmittag zum Kaffee kommen. Also volles Programm kann ich Ihnen sagen. Tja und dann habe ich ja noch Freunde und Kumpels, die kommen auch. Da mischt sich dann mal alles. Ich denke aber, das wird schon gut. Die, die ich nicht so ganz klasse finde aus der Verwandtschaft, gehen da unter, denn wenigstens die Freunde sind ja alle selber ausgesucht.“

Als ein besonderes oder sogar als das Familienfest schlechthin gilt das Weihnachtsfest. Hier existieren bei den meisten Befragten feste Regelungen, wie und wo diese Tage verbracht werden. Deutliche Unterschiede sind zu erkennen zwischen Paaren mit und ohne Kindern. Paare ohne Kinder teilen die Weihnachtstage in der Regel zwischen den jeweiligen Eltern auf, was den Aussagen zufolge zum einen dem eigenen Wunsch entspricht, zum anderen aber auch als Erwartung implizit oder explizit von den Eltern und/oder Geschwistern geäußert wird. Ersteres hängt u.a. damit zusammen, dass sich die Paare häufig noch nicht als eigenständige Familie (siehe auch Kap. 5.1.1.1) mit einem eigenen Wunsch nach einer Weihnachtsfeier definieren, letzteres, da sie von außen in vielen Fällen auch nicht als solche wahrgenommen werden. Es wird erwartet, dass die Eheleute entweder bei dem einen oder anderen Elternpaar zumindest den Heiligen Abend verbringen, und der Erklärungs- oder Rechtfertigungsdruck wird von den Paaren als immens groß empfunden, wenn es doch mal für sich allein feiern will. Dies wird 
deutlich anhand der beiden folgenden Aussagen von Helga Kollo (29) und Johann Rudlack (34):

„Da wir noch keine Kinder haben, können unsere Eltern das nicht verstehen, wenn wir alleine Weihnachten feiern wollen. Wir haben es eigentlich schon zweimal überlegt, ob wir nicht mal ganz für uns bleiben sollen. Aber ein bisschen komisch ist das für uns selbst auch, denn Weihnachten ist ja schon ein Fest der Familie. Und dann sind wir hier nur zu zweit. Wir konnten uns noch nicht so richtig durchringen. Und meine Mutter möchte auch unbedingt, dass wir kommen, denn meine Schwester kommt ja nun nicht mehr jedes Jahr. Sie hat jetzt zwei kleine Kinder. Da kann meine Mutter das übrigens problemlos akzeptieren, dass die unter sich sein wollen, da die ja jetzt eine eigene Familie haben, wie sie sich immer ausdrückt. Es tut mir weh, wenn sie das sagt, zum einen hätte ich das ja auch gern, zum anderen frage ich mich dann manchmal, ob sie mich überhaupt ernst nimmt. Aber eigentlich meint sie es nur gut mit uns und möchte nicht, dass wir da so alleine sind." (Helga Kollo)

„Wir hatten jahrelang eine Aufteilung. In einem Jahr zu meinen Eltern, in dem anderen Jahr zu ihren, damit sich keiner zurückgesetzt fühlt. Das hat auch eigentlich immer ganz gut geklappt. [...] Letztes Jahr haben wir gesagt, wir wollen das mal anders machen und sind dann über die Weihnachtstage zu zweit in den Schnee gefahren. Wir wollten mal unter uns sein. Das war auch nett, aber für mich war es zu wenig. Ich habe gemerkt, ich brauche den familiären Kontakt. Das gehört für mich irgendwie dazu. Das sitzt emotional ganz tief, so bin ich erzogen worden, dass mir da was fehlt. Und deshalb werden wir dieses Jahr zu der herkömmlichen Regelung zurückkehren. Aber außerdem lag auch ein mächtiger Druck auf uns, da wir allen genau erklären mussten, warum wir nun mal allein sein wollen. Es wollte keiner so richtig verstehen. Dieses ständige Rechtfertigen und die fast beleidigten Blicke haben es uns natürlich auch nicht gerade leicht gemacht, unser Alleinsein zu genießen. Das klingt jetzt ein bisschen anklagend von mir, aber das soll es gar nicht. Ich konnte es ja irgendwie verstehen und es hätte mir auch ohne die Reaktion unserer Eltern was gefehlt, da bin ich mir ziemlich sicher.“ (Johann Rudlack)

Anhand der Interviews zeigt sich, dass im Unterschied zu individuellen Festen wie „normalen“ Geburtstagen, zu denen Egos Eltern und Schwiegereltern auch zum Teil zusammen eingeladen werden oder vorbeikommen, Weihnachten getrennt (in den jeweiligen Herkunftsfamilien) verbracht wird. $\mathrm{Zu}$ Weihnachten sind Familientraditionen hinsichtlich bestimmter Abläufe, des Essens u.a.m. von überragender Bedeutung, die von Familie zu Familie verschieden sind und für die befragten Paare ohne Kinder eine große emotionale Rolle spielen. In den überwiegenden Fällen wird Weihnachten als das Familienfest genannt, an dem sich Egos Eltern sowie die Geschwister mit Partnern und Kindern zumindest an einem Tag treffen. Bei größerer räumlicher Distanz kommt es auch vor, dass die Paare abwechselnd jedes Jahr die ganzen Weihachtstage bei einem Elternpaar verbringen, da die Fahrwege zu lang sind, um beide zu besuchen, wie Birgit und Lothar Franz (31 und 34 Jahre) erzählten:

B.F.: „Wenn einer von uns beiden Geburtstag hat, dann sind beide Elternpaare da. Aber bei Weihnachten sieht die Sache ganz anders aus (lacht). Weihnachten ist das getrennt.“ 
L.F.: „Ja Weihnachten muss man sich entscheiden, wo gehen wir hin. Das ist ungeschriebenes Gesetz.“

B.F.: „Ja wo gehen wir hin. Jetzt gehen wir zu meinen Eltern oder meine Eltern kommen zu uns, und seine Eltern bleiben zuhause. Das wird dann so aufgeteilt, wir haben es ja die letzten Jahre so gemacht, dass meine Eltern und Geschwister Heilig Abend hier waren. Und am ersten Feiertag und am zweiten sind wir dann zu seiner Familie gegangen und haben uns da mit seinen Eltern und Geschwistern getroffen. Aber dass dann beide Familien zusammen sind, das ist nicht der Fall.“

Nur in einem Fall wird berichtet, dass Weihnachten mit Freunden gefeiert wird. Alle anderen Befragten sind der Ansicht, dass zu Weihnachten alle zu ihren Familien gehörten, sei es die „eigene“ Familie mit Kindern oder die Familie im Sinne der Herkunftsfamilie.

Als Veranstaltungen weitläufigerer Art werden beispielsweise Hochzeitsfeierlichkeiten erwähnt. $\mathrm{Zu}$ diesem Anlass werden wesentlich mehr Personen eingeladen als zu anderen festlichen Ereignissen. Die Gästeliste kann hier sowohl „nahe“ als auch „entfernte“ Verwandte, ebenso Freunde oder auch Arbeitskollegen umfassen. Zu solchen Gelegenheiten existiert auch ein besonderer Einladungsdruck, der deutlich macht, dass Hochzeiten bezüglich Verwandtschaft einen verpflichtenden Charakter haben. Anhand des Zitates aus einem Interview mit Martin Schmitt (33) zeigt sich auch, dass es in diesen Fällen zu Loyalitäts- und Interessenkonflikten kommen kann, da Einladungen an bestimmte verwandte Personen auch als Pflicht und Belastung angesehen werden.

„Meine Güte, als wir geheiratet haben, wer da alles kommen musste. Ich dachte, wir finden nie einen Raum, wo wir feiern können. Meine Frau hat vier Geschwister, ihre Eltern haben viele Geschwister. Bei mir genauso. Dann gibt es da noch Onkels, Tanten, Cousins, die ja auch schon Kinder haben. Dann waren wir im letzten Jahr auf zwei Hochzeiten bei, ich weiß nicht, ich glaube, Cousinen zweiten Grades von mir und von meiner Frau. Tja, die müssen wir natürlich auch wieder einladen, sonst gibt es wieder Krisen in der Verwandtschaft. Manchmal habe ich schon gedacht, wenn es nach mir ginge, würden wir nur in ganz kleinem Kreis feiern. Ist zwar schön, so ein großes Fest, und es ist natürlich auch ein schönes Gefühl, so viele Verwandte zu haben, die daran teilnehmen, aber manchmal finde ich es echt schwierig dieses Auswählen, wer kommt und wer nicht und warum und vielleicht ist der sauer, wenn er nicht eingeladen ist oder die oder oder oder.“

Weitere Gelegenheiten, an denen verwandtschaftlich interagiert wird, sind sogenannte Familientreffen, die regelmäßig stattfinden können. Die Teilnahme dabei scheint freiwilliger Natur zu sein, Verpflichtungen wie bei oben beschriebenen Familienfesten werden in der Regel nicht erwähnt. In diesem Zusammenhang umschreibt der Begriff der ,Familie' einen Personenkreis, der von einem gemeinsamen Vorfahren abstammt, wozu alle noch lebenden Generationen zählen. $\mathrm{Zu}$ diesen Familientreffen werden alle verwandten Personen ebenso wie die angeheirateten Partner eingeladen. Anlass ist zu- 
meist gegenseitiger Austausch, Geselligkeit, Unterhaltung und Kontaktpflege (vgl. dazu auch Kap. 5.1.2).

Zusammenfassend lässt sich feststellen, dass der Kontakt zur Familie und „nahen“ Verwandtschaft eher gepflegt wird als zu der „entfernten“. Aufgrund des in den meisten Fällen relativ häufigen und engen Kontaktes zu ersteren erfolgen auch gegenseitige Einladungen zu bestimmten Festivitäten. Bei den ,entfernten“ Verwandten überwiegt oftmals eher ein Gefühl der Verpflichtung zur Einladung bei gewissen feierlichen Anlässen (vgl. auch Fenner 1984:110-118). Die unangemeldeten Besuche zu Geburtstagen oder auch spontan zu anderen Gelegenheiten zeigen, dass zwischen „,nahen“ Verwandten ein vertraulicher Umgang herrscht. Bestimmte Konventionen wie telefonische Voranmeldungen, genaue Verabredungen gelten für Familienmitglieder oder „nahe“ Verwandte nicht unbedingt. Die Toleranz ist bei letzteren sehr viel größer, da Vertraulichkeiten dieser Art als ,Familienkonvention“ akzeptiert und anerkannt werden ${ }^{141}$, wie Henrike Maywald (35) erzählte:

„Zu unseren Geburtstagen kommt von uns beiden die Verwandtschaft, also die Familie oder auch mal Tante oder Onkel oder eine Cousine. $\mathrm{Zu}$ denen habe ich ein herzliches Verhältnis, und mein Mann liebt seine Oma über alles. Die kommt dann natürlich auch. Das macht schon Arbeit, da muss man ganz schön was herrichten. Besonders wenn es ein runder Geburtstag ist, da sind es noch mehr. Da kommen auch Freunde und Bekannte. Da wird dann auch eingeladen, also die Freunde und Bekannten werden eingeladen, die Verwandtschaft kommt sowieso. Das meine ich jetzt überhaupt nicht negativ, ne, ist eigentlich völlig okay. Freunde und Bekannte kommen so spontan nicht, die muss man schon einladen."

Beziehungen zu der ,entfernteren“"Verwandtschaft werden entweder als freundschaftlicher Natur bezeichnet, womit insbesondere der Aspekt der Freiwilligkeit impliziert ist und betont wird, oder sie werden gar nicht gepflegt. Verwandtschaftliche Beziehungen geben einerseits eine soziale Sicherheit (,Die kommen immer, das ist schon was Besonderes. Man kann sich eben irgendwie darauf verlassen und es gehört dazu“), auf der anderen Seite wird auch deren verpflichtender Charakter und die Freiwilligkeit von Freundschaften gegenüber gestellt. Die Interviewbeispiele zeigen auch, dass das soziale Handeln und die gegenseitigen Verpflichtungen zwischen Ego und dessen jeweiligen Bluts- und Affinalverwandten variieren (können). In der Regel ist Egos Beziehung zur Affinalverwandtschaft abhängig vom Verhältnis des Ehepartners zu dessen Familie; Ist dies schlecht, ist auch Egos persönlicher Kontakt gering. Die Loyalität gegenüber dem

\footnotetext{
$141 \mathrm{Ob}$ und inwieweit körperliche Berührungen - Umarmung oder Händedruck - Teil dieser Vertraulichkeiten sind bzw. ob und auf welche Weise sie zwischen verschiedenen Personen variieren, wurde von den befragten Personen nicht thematisiert. Auf mein Nachfragen erhielt ich nur sehr vage Antworten, so dass an dieser Stelle keine fundierten Aussagen möglich sind.
} 
Ehepartner sowie gegenüber der eigenen Blutsverwandtschaft wird hier stärker gewichtet.

Im Zusammenhang mit sozialen Interaktionen im Alltag werden auch Nachbarn und Freunde erwähnt und in Beziehung zu Familie und Verwandtschaft gesetzt. Das Bildungsprinzip für die Kategorie ,Nachbarn' ist per definitionem die gemeinsame Residenz. Trotz räumlicher Nähe kann man jedoch Nachbarn haben, die man selten sieht. Eine Änderung der Residenz zieht eine Änderung der Nachbarschaften nach sich und kann sich auf nachbarschaftliche Interaktionen auswirken. In den Interviews wird jedoch die prinzipielle Möglichkeit erwähnt, dass aus guter Nachbarschaft auch Freundschaften entstehen können, wobei deutlich wird, dass bei dieser Zuordnung wiederum andere oder weitere Kriterien erfüllt sein müssen. So äußerte sich Marita Baumann (32):

„Meine eine Nachbarin hat zwei kleine Kinder, da helfe ich ihr mal oder kaufe was für sie ein oder mache mal was mit den Kindern. Wir sehen uns eigentlich täglich und sie ist eher wie eine Freundin als eine Nachbarin. Also sie könnte auch woanders wohnen, dann würden wir uns sicher nicht mehr so oft sehen, aber der Kontakt würde bestimmt nicht abbrechen.“

Soziale Interaktionen mit Nachbarn hängen eng mit gegenseitiger Sympathie zusammen. Bestimmte gegenseitige Hilfeleistungen wie Blumen gießen im Urlaub, den Briefkasten leeren, aber auch die Ausleihe von Lebensmitteln in Notfällen werden häufig als die Merkmale von ,gelebter“ Nachbarschaft bezeichnet, die über eine Begrüßung hinausgehen. In den Fällen, in denen die Befragten von Nachbarn sprechen, die fast schon oder schon Freunde sind, gehen die Interaktionen noch weiter. Gegenseitige Besuche, gemeinsame Feste, größere Hilfe bei Renovierungen o.ä. werden hier als feste Bestandteile von Nachbarschaft angesehen und überwiegend als positiv bewertet. Marion Wieger (34) bemerkt jedoch „ganz realistisch“, dass man sich manchmal zuviel verspreche von Nachbarn, auch wenn man viel zusammen unternommen habe. Ihrer Ansicht nach seien Nachbarn eben doch meistens keine Freunde und der Kontakt, egal wie gut er sei, bestehe in erster Linie aufgrund der gemeinsamen Residenz und sei dementsprechend zweckgebunden.

„Also mal ganz ehrlich und realistisch. Ich mag meine Nachbarn sehr, es sind echt tolle Leute. Sind in ähnlichem Alter wie wir, ähnliche Interessen usw. Wir sehen uns ziemlich oft, da wir die Gärten nebeneinander haben. Komme ich von der Arbeit, trinken wir oft mal einen Kaffee, oder meine Nachbarin hat gerade gebacken. Wir grillen mal spontan zusammen oder gucken ein Video. Das ist eben praktisch, dass die sehr nett sind und gleich nebenan wohnen. Aber ich denke trotzdem, wenn die nicht bei uns wohnen würden, sondern jetzt wegziehen würden, dann wäre der Kontakt vorbei. Komisch oder?! Ich meine, ich mag die beiden sehr und sehe die wirklich oft. Wir reden auch über Persönliches. Aber ich bin mir ziemlich sicher, dass mein Mann und ich im Falle eines Umzugs wahrscheinlich nichts mehr mit denen zu tun hätten oder nur noch sehr selten. Ist schon merkwürdig diese Beziehung. Sie ist gut, und der Witz ist, ich 
sehe meine Nachbarn viel öfter als meine Freunde und sogar einige meiner Verwandten, aber die stehen mir gefühlsmäßig viel näher.“

Ebenso wie Nachbarn werden auch Arbeitskollegen beschrieben als Personen, mit denen sehr viel Zeit des Tages verbracht wird. Der häufige Kontakt basiert auf einem gemeinsamen Arbeitsplatz, was zur Folge hat, dass die Variabilität der Beziehungen sehr groß sein kann, da ein Wechsel des Arbeitsplatzes heutzutage relativ häufig vorkommt. Da die Arbeitskollegen einem ,öffentlichen, außerhäuslichen Bereich` zugeordnet werden, besteht oft der Wunsch, das Privatleben gerade nicht mit Kollegen zu teilen, wie das folgende Zitat exemplarisch zeigt:

„Kollegen sind für mich auch noch so ein Stamm. Wie Freunde und Familie, so gibt es auch Kollegen. Unter diesen Kollegen gibt es dann eventuell auch noch Leute, mit denen man mehr zu tun hat. Die tendieren dann eher zu Freunden. Aber ich finde es schon wichtig, überall gute Kontakte zu haben. Weil man auch viel im Job lebt, und wenn man sich da mit den Leuten nicht versteht, ist das schon eine grausame Situation. Ich kenne aber nicht viele Leute, die außerhalb von der Arbeit noch was mit ihren Kollegen machen. Vielleicht ganz junge Leute, die keine eigene Familie haben. Ansonsten hat doch jeder noch genug zu tun. Oftmals möchte man ja auch nicht, dass sich Job und Privatleben überschneiden, weil dann letztendlich doch das Vertrauen in die Leute fehlt, dass sie sich nicht hinterher doch das Maul zerreißen. Und dann hat man vielleicht privat und in der Arbeit Stress. Ne, also ich finde eine gewisse Distanz zu Kollegen schon ganz wichtig mit einigen wenigen Ausnahmen.“(Ute Wille, 36 Jahre)

\subsubsection{3 Ökonomische und moralische Unterstützungen und Verpflichtungen}

Das Konzept von Verwandtschaft beinhaltet im persönlichen Diskurs die Vorstellung von Solidarität und Reziprozität und scheint mit gewissen Einschränkungen eine wichtige Quelle sozialer Sicherheit zu sein. „Nahen“ Verwandten gegenüber fühlen sich die Befragten zu Hilfeleistungen verpflichtet, da das Geben und Nehmen zwischen Personen, die diesen Kategorien zugeordnet werden, in der Regel als selbstverständlich erachtet wird. Das Verpflichtungsgefühl nimmt zumeist ab, je weiter man sich verwandtschaftlich voneinander entfernt fühlt, was mit der genealogischen Distanz, aber auch mit fehlenden persönlichen Beziehungen zusammen hängen kann. In der Regel überwiegt die Auffassung, dass in bestimmten Notsituationen zunächst die engeren Familienmitglieder der hilfebedürftigen Person gefordert sind, was Ira Wagner (33) deutlich zum Ausdruck brachte:

„Für jeden ist erst mal die eigene Familie zuständig, und um die kümmert man sich auch selbst. Das ist doch selbstverständlich, das gehört einfach dazu, wenn man eine Familie ist. Und auch die Familie meines Mannes, da mache ich das auch. Auch mal bei Onkel und Tante oder Cousin und Cousine. Hängt aber davon ab, ob ich mich mit denen gut verstehe. Dann helfe ich da auch mal aus oder andersherum genauso. Aber bei den anderen, also die Verwandtschaft zweiten Grades oder wie das heißt, da hört es dann einfach auf. Die haben ja auch wieder ihre kleine Familie, also die eng zusammengehörenden Personen. Und dann sind die wieder zuständig. So läuft das doch. Irgendwo 
muss da ja auch die Grenze sein. Gut, vielleicht auch mal mit einer Ausnahme, wenn gar kein anderer einspringen kann, aber in der Regel würde ich da die Hilfe ablehnen, da andere dafür zuständig sind.“

Im Zusammenhang mit Fragen nach gegenseitigen Hilfeleistungen differenzieren die Befragten jedoch nicht nur zwischen „nahen“ und „fernen“ Verwandten, sondern unterscheiden auch sehr häufig deutlich zwischen den Kategorien ,Verwandte', ,Freunde' und ,Bekannte', wie bei Barbara und Thomas Berger (33 und 35 Jahre) deutlich wird:

B.B.: „Ich denke, dass sich jemand aus der Familie mehr herausnehmen kann als jetzt ein Außenstehender. Und wenn es noch so ein guter Freund ist.“

T.B.: „Wir können das ja mal spitzfindig ausdrücken. Freunde kann man sich aussuchen, Verwandte nicht. Und die Blutsbande sind manchmal recht stark. Dass man sagen kann, für Verwandte würde man etwas tun oder auch selbstverständlicher tun, wo man sich bei Freunden oder Bekannten schwer tun würde."

I.S.: „Fällt Ihnen da was ein? Oder haben Sie da persönliche Erfahrungen gemacht?“

T.B.: „Ich würde sagen, für Verwandte würde man sich schwerer tun, eine Hilfeleistung abzulehnen. Bei Freunden könnte man das eher mit seinem Interesse vereinbaren. Das ist schwer zu definieren."

In Deutschland bestehen keine gesetzlich geregelte Verpflichtung zu bestimmten Hilfeleistungen mit Ausnahme von Unterhaltspflichten für Verwandte in gerader Linie (Kap. 4.2). Alle anderen Hilfen sind prinzipiell freiwilliger Natur, geregelt durch einen verwandtschaftlichen Verhaltenskodex, der von den Beteiligten je nach sozialen, kulturellen und ökonomischen Gegebenheiten immer wieder neu ausgehandelt werden kann oder muss (vgl. auch Segalen 1997:9). Die Interviews zeigen, dass gegenseitige Hilfe entweder gelegentlich und spontan geleistet oder zuvor abgesprochen wird wie zum Beispiel beim Hausbau, -umbau oder Umzug. Es wird deutlich, dass der reziproke Charakter verwandtschaftlicher Beziehungen sich darin äußert, dass Gegenleistungen nicht sofort erbracht werden müssen, sondern dass lediglich erwartet wird, dass prinzipiell auch umgekehrt geholfen wird. Dieser Aspekt wird auch von Fenner (1984:120f) bestätigt, der darüber hinaus in seiner Studie konstatiert, dass die „Währung“ der verwandtschaftlichen Leistungen überwiegend instrumenteller Natur sei, worunter er beispielsweise Kinder hüten, Hilfe in Haus und Garten, Pflege bei Krankheiten versteht, ebenso wie emotioneller Natur, wozu Ratschläge in verschiedenen Lebenslagen zählten. Diese Elemente lassen sich durch das mir vorliegende Material bestätigen und finden sich auch in der Aussage von Karl Lenz (39):

„Also wenn ich meinen Verwandten helfe, dann erwarte ich kein Geld. Es wäre ziemlich komisch, wenn man sich da gegenseitig bezahlen würde. Da versucht man das doch lieber auf andere Art und Weise wieder gut zu machen. Was anderes wäre es, wenn ich einen Elektriker bräuchte für eine wichtige Sache und hätte einen in der Familie. Ich würde nicht so ohne weiteres verlangen, dass der mir ganz umsonst bei irgendwelchen komplizierten Sachen hilft, die lange dauern oder so. Man müsste das dann eben genau 
besprechen, wie man sich ,bezahlt ${ }^{6}$. Kommt auf die Personen drauf an, kann ja auch mal ein Essen, eine Kneipentour sein, oder dass ich dann eben auch mal viel Zeit und Mühe investiere, wenn er mich braucht. Normalerweise ist da kein Geld im Spiel, jedenfalls nicht direkt. Also er legt Leitungen und ich bezahle, so läuft das nicht, das regeln wir eben anders."

Nach Hilfe im Fall von Krankheiten gefragt, nennt der Großteil der Befragten die eigenen Eltern als diejenigen, die man, soweit die räumliche Distanz nicht zu groß sei, versorgen würde. Diese Selbstverständlichkeit hängt den Aussagen zufolge damit zusammen, dass Eltern immer da gewesen seien, viel für einen getan hätten und man das prinzipiell irgendwann auch zurückgeben wolle. So berichtete Lea Rösch (34):

„Und ich finde, man hat als Kind eine Verpflichtung. Die Eltern waren immer für einen da, und ich könnte das mit meinem Gewissen nicht vereinbaren. Gut, wenn es nicht anders geht, dass man selber körperlich angeschlagen ist und wirklich nicht pflegen kann. Dann geht es halt nicht anders. Aber wenn es irgendwie machbar ist ... Wenn man sieht, wie manche Kinder mit den Eltern umgehen, da kann ich nur sagen, das ist wirklich das letzte.“

Über die Hälfte der Interviewpartner erwähnt einen Unterschied zwischen den eigenen Eltern und den jeweiligen Schwiegereltern, was das Gefühl von Zuständigkeit und Verpflichtung angeht. Hilfe werde zwar angeboten und geleistet, jedoch bleibt, wie die folgende Aussage von Ira Wagner (33) zeigt, das Gefühl bestehen, dass letztlich die „eigenen“ Kinder für die Pflege der Eltern zuständig und verantwortlich seien und nicht sie als Schwiegertochter. Die Selbstverständlichkeit der Hilfeleistung gegenüber der eigenen Mutter wird hier ebenfalls betont und scheint damit zusammen zu hängen, dass das Konzept von Reziprozität zum Tragen kommt, das Frau Wagner im Fall der Schwiegereltern und Schwägerschaft nicht verspürt. Die Einseitigkeit der Hilfeleistungen führt zu dem Gefühl des Ausgenutzt-werdens, so dass sie sich in Bezug auf Hilfeleistungen gegenüber der Affinalverwandtschaft verweigert.

„Von meiner Schwiegermutter ist das erwartet worden. Ich bin da hingefahren, habe ihr die Haare gewaschen, habe ihr geholfen, meinen Schwiegervater zu füttern usw. Es hat sich jeder auf mich verlassen, die Ira macht das schon, hieß es immer. Wie mein Schwiegervater dann gestorben ist, sagte mein Schwager, Wir müssen uns jetzt alle um Mutter kümmern'. Da habe ich ihn nur angeguckt und gesagt, ,Ihr seid fünf Kinder, kümmert euch mal. Was ich im letzten halben Jahr tagtäglich geben musste, jetzt seid ihr mal dran. Ich bin die Schwiegertochter und es sind leibliche Kinder da, die sich kümmern können. Ich kann irgendwann nicht mehr.' Ich fühle mich da total ausgenutzt. Bei meiner Mutter ist es eine Selbstverständlichkeit, dass ich ihr helfe. Sie ist ja auch jederzeit für mich da, wenn sie kann. [...] Ich habe gesagt, ich lasse mich jetzt von niemandem mehr verpflichten. Ich habe das jahrelang gemacht, da wird man ganz schön hart und verbittert. Ich konnte halt nicht nein sagen."

In einigen wenigen Fällen werden auch bestimmte Tanten oder Onkels genannt, bei denen ein Gefühl von Verantwortlichkeit besteht, sie im Fall einer Krankheit zu pflegen. Es lässt sich jedoch feststellen, dass dies sehr von dem persönlichen Verhältnis, der ge- 
genseitigen Zuneigung und den bisherigen geleisteten Hilfeleistungen abhängt. Hilfen wie Kinder hüten, Unterstützung im oder am Haus oder Garten, Einkaufen o.ä. werden auch mal für ,entfernte“ Verwandte geleistet, wenn zu diesen ein persönlicher Kontakt und Sympathie besteht und die Wohnorte nicht zu weit entfernt sind. Die räumliche Nähe bzw. Distanz ist auch bei den Mitgliedern der Familie oder „nahen“ Verwandten relevant, denn Unterstützung bei alltäglichen Dingen wird eher von denen erbracht, die nicht weit voneinander entfernt wohnen. Von den Personen, die lange Anfahrtswege haben, werden diese Alltagshilfen in der Regel gar nicht erst erwartet, wie Marie Flirrau (35) erzählte:

„Das ist gemischt, wen ich frage. Je nachdem, wo es am einfachsten ist. Eigentlich frage ich schon zuerst meine Mutter oder Schwester. Besonders wenn es längerfristige Sachen sind mit dem Hund, dann spreche ich eher meine Schwester an. Wenn es mal um einen Nachmittag geht, dass es mal schwierig wird, dann frage ich auch mal den Nachbarn. Oder meine Freundin fragt, ob ich was vom Supermarkt brauche. Das mache ich für sie auch, dass ich ihr da mal was mitbringe. Ist was anderes, ob es um was längerfristiges geht oder nur mal eben Hilfe für irgendwas. Für einen Nachmittag wäre die Fahrt zu lang für meine Schwester oder meine Mutter.“

Aus den Interviews geht hervor, dass für oben genannte Hilfeleistungen zunächst Mitglieder der Familie oder der „,nahen“ Verwandtschaft gebeten werden, bevor man sich an Freunde wendet. Dieses trifft auch besonders für den Fall längerfristiger Unterstützung zu. Verwandte werden demnach eher für Hilfeleistungen in Anspruch genommen, je besser sie erreichbar sind, je geringer ihre genealogische Distanz zu Ego ist, je geeigneter sie aufgrund ihrer Fähigkeiten als Helfer sind und je besser ihr persönliches Verhältnis zu Ego ist.

Die Situation stellt sich allerdings anders dar, wenn die Familie weiter entfernt wohnt. In diesen Fällen wird ein Netzwerk gegenseitiger (all-)täglicher Hilfe mit Freunden oder Nachbarn bedeutender, wie Martin Schmitt (33) berichtete:

„Ich habe gar keine Verwandten in der Nähe. Die wohnen alle über 200 Kilometer entfernt. Als ich umgezogen bin, haben mir mein Vater und meine Brüder auch geholfen, aber für den Kleinkram wäre das zu weit. Letztens habe ich einen Schrank gekauft und brauchte Hilfe. Da frage ich natürlich meine Freunde hier, und da helfen wir uns gegenseitig. Oder ich war mal krank und kam nicht raus. Da ist auch meine eine Nachbarin mal eingesprungen, hat mir was eingekauft und einmal das Treppenhaus geputzt. Dafür kann ich ja nicht extra meine Mutter oder so kommen lassen.“

Auf Fragen nach emotionaler Hilfeleistung bei zum Beispiel Problemen in der Ehe, mit den Kindern, Entscheidungen etc. werden Personen der Kategorien ,Freunde ${ }^{6}$ und ,Bekannte ${ }^{6}$ häufiger angesprochen als Verwandte. Als Gründe werden angegeben, dass man sich Freunde aufgrund von gegenseitigem Vertrauen und Sympathie ausgesucht habe und sich von diesen oft besser verstanden fühle. Selbst bei Familienmitgliedern oder bei „,nahen“ Verwandten könne man sich oft nicht so offen äußern wie bei Freunden. Erste- 
re wolle man oft lieber aus persönlichen Angelegenheiten heraushalten, um Konflikte zu vermeiden. In der Regel bilden jedoch Mütter sowohl bei instrumenteller als auch bei emotionaler Hilfeleistung eine Ausnahme. Dies bestätigt auch die Untersuchung von Pfeil und Ganzert (1973:377), in der 84\% der Befragten, deren Mutter in einer Wohnentfernung unter 15 Minuten Fahrzeit wohnt, sie mindestens in einem der Fälle als bevorzugte „Hilfeleisterin“ angeben.

Wie mein empirisches Material zeigt, werden Geldangelegenheiten als besonders konfliktträchtig empfunden, da sie Abhängigkeitsverhältnisse besonderer Art schaffen. Einerseits wird versucht, diesen konfliktträchtigen Bereich aus dem Spektrum verwandtschaftlicher Verflechtungen auszuklammern und lieber die Bank zu beleihen. ${ }^{142}$ Andererseits gelten gerade die engen vertikalen verwandtschaftlichen Beziehungen als diejenigen, die als einzige diese Abhängigkeiten und Konflikte aushalten bzw. tragen können, ohne dass es sich grundsätzlich negativ auf die Beziehung auswirkt (vgl. Fenner 1984: 62-65, 155), wie am folgenden Ausschnitt aus einem Interview mit Thomas Berger (35) deutlich wird:

T.B.: „Grundsätzlich würde ich erst mal sagen ,Bei Geld hört die Freundschaft auf‘. Das hat sich bei mir so eingeprägt, dass ich wirtschaftliche Notwendigkeiten unabhängig von Freunden machen will. Und das ich da im Falle eines Falles einen Unterschied machen würde zwischen Familie oder Verwandten und Freunden. Weil ich die Freundschaft nicht auf eine wirtschaftliche Testfrage stellen wollte. Finanzielle Notlagen besprechen vielleicht, aber nicht um Geld bitten, sondern versuchen, es eher aus dem verwandtschaftlichen Umfeld zu bekommen.“

I.S.: „Sie glauben nicht, dass dadurch auch die verwandtschaftliche Beziehung auf eine Probe gestellt würde?““

T.B.: „Nein, ich glaube, das hat was damit zu tun, dass eben mit dieser verwandtschaftlichen Beziehung auch eine Funktion verbunden ist. Und diese Funktion ist eben auch ein Zusammenhalt in solchen Gütern, Abhängigkeiten, die sich unter Umständen auch aus amtlichen Verpflichtungen oder erblichen Verschachtelungen ergibt. Das hat da seinen Ursprung, nehme ich mal an. Bei Gelddingen, wobei ich normalerweise versuche zu vermeiden, dass ich da in Abhängigkeiten von anderen Leuten komme, würde ich auf keinen Fall zu Freunden gehen, sondern erst den Weg über nahe Verwandte suchen. Nur wenn das überhaupt nicht klappen sollte, dann würde ich zu Freunden.“

Im Zusammenhang mit finanziellen Hilfeleistungen geht Herr Berger im obigen Zitat auf die Funktion verwandtschaftlicher Beziehungen ein, die im Zusammenhalt hinsichtlich bestimmter Güter liegt. Er sieht einen direkten Zusammenhang zwischen bestimmten Abhängigkeiten zwischen Verwandten und gesetzlichen Regelungen, die Unterhaltsverpflichtungen und Erbschaftsangelegenheiten betreffen. Diese Einstellung führt

142 Das hat auch Lüschen in seiner Untersuchung nachgewiesen, in der sich 2/3 aller der Befragten bei Geldangelegenheiten lieber an Kreditinstitute als an Verwandte und Freunde (1988:158) wenden würden. 
dazu, dass er zunächst immer die Hilfe von Verwandten in Anspruch nehme, bevor er sich an andere Leute wenden würde. Anhand der persönlichen Berichte über verwandtschaftliche Hilfe in Bezug auf Geldangelegenheiten lässt sich schlussfolgern, dass in diesen Fällen sehr wohl eine gute Kenntnis über die Gesetzeslage, die die gegenseitige Versorgung, jedenfalls in vertikaler Richtung, regelt, vorherrschend und auch handlungsweisend ist (siehe zum Beispiel auch das Wohnen in demselben Haus oder auf demselben Grundstück mit den Eltern, das man später erben wird, ebenso wie das Leihen von Geld). Es zeigt sich, dass dieses Wissen sowohl ein Verpflichtungsgefühl aber auch eine gewisse Erwartungshaltung gegenüber Familienmitgliedern oder „,nahen“ Verwandten implizieren kann.

Gesamthaft betrachtet lässt sich jedoch feststellen, dass bei den Befragten ambivalente Gefühle hinsichtlich verwandtschaftlicher Hilfeleistungen existieren. Betont wird zum einen das Positive, die Selbstverständlichkeit und Verlässlichkeit, andererseits existiert auch ein Gefühl der Verpflichtung, dem man sich nur schwer entziehen kann und das auch dazu führen kann, dass man sich manchmal ausgenutzt vorkommt, wenn die Gegenseite sich nicht erkenntlich zeigt. Anhand des empirischen Materials lässt sich die These von Rosenbaum (1998:29) unterstützen, dass Verwandtschaft eine Beziehung mit einen normativen Charakter ist, die Ansprüche und Verpflichtungen beinhaltet. Der Mechanismus der Solidarität spielt besonders in Krisensituationen eine große Rolle, in denen an die Familie oder die Verwandtschaft Erwartungen gestellt werden, die man ansonsten an niemanden richten würde, d.h. die Beziehungen zu Verwandten als Kategorie wird insbesondere in Krisenzeiten gepflegt, während ansonsten eine Auswahl innerhalb dieser Kategorie erfolgt. Lautermann (1974:28) konstatiert dazu:

„Wenn das Reziprozitätsprinzip durch den Austausch von Geben und Nehmen, von Leistung und Gegenleistung, Beziehungen intensiviert und festigt, wird dadurch auch die Solidarität gestärkt, jedoch nur insofern es sich um positiv gewertete Beziehungen handelt. Solidarität gibt es nur aufgrund von Übereinstimmung mit dem Gruppenziel und aufgrund von Sympathie mit den Emotionen der Gruppe. Solidarität nimmt desto mehr zu, je mehr die Gruppenmitglieder miteinander interagieren.“

Auffallend ist, dass es nur im Fall von Verwandtschaft möglich zu sein scheint, dass die Hilfeleistungen nicht immer gegenseitig sind. Die Toleranz ist bis zu einem bestimmten Punkt größer als bei Freunden oder Bekannten, die man viel eher kritisieren würde oder von denen man sich ausgenutzt fühlen würde. Ebenfalls ist ein Merkmal verwandtschaftlicher Beziehungen, dass sie, obwohl man sie lange vernachlässigt hat, bei Bedarf aktualisiert werden können. Dabei gelten nicht immer die üblichen Normen bezüglich Reziprozität und Toleranz. 


\subsection{4 „Mein Kind ist ein Teil von mir“6}

Wie die vorherigen Kapiteln gezeigt haben, ist das Konzept von Verwandtschaft als spezifische Form von Verbundenheit im persönlichen Diskurs, der im Kontext der Reproduktionsmedizin geführt wird, aufs engste mit dem Körper in seiner leiblichen Dimension verknüpft (vgl. Kap. 2.3). Die Vorstellung von Blutsverwandtschaft als eindeutige, unveränderliche und dauerhafte Beziehung, die auf einer geteilten Körpersubstanz aufbaut und sich über Leiblichkeit konstituiert, bildet wiederum die Grundlage bzw. hat direkten Einfluss auf die soziale Ausgestaltung dieser Beziehung. Auch wenn umgangssprachlich das Konzept des gemeinsamen Blutes im Begriff der Blutsverwandtschaft wirkmächtig ist, hat jedoch im Zeitalter gentechnologischer Forschung das Konzept der gemeinsamen Gene (die auch bestimmte ,Botschaften' enthalten) dieses fast abgelöst. Dem naturwissenschaftlichen Konzept der Genetik zufolge gilt der Körper eines Kindes als das Resultat der Gametenverschmelzung eines Mannes und einer Frau. Bis zur Geburt eines eigenständigen Menschen kommt es im Verlauf einer Schwangerschaft zu weiteren Zellteilungen und Differenzierungen. Das Wissen über die Weitergabe von genetischem Material wird gleichgesetzt mit dem Wissen über die „natürlichen Fakten“ des Lebens, so dass verwandtschaftliche Beziehungen angesehen werden als „natürliche“ Verbindungen. Die Schaffung von ,natürlicher“"Verwandtschaft als besondere Form sozialer Beziehungen und damit verbunden die Gründung einer Familie mit leiblichen Nachkommen vor dem Hintergrund dieses biogenetischen Zeugungsmodells und die mit einem ,eigenen“ Kind verbundenen Vorstellungen stehen im Mittelpunkt des Denkens und Handelns der von mir interviewten Personen im Kontext der Reproduktionsmedizin, um die es im folgenden Kapitel gehen soll.

\subsubsection{Physische und psychische Ähnlichkeiten}

Allen von mir befragten Personen ist in groben Zügen das naturwissenschaftliche Konzept der Genetik bekannt, das davon ausgeht, dass ein Kind bestimmte genetische Dispositionen aufweist, die auch seine Eltern besitzen, die sie wiederum (in abgeschwächter Form) von ihren jeweiligen Eltern mitbekommen haben. Der Aspekt der Ähnlichkeiten, wozu sowohl Übereinstimmungen in der Physiognomie als auch in Wesensmerkmalen gezählt werden, zwischen ,echten“ Verwandten, spielt in der Vorstellung von einem „eigenen“ Kind eine bedeutende Rolle.

Die Vorstellung, dass Ähnlichkeiten zwischen konsanguinen Verwandten ihren Ursprung in ,der Biologie' haben, und dass diese über den Prozess der Fortpflanzung weitergegeben werden, führt, wie die folgenden Ausschnitte aus Interviews mit Jörn 
Budecker (36) und Barbara Berger (33) ${ }^{143}$ zeigen, in der konkreten Praxis dazu, dass man bestimmte gemeinsame Züge zwischen diesen Personen erkennt. Dieses „Wiederentdecken" und Aufspüren von Gemeinsamkeiten sowohl bei den Agnaten als auch bei den Kognaten führt zu einem besonderen Gefühl von Zusammengehörigkeit. ${ }^{144}$

„Ich finde das schon auffällig, wie sich über mehrere Generationen bestimmte äußerliche Züge genetisch vererben. Man kann das bei uns an vielen Familienfotos sehen. Irgendwo gibt es fast immer eine Ähnlichkeit, entweder über die Seite der Mutter oder des Vaters. Das ist doch eine tolle Sache. Wenn man so zusammensitzt und sich Fotos anguckt und eben solche Ähnlichkeiten feststellt, dann hat man doch ganz stark das Gefühl von Zusammengehörigkeit. Da kommt doch ganz viel hoch, was eben nur unter Verwandten vorhanden ist. Da kann man sich doch irgendwo einordnen, und bei einem Kind ist das dann genauso. Dem kann man dann wieder zeigen, wo es hingehört, oder es sieht es selbst.“ (Jörn Budecker)

„Ich habe einfach das Gefühl, dass es tatsächlich gewisse Veranlagungen gibt, die genetisch bedingt sind, positive und negative. Und ich bin immer wieder beeindruckt, wenn ich Dinge an meinem Sohn feststelle, die ganz offenbar aus meiner Familie kommen. Es gibt bestimmte Ding, wie zum Beispiel das Aussehen vom Kind, das so frappierend dem Aussehen meines Vaters in diesem Alter ähnelt, das man sie fast für Zwillinge halten könnte. Und auch bestimmte vertraute Charaktereigenschaften sind bei ihm drin, die mich ganz ungeheuerlich verwundern." (Barbara Berger)

Ein „eigenes“ Kind zu bekommen, ist das erklärte Ziel aller im Kontext der Reproduktionstechnologien interviewten Personen. Der Wunsch nach einem „eigenen“ Kind hängt sowohl mit der Idee der Weitergabe der eigenen Identität durch genetisches Material als auch mit der Vorstellung von Unsterblichkeit oder Unendlichkeit zusammen. Die Gameten gelten somit als Träger von Identitäten, die von einer Generation an die nächste weitergegeben werden:

„Und dann haben wir gesagt, neugierig sind wir eigentlich schon darauf, was bei so einem Kind rauskäme, was es von wem erbt, wie es sich benimmt. Jetzt mal ein bisschen steril ausgedrückt, dieses Reproduzieren, was jetzt vom wem reinkommt und wie das Kind dann wäre, da waren wir schon sehr neugierig darauf. Und von daher haben wir gesagt, probieren wir es doch mal, es ist doch lohnenswert, wenn da etwas rauskommen kann. Die Gewissheit, dass man dann auch was von sich selbst zurücklässt, wenn man mal nicht mehr ist, ist sowohl ein erhebendes als auch irgendwie ein beruhigendes Gefühl. Also nicht unbedingt in materieller Hinsicht. Das ist das eine, aber es geht da ja noch um mehr, um ein Stück von einem selbst, was bleibt und vielleicht auch noch in

\footnotetext{
143 Einige der in diesem Kapitel verwendeten Interviewzitate sind von mir schon in Hauser-Schäublin et al. (2001) veröffentlicht worden.

144 Diesen Aspekt bestätigt auch die Studie von Edwards über die Vorstellung von Verwandtschaft im Zusammenhang mit Reproduktionstechnologien in England. Die von ihr interviewten Personen sind der Ansicht, dass durch Vererbung sowohl gute als auch schlechte Eigenschaften von den Eltern auf die Kinder weitergegeben werden, übertragen durch geteilte Substanz (sei es Blut oder Gene) (2000:215). Damit zusammenhängend werden Ähnlichkeiten als ein Zeichen von Zusammengehörigkeit gewertet, das weit über die täglichen Interaktionen hinausgeht. Es beinhaltet das Wissen darüber, eine Familie zu sein (vgl. Edwards 2000:221).
} 
weitere Generationen weitergegeben wird.“ (Ehepaar Martin und Tatjana Helming, 38 und 36 Jahre)

Dieses Entdecken des ,Eigenen“, der physischen Ähnlichkeiten sowie charakterlichen Eigenschaften, in dem eigenen leiblichen Kind, führt, wie Marion Wieger (34) erzählte, $\mathrm{zu}$ einem Gefühl von besonderer Vertrautheit und könnte auch dazu beitragen, dass Eltern bereit seien, eventuell ungewöhnliches Verhalten eines Kindes zu entschuldigen bzw. zu akzeptieren.

„Weil man doch unwillkürlich in einem eigenen Kind, in einem leiblichen Kind, nach Ähnlichkeiten sucht, und wenn man dann gewisse Dinge wiederentdeckt, macht es einem so ein Kind auch vertrauter. Nun ist Michael meinem Mann als auch meinem ältesten Schwager sehr, sehr ähnlich, nicht nur optisch, sondern auch in so vielen Kleinigkeiten. Der ist zum Beispiel völlig närrisch auf gelb. Das waren die beiden auch. Das sind solche Sachen, wo meine Schwiegermutter, jetzt wo er größer wird, immer wieder voll Begeisterung Bekanntes entdeckt. Das ist schon etwas ganz Besonderes, das geht eben nur bei dem eigenen Kind. Bei einem Adoptivkind würde man, glaube ich, sobald es Schwierigkeiten gibt, überlegen, wer weiß, wo das herkommt. Man würde sich vielleicht selber gar nicht so die Mühe machen, irgendwelche Probleme zu lösen, sondern würde selber unwillkürlich zu der Entschuldigung greifen, na ja, wer weiß, wo das herkommt.“

Auch von Ute Wille (36) wurde das (potentielle) Problem der ,fremden“ Eigenschaften eines Adoptivkindes im Unterschied zu den vertrauteren eines „eigenen“ Kindes angesprochen:

„Die [Ungewißheit] ist bei einer Adoption extrem groß. Über die macht man sich bei einer Adoption bestimmt bis zu 40\% Gedanken. Wer weiß, ob es [das Kind] Verbrechergene hat oder ob es nicht so intelligent ist, zu intelligent ist, sportlich oder unsportlich ist. Also man macht sich schon Gedanken, was da auf einen zukommt. Weil man von sich selbst ausgehend und dem Partner vielleicht schon eher Eigenschaften abschätzen kann, so wird es [das Kind] vielleicht jähzornig. Das Kind wird das und das. Aber wenn man praktisch die Eltern, die das Kind gezeugt haben, gar nicht kennt, weiß man gar nicht, was da für Wurzeln dahinter stecken. Sensibilität gehört - glaube ich - auch zu den Sachen, die angeboren sind. Es gibt auch Eigenschaften, die angeboren sind, die man nicht erziehen kann. Nicht Ordentlichkeit, nicht so lapidar. Ich meine jetzt schon feste Verhaltensregeln.“

Nicht alle der befragten Personen haben sich schon mit dem Gedanken an eine mögliche Adoption beschäftigt. Es zeigt sich, dass dies sehr davon abhängig ist, in welcher Phase sich das Paar befindet: Hat es gerade erst mit den reproduktionsmedizinischen Behandlungen angefangen und ist noch voller Hoffnung auf ,Erfolg' oder musste sich das Paar schon mit Enttäuschungen abfinden und erfahren, dass die Behandlungen nicht immer zum ,Ziel' führen. Die Nutzung der Reproduktionsmedizin als Behandlung der „ungewollten Kinderlosigkeit" steht hier eindeutig im Mittelpunkt. Eine Adoption kommt für viele wiederum nur dann in Frage, wenn es sich bei dem Kind um einen Säugling handelt. Ein Risiko sei bei einer Adoption immer dabei, da man ja nicht wisse, was das 
Kind mitbringe, zumindest sei aber die Vorprägung bei einem Neugeborenen so gering wie möglich.

Anhand dieser Aussagen lässt sich feststellen, dass das „eigene“ - leibliche - Kind assoziiert wird mit besonders enger Zusammengehörigkeit und Verbundenheit, verstanden als „echte“ Verwandtschaft, die sich besonders über Ähnlichkeiten manifestiert. Physiognomische Ähnlichkeiten zwischen ,echten“ Verwandten sind jedoch nicht nur für die Befragten selbst von besonderer Bedeutung, da sie die Vorstellung haben, dass sie sich aufgrund genetischer Übereinstimmungen in dem Kind wiedererkennen oder spiegeln, sondern lassen auch Außenstehende auf eine gemeinsame ,Biologie' schließen. So berichtete zum Beispiel Martin Bilcher (37): „Schon oft haben uns Leute auf der Straße angesprochen und gesagt , $\mathrm{Na}$, der [der Sohn] gehört aber ganz klar zu Ihnen bei der Ähnlichkeit!“

Diese Vorstellung wird auch von Seiten der Reproduktionsmedizin aufgegriffen und verstärkt. Im Zusammenhang mit der Idee von leiblicher Verwandtschaft wird vor allem die physische und intellektuelle Ähnlichkeit zwischen den Eltern und dem Kind hervorgehoben, was auch im Fall der heterologen Insemination, der Befruchtung mit „Spendersperma", besonders deutlich wird. So wird allergrößter Wert darauf gelegt, dass bestimmte äußerliche Merkmale des „Spenders“ wie Haarfarbe, Augenfarbe und körperliche Statur mit denen des sozialen Vaters des zukünftigen Kindes (dem Ehemann der biologischen Mutter) übereinstimmen. Für die betroffenen Paare ist dies von größter Bedeutung, wie Thomas Berger (35), „steril“ aufgrund einer Erbkrankheit, berichtete:

„Ich musste mich darauf verlassen, was die Ärztin uns gesagt hat. Dass er mir ähnlich sieht. Das war schon wichtig für mich. Man kennt das doch, alle unsere Verwandten suchen dann nach Ähnlichkeiten. Ich hör das doch immer bei Festen ,Ganz der Papa‘. Und wie soll ich denn reagieren, wenn das Kind nun ganz anders aussehen würde als ich. Das wäre doch jedes Mal ein Spießrutenlaufen."

Es wird deutlich, wie intensiv das Bemühen der Eltern (und der Ärzteschaft) ist, zwischen ihnen und ihrem heterolog gezeugtem Kind eine möglichst große Ähnlichkeit herzustellen. Dieses Bestreben hat zum Ziel, die soziale Umgebung in ihrer Annahme zu bestärken, dass es sich um ein leibliches Kind beider Eltern handelt. Aus dem obigen Interviewzitat geht hervor, dass das soziale Umfeld nach Übereinstimmungen in den äußeren Merkmalen zwischen Eltern und Kind sucht. Bei allzu großen Unterschieden, besonders zwischen Vater und Kind, beginnen Verwandte und Bekannte sich zu wundern und zu fragen, woher das Kind dieses und jenes Merkmal habe. Implizit schwingt immer die Vermutung eines Ehebruchs mit - ein Tatbestand, den die heterologe Insemination in sozialer Hinsicht vollständig ausblendet (vgl. auch Strathern 1993b:137f). Unter Rückbezug auf die analytische Trennung von Körper als Objekt der Behandlungen und Leib als subjektivem Empfindungsorgan (Kap. 2.3) lässt sich feststellen, dass 
hier zwar eine körperliche Beziehung zwischen „Spender“ und Empfängerin durch das Zusammenführen der jeweiligen Gameten zum Zweck der Zeugung besteht, jedoch keine leibliche Verbindung. Aus dem bisher Dargelegten wird deutlich, dass körperliche Verwandtschaft im Fall der Befruchtung mit „Spendersperma“ zumindest über ein Elternteil geschaffen werden soll, wobei wie gezeigt bestimmte Mechanismen existieren und Strategien eingesetzt werden, um nach außen die Fiktion gemeinsamer biologischer Elternschaft entstehen zu lassen und Leiblichkeit in Bezug auf beide Elternteile zu konstruieren. Anhand meines empirischen Materials zeigt sich, dass biogenetische Verwandtschaft mit Leib ,gefüllt' ${ }^{6}$ ist, was jedoch im Fall der heterologen Insemination ausgeblendet wird, d.h. die Körpersubstanz des „Spenders“ wird nicht mit leiblichen Eigenschaften assoziiert wie dies im Fall der medizinischen Behandlungsmethoden im homologen System stattfindet. Der körperliche Anteil, der von einem „Samenspender“ stammt, wird demnach sozial unsichtbar gemacht, d.h. der genetische Vater zählt in sozialer Hinsicht nicht.

Strathern hat jedoch für die euro-amerikanische Verwandtschaftskonzeption postuliert, - und mein empirisches Datenmaterials zeigt dies auch für Deutschland - dass die Vorstellung von Fortpflanzung die Annahme der Schaffung von Kontinuitäten zwischen den Generationen beinhaltet, d.h., dass man einen Teil von sich wieder erschafft. Die Fortpflanzung, über die sich „echte“ Verwandtschaft konstituiert, bezieht sich auf körperliche Prozesse. Damit wird dem dominanten Verwandtschaftsmodell zufolge die Entstehung einer Verbindung zwischen Schöpfer (creator) und Erschaffenem (created) assoziiert, was jedoch nichts über die tatsächliche Beziehung aussagt. Strathern stellt fest, dass ,procreation creates“, fragt aber zugleich danach, ob diese Art der Erschaffung auch als Reproduktion konzipiert wird:

„Reproduction commonly means to bring into existence something that already exists in another form. The form may be duplicated (as in making a copy), regenerated (as in making anew), in a symbolic relationship to the original (as in representing it), or recalled (as in bringing it into memory or the imagination). As biology is understood by the lay person, reproduction appears as the process by which an original plant or animal produces individuals similar to itself. Euro-American understandings of the similarities involved in human reproduction are, of course, not at all neutral as to the nature of relationship at issue. A relationship is thought to inhere in a continuity of personal (identity).“ (Strathern 1995:354)

Von Bedeutung ist im Zusammenhang mit der Vorstellung von Reproduktion als Weitergabe oder Wiedererschaffung eines Teils von sich selbst, dass ,the part must be 
known or seen in another specific person to be affective“ (Strathern 1995:354). ${ }^{145}$ Übertragen auf den Umgang mit der heterologen Insemination in Deutschland lässt sich feststellen, dass der „Samenspender“ dem kulturell dominanten biomedizinischen Zeugungsmodell zufolge zwar durch seine Gameten dazu beigetragen hat, ein Kind mit zu ,erschaffen ${ }^{6}$ und damit einen Teil der eigenen Identität durch sein genetisches Material an das Kind weitergegeben hat, seine biologische Reproduktion jedoch aufgrund seiner kompletten sozialen Ausblendung keine Anerkennung findet. „Spender“ können somit sozial als kinderlos gelten, weil ihr genetisches Material nicht zur Geburt ihres Kindes geführt hat: „In short, the procreative act did not lead to reproduction“" (Strathern 1995:355).

\subsubsection{2 „Richtige“ Eltern sein}

Wie ich anhand des empirischen Materials in Kapitel 5.1.1.3 dargestellt habe, gehört zu der Lebensplanung eines verheirateten Paares ein Kind. Damit ist die Vorstellung verknüpft, durch ein Kind zu einem Elternpaar zu werden, das Nachkommen besitzt, und damit sowohl die Blutsverwandtschaft weiterzuführen als auch eine „eigene“ Familie zu gründen. Das ,eigene Kinder bekommen“ wird somit am Körper festgemacht, wobei es um die Weitergabe des eigenen erbbiologischen Materials geht (vgl. Kap. 5.1.4.1). Die Interviews vor allem mit Frauen, die die reproduktionsmedizinische Hilfe in Anspruch nehmen, zeigen jedoch, dass der Wunsch nach einer leiblich erfahrbaren Schwangerschaft betont wird und die Frage der eigenen Identität in Zusammenhang mit der „Fruchtbarkeit“ eine immense Rolle spielt.

„Um was es mir geht? Um ein Kind natürlich, ein eigenes Kind zu haben, das ,Mama“ zu mir sagt, das zu mir gehört. Aber auch ganz stark darum, schwanger zu werden. Ich habe mich schon als Jugendliche im Traum mit schwangerem Bauch gesehen, ich wollte das immer. Zu fühlen, wie es in mir wächst, wie neues Leben entsteht. Das ist doch das Besondere am Frau-sein im Unterschied zum Mann. Dass in mir neues Leben entsteht und wachsen kann. Das ist die totale Erfüllung. Und irgendwann mal Mutter zu sein, das war einfach ein Teil meiner Vorstellung von mir, von meinem Leben. Deshalb bin ich auch bereit, alles dafür zu tun, dass sich dieser Wunsch auch erfüllen lässt.“ (Anja Dietrich, 29 Jahre)

Zur selbstentworfenen Lebensgeschichte, der eigenen Biografie, gehört fast immer die schon in Kindertagen vorgestellte Rolle als Mutter. Ein „eigenes“ leibliches Kind ist im

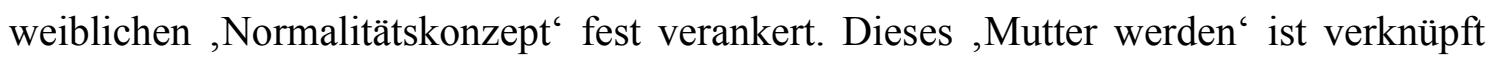
mit der Vorstellung, dass im eigenen Leib ein anderes Wesen heranwächst und sich wohlfühlen kann: „Mutter sein bedeutet, das Wunder der Fortpflanzung in sich selbst zu

145 Dazu auch Strathern (1995:355): „The very idea of continuity between generations is contained in the idea of a downward flow of characteristics. The reverse lies in the desire to trace origins, establish roots, claim inheritance." 
erleben“". Der eigene Leib wird als kreativ, kraftvoll, schützend und nährend vorgestellt und als konstituierend für die eigene Identität empfunden: „Mutter sein bedeutet für mich, den mütterlichen Instinkt ausleben zu können. Das Gefühl zu haben, in mir wächst neues Leben. In meinem Körper. Aus dem geht was Neues hervor.“

Das Austragen und Gebären eines „eigenen“ Kindes markiert auch in sozialer Hinsicht den Übergang von einer Rolle in eine andere, was wiederum direkte Rückwirkungen auf das Selbst und somit auf die Selbst-Identität hat (vgl. Giddens 1991:244). So formulierte Maria Heider (26) sehr deutlich, dass ihrer Ansicht nach eine Frau primär über die körperlichen Prozesse von Schwangerschaft und Geburt zu einer gesellschaftlich anerkannten ,Mutter' werde:

„Wenn man schwanger ist, dann wird man doch erst als Frau in der Gesellschaft anerkannt. Da beweist man auch nach außen, dass man eine richtige Frau ist, also damit meine ich, dass man Kinder bekommen kann. So wird es doch gesehen. Als Schwangere hat man doch einen ganz besonderen Status, man wird nämlich Mutter und das wird anerkannt von der Gesellschaft. Ich will ja selber auch Kinder kriegen, aber es ist auch irgendwie wichtig für andere. Alle fragen mich ständig, wann denn nun ein Kind kommt. Ich will ja auch, aber wenn andere das auch immer wieder toll finden, dann fühlt man sich selbst auch bestätigt. Und es ist ja auch ein tolles Gefühl, wenn man selber was Neues schaffen kann. Ich habe allerdings das Gefühl, dass fast alle in meinem Alter Kinder kriegen. Ich wollte ja schon immer, also schon ziemlich von Anfang an meiner Beziehung mit meinem Mann. Und jetzt steht man ohne Kinder da." (Maria Heider, 26 Jahre)

Es lässt sich feststellen, dass das ,Kinder bekommen“ einen zentralen Stellenwert in der Biografie der interviewten Frauen aufgrund des körperlichen Ausdrucks der eigenen Fruchtbarkeit und der Fähigkeit sich fortzupflanzen erhält. Giddens (1991) bezeichnet den Körper als reflexiven Teil des Selbst, das über den Körper zur Schau gestellt wird (siehe Kap. 2.3.3). Da das Selbst im Körper ,zuhause' ist, sichert dessen Kontinuität auch die Kontinuität der Selbst-Identität (Giddens 1991:55). Die subjektive Empfindungs- und Erfahrungswelt, die für das Selbst konstitutiv ist, ist jedoch der Leib. Damit wird die eigene subjektive - leibliche - Befindlichkeit von der Existenz eines Kindes abhängig, einerseits durch eine Modifizierung (oder Erweiterung) der Selbst-Identität über eine Veränderung des Körpers in seiner leiblichen Dimension, andererseits über die Anerkennung der Frau als Mutter vom sozialen Umfeld. Es wird hier deutlich, wie eng Selbst und Person miteinander verknüpft sind, da beide Aspekte eines Individuums verkörpert sind und körperlich (die Wahrnehmung des Körpers von außen) wie leiblich (die eigene Befindlichkeit) erfahren werden. Das Interviewzitat verweist auch noch auf einen anderen Aspekt: Die soziale Dimension des „Kinderwunsches“. Dem gewünschten Kind wird nicht nur eine hohe Bedeutung im Zusammenhang mit einem individuellen Glücksgefühl zugemessen, das noch nicht existierende Kind ist auch Inhalt zahlreicher Verortungen der „unfruchtbaren“ Frau in einer in ihren Augen „fruchtbaren“ Um- 
gebung. Hier zeigt sich, wie sehr der „Wunsch“ nach einem Kind und damit das reproduktive Verhalten soziale Tatbestände und als solche Formen sozialen Handelns sind. Frau Heider verortet sich nicht nur von sich aus mit ihrer Umgebung. Es wird auch von dieser sozialen Umgebung immer wieder die Tatsache der ausbleibenden Kinder angesprochen, was einen erheblichen Druck verursacht. Auch Stein und Sproll (1995:45) weisen darauf hin, dass Mutterschaft als ,Normalfall' eines gesellschaftlich akzeptierten Frauenbildes gilt und zusammenhängt mit einem sich „,natürlich“ entwickelnden, biologisch determinierten „Kinderwunsch“ der Frauen. So vermittelt die Öffentlichkeit zum Beispiel nicht gerade ein positives Bild kinderloser Frauen (unabhängig davon, ob sie sich selbst als „gewollt“ oder „ungewollt kinderlos“ definieren). Sei es in den Medien oder in der Werbung, kinderlose Frauen gelten oftmals als egoistisch und karrierebesessen, wohingegen unter dem Schlagwort ,neue Mütterlichkeit“ nicht nur aus konservativer Richtung eine Aufwertung der Mutterschaft gefordert wird. ${ }^{146}$ Durch diesen gesellschaftlichen Druck erhält der „Kinderwunsch“ auch eine neue Qualität und bleibt individuell wie gesellschaftlich ein zentrales Moment im Leben der Frau. Die Macht des öffentlichen Diskurses findet sich auch in meinem empirischen Material sowohl in den Aussagen der interviewten Frauen und Männer sowie insbesondere in den Verlautbarungen vieler Reproduktionsmediziner. Die Vorstellungen von weiblicher Identität wird verknüpft mit der Idee eines ,natürlichen Kinderwunsches“ der Frau aufgrund einer „,biologischen Vorherbestimmung“ gekoppelt mit emotionalen Bedürfnissen nach Pflege und Sorge. Dies führt zu der Ansicht, dass Frauen mehr als Männer unter der ,ungewollten Kinderlosigkeit" leiden (müssen), was wie gezeigt direkten Einfluss und Auswirkungen auf die persönlichen Erfahrungen und Erlebnisse der Frauen hat. Hier bieten sich nun die Reproduktionstechnologien an, die weibliche ,Unvollständigkeit ${ }^{\star}$, die sich in der „Sterilität“ ausdrückt, zu beheben, indem sie den Frauen zu einem Kind verhelfen.

Mein Datenmaterial zeigt jedoch, dass der „Wunsch“ nach einem Kind sowohl eine individuelle als auch eine paarbezogene Dimension sowie eine soziale Orientierung hat. So betonen die befragten Personen immer wieder, dass sie sich aus ihrer Liebesbeziehung und/oder ihrer persönlichen Lebenssituation heraus ein Kind wünschen, wobei die Vorstellung eines „eigenen“ Kindes eng verknüpft ist mit dem Bedürfnis nach der Transformation in eine bürgerliche Kleinfamilie. Es ist deutlich geworden, wie sehr der „Kinderwunsch“ für die interviewten Frauen mit dem leiblichen Erleben von Schwangerschaft und Geburt gekoppelt ist. Aber auch die damit einhergehende öffentliche Präsentation von „Fruchtbarkeit“ beider Ehepartner ist ein bedeutender Aspekt, insbeson-

\footnotetext{
146 Siehe dazu beispielsweise den Titel „Der neue Mutterstolz. Kinder statt Karriere“ und den Bericht „Immer mehr Frauen entdecken die Mutterrolle“ im SPIEGEL Nr. 29, 16.07.2001.
} 
dere im Zusammenhang mit der Methode der heterologen Insemination. Im Unterschied zur Adoption eines schon geborenen Kindes wird von Seiten der Ärzteschaft, aber auch von den betroffenen Paare eindeutig die gemeinsame Schwangerschaft als Vorteil der heterologen Insemination hervorgehoben. Barbara (33) und Thomas Berger (35), die mit Hilfe der heterologen Insemination 1995 einen Sohn bekamen, schilderten ihre jeweilige Sichtweise:

B.B.: „Ich bin davon überzeugt, dass die Möglichkeit einer gemeinsamen Schwangerschaft, also dass man schwanger sein kann als Paar, ein Riesenvorteil gegenüber einer Adoption ist. Ich bin sicher, dass Vater werden dadurch erst möglich wird. Viel besser möglich wird. Dass es auch für den nicht zeugungsfähigen Mann eine unheimliche Erleichterung ist, eine Beziehung zu dem Kind aufzubauen.“

T.B.: „Ja, das unterschreibe ich so. Wenn die eigene Frau schwanger ist und irgendwann dieses Kind zur Welt bringt, dann ist man eben Vater. Für sich selbst und für alle anderen auch. Da gibt es keine Frage nach Abstammung, da ist klar, das ist mein Kind. Und alle Welt erklärt Dir, dass er [der Sohn] dir völlig aus dem Gesicht geschnitten ist.“

Diese Zitate stehen exemplarisch für alle Interviews mit Paaren, die die heterologe Insemination nutzen, da alle Männer, die als ,zeugungsunfähig“ gelten, die Schwangerschaft ihrer Ehefrau als besonders bedeutend im Hinblick auf das ,Vater werden' eines „eigenen“ Kindes nennen. Dies lässt die Schlussfolgerung zu, dass die neue soziale Existenzform als Vater für den Mann in der Öffentlichkeit über die sexuelle Performanz läuft, die wiederum auf die Selbst-Identität zurückwirkt. ${ }^{147}$ Die öffentliche Darstellung der eigenen Fruchtbarkeit und der Fähigkeit sich fortzupflanzen, ${ }^{148}$ und somit der Anerkennung als ,Vater' läuft hier über den (sich verändernden) Körper der Frau. Durch die öffentliche Anerkennung als werdende Kernfamilie, die idealerweise eine biologische und soziale Einheit darstellt, entwickelt sich der reflexive Prozess des Selbst, der die Selbst-Identität als ,leiblicher“ Vater des Kindes trotz „Sterilität“ möglich werden lässt.

Im Sinne von Giddens (1991), der postuliert, dass die Selbst-Identität ein reflexiver Prozess zwischen innen und außen ist, der an die Kontinuität der eigenen Biografie geknüpft ist, kann hier festgestellt werden, dass die von mir interviewten Frauen wie Männer eine Veränderung oder Erweiterung ihrer Identität als Mutter oder Vater direkt oder indirekt über den Körper (und den Leib!), den reflexiven Teil des Selbst, anstreben und aus diesem Grund die Hilfe der reproduktionsmedizinischen Möglichkeiten in Anspruch nehmen. Die Reproduktionstechnologien, die eine ,Überbrückung‘ der „Un-

\footnotetext{
${ }^{147}$ Siehe dazu auch Kapitel 4.1.3 zu den kulturspezifischen Konstituenten von Vaterschaft und Mutterschaft im deutschen Recht. Strathern spricht auch von der sozialen Konstruktion von Vaterschaft über die soziale Anerkennung (1992c:163).

148 Weit verbreitet ist auch die Meinung über die Zusammengehörigkeit von Reproduktion und Potenz, die bei der Frau durch den Einsatz von Kontrazeptiva schon lange getrennt werden kann.
} 
fruchtbarkeit" ermöglichen (können), dienen somit dazu, die Selbst-Identität nach außen aufrecht zu erhalten, d.h. die eigene Lebensgeschichte trotz der „Unfruchtbarkeit“ kontinuierlich zu gestalten. Mit dieser Perspektive wird auch das Bemühen der Paare um die Konstruktion von möglichst leiblich-ähnlichen Kindern im Fall der heterologen Insemination einsichtig. Dabei geht es primär um die Kontinuität der Biografie des Selbst des sozialen Vaters, die in dieser Verwandtschaftskonstruktion dominant wird. Wie schon in Kapitel 4.1 gezeigt, lässt die deutsche Gesetzgebung eine kulturelle Konstruiertheit bezüglich Mutterschaft nicht zu: Die das Kind gebärende Frau gilt als die Mutter sowohl in biologischer als auch sozialer Hinsicht.

\subsubsection{Die Betonung der Körperlichkeit}

Im Zusammenhang mit dem Konzept eines „eigenen“ Kindes wird von den befragten Paaren immer wieder, besonders als Kontrast zu Adoptionen oder Pflegschaften, der Aspekt der Zugehörigkeit genannt: Ein „eigenes“ Kind ist eine Person, die zu einem gehört - körperlich (leiblich), emotional und rechtlich. Obwohl zumindest adoptierte Kinder, rechtlich gesehen, den biologischen Kindern vollständig gleichgestellt sind, spielt hier dennoch der Aspekt der biologischen Zugehörigkeit eine bedeutende Rolle. Bei einem ,eigenen“ Kind gibt es keine anderen biologischen Wurzeln und damit ,richtige“ Eltern, die - erzählt man dem Kind von der Adoption - immer eine Rolle spielen werden. Dazu nahm auch Ute Wille (36) Stellung:

„Wir haben auch über Adoption gesprochen, aber letztendlich habe ich gesagt, ich will das nicht, weil es nicht von mir ist. Da komme ich auf Familie und Blutsverwandtschaft zurück. Man stellt sich da ja bestimmte Sachen vor, das heißt, dass man vielleicht stolz sein kann auf sein Kind und will, und dann ist es von jemandem Fremden, und das widerspricht sich mir. Das muss sich nicht grundsätzlich widersprechen, aber bei mir ist das so. Und wenn ich mir dann vorstelle, ich tue alles mögliche für das Kind und irgendwann kommt auf jeden Fall die Frage ,Wo komme ich her?` Ich denke mir, dass das so ein Kind auch in Schwierigkeiten bringt. Und dass einen das selbst in Schwierigkeiten bringt, weil man dann zulassen können muss, dass es sich anders orientiert, weil es sagt ,Ich will zu meinen richtigen Eltern'. Oder zumindest versuchen wird dahin zu kommen, wenn es auch nicht unbedingt möglich ist."

Das Gefühl, man müsse sich mit den „richtigen“ Eltern gegebenenfalls zu einem gewissen Zeitpunkt konkret auseinandersetzen und die Angst vor dem, was dann geschehen könnte, beschäftigt die befragten Paare sehr. Die Vorstellung einer unauflöslichen, kontinuierlichen Beziehung zwischen biologischen Eltern und ihrem Kind und deren ungeteilte Zugehörigkeit wird auch in dem folgenden Interviewausschnitt deutlich, indem Henrike Ludwig (32) sich mit der Möglichkeit einer Adoption auseinandersetzt und ihre zwiespältigen Gefühle zum Ausdruck bringt:

„Wenn man dann dem Kind irgendwann erzählt, dass es adoptiert worden ist, will es sicher die richtigen Eltern kennenlernen. Und das wäre ja auch nichts Schlimmes, wenn 
man dann trotzdem die Eltern bleibt, auch wenn man nicht die leiblichen Eltern ist. Ich würde ihm dabei auch helfen, wenn es geht. Weil die leiblichen Eltern werden doch immer Fremde sein, denke ich mir. Aber das ist natürlich jetzt so theoretisch gesagt. Ich glaube ganz ehrlich, dass ich wahnsinnige Angst hätte vor dem was passiert, wenn mein Kind seine leiblichen Eltern treffen würde. Und wie reagieren die dann. Vielleicht wollen die ja dann eine Beziehung zu dem Kind, selbst wenn das Kind es vielleicht gar nicht so will. Wie soll man dann reagieren? Das ist alles so schwierig. Deshalb wollen wir eben auf jeden Fall erst einmal versuchen, ein eigenes leibliches Kind zu kriegen, von dem wir dann die Eltern sind. Und dann auch immer sein werden. Ich meine, natürlich gibt es Probleme zwischen Eltern und Kindern, das ist ja klar. Dass man sich streitet und nicht einer Meinung ist. Aber man gehört doch irgendwie zusammen, also das Kind gehört einfach zu mir als Mutter, und man muss nicht befürchten, dass man getrennt wird, also dass da sozusagen Gefahr von außen droht. Diese Beziehung zwischen Eltern und Kind kann eben keiner trennen, die dauert einfach ein Leben lang an und man gehört immer zusammen. So sehe ich das. Ist nicht einfach, aber das sind die Gründe, warum wir eben jetzt auch hier sind [in der Klinik für Reproduktionsmedizin].“

Noch präsenter sind die biologischen Eltern im Falle von Pflegekindern, die in der Regel nur für einen begrenzten Zeitraum zur Pflegschaft von diesen getrennt werden, später aber wieder zu diesen zurückkehren sollen (vgl. dazu Kap. 4.3.2). Diese Bedingungen sind für alle Interviewpartner unerträglich, weshalb sie Pflegschaften ablehnen. Dazu äußerten sich auch Ilona und Christoph Ottmers (30 und 33 Jahre):

I.O.: „Ich würde zum Beispiel kein Pflegekind nehmen. Obwohl ich vielleicht später mal Tagesmutter machen würde, aber ein Pflegekind würde ich nicht nehmen, weil ich das nicht verkraften könnte, wenn die mir das Kind wieder wegholen nach einer gewissen Zeit. Das könnte ich nicht. Die suchen ja überall nach Pflegeeltern. Aber ich denke, dass wir da nicht die einzigen sind, die damit ein Problem haben, das Kind wieder herzugeben. Deshalb finden die auch keine Pflegeeltern mehr. Die kriegen die Kinder ja einfach wieder weggenommen."

C.O.: „Da kommen dann die leiblichen Eltern, und die kriegen dann auch noch recht.“

Anhand der Aussagen wird deutlich, dass das ,eigene“ Kind im Kontext der Reproduktionstechnologien verschiedene Aspekte in sich vereint. Es ist Teil des eigenen Körpers (und des Leibes), d.h. die Beziehung zwischen Eltern und Kind konstituiert sich über Leiblichkeit. Diese Leiblichkeit bzw. die leiblichen Eigenschaften des Kindes führen zu dem Gefühl des Vertraut-seins und (Wieder-)Erkennens, die eine besondere Verbundenheit zur Folge hat (vgl. auch Kap. 5.1.4.1). Weiterhin beinhaltet die Weitergabe von biogenetischem Material eine Vorstellung der Kontinuität zwischen den Generationen, in dem es vergangene soziale Beziehungen beinhaltet und transferiert, die eine Person auch in der Gegenwart ausmachen. Mit einem „,eigenen“ Kind wird eine unauflösliche 
Beziehung assoziiert: Ein Kind zu haben wird damit gleichgesetzt mit einer Person, die immer zu einem gehört. ${ }^{149}$

Das biologische Kind ist demnach verbunden mit Vorstellungen enger und unauflöslicher Zugehörigkeit und Verbundenheit, verstanden als „echte“ Verwandtschaft. Dies lässt auch einen Rückschluss auf Schneider zu, der für das amerikanische Verwandtschaftskonzept festgestellt hat, dass die kognatische Liebe, die Eltern und Kinder verbindet, „unity, identity, oneness, togetherness, belonging“ symbolisiert (1980 [1968]:52), was durch die geteilten genetischen Substanzen noch bekräftigt wird. Auch Strathern (1992a) hat gezeigt, dass die Bedeutung des Zurückverfolgens der ,natürlichen“ Verbindungen einen bedeutenden Wert in der englischen Verwandtschaftskonzeption darstellt, da die Identität von Eltern zu Kindern ,fließt‘. Diese Idee basiert auf der Vorstellung sich zu reproduzieren und damit einen Teil seiner selbst wieder zu erschaffen: „That is why who the real parent is matters“ (Strathern 1992a:52). Der damit verbundene deszendente Fluss von ,Leben', von den Vorfahren auf die Nachkommen, führt zu der herausragenden Bedeutung der biologischen Eltern. Edwards (2000) arbeitet in ihrer Studie über Verwandtschaft und Reproduktionstechnologien in England weiterhin heraus, dass das Gefühl von Zusammengehörigkeit, insbesondere von Eltern und Kindern, über das Wissen um die genetischen Ursprünge des Kindes hinaus auch mit der eindeutigen Einordnung einer Person in die Vergangenheit und Gegenwart zusammenhängt. So stellt sie fest (2000:245): „To know a person fully requires more than a knowledge of them as an individual, it requires knowledge of them as a person embedded in, and embodying, kinship. Closeness results as much from knowing as from relating."

Gesamthaft betrachtet soll mit Hilfe der Reproduktionstechnologien ein Kind geschaffen werden, dass alle Kriterien der dominanten Verwandtschaftsvorstellungen, wie sie für Euro-Amerika von o.g. Autoren und bisher von mir für Deutschland herausgearbeitet wurden, erfüllen soll. Das „eigene“ Kind vermittelt eine emotionale Sicherheit und ein Gefühl von Kontinuität, dessen Existenz im Zusammenhang mit Adoptionen und Pflegschaften angezweifelt bzw. kritisch hinterfragt wird. Es ist deutlich geworden, dass hier der Körper im Konstruktionsprozess von Selbst, Person und Verwandtschaft eine bedeutende symbolische Funktion übernimmt.

\footnotetext{
149 Auf die Idee des individuellen Besitzens weist auch Sault in ihrer Analyse des amerikanischen Konzeptes von Elternschaft hin: „When people talk about wanting a child of their own, they mean that the child must be born to them to be real, and born to them to be theirs, their own, or owned. [...] People do not share ownership of a child, for the child is theirs exclusively“ (1994:298; Hervorhebungen im Original).
} 


\subsection{Ein Blick auf die Konstruktion von Zugehörigkeit und Angehörigkeit im Kontext von Adoptionen und Pflegschaften}

Wie ich schon in Kapitel vier dargelegt habe, bietet der deutsche Staat auch die Möglichkeit einer Familiengründung mittels einer Adoption oder der Übernahme einer Pflegschaft eines schon geborenen Kindes. Diese Art der Familien- und Verwandtenbeziehungen konstituieren sich somit nicht über eine geteilte Körpersubstanz, sondern basieren einzig auf rechtlichen Vereinbarungen und legal anerkannten Zugehörigkeiten. Im Folgenden soll es nun um den persönlichen Diskurs von enger Verbundenheit im Kontext von Adoptionen und Pflegschaften gehen unter besonderer Berücksichtigung der Frage, ob Unterschiede in der Konstruktion von Verwandtschaft bei den Personen bestehen, die im Vergleich zum Kontext der Reproduktionsmedizin ohne den Körper auskommen müssen.

\subsubsection{Soziale Interaktionen und der Aspekt der gemeinsamen Lokalität als Basis enger emotionaler Bindungen}

Auch im Kontext von Adoptionen und Pflegschaften gilt die Gemeinschaft von Personen, die als Familie bezeichnet wird, als wichtigste und intimste Form der Zugehörigkeit. Sehr häufig wird ,Familie' von ,Verwandtschaft' abgegrenzt, nicht was die formale Zuordnung von Verwandten als Familienmitglieder betrifft, da die Kategorie ,Verwandte' auch die Familie mit einschließt, sondern in Bezug auf die emotionale Zugehörigkeit. ,Familie“ steht hier für Personen, die miteinander eine affektive Beziehung teilen, die auch das soziale Handeln bestimmt, was Hanna Gerlach (42), die ein Adoptivkind von 12 Jahren und zwei Pflegekinder im Alter von 10 und 13 Jahren hat, sehr deutlich formulierte:

„Familie können für mich zwar Verwandte sein, aber Verwandte sind deshalb noch lange nicht Familie für mich. Verwandt zu sein ist doch nur ein Status, aber was bedeutet das für mein Beziehungsleben? Familie ist doch ein sehr intimer Kreis von Personen, denen ich mich emotional eng verbunden fühle. Mit jemandem verwandt zu sein bedeutet für mich nicht, mit dem Kontakt zu pflegen, oder dass ich was mit dem gemeinsam haben muss. Ich habe viele Verwandte, das sind Fremde für mich. Und nur weil wir verwandt sind, herum zu reisen und uns zu sehen, kommt nicht in Frage. Eigentlich nur bei runden Geburtstagen und Beerdigungen. Familie ist verwandt, aber verwandt ist für mich nicht Familie. Ich habe auch zu meinen Geschwistern nur spärlichen Kontakt. Da stehen mir viele Leute näher, die mir fremd, also die nicht blutsverwandt sind.“

Im Unterschied zu den Paaren, die ich im Kontext der Reproduktionsmedizin interviewt habe, lässt sich feststellen, dass bei Adoptiv- und Pflegeeltern eine Tendenz besteht, bei der Konstruktion von Familie die soziale Komponente besonders hervorzuheben und 
die biologische Zugehörigkeit nur als eine Möglichkeit der Verbundenheit darzustellen. So äußerte sich Anneliese Hildmann (40), Adoptivmutter eines sechsjährigen Kindes:

„Familie ist das Nest, eine Beziehung mit gegenseitigen Abhängigkeiten sowohl emotional, organisatorisch als auch finanziell. Dazu zähle ich schon in erster Linie Eltern und Kinder, aber das sind äußere Faktoren. Ich meine damit, das ist so die klassische Zusammensetzung, und sie stimmt ja auch fast immer. Aber es geht darum, dass eine Familie sich nicht unbedingt aus Blutsverwandten zusammensetzen muss. Wenn beides zusammenkommt, also die leiblichen Eltern sind auch die sozialen, ist es okay und auch gut so natürlich. Aber um was es mir geht, das muss nicht so sein. Deshalb kann man sich einfach nicht lieber haben. Da geht es doch ganz klar um mehr.“

Der Begriff der ,Blutsverwandtschaft', der wie in Kapitel 5.1 gezeigt, auf Abstammung und ,natürliche“ Verbundenheit über Körper rekurriert, wird in 22 Interviews mit Adoptiv- und Pflegeeltern nur dreimal erwähnt, ansonsten überwiegt die Überzeugung, dass gemeinsames Blut keine Grundlage bildet für emotionale Bindungen und Vertrautheit. Anhand diverser Beispiele von der eigenen oft als negativ empfundenen Beziehung zu den leiblichen Eltern wird dieser Aspekt immer wieder in Frage gestellt. Dies führt in sechs Fällen dazu, dass Egos Eltern aufgrund der als schlecht definierten gegenseitigen Beziehungen, was sowohl das Fehlen von emotionaler Unterstützung, von Solidarität und Reziprozität einschließt, explizit nicht als Teil der Familie gelten.

An dieser Stelle muss jedoch erwähnt werden, dass ein wesentlicher Unterschied zwischen den Interviews im Kontext von Reproduktionsmedizin und Adoptionen sowie Pflegschaften hinsichtlich der Perspektive der Befragten bezüglich Definitionen und Zuordnungen bestimmter Personen zu Familie und Verwandtschaft zu erkennen ist. Bei den Paaren, die sich vor oder in reproduktionsmedizinischen Behandlungen befinden, bildet schwerpunktmäßig die Aszendenz, bei Adoptiv- und Pflegeeltern, die zum Zeitpunkt der Interviews alle mit leiblichen, Adoptiv- und/oder Pflegekindern zusammenleben, die Deszendenz den Bezugsrahmen. Dieser Aspekt wird besonders deutlich bei den Paaren, die über lange Jahre Pflegekinder hatten oder noch haben. Es lässt sich feststellen, dass die Definition von Familie und die Zuordnung bestimmter Personen in diese Kategorie eng mit einer gemeinsamen Lokalität zusammenhängt und im Sinne einer Haushaltsgemeinschaft verwendet wird: „Familie, das sind alle, die unter einem Dach leben“, „Alle, die hier im Haus leben oder mal gelebt haben.“ Es zeigt sich allerdings, dass die emotionale Verbundenheit zu den einzelnen Personen dennoch als sehr unterschiedlich bezeichnet wird, die u.a. abhängig von der Dauer des Aufenthaltes der Pflegekinder ist. Auch in diesem Zusammenhang wird die zeitliche Dimension als Kriterium für Zugehörigkeit offensichtlich: Je länger und intensiver man zusammen lebt, desto größer die Verbundenheit. Da eine gemeinsame ,Biologie‘ hier nicht die Grundlage bilden kann, wird besonders betont, dass dieses Gefühl durch das soziale Handeln im Alltag über einen langen Zeitraum entsteht. Dazu äußerte sich Götz Kraus (45) mit ei- 
nem Pflegekind und einem Adoptivkind: „Hat ein Kind bestimmte Lebenszyklen mit uns durchlaufen, gehört es zur Familie dazu, egal ob es ein leibliches Kind ist, ein Pflege- oder Adoptivkind. Auf die gemeinsam verbrachte Zeit kommt es an und wie intensiv diese miteinander durchlebt wurde.“

Auf den ersten Blick scheint die Betonung der sozialen Komponente in der Konstituierung von enger Verbundenheit im Kontext von Adoptionen und Pflegschaften eine wesentlich größere Rolle zu spielen als in den Interviews im Kontext der Reproduktionsmedizin. Bei genauerem Hinsehen fällt jedoch auf, dass Egos Zuordnung von bestimmten Personen zu der Kategorie ,Familie' auch hier kontext- und situationsabhängig ist. Ulrich Küpke (73), in dessen Haus in den letzten 25 Jahren 15 Pflegekinder gelebt haben, berichtete einerseits von seiner großen Familie und erklärte, dass alle, die im Laufe der Jahre mit ihm zusammengelebt hätten, ohne Unterschied zu seiner Familie dazu gehörten. Gleich darauf erzählte er jedoch von einem Familienstammbaum, den er gerade erstellte. Auf meine Nachfrage, welche Personen dort vorkämen, nannte er nur seine leiblichen Kinder sowie seinen Adoptivsohn und deren Kinder, nicht aber die Pflegekinder. Herr Küpke versuchte diese unterschiedliche Zuordnung zu erklären, indem er darauf verwies, dass es seiner Ansicht nach eine ,theoretische Ebene der Familienzugehörigkeit“ und eine „emotionale Ebene von Familie“ gäbe, wobei er zu letzterer seine Pflegekinder immer mit dazu zähle.

An diesen Ausführungen fällt auf, dass die unterschiedlichen Ebenen von Zugehörigkeit auf verschiedene Dimensionen Bezug nehmen. Die von Herrn Küpke ,theoretische Ebene der Familienzugehörigkeit“" genannte Zuordnung von Personen, die in einem von ihm erstellten Familienstammbaum auftauchen, bezieht sich sowohl auf eine geteilte Körpersubstanz durch gemeinsame Abstammung als auch auf eine staatlich legitimierte Zugehörigkeit durch Adoption. Die „emotionale Ebene“ zwischen Pflegekindern und -eltern findet von staatlicher Seite keine Anerkennung bzw. wird im Bürgerlichen Gesetzbuch (BGB) nicht als Verwandtschaft definiert (Kap. 4.1). Meiner Ansicht nach zeigt dieses Beispiel sehr deutlich, dass sich auch in der Definition der Kategorie ,Familie‘, die im persönlichen Diskurs der Adoptiv- und Pflegeeltern auf den ersten Blick sehr viel mehr Personen integriert und den sozialen Aspekt stärker betont als dies im Kontext der Reproduktionsmedizin der Fall zu sein scheint, das kulturell dominante Modell von ,echter“ Verwandtschaft finden lässt, das auf gemeinsamer Abstammung basiert, ergänzt durch staatlich legitimierte Zugehörigkeiten wie Adoptionen. Anhand des empirischen Materials konnte jedoch bisher gezeigt werden, dass kulturelle Vorstellungen über geteilte Körpersubstanz nur ein Teil der verschiedenen Elemente für das Gefühl von enger Verbundenheit darstellen und von den befragten Adoptiv- und Pflegeeltern immer wieder das soziale Handeln im Alltag als ein bedeutender Aspekt hervorgehoben wird, durch welches der eigene Beziehungskreis erweitert oder eingeschränkt 
werden kann. Ein Zusammengehörigkeitsgefühl, das durch soziale Interaktionen im Alltag entsteht, ist abhängig von Kriterien wie Sympathie, Kontakt, Gemeinsamkeiten, Hilfeleistungen und Vertrauen, die immer wieder aktualisiert und modifiziert werden können und müssen. Soziale Beziehungen, die auf diesen genannten Kriterien basieren, werden von den Adoptiv- ebenso wie den Pflegeeltern als wesentlicher Bestandteil des Lebens betont und als essentiell für die persönliche Verortung definiert.

In diesem Zusammenhang wird auch in den Interviews mit Adoptiv- und Pflegeeltern die „Seelenverwandtschaft“ als „Übereinstimmung in der Chemie“ und zur expliziten Betonung einer besonderen emotionalen Verbundenheit erwähnt (vgl. Kap. 5.1.1.1). Dieser Ausdruck wird hier verwendet, um sich von der Idee einer gemeinsamen Verbindung über den Körper als Grundlage enger sozialer Beziehungen zu distanzieren. Das eine schließt das andere zwar nicht aus, wobei primär die „seelische“ Bindung, geprägt von gegenseitiger Zuneigung und gemeinsamen Interessen, als konstituierend für diese Kategorie von Beziehungen hervorgehoben wird und die besondere Qualität und Tiefe einer Beziehung beschreibt. Insbesondere im Zusammenhang mit als Freunden bezeichneten Personen wird diese Wesensübereinstimmung vorausgesetzt, wie Heike Schlote (38), Adoptivmutter eines vierjährigen Kindes, feststellte:

„Mit Freunden, das ist so eine Art Seelenverwandtschaft. Es gibt Leute, da stimmt es einfach. Es gibt Leute, die lernt man kennen, und da stimmt die Chemie. Man hat eine Wellenlänge und versucht, möglichst oft was zu unternehmen und eben auch den Alltag bzw. die Alltagsfreizeit miteinander zu verbringen. Weil man dann oft die gleichen Interessen und Vorlieben hat. [...] Meine eine Schwester lebt in Bonn. Die ruft schon regelmäßig mal an, aber die lebt einfach in einer anderen Welt. Die lebt mitten in der Stadt, ist unverheiratet, hat da ihren Freundeskreis und ihren Job und findet uns hier, glaube ich, total langweilig. Ich denke, so können einem Geschwister auch verloren gehen, egal was einen mal verbunden hat. Mit meiner Freundin halte ich ständig Kontakt, mit der teile ich einfach mehr von meinem Leben.“

Sowohl im Kontext der Reproduktionsmedizin als auch im Kontext von Adoptionen und Pflegschaften spielen Freundschaften eine Rolle im Leben der Befragten. Es zeigt sich jedoch, dass ein wesentlicher inhaltlicher Unterschied der Aussagen in der Gewichtung liegt, die Freunden im eigenen sozialen Beziehungssystem zugesprochen werden. Da Adoptiv- und Pflegeeltern die besondere Bedeutung von Freundschaften im Unterschied zu Verwandtschaft immer wieder betonen, möchte ich im Folgenden auf die Kriterien eingehen, die genannt werden, um Verwandtschaft und Freundschaft gegeneinander abzugrenzen. Lässt sich für den Kontext der Reproduktionsmedizin eher eine die Verwandtschaft ergänzende Rolle von Freundschaften feststellen, ist hier oftmals auch davon die Rede, dass Verwandtschaft durch Freundschaften ersetzt wird. Im Vergleich ist jedoch zu erkennen, dass dabei immer wieder auf bestimmte Aspekte verwiesen wird, die dem Konzept von Verwandtschaft, das auch Vorstellungen über das soziale Handeln umfasst, wie ich es in Kapitel 5.1 für den Bereich der Reproduktions- 
medizin herausgearbeitet habe, zugeschrieben werden. Verwandtschaftliches Handeln ist hier jedoch weiter gefasst und bezieht auch soziale Beziehungen ,nichtverwandtschaftlicher" Art mit ein, die eine bedeutende Rolle im Leben der interviewten Adoptiv- und Pflegeeltern spielen. Als wesentliche Kriterien für die Zuordnung bestimmter Personen zu der Kategorie ,Freunde‘ werden u.a. Zuneigung, Vertrauen, gleiche Interessen, ähnliches Alter und gegenseitiges Verständnis genannt. Deutlich wird hier, dass die Merkmale von Freunden sehr viel homogener sind als diejenigen von Personen, die der Kategorie ,Familie“ oder ,Verwandtschaft' zugeordnet werden. Enge Beziehungen zu Freunden sind grundsätzlich positiv besetzt, was für die Familie nicht unbedingt zutreffen muss. Dies führt beispielsweise dazu, dass persönliche Dinge oft mit Freunden besprochen werden, so auch Probleme, die innerhalb der Familie auftreten. So erzählte Klaus Stellmacher (42), Pflegevater von drei Kindern, dass er die Meinung von Freunden besonders wertschätzen würde, da sie einerseits als Außenstehende die Situation beurteilen könnten, anderseits jedoch aufgrund der gemeinsamen Freundschaft emotional involviert seien.

Die Freiwilligkeit des persönlichen Umgangs und die persönliche Selektion unterscheidet nach Aussagen von Adoptiv- und Pflegeeltern Freundschaften explizit von Verwandtschaft. Es wird betont, dass das normative Element von Verwandtschaft fehle und die soziale Ausgestaltung von Freundschaften wesentlich flexibler sei, da sie zwischen Freunden individuell ausgehandelt werden könne. Hervorgehoben werden insbesondere Gefühle von Nähe und Zuneigung als Voraussetzung für gegenseitige Hilfe und Unterstützung, die nicht abhängig seien vom rechtlich festgelegten Verwandtschaftsstatus. Im Umkehrschluss wird auch darauf hingewiesen, dass gerade aufgrund von gemeinsamen Erlebnissen und gegenseitigen Verlässlichkeiten eine besondere Nähe und Bindung zwischen bestimmten Leuten entstehen könne, die als Freundschaften bezeichnet würden.

\subsection{2 „Alle meine Kinder“6}

Das vorherige Kapitel hat gezeigt, dass auch im persönlichen Diskurs, der im Kontext von Adoptionen und Pflegschaften geführt wird, die soziale Konstruktion von „Blutsverwandtschaft" deutlich wird. Die Konstituenten von als besonders eng konzipierten Formen von Verbundenheit sind hier tendenziell eher sozialer Art. Soziale Interaktionen im Alltag, affektive Bindungen, Vertrauen und besonders eine gemeinsame Lokalität sind Merkmale von Vorstellungen über Zugehörigkeiten und Angehörigkeiten, die sehr oft explizit den biologischen Gemeinsamkeiten gegenüber gestellt werden. Vor diesem Hintergrund stellt sich im folgenden Kapitel die Frage nach den Vorstellungen über Elternschaft und Eltern-Kind-Beziehungen, denen das Kriterium der körperlichen Verbindung, fehlt' und die ,nur' sozial konstituiert sind. 


\subsubsection{1 „Sie ist so sehr meine eigene Tochter, so von ihrem Wesen her.“}

Auch im Zusammenhang mit einem Adoptivkind, das nicht das Resultat der Gametenverschmelzung des Adoptivelternpaares ist und bei dem somit der Aspekt der genetischen Vererbung nicht zutrifft, lässt sich erkennen, dass eine ,Suche“ nach Übereinstimmungen stattfindet und eine wichtige Rolle spielt. Die Bedeutung von Ähnlichkeiten sowohl in physischer Hinsicht als auch in Wesensmerkmalen zwischen Personen, die als Familie kategorisiert werden, wurde schon im Diskurs über das „eigene“ Kind im Kontext der Reproduktionsmedizin dargestellt. So berichtete jedoch auch Astrid Kruse (37), die zwei Adoptivkinder im Alter von 7 und 1,5 Jahren hat:

„Das sind beides unsere eigenen Kinder, unsere Töchter. Das ist überhaupt keine Frage. So sage ich das auch jedem. Lisa passt unheimlich in die Familie meines Mannes rein und Lena ist mein Typ. Ich hole nachher noch mal ein Foto, damit man den krassen Unterschied sieht zwischen den Mädchen. Und Lena ist so sehr meine eigene Tochter, so von ihrem Wesen her, jeder stößt nur an ihre dunklen Augen. Das ist das einzige Kriterium, wo die Leute immer sagen, wo hat sie denn die dunklen Augen bloß her? Und wenn sie jetzt noch mehr meine Augenfarbe hätte, würde das keiner in Frage stellen. Würde das niemand hinterfragen. Da kann man aber sogar sagen, mein Großvater hatte auch ganz braune Augen, mein Schwiegervater hat braune Augen. Da wäre auch wieder eine Parallele. Das sind so die Dinge, die Kinder werden einem, sind einem so ähnlich. Weil man lebt ja auch so viel vor. Du [zu ihrem Mann] sagst oft zu mir, ,Das ist deine Tochter, merkst du das nicht? Sie ist schon so pingelig wie du.' Im Kinderzimmer muss es so und so aussehen. Und sie hat für gewisse Dinge eine besondere Ordnung im Kopf, dass ich denke, oh Gott, so bist du auch. Das hat sie halt nicht mit in die Wiege gelegt bekommen. Aber sie ist zum Beispiel ganz musikalisch. Wir sind das nicht, aber wir unterstützen das und haben ihr ein Klavier gekauft. Das sind so Dinge denke ich, die bekommen sie schon mitgeliefert. Also ich favorisiere immer so den Gedanken, dass Erziehung und Umwelt den Menschen doch wesentlich prägen.“

Wie ich schon in Kapitel 5.1.4.1 beschrieben habe, offenbart sich auch hier die Vorstellung, dass äußere Übereinstimmungen zwischen konsanguinen Verwandten ihren Ursprung in gemeinsamen Genen haben, die über den Prozess der Fortpflanzung an die Nachkommen weitergegeben werden. Anhand des obigen Zitates lässt sich erkennen, dass das Entdecken von bestimmten Ähnlichkeiten auch im nicht-leiblichen „eigenen“ Kind zu einem Gefühl von Vertrautheit und Zusammengehörigkeit führt, jedoch der Einfluss von Sozialisation und Umwelt für die Entwicklung des Kindes hervorgehoben und höher gewichtet wird als die Vererbung. Die Betonung dieser sozialen Aspekte von Verwandtschaft spielt im Konzept eines ,eigenen“ Kindes und in der konkreten sozial gelebten Eltern-Kind-Beziehung im Kontext von Adoptionen eine große Rolle, was ich im Folgenden noch näher zeigen werde. Anhand der bisherigen Darstellungen lässt sich jedoch konstatieren, dass sich die kulturell dominante Vorstellung von „natürlicher“ Verwandtschaft, die sich über Körper konstruiert, auch im Zusammenhang mit Adoption wiederfinden lässt. Ähnlichkeit wird somit als kulturell bedeutendes Zeichen enger Zugehörigkeit in Deutschland verstanden. 
Aus den Interviews mit Adoptiveltern kristallisiert sich auch ein weiterer Aspekt heraus: Die „Freiheit“, die ein Adoptivkind im Vergleich zu einem biologischen Kind mit sich bringt. Aufgrund der mangelnden genetischen Übereinstimmungen und somit der fehlenden Möglichkeit der Vererbung körperlicher Eigenschaften oder Merkmale können sich die Befragten eher von den Kindern distanzieren und diese als ,eigenständigere“ Personen ansehen, ohne nach etwas von sich selbst zu suchen. Sie fühlen sich ausschließlich in sozialer Hinsicht für das Verhalten des Kindes verantwortlich, was ebenfalls oft als schwierige Aufgabe empfunden wird. Dennoch bezeichnete es Ulla Zinke (38), Adoptivmutter ein achtjährigen Tochter, als ein Gefühl großer Befreiung, dass sie zwar ihren Erziehungsstil immer wieder hinterfrage, sich in ihrem „Selbstbild“ jedoch nicht in Frage gestellt sehe, wenn ihr Kind kritisiert werde:

„Ich will ihr Selbstbewusstein aufbauen, das ist meine Aufgabe als Mutter. Wenn es Konflikte gibt mit anderen muss sie für ihren Teil gerade stehen. Vielleicht bin ich in manchen Dingen eine andere Mutter als eine leibliche Mutter. Weil ich das schon bei vielen leiblichen Müttern gesehen habe: Mein Kind würde so was nie tun. Das können sie doch von meinem Kind nicht verlangen. Mein Kind soll das und das, und das würde mein Kind nie sagen oder tun. Mein Kind, das muss ich abholen. Mein Kind kann ich doch nicht so lange allein lassen. Das eigene Kind wird also grundsätzlich in Schutz genommen, und das merken die anderen Kinder. Das hängt damit zusammen meine ich, dass die sich eben alle in irgendeiner Weise in ihren Kindern wiedererkennen oder sich selbst sehen und dann angegriffen fühlen in ihrem Selbstbild, wenn andere ihr Kind kritisieren, oder wenn ihr Kind Probleme mit anderen hat. Das Gefühl habe ich eben nicht. Ich stehe da eher ein bisschen daneben und kann mein Kind auch von außen betrachten. Ich finde, das macht mich auch freier. Ich fühle mich natürlich auch nicht gerade toll, wenn mein Kind irgendwas falsch macht oder sich blöd verhält, aber dann muss ich eben überlegen, wie ich ihm vielleicht was anderes vorleben kann oder muss meine Erziehung mal überdenken. Das stellt mich aber nicht so total in Frage, wie das, glaube ich, bei leiblichen Eltern oft der Fall ist.“

Auch Ingo Kruse (39), Adoptiv- und Pflegevater, äußerte sich zu dem „Stress“, den seiner Ansicht nach leibliche Eltern aufgrund der Vorstellung von der Vererbung eigener genetischer Merkmale sehr häufig hätten:

„Also Eltern haben ja immer das Bestreben, mit ihrem Kind anzugeben. Das geht mir ja teilweise auch so. Muss man ganz ehrlich sagen. Mein Kind ist gut in der Schule, sieht hübsch aus, hat saubere Finger, hat gerade das und das gemacht. Aber Daniel ist nun mal eher ein schwieriges Kind. Da geht es immer nur: ,Oh Gott der Junge, was macht er denn jetzt schon wieder. Wo hat er das bloß her?` Ich kann es nicht mehr hören und will den Jungen davor schützen. Soll ich jetzt immer sagen, von mir hat er es nicht. Kann er ja gar nicht, weil er nicht mein eigen Fleisch und Blut ist. Ziemlich eingeschränkt. Vielleicht kann ich auch lockerer mit den Schwierigkeiten und seinen Macken umgehen, weil es nicht so direkt von mir kommen kann. Nicht so wie bei leiblichen Eltern, die den Stress haben oder sich ihn machen, dass sie womöglich so was vererbt haben. Auf was Gutes wollen natürlich Eltern und Großeltern stolz sein, was Schlechtes verschweigt man. Bei unserem Jungen ist es eben sehr schwierig. Ich gebe ihm mit, was ich kann, Gene sind's ja nicht. Ich versuche alles mögliche mit ihm zu machen und ihm zu helfen. Vielleicht kann ich das eben auch gut mit dem Jungen, weil er eine Prägung 
hat, auf die ich keinen Einfluss hatte. So muss ich ihn nehmen, dafür kann ich aber auch nichts."

Der Unterschied zu biologischen Eltern wird in beiden Zitaten darin gesehen, dass sie als Adoptiveltern mit dem Kind aufgrund fehlender Weitergabe von genetischem Material nicht körperlich verbunden sind. Es lässt sich vermuten, dass sie sich daher nicht in ihrem Selbst, in ihrer verkörperten Identität, in Frage gestellt sehen (vgl. Giddens 1991). Ich habe in Kapitel 2.3. aufgezeigt, wie sehr der physische Körper in euroamerikanischen Gesellschaften als Basis des Selbst erachtet wird (vgl. ScheperHughes/Lock 1987:16-17) und in seiner verleiblichten Form eine besondere Bedeutung für die Selbst-Identität besitzt. Da der Adoptiveltern-Adoptivkind-Beziehung die Konstituente der geteilten Körpersubstanz fehlt, wird das Kind zwar als das „eigene“ - nicht jedoch als das ,eigene“ leibliche - Kind definiert und ist nicht verbunden mit der Vorstellung der Weitergabe von leiblichen Eigenschaften. Es bestehen zwar körperliche Beziehungen in Form von sinnlichen Berührungen (Umarmungen, Hand halten u.a.m.), die über den eigenen Leib erfahren werden, jedoch werden diese körperlichen Ausdrükke enger Beziehungen nicht als leibliche Verbindungen zwischen Eltern und Kindern konzipiert. Diese fehlende körperliche und leibliche Verbindung im Fall von Adoptionen führt dazu, dass man sich zwar zum einen nicht auf äußerliche Merkmale als erkennbare soziale Zeichen von Zugehörigkeit berufen kann, sich aber zum anderen nicht im eigenen individuell verkörperten Selbst und dessen Wahrnehmung über den Leib in Frage gestellt sieht und vom (anderen) Selbst des Kindes abgrenzen kann. Diese Überlegungen stehen im Gegensatz zu zahlreichen Ausführungen von Befragten im Kontext der Reproduktionsmedizin, die der Ansicht sind, dass gerade die bekannten Eigenschaften eines leiblichen Kindes im Unterschied zu den ,fremden' eines Adoptivkindes ein Gefühl von besonderer Vertrautheit und Verständnis auch für ungewöhnliches Verhalten des Kindes schaffen. Da diese Möglichkeit der Weitergabe des eigenen Selbst, das direkt verknüpft ist mit Identität, von Adoptiveltern auf Adoptivkind ausgeschlossen ist, scheinen daher hier die sozialen Komponenten von Elternschaft, geprägt von Fürsorge, Erziehung, Schutz und Beistand für die Entwicklung des Kindes höher gewichtet als die biologischen.

\subsubsection{2 „Eltern wird man aus durchwachten Nächten, durch Wickeln und Freude an der Entwicklung des Kindes“"}

Anhand meines empirischen Datenmaterials kann ich feststellen, dass auch im Kontext von Adoption und Pflegschaften zu der Biografie eines verheirateten Paares ein Kind gehört. Auch im Zusammenhang mit Adoptionen berichten alle Befragten von einem 
Bruch in der Lebensplanung, als sich herausstellte, dass sie zwar fortpflanzungswillig, nicht aber fortpflanzungsfähig waren. ${ }^{150}$ Anhand der Interviews lässt sich feststellen, dass insbesondere in der Lebensplanung der Frauen schon früh eine Lebensgeschichte entworfen wurde, in der sie irgendwann die Rolle als Mutter übernehmen würden. Dorothea Klein (38) erzählte in der Retrospektive, dass der Wunsch nach einer Schwangerschaft nicht unbedingt der vorrangige Grund war:

„Ich wollte schon immer gern Kinder um mich herum haben, wollte immer für viele Kinder Mutter sein. An Schwangerschaft habe ich dabei gar nicht so sehr gedacht. Das war nicht das Wichtigste. Auch wenn ich ein leibliches Kind gehabt hätte, hätte ich noch eins adoptieren wollen oder Pflegekinder haben wollen. Ich wollte immer viele Kinder im Haus.“

Zwei Frauen wussten schon im Alter von Anfang 20, dass sie aufgrund medizinischer Indikationen keine Kinder austragen und gebären können. Sie mussten sich schon sehr früh damit auseinandersetzen, dass die Vorstellung vom ,Mutter werden“ nicht über den eigenen Leib möglich sein würde. Die Frage nach der Veränderung oder Erweiterung der eigenen Identität als Mutter musste dementsprechend unabhängig vom Körper betrachtet werden, wie Evelyn Behler (33) erzählte:

„Ich war nie ganz wild auf diese Gefühl, dass da was in mir wächst, wie ich das so aus meinem Freundeskreis kenne. Aber ich denke, das hat was damit zu tun, dass ich es eben so früh wusste, mit 20 stand das für mich fest, dass ich eben keine Kinder bekommen kann. Vorher habe ich natürlich auch gedacht, dass das mal alles ganz normal klappt. Das setzt man voraus. Aber dann konnte oder besser musste ich mich darauf einstellen, dass aus meinem Körper eben kein Kind kommen kann und dass ich trotzdem keine schlechtere Frau bin.“"

Mit Ausnahme der beiden oben genannten Fälle bemerkten alle anderen der interviewten Paare erst nach einiger Zeit mit regelmäßigem Geschlechtsverkehr, dass sich eine erhoffte Schwangerschaft nicht einstellte. Fünf Paare entschlossen sich relativ bald für eine Adoption ohne medizinische Interventionen, alle anderen begaben sich zunächst in medizinische Behandlung. Verschiedene Möglichkeiten der körperlichen Untersuchung wie Bauchspiegelungen oder Spermiogramme, gefolgt von Hormongaben, Inseminationen, In-vitro-Fertilisation (IVF) oder Mikroinjektion (ICSI) zur Behandlung der „Sterilität" wurden in Anspruch genommen, die allerdings aus den verschiedensten Gründen abgebrochen wurden. Keines der interviewten Paare hielt die Behandlungen bis zum Ende durch, um erst als letzte Möglichkeit ein Kind zu adoptieren. Die Entscheidung

\footnotetext{
${ }^{150}$ Hier möchte ich unbedingt darauf hinweisen, dass diese Aussagen nicht allgemeingültig sind für Adoptionen. Ich beziehe mich hier auf mein empirisches Material, aus dem ich diese Schlussfolgerungen ziehen kann. Einzelpersonen oder Paare, die zwar fortpflanzungsfähig waren, sich jedoch prinzipiell für eine Adoption entschlossen, haben sich nicht auf meinen Zeitungsaufruf gemeldet und konnten somit nicht befragt werden.
} 
fiel zumeist schon viel eher als Konsequenz aus der Ablehnung der Reproduktionsmedizin.

„Wir wollten es zuerst schon mal probieren mit der Medizin, um ein Kind zu bekommen. Mein Frauenarzt hat mir auch gut zugeredet. Und natürlich war die Vorstellung auch schön, mit meinem Mann ein Kind zu haben und vorher selbst schwanger gewesen zu sein. Und dann von Anfang an mit dem Kind zusammen zu sein. Aber ich muss Ihnen ganz ehrlich sagen, schon nach meinem ersten Gespräch in der Klinik da, als ich diese ganzen technischen Begriffe hörte und wusste, ich muss jetzt ständig hierher, total nach Plan leben, mich dem Wissen der Ärzte und der Technik ergeben. Das war mir alles zu künstlich und zu technisch. Klingt vielleicht ein bisschen komisch, aber das ging damals in mir vor. Ich habe lange mit meinem Mann gesprochen, aber mir selbst war sehr schnell klar, dass das nicht mein Weg und damit ja letztlich auch nicht unser Weg sein kann. Ich wusste, dass ich so niemals schwanger werden würde. Mein Mann hat das auch verstanden und mich da zu nichts gedrängt. Ich glaube, im Grunde war er auch froh, dass wir uns da nicht in die Mühlen der Fortpflanzungsmedizin begeben haben. Wir haben uns dann gleich zu einer Adoption entschlossen, das heißt, wir wollten es probieren, ein Kind zu adoptieren. Und das war für uns nicht die schlechtere Alternative, das können sie mir glauben.“ (Bianca Bechmann, 36 Jahre)

Zum Zeitpunkt der Interviews hatten vier Paare zusätzlich zum Adoptivkind ein leibliches Kind, dass in allen Fällen nach der Adoption zur Welt gekommen war. Durch die Adoption war der Übergang demnach von einer sozialen Rolle in eine andere sowohl vom sozialen Umfeld als auch von den Betroffenen selbst schon vollzogen. Die Konstituierung von ,Mutter' fand hier über die Beziehungen $\mathrm{zu}$ anderen statt, und der Aspekt der Schwangerschaft wurde im Zusammenhang mit dem leiblichen Kind, besonders von den Frauen, eher heruntergespielt. Sowohl von Seiten des Mannes als auch der Frau wurde immer wieder betont, dass das Gefühl Vater oder Mutter zu werden und der damit verbundene Rollenwechsel nicht in direktem Zusammenhang mit der Schwangerschaft und dem Gebären des Kindes stand. So erzählte Julia Wahren (38), ein Adoptivkind von sieben und ein leibliches Kind von zwei Jahren:

„Was sollte ich darüber jammern. Ich habe keinen dicken Bauch gehabt und bin dann mit einem Kind durch die Gegend gelaufen. Da ich es nicht im Supermarkt geklaut habe, wo soll es also herkommen. Und wenn man dann offen und ehrlich ist, gibt es überwiegend positive Resonanzen. Und beim ersten Kind [dem Adoptivkind] kamen dann die Nachbarsleute mit Blumen, als wenn eine normale Geburt stattgefunden hätte. War nach der Geburt des zweiten Kindes nicht anders, als ich aus dem Krankenhaus kam. Und für mich war es auch nicht anders. Natürlich war es schön, auch mal eine Schwangerschaft selbst zu erleben. Das würde nicht stimmen, wenn ich da was anderes sagen würde. Ist schon ein tolles Gefühl. Mutter sein bedeutet für mich allerdings nicht schwanger zu sein. Die Schwangerschaft ist für mich untergeordnet, außerdem war sie auch nicht so toll. In dem Augenblick, wo ich schwanger war, fand ich es schön. Aber nach einer gewissen Zeit, nachdem ich mich damit arrangiert hatte, spielte es keine Rolle mehr im Vergleich zu unserem Adoptivkind. Eltern wird man nicht aus Zeugung und Schwangerschaft, sondern aus durchwachten Nächten, durch Wickeln und Freude an der Entwicklung des Kindes.“ 
Aus der Perspektive der mit Adoptiv- und/oder Pflegekindern lebenden Interviewten nimmt das ,Kinder haben“, also das Leben mit den Kindern, den wesentlichen Stellenwert ein. Es lässt sich feststellen, dass der Konstituierung von Elternschaft über körperliche Prozesse durch das ,Kinder bekommen' und damit zusammenhängend auch einer öffentlichen Darstellung von „Fruchtbarkeit“ keine primäre Bedeutung eingeräumt wird. Deutlich wird, dass auch im Zusammenhang mit Adoptionen die Kontinuität der eigenen Biografie als Mittel zur Aufrechterhaltung der Selbst-Identität (vgl. Giddens 1991) eine wesentliche Rolle spielt. So wird versucht, disparate Ereignisse wie die Erkenntnis, sich nicht fortpflanzen zu können, in die Lebensgeschichte zu integrieren und eine Veränderung oder Erweiterung der eigenen Identität als Mutter oder Vater zu erreichen. Das Selbst muss hier jedoch ohne die biologische Dimension der Fortpflanzung konstruiert werden, d.h. der Prozess der Selbst-Reflexivität (Giddens 1991) läuft hier nicht primär über den Körper, worauf auch die deutliche Ablehnung der medizinischtechnischen Möglichkeiten zur Überbrückung der „Sterilität“ hinweist. Dies wird auch bestätigt durch die folgenden Zitate von den Adoptiveltern Astrid und Ingo Kruse (37 und 39 Jahre), in denen sie die Vorteile einer Adoption für eine Symmetrie der Elternschaft betonten:

A.K.: „Der eindeutige und für uns ganz klare Vorteil einer Adoption gegenüber einer Befruchtung mit Spendersamen ist doch ganz klar, dass wir beide die gleichen Voraussetzungen haben. Wir sind beide nicht die leiblichen Eltern. Das ist doch ein totales Ungleichgewicht, wenn nur die Frau die leibliche Mutter ist und der Mann nicht der leibliche Vater."

I.K.: „Ich lehne die Blutsideologie völlig ab. Ich denke, dass es keine Rolle spielt, in welchem Bauch das Kind war und von wem es die Gene gekriegt hat, sondern zu wem es Bindungen entwickelt. Wir wollen dem Kind beide was mitgeben, ihm Vorbild sein. Gene spielen dabei keine Rolle. Für uns hat die Adoption es auch ermöglicht, dass wir beide auf die gleiche Art und Weise Eltern werden. Wissen Sie, was ich meine? Wir haben beide das gleiche erlebt, und es sind die gleichen Bedingungen."

Im Unterschied zu den Aussagen der Paare, die die heterologe Insemination nutzen, um zumindest über die Schwangerschaft der Frau die genetische Zugehörigkeit zu einem Elternteil zu schaffen, tritt hier die Bedeutung der biologischen Elternschaft im Vergleich zu der sozialen in den Hintergrund. Dies wird auch deutlich an der Tatsache, dass alle Adoptiveltern offen mit der Tatsache der Adoption umgehen und es ihrem Kind zu gegebener Zeit mitteilen wollen bzw. schon mitgeteilt haben (je nach Alter des Adoptivkindes). Da die nähere soziale Umgebung aufgrund der fehlenden Schwangerschaft in fast allen Fällen von der Adoption weiß, besteht hier kein Bestreben, diese in der Annahme zu bestärken, dass es sich um ein ,eigenes“ leibliches Kind handelt. Auch wenn die strukturelle Einheit einer Kernfamilie sich in den Personenkonstellationen einer Adoptivfamilie widerspiegelt und somit öffentliche Anerkennung findet, wird seitens der Adoptiveltern nicht das Verheimlichen der, gespaltenen“ Elternschaft angestrebt 
(wie beispielsweise bei der heterologen Insemination, bei der die Kinder in der Regel nichts von ihrer Erzeugung erfahren sollen, um auch den Schein einer genetischen Einheit der Kernfamilie zu bewahren). Die öffentliche Akzeptanz einer Adoption im Unterschied zur heterologen Insemination liegt meiner Ansicht nach daran, dass es sich um ein nicht-körperliches und nicht-leibliches Kind beider Ehepartner handelt und somit auch losgelöst von der leiblichen Dimension einer Paarbeziehung, der sinnlichen ganzheitlichen Wahrnehmung und Empfindung, was auch intime sexuelle Beziehungen beinhaltet, konzipiert werden kann. Im Fall der heterologen Insemination schwingt durch die körperliche Verbindung zwischen der Frau (der biologischen Mutter des Kindes) und dem „Samenspender“ (dem biologischen Vater) über Körpersubstanzen implizit auch immer die Vermutung eines Ehebruchs mit, was dazu führt, dass versucht wird diese Zusammenhänge auszublenden (siehe dazu auch Kap. 6).

\subsubsection{3 „Das waren sofort unsere Kinder“6}

Wie im Kontext der Reproduktionsmedizin wird auch in den Interviews mit den Adoptiv- und Pflegeeltern der Aspekt von Zugehörigkeit erwähnt. Paare, die sich vor oder in reproduktionsmedizinischen Behandlungen befinden, betonen gerade den Aspekt der „richtigen“ Zugehörigkeit eines „,eigenen“ Kindes und verstehen darunter die körperliche, leibliche, emotionale und rechtliche Zugehörigkeit (Kap. 5.1.4.3). Obwohl das Adoptivkind einem leiblichen Kind rechtlich in jeder Hinsicht gleichgestellt ist (vgl. Kap. 4.1 und 4.3), ruft doch die Vorstellung, dass das adoptierte Kind andere biologische Wurzeln habe und damit irgendwo ,richtige“ Eltern seien, die gegebenenfalls irgendwann von Bedeutung sein könnten, auch insbesondere bei Adoptiveltern Ängste und Unsicherheiten hervor. In den Gesprächen spielt dieser Aspekt immer wieder eine Rolle, wie ich im Weiteren noch zeigen werde, allerdings wird von Ingo und Astrid Kruse (39 und 37 Jahre) auch gerade das Gefühl einer besonderen emotionalen Zugehörigkeit zum „eigenen“ adoptierten Kindes besonders betont. ${ }^{151}$

A.K.: „Wir haben sie zwei Tage nach der Geburt das erste Mal gesehen und wurden im Krankenhaus auch gleich als die Eltern vorgestellt. Eine Geburt kann wirklich nicht schöner sein, es war ja im Prinzip auch eine Geburt für uns. Dieses Kind wurde uns dann geschenkt, und das Leben hat sich stetig verändert für uns. Natürlich beginnt dann mit einem selber ja auch ein Reifeprozess, wenn ein Kind im Haus ist. Und man muss sagen, bei beiden Kindern, gefühlsmäßig, das waren sofort unsere Kinder. Deswegen, wenn ich gefragt werde nach eigenen Kindern, muss ich immer erst überlegen, was die Leute denn eigentlich meinen. Weil es sind unsere Kinder.

I.K.: „Das war von der ersten Sekunde an für uns kein Problem, dass sie nicht unsere leiblichen Kinder sind. Und auch für den Rest der Familie nicht [...].“

151 Siehe dazu auch Modell (1994) in ihrer Untersuchung über Adoptionen in den USA. 
Es lässt sich allerdings feststellen, dass die auftretenden Fragen und Probleme bei Adoptiv- und Pflegeeltern nicht identisch sind. Gilt das adoptierte ebenso wie das leibliche Kind auch rechtlich als das ,eigene“ Kind der Adoptiveltern mit allen Elternrechten und -pflichten, so ,gehört‘ das Pflegekind auch weiterhin zu den biologischen Eltern mit dem Ziel, in absehbarer Zeit mit diesen wieder zusammen zu leben (siehe Kap. 4.3.2).

In den Gesprächen ist deutlich geworden, dass dieser rechtliche Unterschied auch Auswirkungen auf die Gefühle von Zugehörigkeit und Zusammengehörigkeit von Eltern zu den Kindern hat. Sprechen Adoptiveltern von ihrem „eigenen“ Kind oder ihren ,eigenen“ Kindern, dann handelt es sich immer um das adoptierte bzw. um das leibliche sowie das adoptierte Kind. Im Konzept des „,eigenen“ Kindes spielen hier demnach die legal anerkannte Übernahme der sozialen Elternschaft und damit auch die rechtliche Zugehörigkeit des Adoptivkindes eine wesentliche Rolle ebenso wie Fürsorge und Erziehung, also die soziale Interaktion in einem gemeinsamen Lebensalltag. Pflegeeltern sprechen zwar von ihrer Familie und schließen in der Regel die Pflegekinder in diese Kategorie mit ein (Kap. 5.2.1), differenzieren jedoch zwischen den leiblichen und den Pflegekindern, indem sie nur die leiblichen (oder auch ein Adoptivkind) als ihre ,eigenen“ Kinder bezeichnen. Meiner Auffassung nach hängt diese Unterscheidung auch mit der Tatsache zusammen, dass Pflegschaften in der Regel zeitlich begrenzte Gemeinschaften darstellen und per Gesetz definiert sind als „familienergänzend“ oder „familienersetzend“ (InfoPflege 1996:9). Referenzpunkt bei dieser Definition von Pflegschaften sind die biologischen Eltern als „natürliche“ Eltern im Sinne von Herkunftseltern, die über kurz oder lang die Erziehung und Fürsorge für ihre „eigenen“ (leiblichen) Kinder wieder übernehmen sollen. Der Erhalt bzw. die Wiederherstellung der Einheit von biologischer und sozialer Elternschaft stellt das erklärte Ziel von Pflegschaften dar. Diese Idee beinhaltet jedoch auch ein zentrales Problem hinsichtlich des Gefühls von Zugehörigkeit und Angehörigkeit seitens der Pflegeeltern zu den Pflegekindern. Es entstehen zwar affektive Bindungen, besonders aufgrund einer gemeinsamen Residenz und den sozialen Interaktionen im Alltag, jedoch existieren, wie im folgenden Zitat zu lesen ist, gewisse Mechanismen, die die Diskrepanz zwischen den „eigenen“ und den Pflegekindern deutlich werden lassen. Es zeigt sich, dass das Wissen um die rechtliche Zuordnung der Pflegekinder zu anderen ,richtigen“ Eltern dazu führt, dass Pflegeeltern versuchen, um sich im Fall einer Rückführung zu den Herkunftseltern vor zu großen Enttäuschungen und Trauer zu schützen, eine gewisse emotionale Distanz zu den Kindern aufrecht zu erhalten. Dies wird auch im folgenden Interviewausschnitt deutlich:

„Mit dem Gedanken, das Kind geht wieder weg, ist es schwierig, das zur Familie zu zählen. Es gehört ja nicht ganz dazu. Wobei ich schon, wenn mich jemand fragt, wer zu meiner Familie gehört, auch meine Pflegekinder dazuzähle. Mit denen lebe ich ja zusammen und verbringe meine Tage, was sehr wichtig ist. Dennoch steht ihr Name nicht an der Tür. Da ist einfach immer der Hintergedanke, sie gehen wieder, was meine eigenen Kinder eben nicht müssen. Meine Frau versucht da nicht dran zu denken. Kann man 
ja auch gar nicht im täglichen Leben. Aber für uns als Eltern ist das manchmal sehr belastend. Ich denke da mehr an die Kinder, dass wir denen eben was mitgeben können, dass sie zumindest für eine Zeit, und unsere beiden Pflegekinder sind ja schon drei Jahre da, also für eine Zeit lang glücklich waren und in einer schönen Gemeinschaft leben konnten.“ (Achim Wellenreuther, 43 Jahre, Adoptiv- und Pflegevater)

Auch für Ingeborg Dammers (44) gehören ihre Pflegekinder aufgrund des gemeinsamen Lebens zu ihrer Familie dazu, wobei jedoch auch für sie die fehlende rechtliche Zugehörigkeit des Pflegekindes im Vergleich zu ihrem Adoptivsohn den wesentlichen Unterschied darstellt: Dieser ,gehört“ zu den Adoptiveltern und damit auch zu deren Verwandtschaft:

„Bei Klaus ist es eben anders. Er ist unser Kind. Er ist nicht unser leibliches, aber er gehört auch rechtlich zu uns. Während das bei den anderen Kindern nicht ist. Aber für mich ist klar, die leben hier, die machen alles wie wir, die leben mit unserer Familie, sind Teil unserer Familie. Aber es ist schon anders, dass Klaus wirklich in jeder Hinsicht Teil unserer Familie ist, das heißt, es gibt keine rechtlichen Verbindungen mehr zu seiner früheren Verwandtschaft, also der Blutsverwandtschaft. Auch was das angeht, gehört er zu uns. Bei den anderen sind da immer noch andere Personen, also die leiblichen Eltern, die mal mehr und mal weniger auftauchen oder von sich hören lassen."

Ein weiterer Aspekt, der in den Interviews mit Adoptiveltern thematisiert wird, sind die Situationen, in denen die adoptierten Kinder - zum Beispiel durch die Begegnung mit schwangeren Frauen oder durch Kinderbücher zum Thema Schwangerschaft und Geburt - schon sehr früh Fragen nach ihrer Herkunft stellen (,War ich auch mal in deinem Bauch?"“). Schon zu diesem Zeitpunkt muss überlegt werden, was und wie dem Kind die ,Wahrheit' mitgeteilt wird, wobei alle Adoptiveltern der Ansicht sind, dass es wichtig sei, in altersgerechter Weise ehrlich auf dieses für das Kind wichtige Thema einzugehen. Weiterhin wird versucht dem Kind zu erklären, dass es da noch ,andere Eltern“ gebe, wobei die meisten von „leiblichen Müttern oder Vätern“" sprechen, eine auch von „der Frau, die dich geboren hat“. Die Bezeichnung „richtige Eltern“ verwenden Adoptiveltern in den von mir geführten Interviews nicht, was meiner Ansicht nach mit der Tatsache zusammenhängt, dass sie - wie in dem vorherigen Kapitel gezeigt - die soziale Elternschaft als wesentlich bedeutsamer definieren als die biologische und sich somit als die „richtigen“ Eltern empfinden, auch wenn noch leibliche Eltern existieren. ${ }^{152} \mathrm{Im}$ rechtlichen Diskurs in Deutschland gilt die das Kind austragende und gebärende Frau als die (,natürliche“) Mutter (Kap. 4.1), was bedeutet, dass die biologische Mutter weiterhin ,Rechte“ als Geburtsmutter behält. ${ }^{153}$ Mit dieser Definition fühlen sich Adoptiv-

\footnotetext{
152 Die Betonung von Pflege und Erziehung als Ausdruck von Mutterschaft seitens der Adoptivmütter zeigt auch Ragoné $(1994,1998)$ im Zusammenhang mit Leihmutterschaft auf. Mutterschaft kann demnach angenommen werden - durch die Adoptivmutter - oder verweigert werden - durch die Leihmutter - (hier durch die leibliche Mutter).

153 Siehe dazu auch Strathern (1995:349): „Indeed it is one of the 'facts of life' that Euro-Americans take for granted. Whoever acts the part of parent, a biogenetic tie with genitor or genetrix in and of itself also indicates parenthood.“
} 
mütter sehr oft benachteiligt, weil die biologische als „richtige“ Mutterschaft dadurch eine besondere - ihrer Ansicht nach nicht gerechtfertigte - Betonung erfährt, wie auch Astrid Kruse (37) deutlich machte:

„Es war immer klar, dass sie wissen soll, dass sie adoptiert ist. Ich glaube, sie hat das als Erste angeschnitten. Sie wurde wach und fragte mich: ,Bin ich eigentlich aus deinem Bauch geboren?` Da war sie so drei, dreieinhalb Jahre vielleicht. Und dann ist sie darüber wieder eingeschlafen. Es war mal so eine Zeit, da war ich ein bisschen traurig, weil ja irgendwie die Stunde der Wahrheit kam. Gerade die Frage: War ich in deinem Bauch? ist manchmal so schlimm. Eigentlich gar nicht für mich selbst, denn ich finde es nicht wichtig, dieses Kind geboren zu haben. Aber ich weiß nicht, ob das letztlich für mein Kind eine Bedeutung hat. Ich meine langfristig. Erst einmal beschäftigt sie sich damit, wo Babys herkommen und will natürlich auch wissen, wo sie herkommt. Ist doch ganz klar. Eigentlich hatten wir diese Fragen ganz gut abgeklärt und waren beide zufrieden. Es ist aber immer wieder hochgekommen durch verschiedene Anlässe. Zum Beispiel haben sie andere Leute oder Kinder angesprochen und gefragt, ob sie ihre richtige Mutter kennt. Das ist bitter für mich, denn wer ist denn die richtige Mutter? Diejenige, die wickelt, sich kümmert, füttert, liebt und sich sorgt oder diejenige, die das Kind zwar neun Monate mit sich rumträgt, es dann aber weggibt. Womit ich das um Himmels willen nicht verurteile. Ich wehre mich nur gegen dieses Besondere der richtigen Mutter im Unterschied zu mir, die ich ja nur Adoptivmutter bin. Das tut weh. Aber das kommt nicht aus mir selbst und auch nicht direkt von meinem Kind, sondern das bringt die Gesellschaft mit und soweit ich weiß, ist das ja jetzt sogar gesetzlich festgehalten, wer die richtige Mutter ist. Manchmal bin ich darüber echt frustriert und empfinde das als eine totale Diskriminierung von uns Adoptivmüttern.“

Auch das folgende Interview zeigt, dass die Auseinandersetzung mit dem möglichen Verhalten des Adoptivkindes bei Kenntnis über eine andere (genetische) Herkunft immer wieder einen großen Raum einnimmt. Ulla Zinke (37) lässt diesen Zeitpunkt auf sich zukommen und will es ihrem Kind überlassen $\mathrm{zu}$ entscheiden, welche Rolle sie dann übernehmen soll. Diese Beschäftigung mit der Perspektive des Kindes stellt einen wesentlichen Aspekt in den Gesprächen mit Adoptiveltern dar. Sie haben ihre eigenen Wünsche, Vorstellungen und Hoffnungen über die Beziehung zu ihrem Kind, ziehen jedoch (wenn auch oft sehr angstvoll) in Betracht, dass durch das Handeln oder die Entscheidungen des Kindes , die Würfel neu gemischt werden' und nach langen Jahren des Zusammenlebens und enger emotionaler Bindungen die biologischen Eltern plötzlich wichtiger werden könnten, allein aufgrund der Tatsache, dass es sich dabei eben um die „richtigen“ Eltern handelt. So erzählte Frau Zinke:

„Ich denke da nicht viel an die biologischen Eltern. Irgendwann wird der Punkt kommen, wenn Frederike in der Pubertät ist, dass sie dann vielleicht nachforschen will. Da werde ich ihr nicht im Weg stehen. Sicherlich werde ich ihr auch nicht unbedingt helfen, in irgendwelchen Akten zu wühlen, um zu wissen, wo die leibliche Mutter ist. Das muss sie selber machen. Ich würde sie mit dem Auto hinfahren, wo sie möchte, aber ich würde nicht den Kontakt für sie aufnehmen. Ich werde ihr kein Brett in den Weg legen. Das habe ich von Anfang an nicht gemacht. Ich habe ihr auch freigestellt und stelle ihr immer wieder frei, ob sie nur meine Freundin sein will, oder ob sie mich als Mama ak- 
zeptiert. Das muss sie selber entscheiden. Das ist ganz allein ihr Ding, ob sie mich als Mutter akzeptiert irgendwann. Jetzt ist es ja mehr, weil sie es nicht anders kennt. Aber wenn sie mal zwölf ist und das richtig kapiert, was da eigentlich gelaufen ist, ob sie mich dann noch als ihre richtige Mutter akzeptiert oder nur als Freundin, das überlasse ich ihr. Da würde ich sie nie zwingen oder druckmäßig dahinterstehen. Ich war mir ja im vorneherein im klaren, dass irgendwann das Gespräch darauf kommt und dass ich niemals ihre leibliche Mutter sein kann, auch wenn sie für mich mein Kind ist. Dennoch muss ich gestehen, habe ich insgeheim auch Ängste, dass jemand anders plötzlich wichtiger werden könnte als ich und unsere ganzen gemeinsamen Jahre im Vergleich zu der leiblichen Mutter weniger wert sind.“

Ständig präsent sind die biologischen Eltern im Falle von Pflegekindern, die in der Regel nur für einen begrenzen Zeitraum zur Pflegschaft von diesen getrennt werden, später aber wieder zu diesen zurückkehren sollen. So konnte ich schon zeigen, dass alle Befragten emotionale Probleme mit der Situation nennen, dass die Kinder über kurz oder lang wieder zu den leiblichen Eltern zurückkehren sollen (vgl. Kap. 5.1.4.3). Für die interviewten Pflegeeltern ist jedoch sehr klar, dass sie trotz dieser engen affektiven Bindungen zu ihren Pflegekindern auf keinen Fall die Rolle der „richtigen“ Eltern übernehmen oder diese ersetzen wollen (vgl. auch Modell 1994:47ff). Sie empfinden sich als „Vertreter der Mutter/des Vaters/der Eltern“ (je nachdem, in welchen familiären Konstellationen das Kind vorher gelebt hat) oder als „Mutter bzw. Vater auf Zeit“. Kontakte zu den leiblichen Eltern, auch wenn sie von diesen oftmals nicht gepflegt werden bzw. sich als sehr schwierig gestalten, machen allen Beteiligten immer wieder bewusst, dass sie als Pflegeeltern nur vorübergehend einspringen. Wie ich in Kapitel 4.3.2 dargelegt habe, liegt die elterliche Sorge in der Regel bei den Herkunftseltern. Den Herkunftseltern steht auch während der Pflegschaft das elterliche Umgangsrecht zu. Mein Datenmaterial zeigt, dass diese Situation in zahlreichen Fällen zu Problemen zwischen Pflegeeltern und leiblichen Eltern führt, besonders wenn das Kind diese nach langer Zeit der Pflegschaft nicht mehr sehen will, den Kontakt abbrechen und ein Kind der Pflegeeltern sein möchte. Manche - besonders lang andauernde - Pflegschaften werden nach Zustimmung der leiblichen Eltern zu Adoptionen. Jedoch kommt es immer wieder zu dramatischen Szenen, wenn die leiblichen Eltern die Kinder nach Jahren wieder zu sich nehmen wollen, wie Margot Hansen (48), vier Pflegekinder, berichtete: ${ }^{154}$

„Die leiblichen Eltern haben da mehr Probleme. Zum Beispiel bei der Konfirmation von Ilona waren die richtigen Eltern da. Ich habe aber eine Rose von dem Kind bekommen. Die Mutter war total beleidigt. Dass mir ihre Tochter eine Rose gibt, aber sie das Kind zur Welt gebracht hat. Da muss die Mutter mit fertig werden. Terror haben die Eltern gemacht. Die Eifersucht von der Elternseite ist riesig, besonders von der Mutter. Also

\footnotetext{
${ }^{154}$ In mehreren Interviews erzählten Pflegeeltern von Konflikten mit den leiblichen Eltern, denen die Kinder beispielsweise aufgrund von emotionaler oder körperlicher Vernachlässigung zum Teil unter Zwang weggenommen wurden. So war die Rede von nächtlichen beleidigenden Anrufen der leiblichen Mutter/Eltern, Drohungen das Kind abzuholen, Eifersüchteleien u.a. Auf diesen Aspekt soll allerdings in dieser Arbeit nicht näher eingegangen werden.
} 
ich denke, dass die biologische Elternschaft da überhaupt nichts bedeutet. Die Mutter hat das Kind zur Welt gebracht und irgendwann abgestoßen. Für mich ist das Kind genauso lieb, ob das jetzt aus meinem Bauch kommt oder nicht. Ich sehe da keinen Unterschied. Die Probleme hat man mit den leiblichen Eltern, die dann plötzlich glauben, sie haben einen Anspruch. Und dann drohen, sie holen das Kind ab oder es tatsächlich irgendwann wieder abholen. Und das wird auch noch vom Jugendamt unterstützt. Die wollen das ja am liebsten, dass die Kinder alle wieder zu ihren Eltern zurückgehen. Das ist alles ein Riesenstress für uns und die Kinder. Aber da kann man nichts gegen machen, so hat der Staat das eben festgelegt. Weil die meinen, der Kontakt zwischen den Kindern und den Eltern muss bestehen bleiben. Ich kann ihnen sagen, das ist doch oft reine Theorie, was die sich da ausdenken und gesetzlich festlegen. Ich sehe immer nur Probleme mit diesen Situationen, und was dann daraus wird.“

Anhand dieses Zitates fällt auf, dass der Aspekt der körperlichen Zugehörigkeit eine bedeutende Rolle auch im Fall von Pflegeelternschaft von Seiten der staatlichen Jugendämter spielt. Das im Grundgesetz festgelegte Elternrecht, das den biologischen Eltern das Recht und die Pflicht zuweist auch die soziale Elternschaft auszuüben, bestätigt die kulturell dominante Vorstellung vom Ideal der Einheit von biologischer und sozialer Elternschaft, die ihren Ausdruck in der Kernfamilie findet, auch im staatlichen Umgang mit Pflegschaften. So wird immer wieder versucht, die Kinder zu den leiblichen Eltern zurückzuführen mit der Begründung des „Kindeswohles“ (vgl. Kap. 4.3.2). Dieser Punkt wird von vielen Pflegeeltern scharf kritisiert. Oft sei schon vorher absehbar, dass es nur eine Frage der Zeit sein werde, bevor die Kinder wieder von den leiblichen Eltern weggebracht werden müssten, da sich an deren schlechter psychischer oder physischer Situation nichts verändert habe. Ihrer Ansicht nach stehe ein theoretisches Modell im Vordergrund, das das Zusammenleben von Kindern und leiblichen Eltern betone und als Ideal ansehe, was sie jedoch für sehr realitätsfremd hielten, da es in vielen Fällen gerade nicht zum „Wohl des Kindes“ sei, das immer propagiert werde.

Das hier Dargelegte macht das Spannungsverhältnis zwischen Vorstellungen von determinierter Blutsverwandtschaft und der ,Aushandelbarkeit‘ sozialer Beziehungen in der Konstruktion von Verwandtschaft in Deutschland deutlich. Im Kontext von Adoptionen und Pflegschaften erfährt die soziale Komponente von Elternschaft eine höhere Gewichtung als die biologische. Die dominante kulturelle Vorstellung von der Unauflösbarkeit der Beziehung zwischen biologischen Eltern und Kindern findet sich zwar auch hier wieder beispielsweise in den Ängsten vor dem Treffen zwischen dem Kind und den biologischen Eltern und der damit verbundenen Frage, wie wichtig denn vielleicht letztlich doch die biologischen Wurzeln seien. Im alltäglichen Leben werden diese Überlegungen jedoch ausgeblendet. In der täglichen sozialen Interaktion wird das gemeinsame Leben, Fürsorge, Liebe, und Unterstützung als elterliche Werte betont, die eine bedeutendere Rolle spielen als die Gene. Die Bedeutung biologischer Wurzeln für ein Individuum wird zwar anerkannt. Abgelehnt wird jedoch die Überzeugung, dass diese für ein Kind letztendlich von höherer Relevanz sind als die sozialen Einflüsse . 


\section{6 „Zeugung“ im Schnittpunkt verschiedener Diskurse}

Aus der bisherigen Analyse der Konstruktionsprozesse von Verwandtschaft im Rahmen der Selbst-Reflexivität des Körpers in verschiedenen sozialen Kontexten hat sich gezeigt, dass die Diskurse über Verwandtschaft, Körper, Selbst und Person nicht eindeutig gegeneinander abzugrenzen sind, sondern dass sie Einfluss nehmen auf die persönlichen Sichtweisen und Auswirkungen haben auf das soziale Handeln. So wird in der Konstruktion von Verwandtschaft „echte“ Verwandtschaft als „,natürlich“ über die Idee geteilter Körpersubstanzen konstruiert, wobei die Zeugung des menschlichen Lebens im Schnittpunkt dieser kulturspezifischen Vorstellungen steht. Es ist deutlich geworden, dass Verwandtschaft sozial über Körper und Körpersubstanzen definiert wird, und die Kongruenz von biologischer und sozialer Verwandtschaft eine zentrale Bedeutung erfährt. Unter den Bedingungen der Reproduktionstechnologien hat sich der Prozess der Fortpflanzung und damit die Möglichkeit der Erschaffung neuen Lebens verändert. Der Geschlechtsverkehr bildet nicht mehr die Voraussetzung für das Verschmelzen der Zeugungssubstanzen, sondern kann durch die außerkörperliche Zusammenführung von Eiund Samenzellen ersetzt werden. ${ }^{155}$ Die reproduktionsmedizinische Praxis ,assistiert“ jedoch nicht nur bei der Zeugung, sondern greift kulturelle Konstruktionen der Verwandtschaftsvorstellungen auf, in dem sie vorgibt, „natürliche“ Verwandtschaft zu schaffen, zumindest über den Körper eines Elternteils. Somit greifen die Reproduktionstechnologien direkt in den Bereich der ,Natur' ein. Zum einen wird die Erzeugung von Kindern räumlich und zeitlich von der Sexualität und Intimität des Paares herausgelöst, was beinhaltet, dass die Zeugungssubstanzen als vom Körper und von sozialen Beziehungen losgelöste Einheiten betrachtet werden und damit ,zielgerichtet ${ }^{`}$ verwendet und manipuliert werden können. Es ist deutlich geworden, dass der materielle Umgang mit Körpersubstanzen und die zentrale Bedeutung des Körpers in seiner verleiblichten Dimension kulturelle Vorstellungen von Selbst (und damit Identität), Person und soziale Beziehungen, vor allem Verwandtschaft, berührt. Ich konnte zeigen, dass Leiblichkeit ein Ausdruck „natürlicher“" verwandtschaftlicher Beziehungen darstellt und welche leiblichen Eigenschaften einem ,eigenen“ Kind zugeschrieben werden. Im Zusammenhang mit der ,assistierten“ Fortpflanzung kommt es nun auch zu direkten und indirekten Körperbeziehungen zwischen dem Paar und anderen an der Erzeugung des Kindes beteiligten Personen, sei es die Behandlung des Körpers (insbesondere der Frau) durch

\footnotetext{
155 Der Prozess der Fortpflanzung im Kontext der Reproduktionsmedizin ist zwar nicht gänzlich von Sexualität losgelöst, da zumindest der Mann durch Masturbation sein Sperma gewinnen muss, jedoch kann er unabhängig von einer gemeinsamen sexuellen Beziehung des Paares in Gang gebracht werden.
} 
den Arzt oder die Verwendung fremder Körpersubstanz wie im Fall von „Spendersperma“. Die Erzeugung menschlicher Körper bedeutet auch die Schaffung von „Verwandten“ und „Verwandtschaften“, die im Kontext der Reproduktionstechnologien von einem privaten zu einem öffentlichen Ereignis mit Unterstützung verschiedenster Personen wird. So sind die Ärzte an der Erzeugung leiblicher Nachkommen eines Paares beteiligt, die ,traditionell‘ mit Sexualität und geschlechtlicher Fortpflanzung assoziiert wird. Sie werden somit Teil des ,Zeugungsvorganges' und ,Vermittler' von Verwandtschaft, gelten aber als soziale Nicht-Verwandte und haben letztlich keinen Anteil an der sozialen Elternschaft. Es stellen sich somit Fragen nach Veränderungen von ,traditionellen' Konzepten von Zeugung sowie deren Auswirkungen auf Vorstellungen von Verwandtschaft, da beispielsweise auch Annahmen über ,natürliche“ Elternschaft in Deutschland durch Methoden wie die heterologe Insemination in Frage gestellt werden können. So weist der körperliche Beitrag eines „Samenspenders“ zur Zeugung ihn als biologischen Vater des Kindes aus (Kap. 2.2.2), was dem rechtlichen Verwandtschaftsdiskurs in Deutschland zufolge eine (bluts-)verwandtschaftliche Beziehung zwischen dem Kind und dem „Samenspender“ herstellt und idealerweise auch die Übernahme der sozialen Vaterschaft beinhaltet (vgl. Kap. 4). Die Person des „Samenspenders“ als ,Erzeuger‘ wird jedoch sozial vollständig ausgeblendet (vgl. Kap. 5.1.4), und nur das behandelte Ehepaar tritt die soziale Elternschaft an. So werfen die reproduktiven Technologien neue Fragen auf hinsichtlich des Spannungsverhältnisses von determinierter Blutsverwandtschaft, Verhandelbarkeit sozialer Beziehungen, Körper und Leiblichkeit.

Im folgenden Kapitel, von dem ich Auszüge bereits wörtlich oder sinngemäß in HauserSchäublin et al. (2001) veröffentlicht habe, wende ich mich den Konsequenzen der bisher in meiner Dissertation analysierten Konstruktionen zu mit dem Schwerpunkt auf der Zeugung als Gegenstand unterschiedlicher Diskurse. Das Ziel aller Beteiligter im Kontext der Reproduktionsmedizin ist die Erzeugung des „eigenen“ leiblichen Kindes eines Paares. Anhand meines empirischen Materials wird jedoch ersichtlich, dass von der Ärzteschaft und den Paaren zur Beschreibung des Vorgangs nicht nur unterschiedliche Termini verwendet werden, sondern dass damit auch andere Vorstellungen verbunden sind. Da ich bisher herausgearbeitet habe, dass der Körper einmal als symbolische Repräsentation von Verwandtschaft gilt und zum anderen mit der Erfahrung von Verwandtschaft verbunden ist, ermöglicht die analytische Trennung zwischen ,Körper ${ }^{\star}$ und ,Leib', dem Körper als Behandlungsgegenstand, als objektiviertem sowie materiellen Leib, und dem subjektiv empfundenen Leib, Brüche und Widersprüche aufzuzeigen und zu analysieren, die sich aus unterschiedlichen Perspektiven der Menschen ergeben, die direkt von den Technologien betroffen sind oder diese anwenden. 


\subsection{Die innermedizinische Perspektive}

\subsection{1 „Man muss sich auch mal von dem Mythos Zeugung verabschieden}

\section{können“6}

Schlägt man unter „Zeugung“ im Wörterbuch nach, findet sich einzig der Hinweis auf den Begriff der Fortpflanzung, die definiert wird als „Erzeugung von Nachkommen bei Mensch, Tier und Pflanze; dient der Arterhaltung, wobei Anlagen der Elternindividuen an das neue Individuum weitergegeben werden“ (Universal Lexikon 1993). In der konkreten empirischen Forschung brachte meine Frage nach ,Zeugung' allerdings einige der interviewten Ärzte ins Grübeln. Es schien eine Frage zu sein, mit der sie sich im Rahmen ihrer professionellen Tätigkeit als Reproduktionsmediziner nicht näher beschäftigt oder die sie sich bisher so noch nicht gestellt hatten (,Was Zeugung ist? Da habe ich mir noch nie Gedanken drüber gemacht."). So äußerte sich beispielsweise auch Dr. Eva Bircher (39):

„Die Frage, was Zeugung für mich ist, ist gar nicht so leicht zu beantworten. Sie haben mich ja gestern schon mal angesprochen, da konnte ich nun schon ein bisschen drüber nachdenken, soweit das ging, und da habe ich plötzlich gemerkt, dass es mir gar nicht so klar ist, wie ich wahrscheinlich immer gedacht habe, dass es mir klar erscheinen würde. Ich weiß nicht, ob das für Sie verständlich ist. Jetzt so spontan, was ich damit assoziiere, das wäre Entstehung neuen Lebens, im weitesten Sinne eine Urkraft.“

Frau Dr. Bircher versteht hier unter Zeugung etwas Abstraktes, die Entstehung neuen Lebens, ohne direkten Bezug zu nehmen auf die Art und Weise, den Ort oder die Zeit der Entstehung. Diese abstrakte Vorstellung wurde auch nach einiger Überlegung von Dr. Peter Heise angesprochen, nach dessen Einschätzung der Begriff der Zeugung auf Fachtagungen oder in Fachzeitschriften zur Reproduktionsmedizin in Deutschland vermieden werde. Seiner Meinung nach könne dies damit zusammenhängen, dass Zeugung nicht genau definiert sei und ,irgendwie vielleicht sogar was ,Mythisches“ hat", womit man doch im (reproduktions-)medizinischen Alltag nichts zu tun habe. In der Reproduktionsmedizin gehe es doch in erster Linie um das Eindringen der Samenzelle in die Eizelle sowie deren Verschmelzung und damit um die Befruchtung. ${ }^{156}$

Auch in der konkreten reproduktionsmedizinischen Praxis, also in der Interaktion zwischen Arzt und Paar, wird der Begriff der Zeugung weitgehend vermieden ${ }^{157}$ (außer im

\footnotetext{
156 Unter Befruchtung wird das Verschmelzen von Samenzelle und Eizelle verstanden. Es gibt eine unterschiedliche zeitliche Befruchtungsfähigkeit der Gameten: für die Samenzellen zwei bis drei Tage nach der Ejakulation, für die Eizellen ca. 12 Stunden nach der Ovulation (vgl. Martins/Breckwoldt/Pfleiderer 1994:43). Nach der Befruchtung findet in den folgenden 24 Stunden die Teilung in Mehrzeller statt, die im Rahmen der IVF/ICSI-Behandlungen beim Embryotransfer in die Gebärmutter der Frau übertragen werden.

157 Diese Feststellung basiert auf eigener teilnehmender Beobachtung und Gesprächsprotokollen während täglicher Sprechstunden in den reproduktionsmedizinischen Kliniken.
} 
Zusammenhang mit der Diagnose „männlicher Zeugungsunfähigkeit“). In den folgenden Interviewausschnitten wird deutlich, dass es nach Ansicht der Ärzte im Rahmen ihrer beruflichen Tätigkeit entweder gar nicht um Zeugung geht, oder dass sie nur die technischen Voraussetzungen schaffen, damit Zeugung stattfinden könnte. So erzählte Dr. Frank König (43):

„Ja, das ist sicher eine schwierige Frage, das gebe ich zu. Was ist denn überhaupt Zeugung? $\mathrm{Na}$, es hat doch normalerweise was mit dem Zeugungsakt zu tun, also Geschlechtsverkehr. Und dass ist eine Sache der Zweisamkeit, eine Sache des Paares. Wobei dem Ganzen oft zuviel an Pathos beigemessen wird, finde ich. Nun, hier sind jetzt natürlich Paare, die nicht durch Geschlechtsverkehr schwanger werden können. Wir verhelfen dem Paar zu einer Schwangerschaft mit unseren Mitteln, die die natürlichen Vorgänge nachempfinden. Geht es dann um Zeugung oder nur in einem übertragenen Sinne? Verstehen Sie, was ich meine? Was wir hier machen bzw. wozu wir beitragen, ist die Verschmelzung von Ei- und Samenzellen zu erreichen, also die Befruchtung bzw. die Entstehung neuen Lebens. Das findet ja in der Petrischale statt. Da kann keiner was zu tun, also niemand kann das beeinflussen, ob sich Ei- und Samenzelle treffen. Dass da was entsteht oder gezeugt wird, läuft dann von ganz alleine oder eben auch manchmal nicht.“

Ganz explizit trennte Dr. Stefan Priesmeier (46) zwischen Zeugung und Befruchtung und verwies auch auf seine unterschiedliche Meinung als Privatperson und im Rahmen seiner beruflichen Tätigkeit:

S.P.: „Was die Zeugung ist? Na, sie stellen Fragen. Wollen Sie jetzt meine persönliche Meinung hören oder meine als Arzt?““

I.S.: ,Sowohl als auch.“

S.P.: „Also Zeugung ist das Zusammenkommen von Ei- und Samenzelle und die Entstehung neuen Lebens. So mal als prinzipielle Aussage vorweg. Als Privatperson verbinde ich mit Zeugung auch Sexualität, also so was wie einen Zeugungsakt. Der hat was mit Gefühlen zu tun und hängt eng mit der Partnerbeziehung zusammen. Ja, und dann muss da natürlich auch noch der Wunsch nach einem Kind sein. Wenn man verhütet, dann hat man auch Geschlechtsverkehr, aber das wäre dann kein Zeugungsakt. Weil die Zeugung ja gar nicht möglich wäre. Wenn ich jetzt aber über Zeugung hier im Job sprechen sollte ... Also, zunächst gebe ich zu, dass ich darüber gar nicht rede oder es zumindest umgehe, wenn nicht gerade ein Paar das Gespräch darauf bringt. Aber was ich hier mache, das ist zwar neues Leben schaffen, aber ... (zögert). Meine Güte, können Sie nicht was Leichteres fragen. Also mal ganz deutlich: Hier arbeite ich mit fremden Eizellen und Samenzellen, und wenn ich Glück habe, befruchten die sich, zuhause im Ehebett zeuge ich. Das bringt es wohl so ungefähr auf den Punkt.“

Der Arzt Rolf Wellmann (45) war der Auffassung, dass unter dem Mikroskop zwar gezeugt werde im Sinne der prinzipiellen technischen Voraussetzung, dass Ei- und Samenzelle sich verbinden könnten, wies aber auch darauf hin, dass Zeugung letztlich doch eher die Entscheidung eines Paares für ein Kind sei, die an anderer Stelle und zu anderen Gelegenheiten getroffen werde. Er sah die Klinik nicht als die passende Umgebung für eine Zeugung und besonders nicht für einen Zeugungsakt: 
„Zeugung an sich würde ich nicht unbedingt auf einen Zeitpunkt beschränken. Das ist für mich höchstens der formelle Zeitpunkt, wo dann das Leben in Gang gesetzt wird durch eine Samenzelle und eine Eizelle. Wir zeugen aus meiner Sicht letztlich unter dem Mikroskop. Wenn man das Technische sieht. Aber eigentlich ist die Zeugung die Entscheidung, nichts mehr dagegen zu tun, dass man sich fortpflanzt. Ich würde das als den Punkt nehmen, denn das ist eigentlich ein Konsens, den die beiden Beteiligten haben. Mir wäre die Vorstellung ganz lieb, dass in dem Moment auch wirklich Einigkeit besteht. Ich habe nicht die große Hoffnung, dass das besonders häufig der Fall ist, wenn wir das gerade unter dem Mikroskop machen. Da bin ich mir nicht sicher, was das Paar macht. Der eine liegt im Bett, halb betäubt von der Narkose und der andere blättert lustlos in einer Zeitung herum. Ich weiß nicht, was das für ein Zeugungsakt ist. Ich würde mich dann lieber darauf zurückziehen, dass sie mal eine gemeinsame Entscheidung getroffen haben und den Zeugungsakt so ein bisschen vorverlegen. Wohlwissend, dass das formal natürlich ganz anders ist. Und das ist auch etwas, was man in eine Behandlung mit hineinnehmen kann, wo die Besonderheit ist, dass das von der Sexualität, von der Erotik und von der Beziehung zwischen den beiden Menschen getrennt wird oder ein Stück weit zumindest.“

Es wird deutlich, dass Rolf Wellmann sich nur ungern auf einen Zeugungszeitpunkt festlegen will. Er sieht Zeugung zwar auch ähnlich wie schon Dr. Eva Bircher als Beginn neuen Lebens, der auch unter dem Mikroskop stattfinden kann, möchte jedoch lieber eine Trennung vollziehen zwischen der Technik, die die reproduktionsmedizinischen Behandlungen bietet, und der privaten Entscheidung eines Paares sich fortpflanzen zu wollen. Für ihn ist Zeugung somit eher ein Prozess, der sich über einen längeren Zeitraum hinziehen kann, zu dem eine gemeinsame Entscheidung ebenso wie eine sexuelle Beziehung (der „Zeugungsakt") gehört. Er schränkt jedoch ein, dass aufgrund der formalen Kenntnis über die biologische Fortpflanzung der Zeugungsakt als Grundlage für das Zusammenkommen von Ei- und Samenzelle zeitlich nicht verlegt werden könne, würde es in der konkreten reproduktionsmedizinischen Praxis allerdings begrüßen, wenn dennoch die persönliche sexuelle Beziehung des Paares im Vordergrund des Zeugungsprozesses stünde.

Als Arzt im konkreten Umgang mit den Patienten wird versucht, den „Mythos Zeugung“ aus den Behandlungen herauszulassen („Man muss sich auch mal von dem Mythos Zeugung verabschieden können"). Hier geht es in erster Linie um die Befruchtung, d.h. um Zellen und deren (erwünschte) Verschmelzung. Den intimen emotionalen Teil einer Paarbeziehung wollen die Ärzte nicht berühren. Der Arzt sieht seine Aufgabe darin, „mechanische“ oder ,technische“ Hilfestellung bei der Befruchtung zu geben und möchte sich aus der Zeugung, die, wie im folgenden Zitat zu lesen ist, auch an die Vorstellung von Sexualität gekoppelt ist, heraus halten. Durch die Terminologie der eher nüchternen ,Befruchtung im Unterschied zur emotionaleren ,Zeugung“ drückt sich nicht nur ein Versuch der Objektivierung der Vorgänge aus, sondern zugleich eine emotionale Distanzierung, die, wie Dr. Eva Bircher ebenfalls erzählte, auch erwünscht 
sei, da eine zu intensive persönliche Auseinandersetzung mit den Paaren auch für die Ärzte eine zu große emotionale Belastung darstelle:

„Das ist zum Beispiel auch bei einer Geburt so, dass das auch immer wieder empfohlen wird, der Arzt macht das, was Medizinisches anfällt, und ist aber ansonsten mehr eine Randfigur. Er soll das Paar, oder diese Intimität, diese Familienbildung, oder was auch immer, möglichst wenig stören, wenn nicht irgendwas ist, wobei er eingreifen muss. Und auch da hat wahrscheinlich der Arzt das Bedürfnis, dass er in der Vorstellung eben nicht mit in die Zeugung, in diese Intimität, oder sogar noch in die Sexualität einbezogen wird. Ich glaube, da will er sich ganz deutlich abgrenzen: Er macht die Mechanik am Rande und das andere tangiert er nicht. Vielleicht hat er das Bedürfnis, sich stärker abzugrenzen. In der Realität geht das nicht immer. Aber man will nicht emotional mit hinein gezogen werden."

\subsubsection{Der Arzt als (Mit-)Erzeuger?}

Wie ich zeigen konnte, verstehen die Ärzte im Rahmen ihrer beruflichen Tätigkeit unter Zeugung im weitesten Sinne die Schaffung neuen Lebens, die entweder im Labor stattfinden kann und/oder Teil einer (körperlichen und emotionalen) Beziehung des Paares ist, die außerhalb der Klinik oder Praxis gelebt wird. Sie vermeiden den Begriff der Zeugung und verwenden dafür lieber den Begriff der Befruchtung, der sich auf die körperlichen Vorgänge bei der Entstehung menschlichen Lebens bezieht. Im Zusammenhang mit der Vorstellung von Zeugung stellte ich in den Interviews auch explizit die Frage nach einem Zeugungszeitpunkt. Anhand der IVF/ICSI-Methode wird deutlich, wie viele verschiedene Stadien es bei der Schaffung neuen Lebens geben kann: Zunächst werden nach einer Hormonbehandlung Eizellen entnommen, die danach entweder im Reagenzglas mit Samenzellen zusammengeführt oder direkt in die Eizelle eingebracht werden (Kap. 4.3.1.2). Hat eine Befruchtung stattgefunden, werden kurz darauf die Embryonen auf die Frau übertragen, wobei auch die Möglichkeit der Kryokonservierung von befruchteten Eizellen (Vorkernstadien), die in einem späteren Zyklus transferiert werden sollen, gegeben ist. Nach Ansicht des Arztes Rolf Wellmann beschränkt sich Zeugung nicht auf einen einzigen Moment, wobei er allerdings das „technische“ Zusammenkommen von Ei- und Samenzelle formal als Zeitpunkt der Zeugung im Sinne von Lebensbeginn bezeichnet (Kap. 6.1.1). Da das Zusammenführen der Körpersubstanzen jedoch nur möglich werden kann durch den beruflichen Einsatz verschiedener Experten, insbesondere des Arztes, stellt sich bei dieser Vorstellung von Zeugung auch die Frage nach dessen Rolle. Im folgenden Interview sprach Dr. Norbert Krüger (43) von der Befruchtung, ,als symbolischem Zeugungsakt" und vom Embryotransfer als einem Zeitpunkt, der symbolhaft einen hohen Stellenwert für die Paare trage und ging auf Nachfragen auch auf die Rolle als ,Erzeuger‘ ein.

N.K.: „Es findet natürlich eine gewisse Entkoppelung [zwischen dem Zeitpunkt der Befruchtung und dem des Embryotransfers] statt, das ist korrekt. Gerade wenn man das 
mit der Kryokonservierung hier mit einbringt. Für die Paare ist es dann meist so, dass der Zeitpunkt der Übertragung ein sehr wichtiger Zeitpunkt ist und eigentlich erst in dem Moment das Gefühl bei vielen Paaren aufkommt, so jetzt ist das meins, jetzt gehört es mir wieder, und von den Paaren, die zumindest IVF machen, ist dieser Embryotransfer ein Zeitpunkt, der symbolhaft einen hohen Stellenwert eben auch trägt.“

I.S.: „Es gibt da allerdings auch ganz unterschiedliche Aussagen. Einige Paare sagen, Zeugung ist für sie das rein Technische, die Verschmelzung. Das macht es für sie auch nicht mehr so schwierig damit umzugehen, weil das bei der normalen Zeugung genauso wie bei der sogenannten künstlichen passiert. Da versucht man dann, dieses Problem irgendwie zu lösen.“

N.K.: „Ich denke zum Beispiel jemand, der aus der katholischen Glaubenslehre kommt, der sieht das auch noch wieder ganz anders. Auch die Penetration im engeren Sinne ist schon ein Teil des Zeugungsaktes und das ist auch ein längerer Prozess: Aber in der heutigen Zeit können sie durch die Anwendung von Verhütungsmitteln auf der einen Seite ihre Sexualität ausleben, ihre Gefühle ausleben, auf der anderen Seite eben auch terminiert ihren Kinderwunsch erfüllen, wenn sie es gerade möchten, oder wenn sie eben denken, dass es zeitlich günstig ist. Für sie persönlich als Paar [...].“

I.S.: „Wenn, wie Sie sagen, der Embryotransfer für die Paare eine besondere Situation ist oder im weitesten Sinne symbolisch die Zeugung, wie sehen Sie da ihre Rolle?“

N.K.: „Im juristischen Sinn bin ich der Erzeuger. Das bin ich auch schon in dem Moment, wo ich eine Insemination durchführe, weil eben diejenige Person, die der Frau beiwohnt innerhalb einer gewissen Zeit vor der Entbindung - und das kann im medizinischen Bereich ein Mann als auch eine Frau sein, die der Frau beiwohnt - Erzeuger des Kindes ist, was auch gewisse unterhaltsrechtliche Dinge nach sich ziehen kann. Manchmal kommt mir die Situation ein bisschen vor, wie einst bei der Jungfrau Maria mit der unbefleckten Empfängnis. Es ist die Entkoppelung irgendwo da, weil der Akt der Zeugung außerhalb des Körpers stattfindet, zumindest auf IVF bezogen. Ich versuche das eher so zu betrachten, dass ich mich in den Dienst des Paares stelle. Allerdings ist es eine Gemeinschaftsaktivität, weil noch eine Helferin mit dabei ist, die dann tatsächlich den Stempel auch drückt, ${ }^{158}$ und dann die Flüssigkeit mit den Embryonen eingibt. So gesehen, muss man das eher im Team sehen, wenngleich ich mir bewusst bin, dass ich da eine herausgehobene Position einnehme, und ich weiß nicht, ob es vielleicht auch manchmal so ist, dass es für viele dann, nicht dass ich ein Gott bin, das möchte ich mir auch nicht anmaßen, aber dass man vielleicht auch in so eine Rolle hineingedrängt wird, oder dass das so gesehen wird, dass man eine gewisse Allmacht hat.“

Auch Dr. Jan Hagemann (47) wies die Rolle als ,Erzeuger` von sich:

„Ich sehe mich nicht so. Ich sehe mich eigentlich auch nicht als jemand, der Dinge möglich macht, die dem Herrgott vorbehalten sein sollen, aus welcher Religion man auch kommen mag. Sondern ich sehe eigentlich, dass ich jemand bin, der aufgrund seiner Ausbildung in der Lage ist, dieses Problem in seiner Komplexität zu erfassen und es so zu therapieren, dass das, was durch mechanische oder organische oder sonstige Ursa-

\footnotetext{
158 Der Embryotransfer erfolgt durch die Scheide, indem durch den Arzt ein dünner Plastikkatheder in die Gebärmutter eingeführt wird. Eine medizinische Mitarbeiterin drückt die Flüssigkeit mit den Embryonen dann direkt in die Gebärmutter hinein.
} 
chen nicht möglich ist, doch möglich wird. Der Zeugungsakt selber ist für mich, und das hat sich ja in den letzten dreißig Jahren auch nicht geändert, das ist für mich eine faszinierende Sache. Wir wissen viel zu wenig davon. Gucken Sie, ich bin gelernter Biochemiker, wenn ich mir überlege, wie wenig wir über das wissen, was wir letztendlich hier machen, kann ich sagen, eigentlich bin ich ein Höhlenbewohner, der einen Chagall oder Picasso beschreiben soll.“

Anhand dieser Interviews wird deutlich, wie verschiedenartig die Vorstellungen von Zeugung sind. Im ersten Ausschnitt spricht Dr. Krüger vom Geschlechtsakt als Teil des Zeugungsaktes, aber auch davon, dass die Zeugung außerhalb des Körpers stattfindet, womit er hier den Embryotransfer meint, bei dem sowohl er als auch Helferinnen beteiligt sind. Dr. Hagemann bezeichnet den Zeugungsakt als faszinierende Sache, wobei er damit nach meiner Interpretation auf die Verschmelzung von Ei- und Samenzelle verweist und nicht auf den Geschlechtsverkehr. Es ist offensichtlich, dass im innermedizinischen Diskurs und im konkreten Berufsleben die Betonung in erster Linie auf dem (technischen) Vorgang der Befruchtung liegt. Der Begriff der Zeugung wird überwiegend nicht verwendet, denn er beinhaltet sowohl soziale als auch körperliche und leibliche Beziehungen, die seitens der Ärzteschaft im Namen ihres Amtes zu den Paaren und den entstandenen Kindern nicht gewünscht werden. So äußerte sich Dr. Norbert Krüger:

„Ich denke, dass ich für einige Paare eine Position einnehme, dass ich derjenige bin, der diesem Paar zu dem Kind verholfen hat. Dass ich in deren Empfinden eine gewisse allmächtige Position habe. [...] Ich habe eine gute Distanz dazu, dadurch dass ich selber zwei Kinder habe. Ich glaube, das ist auch sehr wichtig für jemanden, der in diesem Bereich tätig ist, dass er selber Kinder hat. Ich glaube, das hilft einem, diese Distanz dann auch zu wahren und zu sagen, das ist wirklich mein Kind, biologisches Kind, selbst erzeugt, und das sind jetzt die Kinder von den Paaren. Ich war eine Zeitlang daran beteiligt, aber ich kann dann auch, wenn ich so ein Kind auf den Arm nehme und das mal herumtrage und vielleicht auch ein Foto von mir mit dem Kind gemacht wird, auch gut sagen, hier, nehmt euer Kind wieder, denn das ist euer Kind. Man darf vor allen Dingen auch nicht vergessen, dass viel von der Arbeit ja gar nicht von mir allein erledigt wird, sondern da sind die Helferinnen an der Rezeption, die Mitarbeiterinnen im Labor, die mit zu dem Gelingen einer Behandlung beitragen. Als Frauenarzt ist man da sicherlich in einer exponierten Stellung, weil man am häufigsten Kontakt hat mit dem Paar, aber die anderen sind ja genauso beteiligt.“

Auch der Arzt Rolf Wellmann ging auf den Aspekt der persönlichen Beziehungen ein, die die Paare (und hier besonders die Frauen) aufgrund seiner Mithilfe bei der Zeugung an ihn herantragen und betont besonders die Ungleichgewichtigkeit. Er freut sich an den geborenen Kindern, fühlt sich aber in keiner Weise für diese zuständig.

„Ein nicht unerheblicher Teil unserer Tätigkeit ist das Showgeschäft. Und wenn sie sich noch so wenig für das Ergebnis dieser Schwangerschaft, die Sie in Gang gebracht haben, interessierten, könnten Sie das dem Paar doch nicht zu verstehen geben, weil sich das Paar gekränkt fühlen würde. Die Mutter, die dieses Kind dem 30 bis 40 prozentigen Vater [meint sich selbst] auch noch vorführen will, will ja auch was dafür. Ich meine, wenn ich mir soviel Mühe gegeben habe, sie schwanger zu bekommen, dann will sie ja 
zeigen, dass sie ihren Teil auch gut gemacht hat. Und das muss man wohl akzeptieren. Ich muss ganz ehrlich sagen, die Kinder interessieren mich eigentlich nicht so sehr. Denn sie unterscheiden sich nicht von anderen Kindern und nicht dadurch, dass ich das war. Für mich ist das Paar entscheidend. Ich habe die Beziehung zum Paar aufgebaut im Idealfall. Das ist mir wichtig. Dass dann zum Schluss auch noch das schönste Kind auf die Welt kommt, kann ich nicht nachvollziehen. Das habe ich bei meinen Kindern. Aber ich würde das Paar kränken, wenn ich nicht vermitteln würde, dass ich mich besonders über dieses Kind freuen würde. [...] Und es ist auch notwendig, denn die Paare wollen alle eine Rolle für sich. Sie wollen nicht in der Masse untergehen. Das ist auch irgendwie verständlich. Ich freue mich wirklich über meine Paare. Das spiele ich denen nicht vor. Sie kriegen meine volle Zuwendung. Nicht nur fachlich, das sowieso. Den Anspruch hat jeder, der irgendwo hingeht, dass da jemand sitzt, der weiß, was gemacht wird oder eben nicht. Die Kinder sind mir, ehrlich gesagt, hinterher wurscht, weil ich mich dafür nicht zuständig fühle. Ich fühle mich absolut nicht als Vater.“

Gesamthaft betrachtet zeigen die Aussagen in den Interviews, dass die Definitionen von Zeugung komplex, heterogen und kontextspezifisch sind und sich nicht allein auf die Beschreibung der geschlechtlichen Fortpflanzung beziehen. Zeugung hängt für die Ärzte in unterschiedlichem Ausmaß mit Intimität und Emotionen zusammen. Es lässt sich feststellen, dass die verwendete Begrifflichkeit unterschiedliche Perspektiven zwischen Beruf und Privatleben ausdrückt. Geht es im Beruf um den Umgang mit Körper als objektiviertem und materiellen Leib als Gegenstand der reproduktionsmedizinischen Behandlungen, steht im Privatleben der Ärzte der Leib als subjektives Empfindungsund Erfahrungsorgan, mit dem sie ihre eigene Lebenswelt wahrnehmen, im Zentrum. In diesem Kontext ist die Vorstellung von Zeugung direkt verknüpft mit einer emotionalen sexuellen und somit leiblich erfahrbaren Paarbeziehung ebenso wie mit Gedanken an das leibliche Kind, das entstehen soll. Diesen Aspekt möchte ich anhand eines Interviewausschnitts mit Rolf Wellmann noch verdeutlichen:

„Aus eigener Erfahrung kann ich sagen, Zeugung ist schon ein eher lustvoller Akt. Meine Frau ist immer im zweiten nicht verhüteten Monat schwanger geworden. Von daher kann ich die Trennung von dem, was ich zielgerichtet tue, um meinen Wunsch zu erfüllen, also ein Kind zu haben, und lustvoller Sexualität, nicht so auseinanderhalten, während das ja bei unseren Patienten hier was ganz anderes ist. Das eine verdrängt das andere ja offensichtlich völlig.““

Bei allen Methoden der ,,assistierten“ Fortpflanzung arbeiten die Ärzte im Namen ihres (bezahlten) Amtes oder ihres beruflichen Auftrages und reduzieren ihren Beitrag auf die Unterstützung bei der Befruchtung von Körperzellen (bzw. heben diesen Beitrag besonders hervor). Als ,professionelle Zeugungshelfer ${ }^{\star}$ bleiben sie sozial unsichtbar, obwohl sie den Anstoß für die Befruchtung und damit für das Entstehen eines Kindes geben. Durch die Konzentration auf den Befruchtungsvorgang und damit den rein körperlichen Teil der Fortpflanzung sehen sie sich nicht als ,(Mit-)Erzeuger', da diese Rolle ihrer 
Ansicht nach auch die Teilhabe an der sozialen Elternschaft bedeutet. ${ }^{159}$ Zeugung wird hier demnach assoziiert mit einer körperlichen und leiblichen Erfahrungswelt. „Der Natur auf die Sprünge helfen“

In Kapitel vier habe ich die gesellschaftlichen Rahmenbedingungen für die Forschung und Anwendung der reproduktiven Technologien beschrieben, die in den Gesetzestexten sowie den Richtlinien für die Ärzte als „,künstliche Befruchtung“ bezeichnet werden und damit ein Gegensatzpaar mit der „,natürlichen Befruchtung“ bildet. $^{160}$ In meinen Gesprächen mit den Ärzten thematisierte ich diese Gegenüberstellung von ,natürlich“ und „künstlich“, da letzterer Begriff schließlich ihre Tätigkeit beschreibt: die Durchführung von „künstlicher“ Befruchtung. In der reproduktionsmedizinischen Praxis, insbesondere in der Interaktion mit den Paaren, wird die Verwendung dieser Unterscheidung in „,natürlich“ versus „künstlich“ ebenso wie das Hervorheben der technischen Eingriffe seitens der Ärzteschaft vermieden. In den Gesprächen mit den Paaren betonen die Ärzte häufig, dass die fortpflanzungsmedizinischen Behandlungen gerade nichts „Künstliches“ seien. ${ }^{161}$ Die Ambivalenz der Terminologie wird in einem Interview mit dem Arzt

159 Die Frage nach der Rolle des Arztes als ,Erzeuger` im Zusammenhang mit der Nutzung der Reproduktionstechnologien von Frauen, die zwar Babys, aber keine sexuelle Beziehung wollen, diskutiert Strathern (2001:359-395) aus kulturanthropologischer Perspektive. So verweist sie u.a. auf die Witze über die „,äterliche“ Rolle des Klinikers, der durch seine Anstrengung ein Kind ,hervorgebracht" hat und sieht sie als eine Art kulturellen Kommentar über die Beziehung zwischen technologisch unterstützten und natürlichen Empfängnisprozessen, die aus den involvierten Personen in euro-amerikanischen Gesellschaften Eltern machen. In Situationen, in denen ein zukünftiger Vater vorhanden ist - gleich ob ein bevollmächtigter oder ein genetischer Vater - handelt der Kliniker selbst bloß als Stellvertreter. Problematisch wird es dann, wenn Frauen eine reproduktionsmedizinische Behandlung wünschen, ohne dass ein Vater vorhanden ist und den Arzt somit in eine „quasi-sexuelle“ Rolle drängen, da der Sexualakt eine zentrale Bedeutung innerhalb einer ehelichen Gemeinschaft hat und den Bund der Partner und ihre Liebe zueinander symbolisiert (vgl. Strathern 2001:360, 372). Wie auch in meiner Arbeit deutlich geworden ist, werden im Kontext der Reproduktionsmedizin jedoch alle Anstrengungen unternommen, Beziehungen zu umgehen. Ich möchte hier allerdings erwähnen, dass verschiedene reproduktionsmedizinische Kliniken Treffen organisieren, zu denen alle ,erfolgreich“ behandelten Paare mit ihren Kindern eingeladen werden. In diesem Zusammenhang wird oftmals - meiner Ansicht nach werbewirksam - von den männlichen Ärzten eine ,symbolische Vaterschaft ‘übernommen, in dem darauf verwiesen wird, dass es ,alles unsere Kinder“ sind. Damit wird den Paaren und der über die Medien erreichten Öffentlichkeit eine Form von sozialer Beziehung zu den ,gezeugten' Kindern suggeriert, die jedoch kontext- und situationsspezifisch ist und letztlich keine sozialen Konsequenzen beinhaltet. Mir ist bisher kein Fall einer öffentlichen Präsentation von ,symbolischer Mutterschaft‘ bekannt, auch wenn zahlreiche „Sterilitätsbehandlungen“ von Ärztinnen durchgeführt werden. Eine Erklärung liegt meiner Ansicht nach in den unterschiedlichen Kriterien für die Konstituierung von Mutterschaft und Vaterschaft in Deutschland, die Vaterschaft im Gegensatz zu Mutterschaft ,verhandelbar macht und die nicht durch Schwangerschaft und Geburt offensichtlich ist (vgl. Kap. 4.1.3). Ich möchte auf diesen Aspekt hier zwar verweisen, werde ihn aber aufgrund mangelnden Datenmaterials in meiner Arbeit nicht weiter bearbeiten.

${ }^{160}$ Ich möchte hier noch mal explizit auf die sprachliche Begrifflichkeit der ,Befruchtung ‘ und nicht der ,Zeugung ‘ in den Gesetzestexten zum Umgang mit den Reproduktionstechnologien hinweisen.

161 Diese Aussage beruht auf meiner teilnehmenden Beobachtung von Interaktionen zwischen Paaren und Ärzten in den reproduktionsmedizinischen Kliniken. Auch die Literatur zeigt, dass diese Ansicht unter Reproduktionsmedizinern dominiert. So äußert sich zum Beispiel Beier (1997:9) zum Begriff der „künstlichen Befruchtung“ und betont, wie verwirrend diese Bezeichnung für Mediziner und Laien gleichermaßen sei, denn die therapeutisch erstrebte und erzielte genetische Vereinigung der Keimzellen sei im echten Sinne des Wortes nicht künstlich. Beier ist der Ansicht, dass der Gesetzgeber bei der Formulierung des Embryonenschutzgesetzes von „künstlicher Befruchtung“ gesprochen habe, da er damit lediglich die durch ärztliche Kunst, d.h. durch ärztlichen Eingriff gebahnte und unterstützte Befruchtung gemeint habe. Der biologisch definierte Befruchtungsvorgang selbst bleibe bezüglich der Vereinigung der haploiden Chromosomensätze von Vater und Mutter auch bei der „künstlichen“ Befruchtung ein genetisch völlig 
Rolf Wellmann sehr deutlich, indem es sich seiner Ansicht nach sehr wohl um ein „künstliches“ Verfahren handele, das prinzipiell jedoch nicht von großer Bedeutung sei. So solle man das auch den Patienten vermitteln, damit sie sich angstfreier in die Behandlungen hineinbegeben könnten. Die technische Seite der Behandlung werde seiner Ansicht nach von Kollegen dann hervorgehoben, wenn diese sich selbst als „HightechKinderproduzierer“" definierten und daraus ihr Selbstbewusstsein bezögen.

„Es ist etwas Künstliches, darüber brauchen wir nicht zu diskutieren. Das Problem ist aus meiner Sicht ein anderes. Wenn ich mich als Hightech-Kinderproduzierer definiere, dann werde ich in den Vordergrund stellen, welch schwierige hormonelle Konstellationen wir bewältigen müssen, welche technisch aufwendigen Ultraschalluntersuchungen, und dass wir dann auch noch mit Mikroinjektionsapparaten hochsensibles Zellmaterial manipulieren. Das ist die eine Sache. Das liegt an mir. Wenn das für mich wichtig ist, und ich das brauche, es so hoch zu spielen, dann werde ich es als künstliche Befruchtung sehen. Ich sage mir, wir überwinden oder wir helfen an Stellen nach, die in der Natur normalerweise sowieso genauso ablaufen. Wir beheben keine Störung, wir machen in dem Sinne keine Medizin, sondern wir machen ein bisschen Mechanik, ein bisschen Feinmechanik. Wenn man es auf die Mikroinjektion bezieht. Wir helfen ein bisschen nach an den Stellen, wo es bei den Paaren hakt. So sehe ich es! Ich meine, wenn ich für meine Definition der Tätigkeit und für meine Wichtigkeit brauche, es hoch zu spielen, dann muss ich auch lernen, dass ich bei den Patienten Widerstände aufbaue, die sich dann natürlich dagegen wehren und Angst davor haben, ob sie das nun aussprechen oder nicht. Sie werden auf jeden Fall sehr viel kritischer und angstbesetzter damit umgehen. Ich rufe die Geister selber, und wenn ich denen sage, natürlich ist das technisch aufwendig, aber im Grunde genommen tun wir das Sperma da hin, wo es sowieso von der Natur aus hingehört, dann fühlen sich die Patienten meistens auch ein bisschen lokkerer und sagen: ,Eigentlich haben sie recht. ' (lacht). Das kommt immer darauf an, warum man etwas macht, und was man selber davon hat. Und den Patienten nützt das Hochspielen schon gar nicht. Da werden sie nur mehr Widerstände haben, und wenn sie dann einen einfachen Vorgang wie einen Transfer auch noch bei einer verspannten Patientin erleben müssen, dann ist das für sie [die Frau] in höchstem Maße unerfreulich ist und wird wahrscheinlich auch nicht zum Erfolg führen. Was hinterher aber nur als Beweis angesehen wird, wie schwierig es wirklich ist. Man kann sich das alles immer selber begründen. [...] Doch, es ist im höchsten Masse künstlich, was wir machen, aber ich würde die Wertigkeit, dass es künstlich ist, nicht so hoch hängen, weil es im Prinzip in der Natur auch nicht soviel anders läuft. Das Prinzip ist ja im Grund genommen das Gleiche, wir bringen eine Eizelle und eine Samenzelle zusammen und von da ab tun wir gar nichts mehr, und mehr macht die Natur auch nicht. Nur dass wir dafür sorgen müssen, dass bei verklebten Eileitern das Sperma einen anderen Weg nehmen kann, schön und gut, aber ich würde diese Künstlichkeit als sinnvoll ansehen, sonst würde es nicht gehen. Und ich habe überhaupt kein Verständnis dafür, dass eine Frau, die eine Unter-

natürlicher Vorgang ohne Beeinträchtigung und ohne „Manipulation“ dieses entscheidenden Prozesses. Beier plädiert für die Bezeichnung ,assistierte Reproduktion“ als zutreffendsten Begriff. Hier ist jedoch kritisch anzumerken, dass auch der Begriff der Reproduktion nicht wertneutral ist, sondern dass es sich dabei um einen Terminus handelt, der sich in Anlehnung an die kapitalistische Wirtschaftsform in der Mitte des 19. Jahrhunderts herauskristallisiert hat. So verweist auch Duden (1987:43) darauf, dass sich der heutige Begriff der Reproduktion an den modernen Wissenschaften und am Warenbegriff der „Produktion“ orientiere. 
leibsentzündung gehabt hat und nun verschlossene Eileiter hat, keine Schwangerschaft erleben soll, weil es ach so künstlich ist."

Es ist schon in den vorangegangenen Kapiteln deutlich geworden, dass im medizinischen Diskurs eine Konzentration bzw. Reduzierung auf die körperlichen Vorgänge der Zeugung stattfindet bzw. die Befruchtung als wesentlicher Teil der Zeugung angesehen wird, die im Reagenzglas genauso stattfinden kann wie im Körper der Frau. Mit dieser Perspektive, die den objektivierten Körper in den Mittelpunkt rückt, und der in diesem Zusammenhang verwendeten sprachlichen Begrifflichkeit lässt sich auch das Argument vertreten, dass es nur darum geht, „,der Natur auf die Sprünge zu helfen“ und mit Hilfe der Medizin die Vorgänge durchzuführen bzw. zu unterstützen, die in der ,Natur' im ,Normalfall‘ auch stattfinden.

Der Aspekt des Eingreifens in die ,Natur' findet sich pointiert auch an anderer Stelle. So konstatierte Prof. Dr. Peter Heise im Gespräch explizit, dass für ihn die gesamte Medizin ein Eingriff in die Natur darstelle, insofern sie sogenannte ,natürliche Abläufe“ zu korrigieren versuche. Auch eine Blinddarmentzündung stelle seiner Ansicht nach einen „natürlichen“ Ablauf dar, nämlich die Auseinandersetzung zwischen einem Organismus und Bakterien. Dennoch werde sie nicht schicksalhaft hingenommen, sondern es werde vielmehr von der Medizin erwartet, hier korrigierend im Sinne einer Verbesserung der Gesundheit bzw. einer Wiederherstellung einzugreifen. Medizin sei prinzipiell „,künstlich“, auch auf der Intensivstation eines Krankenhauses werde beispielsweise Leben „künstlich“ verlängert. Die Probleme der Menschen mit der Geschwindigkeit der technologischen Entwicklung wurde als ein weiterer Aspekt von Prof. Dr. Peter Heise angesprochen: Die In-vitro-Fertilisation (IVF), vor 15 Jahren noch revolutionär, gelte heute (auch für die medizinischen Gutachter der Krankenkassen) als eine bewährte Methode zur Behandlung von weiblicher „Sterilität“ (siehe Kap. 4.3.1.2). Diese Entwicklung zeige seiner Ansicht nach, dass medizinische Eingriffe, die damals noch als bahnbrechend galten, heute als Routineeingriffe angesehen und von sehr vielen Leuten so eingeschätzt würden:

„Man gewöhnt sich doch an alles. So ist der Mensch strukturiert. Alles Neue macht erst mal Angst und man regt sich vielleicht auch auf, aber dann erkennt man den Wert oder gewöhnt sich einfach daran. Sie sehen das doch mit der Klonfrage ganz genauso. Noch schreit alles, aber warten sie doch noch mal ein bisschen ab. Und fragen Sie im übrigen die Leute, die jetzt schreien, wobei ich nicht für das Klonen bin, ich beziehe mich hier in erster Linie auf die Sterilitätstherapien, auch mal, was sie machen würden, wenn sie persönlich betroffen wären. Das ist doch immer wieder so. Wenn man selber medizinische Hilfe braucht, denkt man doch in den wenigsten Fällen über Ethik und Moral nach, da will man doch alles nutzen, was in der Medizin schon angewendet wird. Sei es künstlich, sei es technisch.“

Anhand der obigen Aussagen werden mehrere Aspekte besonders deutlich. Im ersten Interviewausschnitt verweist Rolf Wellmann darauf, dass die Reproduktionsmedizin nur 
an den Stellen nachhelfe, die in der ,Natur' derart auch ablaufen, und somit die ,natürlichen“ Vorgänge nur unterstütze. Er nimmt somit eine Angleichung oder Gleichsetzung „natürlicher“ und „künstlicher“ Vorgänge vor und versteht unter „natürlich“ allein die körperlichen Prozesse, die ab einem bestimmten Zeitpunkt von allein ablaufen, auch ohne das Zutun des Arztes bzw. der reproduktionsmedizinischen Verfahren. Diese seien zwar unterstützend, könnten aber nichts gegen den ,Willen der Natur ${ }^{6}$ erreichen. Ab einem bestimmten Punkt könne der Mensch (der Arzt) nichts mehr für bzw. gegen die ,Natur' tun, da sie nach ihren eigenen Gesetzen funktioniere.

So nimmt „natürlich“ Bezug auf den ,normalen“, ohne Einsatz der Medizin, ablaufenden Prozess der Fortpflanzung und wird auf den genetischen Zeugungsvorgang reduziert. Auch hier wird offenbar, dass sich das professionalisierte Handeln auf den körperlichen Aspekt von Zeugung, d.h. die Zusammenführung von Ei- und Samenzelle, bezieht, der Leib als Ort der subjektiven Wahrnehmung und der sozialen Interaktionen in der beruflichen Praxis jedoch ausgeblendet wird, was auch durch die ausschließliche Verwendung des Begriffes der (,natürlichen“ bzw. „,künstlichen“) Befruchtung deutlich wird. Sexualität und soziale Beziehungen und damit Leiblichkeit finden im professionalisierten Diskurs über Befruchtung und im konkreten Umgang in der reproduktionsmedizinischen Praxis keine Erwähnung mehr, was den Ärzten erlaubt, „natürlich“ mit „künstlich“ gleichzusetzen oder beides aneinander anzugleichen. Die Betonung der genetischen Prozesse als „natürliche“ Fakten ermöglicht den Reproduktionsmedizinern zu zeigen, dass man , der Natur nur auf die Sprünge hilft“ ebenso wie den Verweis auf die ,Hilflosigkeit‘ der Ärzte, wenn die Behandlungen nicht zum ,Erfolg‘ führen: „In der Natur ist alles möglich, da kann es mal so und mal so passieren. So etwas kann man nicht erklären und hat keinen Einfluss darauf, man kann nur die besten Bedingungen schaffen“. Aufgrund der prinzipiellen ,Launenhaftigkeit der Natur", die auch bei der den genetischen Prozessen des ,natürlichen“ Zusammenkommens von Ei- und Samenzellen zu Fehlentwicklungen und Aborten führt, lässt sich die Anwendung der Reproduktionstechnologien als „Hilfe an der Natur“ in den reproduktiven Prozess einbinden. Ebenso lassen sich dadurch auch die möglichen ,Fehler' oder ,Misserfolge“ der reproduktionsmedizinischen Anwendungen erklären (vgl. Franklin 1995:331, 1997:144f), da ,Erfolge' und ,Misserfolge“ in der, Natur' der Sache liegen und der Arzt ebenso wie die Paare dieser letztlich , ausgeliefert' sind:

„[...] Für alles, was gemacht wird, muss auch eine Erklärung angeführt werden. Keiner hier kann mehr schicksalhafte Verläufe akzeptieren. Der Erklärungsnotstand ist vor allem dann sehr groß, wenn eine Behandlung nicht geordnet abläuft, wenn nach einer Eizellentnahme statt vier Eizellen nur eine Eizelle aufgefunden wird. Wenn nach Auffinden von fünf oder sechs Eizellen nur eine befruchtet ist; oder ursprünglich drei befruchtete sich nicht weiter teilen und vielleicht nur eine oder gar keine befruchtet ist. Dann wird auch gleich gesagt, es muss doch irgendwelche Ursachen geben. Es wird aus meiner Sicht heute zu wenig akzeptiert, dass man bestimmte Ursachen auch nicht be- 
nennen kann, dass das auch nicht von mir möglich ist, und das hilft einem auch dabei, seine eigene Macht zu sehen und zu sehen, dass es keine Allmacht ist. Dass man auch hier nur eine gewisse Begleitung und Unterstützung anbieten kann Aber letztlich kann ich doch genauso wie die Paare gar nichts gegen die Natur ausrichten, die sich vielleicht entschließt, bestimmte Zellentwicklungen nicht weiter stattfinden zu lassen."(Dr. Norbert Krüger)

Prof. Peter Heise betont noch einen weiteren Aspekt, in dem er nicht nur die Reproduktionsmedizin mit ihren Methoden als „künstlich“ definiert, sondern weitaus umfassender die ganze Medizin als Eingriffe in die Natur darstellt, die ,natürliche Abläufe“ zu korrigieren oder wiederherzustellen versucht. Er hebt besonders die gesellschaftliche Veränderung in der Einschätzung und Akzeptanz medizinischer Technologien hervor und verweist auf die veränderte Einstellung zur Anwendung der In-vitro-Fertilisation. In diesem Zusammenhang erwähnt er auch die seiner Ansicht nach häufige Überzeugung der Paare, ihren „Kinderwunsch“ zeitlich determinieren zu können, wenn es ihnen passe. So lange man sich gesund und körperlich nicht beeinträchtigt fühle und denke, man könne sich den „Kinderwunsch“ erfüllen, wann man wolle und es zeitlich günstig sei, werde die Fortpflanzungsmedizin oftmals als technisch und „künstlich“ abgeurteilt. Wenn dann aber die Situation eintrete, dass anders eben kein Kind komme, dann sei keine Rede mehr von zu „künstlich“. Dann wolle man nur noch alle Möglichkeiten nutzen und nicht aufgeben (vgl. auch Kap. 6.2). Es zeigt sich hier, dass von professioneller Seite die Einschätzung von ,natürlich“ und ,künstlich“ als situations- und kontextspezifisch verschiebbar und aushandelbar angesehen wird: Nach Überzeugung von Prof. Heise passt sie sich zum einen den wandelnden Bedürfnissen der Gesellschaft an, zum anderen ist sie abhängig von der persönlichen Betroffenheit. Was in einer Lebenssituation noch als „künstlich“ und zu technisch abgelehnt wird, kann in einer anderen Lebenssituation plötzlich als akzeptabel gelten oder sogar ,der Griff nach dem letzten Strohhalm“ sein.

In den folgenden Kapiteln soll es nun um die Perspektive der Paare gehen, die zum Zweck der Erzeugung von leiblichen Nachkommen reproduktionsmedizinische Hilfe in Anspruch nehmen. Zunächst werde ich auch hier der Frage nach der Vorstellung von Zeugung nachgehen und die verschiedenen Positionen, die sich aus meinen Interviews herauskristallisiert haben, darstellen. Des weiteren möchte ich untersuchen, ob sich der innermedizinische Diskurs bezüglich der spezifischen Sichtweise und Veränderbarkeit der Vorstellungen von ,natürlich“ und ,künstlich“ in den Einschätzungen der ,ungewollt kinderlosen“ Paare finden lässt. Es ist deutlich geworden, dass für die Ärzte der professionalisierte Umgang mit Körpern im Zentrum ihres Berufes steht und nicht die eigene Lebenswelt und Leiblichkeit als Privatperson. Die Paare sind jedoch von den konkreten Behandlungen sowohl in ihrer Körperlichkeit als auch in ihrer Leiblichkeit betroffen. Gerade auch sie sind mit dem öffentlichen Diskurs konfrontiert, in dem die 
Dichotomie „,natürlich“ versus „künstlich“ thematisiert wird, und der sich häufig in den Meinungen und Reaktionen des sozialen (nicht-betroffenen) Umfeldes widerspiegelt.

\subsection{Die Sichtweise der Paare}

\subsubsection{Zeugung als Prozess}

In 20 von 45 Interviews wird deutlich, dass sich die von mir befragten Paare mit dem Begriff der Zeugung schwerpunktmäßig auf ihre Partnerschaft beziehen und die Gemeinsamkeit betonen, die für sie die Voraussetzung bildet für die Erzeugung eines Kindes. Das Zusammensein der Partner während der Behandlungen, die emotionale Nähe, das Zusammenleben im Alltag sowie die gemeinsame Entscheidung für ein Kind wurden als wichtige Aspekte genannt, die die Voraussetzungen bilden für die „,biologische Zeugung“ im Sinne des Verschmelzens von Ei- und Samenzelle. So äußerte sich Maria Heider (26), die zum Zeitpunkt des Interviews den zweiten IVF/ICSI-Versuch unternahm, folgendermaßen:

„Zeugung, das ist zum einen biologische Zeugung. Aber auch das Zusammenkommen von zwei Menschen mit einer bestimmten Vorstellung, ein Kind zu bekommen. Der Gedanke, der Wunsch an sich, ist für mich zwar nicht schon die halbe Miete, aber das andere, wie das dann stattfindet, ist im Endeffekt nicht mehr ausschlaggebend. Das eine ist die Voraussetzung für das andere.“

Auch Elisabeth Leinemann (34), die durch ICSI schwanger wurde, betonte das Zusammensein und die emotionale Nähe als wichtigste Kriterien:

„Der Begriff Zeugung ist verwischt, Schöpfung ist auch ein schlechtes Wort, denn es ist schon die ganze Zeit, während man auf die Punktion hinarbeitet. Das sind so 14 Tage, wo mein Mann mir jeden Abend einen Schmerz zufügt, weil er mir eine Spritze in den Hintern wirft. ${ }^{162}$ Aber jedes Mal sagen wir, das ist wieder ein Schritt hin zum Kind. Und das ist letztendlich der Überbegriff von Zeugung. Der ganze Weg ist es schon, und der Moment selber, wo mein Mann da ins Zimmer muss und Sperma abgeben. Das ist für uns Nebensache, das ist wieder nötig. Aber das ist nicht abwertend, dass dieses Kind nicht in Liebe, im Bett oder sonst wie gezeugt wurde. Das eine schließt das andere nicht aus. Aber es ist schon was Getrenntes, es hat nichts miteinander zu tun. Liebhaben findet eben woanders statt. Das Zusammensein und auch die Zärtlichkeit irgendwo ist das Wichtigste und die Voraussetzung für alles andere. Das ist ein Zeugungsprozess. Und der zieht sich bis in die schlimme Phase nach dem Transfer, wenn die Blutungen einsetzen oder wenn man überfällig ist. Das ist so ein Riesenkomplex die Zeugung.“

\footnotetext{
162 Der Ehemann ist selber Arzt von Beruf und das Verabreichen von Spritzen ist für ihn Routine. Daher spritzt er auch seiner Frau die tägliche Hormondosis. In fast allen anderen Fällen von IVF und ICSI, bei der eine hormonelle Stimulierung notwendig ist, mussten sich die Frauen die Spritzen selbst geben.
} 
Hier wird deutlich, dass Zeugung nicht an einen bestimmten Zeitpunkt geknüpft ist, sondern die Vorstellung eines längerandauernden Zeugungsprozesses dominiert. Er beginnt mit der Entscheidung eines Paares für ein gemeinsames Kind, beinhaltet aber auch medizinische Eingriffe sowie die Zusammenführung von Körperzellen im Reagenzglas. Die leiblich erfahrbare Beziehung zwischen den Partnern steht hier jedoch im Vordergrund. Maria Heider drückt explizit aus, dass für sie nicht mehr ausschlaggebend sei, wie die „biologische Zeugung“ zustande komme, wenn zwei Menschen sich gefühlsmäßig einig seien und ein Kind bekommen möchten. Dieser „biologische“ Teil der Zeugung findet unter Zuhilfenahme der Medizintechnik statt, wobei es in erster Linie um die Produktion, Gewinnung und Zusammenführung von Körperzellen geht. Durch den Einbezug verschiedener Stadien von Zeugung und die primäre Betonung der Intimbeziehung des Paares als Voraussetzung für die Schaffung eines Kindes wird hier versucht, den ausschließlich körperlichen Teil als Gegenstand der Behandlungen nicht so hoch zu bewerten wie den leiblichen im Sinne der subjektiven sinnlichen Erfahrungswelt.

Die durch die reproduktionsmedizinischen Verfahren vollzogene Trennung von Geschlechtsverkehr und Fortpflanzung spiegelt sich auch bis zu einem gewissen Grad in der Unterscheidung zwischen Zeugung und Befruchtung wider. Wie ich oben schon zeigen konnte, werden Sexualität und Zeugung mit intimen sozialen Beziehungen assoziiert, während die Fortpflanzung und damit Befruchtung aus der Paarbeziehung heraus in die Klinik bzw. ins Labor verlegt und an den Arzt abgegeben werden. Dieses Loslösen des körperlichen Teils vom leiblichen Teil der Erzeugung eines Kindes führt in vielen Fällen zu permanenter Anspannung. So schilderte Maria Heider (26), die sich in einer ICSI-Behandlung befand, ihre Erfahrungen:

„Bei einer künstlichen Befruchtung ist sechs Wochen permanenter Stress. Weil das Romantische, dieses Natürliche und Lockere, ,Ach Schatz, ich bin schwanger', das ist nicht gegeben. Zeugung hat mit Intimität und Gefühl zu tun. Wäre auf jeden Fall schöner. [...] Ich verlagere die Zeugung nicht in die Klinik. Auf keinen Fall. Wenn es so wäre, dann müsste auch die Klinik der Vater sein. Es kommt auf das Drumherum an, auf die Partnerschaft, auf das Zueinander. Es ist eine wahnsinnige Belastung, eine extreme Zeit für beide. Das ist wesentlich wichtiger als der Zeitpunkt, wo die Eizelle sich geteilt hat. Auch wenn was wächst, hat man noch lange keine Garantie, dass davon auch irgendwann mal ein Kind auf ihrem Schoß sitzt. Mein Mann hat mal gesagt, nachdem wir uns mal furchtbar gekracht haben nach dem Transfer ,Das ist wirklich hervorragend. Man kann heute Kinder zeugen, ohne sich zu mögen.' So ist es halt. Und das ist der Punkt, den ich wahnsinnig schade finde. Das ist wahrscheinlich auch der einzige Punkt, unter dem ich leide.“

Der oben erwähnte Aspekt, dass ein Paar heute Kinder zeugen könne, ohne sich gerade gut verstehen zu müssen, findet sich auch in anderen Interviews wieder. Es wird als „,irgendwie blödsinnig oder paradox“ bezeichnet, dass man in die Klinik gehen und sich 
dort schwanger machen lassen könne, obwohl die Ehe gerade nicht funktioniere. Wenn man sich nicht verstehe, würde man „,normal“ ja auch nicht miteinander ins Bett gehen.

Anhand meines empirischen Materials lässt sich feststellen, dass der Trennung von Zeugung und Befruchtung auch die Trennung zwischen Zuhause und Paarbeziehung versus Klinik und Labor entspricht. Zeugung, die private Umgebung und der Lebenspartner werden mit Intimität und sozialen Beziehungen in Verbindung gebracht, wohingegen mit Befruchtung ein medizinisch-technisches Umfeld assoziiert wird. Für Mathilde Schumann (37), die 1996 mit Hilfe der In-vitro-Fertilisation einen Sohn zur Welt brachte, habe Zeugung mit Liebe und einem schönen Gefühl zu tun, das man empfinde, wenn man mit dem Wunsch ein Kind zu bekommen, Geschlechtsverkehr habe. ${ }^{163}$ Die Situation in der Klinik nach dem Embryonentransfer empfand sie als unangenehm, besonders weil sie sich körperlich sehr schlecht fühlte, als sie ihren Sohn „empfing“. In der Retrospektive wurde dieser Moment jedoch von ihr heruntergespielt und der ,Erfolg' der Behandlung, dass sich ein Embryo in der Gebärmutter eingenistet hat und es zu einer Schwangerschaft gekommen ist, betont:

„Ich weiß nicht, aber im Normalfall macht man zusammen Liebe, wie es so schön heißt, und dann entsteht ein Kind. Da ist eben eine Menge Gefühl im Spiel. Und wenn ich daran denke, wie ich mich gefühlt habe, als ich letzten Endes meinen Sohn empfangen habe, also nach dem Retransfer, da habe ich mich so schlecht wie selten in meinem Leben gefühlt. Ich konnte vor Schmerzen nicht liegen, habe mich nicht getraut mich zur Seite zu drehen aus Angst, irgendwas Kostbares könnte verrutschen oder so ähnlich. Und habe krampfhaft immer nur gedacht, hoffentlich wird es was. Das macht man doch im Normalfall nicht, also wenn man eben nicht in die Klinik muss für ein Kind. Das war eine schrecklich unangenehme Situation. Aber das spielt jetzt keine Rolle mehr. In dem Moment habe ich auch nicht an ein Kind gedacht, sondern an eine Eizelle und eine Samenzelle. Das ist einfach was anderes, verstehen sie?! Das hat eben der Arzt oder besser die Ärzte bewirkt, die uns da betreut haben die ganze Zeit. Das war schon sehr freundschaftlich zum Glück mit denen und ich bin ihnen unendlich dankbar. Aber irgendwie meine ich, dass wir meinen Sohn woanders gezeugt haben als in der Klinik, auch wenn er letztlich dort entstanden ist mit Hilfe der Ärzte, so dass Ei und Samenzelle eben zusammenkommen konnten. Aber eben für uns nicht unbedingt. Dass es so gefühlsmäßig richtig schön war, das war dann eben doch irgendwann mal zuhause, auch wenn es vom Zeitpunkt nicht so hin kommt.“

Ich möchte an dieser Stelle darauf verweisen, dass der Arzt Rolf Wellmann mir in einem Gespräch mitteilte, dass er den Paaren vorschlage, den „Zeugungsakt“ zuhause nachzuholen, auch wenn alle wüssten, dass es „,biologischen Kenntnissen“ zufolge nicht möglich sei. Es bestätigt meine bisherigen Aussagen, dass in der Ideologie das eigene

\footnotetext{
163 Ich möchte hier auf das in vielen Interviews angesprochene idealisierte Bild von ,normaler` Zeugung als gefühlvollen Akt zweier Menschen, die sich lieben, verweisen. Die gesellschaftliche Realität zeigt jedoch, dass die Erzeugung menschlichen Lebens auch durch eine Vergewaltigung oder im Rahmen eines Geschlechtsaktes stattfinden kann, der mit Liebe und Gemeinsamkeit nichts zu tun haben muss.
} 
Kind als Ausdruck der intimen sozialen Beziehung eines Paares angesehen wird. ${ }^{164}$ Die Vorstellung von Zeugung hängt hier eng zusammen mit dem sinnlich erfahrbaren Leib sowie Emotionalität und Intimität, also mit individuellen Empfindungen und Erfahrungen, die sich an der Praxis bricht, in der gerade Intimität und Nähe von der Erzeugung eines Kindes getrennt werden. Die Befruchtung im Sinne der Zusammenführung von Ei- und Samenzelle und deren Verschmelzung berührt im Rahmen der Reproduktionsmedizin primär die Körperlichkeit der Paare. Die deutlich gewordene situative und kontextabhängige Betonung oder Vernachlässigung von Körper bzw. Leib bringt spezifische Probleme für die betroffenen Paare mit sich. Eine Paarbeziehung beinhaltet beide Dimensionen, sowohl Körperlichkeit als auch Leiblichkeit, und es zeigt sich, dass mit der symbolischen Konstruktion des Körpers als Biokörper und damit als Repräsentation von „echter“ Verwandtschaft die leiblichen Erfahrungen nicht erklärt werden können. Letztere müssen in den Behandlungen ausgeblendet werden, obwohl gerade die Schaffung eines leiblichen Kindes das erklärte Ziel darstellt.

Ich habe schon an anderer Stelle gezeigt (Hauser-Schäublin et al. 2001:109ff), dass die Trennung zwischen Befruchtung und Zeugung insbesondere im Falle einer nicht eingetretenen Schwangerschaft mit erheblichen Schwierigkeiten verbunden ist. In diesen Situationen wird offensichtlich, dass es sich nicht nur um Körperzellen und einen ausschließlich technischen Vorgang handelt, sondern wie sehr dieser mit dem eigenen Leib verknüpft ist. Die Sichtbarmachung der einzelnen Schritte des Fortpflanzungsvorganges kann zwar zur Abschaffung der Sinnlichkeit und zu einer distanzierten Betrachtungsweise führen (vgl. Schindele 1990:44), da dieses Objektivieren auch zu einer Entfernung vom Selbst führt. Es ist jedoch auch deutlich geworden, dass gerade dieser Akt der Visualisierung zu einer besonderen Bindung an die Körperzellen führen kann, wie Ute Wille (36) erzählte: „Jedes Mal dachte ich, toll, das sind deine Eizellen. Wie viele sind es? Toll! Je mehr desto besser. Wir kannten uns schon richtig gut". Mein Datenmaterial zeigt, dass die Zerlegung des Zeugungsprozesses in immer kleinere Sequenzen und die Visualisierung auch von nicht wahrnehmbaren Vorgängen im Körperinneren dazu führt, dass diese einzelnen Abschnitte zu leiblich erfahrbaren Ereignissen werden können. ${ }^{165}$

Siehe dazu auch Strathern (2001:372) über die Vorstellung von Sexualität zwischen Eltern als Basis der elterli-
chen Liebe und der Liebe innerhalb einer Familie in euro-amerikanischen Gesellschaften: „Sex gilt damit als Symbol
für die Natürlichkeit der Bande zwischen den Ehepartnern, als deren ebenso natürliches Ergebnis das Kind angesehen
wird. Zwar ist Geschlechtsverkehr nicht auf die Ehe beschränkt, aber das ist nicht der wesentliche Punkt. Es geht
darum, dass er innerhalb einer ehelichen Gemeinschaft eine wichtige symbolische Aufgabe erfüllt. Er steht einerseits
für die biologische Notwendigkeit und soll andererseits auch die Liebe der Eltern stimulieren, die auch die Liebe der
Eltern zu ihren (eigenen) Kindern ist.“
165 Insbesondere zu leiblich erfahrbaren Erlebnissen von Frauen, da per Ultraschall ausschließlich ein Blick in ihr
Körperinneres möglich wird. Siehe dazu auch Duden (1991), die bei der Kontroverse um die reproduktiven Techno-
logien die Frage aufgreift, ob es einen Authentizitätsverlust der Körpererfahrung gibt, wenn die haptische (= den
Tastsinn betreffende) durch eine technische Körpererfahrung ersetzt wird. Sie hat sich insbesondere mit Prozessen
der Sichtbarmachung vor allem von Schwangerschaften beschäftigt. Ihrer Auffassung nach wird nur das als Realität
anerkannt, was gesehen werden kann. Strathern (1992a:49ff) postuliert, dass die Visualisierung des Körperinneren 
Durch die Visualisierung kommt es zu einer Transformation des Körpers in den Leib, da eine starke Bindung an die Körperzellen entsteht; Genau diese leibliche Dimension des Körpers muss jedoch ansonsten in den Behandlungen ausgeblendet werden. Dieses leibliche Erfahren und Empfinden führt auch zu Gefühlen von Trauer und Verlust über das potentielle (leibliche) Baby.

„Man verlässt schon halbschwanger die Klinik. Wie ein rohes Ei. Das erste Mal war es bei mir sehr schlimm und dieses Mal habe ich mir gesagt, verflucht nochmal, wenn jemand dableiben will, soll er dableiben und wenn er gehen will, soll er gehen. Jetzt habe ich es anders gemacht. Psychisch geht es mir jetzt besser als beim ersten Mal. Da war ich ein Seelchen und habe gelitten, besonders an dem Tag in die Klinik zu gehen und zu hören, dass man doch nicht schwanger ist. Das war eine Katastrophe. Dieses Mal habe ich normal weitergearbeitet, habe normal mein Heu gemacht. Mal schauen, was rauskommt. Diese zwei Wochen sind mir leichter gefallen als das letzte Mal. Man kann sich selbst nicht beeinflussen und sich sagen, lebe normal weiter, in zwei Wochen weiß man ja Bescheid. Aber irgendwo im Hinterkopf ist es doch und je näher der Termin rückt, um so komischer wird es. Ich habe die Embryonen doch am Bildschirm gesehen." (Maria Heider, 26 Jahre)

Die Gratwanderung zwischen Körper und Leib wird immer wieder deutlich. Einerseits versuchen die Paare, möglichst wenig Gefühl zu investieren und sich klarzumachen, dass zwar neues Leben aus der Verschmelzung ihrer Körperzellen entstanden ist, es sich hierbei jedoch noch nicht um die (leiblichen) Kinder handelt, die sie später auch im Arm halten werden, sondern um Zellkombinationen, die im Wachstum begriffen sind. Andererseits scheint es oftmals kaum möglich zu sein, diese Gefühle auf Dauer zu unterdrücken oder erst gar nicht entstehen zu lassen. Besonders bei negativem Verlauf der fortpflanzungsmedizinischen Behandlung (keine Befruchtung der Eizellen, keine Einnistung in die Gebärmutter), die direkt am weiblichen Leib erfahren wird, wird diese Transformation besonders deutlich, da die erhoffte Leiblichkeit in Form eines eigenen Kindes nicht zustande gekommen ist. Wenn sich auch der Körper der Frau regeneriert und bald wieder in der Lage ist, einen neuen Behandlungsversuch zu beginnen (oft sogar schon im folgenden Zyklus), bleiben die Schmerzen am Leib jedoch fast immer sehr viel länger.

mit Hilfe von Ultraschall in der Pränatalmedizin dazu beitragen soll, eine ,echte` Beziehung zwischen Mutter und Fötus von Individuum zu Individuum entstehen zu lassen. Das Sichtbarmachen eines Bildes des Fötus ziele ihrer Ansicht nach darauf ab, eine Person darzustellen. Sie verweist dabei auf die englische Vorstellung von Person, d.h. eine Person agiert als Subjekt im Kontext von Beziehungen und ist darüber hinaus sichtbar, was bedeutet, man kann eine Person sehen, wenn diese als Individuum erscheint, und ein Individuum wird über den Körper konzipiert (1992a:50). Übertragen auf den von mir untersuchten Kontext lässt sich postulieren, dass hier das Herstellen einer ,Beziehung` zu den Eizellen und dem befruchteten Embryo stattfindet. 


\subsubsection{Die Gleichsetzung von Zeugung und Befruchtung}

Anhand von 25 Interviews lässt sich jedoch auch feststellen, dass eine Vorstellung von Zeugung dominiert, die sich auf die Verschmelzung von Ei- und Samenzelle bezieht und Zeugung und Befruchtung somit gleichsetzt. Inge Groß (38) ist der Auffassung, dass Zeugung und Befruchtung zwar verschiedene Begriffe sind, letztlich jedoch das gleiche Phänomen beschreiben:

„Zeugung ist das, was bei uns jetzt nicht mehr klappt, wo wir uns helfen lassen müssen. Das ist für mich die Zeugung, ja also die künstliche Befruchtung. Verschiedene Namen, aber letztlich geht es um das gleiche. Aber das hat nichts mit Sexuellem zu tun, das ist, wie wenn ein Mechanismus kaputt ist.“

Diejenigen, die Zeugung und Befruchtung nicht unterscheiden, sind der Ansicht, dass die Zeugung gleichermaßen im Körper der Frau oder im Reagenzglas stattfinden könne, was letztlich für das ,Ergebnis‘ keine große Rolle spiele. Aus diesem Grunde haben sie auch keine größeren Schwierigkeiten damit, medizinische Hilfe in Anspruch zu nehmen. ${ }^{166}$ Wie die Befruchtung letztendlich zustande komme und wo sie stattfinde, sei nicht entscheidend.

Ein wichtiger Aspekt scheint hier zu sein, dass die reproduktiven Techniken den Zeugungsort- und -zeitpunkt genauer fassbar machen können. Bei der In-vitro-Fertilisation (IVF) werden die Körperzellen im Reagenzglas zusammengeführt, und bei der Mikroinjektion (ICSI) wird die Samenzelle unter dem Mikroskop direkt in die Eizelle eingebracht. Danach folgt eine Wartezeit von 24 Stunden, nach der festgestellt werden kann, ob eine Verschmelzung der Zellen stattgefunden hat. Die Interviews zeigen, dass bei beiden Techniken der ,Zeugungszeitpunkt“ (Befruchtung) und ,Zeugungsort" (Labor) aufgrund der Gleichsetzung von Zeugung und Befruchtung für die Paare eindeutig zu sein scheint. Wie ich schon in Kapitel 4.3.1.2 beschrieben habe, besteht die Möglichkeit, Vorkern-Stadien einzufrieren (Kryokonservierung) und erst nach einem längeren Zeitraum aufzutauen, um sie in die Gebärmutter der Frau zu transferieren. In diesem Fall ,verlegen' viele Paare den Zeugungszeitpunkt und datieren ihn vor, so als ob das Verschmelzen der Zellen und ihre Teilung erst 48 Stunden zuvor stattgefunden hätte. ${ }^{167}$ So berichteten Juliana und Tim Bergmann (33 und 38 Jahre):

J.B.: „,Der Zeitpunkt der Zeugung ist, wenn die Eizelle in die Samenzelle eindringt. Wir haben damals gesagt, am 31. August haben wir unser Kind gemacht. Das war der Tag der Punktion, und da werden sie dann auch zusammengeführt im Reagenzglas."

T.B.: „Denk ich auch. Wobei bei uns, gerade wo wir jetzt ein Vierteljahr dazwischen haben [zwischen zwei Embryonentransfers], die Kühlschrankzeit mal abziehen würde.

\footnotetext{
166 Siehe dazu auch Kapitel 6.2.3.

167 Die Zeitspanne, nach der die Embryonen ohne Kryokonservierung transferiert würden (siehe Kap. 4.3.1.2).
} 
Im Grunde genommen wäre das also gestern gewesen. Die Befruchtung des Eies mit dem Samen. Die Gefrierzeit würde ich jetzt mal abziehen. Die kann man ja vernachlässigen, also spielt ja auch keine so wichtige Rolle.“

Die Hervorhebung der Bedeutung des Befruchtungszeitpunktes seitens der Betroffenen macht es somit ermöglicht, die Zeit der Kryokonservierung gedanklich von der Zeugung abzuziehen.

Bei der Methode der Insemination findet die Befruchtung im Körper statt, was ca. zwei bis drei Tage nach dem Einbringen des Spermas in die Gebärmutter sein kann, also nachdem die Frau/das Paar die Klinik verlassen hat und sich wieder zuhause befindet. Es kristallisiert sich aus den Interviews heraus, dass dem Moment der Insemination ${ }^{168}$ im homologen System häufig eine besondere Bedeutung zuteil wird. So sprach das Paar Christine und Karl Lenz (30 und 39 Jahre) von ihren widersprüchlichen Empfindungen:

C.L.: „Zeugung ist Befruchtung, also Verschmelzung von seinen und meinen Zellen. Das findet ja z.B. bei Inseminationen im Körper statt, vielleicht eine Stunde später. Da wird dann erst was gezeugt und nicht im Moment der Insemination. Ist doch beim Geschlechtsverkehr auch nicht so, dass in dem Moment die Befruchtung stattfindet, sondern das kann viele Stunden später sein. Aber (zögert) der Moment der Insemination war doch schon was besonderes, also da wird mir das Sperma von meinem Mann eingeführt. Das hat nun wirklich nicht weh getan. Aber wie soll ich das erklären, obwohl wir ja schon einiges mitmachen für unseren Kinderwunsch, also auch so technische Sachen und so. Mit denen man sich ja auch abgefunden hat. Na ja, da denkt man dann doch daran, dass so was sonst woanders passiert, also wenn man normal ein Kind zeugen kann.“

K.L.: „Also ich komme damit auch gut klar. Ist für uns prinzipiell kein Problem, das wir das jetzt hier so machen lassen. Aber Sie können sich das sicher nicht so vorstellen, dass man da so sitzt und sieht, dass da gerade mein Sperma inseminiert wird. Also irgendwie sitzt man so distanziert oder so als Betrachter dabei, während der Arzt da mit dem eigenen Sperma hantiert, so personslos irgendwie. Also, dem ist das ja egal, ob er da nun meins einführt oder von jemandem anders. Ich meine, die sind hier schon freundlich, das war vorher in der großen Klinik ganz anders, da war alles völlig steril und ich hatte quasi keinen Zugang. Aber ich bin eben doch nur der Statist am Rande. Wenn es dann hinterher klappt, wäre es mir aber sicher total egal, dann wäre es ja mein eigenes Kind, also dann kommt ja das Gleiche dabei raus wie normal auch.“

C.L.: „Es gibt viele Frauen, das weiß ich, die wollen ihre Männer gar nicht dabei haben, um sie aus dem Ganzen herauszuhalten. Ich bin aber schon ganz froh, wenn mein Mann dann noch da ist. Sonst wäre es so ganz getrennt von ihm, also eben nur Samen und Technik, verstehen Sie?““

Hier wird deutlich, dass die persönliche Definition von Zeugung sich auf die Befruchtung bezieht, die im Fall einer Insemination im Körper der Frau stattfinden kann, also

168 Die heterologe Insemination wird in Kapitel 6.3 noch ausführlicher diskutiert. 
räumlich und zeitlich getrennt von der Person des Arztes und der Klinik. ${ }^{169}$ Dennoch ist auch ersichtlich, dass eine wesentliche Schwierigkeit für beide Partner ${ }^{170}$ gerade darin besteht (und ähnliches wird auch in neun weiteren Interviews genannt) damit umzugehen, dass es im Moment der Insemination einzig um Körperzellen, nämlich das im Labor aufbereitete Sperma, geht, die losgelöst von der eigenen Leiblichkeit vom Arzt ${ }^{171}$ „vor Ort“ gebracht werden. Herr Lenz empfindet sich als Statist und nur am Rande beteiligt, da er persönlich nichts beitragen kann und nichts (er)spürt. Auch spricht er die Austauschbarkeit des Spermas an, indem er darauf hinweist, dass es für ihn zwar eine sehr intime und ,einzigartige‘ Substanz sei, die in die Gebärmutter seiner Frau eingeführt werde, es sich jedoch für den Arzt um nur eine von vielen in der täglichen Berufsroutine handele und damit entpersonalisiert werde. Diese Aussage verdeutlicht, dass zwar einerseits seitens der Paare versucht wird, Sperma wie Eizellen als Körpersubstanzen zu sehen, die auch losgelöst vom eigenen Leib verwendet werden können, um eine Befruchtung zu erreichen (letztlich zu ,zeugen'). Doch lässt sich auch feststellen, dass eben diese Vorstellung der Loslösbarkeit der Körperzellen nur schwer gelingt, insbesondere da das Ziel dieser Behandlung des Körpers die Schaffung von Leiblichkeit im Sinne eines leiblichen Kindes ist. Der Austausch von Körpersubstanzen zwischen Ehepartnern wird überdies ,normalerweise" mit einer sexuellen körperlichen und leiblichen Beziehung assoziiert, was an der Aussage von Frau Lenz deutlich wird, der die Anwesenheit ihres Mannes bei der Insemination wichtig ist.

Es lässt sich vermuten, dass auch hier die Vorstellung von Zeugung nicht ausschließlich auf körperliche Vorgänge reduziert und von der eigenen Leiblichkeit losgelöst werden kann, wie im Interview zunächst angesprochen. Der Zwiespalt des Paares wird deutlich, die zwar einerseits Zeugung als einen Vorgang beschreiben, der auch problemlos an einen anderen Ort bzw. in einen anderen Kontext verlagert werden kann, auf der anderen Seite allerdings den ,Weg zur Zeugung' thematisieren, auf dem die Transformation

\footnotetext{
169 Die raumzeitliche Organisation von reproduktionsmedizinischen Behandlungen kann ausführlicher in HauserSchäublin et al. (2001:70-73) nachgelesen werden.

170 Eine detailliertere Diskussion der geschlechtsspezifischen Wahrnehmungsunterschiede soll hier nicht erfolgen. Deutlich wird, dass beide Partner Schwierigkeiten haben. Ob die Frau eine andere Perspektive hat als ihr Mann und die Situation weniger ,fremd ' empfindet, da sie das Sperma ihres Mannes wie beim Geschlechtsverkehr eher ,passiv empfängt (wobei ich ausdrücklich darauf hinweise, dass ich hiermit nicht das passive sexuelle Verhalten meine, sondern mich ausschließlich auf die Samenübertragung beziehe im Sinne von, etwas in sich aufnehmen'), kann hier nicht beantwortet werden. Ebenso kann über die Möglichkeit, dass der Mann sich hier gerade deshalb so sehr als Statist empfindet, da er in diesem Fall passiv daneben sitzt, wohingegen er im Geschlechtsakt ,aktiv' handelt (im Sinne von ,etwas verteilen/weitergeben'), nur spekuliert werden.

171 Auf die Rolle des männlichen Arztes, der die Insemination durchführt und dadurch aktiv ,zeugend` tätig ist, während der Ehemann eher unbeteiligt am Rande sitzt, wird hier nicht näher eingegangen, da ein mögliches Insuffizienzgefühl gegenüber dem Arzt bei der homologen Insemination seitens der Interviewten nicht angesprochen wurde und somit nur Vermutungen angestellt werden könnten (siehe dazu auch Hauser-Schäublin et al. 2001:278ff). Feststellen kann ich jedoch, dass dieser Aspekt im Zusammenhang mit den von Ärztinnen durchgeführten Inseminationen nicht thematisiert wurde.
} 
von Leib zu Körper vollzogen werden muss. Diese Hypothese soll durch einen weiteren Interviewausschnitt gefestigt werden, in dem Marion Wieger (34) schilderte, warum ihr Mann bei den Inseminationen nicht dabei war. Trotz der Definition von Zeugung als einem „medizinischen Vorgang“ mittels medizinischer Eingriffe ist erkennbar, dass auch sie Klinik und Ärzte von Zuhause und Ehemann und damit von Sexualität und intimen Beziehungen trennen möchte. ${ }^{172}$

„Zeugung ist für mich ein rein medizinischer Vorgang. Mehr ist es nicht. Da befruchten sich Zellen, was zwar ein Wunder ist, aber dennoch ein rein medizinischer Vorgang schlussendlich. Und so sehe ich das auch. Habe da kein Problem mit. [...] Mein Mann geht nicht mit zu den Inseminationen. Ich finde es auch eigentlich gar nicht verkehrt. So belastet es ihn weniger und hat nicht so viele Auswirkungen auf unsere Beziehung. Also, na ja, auch was das Miteinanderschlafen angeht. Ich finde, man lässt da schon viele Illusionen. Ich will das gar nicht unbedingt, dass er da mitkommt zu den gynäkologischen Untersuchungen. Manchmal ist es so einfacher, gerade in Situationen, wo es so frustrierend ist oder wo man so mit sich selber hadert. Das braucht er ja nicht alles mitzukriegen. Er weiß ja, dass es belastend ist und dass es mich belastet und dass es uns belastet, aber er braucht ja nicht überall mit hin zu dackeln.“

Wie ich schon erwähnt habe, bezeichnen auch Paare, die die Methoden der IVF und ICSI nutzen, als Zeugung die Verschmelzung von Ei- und Samenzelle und legen diese somit auf einen bestimmten Moment fest. Dass diese im Labor stattfindet, während beide Partner sich an anderen Örtlichkeiten aufhalten, und somit aus der Intimbeziehung des Paares herausgenommen wird, wird zwar in zwei Interviews thematisiert, allerdings nicht als Problem empfunden. ${ }^{173}$ In vielen Gesprächen geht es hingegen um die Beteiligung der zahlreichen medizinischen Mitarbeiter, insbesondere um die Rolle des Arztes, „ohne den die Zeugung ja gar nicht erst zustande kommen könnte“. ${ }^{174}$ Da Zeugung hier jedoch als „biologische Zeugung“ definiert wird, die medizinische Unterstützung braucht, sehen die Paare den Arzt als jemanden ,der so eine Art Mechanik macht“, weil er das gelernt habe. Es fällt auf, dass hier der medizinische Diskurs übernommen wird, da diese Beschreibungen mit den Eigendefinitionen der Ärzte übereinstimmen ebenso wie die gleiche sprachliche Begrifflichkeit verwendet wird (siehe Kap. 6.1.2). Feststel-

\footnotetext{
172 Auf die konkreten Gründe der Abwesenheit von den Männern ist keine der Frauen direkt eingegangen. Erst auf mein Nachfragen wurden Überlegungen angestellt. Zumeist wurden jedoch zeitliche Probleme und Schwierigkeiten mit dem Arbeitgeber genannt, in keinem Fall persönliche Widerstände. Auffällig war jedoch, dass die genannten Probleme ebenso bzw. stärker die Frauen betreffen, die ja noch viel mehr Zeit in den Kliniken verbringen müssen. Diese Diskrepanz ist offensichtlich, scheint allerdings aus Gründen, die hier nicht näher untersucht worden sind, bei den Frauen eher als zwar problematischer aber dennoch notwendiger, Teil' der Behandlung akzeptiert zu werden.

173 Gedanken über den Aspekt, dass mit Hilfe der Technik Kinder ,gezeugt‘ werden können, obwohl sich das potentielle Elternpaar gerade nicht gut versteht (siehe dazu Kapitel 6.2.1), sind nicht angesprochen worden.

174 Diese Art von Aussagen tauchen in Interviews mit den Paaren, die Zeugung als Prozess definieren, nur selten auf. Da Zeugung als komplexer Vorgang, der sich über einen langen Zeitraum hinziehen kann, vorgestellt wird, und eng mit einer emotionalen Intimbeziehung des Paares verknüpft ist, finden sich dort eher Äußerungen darüber, dass es ohne eine starke emotionale Paarbindung nicht zu einer Zeugung kommen könne unabhängig vom Einsatz des Arztes oder der Ärzteschaft (siehe dazu Kapitel 6.2.1).
} 
len lässt sich, dass sich die Paare von dem sie betreuenden Arzt oder den Ärzten Freundlichkeit und Verständnis wünschen und froh sind, wenn eine ,gute Beziehung“ zwischen ihnen vorherrscht. Jedoch wurde auf meine Nachfrage deutlich, dass der Arzt zwar „so eine Art Mit-Erzeuger ist“, weil er alles dazu tut, damit eine Befruchtung zustande kommen könne, nähere Beziehungen während der Behandlungen jedoch nicht unbedingt erwartet würden. So äußerten sich auch Ilona und Christoph Ottmers (30 und 33 Jahre), die den zweiten ICSI-Versuch unternahmen:

I.S.: „Dr. Hagemann hat vorhin, als er die Embryonen in die Gebärmutter eingebracht hat, zu ihnen gesagt, ,Vater wird's schon richten“. Was haben Sie denn dabei gedacht?“،

C.O.: „Sicher ist er von keinem Kind der richtige Vater, aber der Macher ist er schon irgendwie. Allerdings finde ich, der eine repariert ein Auto, der nächste einen Arm, und er macht halt Kinder. Da ist eine Dienstleistung. Aber sonst bringt das keinen Bezug. Wenn ich meinem Kind das mal erzählen sollt, würde ich sagen, der da hinten hat ein bisschen nachgeholfen bei der Zeugung. Aber wen soll ich denn da überhaupt zuordnen, hier rennen doch so viele herum, und wir sind ja hier auch nicht gerade das einzige Paar mit einem Problem. An unserer Geschichte haben schon so viele herumgemengt, da wird es ein bisschen unübersichtlich. Aber die haben eben alle ihre Lebensberechtigung, ihr Einkommen.“

I.O.: „Natürlich ist er kein Vater, meint er wahrscheinlich so im übertragenen Sinne, weil er so viel an der ganzen Sache beteiligt war. Klingt ja auch irgendwie nett und persönlich, und wir hatten ja auch eine gute Beziehung im weitesten Sinne. Aber mehr verbindet uns ja nicht. Ich meine, ohne ihn hätte es keine Befruchtung gegeben, wäre eben kein Kind gezeugt worden, wobei man ja hier nicht den Biologen vergessen darf, der das ja schließlich alles im Labor macht. Man darf sich da nicht so reinhängen, muss das abgeklärter sehen.“

Das Paar ist hier übereinstimmend der Ansicht, dass sich die Ärzte kraft ihres Amtes um sie kümmern und freundlich sind, ansonsten eine bezahlte Dienstleistung vollbringen wie in anderen Berufssparten auch. Mein empirisches Material zeigt jedoch, dass es geschlechtsspezifische Unterschiede gibt, was die Erwartung an die Beziehung zu einem Arzt angeht. Frauen entwickeln oftmals engere Bindungen an den Arzt, da sie regelmäßige Termine zu Ultraschallkontrollen haben und somit mehr Zeit in der Klinik verbringen als ihre Männer (vgl. Kap. 4.3.1.1). Hinzu kann gerade bei einem männlichen Arzt das Gefühl von Vertrauen, Verständnis und intensiver Unterstützung bei der Erfüllung des „Kinderwunsches“ entstehen. Wie ich schon an anderer Stelle gezeigt habe (vgl. Hauser-Schäublin et al. 2001:280f), bestehen die Beziehungen seitens der Ärzte zum Paar, auch nach Geburt eines Kindes, in erster Linie im Namen ihres Berufes, der sich mit der ,Reparatur' von Körpern und nicht mit Leibern beschäftigt. Bei einer Trennung von Zeugung und Befruchtung und damit zwischen Intimität und sozialen Beziehungen versus der beruflichen Beschäftigung am und mit Körper ist es möglich, sowohl seitens der Ärzte (vgl. Kap. 6.1.1) als auch seitens der Paare, die Rolle des Mediziners auf einen Raum und eine Zeit festzulegen, die mit der Privatsphäre aller 
Beteiligten nichts zu tun haben muss. Mein Material zeigt, dass diese Trennung theoretisch seitens der Paare zwar vollzogen wird, dass es im konkreten Umgang jedoch immer wieder zu Brüchen kommen kann und es sich oftmals eher um ein Oszillieren zwischen beiden Aspekten handelt.

\subsection{3 „Natürlich“ versus ,künstlich“?}

In den Interviews über „Zeugung“ fallen auch die Begriffe „natürlich“ und „künstlich“, eine Dichotomie, mit der die Paare im Kontext der Reproduktionsmedizin immer wieder konfrontiert werden. Besonders im öffentlichen Diskurs findet sich diese Gegenüberstellung, und insbesondere diejenigen Paare müssen sich damit auseinandersetzen, die ihre soziale Umgebung über die reproduktionsmedizinische Behandlung, in die sie sich begeben haben, informieren. Die Reaktionen des Umfeldes auf diese Nachricht sind oft kritisch oder ablehnend bezüglich einer ,künstlich“ herbeigeführten Befruchtung. Sehr häufig wird daher die Behandlung verschwiegen, um sich nicht negative Bemerkungen anhören zu müssen, wie Tim Bergmann (38), im zweiten IVF-Versuch, berichtete:

„So richtig bewusst geworden, dass es sich um was Künstliches handelt, ist es uns erst, als wir das einigen Verwandten und Freunden erzählt haben. Ach du liebe Güte, da gab es Horrorvorstellungen von dem, was wir da jetzt machen lassen. Besonders die ältere Generation hat ja gar keine Ahnung. Die denken ja, das ist Teufelszeug, was da passiert. Aber selbst unsere ach so aufgeklärten Freunde meinten zum Teil nur: ,Ihr müsst es ja wissen, was ihr macht. Aber wenn ihr mich fragt, man sollte sich doch überlegen, ob das nicht von irgendwo her so gewollt ist oder dass es nicht sein soll. Da pfuscht man doch der Natur ins Handwerk.' Solche Sprüche haben wir uns da anhören müssen. Also ob wir uns nicht auch Gedanken darüber gemacht haben. Aber das ist doch Blödsinn, dass man die Natur künstlich zu etwas zwingt, wie behauptet wird. Wenn es nicht sein soll, dann wird es sich nicht befruchten oder einnisten, egal was man vorher versucht. Aber warum soll man es nicht erst mal versuchen, es ist doch nichts anderes, als was in der Natur auch passiert. So haben uns das die Ärzte auch erklärt und es stimmt ja auch. Aber wir sind jetzt schlauer und machen das nur für uns selbst. Mit Außenstehenden reden wir nicht mehr darüber.“

Die meisten Paare lehnen spontan die Unterteilung in „,natürliche“ versus „,künstliche“ Befruchtung ab mit ähnlichen Begründungen, wie sie Herr Bergmann im obigen Interviewausschnitt gibt. Alle finden übereinstimmend die spontane ,natürliche“ Befruchtung schöner und wären froh, wenn es auf „,natürlichem“ Wege klappen würde. Da dies jedoch bei ihnen nicht der Fall sei, würden sie dafür eben Hilfe in Anspruch nehmen. Sie betonen immer wieder, dass es doch gar nichts „Künstliches“ sei, da es sich um die Körperzellen beider Partner handele, die statt im Körper im Reagenzglas zusammenkämen. Wenn das Kind dann da sei, würde doch ,normal“" auch niemand fragen, wie es entstanden sei. Diese Aussagen und die verwendeten Begrifflichkeiten zeigen auf den ersten Blick eine Übereinstimmung mit der Perspektive der Ärzte, in dem auch hier bei 
der „natürlichen“ Befruchtung der Körper bzw. körperliche Vorgänge in den Mittelpunkt gerückt werden und der subjektiv erfahrbare Leib keine Erwähnung findet. Es wird jedoch deutlich, dass die Paare, trotz ihrer Definition von ,natürlich“, dazu tendieren, ihre reproduktionsmedizinischen Behandlung dem sozialen Umfeld aufgrund der negativen Erfahrungen durch Unverständnis oder Ablehnung zu verschweigen. So scheint durch das Verschweigen der Inanspruchnahme der Medizin versucht zu werden, Dissonanzen, die durch einen Bruch zwischen der Konstruktion von ,Natürlichkeit' im Zusammenhang mit Fortpflanzung in der Ideologie und der Konstruktion von ,Natürlichkeit' in der reproduktionsmedizinischen Praxis auftauchen, zu glätten.

Anhand der Aussagen lassen sich jedoch unterschiedliche persönliche Positionen herausarbeiten, die einerseits abhängig sind von der Art der medizinischen Indikation (ob zum Beispiel von Anfang keine andere Möglichkeit bestand eine Schwangerschaft herbeizuführen), welche Behandlungsmethode zur Zeit des Interviews durchgeführt wurde oder wie lange sich ein Paar schon in einer Therapie befand:

D.L.: „Was soll das Gerede darum, wir haben doch sowieso keine andere Chance. Natürlich nehmen wir jede Hilfe, die wir kriegen können. Bei uns geht es eben nur auf diesem Wege.“

K.L.: „Über so was kann man sich nur Gedanken machen, wenn man eine Wahl hat. Die haben wir doch gar nicht. Also gibt es für uns überhaupt keine Künstlichkeit in dem Sinne. Wir nehmen alle Möglichkeiten, die uns von der Medizin geboten werden." (Ehepaar Karin und David Lausitz, 28 und 32 Jahre, im dritten IVF/ICSI-Versuch)

Die Meinungen verändern sich oftmals in verschiedenen ,Stadien“ der Therapie. Was zunächst noch als ,künstlich“ beispielsweise im Sinne von äußeren medizinischtechnischen Eingriffen in den Fortpflanzungsprozess und außerkörperlichen Vorgängen bewertet und persönlich abgelehnt wird, wird im Verlauf der Behandlung und zunehmender Frustration immer ,normaler“. Besonders offensichtlich wird dies an den folgenden Interviewausschnitten mit Constanze Hebler (30), die im zeitlichen Abstand von einem Jahr aufgenommen wurden:

„Wir sind heute zum ersten Mal hier in der Praxis. Ich war zwar schon mal beim Frauenarzt und der meinte, man müsse mir wahrscheinlich Hormone geben. Jetzt sind wir hier und wollen das noch mal von anderer Seite hören, was man da machen kann, damit ich schwanger werde. Ich finde solche Hormonbehandlungen auch ganz natürlich. Das ist doch ähnlich wie mit der Antibabypille. Die nimmt man, um zu verhüten und jetzt nehme ich eben ein paar andere Hormone, um schwanger zu werden. Solange ich noch mit meinem Mann schlafe, ist das doch alles ganz natürlich. So etwas wie Inseminationen ist für mich ein künstlicher Eingriff von außen. Da braucht es ja keinen Geschlechtsverkehr mehr.“ (Januar 1997)

Nach einem Jahr hatte sich ihre Einschätzung jedoch sehr verändert, wie sie mir gegenüber zum Ausdruck brachte: 
C.H.: „Jetzt reden wir schon wieder miteinander. Schön sie mal wiederzusehen, aber schlecht, dass ich immer noch hier bin. Es hat bei uns eben immer noch nicht geklappt. Als wir mit Hormonen anfingen, da dachte ich, ist ja nur eine kleine Unterstützung, in dem Sinne eine Pille wie die Antibabypille, eben nur jetzt andere Hormone. Also Tabletten ist man ja gewohnt. Dann hat der Arzt gesagt, wir könnten es mal mit Inseminationen versuchen, aber es wird wahrscheinlich auf IVF hinauslaufen. Und jetzt habe ich gerade meinen ersten Versuch hinter mir und warte, ob was passiert, also ob ich schwanger bin. Meine Güte, bin ich froh, dass ich weitermachen kann und nicht aufhören muss. Früher habe ich das mal anders gesehen, da war ich der Meinung, das wäre alles zu unnatürlich, zu künstlich. Aber das ist ja Blödsinn. Es heißt zwar künstliche Befruchtung, aber da ist doch nichts Künstliches bei. Nachdem ich nun schon ein Jahr immer in der Klinik war und das alles auch schon gewohnt war, war das eigentlich dann nur ein kleiner Schritt.“

I.S.: „Sie hatten beim letzten Mal davon gesprochen, dass künstlich für sie wäre, wenn sie nicht mehr mit ihrem Mann schlafen müssten, um schwanger zu werden. Solange sie Geschlechtsverkehr hätten oder haben müssten, wäre es für sie ganz natürlich. Wie im Fall der Hormonbehandlungen, mit denen Sie begonnen hatten.“

C.H.: „Ja, stimmt, das habe ich damals wohl so gesagt. Aber Meinungen ändern sich. Man muss sich eben mit dem Ganzen auseinandersetzen. Man gewöhnt sich außerdem auch an vieles hier [in der Klinik]. Es ist auch schon so, dass mein Kinderwunsch sehr groß ist und im Laufe der Zeit eigentlich immer größer wird. Man wird ja nicht jünger und die Zeit vergeht, eine Behandlung folgt der nächsten. Wenn dann eben der nächste Schritt helfen könnte oder die Chancen auf ein Kind damit größer sind, dann gehen wir diesen Weg. Jetzt kann ich nicht mehr aufgeben, sonst mache ich mir ja ewig Vorwürfe. Außerdem schlafe ich ja heute auch noch mit meinem Mann. Schwanger kann ich davon aber nicht werden. Wie ich schon gesagt habe, das ist alles gar nicht künstlich, ist eben Hilfe von außen, aber alles andere läuft doch ganz normal ab. Das ist ja nicht zu vergleichen mit dieser anderen Methode, dieser ICSI, wo die Samenzellen gar keine Wahl haben. Die werden eingespritzt in die Eizelle, und die müssen zusammenkommen. Das ist für mich eine künstliche Methode. Ich meine, auf diese Weise [IVF] ist den Zellen ja noch die Möglichkeit gegeben, sich zu vereinigen oder nicht. Das ist zwar außerhalb von meinem Körper, weil die Eileiter nicht funktionieren, wie sie müssten. In meinem Fall wird der Weg jetzt umgangen. Aber es sind doch meine Eizellen und seine Samenzellen, aus denen ein Kind entstehen würde. Und das wäre dann ganz normal unseres, egal wie es zustande gekommen ist.“ (Januar 1998)

Insbesondere aus dem zweiten Zitat geht hervor, wie sich die Grenzen im Verlauf eines Jahres verschoben haben zwischen noch „,natürlichen“ und schon „künstlichen“ Behandlungsmethoden. Dies beruht zum einen auf einem gewissen Gewöhnungseffekt, der aufgrund des routinisierteren Umgangs mit den medizinischen Eingriffen in den Körper eingetreten ist, zum anderen auf dem immer größer werdenden „Kinderwunsch“ und der Kompensation kognitiver Dissonanzen. Zum Zeitpunkt des zweiten Interviews hat sich die Einstellung von Frau Hebler relativiert und sie empfindet die Eingriffe von außen in den Fortpflanzungsprozess nicht mehr als so einschneidend wie noch im Jahr zuvor zu Beginn der Therapie(n). Sie ist der Ansicht, dass ihr keine Wahl bleibe, wenn sie ihren „Kinderwunsch“ erfüllen möchte, der, wie oben deutlich erwähnt, immer größer wird, je 
mehr Zeit vergeht und je größer die Konzentration auf ein potentielles Kind wird; Je mehr ,Misserfolge' durchlebt worden sind, desto stärker die Hoffnung auf Erfolg mit einer anderen Behandlungsmethode (vgl. Franklin 1998). ${ }^{175}$ Der Aspekt der „Wahl“ wird in zahlreichen Interviews thematisiert, meist im Zusammenhang mit der Überzeugung, keine andere Wahl zu haben als die Reproduktionsmedizin, da es ,,auf normalem Wege“ nicht zu einer Schwangerschaft kommt. Alternativen wie beispielsweise Adoptionen fallen hier nicht unter die Vorstellung von Auswahl, was deutlich werden lässt, dass einzig die Reproduktionsmedizin als zuständig für die Erfüllung des „Kinderwunsches“" angesehen wird. Im Verlauf der zeit- und energieaufwendigen Therapien (Kap. 4.3.1.2) konzentriert sich das Leben der Frauen jedoch immer mehr auf die „Unfruchtbarkeit“ bzw. den „Kinderwunsch“ und die Therapie nimmt eine Eigendynamik an, die es ihnen schwer macht auszusteigen, selbst wenn sie sich vorher ein Zeitlimit gesetzt haben. Fast alle der interviewten Frauen, die sich längere Zeit in reproduktionsmedizinischer Behandlung befinden, erwähnen die Bereitschaft, sämtliche Methoden zu nutzen, um ein „eigenes“ Kind zu bekommen. Wenn es weitere Möglichkeiten - und damit noch „Hoffnungen“ - gebe (wie bei Frau Hebler von Inseminationen zu IVF), werde man diese auch in Anspruch nehmen: „Früher war das für mich ein Unterschied, jetzt nicht mehr. Das ist wie eine Krankheit, die man behandeln muss, man sucht nach immer neuen Wegen und will nicht aufgeben." Insbesondere die Frauen, deren Körper im Mittelpunkt der reproduktionsmedizinischen Behandlungen stehen, fühlen sich verpflichtet alles zu versuchen; Es könnte ja sein, dass sie vor dem ausschlaggebenden Versuch aufgeben (vgl. auch Kap. 5.1.4.2, Franklin 1997:169ff).

Anhand der Interviews zeigt sich, dass „künstlich“ seitens der befragten Paare im Sinne von externer Hilfe verstanden wird, die erforderlich ist, damit eine Befruchtung zustande kommen kann. Diese kann variieren, je nachdem ob ,nur' eine Insemination notwendig ist, bei der die Befruchtung noch im Körper stattfindet, oder IVF/ICSI, bei der sämtliche Vorgänge mit Ausnahme der Ei- und Spermienproduktion außerhalb des Körpers ablaufen (wobei im Falle der vermehrten Eizellgewinnung starke Hormongaben an die Frau unerlässlich sind). Mit „,natürlich“ werden hier alle körperlichen (biogenetischen) Vorgänge definiert, die ohnehin bei der Fortpflanzung ablaufen, was bedeutet, dass für die meisten Paare die reproduktionsmedizinische Behandlung zwar einen „externen“ Eingriff darstellt, es sich aber ansonsten um „eine Hilfe an der Natur“ handelt, da auch auf „natürlichem“ Wege Ei- und Samenzellen verschmelzen, damit ein Kind entstehen kann. „Natürlich“ wird demnach verstanden als etwas, was ohne medi-

\footnotetext{
175 Meine Beobachtungen haben gezeigt, dass diese ,Hoffnungen“ durch den Einfluss der Ärzteschaft aufrecht erhalten und immer wieder unterstützt werden. „Wir machen weiter“, „Das ist noch nicht das Ende, da gibt es ja noch andere Möglichkeiten“, ,Jetzt nur nicht aufgeben“ sind Äußerungen, die ich im Zusammenhang mit dem Überbringen von,schlechten` Behandlungsergebnissen an die Paare immer wieder gehört habe.
} 
zinische Eingriffe in anderen Kontexten als Teil des Fortpflanzungsprozesses auch abläuft.

Unter Rückbeziehung auf die eingangs vollzogene analytische Trennung von Körper und Leib kann hier jedoch auch festgestellt werden, dass die Vorstellung von „,natürlich“ in der Ideologie in direktem Zusammenhang steht mit der Vorstellung einer körperlichen und leiblichen Paarbeziehung (,Wenn es auf natürlichem Wege geht, ist es auf jeden Fall schöner, irgendwie gefühlvoller eben“), die sich, wie ich in Kap. 6.2.1 zeigen konnte, an der konkreten reproduktionsmedizinischen Praxis bricht. Die Trennung von Sexualität und Fortpflanzung ist jedoch schon länger möglich, und nicht erst seit der Anwendung empfängnisfördernder Reproduktionstechnologien Ende der 1970er Jahre. Wie im Interview mit Frau Hebler zu erkennen, zählen beispielsweise Hormongaben in Form von Tabletten nicht zu ,künstlichen“ Eingriffen mit dem Hinweis, daran sei man (als Frau) ja bereits durch die Antibabypille gewöhnt. Auch in diesem Fall werden dem weiblichen Körper über einen längeren Zeitraum Hormone zugeführt, um Sexualität unabhängig von einer möglichen Schwangerschaft zu erleben. Insofern nicht schon vorher die „Sterilität“ eines Partners bekannt ist, gehen beide Partner davon aus, zu einem späteren Zeitpunkt die Kontrazeption abzubrechen, ,normal' zu funktionieren und somit auf „natürlichem“ Wege mittels Geschlechtsverkehr Kinder zu zeugen. ${ }^{176}$ Diese Vorstellung hält sich solange, bis klar wird (und die Gründe sind hier sehr verschieden), dass es auf diese Weise nicht funktioniert und medizinische Hilfe für die Fortpflanzung in Anspruch genommen werden muss oder kann. Es werden nun Interventionen vorgenommen, die von der körperlichen und leiblichen Beziehung des Paares separiert werden und die die Trennung zwischen Körper als Gegenstand von Behandlungen und dem Leib als subjektivem Empfindungsorgan beinhalten. Nicht nur zur Verhütung einer Schwangerschaft, sondern auch zum Erreichen einer Schwangerschaft und damit zur Überwindung der ,ungewollten Kinderlosigkeit“ eines Paares wird primär der Körper der Frau behandelt. Dabei wird sowohl im medizinischen als auch im öffentlichen Diskurs, insbesondere in den Medien, gerade die ,reproduktive Wahlfreiheit“ der Frauen als Preis für ihre „Sterilität“ gehandelt: Frauen wollen zu spät Kinder, haben zu lange Verhütungsmittel genommen oder sogar eine Schwangerschaft abgebrochen. Gewollte und ungewollte Kinderlosigkeit werden dabei verknüpft. Frau Hebler scheint diese weit verbreitete Ansicht im obigen Interviewausschnitt internalisiert zu haben, in dem sie explizit darauf verweist, dass sie ja vielleicht selber schuld sei an der „ungewollten Kinderlosigkeit“ und dass die körperlichen Eingriffe, die jetzt notwendig

\footnotetext{
176 Die Frauen und Männer, die ich befragte, hatten in den seltensten Fällen an ihrer Fruchtbarkeit gezweifelt und ein Kind in ihre Vorstellung über ihr Leben fest eingeplant. Oft wurde die „Sterilität“ anderen Personen gegenüber verschwiegen, um nicht den Eindruck zu erwecken, man wäre , anormal' oder ,krank' (siehe dazu Hauser-Schäublin et al. 2001:138-143).
} 
seien, nur eine weitere ,Überlistung' ihres Körpers bedeuteten. Mit dieser Erklärung stellt sie ihren Körper als behandelbares und reparierbares Objekt in den Mittelpunkt, was sich mit den Aussagen der Ärzte deckt, wie ich sie in Kap. 6.1 dargelegt habe. Sie schildert sich selbst als aktive Mitgestalterin der Situation, d.h. so wie sie sich früher für die Verhütung entschieden und dafür die Antibabypille eingenommen hat, so hat sie jetzt den Entschluss für die Inanspruchnahme der Reproduktionstechnologien gefasst und ist bereit, mit den Ärzten in jeder Hinsicht zusammen zu arbeiten. Diese Einwilligung, auch compliance genannt, ist ein wesentlicher Aspekt in der reproduktionsmedizinischen Praxis. Der häufige Kontakt mit dem Arzt oder der Klinik und das selbständige Spritzen von Hormonen zuhause (siehe Kap. 4.3.1.2) scheint das Gefühl einer aktiven Beteiligung an der Überwindung der ,ungewollten Kinderlosigkeit“ zu unterstützen. Den Paaren, speziell den Frauen, wird in der konkreten reproduktionsmedizinischen Behandlung auch vermittelt, dass sie die Situation mitgestalten und ohne ihren persönlichen Einsatz durch die tägliche Selbstmedikation von Hormonspritzen die ,natürlichen" Vorgänge gar nicht ablaufen könnten. Da die Frauen im Fall der Diagnose „Sterilität“" vor allem darunter leiden, die Kontrolle über ihren Körper verloren zu haben und sich in ihrer Leiblichkeit beeinträchtigt fühlen (Hölzle 1987:24, Hauser-Schäublin et al. 2001:138ff), erscheint es zunächst als Widerspruch, wenn sie sich aus diesem Grund in die Verantwortung von Ärzten begeben. Sie wollen den Wunsch nach (Wieder-)Herstellung der Kontrolle über ihren Körper realisieren, indem sie die Ärzte als Sachverständige für den Körper und die ,nicht-funktionierenden' Körperteile anerkennen, wobei das eigene Leibempfinden eine zu vernachlässigende Größe darstellt. Die Ärzte vermitteln jedoch u.a. durch eine anteilige Kompetenzübertragung den Anschein, dass sie den Frauen die Kontrolle über ihren Körper durch eine Schwangerschaft zurückgeben können. Die ärztliche Aktivität konzentriert sich dabei allerdings auf technisch vermittelte oder medikamentöse Eingriffe am Körper und vernachlässigt die leibliche Dimension des Frauenkörpers, die in den meisten Fällen primär von der „Unfruchtbarkeit" betroffen ist.

An dem bisher Dargelegten wird deutlich, dass in der Konstruktion von „Natürlichkeit“ in der reproduktionsmedizinischen Praxis die ,natürlichen Tatsachen“ immer weiter auf körperliche (biogenetische) Prozesse reduziert werden. So definieren die befragten Ärzte (zumindest kraft ihres Amtes) sowie die Frauen/Paare die reproduktionsmedizinischen Maßnahmen nicht als „,künstlich“, sondern als Hilfe und Unterstützung der „natürlichen" Vorgänge und beziehen sich damit auf das Zusammenkommen und Verschmelzen von Ei- und Samenzellen. Franklin hat in ihren Arbeiten für den euroamerikanischen Kontext gezeigt, dass unter den Bedingungen der Reproduktionstechnologien in den 1990er Jahren nicht mehr Befruchtung und Empfängnis als synonym und gleichzeitige Vorgänge verstanden werden, sondern dass die neuen Technologien $\mathrm{zu}$ einer zeitlichen Aufteilung und Neubestimmung der Begriffe als physische Ver- 
schmelzung von Ei- und Samenzelle einerseits und als genetische Verschmelzung andererseits geführt haben (vgl. Franklin 1993b:534, 1995:331). Die medizinische Intervention schafft immer neue visuelle Eindrücke und erweitert das naturwissenschaftliche Verständnis über die ablaufenden Zeugungsprozesse, wodurch das biogenetische Zeugungsmodell immer differenzierter wird und ihrer Ansicht nach zu Veränderungen des gesellschaftlichen common sense über die „,natürlichen Tatsachen“ des Lebens und damit über die Zeugung menschlichen Lebens (vgl. Franklin 1992) führt. ${ }^{177}$ Franklin legt dar, dass durch die Konzentration auf die genetischen Prozesse, die während der Zeugung ablaufen, diese als grundlegende „,natürliche Fakten“ anerkannt werden und damit ins Zentrum der Zeugungsvorstellungen rücken. Franklins Analysen lassen sich auch auf meine Untersuchung in Deutschland übertragen. So hat sich gezeigt, dass unter den Bedingungen der Reproduktionstechnologien sowohl von professioneller Seite als auch von Seiten der ,ungewollt kinderlosen“ Paare zwar nicht eindeutig zwischen Befruchtung und Konzeption unterschieden wird, die biogenetischen Prozesse der Zeugung (hier als „Befruchtung“ bezeichnet) jedoch in der konkreten reproduktionsmedizinischen Praxis eindeutig in den Mittelpunkt rücken und immer mehr zum tatsächlichen ,Zeugungszeitpunkt und -akt‘ werden und damit losgelöst werden von direkten sozialen Interaktionen. Sind die Diskurse über Zeugung und Leiblichkeit für die Ärzte Teil ihres Privatlebens, konzentrieren sie sich im Berufsleben auf Diskurse über Befruchtung und Körper, was sie in die Lage versetzt zwischen beiden Bereichen zu trennen. Die betroffenen Paare können diese Trennung nicht vollziehen, da ihre eigene Lebenswelt und die eigene Leiblichkeit aufgrund der „ungewollten Kinderlosigkeit“ direkt betroffen sind und deren Überwindung durch die Schaffung eines Kindes mit Hilfe medizinischtechnischer Behandlungen des Körpers angestrebt wird. Dadurch ergeben sich Widersprüche und Brüche zwischen Ideologie und Praxis für die Paare, und es ist deutlich geworden, dass dabei ein ständiges ,Pendeln` zwischen objektiviertem Körper und subjektiv erfahrenem Leib je nach Kontext und Situation stattfindet.

Die neue Gewichtung im biogenetischen Zeugungsmodell hat auch zu einem wachsendem Bewusstsein über Risiken im Zusammenhang mit den ,natürlich“ ablaufenden genetischen Prozessen geführt. Mit der Betrachtung von der Zeugung menschlichen Lebens an sich als „obstacle course“ (Franklin 1995:331, 1997:105ff) aufgrund der Unberechenbarkeit der ,Natur' können damit die „natürliche“ und die „künstliche“ aneinander angeglichen werden. Schwierigkeiten und Misserfolge der Reproduktionstechnologien werden denen der ,Natur' gleichgesetzt, da beide Bereiche durch hohes Versagen charakterisiert sind. Somit legitimieren sich die reproduktiven Technologien aufgrund

\footnotetext{
177 Siehe dazu auch Franklin (1997:10): „This does not mean reproduction is no longer seen as ,natural': to the contrary, new forms of reproductive technology are ubiquitously re-naturalised. [...] The instrumentalisation of conception $[\ldots]$ indexes the changing meanings of 'the facts of life' in such a context."
} 
der Defizite der ,Natur“ („Der Natur auf die Sprünge helfen“) und binden sich selbst als notwendigen Faktor in den reproduktiven Prozess ein: „The importance of this shift is in its legitimation and naturalization (indeed legitimation through naturalization) of assistance to the reproductive process.“ (Franklin 1995:334; Hervorhebung im Original)

Im folgenden Kapitel werde ich die heterologe Insemination ins Zentrum meiner Betrachtungen stellen. Diese Methode wird in einigen Interviews als „künstlich“ bezeichnet im Unterschied zur homologen Insemination mit der Begründung, dass es sich dabei ja nicht mehr um die Körpersubstanzen der Partner handele, und somit nicht mehr „um eine Hilfe an der Natur", sondern dass hier fremdes Sperma verwendet werde, das auf „natürlichem“ Wege nie in die Gebärmutter der Frau gelangen würde. ${ }^{178}$ Bei diesen Interviews handelt es sich allerdings um Personen, für die diese Methode aufgrund ihrer persönlichen medizinischen Indikation nicht infrage kommt oder um solche, die sie trotz männlicher „Sterilität“ ablehnen. Die Paare, die sich für die heterologe Insemination entschieden haben, bezeichnen sie selbst nicht als „künstlich“, sondern heben gerade die „Natürlichkeit“" der Befruchtungsvorgänge hervor, die im Körper der Frau und nicht im Reagenzglas stattfinden könne. Die Tatsache, dass es sich bei der „natürlich“ stattfindenden Befruchtung und Verschmelzung nicht um die Körpersubstanzen beider Ehepartner handelt, wird in diesem Zusammenhang nicht erwähnt. Auf diesen Aspekt werde ich im Folgenden näher eingehen.

\subsection{Fallbeispiel: Zeugung mit ,Spendersperma“6}

Mit der heterologen Insemination kann mit Hilfe eines anonymen „Samenspenders“ ein „eigenes“ Kind eines Ehepaares geschaffen werden, wobei sich nur die Beziehung zwischen Mutter und Kind über Körper (und Leib) konstituiert. Der Ehemann der Mutter übernimmt die soziale Vaterschaft, und der biologische ,Zeugungsanteil‘ des „Spenders" wird sozial ausgeblendet. Diese Behandlungsmethode kann dann in Anspruch genommen werden, wenn mit den Samenzellen des Ehemannes die Befruchtung der Eizelle nicht möglich ist. Die Frau ist jedoch zumeist körperlich „,fruchtbar“ und könnte demnach mit dem Sperma eines anderen Mannes schwanger werden, sei es durch Geschlechtsverkehr oder Insemination.

Anhand meiner Darstellungen verschiedener Perspektiven zur Definition und zum Umgang mit Zeugung in den letzten Kapiteln lässt sich feststellen, dass im Kontext der Reproduktionsmedizin entweder eine Trennung vollzogen wird zwischen Zeugung im Sin-

\footnotetext{
178 An diesen Aussagen wird meiner Ansicht nach sehr deutlich, dass der „,natürliche“ Weg idealerweise eine körperliche und leibliche Komponente der ,Zeugungsparteien' beinhalten sollte, auch wenn kontext- und positionsspezifisch die „,natürlichen“ Tatsachen immer weiter auf körperliche (biogenetische) Prozesse reduziert werden.
} 
ne von intimen sozialen Beziehungen und Befruchtung als körperlichem Teil der Fortpflanzung, oder dass Zeugung als biologische Zeugung definiert und der Befruchtung gleichgesetzt wird. Die Befruchtung und das Verschmelzen von Ei- und Samenzellen kann entweder im Körper der Frau nach Einbringen der Spermien des Ehemannes durch den Arzt (Insemination) oder im Labor durch Zusammenführen der Gameten im Reagenzglas (IVF/ICSI) stattfinden, d.h. es kommt zu direkten und indirekten Körperbeziehungen durch den Austausch der Zeugungssubstanzen sowohl zwischen den ,Zeugungsparteien' als auch zwischen diesen und dem Arzt, wobei nur die Paarbeziehung sowohl eine körperliche als auch eine leibliche Dimension beinhaltet (Kap. 6.2). Im Fall der heterologen Insemination kommt ein weiterer Aspekt hinzu, nämlich die Verwendung von „Spendersperma“, um die Befruchtung der Eizellen einer Frau zu erreichen; Eine dritte (unbekannte) Person kommt somit ins Spiel. Aufgrund der „Spende“ von Samenzellen ist sie direkt am Zeugungsprozess beteiligt bzw. macht eine Befruchtung und somit eine Schwangerschaft überhaupt erst möglich. Damit besteht zwar eine körperliche Verbindung zwischen dem „Samenspender“ und der „Empfängerin“ ebenso wie zwischen dem „Spender“ und dem Kind, die jedoch losgelöst von Leib und Leiblichkeit sozial unsichtbar gemacht wird.

In Kapitel 4.3.1.3 habe ich mich als Teil des rechtlichen Verwandtschaftsdiskurses in Deutschland mit den zivilrechtlichen Konsequenzen der heterologen Insemination in Bezug auf die Zuordnung, Rechte und Pflichten der beteiligten Personen beschäftigt. Im Folgenden möchte ich den konkreten sozialen Umgang mit der fremden Körpersubstanz untersuchen, die zur Zeugung des ,eigenen“ Kindes eines Paares notwendig ist. In meiner Arbeit ist deutlich geworden, dass im Zentrum der deutschen Ideologie von ,natürlicher" Verwandtschaft die Idee biogenetischer Verwandtschaft steht. Zeugung gilt dabei als Grundlage für „,natürliche“ und soziale Verwandtschaft. Den männlichen und weiblichen Zeugungsbeiträgen, Sperma und Ei, werden dabei grundlegende Bedeutungen beigemessen, weil sich „natürliche“ Verwandtschaft über Körper und Körpersubstanzen konstituiert. Im Zusammenhang mit dem Konzept eines „eigenen“ Kindes im Kontext der Reproduktionsmedizin (Kap. 5.1.4) wurde deutlich, welche Vorstellungen mit der Weitergabe von Körpersubstanz verknüpft sind, was sich insbesondere auf physische und psychische Merkmale bezieht, und welche leiblichen Eigenschaften dem „eigenen“ Kind zugesprochen werden. Ich möchte hier nun der Frage nachgehen, inwieweit diese kulturell dominanten Vorstellungen das soziale Handeln in Bezug auf die Zeugung mit Hilfe der heterologen Insemination beeinflussen, da hier die Körpersubstanz einer dritten Person losgelöst von sozialen Beziehungen beteiligt ist, und deren ,Verwendung' zum Zweck der Erzeugung eines Kindes keine Folgen hat für jene Beziehungen, die sich aus der Fortpflanzung ergeben. Welche Rolle spielt in diesem Zusammenhang der materielle Körper bei der Konstituierung von Verwandtschaft, und wie 
wird mit Elementen, die die ,traditionellen“ Vorstellungen der Zeugung von „eigenen“ Kindern stören oder diesen widersprechen, umgegangen?

Im rechtlichen Diskurs über Verwandtschaft ist deutlich geworden, dass es bei den homologen Inseminationen sowie bei den Methoden der IVF und ICSI eine eindeutige personenstandsrechtliche Zuordnung des entstandenen Kindes gibt. Per Gesetz (siehe Kap. 4.1.3) ist festgelegt, dass die im Rahmen einer Ehe geborenen Kinder dem Ehemann der Mutter zugeordnet werden und somit staatlicherseits davon ausgegangen wird, dass es sich um die Übereinstimmung von biologischer und sozialer Vaterschaft handelt (ungeachtet der „tatsächlichen“ biologischen Abstammung). Im Fall der heterologen Insemination, bei der es sich bei dem biologischen Vater um einen „Samenspender“ handelt, wird vor Durchführung der Behandlung durch einen Vertrag zwischen der Klinik und dem Ehemann festgelegt, dass dieser die Vaterschaft anerkennt und damit auch unterhaltsrechtliche Verpflichtungen eingeht. Er unterschreibt, dass er den Akt der Insemination als seinen eigenen anerkennt, d.h., dass er der ,Erzeuger' ist, was auch beinhaltet, dass er verneint, dass jemand anders sexuellen Kontakt zu seiner Frau hatte, der zur Schwangerschaft geführt haben könnte. ${ }^{179}$ Es wird somit zum einen per Kontrakt geregelt, dass es keine sexuelle Beziehung zwischen der Ehefrau und dem „Spender“ gegeben hat und gleichzeitig schriftlich bestätigt, dass eine Sexualbeziehung zwischen den Ehepartnern existiert, durch die es theoretisch auch zur Zeugung hätte kommen können. ${ }^{180}$ Im folgenden Interview mit Dr. Norbert Krüger wird jedoch deutlich, dass trotz aller schriftlichen Fixierungen jeder Mann bei berechtigten Zweifeln innerhalb von zwei Jahren das Recht hat, die Vaterschaft anzufechten (vgl. Kap. 4.1.3). Aufgrund der vorherigen Einverständniserklärung zu der Behandlung wäre diese Anfechtung vermutlich nicht ganz einfach, jedoch prinzipiell zulässig. Bei erfolgreicher Vaterschaftsanfechtung könnte es dazu kommen, dass die Ärzte gezwungen wären, die persönlichen Daten des biologischen Vaters aufzudecken, damit dieser seinen unterhaltsrechtlichen Verpflichtungen nachkommt. ${ }^{181}$

\footnotetext{
179 Diese Aussagen beruhen sowohl auf einem Vertrag, der mir persönlich aus einer Klinik vorliegt, als auch auf persönlichen Gesprächen mit verschiedenen Ärzten während meiner Feldforschung.

${ }^{180}$ Nach Darstellung einiger Ärzte besteht bei den anderen Methoden der ,assistierten“ Reproduktion im homologen System zumindest trotz allem theoretisch die Möglichkeit, dass es aufgrund einer (angenommenen) sexuellen Beziehung zwischen den Ehepartnern zur Zeugung auch ohne Hilfe von außen kommen könnte. Das unterscheidet diese von der heterologen Insemination, bei der ohne die Unterstützung eines Arztes eine Befruchtung der Eizelle nicht möglich wäre. Der Arzt Rolf Wellmann äußerte mir gegenüber in einem persönlichen Gespräch, dass es trotz eindeutiger medizinischer Indikation der männlichen „Sterilität“ immer wieder vorkäme, dass Ehemänner bei einer eingetretenen Schwangerschaft nach heterologer Insemination daran glaubten, dass sie vielleicht doch die biologischen Väter seien, da sie ja weiterhin mit ihrer Ehefrau Geschlechtsverkehr hatten: „Und die denken dann einfach manchmal, sie sind doch die biologischen Väter und nicht der Samenspender. Wenn auch medizinisch alles dagegen spricht, aber sie denken, es könnte ja dennoch einmal geklappt haben.“ Dieser Aspekt soll hier zwar erwähnt werden, kann jedoch mit dem empirischen Material nicht weiter belegt und diskutiert werden.

181 Im gegenwärtigen Recht ist der „Spender“ Vater i.S.v. § 1592 Br. 3 BGB und daher feststellbar (§ 1600 BGB). Ist der „Samenspender“ als Vater des fremdgezeugten Kindes gerichtlich festgestellt worden, stellt sich die Frage nach
} 
I.S.: „Sie haben vorhin gesagt, juristisch gesehen sind Sie der Vater, weil Sie praktisch der Frau beiwohnen. Allerdings ist es doch rechtlich gesehen so, dass das Kind, das im Rahmen einer Ehe zur Welt kommt, automatisch dem Ehemann zugeordnet wird, dass also der Ehemann zunächst grundsätzlich als der Vater gilt.“

N.K.: „Das ist korrekt. Das trifft zu, solange biologische und soziale Elternschaft nicht getrennt sind. Bei einer heterologen Behandlung, wo auf einen Samenspender zurückgegriffen wird, haben Sie ja eine Trennung von biologischer und sozialer Elternschaft. Wir führen deshalb die Behandlung nur bei Paaren durch, die verheiratet sind, und durch den vorherigen Abschluss des Notarvertrages verpflichtet sich das Paar ja auch, oder der Vater, hier die Elternschaft anzuerkennen. Bei heterologen Behandlungen lassen wir die Paare vorher einen Notarvertrag abschließen. Der Mann erklärt dadurch gegenüber seiner Frau, dass er den Akt der Zeugung, d.h. die Samenübertragung, automatisch als seine Handlung ansieht, dass er auch voll dahinter steht. Und das begründet dann auch eine unterhaltsrechtliche Verpflichtung gegenüber der Ehefrau und dem Kind. Allerdings kann hier die Elternschaft innerhalb von zwei Jahren nach der Geburt eines Kindes angefochten werden, wenn gewichtige Gründe vorliegen. Bei jemandem, der keine Samenzellen im Ejakulat hat, können gewichtige Gründe vorliegen.“

I.S.: „Aber der soziale Vater unterschreibt vor der Behandlung, dass er dieses Kind als seines anerkennt mit allen Konsequenzen. Wäre das nicht ein Problem, wenn er anfangen würde, die Vaterschaft anzufechten?“

N.K.: „Grundsätzlich verzichtet er auf der einen Seite in diesem Notariatsvertrag die Vaterschaft anzufechten, auf der anderen Seite hat er nach BGB zwei Jahre nach Geburt des Kindes das Recht dazu. Wenn die zwei Jahre um sind, hat er das nicht mehr. Jeder Mann in Deutschland hat innerhalb von zwei Jahren nach Geburt eines Kindes die Möglichkeit, die Vaterschaft anzufechten.“

I.S.: „Ob das nun vorher notariell festgehalten wurde oder nicht?“

N.K.: „Theoretisch ja. Er würde dann sicher aufgrund des Passus in dem Notariatsvertrag Probleme haben damit durchzukommen. Aber das Grundrecht können sie nicht durch einen Vertrag aufheben. Grundsätzlich ist der Arzt verpflichtet, die Herkunft der Samenzellen zu dokumentieren. Und da sie dann in den Krankenunterlagen sind, sind sie verpflichtet, diese zehn Jahre lang aufzuheben. Diese Daten unterliegen erst mal der ärztlichen Schweigepflicht. Es wäre denkbar, wenn man mal wieder auf die rechtliche Schiene geht, dass ein Jugendamt per Gerichtsverfahren die biologische Vaterschaft abklären lassen könnte. Dann könnte ein Richter einen Arzt von der Schweigepflicht entbinden. Es heißt also, dass jeder, der einem Samenspender in Deutschland absolute Anonymität zusichert, lügt. Weil er das nicht kann.“

Wie ich schon an anderer Stelle gezeigt habe, wird der Ehemann der Frau solange legal als Vater anerkannt und die „tatsächliche“ Abstammung durch den deutschen Staat nicht in Frage gestellt, solange sich das Ehepaar einig ist (Kap. 4.1.3). Auch seitens der Ärzteschaft und insbesondere seitens der interviewten Paare wird der „Samenspender“ in sozialer Hinsicht vollständig ausgeblendet und tritt nicht in Erscheinung. Einerseits,

der Unterhaltsverpflichtung. Wenn der Ehemann der heterologen Insemination zugestimmt hat, ergibt sich für ihn als sozialen Vater die Pflicht, für das Kind wie ein ehelicher Vater zu sorgen. Ist der soziale Vater jedoch mittellos, kann der „Samenspender“ für den Unterhalt des Kindes herangezogen werden (vgl. Hauser-Schäublin et al. 2001:273). 
indem die Zeugung durch die Vermittlung des Arztes ohne Geschlechtsverkehr zustande kommt, was bedeutet, dass zwar eine körperliche, nicht aber eine direkte leibliche Beziehung zwischen „Spender“ und Empfängerin bestand. Andererseits, indem der „Spender" anonym bleibt und der Ehemann mit dem Transfer des fremden Spermas einverstanden ist. Der Mann erklärt vor der Durchführung schriftlich gegenüber seiner Frau und den Ärzten, dass er die Samenübertragung (,den Akt der ,Zeugung““), als seine Handlung ansieht und das Kind dadurch als sein „eigenes“ anerkennt. Durch das Verschweigen der Erzeugung des Kindes sowohl dem sozialen Umfeld als auch in allen mir bekannten Fällen dem Kind gegenüber, definiert sich das Paar mit seinen Nachkommen als „eigene“ Familie (vgl. Kap. 5.1.4.1) und wahrt somit den Schein einer ,normalen“ reproduktiven Kleinfamilie. ${ }^{182}$ Wird die persönliche Anerkennung vom sozialen Umfeld bestätigt, ist die Konstruktion eines „eigenen“ leiblichen Kindes geglückt. Als leiblich gelten in der Konstruktion von Verwandtschaft in Deutschland somit auch Kinder, deren Mutter die leibliche, der Vater jedoch ,nur' der soziale ist - jedenfalls solange, bis das volljährige Kind bei Kenntnis der gespaltenen Vaterschaft Auskunft über den biologischen Vater verlangen sollte.

Die Kontrolle der Zeugung durch Ärzte ohne sexuelle Beziehungen der ,Zeugungsparteien' versucht darüber hinaus die sozialen Probleme, die sich daraus ergeben könnten, $\mathrm{zu}$ vermeiden. Eine Liebesbeziehung einer verheirateten Frau mit einem Liebhaber, aus der ein Kind entsteht, erfährt sozial eine grundlegend andere Bewertung als die Befruchtung der Eizellen einer Frau mit „Spendersamen“ in einer Klinik. Beide Wege können zu einer Schwangerschaft führen, jedoch gilt ersterer als Ehebruch, zweiterer als „assistierte“ Reproduktion, obwohl in beiden Fällen ein ,fremdgezeugtes' Kind entsteht. Dazu äußerte sich explizit Lea Jäger (36), die auch noch einen weiteren Aspekt in Bezug auf das „Spendersperma“ erwähnt: Den durch die Bezahlung entstehenden Warencharakter.

„Ich bin da ganz realistisch. Die Vorteile von einer Befruchtung mit Spendersperma gegenüber einer Affäre liegen doch ganz klar darin, dass es sich dabei um ein Geschäft handelt. Ich bezahle für eine Ware, das hat nichts mit Gefühlen zu tun. Ich freue mich über das, was ich gekauft habe, weil es mir ganz wichtig ist, aber es ist doch egal, von wem ich es gekauft habe. Ich mache mir da keine Gedanken über den Spender. Er soll nicht ganz blöd sein, nicht unbedingt ein Krimineller und meinem Mann ähnlich sehen. Wenn ich mir vorstelle, ich würde mir jetzt einen Geliebten oder einen One-night-stand suchen für ein Kind und würde dann schwanger. Das könnte ich einfach nicht. Zum einen kann ich nicht einfach mit jedem X-beliebigen Deppen ins Bett steigen, er müsste mir also auf jeden Fall sympathisch sein. Und vielleicht müsste ich dann doch immer wieder an ihn denken, wenn ich dann schwanger wäre. Ob das Kind ihm wohl ähnlich

\footnotetext{
182 So konstatiert auch Strathern (2001:373): „Euro-Amerikaner/innen sind also der Auffassung, dass die Fortpflanzung im Rahmen einer Beziehung stattfinden sollte, und dass unter normalen Umständen diese Beziehung eine sowohl eheliche wie sexuelle ist: Der soziale Partner ist auch ein biologischer Partner.“
} 
sehen würde usw. Ich kann mir ehrlich gesagt auch nicht vorstellen, dass das eine Partnerschaft überlebt, obwohl ich weiß, dass es eine Menge Kinder gibt, von denen die Männer glauben, dass es ihre eigenen sind und es stimmt gar nicht. Da konnten die Frauen das ja auch verdrängen bzw. mit einer Lüge leben. Aber ich kann das nicht und mein Mann würde natürlich durchdrehen, wenn ich mit einem anderen Sex hätte. Und heimlich käme ja wieder nicht in Frage. Also geht es nur so, und für uns beide ist es so am besten. Es hat mit unserer Beziehung, also auch der sexuellen, eben nichts zu tun. Ich denke in solchen Momenten überhaupt nicht an den anderen Mann, der interessiert mich doch im Grunde gar nicht. Es geht einzig und allein darum, dass sich Zellen befruchten, damit wir zu unserem Kind kommen. Und dabei helfen die [die Ärzte] uns hier eben. So sehe ich das! Klar klingt das jetzt alles ziemlich pragmatisch, aber es geht doch nur darum, dass sich was befruchtet. Wenn die Samen bei mir eingeführt werden, dann ist das zwar irgendwie der Zeugungsakt, aber eben nur ganz formell.“

Sowohl aus diesen Aussagen von Frau Jäger als auch anhand des Interviews mit Dr. Norbert Krüger, in dem er auf den schriftlichen Vertrag zwischen der Klinik und dem Ehemann hinweist, in dem dieser die Insemination als seinen ,Zeugungsakt anerkennt, lässt sich vermuten, dass Zeugung im Fall der heterologen Insemination als Moment der Samenübertragung gilt. Ich habe schon in Kapitel 6.2.2 dargelegt, dass trotz der Definition von Zeugung als Verschmelzung von Ei- und Samenzelle und der damit zusammenhängenden Konzentration auf den körperlichen Vorgang der Befruchtung, der Moment der (homologen) Insemination von Christine und Karl Lenz als ein besonderer Augenblick bezeichnet wird. Es ist auch deutlich geworden, dass im Kontext der medizinischen Behandlung sowohl die Ärzteschaft aber auch die Paare bemüht sind, Sperma wie Eizellen als vom Körper und vom Leib losgelöste Entitäten zu sehen, die dadurch auch veräußerbar werden. Für erstere ist diese Loslösung Teil des Berufes und erfolgt kraft ihres Amtes, letzteren gelingt die Trennung der Körperzellen von der eigenen Leiblichkeit oftmals jedoch nur schwer, da ein Austausch von Körpersubstanzen mit dem Ziel ein Kind zu zeugen mit einer aktiven sexuellen körperlichen und leiblichen Beziehung assoziiert wird. Im Fall der heterologen Insemination muss dieser Zusammenhang jedoch völlig ausgeblendet werden. Die Konzentration auf Körperzellen, die von der Person des „Spenders“ und somit von dessen Leiblichkeit getrennt werden können, lässt sich im Interview mit Frau Jäger (s.o.) erkennen. Dadurch eröffnet sich die Möglichkeit, das Fremdsperma als Ware anzusehen, die veräußert werden kann. ${ }^{183}$ Diese Transaktion wird sowohl seitens der Paare als auch der Ärzte, so wie mir in persönlichen Gesprächen wiederholt mitgeteilt wurde, als „Geschäft“ angesehen, das nach der Bezahlung als abgeschlossen gilt. So hat beispielsweise für Frau Jäger diese Transaktion nichts mit persönlichen Gefühlen und intimen sexuellen Beziehungen zu tun wie die

\footnotetext{
183 Die zu analytischen Zwecken vorgenommene Trennung von Körper und Leib lässt auch deutlich werden, dass das medizinisch geprägte Körperkonzept zur Auffassung des Körpers als gesellschaftlich verwertbare Ressource führen kann (siehe dazu auch Hauser-Schäublin et al. 2001:288ff). Besonders deutlich wird dies auch in den rezenten Diskussionen zur Stammzelltherapie mit embryonalen Stammzellen.
} 
Vorstellung eines Ehebruchs. Strathern hat darauf hingewiesen, dass der Samen an sich nicht die soziale Identität des „Spenders““,verrät‘ (1997:300), so dass die Befruchtung der Eizellen nicht notwendigerweise zu einer Beziehung zwischen den Personen führen muss, von denen die Substanzen stammen. Auch mein empirisches Material zeigt, dass die befragten Paare keinerlei Verpflichtung gegenüber dem „Spender“ empfinden, da sie sein Sperma käuflich erworben haben. Durch die finanzielle Abgeltung wird das Sperma zu einer Ware, die nach der Transaktion den Besitzer wechselt und nicht zu sozialen Beziehungen zwischen Geber und Nehmer jenseits der Transaktion beiträgt. ${ }^{184}$ Es wird deutlich, dass die heterologe Insemination ein Verfahren darstellt, das es gerade erlaubt Beziehungen zu umgehen. Personen müssen nicht mehr miteinander agieren, um Samen zu „spenden“ oder diesen zu empfangen oder besser gesagt, ihre Interaktionen müssen keine Folgen für jene Beziehungen haben, die sich aus der Fortpflanzung ergeben.

Obwohl ich darlegen konnte, dass im Zusammenhang mit der heterologen Insemination Zeugung auch seitens der Paare überwiegend auf das Zusammenführen von Körperzellen beschränkt wird, möchte ich hier jedoch darauf verweisen, dass diese Reduzierung auf körperliche Vorgänge an verschiedenen Punkten zu brechen scheint bzw. nicht durchgängig durchzuhalten ist. So waren in vielen Fällen (40 von 62 teilnehmend beobachteten heterologen Inseminationen) die Ehemänner bei der Übertragung des Fremdspermas nicht anwesend. Müssen sie bei der homologen entweder das Zuhause gewonnene Sperma in der Klinik abgeben oder es selbst vor Ort gewinnen, wird für die heterologe Insemination kryokonserviertes „Spendersperma“ verwendet. Befragt nach dem Grund der Abwesenheit des Ehemannes nannten die meisten Frauen berufliche Gründe. Eine andere Erklärung wurde nicht abgegeben, auch nicht in längeren Interviews, bei denen der Ehemann selbst zu Wort kam. Die behandelnden Ärzte waren jedoch der Ansicht, dass viele Männer den Inseminationen bewusst wegblieben, da sie aufgrund der diagnostizierten „Zeugungsunfähigkeit“ Schuldgefühle empfänden. Das Gefühl der Insuffizienz sei bei der persönlichen Anwesenheit besonders groß, da sie nicht nur mit dem Fremdsperma direkter konfrontiert seien, sondern auch damit, dass eine dritte Person diese Übertragung vornähme (insbesondere wenn es sich dabei um einen männlichen Arzt handele). Nach Meinung der Ärzte werde die Zeugung hier einzig den Frauen überlassen, und die Männer würden sich erst wieder daran beteiligen, wenn ihre Frauen schwanger seien (siehe auch Kap. 6.2.2).

\footnotetext{
184 Im Unterschied zu Tauschhandlungen, die in der Ethnologie als „Gabentausch“ bezeichnet werden. Die Annahme einer Gabe erfordert auch das Geben einer Gegengabe (Mauss (1990 [1968]), wodurch ein Kontinuum von wechselseitigen Beziehungen entsteht, bei denen ein Geber immer wieder auch selbst zum Nehmer wird (es kann sich um die gleiche oder um eine andersartige Gabe handeln).
} 
Die Betrachtung dieser Aussagen im Zusammenhang mit meinen bisherigen Analysen in diesem Kapitel bringt mich auf dem Hintergrund meines theoretischen Ansatzes zu der Hypothese, dass den ,zeugungsunfähigen“ Ehemännern im Fall der heterologen Insemination die Loslösung der Körpersubstanzen von der Leiblichkeit des „Spenders“ nicht oder nur sehr schwer glückt. Ebenso wenig scheint das Ausblenden der Vorstellung von intimen sexuellen Beziehungen zwischen zwei Personen im Zusammenhang mit dem Austausch von Körpersubstanzen zum Zweck der Fortpflanzung zu gelingen. Meiner Ansicht nach liegt auch ein Grund für diese Schwierigkeit in der Vorstellung, dass der Moment der Insemination als „Zeugungsakt“ bezeichnet wird, der zwar einerseits vom Ehemann schriftlich als sein eigener anerkannt wird, wobei ihm aber im konkreten Moment der Übertragung von Fremdsperma, das in einer Spritze sichtbar ist, deutlich wird, dass er hier definitiv nicht derjenige ist, der das Kind ,zeugt‘. Es scheint, dass den betroffenen Frauen die Trennung der Zeugungssubstanzen von der Leiblichkeit des „Spenders“ besser gelingt, wie im Interview mit Frau Jäger zu lesen ist, und was auch durch weitere Interviewbeispiele bestätigt werden kann. ${ }^{185}$

Gesamthaft betrachtet wird deutlich, dass die Paare versuchen, dem Idealbild einer ,normalen‘ Kleinfamilie in Deutschland zu entsprechen und andere in dem Glauben zu lassen, dass es sich um ein leibliches Kind beider Elternteile handelt (vgl. Kap. 5.1.4). Alle Befragten teilten mir übereinstimmend mit, dass ihr Kind niemals erfahren solle, dass es mit Fremdsperma gezeugt wurde. ${ }^{186}$ Thomas Berger (35) machte seine Ansicht darüber explizit deutlich:

„Unsere Gesellschaft ist eben noch nicht so weit, dass man das sagen könnte. Das wird doch total tabuisiert. Was in den Köpfen von anderen Leuten da vor sich geht, möchte ich lieber nicht wissen. Und dann wird unser Kind vielleicht noch blöd angeguckt oder besonders unter die Lupe genommen, oder sogar von anderen gehänselt. Nein, solange die Leute damit nicht umgehen können, wird es unser Kind niemals erfahren. Ist doch gut, dass seine Mutter mit ihm blutsverwandt ist. Da ist man sich über die ein Seite doch ganz sicher. Aber wir sind seine Eltern, und das sollen auch alle glauben. Ich habe für mich selbst immer gesagt, und das sehen wir auch als Paar so, dass die soziale Elternschaft doch viel wichtiger ist als die biologische. Was ja viel wichtiger ist als alles andere ist ja, dass man als Vater für das Kind da ist, so oft es geht, ihm was mitgibt für das Leben und für es sorgt. Das zählt doch am meisten!“‘

\footnotetext{
185 Trotz meiner Interpretationen in Bezug auf die von mir beobachtete auffällige Abwesenheit der Ehemänner bei der heterologen im Vergleich zu der homologen Insemination, möchte ich betonen, dass ich meine Interviews mit Paaren führte, die schon ein Kind geboren hatten und über einige Jahre mit diesem zusammen lebten. In diesen Gesprächen wurde der „Spender“ von beiden Gesprächspartnern nicht näher thematisiert, ebenso wenig die Art der Erzeugung. Die Paare hoben besonders hervor, wie glücklich sie über ihr „eigenes“ Kind seien und stellten die positiven Entwicklungen des Kindes heraus. Besonders hervorgehoben wurde die soziale Verbindung zwischen dem Kind und dem erziehenden Vater, und der „Spender“ spielte den Aussagen zufolge keine Rolle mehr, wenn das Kind erst einmal da war.

186 Siehe dazu auch Haimes (1990:154-173), die sich mit den Interessen und Motivationen der Geheimhaltung von den an der Erzeugung eines Kindes mittels „Spendersperma“ beteiligten Personen („Spender“, Paar, Kind) beschäftigt.
} 
Es lässt sich feststellen, dass die Schaffung von leiblichen Nachkommen - zumindest über die Mutter - und die sich aufgrund der Ehe ergebende staatliche Anerkennung einer Kernfamilie die auslösende Motivation für die Anwendung und Nutzung der heterologen Insemination ist (vgl. Kap. 5.1.4.2). Dennoch ist in den von mir geführten Interviews das Herunterspielen gerade einer genetischen Verbindung und die besondere Betonung der sozialen Elternschaft nach der Geburt eines Kindes durch alle Beteiligten auffällig. Die scheinbar geringe Bedeutung, die Herr Berger im obigen Interviewausschnitt der genetischen Verwandtschaft beimisst, kontrastiert mit der hohen Geheimhaltungsstufe, wie sie im Zusammenhang mit der heterologen Insemination seitens der Paare (wie auch seitens der Ärzteschaft) praktiziert wird. Hier findet sich ein Bruch zwischen der präsentierten ,abgeklärten' Distanz zur Bedeutung der genetischen Verwandtschaft und ihrer Akzeptanz im Rahmen des Umgangs mit dem sozialen Umfeld. Diese Art und Weise des Umganges scheint es den Paaren jedoch zu ermöglichen, die heterologe Insemination für sich persönlich in Einklang zu bringen mit den kulturell dominanten Werten über die Beziehung zwischen Eheleuten, über Elternschaft und Familie in Deutschland, wie ich sie bisher in meiner Untersuchung herausgearbeitet habe, womit sie der Anerkennung der Konstituierung von Verwandtschaft durch genetische Abstammung und gleichzeitig dem Wertkonzept der Kernfamilie verhaftet bleiben. Gerade im Fall der heterologen Insemination wird dadurch offenbar, dass die Maßnahmen der Reproduktionstechnologien darauf ausgerichtet sind, und in der konkreten Praxis mit allen Mitteln versucht wird, „Blutsverwandte“ zu schaffen, jedoch nicht im biogenetischen Sinn, sondern kulturell konstruierte Blutsverwandte (vgl. Hauser-Schäublin 1998:65). Die Aspekte, die die traditionellen Vorstellungen der Zeugung von „eigenen“ Kindern stören oder diesen widersprechen würden, werden weitgehend ausgeblendet. 


\title{
$7 \quad$ „Verwandtschaft“" und Reproduktionstech- nologien in Deutschland aus ethnologischer Perspektive
}

\begin{abstract}
„Insofar as kinship is thought of as combining social and natural domains, and is thus the place of overlap between them, the recognition of one component without the other always gives the people pause. What is new is the assistance being given to each domain. The natural facts of procreation are being assisted by technological and medical advances. The social facts of kin recognition and relatedness are being assisted by legislation. Kinship is doubly assisted. There is a further outcome in such assistance, for it takes away the very concept that made kinship itself a distinctive domain. There is little now to be taken for granted.“ (Strathern 1992b:20)
\end{abstract}

In dem Schlusskapitel meiner Arbeit möchte ich noch einmal die wesentlichen Ergebnisse der einzelnen Kapitel zusammenfassen und abschließend interpretieren. Schon in der Einleitung habe ich darauf hingewiesen, dass meine Studie ein situations- und kontextspezifisches Bild von Verwandtschaft in Deutschland bietet. Die Darstellung der Konstruktionsprozesse von Verwandtschaft in verschiedenen Diskursen unter den Bedingungen der Reproduktionstechnologien bietet zwar ein effektives Bild, das jedoch nicht ohne den gesamtgesellschaftlichen Kontext betrachtet werden kann. Auf diesen Aspekt werde ich im Verlauf dieses Kapitels Bezug nehmen.

\subsection{Die soziale Konstruktion von Blutsverwandtschaft und ihre spezifischen Konsequenzen in verschiedenen Kontexten vor dem Hintergrund der reproduktiven Technologien}

\section{Der rechtliche Diskurs}

Die Betrachtung des rechtlichen Diskurses von Zugehörigkeit und Angehörigkeit in Deutschland (Kap. 4) hat gezeigt, dass nach § 1589 des Bürgerlichen Gesetzbuches (BGB) als „Verwandtschaft“ einzig Abstammungsbeziehungen sowie Beziehungen, die durch eine Adoption gegründet werden, definiert werden. Somit konstituiert sich Verwandtschaft primär über die Fortpflanzung, und im Zentrum von Vorstellungen von „natürlicher“ Verwandtschaft steht die Idee biogenetischer Verwandtschaft. Daher „hat“ 
man in Deutschland eine bestimmte Verwandtschaft durch die Geburt. Diese ,existiert unabhängig vom individuellen Wollen und Wünschen“ (Rosenbaum 1998:29). „Natürliche" Verwandtschaft und die daraus resultierenden Beziehungen werden, im Unterschied zu ,nur' sozialer Verwandtschaft (z.B. durch Heirat oder Adoption), als unauflöslich verstanden. Obwohl Abstammungsbeziehungen im deutschen Recht als eindeutig festgelegt und damit unauflösbar gelten, ist im Fall von Adoptionen eine personenstandsrechtliche Neuzuordnung von Personen zulässig. Im Fall von adoptierten Minderjährigen bedeutet dies, dass $\mathrm{zu}$ der bisherigen Verwandtschaft das rechtliche Verwandtschaftsverhältnis erlischt. Abstammungsbeziehungen können darüber hinaus durch die Eheschließung zweier Personen erweitert werden, da Ego durch die Heirat mit den Verwandten des Ehepartners verschwägert wird. Eheliche Beziehungen werden durch einen freiwilligen Willensentscheid beider Ehepartner eingegangen und können unter bestimmten Gründen durch ein gerichtliches Urteil wieder aufgelöst werden. Zum Status des Ehepartners äußert sich das Bürgerliche Gesetzbuch (BGB) nicht, jedoch kommt im Strafgesetzbuch (StGB) die Kategorie ,Angehörige‘ zum Tragen, die sowohl Abstammungs- als auch Affinalbeziehungen ebenso wie soziale Beziehungen durch Pflegschaften beinhaltet (Kap. 4.1). Die Ehescheidung betrifft allerdings in rechtlicher Hinsicht nur die Verbindung zwischen den beiden Ehepartnern (die somit füreinander nicht mehr als Angehörige im Sinne des StGB gelten), nicht aber die durch die Eheschließung entstandenen Schwägerschaften; Sie bleiben laut BGB weiterhin bestehen.

Es ist deutlich geworden, dass die im BGB definierte Kategorie von Verwandtschaft mit bestimmten normativen Handlungsanweisungen bezüglich Unterhalts- und Erbschaftsangelegenheiten ausgestattet ist. Auch für die Institution der Ehe gelten bestimmte rechtliche Wirkungen, die primär den gegenseitigen Schutz und die Versorgung der Ehepartner garantieren sollen (Kap. 4.2). Aufgrund des Angehörigenstatus von Personen existieren in strafrechtlicher Hinsicht gewisse Begünstigungen und Beschränkungen wie beispielsweise Eheverbote zwischen Verwandten der geraden Linie sowie Inzestverbote zwischen direkten leiblichen Verwandten in der Aszendenz und Deszendenz.

Den Rechtsbeziehungen zwischen Eltern und Kindern wird in Deutschland eine zentrale Bedeutung beigemessen. Das Elternrecht ist im Grundgesetz (Art. 6 II, III GG) geschützt und wird als ein im Interesse des Kindes pflichtgebundenes Recht verstanden. Anhand des Kindschaftsreformgesetzes von 1998 (Kap. 4.1.3) ist zu erkennen, dass „natürliche Abstammung“ den zentralen Aspekt darstellt, der primär die personen- und vermögensrechtliche Zuordnung zwischen Eltern und Kind(ern) bestimmt. Damit wird deutlich, dass die Fortpflanzung und damit die geteilte Körpersubstanz als Symbol für die ,Natürlichkeit‘ der Bande zwischen Eltern und Kindern angesehen wird. Anhand der Analyse des rechtlichen Diskurses konnte ich jedoch auch zeigen, dass ,natürliche“ Zuordnungen kontext- und motivationsspezifisch ,verhandelbar' und modifizierbar sein 
können und Abstammung eine weitgehende „Rechtsregel“ (vgl. Lüderitz 1999:244ff) darstellt, über die sich durch Rechtsgeschäfte (Anerkennung) oder ihr Unterlassen (Anfechtung) hinweggesetzt werden kann. Die Statusbeziehung eines Kindes zur Mutter ist in Deutschland eindeutig geregelt, in dem Schwangerschaft und Geburt als die Kriterien für Mutterschaft festgelegt sind, wohingegen die Kriterien für die Zuordnung zu einem Vater heterogener sind. Damit definiert das werdende Kind die Mutter, da der Entwicklung des Kindes im Leib der schwangeren Frau eine besondere Aufmerksamkeit zuteil wird. Biologische Vaterschaft ist zwar mit der Möglichkeit verbunden, sie mit sozialer Vaterschaft zu verknüpfen, doch besteht diesbezüglich keine eindeutige Regelung. Ebenso muss keine Verknüpfung zwischen der biologischen und legalen Anerkennung der Vaterschaft bestehen. Die rechtliche Vaterschaftsanerkennung ist zum einen an die Institution der Ehe gebunden und impliziert somit eine sexuelle Beziehung zur Mutter, zum anderen richtet sie sich im Fall der Geburt eines Kindes außerhalb einer Ehe nach den Angaben der Kindsmutter. In den Fällen, in denen eine eindeutige Statusbestimmung des Kindes und somit Zuordnung zu einer bestimmten Verwandtschaft möglich ist, ist für den deutschen Staat die Frage nach der ,tatsächlichen“ Abstammung nicht von primärer Relevanz. Hieraus lässt sich ableiten, dass im deutschen Rechtssystem die genetische nicht mit der personenstandsrechtlichen Zugehörigkeit übereinstimmen muss und somit eine Einheit von biologischer und sozialer Elternschaft, insbesondere Vaterschaft, staatlicherseits für die formalrechtliche Zuordnung von Personen zu Verwandtschaft und damit deren legale Bestätigung nicht maßgeblich ist. Die Anerkennung der Vaterschaft eines (Ehe-)Mannes kann als eine willentliche Entscheidung bezeichnet werden, deren Motivation unterschiedlicher Natur sein kann, die jedoch den Status des Mannes sowie des Kindes definiert. Diese Möglichkeit des ,Aushandelns“ sozialer Vaterschaft nimmt Bezug auf das „Kindeswohl“, das u.a. eine Kontinuität von sozialen Beziehungen (vgl. Kap. 4.3.2) beinhalten soll. Die Feststellungen von Strathern (2001), dass zwar in der euro-amerikanischen Vorstellung die genetischen Entwicklungen als Angelegenheit eines biologischen Prozesses betrachtet würden, der sich nach der Vereinigung der elterlichen Gameten automatisch entfalte, dass in diesem Verwandtschaftssystem jedoch ständig an Beziehungen ,gearbeitet“" werden müsse, lassen sich auch auf Deutschland übertragen. So wird auch hier ein Kind nicht axiomatisch in ein Gefüge von Beziehungen hineingeboren, sondern es tritt, obwohl zu seiner Zeugung eine Beziehung notwendig war, selbst als eigenständige Person mit einem ,Bedürfnis“ nach Beziehungen auf. Daraus folgt nach Strathern, dass das Kind, das ohne Zweifel mit einem genetischen Vater geboren wird (wer immer das auch ist, es gab ihn einmal), immer noch einen sozialen Vater benötigt (2001:391), was auch im bundesdeutschen rechtlichen Diskurs über Elternschaft und Verwandtschaft deutlich geworden ist. So hat nach deutschem Recht ein Mann keinen Anspruch darauf, die (biologische) Vaterschaft einzuklagen, solange diese entweder vom Ehemann der Mutter anerkannt bzw. nicht 
angefochten oder von einem anderen Mann mit Zustimmung der Mutter anerkannt wurde. Auch hier zeigt sich, dass es für den deutschen Staat letztlich nicht ausschlaggebend ist, wie eine rechtlich als Kernfamilie definierte und anerkannte Einheit gegründet wird. Relevant sind eindeutige legale Zuordnungen und damit Zugehörigkeiten und Angehörigkeiten von verwandten Personen, was bestimmte Rechte und Pflichten mit sich bringt. Im rechtlichen Diskurs von Verwandtschaft in Deutschland zeigen sich somit zwei dominante Konzepte: Einerseits das Primat von Blutsverwandtschaft, andererseits das Primat der strukturellen Integrität der Kernfamilie. Im Idealfall sind beide Prinzipien miteinander verwoben in der Familie bestehend aus Vater, Mutter und leiblichen Kindern. In den vom Ideal abweichenden Fällen bietet die Gesetzgebung die Möglichkeit einer rechtlichen ,Nachregulierung', so dass kulturell etablierte Vorstellungen von der Integrität der Kernfamilie - in ideologischer und struktureller Hinsicht die Einheit aus Eltern und Kind(ern) - mit Hilfe der Gesetzgebung verstärkt werden, auch wenn in genetischer Hinsicht das Bild der ,normalen` Familie nicht zutrifft.

Die ,Abweichung ${ }^{6}$ vom Ideal und die Spannung zwischen den eindeutigen, ausschließlichen und als dauerhaft konzipierten Vorstellungen von biologischer Verwandtschaft und den sozialen ,Dissonanzen“ zeigt sich in zahlreichen Familienformen der gesellschaftlichen Realität wie beispielsweise Adoptivfamilien, Pflegefamilien, Stiefelternschaften oder neu zusammengesetzte Familien, in denen Kinder mehrere soziale Elternteile haben. So werden sie in der Regel sprachlich gekennzeichnet, was die Abweichung oder die ,Ersatzform‘ der Familienbildung verdeutlicht (vgl. Hoffmann-Riem 1989). Insbesondere Stiefelternschaften sind in der gegenwärtigen sozialen Realität, in der ein Auseinanderbrechen von Paarbeziehungen und Neuzusammensetzungen von Familien, die nicht über die biologische Reproduktion entstanden sind, weit verbreitet ist, ein häufig vorkommendes Phänomen. In diesem Kontext gerät jedoch die ,Aushandelbarkeit' von sozialer Verwandtschaft in Spannung mit den beiden oben aufgezeigten Polen des rechtlichen Diskurses. So handelt es sich im Fall der Stieffamilien zwar in struktureller Hinsicht um eine Familie, deren Gründung staatlich legitimiert ist, die jedoch keine legale Anerkennung der Vater- oder Mutterschaft des Stiefelternteils beinhaltet. ${ }^{187}$ Die rechtlich anerkannte Mutter- bzw. Vaterschaft verändert sich im Fall einer Wiederheirat nach Scheidungen in der Regel nicht. Der jeweilige Stiefelternteil hat auch bei gemeinsamer Residenz und geteiltem Alltag - keine Entscheidungsbefugnis und Elternrechte bezüglich des Kindes des Ehepartners. Auch wenn der geschiedene Elternteil des Kindes sein Umgangsrecht nicht wahrnimmt und kein Kontakt zwischen ihm und dem Kind besteht, bleibt der legal anerkannte verwandtschaftliche Status des

\footnotetext{
187 Eine Ausnahme bildet die Adoption des Stiefkindes durch den Stiefelternteil, da in diesem Fall sämtliche Rechte und Pflichten des biologischen Elternteils erlöschen.
} 
Kindes unverändert. Da die gesellschaftliche Realität zeigt, dass immer weniger Kinder ihre gesamte Kindheit mit ihren leiblichen Eltern verbringen, sondern zum Teil sogar ein mehrmaliger Wechsel der Familienzusammensetzungen erfolgt, scheint für den deutschen Staat die eindeutige Zuordnung einer Person in ein verwandtschaftliches Beziehungssystem zumindest formal unerlässlich, da, wie ich in Kapitel 4.2. gezeigt habe, ein verwandtschaftlicher Status mit bestimmten Rechtsfolgen verbunden ist. Im Fall von Stiefelternschaften kann somit zwar die soziale Verwandtschaft im Sinne eines persönlichen Zusammengehörigkeitsgefühls seitens des Stiefelternteils sowie des Kindes ,ausgehandelt' werden, was jedoch keinen Einfluss auf die gesetzliche und legal anerkannte Form von Zugehörigkeit und Angehörigkeit hat (siehe auch Le Gall/Martin 1990).

Auch im Fall der Diskrepanz zwischen genetischer Herkunft und legal anerkannter Verwandtschaft wird deutlich, in welches Spannungsverhältnis die ,Aushandelbarkeit gerät. Unabhängig vom rechtlichen Verwandtschaftsstatus einer Person wird jedem Menschen als Teil des Persönlichkeitsrechts des Grundgesetzes (Art 2 Abs. 1 GG) das Recht zuerkannt, Kenntnis über seine genetische Herkunft zu erlangen. Es hat sich gezeigt, dass die deutschen Gesetze die besondere Bedeutung der Aszendenz für die Entwicklung und Persönlichkeitsfindung des Kindes hervorheben. Dem kulturell dominanten biogenetischen Zeugungsmodell zufolge (Kap. 2.2.2) wird durch die biologische Reproduktion dem Kind seine genetische Identität über beide Elternteile verliehen, egal ob es seine Eltern kennt oder nicht (vgl. Franklin 1991:197; Strathern 1992a:24, 1993a:14). Durch die Anerkennung von Verwandtschaft als primär über den Körper konstituiert, räumt der Staat einem Kind somit unabhängig vom rechtlich anerkannten Verwandtschaftsstatus die Möglichkeit ein, seine biologische Verwandtschaft zu bestimmen. Im Fall von Adoptionen, bei denen die Abstammungsbeziehungen rechtlich nicht mehr relevant sind und weder seitens der biologischen Eltern noch seitens des Kindes eingeklagt oder angefochten werden können, liegt es an den Adoptiveltern, ob sie dem Kind die ,Wahrheit‘ über seine genetische Herkunft erzählen. Hat das Kind Kenntnis davon, dass es andere biologische Eltern hat, steht es ihm rechtlich zu, alle Möglichkeiten zu nutzen, um über diese Informationen einzuholen und zu diesen Kontakt aufzunehmen.

Ein weiterer Kontext, in dem die Spannungsverhältnisse zwischen eindeutiger biologischer Verwandtschaft und ,verhandelbarer' sozialer Verwandtschaft im Zusammenhang mit dem Ideal der Kernfamilie neue Fragen aufwerfen, ist die staatlich legitimierte Möglichkeit der ,assistierten“ Familienbildung mit Hilfe der Reproduktionstechnologien. In Kapitel vier hat sich gezeigt, dass die legal anerkannte Vaterschaft eines (Ehe-)Mannes weitgehend, aushandelbar' ist. Diese Regelung macht auch die Durchführung der heterologen Insemination mit Hilfe der Reproduktionsmedizin, die die Schaffung eines Kindes mit „Spendersperma“ zum Ziel hat, in Deutschland möglich, 
kann jedoch auch zu spezifischen Problemlagen und Streitfragen führen. So können zwar notariell beglaubigte oder auch inoffizielle Verträge zwischen den Ärzten und Paaren abgeschlossen werden, in denen der Ehemann als zukünftiger sozialer Vater das mit Hilfe von „Spendersperma“ gezeugte Kind als sein leibliches anerkennt, jedoch wird ihm per Gesetz die Möglichkeit einer Vaterschaftsanfechtung zugestanden (Kap. 4.1.3, 4.3.1.3). In struktureller Hinsicht entsteht eine Kernfamilie und wird vom Staat (der über deren Entstehung offiziell keine Kenntnis hat bzw. haben muss) unabhängig von den „tatsächlichen“ Abstammungsbeziehungen als solche anerkannt und durch das Grundgesetz geschützt. Da jedoch die Kenntnis über die biogenetische Herkunft als unverzichtbarer Bestandteil der individuellen Identität gilt, ${ }^{188}$ bleibt das Recht des Kindes auf Kenntnis seiner Verwandtschaft im Sinne von $\S 1589$ BGB bestehen. Es ist somit befugt, mit Erreichen der Volljährigkeit bei berechtigten Zweifeln die Vaterschaft anzufechten oder in Vertretung durch die Mutter schon früher anfechten zu lassen, was zu spezifischen Problematiken hinsichtlich einer personenstands- aber auch unterhaltsrechtlichen Zuordnung führen kann, ganz zu Schweigen von persönlichen emotionalen Schwierigkeiten insbesondere des Kindes. Aufgrund der Konstruktion des Primats von Blutsverwandtschaft bzw. der Vorstellung von ,natürlicher“ Verwandtschaft als biogenetische Verwandtschaft stellt das Kind über die körperliche Verbindung eine ,verwandtschaftliche“ Beziehung zu dem „Samenspender" her, die in rechtlicher Hinsicht allerdings zugunsten einer strukturellen Integrität der Kernfamilie nicht anerkannt wird. Da ,natürliche“ Verwandtschaft jedoch als unveränderlich und selbstverständlich erachtet wird (vgl. auch Schneider 1980 [1968]:26; Strathern 1992b:19), impliziert diese Idee, dass das Kind auch weiterhin zwei „Väter“ hat. Strathern schlussfolgert daraus meiner Ansicht nach folgerichtig: „If mothers, fathers and parents are still kin, then procreation continues to produce kinship“ (1995:352). Als Beispiel führt sie die Verwendung der Verwandtschaftstermini an, die trotz Reduzierung der Zeugungsbeiträge auf die Körpersubstanz auch weiterhin Bestand haben. Auch bei Unkenntnis des „Samenspenders“ bleibt er der biologische Vater des Kindes. Strathern bezeichnet dieses Phänomen auch als „dispersed kinship“ (1995:152), d.h. die biologischen Elternteile existieren weiter, auch wenn sie legal und sozial ausgeblendet werden. ${ }^{189}$

Betrachtet man nun die staatlich legitimierten Wege und Möglichkeiten der ,,assistierten“ Familienbildungen in Deutschland genau, lässt sich feststellen, dass sich die Reproduktionsmedizin mit ihren spezifischen Behandlungsmethoden im homologen Sy-

\footnotetext{
188 Dieser Aspekt hat Eingang in die Gesetzgebung gefunden und findet im Zusammenhang mit immer wieder diskutierten Gesetzesentwürfen zur heterologen Insemination und der Aufdeckung der Anonymität des „Spenders“ eine besondere Beachtung.

189 Strathern gibt in diesem Zusammenhang auch das Beispiel der Leihmutter an, die, obwohl legal und sozial nicht anerkannt, weiterhin mit dem Terminus Mutter bezeichnet wird.
} 
stem, Adoptionen, Pflegschaften oder auch Stiefelternschaften aufgrund der eindeutigen legalen Zuordnungen von Personen trotz der Diskrepanz zwischen biologischer und sozialer Elternschaft in den rechtlichen Verwandtschaftsdiskurs integrieren lassen, da der Wertzusammenhang der Kernfamilie erhalten werden kann. Um die sozialen Konsequenzen, die sich aus der bewussten Entscheidung für die Anwendung bestimmter Methoden reproduktionsmedizinischer Technologien, in die Körpersubstanzen dritter Personen involviert sind, ergeben (können), zu vermeiden, ist das vorrangige Ziel der bundesdeutschen Gesetzgebung, nur die Gründung einer Familie zuzulassen, die auf „,natürlicher“ Abstammung beruht. ${ }^{190}$ Die staatlich legitimierten Wege und Möglichkeiten einer ,assistierten“ Familiengründung mit Hilfe der Reproduktionsmedizin sind daher auf eheliche und nicht-eheliche Paare begrenzt (Kap. 4.3.1.1). ${ }^{191}$ Alleinstehende Frauen und Männer sowie homosexuelle Paare können sich nicht auf das ,Naturrecht“ des „Kinderwunsches“ und auf das Recht auf Fortpflanzung berufen, da sie nicht die notwendigen Kriterien für Elternschaft erfüllen. Das Konzept von biologischer Elternschaft wird hier mit der Einheit von Ehe und Familie verwoben und gilt damit auch als Grundlage für soziale Elternschaft. Argumentiert wird mit dem Begriff des „Kindeswohls“, das keine bewusste Herbeiführung gespaltener Elternschaften zulässt, da diese Form nur als „Reparaturmodell“ (siehe dazu Petersen 2000:100-110) für eine Einheit zwischen Eltern und Kindern, die sich über den Prozess der biologischen Reproduktion konstituiert und legitimiert, angesehen wird. Da die „natürlichen Fakten“ das Kind den biologischen Eltern a priori zuordnen, sind diese primär für die weitere Pflege und Erziehung verantwortlich (siehe Art. 6 II, III GG). Mit Strathern lässt sich demnach auch für den rechtlichen Verwandtschaftsdiskurs in Deutschland feststellen, dass ,social constructions follow biology“ (1992b:27), da die Anerkennung der „,natürlichen“ Fakten des Lebens als unveränderlich und selbstverständlich eine rationale Begründung für die ,Natürlichkeit‘ von Familien- bzw. Eltern-Kind-Beziehungen liefern. Ein Paar wird aufgrund der biologischen Prozesse zu Eltern und die soziale Anerkennung folgt den „natürlichen“ Fakten, da diese sich dem menschlichen Zugriff entziehen (Schneider 1980 [1968]:23ff; Strathern 1992b:24). Anhand der kulturellen Konstruktion von Verwandtschaft im rechtlichen Diskurs wird deutlich, dass trotz zunehmender gesellschaftlicher Veränderungen und sozialer ,Abweichungen“ die Kernfamilie als kleinste „,natür-

\footnotetext{
190 Dazu Lucke (1998:73-74): „Durch das Recht werden verwandtschaftliche Sozialbeziehungen abgesichert, kanalisiert, kontrolliert, ideologisch - zum Beispiel über die von ihm transportierten Leitbilder des Familien- und Verwandtschaftslebens - legitimiert und überhaupt erst mal registriert und offiziell festgehalten.“

191 Wobei es sich hierbei nicht um eine gesetzliche, sondern um eine standesrechtliche Regelung des deutschen Ärztetages handelt (vgl. Kap. 4.3.1.1). Nach Genehmigung durch die Landesärztekammer darf der Arzt homologe Inseminationen auch bei nicht-ehelichen Gemeinschaften durchführen.
} 
liche“ Verwandtschaftseinheit immer noch als „Keimzelle“ des Staates gehandelt wird. $^{192}$

Das Konzept von Blutsverwandtschaft findet seinen Widerhall auch im Embryonenschutzgesetz von 1991, das als ein zentrales Ziel verfolgt, gespaltene Mutterschaften durch das Verbot der Eizellen- und Embryonenspende schon im Vorfeld zu verhindern. Leihmutterschaften werden in Deutschland per Gesetz verboten und unter Strafe gestellt. Zur gespaltenen Vaterschaft macht das Embryonenschutzgesetz keine direkten Aussagen und schließt diese somit nicht aus. Wie ich in Kapitel vier gezeigt habe, wurde 1998 im Kindschaftsreformgesetz Mutterschaft zum ersten Mal eindeutig definiert. Nach $§ 1591$ BGB gilt die Frau rechtlich als Mutter, die das Kind geboren hat. Somit ist Mutterschaft im Gegensatz zu Vaterschaft nach deutschem Recht nicht anfechtbar und damit auch nicht, verhandelbar'. Die eindeutige Definition von Mutterschaft steht meiner Ansicht nach in direktem Zusammenhang mit den neuen reproduktiven Technologien, deren Gelegenheiten zur Kombinierbarkeit von Körpersubstanzen und die dadurch mögliche Fragmentierung in ,eizellspendende“, austragende sowie soziale Mutterschaft. Vor dem Einsatz der medizinisch-technischen Methoden wurde Mutterschaft immer verstanden als eine Erfahrung, die soziale und biologische Aspekte in sich vereint, da die soziale Mutterschaft als ,natürliche“ Konsequenz der körperlichen Verbindung während Schwangerschaft und Geburt galt (vgl. Schneider 1980 [1968:170). Durch die Einheit von genetischer, körperlicher und sozialer Mutterschaft entstand ein „natürliches“ Kontinuum von der Zeugung über Schwangerschaft und Geburt bis zur Pflege und Erziehung des Kindes. Vaterschaft nahm in euro-amerikanischen Gesellschaften schon immer eine ,doppelte Identität‘ ein (vgl. Dolgin 1995:49ff) und war und ist auch im bundesdeutschen Recht nicht eindeutig geregelt. ${ }^{193}$ Mit der Auftrennung zwischen psycho-sozialen und biologischen Elementen im Zusammenhang mit Leihmutterschaften kann nun auch Mutterschaft diese ,doppelte Identität ${ }^{\star}$ annehmen, was jedoch von der deutschen Gesetzgebung wegen ihrer „Unnatürlichkeit“ untersagt wird (vgl. Petersen 2000:106f). ${ }^{194}$ Unter den bisherigen gesellschaftlichen Rahmenbedingungen bestand kein Bedarf zu regeln, wer die Mutter ist. In Anlehnung an Giddens, der das Überprüfen und Anpassen der gesellschaftlichen Verhältnisse entsprechend der neuesten wissen-

\footnotetext{
192 So hält Lucke beispielsweise gerade das Familienrecht (FamR) als Teil des Bürgerlichen Gesetzbuches für besonders konservativ und neueren gesellschaftlichen Entwicklungen gegenüber wenig aufgeschlossen (1998:76). Sie verweist darauf, dass das FamR seit dem Gesetzbuch der Jahrhundertwende (BGB 1900) nur wenige Reformen erfahren hat, zu deren wichtigsten das Gleichberechtigungsgesetz (GlberG 1957) und das Erste Ehereformgesetz (1.EheRG 1976) zählen.

193 Kettner (2001:37-39) beschäftigt sich in seinem lesenswerten Artikel zur gespaltenen Elternschaft mit der Frage, warum die „Eizellenspende“ in Deutschland widerrechtlich, die „Samenspende“ aber zulässig ist. Er analysiert die aus der Sicht der Gesetzgebung geäußerten Befürchtungen und kommentiert sie kritisch.

194 So wird argumentiert, dass die „Samenspende“ „,natürliche“ Vorgänge nur nachahme, wohingegen sich die „Eizell- oder Embryonenspende“ weit von der „,natürlichen“ Fortpflanzung entferne und somit auch zu einer doppelt gespaltenen Elternschaft führen könnte (Petersen 2000:78, 106).
} 
schaftlichen Kenntnisse als ein Kennzeichen von Gesellschaften der Moderne beschreibt (vgl. 1995:54), ${ }^{195}$ lässt sich feststellen, dass die Selbst-Reflexivität der Gesetzgebung dort zutage tritt, wo durch die Etablierung und Anwendung der Reproduktionsmedizin neue gesetzliche Regelungen erforderlich werden. Dadurch wird offenbar, dass unter den Bedingungen der Reproduktionstechnologien kulturelle Selbstverständlichkeiten in Frage gestellt werden, auf die mit neuen bzw. veränderten gesellschaftlichen Rahmenbedingungen reflexiv reagiert wird, die wiederum Einfluss auf kulturelle Orientierungs- und Wertvorstellungen haben. In Bezug auf die 1998 gesetzlich festgelegte Definition von Mutterschaft lässt sich eine Konzentration auf biologische Faktoren wie Schwangerschaft und Geburt als die Kriterien, die die Beziehung zwischen Mutter und Kind definieren, feststellen. ${ }^{196}$ Somit nimmt in der kulturellen Konstruktion von Leiblichkeit in Deutschland die Mutter die zentrale Rolle ein. ${ }^{197}$

\section{Der persönliche Diskurs}

Im persönlichen Diskurs über Verwandtschaft (Kap. 5), den ich anhand von Interviews im Kontext der Reproduktionsmedizin dargelegt habe, findet sich die im rechtlichen Diskurs herausgearbeitete kulturell dominante Konstruktion von „echter“ Verwandtschaft über eine geteilte Körperlichkeit wieder. Mein empirisches Material (Kap. 5.1) hat gezeigt, dass die eigene Verortung in eine Familie als die bedeutendste und identitätsstiftendste definiert wird. Im Kontext der Reproduktionsmedizin assoziieren die Befragten mit dem Begriff der Familie primär eine Gemeinschaft, die über den Prozess der Fortpflanzung durch eine gemeinsame Körpersubstanz verbunden ist, und die dadurch auch die ,Natürlichkeit‘ dieser Verbindungen impliziert. Die Mitglieder einer Familie werden auch als blutsverwandt bezeichnet; Eine Definition, die abhängig vom jeweiligen Referenzpunkt mit der gesetzlichen Festschreibung von Verwandtschaft übereinstimmt (§ 1589 BGB). Bezieht sich die Definition von Familie zum Zeitpunkt der Interviews auf die Herkunftsfamilie, dann handelt es sich ausgehend von Ego um Blutsverwandtschaft. Bezeichnet ,Familie‘ jedoch die Fortpflanzungsfamilie bestehend aus Ego als einem Elternteil, handelt es sich um eine Verbindung von Affinalität und Filiation,

\footnotetext{
195 Dies wird auch deutlich an den rezenten politischen Entscheidungen zur embryonalen Stammzellforschung. Das gesetzliche Verbot der Herstellung von Embryonen außer zum Zweck der Fortpflanzung in Deutschland wird ab Februar 2002 umgangen durch die dann zulässige Einführung von (überzähligen) Embryonen aus dem europäischen Ausland zum Zweck der medizinischen Forschung. Langfristig steht auch eine Änderung des Embryonenschutzgesetzes zur Debatte (DIE ZEIT 18.10.2001). Auch die Änderung des Gesetzes hinsichtlich einer Anwendung der noch untersagten Präimplantationsdiagnostik (zu therapeutischen Zwecken) ist ein weiterer Diskussionspunkt.

196 Andere Konstruktionen von Verwandtschaft wie zum Beispiel über Ammenschaft, bei der eine Frau, die gerade ein Kind geboren hat, ein weiteres Kind betreut und stillt, fanden keinen Eingang ins deutsche Recht.

197 Was auch darauf hinweist, warum die Befruchtung mit „Spendersperma“ seitens der Reproduktionsmedizin propagiert wird als Möglichkeit zur Schaffung eines „eigenen“ Kindes, da es öffentlich und rechtlich über die Schwangerschaft der Frau als leibliches Kind (beider Elternteile) anerkannt wird (siehe Kap. 5.1.4.1, 6.3 und 7.2).
} 
wobei nur letzteres Verwandtschaft im Sinne des Bürgerlichen Gesetzbuches (BGB) darstellt (Konsanguinität). In den Interviews werden die Begriffe ,Familie‘ und ,Verwandtschaft' sehr häufig alternativ oder synonym verwendet, wobei in Kapitel 5.1.1.1 deutlich geworden ist, dass die Befragten dennoch differenzieren, einerseits auf der Ebene der persönlichen formalen Zuordnung bzw. Abgrenzung von Personen, andererseits auf der Emotions- und Handlungsebene. In den Interviews aus dem Kontext der Reproduktionsmedizin bildet in erster Linie die Herkunftsfamilie den Bezugsrahmen, da sich ein Paar ohne Kinder noch nicht als Familie definiert. Nach der Geburt eines Kindes liegt der Bezug schwerpunktmäßig auf der „eigenen“ Familie, bestehend aus Ehepartner und Kind.

Es hat sich gezeigt, dass eine gemeinsame Abstammung, ergänzt (in seltenen Fällen ersetzt) durch Affinalität, ein dominantes Merkmal bei der persönlichen Definition von Familie und der Zuordnung verschiedener Personen zu dieser Kategorie darstellt. In Kapitel 5.1.1.1 wurde deutlich, dass Ego Personen, die diese Kriterien erfüllen, unter ,Familie‘ zusammenfasst, oder, was sehr viel häufiger der Fall ist, Familie je nach Kontext und individueller Situation unterschiedlich definiert. Das persönliche Netzwerk von Zugehörigkeiten und Angehörigkeiten ist demnach nicht statisch, sondern verändert sich im Verlauf des Lebens stetig. So kann der Status als Tochter oder Sohn in einem Beziehungssystem später zum Status als Mutter oder Vater in einem anderen Kreis von Beziehungen werden. ,Familie' ist sowohl im rechtlichen als auch im persönlichen Diskurs somit keine eindeutig begrenzte statische Einheit, d.h. auch wenn Ego in einer ,eigenen“ Familie mit Partner bzw. Partnerin und Kind(ern) lebt, bleibt er weiterhin formalrechtlich durch Filiation und Geschwisterschaft an Mitglieder der Geburtsfamilie gebunden. Auch die persönliche Bedeutung von Familie und Verwandtschaft ist im Lebenszyklus von unterschiedlicher Relevanz und kann sich in verschiedenen Lebenssituationen und Konstellationen verändern (vgl. auch Rosenbaum 1998:29). Jedoch ist deutlich geworden, dass ,Familie“ nicht nur ein Begriff ist, der eine bestimmte Kategorie von Verwandten eingrenzt oder ausschließt, sondern insbesondere eine starke affektive Bedeutung beinhaltet.

Aufgrund des in der Verwandtschaftsethnologie als bilateral kategorisierten Verwandtschaftssystems verfügt Ego über zahlreiche Verzweigungen und Verbindungen in der Aszendenz sowie in der Deszendenz. Mein empirisches Material hat gezeigt, dass sich in der Anerkennung von bestimmten Personen als Verwandte eine gewisse soziale Erschöpfung einstellt, die im täglichen Leben zu einer Auswahl und Begrenzung der weiten Verzweigungen führt. So bricht Ego Verbindungen ab oder fügt weitere Personen zu seinem Beziehungssystem hinzu, indem kontext- und situationsabhängig unterschiedliche Kriterien betont oder vernachlässigt werden (Kap. 5.1.2 und 5.1.3; siehe auch Rosenbaum 1998:20). Die Kenntnis über die genealogische Position stellt beispielsweise 
ein bedeutendes Merkmal dar zur Positionierung von Personen in Vergangenheit und Gegenwart, jedoch wird nicht unbedingt jeder aufgrund der genealogischen Verortung als verwandt bezeichnet. So differenzieren die Befragten deutlich in „nahe“ und „entfernte" Verwandtschaft, wobei die Kriterien für diese Unterscheidung sehr unterschiedlich sind. Die Auswahl der Verwandten, mit denen Ego interagiert, kann sich zum einen nach der Erreichbarkeit (räumliche Nähe bzw. Distanz) richten, nach dem persönlichen Verhältnis, dem Grad der Verpflichtung, die Ego aufgrund von Interaktionserwartungen seitens der Verwandten aus moralischen Gründen empfindet, sowie nach der Art verwandtschaftlicher Treffen.

Da durch eine Heirat neue soziale (und legal anerkannte) Zugehörigkeiten geschaffen werden, muss bzw. kann Ego nun aus einer noch größeren Anzahl von verwandten Personen selektieren. Mein Datenmaterial hat gezeigt, dass Affinalverwandtschaft den bereits bestehenden Verwandtschaftsbeziehungen innerhalb der Herkunftsfamilie hinzugefügt wird, wobei in den Vorstellungen der interviewten Personen eine Unterscheidung vorgenommen wird zwischen „natürlicher“ Verwandtschaft (Blutsverwandtschaft) und Affinalverwandtschaft (vgl. auch Marbach 1998:113). Auch Schneider leitet aus der Grundannahme „kinship is the blood relationship, the fact of biogenetic substance“ (1980 [1968]:107) zwei Kategorien von Verwandten ab: „relatives by blood“ und ,relatives by law“ (1980 [1968]:25). Seiner Ansicht nach teilen Affinalverwandte keine „natürliche“ Substanz miteinander wie Blutsverwandte, sondern nur eine gemeinsame Beziehung, so dass ,genealogisch ‘ und, sozial' alternative Unterscheidungskriterien für verwandtschaftliche Beziehungen darstellen. Auch Edwards und Strathern (2000:150) konstatieren als distinktives Element des euro-amerikanischen Verwandtschaftssystems die Teilung und Kombination von sozialen und biologischen Fakten. Soziale und biologische Aspekte, in sich unendlich vielschichtig und komplex, dienen gleichwertig sowohl zur Verbindung und Unterstützung als auch zur Abwertung des anderen: ,[...] the divide (between biological and social kinship) is also a combination or association (of disparate elements), and belongs to a whole range of possibilities for the inclusion and exclusion of persons“. Diese Feststellungen lassen sich auch auf meine Untersuchung über die Konstruktion von Verwandtschaft im Kontext der Reproduktionsmedizin in Deutschland übertragen. Geteilte Körpersubstanzen per se bilden das maßgebende konstituierende Element für „,natürliche“ und damit ,echte“ Verwandtschaft. Somit konstituiert die genetische Abstammung bestimmte Formen sozialer Beziehungen; Diejenigen sozialen Beziehungen, die auf genetischer Abstammung beruhen, gelten in Deutschland primär als verwandtschaftliche Beziehungen. Das Konzept von Konsanguinität beinhaltet jedoch nicht nur geteiltes Blut (oder Gene), sondern auch Vorstellungen über geteilte leibliche Eigenschaften und Emotionen, die über Körpersubstanzen weitergegeben 
werden können. Affinalbeziehungen als ,nur' soziale Beziehungen beinhalten nicht „natürlicherweise“ eine emotionale Bindung. ${ }^{198}$ Die emotionale Bindung an die Mitglieder der Herkunftsfamilie ist diejenige, der am meisten Bedeutung beigemessen wird (wobei die Eltern vor den Geschwistern genannt werden). Erwähnt werden in diesem Zusammenhang insbesondere die geteilte Vergangenheit, die gemeinsame Lokalität und die gemeinsamen Erfahrungen, die der Blutsverwandtschaft eine besondere Grundlage und Qualität verleihen. Des weiteren signalisiert eine gemeinschaftliche Abstammung einen Bezug auf eine gemeinsame Geschichte und Genealogie, die eine besondere zeitliche Dimension dieser Form von Zugehörigkeit mit sich bringt. Es lässt sich somit feststellen, dass im persönlichen Diskurs von den Befragten differenziert wird zwischen „natürlichen“ und somit ,gegebenen“ sowie sozialen ,verhandelbaren“ Verwandtschaftsverbindungen, die freiwillig ausgewählt und eingegangen werden, wozu auch die Affinalbeziehungen zählen. Diese konstituieren sich über die Heirat zweier Ehepartner. Im persönlichen Verwandtschaftsdiskurs hat sich die dominante Meinung gezeigt, dass die offizielle Zugehörigkeit zur Affinalverwandtschaft mit einer Ehescheidung beendet ist; Weiterer Kontakt ist ausschließlich abhängig vom persönlichen Verhältnis der Beteiligten. In den Fällen, in denen ein Paar schon mit einem gemeinsamen Kind zusammenlebt, wird deutlich, dass sich mit der Geburt eines Kindes der Referenzpunkt ändert: Für die jeweiligen Elternteile bleibt die Unterscheidung zwischen Blutsverwandtschaft und Affinalverwandtschaft weiterhin rechtlich und persönlich bestehen, für das Kind ändert sich jedoch der Bezugsrahmen, da sowohl die mütterliche als auch die väterliche Linie zur Blutsverwandtschaft wird.

Anhand des Blickes auf die Konstruktion von Zugehörigkeit und Angehörigkeit im Kontext von Adoptionen und Pflegschaften im Sinne einer Vergleichsgruppe fiel auf, dass das Gefühl von Zugehörigkeit zu einer Familie oder die Zuordnung von Personen zur ,eigenen“ Familie nicht primär durch eine gemeinsame ,Biologie“ konstituiert wird (Kap. 5.2). Alle Befragten lebten mit Kindern zusammen und so bezogen sich die Aussagen zu ,Familie“ primär auf die Fortpflanzungsfamilie. ${ }^{199}$ Hier werden sowohl soziale Interaktionen als Grundlage emotionaler Nähe und Bindung als auch die gemeinsame Lokalität besonders betont, da das gemeinschaftliche Wohnen häufige und intensive Begegnungen mit sich bringt. Dies führt wiederum den Aussagen zufolge zu gegenseitiger Unterstützung und der Fähigkeit, sich aufeinander verlassen und vertrauen zu können. Es stellte sich heraus, dass von den Adoptiv- und Pflegeeltern Formen von Bezie-

\footnotetext{
198 Mit Bezug auf Schneider (1980 [1968]) stellt auch Strathern (1992c:154) fest, dass ein eindeutiger Verwandter in Euro-Amerika derjenige ist, mit dem Ego durch Blut und durch eine gemeinsame Beziehung geprägt von Liebe und Fürsorge verbunden ist.

199 Wobei ich hier explizit erwähnen möchte, dass dieser Begriff sich zwar auf die sexuelle Fortpflanzung bezieht, ich darunter jedoch auch Familien subsumiere, die durch Adoption gegründet wurden.
} 
hungen, die primär durch gemeinsames Leben und Arbeiten, gegenseitigen Zuneigungen und/oder gemeinsamen Interessen entstehen, tendenziell höher wertgeschätzt werden als diejenigen, die , unfreiwillig' primär von geteilter Substanz abgeleitet sind. So lässt sich hier die Tendenz feststellen, diese Beziehungen in emotionaler Hinsicht höher zu bewerten als Blutsbeziehungen oder diese sogar zu ,ersetzen'. Es ist jedoch auch in Kapitel 5.2.1 offensichtlich geworden, dass zwischen ausschließlich sozialen Zugehörigkeiten und denen durch Geburt oder Adoption differenziert wird. Formalrechtlich gibt es zumindest zwischen Adoptiv- und leiblichen Kindern eines Elternpaares keinen Unterschied, wobei Pflegekinder, auch bei langandauernder räumlicher Trennung, immer den leiblichen Eltern zugeordnet bleiben. Diese rechtliche Differenzierung findet sich auch im persönlichen Diskurs im Kontext von Adoptionen und Pflegschaften wieder, indem die Kinder bei den befragten Pflegeeltern zwar einen hohen emotionalen Stellenwert einnehmen und in dieser Hinsicht als Teil der Familie gelten, dennoch das Wissen über die Existenz leiblicher Eltern und den bestehenden Verwandtschaftsstatus des Pflegekindes dazu führen, dass sie in der formalen Zuordnung von den leiblichen und/oder Adoptivkindern unterschieden werden. Anhand meiner Ausführungen wird deutlich, dass ,Familie“ auch hier kontextabhängig definiert wird und abhängig ist vom jeweiligen Bezugsrahmen. Auch wenn ,Familie‘ als soziale Einheit betont wird, die für das tägliche Leben von großer Relevanz ist, findet sich in anderen persönlichen Kontexten die kulturell dominante Vorstellung von „echter“ Verwandtschaft über Prokreation, die durch Adoptionsverwandtschaft erweitert werden kann.

Zusammenfassend lässt sich festhalten, dass die Konstituenten für Formen sozialer Beziehungen, die im persönlichen Diskurs in Deutschland als Verwandtschaft konzipiert werden, eine Kombination aus Körpersubstanz, Gefühl, Pflege, Fürsorge und Erziehung bilden. Diese Elemente können kontext- und situationsspezifisch unterschiedlich präsent und ausgeprägt sein. So werden beispielsweise die konsanguinen den affinalen Beziehungen hinsichtlich der Kriterien wie geteilte Körpersubstanz, gemeinsame Lokalität, ökonomische und moralische Verpflichtungen und Emotionen einander gegenüber gestellt. In Fällen, in denen das Kriterium der gemeinsamen Residenz bei der Affinalverwandtschaft existiert, beinhaltet es auch häufige soziale Interaktionen, die besonders wertgeschätzt werden, was wiederum zu dem Gefühl enger Verbundenheit führen kann (vgl. auch Carsten 2000a:22). Im täglichen Leben werden verschiedene Kriterien der Vorstellung über Beziehungen und Verbundenheit betont oder vernachlässigt- biologische Verbindungen, zu einer bestimmten Familie, zu einem Ort oder Haus zu gehören, gemeinsame Namen, gleiche Interessen etc. - und mit unterschiedlichen Bedeutungen versehen, was direkte Auswirkungen auf das soziale Handeln hat. Mit Bezug auf die aktuellen Arbeiten von Edwards (2000) sowie von Edwards und Strathern (2000) kann ich anhand meiner Untersuchung feststellen, dass auch in den Konstruktionsprozessen von Verwandtschaft in Deutschland unter den Bedingungen der Reproduktionstechno- 
logien biologische und soziale Kriterien die zentralen Elemente darstellen und - wiederum in ihrer kulturellen Konstruiertheit - zur gegenseitigen Verstärkung oder Ersetzung dienen. ${ }^{200}$

\subsection{Das „eigene“ Kind im Schnittpunkt von Körper, Selbst und Person}

Im Kontext der Reproduktionsmedizin ist das vorrangige Ziel aller Beteiligten die Schaffung von „eigenen“ leiblichen Kindern (5.1.4). Es hat sich gezeigt, dass bei den Befragten die vorherrschende ideale Vorstellung diejenige von der sozialen und biologischen Einheit der Kernfamilie bestehend aus einem Elternpaar und den leiblichen Nachkommen ist. Diese substantielle Einheit gilt auch als emotionale Einheit mit engen und intimen Bindungen, was von Schneider als „diffuse enduring solidarity“ (1980 [1968]:50) beschrieben wurde. In den Interviews wird immer wieder ein gemeinsames leibliches Kind als ein „Zeichen der Liebe“ und als Ausdruck einer besonders engen affektiven Bindung zwischen den Ehepartnern bezeichnet. Kinder gelten somit als symbolische Repräsentationen der Liebe und Einheit eines Paares und der „Kinderwunsch“ kann verstanden werden als das Bemühen eines Paares, ihre Ehe mit dem ,fehlenden Teil‘ auszustatten. Diese intensiven persönlichen Beziehungen, die Luhmann (1994 [1982]) auch „Intimbeziehungen““201 nennt, sind ein wichtiges Argument in der reproduktionsmedizinischen Praxis (vgl. Hauser-Schäublin 1995:167), zum einen seitens der „ungewollt kinderlosen“ Paare, für die die Schaffung von Nachkommen mit dem gewählten Liebespartner das erklärte Lebensziel darstellt (Kap. 5.1.1.3, 5.1.4), zum anderen seitens der Anbieter, die die fortpflanzungsmedizinischen Behandlungsmöglichkeiten verwirklichen wollen.

Ein wesentlicher Aspekt, der sich aus den Interviews im Kontext der Reproduktionsmedizin im Zusammenhang mit dem Konzept eines „eigenen“ Kindes herauskristallisiert hat, ist die Vorstellung von der Weitergabe der eigenen Gene und der Idee von Unsterblichkeit. Das Entdecken des ,Eigenen“ im Kind, die physischen und charakterlichen Ähnlichkeiten, schafft eine besondere Vertrautheit und unterstützt die Vorstellung, dass das leibliche Kind ein Teil von einem selbst ist. Warum begreifen die befragten Paare

\footnotetext{
200 Siehe dazu auch Edwards und Strathern (2000:157): „But ideas of shared substance are only ever one side of the story; connections are also broken through lack of attention.“ $[\ldots]$,Blood ties might theoretically allow connections through genealogy and procreation to be traced in ever widening circles, but if it comes to recognizing persons as relatives, then in the English experience social exhaustion intervenes.“ (2000:159-160)

201 Luhmann spricht auch von einer Spezialisierung auf emotionale Bedürfnislagen, die daraus resultiert, dass die Grunderfahrung im Leben jedes Individuums die Differenz von persönlichen und unpersönlichen Beziehungen sei (1994 [1982]:193-195). Die meisten Anforderungen des Lebens könnten nur noch in unpersönlichen Beziehungen sichergestellt werden, was zugleich zu einer Intensivierung und Emotionalisierung von persönlichen Beziehungen geführt habe. Liebe werde dabei zu einem zentralen Kommunikationsmedium.
} 
jedoch den Körper ihres Kindes als Teil ihres eigenen Selbst bzw. ihrer Identität? Ich habe gezeigt, dass aus biomedizinischer Sicht Körper und Selbst voneinander unterscheidbare und trennbare Entitäten darstellen (Kap. 2.3), so dass der Körper behandelt werden kann, ohne dass das Selbst oder die Identität davon betroffen sein muss. Es ist aber auch deutlich geworden, dass damit ein zentraler Wert der spätmodernen westlichen Gesellschaft, das individuell verkörperte Selbst, in Frage gestellt wird. Der Soziologe Giddens hat postuliert, dass auf der Grundlage dieser Annahmen das Selbst nicht als unveränderlich und gegeben konzipiert wird, sondern immer neu bestätigt und reflexiv an neue Gegebenheiten und Informationen aus dem soziokulturellen Umfeld angepasst werden muss (1991:52). Damit bildet der Körper in der Spätmoderne die Grundlage des Selbst und stellt gleichzeitig dessen Ausdrucksmedium dar. Ebenso wie das Selbst ist auch der physische Körper keine festgelegte Entität mehr, sondern eingebunden in die Reflexivität der Spätmoderne (Giddens 1991:218). Ich habe dargelegt (Kap. 2.2.2), dass sich dem biomedizinischen Zeugungsmodell zufolge die genetische Identität eines Individuums aus den körperlichen Zeugungsbeiträgen beider Elternteile zusammensetzt. Die Vorstellung von der Weitergabe der eigenen Identität ist somit direkt geknüpft an den eigenen Körper, der in seiner verleiblichten Dimension als subjektive Empfindungs- und Erfahrungswelt konstitutiv für das Selbst ist (Kap. 2.3.2, 2.3.3). Aufgrund der erläuterten Basis des Selbst im Körper und seiner Wahrnehmung über den Leib lässt sich in den leiblichen Eigenschaften eines „eigenen“ Kindes das eigene Selbst wieder entdecken und somit über das leibliche Kind die eigene Identität über die eigene Generation hinaus verlängern. Es hat sich weiterhin gezeigt, dass das Austragen und Gebären eines „eigenen“ Kindes und damit die körperliche Repräsentation der eigenen „Fruchtbarkeit“ eine bedeutende Rolle bei der Konstituierung der Selbst-Identität (siehe Giddens 1991:5) als Frau und Mutter spielt. Die Kontinuität der Selbst-Identität ist jedoch nur gegeben, wenn die selbstentworfene Lebensgeschichte ohne Brüche verläuft bzw. wenn Dissonanzen integriert werden können und damit die Kontinuität der eigenen Biografie gesichert ist. „Sterilität“ und ,ungewollte Kinderlosigkeit“" können existentielle Lebenskrisen auslösen, da in den Biografien der befragten Paare, und insbesondere der Frauen, ein Kind fest eingeplant war. Die „ungewollte Kinderlosigkeit“ stellt somit auch die Selbst-Identität in Frage. In Anlehnung an Giddens lässt sich für den Kontext der Reproduktionsmedizin postulieren, dass die leiblich erfahrbare Schwangerschaft für das Selbst der Frauen von zentraler Bedeutung ist, da Selbst und Körper (Leib!) direkt miteinander verbunden sind. Die Eigen- und Fremdwahrnehmung als „fruchtbare“ Frau, die durch die Überbrückung der „Unfruchtbarkeit“" (sei es die eigene oder die diagnostizierte „Sterilität““ des Mannes) mit Hilfe der Reproduktionstechnologien möglich wird, verhilft dazu, die Selbst-Identität als Frau und Mutter nach innen (das Selbst) und außen (als Person) aufrecht zu erhalten. Auch die Veränderung oder Erweiterung der Selbst-Identität des Mannes als Vater ist direkt an den Körper 
geknüpft, da er durch die Schwangerschaft der Frau seine „Fruchtbarkeit“" und Fortpflanzungsfähigkeit unter Beweis stellt. Durch die Anerkennung des soziokulturellen Umfeldes der Vaterschaft über Körper und Körperlichkeit findet der Übergang von einer sozialen Rolle in eine andere statt, was wiederum direkte Rückwirkungen auf die Selbst-Identität hat. Mit Bezug auf Giddens (1991) zeigt sich, dass eine Veränderung am Körper hier zu einer Veränderung des individuellen leiblich erfahrbaren Selbst führt, und dass sich durch diesen reflexiven Prozess zwischen innen und außen, zwischen Selbst und Person die Selbst-Identität konstituiert. Beide Aspekte eines Individuums sind eng miteinander verknüpft sind, da beide verkörpert sind und körperlich (die Wahrnehmung des Körpers von außen) wie leiblich (die eigene Befindlichkeit) erfahren werden. Somit wird die Konstituierung einer Selbst-Identität als Vater oder Mutter möglich und trotz der Krise durch die Diagnose „Unfruchtbarkeit“ und deren Syndrom der „ungewollten Kinderlosigkeit“ kann die Kontinuität der eigenen, selbst entworfenen, Biografie aufrecht erhalten werden (Kap. 5.1.4).

Die symbolische Funktion und die Bedeutung, die dem Körper (über geteilte Körpersubstanz) bei der Konstituierung von ,echter“ Verwandtschaft zugewiesen wird und die enge Verbindung des Körpers mit der Erfahrung des Selbst und dem Erleben sozialer Beziehungen, in denen das Individuum zur Person wird, findet sich auch bei der heterologen Insemination, bei der körperliche Verwandtschaft letztlich jedoch nur über ein Elternteil, die Mutter, geschaffen wird. Obwohl das Kind keine genetische Verbindung zu dem sozialen Vater besitzt und sich dieser demzufolge körperlich (biogenetisch) nicht reproduziert, d.h. an der genetischen Identität des Kindes keinen Anteil hat, erfolgt dennoch über die Schwangerschaft der Ehefrau und die damit einhergehende Veränderung ihres Körpers eine öffentliche Darstellung seiner Fortpflanzungsfähigkeit (s.o.). Die Entwicklung der Selbst-Identität als Vater verläuft auch hier über den Körper, wenn auch nicht über den eigenen, so doch indirekt über den Körper der Frau. Ich konnte anhand meines Datenmaterials allerdings zeigen, dass Mechanismen existieren, diese Brüche zwischen Ideologie und Praxis in Übereinstimmung zu bringen und zumindest das soziale Umfeld davon zu überzeugen, dass es sich um ein leibliches Kind beider Eltern handelt. So streben die Eltern danach, eine möglichst große körperliche Ähnlichkeit zwischen ihnen und ihrem heterolog gezeugtem Kind zu erzielen, um der Vermutung eines Ehebruchs auszuweichen (Kap. 5.1.4.1, 6.3). Sexualität gilt in der Konstruktion von Verwandtschaft in Deutschland als Symbol für die ,Natürlichkeit' der Verbindungen zwischen Eheleuten, als deren ebenso ,natürliches“ Ergebnis das Kind angesehen wird (Kap. 6.2; vgl. auch Schneider 1980 [1968]:43, Strathern 2001:372). Der Geschlechtsverkehr erfüllt somit innerhalb der ehelichen Gemeinschaft eine wichtige symbolische Aufgabe, und Ehebruch wird assoziiert mit sexuellen, d.h. sowohl körperlichen als auch leiblichen, Beziehungen zwischen einem Ehepartner und einer dritten Person. Bei der Anwendung und dem Umgang mit der heterologen Insemination wird die Leib- 
lichkeit (und damit auch das Selbst des „Spenders“) in sozialer Hinsicht vollständig ausgeblendet. Diese beabsichtigte Anonymität zielt auf ein Unsichtbarmachen und das Verhindern von sozialen Beziehungen ab. Wird die Ähnlichkeit zwischen Eltern und Kind vom sozialen Umfeld bestätigt, ist auch das Ziel, die gesellschaftliche Anerkennung der sozialen und biologischen Vaterschaft, erreicht. Es lässt sich demnach postulieren, dass die wesentlichen Kriterien für die Bestätigung der Vaterschaft des sozialen Umfeldes zum einen dessen Beziehung zur biologischen Mutter ist, da durch die Schwangerschaft das Kind als Zeichen eines reproduktiven Prozesses anerkannt wird, auch wenn der Mann sich selbst nicht biogenetisch reproduziert hat. ${ }^{202}$ Zum anderen erfolgt die Anerkennung der Vaterschaft über seine Performanz, d.h. das soziale Handeln als ,Vater“. ${ }^{203}$ Auch im Fall männlicher „Sterilität“ kann die eigene Lebensgeschichte somit aufgrund der erfüllten Eigen- und Fremderwartungen an die soziale und geschlechtliche Rolle nach außen hin ohne Bruch verlaufen, was reflexiv auf das Selbst zurückwirkt und auch hier die Kontinuität der Selbst-Identität sichert. ${ }^{204}$

Für den Kontext der Reproduktionsmedizin lässt sich schlussfolgern, dass das „eigene“ leibliche Kind zu gleichen Teilen die Beiträge der Eltern verkörpert, mit denen es genetisch verbunden ist, und somit alle Kriterien einer verwandtschaftlichen (Bluts-)Beziehung im Gegensatz zu einer Adoption erfüllt. Dieses Konzept von leiblicher Elternschaft beinhaltet die Vorstellung einer unauflöslichen Bindung zum Kind. Ein ,eigenes“ Kind gilt als eine Person, die sowohl in leiblicher, emotionaler sowie rechtlicher Hinsicht zu einem gehört. Obwohl zumindest adoptierte Kinder, rechtlich gesehen, den leiblichen Kindern vollständig gleichgestellt sind, spielt dennoch der Aspekt der biologischen Zugehörigkeit eine bedeutende Rolle. Bei einem „,eigenen“ Kind gibt es keine anderen biologischen Wurzeln und damit „,richtige“ Eltern, die - erzählt man dem Kind von der Adoption - immer eine Rolle spielen werden. ${ }^{205}$ Das leibliche Kind wird demnach aufgrund der „,natürlichen Fakten“ der biologischen Reproduktion assoziiert mit besonders enger Zusammengehörigkeit und Verbundenheit, verstanden als „echte“

\footnotetext{
202 Siehe dazu auch Kap. 5.1.4.1, in dem ich mit Bezug auf Strathern (1995:354) gezeigt habe, dass sich die „Samenspender" in Deutschland aufgrund des biomedizinischen Zeugungsmodells zwar genetisch fortpflanzen, sich jedoch nicht aufgrund ihrer kompletten sozialen Ausblendung reproduzieren und gesellschaftlich weiterhin als kinderlos gelten. Im Gegensatz dazu wird hier der Ehemann als Vater vom sozialen Umfeld als sich reproduzierend bestätigt, auch wenn er sich nicht genetisch fortgepflanzt hat.

203 Siehe dazu auch den Aufsatz von Hagström (1996) „Becoming a Father and Establishing Paternity“.

204 Vgl. dazu auch die erste große Untersuchung über die Auswirkungen der heterologen Insemination von Snowden et al. (1983), die beschreibt, dass die Ehe und die Psyche des Mannes durch die ,erfolgreiche' heterologe Insemination stabilisiert würden.

205 Siehe dazu das Recht auf Kenntnis der genetischen Abstammung im bundesdeutschen Grundgesetz (Art. 2 Abs. 1 GG) sowie für den amerikanischen Kontext die Untersuchung von Modell (1994), in der sie zeigt, wie viel Bedeutung der biologischen Verwandtschaft im Vergleich zu der ,nur' sozialen beigemessen wird. Dies wird auch daran deutlich, dass es Vereinigungen von Menschen gibt, die als Kinder adoptiert wurden, sich jedoch auf die Suche nach ihren ,richtigen' Eltern begeben.
} 
Verwandtschaft. Damit behält auch Schneiders Feststellung hinsichtlich der amerikanischen Verwandtschaftsvorstellung, dass „kinship is a fact of shared biogenetic relationship“ für Deutschland seine Gültigkeit (1980 [1968]:107). Diese von mir in Kapitel vier und fünf meiner Arbeit herausgearbeitete kulturelle dominante Konstruktion von Blutsverwandtschaft in der Ideologie findet sich auch in der konkreten reproduktionsmedizinischen Praxis. Auf der Grundlage dieses Konzeptes legitimiert sie ihre Anwendung und wirkt gleichzeitig wieder darauf zurück, in dem sie die Vorstellungen von „echter“ im Sinne von leiblicher Verwandtschaft noch verstärkt und diese mit einem enormen emotionalen Potential aufgeladen wird.

Mein empirisches Material hat gezeigt, dass Ähnlichkeit, ungeachtet der genetischen Information, als kulturell bedeutendes Zeichen enger Zugehörigkeit verstanden wird. Auch im Kontext von Adoptionen und Pflegschaften lässt sich die Vorstellung wiederfinden, dass äußere Übereinstimmungen zwischen konsanguinen Verwandten ihren Ursprung in gemeinsamen Genen haben und „echte“ Verwandtschaft sich somit über den Körper konstruiert (Kap. 5.2.2.1). Es ist jedoch deutlich geworden, dass das Entdecken von bestimmten Ähnlichkeiten auch im nicht-leiblichen „eigenen“ Kind zu einem Gefühl von Vertrautheit und Zusammengehörigkeit führt, wobei allerdings der Einfluss von Sozialisation und Umwelt für die Entwicklung des Kindes betont und höher gewichtet wird als die Genetik. Wie ich in meiner Arbeit gezeigt habe, werden von Adoptiv- und Pflegeeltern tendenziell die sozialen Aspekte von Verwandtschaft besonders betont, was sich auch im Konzept des „eigenen“ Kindes widerspiegelt. Adoptivkinder gelten alle als ,eigene“ Kinder, wohingegen Pflegekinder zwar gefühlsmäßig ein Teil der Familie sind, jedoch nicht als „eigene“ Kinder bezeichnet werden. Diese Bezeichnung ist demnach reserviert für leibliche Kinder und nicht-leibliche Adoptivkinder, was dem kulturell dominanten Konzept von „,natürlicher“ Verwandtschaft entspricht.

Von Adoptiveltern wird darüber hinaus gerade die „besondere Freiheit“" betont, die man hat, da das Kind eben nicht von einem selbst abstammt und man somit genetisch auch nichts vererbt haben kann. Diese Einschätzungen lassen wieder einen Bezug zu Giddens (1991) herstellen, mit dem argumentiert werden kann, dass sich leibliche Eltern, dadurch dass ein Teil des Körpers (und des Leibes!) im leiblichen Kind steckt, bei ,Misserfolgen' des Kindes in ihrem Selbst angegriffen fühlen (vgl. auch Kap. 7.1). Anhand der Aussagen von Adoptiveltern (Kap. 5.2.2.1) lässt sich daher die Vermutung aufstellen, dass diese sich bei auffälligen Entwicklungen und Verhaltensweisen des Kindes in der Regel in ihrem Selbst nicht in Frage gestellt sehen, da das Kind nicht über die Fortpflanzung an ihre eigene Körperlichkeit gebunden ist, sondern eine Abgrenzung zum (anderen) Selbst des Kindes stattfinden kann. Aus der Perspektive der mit Adoptivund/oder Pflegekindern lebenden interviewten Personen nimmt das ,Kinder haben', also das Leben mit den Kindern, den wesentlichen Stellenwert ein. Elternschaft wird hier 
somit nicht über den Körper, den selbstreflexiven Teil des Selbst, konstruiert. Dies wird auch bestätigt durch die Aussagen, in denen gerade die Vorteile einer Adoption für eine gemeinsame gleichwertige soziale Elternschaft betont werden. Dennoch zeigte sich, dass auch im Kontext von Adoptionen durch eine „ungewollte Kinderlosigkeit“ das Selbst der Paare in eine Lebenskrise gerät und daran gearbeitet werden muss, die eigene Biografie weiter zu entwickeln bzw. die Kontinuität der eigenen Biografie und damit die Kontinuität der Selbst-Identität aufrecht zu erhalten. Auch hier besteht das Bestreben, eine Veränderung oder Erweiterung der eigenen Identität als Mutter oder Vater zu erreichen. Dennoch läuft der Prozess der Selbst-Reflexivität nicht primär über den Körper, was die deutliche Ablehnung der medizinisch-technischen Möglichkeiten zur Überbrückung der „Sterilität“ zeigt (Kap. 5.2.2.2; vgl. auch Wild 1998:273). Die Eigenwahrnehmung (die Erfahrung des Selbst) ebenso wie die Fremdzuschreibung (die Person) als ,Mutter' und ,Vater' findet im Fall einer Adoption über das soziale Handeln, über die Beziehung zur Umwelt und zum Kind statt.

Anhand meiner Darstellungen zeigt sich, wie situations- und positionsspezifisch Konzepte von ,eigenen“ Kindern sind und wie des weiteren eine systematische Auswahl der diskursiven Möglichkeiten je nach sozialem Kontext erfolgt. Ich habe herausgearbeitet, dass die dominante kulturelle Vorstellung von der Unauflösbarkeit der Beziehung zwischen biologischen Eltern und Kindern sowohl im Zusammenhang mit der Reproduktionsmedizin als auch bei Adoptionen und Pflegschaften immer wieder durchschimmert, jedoch abhängig von der persönlichen Betroffenheit und dem spezifischen Kontext eine unterschiedliche Gewichtung und Auswahl konstitutiver Elemente von Elternschaft erfolgt. So kann ein Element zum anderen führen oder gegen das andere ausgespielt werden und man kann entscheiden, inwieweit man diese Verbindungen zu beanspruchen oder abzulehnen wünscht (vgl. Edwards und Strathern 2000:159). ${ }^{206}$ Es ist jedoch in meiner Arbeit über Konstruktionsprozesse von Verwandtschaft offensichtlich geworden, dass leibliche Verwandtschaft als innigste und engste Form der sozialen Beziehung in Deutschland gilt. Im Zuge der reproduktionsmedizinischen Technologisierung gewinnt die genetische Elternschaft immer mehr an Gewicht, wobei die Aspekte der Anerkennung von Verwandtschaft, die mit geteilter Körpersubstanz zu tun haben, noch verstärkt werden, nicht zuletzt, weil die Genetik als Wissenschaft Vorstellungen von Blutsverwandtschaft weiterführt und verstärkt. So kann auch ein adoptiertes oder in Pflegschaft genommenes Kind einen „Kinderwunsch“ erfüllen, jedoch ist bei der Nach-

\footnotetext{
206 Als prägnantes Beispiel wird von Edwards und Strathern (2000:159) die Leihmutterschaft genannt, bei der die „Spende“, die „Spender“ und Empfänger verbindet, sie gleichzeitig auch trennt. Für meine Untersuchung in Deutschland lässt sich dieser Aspekt auf die „Samenspende“ übertragen. Eine potentielle zukünftige Beziehungskette und Ansprüche, die durch die Verbindung des Kindes zu dem „Samenspender“ entstehen könnten, werden abgeschnitten und für die Zukunft deaktiviert, indem eine Wahrheit (,biologisch') gegen die andere (,sozial') ausgespielt wird.
} 
frage nach den reproduktionsmedizinischen Verfahren der Wunsch nach einem „eigenen“ leiblichen Kind ausschlaggebend. Das dominante kulturelle Konzept vom ,eigenen“ Kind ist verknüpft mit dem Idealkonzept von Elternschaft, da die leiblichen Eltern über den biologischen Prozess der Fortpflanzung untrennbar mit dem Kind verbunden sind und nur diese Verbindung auch als emotional und rechtlich unauflöslich verstanden wird. Andere Formen von Verwandtschaft erfahren eine nachgeordnete kulturelle Bedeutung (vgl. Kap. 7.1), da sie sich nicht auf den ,natürlichen Fakten“ gründet. ${ }^{207}$ Daher fühlen sich auch Adoptiveltern oftmals als ,nur' soziale Eltern und damit gesellschaftlich zu Eltern zweiter Klasse degradiert, da in Deutschland die biologischen Eltern aufgrund der Abstammung auch weiterhin die Rechte als Geburtseltern behalten, auch wenn diese legal nicht anerkannt werden. ${ }^{208}$

\subsection{Die Konstruktion der Reproduktion}

Wie ich in meiner Untersuchung über Konstruktionen von Zugehörigkeit und Angehörigkeit, die als Verwandtschaft konzipiert werden, gezeigt habe, hängt das Konzept von Verwandtschaft als besondere Form sozialer Beziehungen in Deutschland aufs engste mit dem Körper zusammen. Durch den Austausch von Körpersubstanzen im Prozess der Fortpflanzung wird die biogenetische Identität zwischen Eltern und ihren Kindern hergestellt. Gleichzeitig wird die soziobiologische Einheit der Kernfamilie aus dem Paar mit seinen leiblichen Nachkommen begründet. Es ist deutlich geworden, dass der materielle Umgang mit Körpersubstanzen, wie sie in der reproduktionsmedizinischen Praxis vollzogen wird, daher kulturelle und gesellschaftliche Vorstellungen von Selbst/Identität, Person und sozialen Beziehungen, vor allem Verwandtschaft, berührt.

In der spätmodernen Gesellschaft (vgl. Giddens 1991:1) wird im biomedizinischen Körperverständnis der Körper als Objekt und damit als teilbar, formbar, reparierbar und abtrennbar vom subjektiv erfahrbaren Leib konzipiert. Andererseits wird er aber auch als geschlossene Einheit betrachtet, der nicht nur eine physische Größe, sondern auch

\footnotetext{
207 Wie ich in Kapitel 2.2.1 beschrieben habe, hat der englische Sozialanthropologe Jack Goody (1989 [1983]) nachgewiesen, dass sich im Verlauf von Jahrhunderten Vorstellungen und Bewertungen von leiblichen und nichtleiblichen Kindern in unserer Kultur verändert haben. Mit der Vereinheitlichung nach dem germanischen Berechnungssystem im 7. Jahrhundert setzte die Kirche eine Konstruktion von Blutsverwandtschaft durch, die die Deszendenz als Hauptprinzip betonte. Die Idee von Blutsverwandtschaft als „echte“ Verwandtschaft löste andere Erklärungsmodelle für soziale Beziehungen ab. Nicht-leibliche Verwandtschaft wie beispielsweise im Fall von Adoptionen und Pflegschaften erfahren somit eine andere kulturelle Bewertung.

208 Siehe dazu auch die Arbeit von Schmitt (2000) über Herkunftsmutterschaft in Deutschland, in der sie zeigt, dass Frauen, die ihr Kind zur Adoption freigegeben haben, sich zunehmend auf die Tatsache berufen, dass sie biologische Mütter ihrer Kinder seien. Im Zusammenhang mit offenen Adoptionen fordern sie verstärkt eine soziale Anerkennung als Geburtsmütter und betonen damit gerade die biogenetische Beziehung, die auch die Reproduktionstechnologien so hervorhebt. Als Folge der sozialen Anerkennung wollen sie allerdings auch eine ,anteilige soziale Mutterschaft" mit den Adoptivmüttern ihrer Kinder leben. Bisher ist dieser Fall, dass mehrere Frauen eine gesellschaftlich anerkannte soziale Mutterschaft ausüben, in Deutschland jedoch rechtlich nicht zulässig.
} 
verkörpertes Selbst ist (Kap. 2.3.3). In diesem Spannungsfeld einerseits der Teilbarkeit und Zusammensetzbarkeit von physischen Körpern und damit der Loslösbarkeit von Zellen und Gameten und andererseits der integralen Verkörperung eines leiblichen Verwandten arbeitet auch die Reproduktionsmedizin (vgl. Hauser-Schäublin 1998:65).

Mit meiner Analyse habe ich gezeigt, dass in der bundesdeutschen Gesetzgebung durch den Einfluss der Reproduktionsmedizin bisher keine Veränderungen von ,traditionellen“ Konzepten feststellbar sind, sondern dass vielmehr bestehende Konzepte von ,natürlicher" Verwandtschaft bestätigt und verstärkt werden. ${ }^{209}$ Die neuen Fortpflanzungsmöglichkeiten werden in ,alte' Modelle integriert, und besonders im persönlichen Diskurs werden Teilaspekte im Sinne der alten Ideale umkomponiert sowie ,Dissonanzen` geglättet, was ich im Zusammenhang mit der Analyse eines ,eigenen“ Kindes (5.1) und von Zeugungskonzepten (Kap. 6) darlegen konnte. Das biomedizinische Körpermodell, das von der prinzipiellen Teilbarkeit und Reparierbarkeit des Körpers ausgeht, beinhaltet auch die Überzeugung der Loslösbarkeit von Ei- und Samenzellen sowie von Embryonen vom Körper und ihre Neuzusammensetzbarkeit und Manipulierbarkeit. Dieses Körperkonzept macht die Anwendung und Nutzung der Reproduktionstechnologien überhaupt erst möglich und muss daher sowohl von Seiten der Ärzte (zumindest kraft ihres Amtes) sowie von den ,ungewollt kinderlosen“ Paaren in der konkreten medizinischen Praxis anerkannt werden. In Kapitel sechs konnte ich jedoch zeigen, dass diese Reduzierung der ,Zeugungsbeiträge' auf eine reine Körpersubstanz nur möglich ist, in dem ,traditionelle' Vorstellungen von Zeugung, die an eine körperliche und leibliche Paarbeziehung geknüpft sind, modifiziert werden. Mit der Konstruktion des biomedizinischen Körperkonzeptes lassen sich leibliche Erfahrungen und Empfindungen nicht erklären. Anhand meines empirischen Materials sind die Widersprüche und Brüche deutlich geworden, die das Zusammentreffen von reproduktiven Technologien und deren Konzentration auf den Körper als Objekt von Behandlungen und der Leiblichkeit der betroffenen Paare mit sich bringen. Unter den Bedingungen der Reproduktionstechnologien verliert der Geschlechtsakt, der nach Schneider (1980 [1968]:40) das zentrale Symbol der amerikanischen Verwandtschaftsvorstellung darstellt und die Einheit der Familie schafft und definiert, seine Gültigkeit, da nicht mehr Zeugung im Sinne eines Geschlechtsaktes, sondern das konkrete Zusammenführen von Körpersubstanzen eines Paares und deren Verschmelzung als konstituierend für Leiblichkeit definiert wird (vgl. auch Franklin 1995). Die Angleichung der „künstlichen“ an die „,natürliche“ Zeugung wird auch offenbar in den verwendeten sprachlichen Begrifflichkeiten, da es in der konkreten reproduktionsmedizinischen Praxis um „Befruchtung“ und damit die körperli-

\footnotetext{
209 Wobei sich auch gezeigt hat, dass gerade die heterologe Insemination ,traditionelle“ Muster und Wertvorstellungen aufgreift, in dem sie insbesondere die dadurch mögliche Schwangerschaft der Frau betont und den Wertzusammenhang der ideologischen und strukturellen Einheit einer Kernfamilie festigt (vgl. auch Haimes 1990:154-173).
} 
chen Prozesse, und nicht primär um „Zeugung“ als leiblich-körperliche Erfahrung geht (Kap. 6.1). „Natürliche“ Befruchtung beschreibt den genetischen Befruchtungsvorgang, der nach Meinung der Mediziner bei der „,künstlichen“ ebenso unangetastet bleibt wie bei der „natürlichen“ Befruchtung. Mit dieser Einschätzung greifen technologische Verfahren demnach nicht in ,natürliche“ Vorgänge ein. Sie produzieren „,natürliche“ Vorgänge mit Hilfe der Technik und lassen sich somit in den reproduktiven Prozess einbinden; „Künstlich“ herbeigeführt werden nur die Voraussetzungen für eine „natürliche“ Befruchtung, die durch das Zusammenkommen von Ei- und Samenzellen stattfindet. ${ }^{210}$ ,Natur' wird zunehmend zum Synonym mit Biologie, der zwar eine eigene generative Macht zugesprochen wird, die jedoch durch die Reproduktionstechnologien unterstützt werden kann (und muss). Hier wird die Herstellung eines Kontinuums zwischen normaler „Fruchtbarkeit“ und „Unfruchtbarkeit“" in einer Weise vollzogen, die die Notwendigkeit der wissenschaftlichen Assistenz menschlicher Reproduktion offenbart. Mit Bezug auf Strathern (1995:349) kann hier konstatiert werden, dass die für westliche Gesellschaften so zentralen kulturellen Annahmen, dass „,natürliche Fakten“ die Grundlagen für menschliche Beziehungen bilden und Verwandtschaft als Resultat „natürlicher Fakten" durch Zeugung und Geburt verstanden wird, Einfluss nehmen auf den Umgang mit den Reproduktionstechnologien. Gleichzeitig findet jedoch unter den Bedingungen der Forschung und Anwendung der reproduktiven Technologien eine zunehmende Konzentration auf körperliche (biogenetische) Prozesse (= Befruchtung und Verschmelzung) statt, so dass ,natürliche Fakten“ als getrennte Einheiten betrachtet werden können, ohne Bezug zu nehmen auf soziale Beziehungen (= Zeugung).

In diesem Zusammenhang möchte ich auf dem Hintergrund kulturvergleichender Studien zu Vorstellungen von Verwandtschaft und Zeugung (vgl. auch Kap. 0) hier noch einmal einen meiner Ansicht nach zentralen Aspekt der kulturspezifischen Konstruktion von Zeugung herausstellen, der sich aus meiner Untersuchung unter den Bedingungen der Reproduktionstechnologien in Deutschland herauskristallisiert hat. So zeigen nichtwestliche Zeugungstheorien in erster Linie soziale Beziehungen auf, in die ein Kind hineingeboren wird und an denen es in besonderer Weise durch Rechte und Pflichten teilnimmt (vgl. Hauser-Schäublin 1991, 1995, Strathern 1992a, 2001). Wird Geschlechtsverkehr direkt mit Fortpflanzung in Verbindung gebracht, findet sich oftmals die Auffassung, dass nicht nur ein sexueller Akt, sondern eine kontinuierliche sexuelle

\footnotetext{
210 Mit der „Re-Naturalisierung“ der neuen Formen reproduktiver Technologien beschäftigt sich auch Franklin und stellt fest: „, The instrumentalisation of conception [...] indexes the changing meanings of the 'facts of life' in such a context" (1997:10). Eine feministische Perspektive auf die Auswirkungen neuer Technologien für das Verständnis menschlicher ,Natur' und den Naturbegriff der heutigen Wissenschaften liefern auch die Arbeiten von Haraway $(1995,1997)$. Dabei kritisiert sie insbesondere den Rekurs auf ,Natur' als ethische Orientierung. Ein wichtiger Einwand ist, dass ,Natur' keine Konstante, sondern eine soziale Kategorie darstelle, und immer in einer historisch, kulturell und patriarchal geprägten Form vorliege (siehe auch Duden 1987, Bayertz 1987).
} 
Beziehung die Voraussetzung für die Entstehung eines Kindes bildet. Hauser-Schäublin (1995:173) hat gezeigt, dass dies mit Vorstellungen darüber zusammenhängt, dass für den Aufbau des Kindes eine Akkumulierung von Körpersubstanzen, meist als Blut oder Sperma bezeichnet, notwendig ist. Gleichzeitig, so konstatiert sie, werde damit symbolhaft die Notwendigkeit einer kontinuierlichen (sozial anerkannten) Beziehung zwischen einem Mann und einer Frau für die Zeugung eines Kindes ausgedrückt. Basierend auf dem kulturell dominanten biomedizinischen Zeugungsmodell (Kap. 2.2.2) wird in euroamerikanischen Zeugungsvorstellungen die Entwicklung des Embryos jedoch als ein spontaner Prozess betrachtet, der auf den Augenblick der Empfängnis folgt, für den ein einziger Geschlechtsakt genügt. ${ }^{211}$ Das Wachstum des Kindes ist Teil eines biologischen Prozesses, der seinen eigenen Verlauf nimmt. Das Erscheinen des Kindes demonstriert nur die ursprüngliche Vereinigung der elterlichen Gameten. Mit der Verschmelzung der Gameten beider Elternteile ist ein neues Individuum als haut- und körpergebundene Einheit entstanden, dessen genetische Identitätsbildung abgeschlossen ist (siehe auch Petersen 2000:53-56). Das Individuum wird somit von Individuen geboren, zu dessen Entstehung zwar eine Beziehung notwendig ist, die jedoch darüber hinaus nicht andauern muss. So stellt Strathern für den englischen Kontext fest, was sich auch auf Deutschland übertragen lässt: „The child was there, according to this view, as an outcome of the acts of other individuals, whatever relations they may claim afterwards. From such a perspective, then individuals reproduce individuals [....] Relationships, in this English mode, were not reproduced in the very act of procreation (1992a:53; Hervorhebung im Original). Im Vordergrund dieser Vorstellung von Zeugung steht die Betonung des Individuums und dessen körperliche Konstituierung aus einem Set von elterlichen Genen. Diese körperlichen ,Interaktionen` zum Zweck der Erzeugung eines Kindes können durch die reproduktiven Technologien gänzlich von sozialen Beziehungen gelöst werden und erlauben, wie ich zeigen konnte, gerade das Umgehen von Beziehungen (Kap. 6.1.2, 6.3).

Die Reproduktionstechnologien arbeiten, so ist in meiner Arbeit deutlich geworden, im Spannungsfeld von einerseits der Loslösung von Zellen und Gameten von Fragen nach sozialen Beziehungen und andererseits dem im individuellen Leib symbolisch verorteten Blutsverwandten. Die spezifische Problematik der Nutzung dieser Technologien besteht darin, dass die „ungewollt kinderlosen“ Paare das Ziel haben, „natürliche“ Verwandtschaft, die sich über geteilte Körpersubstanz konstituiert, durch ein leibliches Kind zu schaffen. Die reproduktionsmedizinischen Verfahren behandeln jedoch primär

\footnotetext{
211 Siehe dazu Strathern (1999:78f): "However, the idea that the biological tie can be created in a moment, through the conception at a single point in time, also appears to be of another order of fact from the continuing nurture of the child. It belongs to a natural order open to scientific investigation. It has the character of a constitutive finality that cannot be modified, that once known cannot be laid aside."
} 
den Körper als Objekt und klammern den Leib als individuelles subjektives Empfindungs- und Wahrnehmungsorgan aus. Es hat sich gezeigt, dass gerade hier die besondere Schwierigkeit für die Paare liegt, da der Körper eben nicht nur Materie, sondern gleichzeitig Leib und verkörpertes Selbst ist. Um mit dieser Gratwanderung umzugehen, lassen sich bestimmte Mechanismen feststellen, die medizinisch-technischen Behandlungen, mit deren Hilfe zwar leibliche Kinder entstehen können, deren Erschaffung jedoch unter Beteiligung zahlreicher Personen durch ausschließliche Eingriffe am Körper stattfindet, aus der körperlichen und leiblichen Paarbeziehung herauszuhalten. Werden im homologen System die an der ,Zeugung' beteiligten Personen wie die Ärzte in sozialer Hinsicht ausgeblendet, d.h. sie haben keinen Anteil an der sozialen Elternschaft, spielt jedoch bei der Methode der heterologen Insemination die Körpersubstanz einer dritten Person eine Rolle. Diese Person leistet zwar dem biomedizinischen Modell zufolge einen Zeugungsbeitrag, übernimmt jedoch keinen sozialen Anteil an der durch seine Körpersubstanz geschaffenen Verwandtschaft. Mit Hilfe der Körper-LeibTerminologie war es mir möglich, die besondere Problematik dieser Behandlungsmethode aufzuzeigen, wie sie zwischen Paaren und Ärzten nicht thematisiert wird (und auch nicht in der Paarbeziehung, wie meine Interviews vermuten lassen). Das Paar kommt in die Klinik mit dem Ziel, ein leibliches Kind zumindest über ein Elternteil zu schaffen und die soziale Akzeptanz des Kindes als Ausdruck eines reproduktiven Prozesses des Ehepaares durch das soziale Umfeld zu erreichen, d.h. mit den verschiedensten Mitteln soll Leiblichkeit sozial konstruiert werden. Obwohl die biogenetische Verbindung einen zentralen Aspekt für die Nutzung der heterologen Insemination ist, wird er im Prozess der Fortpflanzung gleichzeitig heruntergespielt oder ausgeblendet bzw. es werden die Aspekte hervorgehoben, die mit der dominanten Verwandtschaftsideologie und ,traditioneller' Reproduktion übereinstimmen. Die Paare entwickeln Strategien, um das Ungleichgewicht der Beziehung auszugleichen, indem sie die soziale Vaterschaft besonders betonen und höher wertschätzen als die biologische Verbindung zum „Spender" und diese ausblenden. ${ }^{212} \mathrm{Zu}$ diesem Zweck muss eine soziale Dekontextualisierung, d.h. eine konzeptuelle Loslösung der Zeugungsbeiträge von sozialen Beziehungen, und eine Reduzierung auf den Körper erfolgen, was sich in bestimmten Situationen jedoch (beispielsweise dem Zeitpunkt der Insemination, siehe Kap. 6) als besonders schwierig erweist. Insbesondere im Zusammenhang mit der heterologen Insemination offenbart sich somit das kulturelle Paradox zwischen der Betonung der Biologie und der biogenetischen Prozesse einerseits als „,natürliche Fakten“, die die Grundlage von Vorstellungen über „,natürliche“ Verwandtschaft in der Ideologie bilden, und andererseits der Versuch, diese „natürlichen“ Vorgänge als Grundlage für Beziehungen, die sich aus

${ }^{212} \mathrm{Zu}$ ähnlichen Strategien und Mechanismen im Umgang mit asymmetrischer Elternschaft für den Fall von Leihmutterschaft in den USA siehe Ragoné (1994). 
der Fortpflanzung ergeben, in der reproduktionsmedizinischen Praxis herunterzuspielen und die genetischen Zeugungsanteile des „Spenders“ auszublenden (vgl. auch Strathern 1995:359). ${ }^{213}$

Auf die kulturelle Konstruktion des „Kinderwunsches“ habe ich schon an anderer Stelle die ethnologische Brille gerichtet ${ }^{214}$, doch möchte ich hier noch einmal auf den kulturspezifischen Umgang mit „Sterilität“ bzw. ,ungewollter Kinderlosigkeit“ eingehen, da, wie ich gezeigt habe, gerade in diesem Bereich Körper und Selbst fundamental miteinander verbunden sind. Durch die gesellschaftliche Anerkennung von „Kinderlosigkeit“ als ,Krankheit‘ in Deutschland (Kap. 4.3.1.1) wird auch deren Behandlung staatlich legitimiert und von den Krankenkassen nach medizinischer Indikation finanziert. Mit der Durchsetzung der reproduktiven Technologien wurden immer mehr ,Auswahlmöglichkeiten“ zur Überwindung der ,ungewollten Kinderlosigkeit“ über Eingriffe am Körper geschaffen. Primär wird dabei Bezug genommen auf das psychische Leid der Betroffenen, dass durch den Einsatz der Medizin überwunden werden könnte. ${ }^{215}$ Diese ,Wahlmöglichkeiten', die im Zusammenhang mit den Reproduktionstechnologien propagiert werden, scheinen jedoch eher dazu beizutragen, dass alternative Umgangsformen mit „ungewollter Kinderlosigkeit“ nur sehr schwer entwickelt werden können. Die ,Qual der Wahl' führt zu einem Gefühl der Verpflichtung, so dass ,ungewollt kinderlose“ Paare sich erst zur Akzeptanz der Kinderlosigkeit berechtigt fühlen, wenn sie alle medizinischen Möglichkeiten ausgeschöpft haben (siehe auch Kap. 5.1.4.2). Heutzutage muss sich ein „ungewollt kinderloses“ Paar (und insbesondere eine „ungewollt kinderlose“ Frau) legitimieren, wenn es die Methoden der Reproduktionstechnologien nicht nutzt. So schildern von mir befragte Frauen, dass sie reproduktionsmedizinische Behandlungen in Anspruch genommen haben, um sich nicht irgendwann vorwerfen zu müssen, sie hätten nicht alles versucht. In Anlehnung an Giddens (1991:220) lässt sich feststellen, dass die biologische Reproduktion in der spätmodernen Gesellschaft somit

\footnotetext{
213 In zahlreichen außereuropäischen Konstruktionen von Zeugung und Fortpflanzung, in denen die Vorstellung dominiert, dass neues menschliches Leben nur durch häufigen Geschlechtsverkehr entstehen kann, da das Kind im Mutterleib durch die vereinigte Körpersubstanz von Mann und Frau allmählich aufgebaut wird, gilt derjenige Mann als genitor des Kindes, der mit der Mutter häufig und regelmäßig Geschlechtsverkehr hatte, und nicht etwa ein anderer Mann, mit dem sie vielleicht nur ein einziges Mal Verkehr hatte - auch wenn letzterer vielleicht der genetische Vater des Kindes ist. Genitor und genetrix sind demnach diejenigen Personen, die aufgrund von kulturspezifischen Zeugungs- und Empfängnistheorien den Status der physischen Eltern innehaben (vgl. auch Barnes 1973). Genetischer Vater und genitor können, müssen aber demnach nicht identisch sein. Anhand meiner Darstellungen lässt sich konstatieren, dass eine Unterscheidung dieser Art im kulturell dominanten Verwandtschaftsdiskurs in Deutschland nicht möglich ist.

214 Siehe dazu Hauser-Schäublin et al. (2001:228-232).

215 Wobei diese Auswahlmöglichkeiten in Deutschland nur bestimmten Personengruppen wie heterosexuellen ehelichen und in Ausnahmefällen nicht-ehelichen Paaren zur Verfügung stehen und somit auch nur in diesen Fällen eine Anerkennung des psychischen Leidens an „ungewollter Kinderlosigkeit“ erfolgt (siehe auch Kapitel 7.2).
} 
zu einer individuellen Entscheidung am individuellen autonomen Körper wird. ${ }^{216}$ Die Berufung auf die ,Natürlichkeit“ und ,Selbstverständlichkeit“ des „Kinderwunsches“ bekräftigt überdies die Idee eines ,Naturrechts' auf Kinder. Über dieses ,Naturrecht` bezieht auch die Reproduktionsmedizin als ,Heilmethode“ ihre Legitimation, wobei sie letztlich die Ursachen nicht heilt, sondern nur zur Überbrückung der , ungewollten Kinderlosigkeit" beiträgt. ${ }^{217}$ Die Gesellschaft stellt den betroffenen Paaren zwar die (medizinischen) Verfahren für eine „Kinderwunsch-Behandlung“ zur Verfügung, überlässt jedoch den Umgang damit den Einzelnen selbst. ${ }^{218}$ In Kapitel 2.3.3 habe ich gezeigt, dass im Diskurs über den Körper in der westlichen Gesellschaft das Selbst einerseits als vom Körper getrennt betrachtet wird, d.h. der Körper kann unabhängig vom Selbst bearbeitet und verändert werden, und andererseits Körper und Selbst als untrennbar miteinander verbunden konzipiert werden. Dem westlichen Konzept des individuellen Selbst, in dem das Selbst fest einem Körper zugeordnet ist, liegt auch das Konzept eines autonomen individuellen Selbst zugrunde und der Vorstellung der eigenen Handlungsfähigkeit (agency). Dieses Konzept von persönlicher Autonomie hinsichtlich Auswahl und Entscheidungen sowie dem Anspruch auf eigene Kontrolle spiegelt sich auch im gesellschaftlichen Umgang mit biologischer Reproduktion wider (vgl. Giddens 1991:219-226). ${ }^{219}$ So sind die von mir im Kontext der Reproduktionsmedizin befragten Paare übereinstimmend der Meinung, die Medizin biete ihnen eine wirkliche Wahlmöglichkeit und ermögliche ihnen, aktiv an der Überwindung ihrer ,ungewollten Kinderlosigkeit" mitzuarbeiten und nicht nur passiv zu warten wie im Fall von Adoptionen (Kap. 6.2.3).

Die Inanspruchnahme der Fortpflanzungsmedizin stellt jedoch nicht nur eine individuelle, von gesellschaftlichen Zusammenhängen losgelöste Handlung dar. Sie trägt vielmehr aktiv zur weiteren Verbreitung und zur Akzeptanz der vorhandenen Technologien als Notwendigkeit bei. Hier zeigen sich auch die Konsequenzen eines mechanistischen

\footnotetext{
216 Siehe dazu Strathern (1992c:156), die anhand eines Beispieles aus Melanesien die spezifische euroamerikanische Vorstellung von Zeugung über körperliche Prozesse hervorhebt: „In Melanesia, for instance, much cultural effort can be expended on making sure that persons conceive in the right relational context: infertility may be attributed to deficiencies in social relations, and facilitation will then attend to people's intentions in the matter. By contrast, Euro-Americans find nothing exceptional in the possibility of facilitating the physical process, an operation regarded as independent of personal or social identity.“

217 Die neuen Techniken der Fortpflanzungsmedizin werden nach Kettner (2001:35) auch immer häufiger als medicine du désir angesehen, d.h. als wunscherfüllende (statt Krankheiten behandelnde) Medizin.

218 So wird propagiert, dass die reproduktionsmedizinische Praxis Frauen und Männern bei der ,Erfüllung` des „Kinderwunsches“ hilft. Wenn die medizinischen Maßnahmen allerdings durchgeführt worden sind - egal, ob ein Kind daraus resultiert oder nicht - ist der medizinische Part am Ende. Die Paare werden danach nicht weiter betreut. Dass sie dann jedoch nicht weniger ,kinderwunschkrank' sind, hat mein empirisches Material gezeigt (Kap. 5.1.4.2, 6.2.3).

219 Dazu auch Strathern (1996:47): „Choice is a significant value to which Euro-Americans give weight in setting up families, and IVF and associated procedures widen the choice that is available to potential parents, regardless of their fertility [...].“
} 
Körperverständnisses und einer Reduzierung von „Unfruchtbarkeit“ und „Fruchtbarkeit“" auf den (weiblichen) Körper als Objekt von Behandlungen. Ebenso wie die gewollte „Unfruchtbarkeit“" bei der Kontrazeption scheinbar beeinflusst und bestimmt werden konnte, so soll nun die „Fruchtbarkeit“ nach der Beendigung der ,gewollt kinderlosen“ Phase wieder hergestellt werden. Durch diesen entpersonalisierten Diskurs und die damit verbundene Loslösung vom Leib wird die Idee von der Plan- und Machbarkeit der menschlichen Fortpflanzung verstärkt, die eine fortschreitende Medikalisierung der „Unfruchtbarkeit“ sowie die wachsende Kontrolle und ,Verrechtlichung“ des „Kinderwunsches“ (der aufgrund seiner ,Natürlichkeit‘ keiner Erklärung bedarf) beinhaltet. ${ }^{220}$ Deutlich wird dies auch an der zunehmenden Verstärkung der gesellschaftlichen Stigmatisierung von „Unfruchtbarkeit““ als etwas ,Unnatürliches“, das von der idealen gesellschaftlichen Norm der Kernfamilie abweicht. Durch meine Untersuchung sind die sozialen Dimensionen der ,ungewollten Kinderlosigkeit“" deutlich geworden: Einerseits die individuell empfundene Enttäuschung, die Paarbeziehung nicht um ein gemeinsames Kind erweitern zu können und somit auch einer Eltern-Kind-Beziehung als Teil der idealen Vorstellung einer Kernfamilie als kleinsten ,natürlichen“ Verwandtschaftseinheit nicht entsprechen zu können. Ein weiterer wesentlicher Aspekt ist die Stigmatisierung insbesondere kinderloser Frauen in unserer Gesellschaft. So zeigen die Interviews mit Frauen, dass es auch heute noch schwer ist, als Frau, ohne Mutter zu sein, ein ,erfülltes‘ Leben zu führen (Kap. 5.1.4.2). Gleichzeitig erfährt die Idee eines „natürlichen Kinderwunsches“ der Frauen eine neue Bewertung. Dies zeigt auch der neue Bedeutungsgewinn von „Fruchtbarkeit“ und Mutterschaft durch den Rückgriff auf bzw. die Verstärkung von Kriterien wie Schwangerschaft und Geburt für die Definition von Mutterschaft in Deutschland im Kindschaftsreformgesetz von 1998 (Kap. 4.1.3).

Wenn man in Betracht zieht, dass in unserer Gesellschaft ein verheiratetes Paar ohne Kinder ,noch nicht wirklich eine Familie ausmacht“ (Kap. 4 und 5.1) und dass ,Kinder bekommen“ als ein „,natürlicher“ und „normaler“ Wunsch anerkannt wird, der auf einem ,Naturrecht` des Menschen zur Fortpflanzung basiert, dessen Nicht-Erfüllung zu einem erheblichen Leidensdruck führt, dann ist es durchaus nachvollziehbar, was ,unfruchtbare“ Paare antreibt, alle in Deutschland zulässigen Möglichkeiten reproduktionsmedizinischer Behandlungen zu nutzen, um ein Kind zu bekommen. Durch die Überbrückung der „Sterilität“ kann ein Paar in eine Familie transformiert werden und sich dadurch

\footnotetext{
${ }^{220}$ Hier wird der Aspekt der Plan- und Machbarkeit des „Kinderwunsches“ deutlich, dem ich bei meiner Untersuchung immer wieder begegnet bin. Siehe dazu auch Lundin (1996:19): ,[...] people's ideas about families and parenthood as being decreed by fate are interwoven with the idea that reproduction can be planned, that we are free to choose by ourselves." Ich möchte auch auf die Diplomarbeit von Eva-Maria Knoll (2001) verweisen, die sich mit dem österreichischen IVF-Diskurs aus ethnologisch-feministischer Perspektive beschäftigt hat. Sie hat festgestellt, dass auf allen von ihr untersuchten Diskursebenen vom Prinzip der Planbarkeit und Machbarkeit in Bezug auf den „Kinderwunsch“ ausgegangen wird.
} 
wieder in die dominante Verwandtschaftskonstruktion einfügen. Der in Kapitel fünf deutlich gewordene intensive Wunsch nach einem „eigenen“ leiblichen Kind lässt sich auch in Bezug setzen zu der zunehmenden Genetisierung der spätmodernen Gesellschaft, da die genetische Identität eines Menschen eine immer größere Gewichtung erfährt. ${ }^{221}$ Mit der Vorstellung, Identität, Individualität und Persönlichkeitsentwicklung seien genetisch begründet, wird die biologische Verbindung zwischen leiblichen Eltern und Kindern im Verhältnis zu den Einflüssen sozialer Interaktionen und Institutionen immer wichtiger. ${ }^{222}$ Die Betonung von Familie und der biogenetischen Grundlage von Verwandtschaft bleibt im rechtlichen Diskurs, der direkte Handlungsanweisungen gibt, unverändert und wird im Kontext der Reproduktionsmedizin noch verstärkt. ${ }^{223}$ Außer im Zusammenhang mit der Methode der heterologen Insemination entstanden keine neuen Diskurse als Resultat der Auseinandersetzung mit den neuen Technologien in Deutschland $^{224}$, da sich alle Behandlungsmethoden im homologen System - und die dadurch geschaffenen biologischen und sozialen Zugehörigkeiten - in den dominanten Verwandtschaftsdiskurs integrieren lassen. Es ist deutlich geworden, dass das erklärte Ziel die Schaffung von Blutsverwandtschaft bzw. Leiblichkeit ist; Dort wo sie körperlich nicht möglich ist, wird sie kulturell konstruiert (vgl. Hauser-Schäublin 1998:65). Gleichzeitig erfolgt mit Hilfe der medizinischen Verfahren die Verfestigung und Absicherung der ,naturalisierten“ sozialen Form der Kernfamilie. Zu diesem Zweck werden von allen Personen, die direkt oder indirekt mit den Reproduktionstechnologien zu tun haben, diejenigen Aspekte betont, die der dominanten Verwandtschaftsideologie in Deutschland entsprechen und diejenigen Aspekte, die mit dieser nicht übereinstimmen, ausgeblendet. ${ }^{225}$ Dadurch wird die Konstruktion von „,natürlicher“ Verwandtschaft in der Ideologie, die sich über Leiblichkeit konstituiert, um eine zweite Konstruktion erweitert, die die körperlichen (biologischen) Komponenten immer mehr ins Zentrum von „Verwandtschaft" rückt.

\footnotetext{
221 Siehe dazu auch den Artikel von Finkler „The Kin in the Gene“, in dem sie sich mit der Medikalisierung von Familie und Verwandtschaft in der amerikanischen Gesellschaft beschäftigt und der Auffassung ist, ,that the emphasis placed on genetic transmission elaborates on the American folk category of bilateral kinship" (2001:247).

222 Siehe dazu auch Coester (1983:354): „Wo das biologische Abstammungsverhältnis in den Mittelpunkt rückt, spielt neben dem Kindeswohl das Recht der leiblichen Eltern eine Rolle.“

${ }^{223}$ Ich stimme Kettner in seiner Auffassung zu, dass derzeitig der Wunsch kinderloser Paare nach einem eigenen Kind ausschließlich adultozentrisch befriedigt wird (2001:39), obwohl das „Kindeswohl“ als Begründung herangezogen wird. Seiner Ansicht nach lässt sich dies daran belegen, dass das Adoptionswesen - im Vergleich zu Aufwand und Aufmerksamkeit bezüglich der Fortpflanzungsmedizin - in politischer, juristischer und ethischer Hinsicht unglaublich vernachlässigt werde.

${ }^{224}$ So gibt es rezente Entwicklungen in den politischen und rechtlichen Diskussionen über eine Aufdeckung der Identität des ,Spenders“.

${ }^{225}$ Mit der Bildung von Familien durch die heterologe Insemination und der besonderen Problematik hinsichtlich deren Geheimhaltung und der Frage nach Anonymisierung von „Samenspendern“ beschäftigen sich u.a. detaillierter Delaisi de Parseval/Janaud (1986 [1983]); Hoffmann-Riem (1989); Haimes (1992); Daniels/Haimes (1998); Thorn (1999).
} 
Meine Studie zeigt ein Bild von „Verwandtschaft“ unter den Bedingungen der Reproduktionstechnologien in Deutschland, das nicht ohne Bezug zur sozialen Realität betrachtet werden kann. Die gelebte Praxis mit ihrer Dynamik und Variabilität von Beziehungen bricht sich zunehmend an dem idealen Konzept der biologischen Fundierung einer auf Dauer zusammenlebenden Kleinfamilie. Zwar wachsen die meisten Kinder nach wie vor in einer Familie auf. Diese ist aber nicht notwendigerweise die zeitlich überdauernde, soziobiologische Kernfamilie aus Eltern und den von ihnen abstammenden Kindern. Im Zuge hoher Scheidungsraten findet ein ständiges Auflösen und Neuzusammensetzen von Familien statt und andere Familienformen wie beispielsweise EinEltern-Familien, Stieffamilien oder sogenannte Patchwork-Familien, in denen Kinder unterschiedlicher Eltern zusammenleben, haben die traditionelle Kleinfamilie ergänzt. Dennoch zeigt sich unter den Bedingungen der Reproduktionstechnologien eine neuerliche Betonung des biologischen Abstammungsverhältnisses für Eltern-KindBeziehungen als Mittel zur Verfestigung ,natürlicher“ Familienbande, unabhängig von sozialen Sachverhalten (vgl. Stacey 1992a:37f). Mit Bezug auf Lucke (1998:70) lässt sich feststellen, dass Sozialverwandtschaften trotz ihrer faktischen Belastbarkeit und realer biografischer Existenz sowie praktischer Relevanz keine gesetzliche Legitimation erhalten, ebenso wenig wie sie die juristische Gleichstellung mit der vom Recht anerkannten Legalverwandtschaft erlangen. In einer Phase, in der persönliche Zugehörigkeiten und Angehörigkeiten häufig wechseln können, scheint damit versucht zu werden, in der Heterogenität der neuen Familienkonstellationen einen Ordnungsfaktor mit Bestandkraft zu fixieren. Dieser liegt gerade nicht, wie ich zeigen konnte, auf einer besonderen Gewichtung der sozialen Verwandtschaft, sondern es erfolgt eine zunehmende Genetisierung und die Betonung der genetischen Determiniertheit des Individuums. ${ }^{226}$ Die Naturwissenschaften der Biologie oder Genetik als symbolische Repräsentationen von Verwandtschaft eröffnen ein neues Verständnis über Konzepte von Verbundenheit. So unterstützen die Reproduktionstechnologien die Vorstellungen von Elternschaft als Konsequenz aus dem biologischen Prozess der Zeugung, was auch eine öffentliche Anerkennung und Bekräftigung des biologischen Prozesses als fundamental für Elternschaft beinhaltet. In diesem Zusammenhang bleibt auch die Idee, dass soziale und/oder rechtlich definierte Beziehungen biologischen Bindungen gleichgesetzt werden könnten, gegenwärtig eine Fiktion. Eine Veränderung der dominanten kulturellen Konstruktion von Verwandtschaft in Deutschland könnte unter den gegenwärtigen gesellschaftlichen Rahmenbedingungen eher die weitere Verbreitung sozialer Familienbeziehungen wie

\footnotetext{
${ }^{226}$ Siehe dazu Coester-Waltjen, Gutachterin des 56. Juristentages: „Betrachtet man [...] die Umstände, die zum Phänomen Elternschaft beitragen, so kommt den Keimzellen in zunehmenden Maße als einzigem Element die Rolle der ,conditio sine qua non' zu: Ohne die Keimzellenverschmelzung kann kein Kind und damit keine Eltern-KindBeziehung entstehen. Alle anderen Elemente wie Schwangerschaft, Geburt, soziale Fürsorge sind - zumindest in nächster Zukunft - für die Begründung, wenngleich nicht für den Bestand der Eltern-Kind-Beziehung, eleminierbar“" (1986:85).
} 
Adoptiv-, Pflege- oder Stieffamilien als die Anwendung der Reproduktionstechnologien bewirken. So könnten beispielsweise durch offene Adoptionen ,neue‘ Familienformen mit gesellschaftlich anerkannter anteiliger Elternschaft geschaffen werden, was das Aufbrechen kernfamiliärer Konzepte beinhalten würde. ${ }^{227}$

\section{Ausblick}

Es ist in meiner Arbeit deutlich geworden, wie eng Körper, Selbst/Identität, Person und soziale Beziehungen, vor allem Verwandtschaft, miteinander verknüpft sind. Eine Personengruppe wurde dabei jedoch ausgelassen: die „Samenspender“. So konnte ich nur über sie schreiben, sie selbst kamen nicht zu Wort. Dieser Umstand liegt in ihrer kompletten sozialen Ausblendung begründet. Es war mir nicht möglich, nähere Informationen über die „Spender“ zu bekommen, geschweige denn persönlich Kontakt zu ihnen aufzunehmen. Die Ärzte verweigerten mir sämtliche Auskünfte mit dem Hinweis auf die den „Samenspendern“ zugesicherte absolute Anonymität. So bilden die „Samenspender“ in Deutschland eine Personengruppe, von deren Existenz man zwar weiß, die jedoch nirgends auftauchen. Aufgrund der von mir herausgearbeiteten kulturellen Konstruktion von Verwandtschaft unter den Bedingungen der Reproduktionstechnologien in Deutschland, unter denen die biogenetische Verbindung zwischen Eltern und Kindern eine verstärkte Betonung und Wertigkeit erfährt, stellen sich mir Fragen nach den Vorstellungen von Verwandtschaft der „Samenspender“, die, obwohl zwischen ihnen, der Empfängerin des Spermas und dem gezeugten Kind körperliche Beziehungen bestehen, nicht in das bestehende Verwandtschaftsmodell integriert werden. Mit Hilfe der Reproduktionsmedizin haben sich „Samenspender“ biogenetisch fortgepflanzt, Nachkommen erzeugt und somit „natürliche“ Verwandtschaft geschaffen, legal und als Person im sozialen Handeln und in sozialen Beziehungen gelten sie jedoch weiterhin als kinderlos. Obwohl eine enge Verbindung von Körper, Selbst und Identität existiert, macht nur die (biomedizinische) Betrachtung des Körpers als losgelöst vom Selbst es möglich, sich von Teilen des Körpers wie beispielsweise Samenzellen zu trennen, ohne dass das Selbst davon betroffen sein muss. Möglicherweise würde eine empirische Untersuchung mit „Samenspendern“ vor diesem Hintergrund eine neue oder veränderte Perspektive eröffnen auf die Betrachtung der komplexen Verhandlungen von Konzepten über Kör-

\footnotetext{
227 Dazu möchte ich abschließend aus der Untersuchung von Modell (1994) über Adoptionen in Amerika zitieren, in der meine Überlegungen explizit aufgegriffen und unterstützt werden: ,[...] the degree of choice offered by open adoption cuts at deep-rooted convictions about the inevitability, the imperative, of parenthood. Carried to a logical end, open adoption 'opens' concepts of parenthood, of mother and father, and of family - thus of kinship altogether. If people who are unrelated, and not otherwise 'bound' to one another, share a child, what does that do to the notion of being a parent? If 'mother' includes the woman who bears but does not raise a child and, with equal significance, the woman who raises but has no birth tie to the child, then 'mother' is no longer absolute - or absolutely linked with nature. A family composed of people with a common interest in a child, a blended family, is not the same as a family extended by genealogy and by marriage“" (Modell 1994:236; Hervorhebungen im Original).
} 
per, Leib, Selbst und soziale Beziehungen im Zusammenhang mit den Reproduktionstechnologien.

Weiterhin stellte sich mir bei der Bearbeitung meines Themas die Frage, wie wohl heterolog gezeugte Kinder in Deutschland, die von ihrer Erzeugung Kenntnis haben, und damit von ihrer körperlichen Verbindung zu einem „Samenspender“, mit diesem Wissen umgehen? Hat die kulturelle Anerkennung der Aszendenz als unverzichtbarer Bestandteil der eigenen Identität und ihre faktische Unauflösbarkeit Auswirkungen auf das konkrete Handeln, d.h. suchen diese Kinder ihre genetische Herkunft (den „Samenspender"), und welche Konsequenzen hat dies auf soziale Beziehungen? Dem Recht auf Kenntnis der Abstammung muss nach geltender Rechtslage nicht notwendig das Recht auf Kennenlernen und sozialen Umgang folgen, was jedoch zukünftig nicht undenkbar wäre. Vom Recht auf Umgang mit dem genetischen Vater könnte sich auch der Weg zu einer anteiligen Personensorge des Vaters ergeben, d.h. eine anteilig gelebte soziale Vaterschaft wie sie auch von Herkunftsmüttern in Deutschland gefordert wird (vgl. auch Stein-Hilbers 1994:203; Schmitt 2000). Meines Wissens existiert keine Untersuchung in Deutschland ${ }^{228}$, die sich mit dieser spezifischen Thematik im Hinblick auf soziale Beziehungen, vor allem Verwandtschaft, beschäftigt.

228 So gibt es zwar für den Fall von Adoption zahlreiche Untersuchungen über Kinder, die Informationen über ihre biologischen Eltern suchen und diese zum Teil auch kennen lernen wollen. Studien über heterolog gezeugte Kinder und über „Samenspender“ existieren beispielsweise von Daniels (1987, 1989, 1998) aus Neuseeland. 
8 Anhang 


\section{Anhang 1: Fragebogen Reproduktionsmedizin}

\section{Fragebogen zu Familie und Verwandtschaft}

Beantworten Sie bitte die folgenden Fragen so ausführlich wie möglich. Sie können die Namen verändern, so dass Ihre Anonymität gewahrt bleibt oder nur die Anfangsbuchstaben der Vorbzw. Nachnamen verwenden.

1. Gibt es für Sie einen Unterschied zwischen Familie und Verwandtschaft?

Wenn ja, welchen?

1.1 Wen zählen Sie zu Ihrer Familie?

1.2 Wen zählen Sie zu Ihrer Verwandtschaft?

2. Zu welchen der o.a. Personen haben Sie die häufigsten Beziehungen?

3. Mit welchen (ev. auch nicht-verwandten) Personen fühlen sie sich emotional besonders verbunden?

3.1 Gehören diese zum überwiegenden Teil zu dem, was Sie als Familie oder Verwandtschaft bezeichnen?

( ) ja Welche sind dies?

( ) nein Um wen handelt es sich (z.B. Freund/Freundin)?

4.Wie sehen die Beziehungen mit den Personen, die Ihnen am wichtigsten sind, aus?

a) persönliche Treffen: mit wem und wie oft? Von wem geht die Initiative aus?

b)telefonischer Kontakt: mit wem und wie oft?

Von wem geht die Initiative aus? Über was wird gesprochen?

c) brieflicher Kontakt (auch Geburtstags-, Weihnachtskarten, Urlaubsgrüße...):

mit wem und wie oft? Was wird den verschiedenen Personen mitgeteilt?

d) Welche Feste werden bei Ihnen gefeiert, und wer wird eingeladen?

e) Gibt es gegenseitige Hilfeleistungen mit bestimmten Personen?

Welche sind dies, und wie sieht die Hilfe aus?

5. Zu welchen der unter 1.1 und 1.2 genannten Personen haben Sie keine oder sogar schlechte Beziehungen?

6. Wie lange haben Sie sich ein Kind gewünscht, bevor Sie medizinische Hilfe in Anspruch nahmen?

7. Wie lange dauerte Ihre Behandlung, und welche Behandlungsmethoden wurden angewendet?

8. Wie alt ist Ihr Kind jetzt?

9. Was bedeutet es für Sie, ein eigenes Kind zu haben?

10. Was bedeutet es für Sie, Mutter zu sein? 
10.1 Was bedeutet es für Sie, Vater zu sein?

10.2 Was bedeutet für Sie die Ehe?

10.3 Was bedeutet für Sie eine Familie?

11. Haben sich Ihre Vorstellungen vom Mutter-, Vater-, Eltern- sein im Verlauf der medizinischen Behandlung verändert und wenn ja, inwiefern?

12.Haben Sie je an Adoption gedacht? ( ) ja ( ) nein Könnten Sie Ihre Antwort bitte kurz erklären!

13. Existiert für Sie ein Unterschied zwischen einer sog. „,natürlichen“ und einer „künstlichen“ Zeugung?

\section{$\underline{\text { Angaben zur eigenen Person }}$}

Geschlecht: ( ) $\mathrm{m} \quad(\mathrm{m}$

Geburtsjahr:

Konfession: ( ) kath( ) ev

Geburtsort:

( ) andere:

Wohnort:

( ) keine

Schulbildung:

Beruf:

(ggf.) ehrenamtliche Tätigkeit:
( ) kirchlich
( ) politisch/gewerkschaftlich

( ) e.V.

\section{Lebens-/Ehepartner/in:}

Geburtsjahr:

Geburtsort:

Wohnort:

Schulbildung:

Beruf:

\author{
Konfession: ( ) kath( ) ev \\ ( ) andere: \\ ( ) keine \\ (ggf.) ehrenamtliche Tätigkeit: \\ ( ) kirchlich \\ ( ) politisch/gewerkschaftlich \\ ( ) e.V.
}

Vielen Dank für die Beantwortung der Fragen. Falls ich mich zu einem Gespräch mit Ihnen treffen darf, schreiben Sie hier bitte Ihren Namen, Ihre Adresse und Ihre Telefonnummer auf. Ich setze mich dann mit Ihnen in Verbindung. 


\title{
Anhang 2: Leitfadeninterview Reproduktionsmedizin
}

\author{
Komplex Familie und Verwandtschaft
}

1. Mit welchen Personen fühlen sie sich besonders verbunden, zu welchen Personen fühlen sie sich zugehörig? Warum?

2.Familie und Verwandtschaft?

Definition/Unterschied/Wer wird dazu gezählt, wer nicht und warum?

Gibt es bei Ihnen einen Familienstammbaum?

Wer führt diesen bzw. vervollständigt ihn?

Familienwappen? -> der väterlichen oder der mütterlichen Seite?

Hat der Familienname eine besondere Bedeutung für Sie?

Familientage -> welche Seite der Verwandtschaft?

orale Familientraditionen?

Erbstücke? Wird etwas vererbt oder weitergegeben?

Namensgebung (von Großeltern, Eltern auf Kinder?)

physische Ähnlichkeiten?

Was bedeutet Blutsverwandtschaft und angeheiratete Verwandtschaft für Sie?

Wo liegt für Sie der Unterschied im Verwandtschaftsverhältnis?

Kennen Sie die Schwiegerverwandtschaft Ihrer Geschwister (Verwandtschaft der Ehepartner Ihrer Geschwister?

Welche der o.g. Personen werden wie bezeichnet (Onkel, Tante, Schwiegermutter..) und wie angeredet? Wie werden Sie angeredet?

Gibt es Personen, die mit Verwandtschaftstermini bezeichnet oder angeredet werden, die in Ihrer Genealogie nicht auftauchen (formal nicht zur Verwandtschaft gehören) wie bspw. Paten, Freunde der Eltern o.a.?

Gibt es Identifikationsfiguren als Kind oder auch jetzt noch? /Ferien früher wo? Zählen Sie auch die geschiedenen EhepartnerInnen zu Ihrer Verwandtschaft?

3. Mit welchen der aufgezählten (und/oder aufgezeichneten) verwandten Personen besteht eine emotionale Verbindung? Warum?

Gibt es besondere Erwartungen an Familie und Verwandtschaft?

$\mathrm{Zu}$ welchen nicht-verwandten Personen (bspw. FreundInnen) besteht eine emotionale

Beziehung?

4.Mit welchen Personen haben Sie im Alltag zu tun?

5.Wie sehen die Beziehungen mit diesen für Sie wichtigen Personen aus?

Mit wem und wie oft? Von wem geht die Initiative aus?

Über was wird gesprochen? Wohnorte der Personen?

a) persönliche Treffen (-> Begrüßungsrituale)

b) telefonischer Kontakt

c) brieflicher Kontakt (Briefe, Karten......)

d) Festlichkeiten?

- Welche werden gefeiert und wer wird eingeladen bzw. nicht eingeladen? Wovon hängt das $a b$ ?

- Wer bekommt welche Geschenke, und welche Geschenke bekommen Sie?

- Verschicken Sie auch Pakete?

- Welche Personen laden Sie von Zeit zu Zeit zum Essen ein, und von welchen Personen werden Sie eingeladen?

e) gegenseitige Hilfeleistungen:

- An welche Personen würden Sie sich im Fall finanzieller Notlagen wenden? An wen auf keinen Fall?

- Wen würden Sie im Fall einer Krankheit versorgen und pflegen?

- Auf wessen Hilfe hoffen Sie im Alter, und für wen würden Sie im Alter sorgen? 
- Wen würden Sie um kleinere Gefälligkeiten bitten wie bspw. Babysitten, Blumen gießen während der Urlaubszeit o.a.?

- Gibt es eine Erwartungshaltung/eine Verpflichtung hinsichtlich bestimmter Personen?

\section{Zu welchen Personen, die formal zu Familie/Verwandtschaft gezählt wurden, bestehen keine oder schlechte Beziehungen?}

Komplex Zeugung/Körper

1. Seit wann wünschen Sie sich ein Kind? Wann begannen Sie, medizinische Hilfe in Anspruch zu nehmen?

2. Haben Sie bereits ein Kind oder mehrere?

3. Welche Behandlungsmethoden wurden/werden (jeweils) angewendet und wie lange dauerte ihre Behandlung insgesamt?

4. Haben Sie sich Grenzen gesetzt? Dauer, Methode?

5. Welche Gründe spiel(t)en für Sie eine Rolle bei der Entscheidung, ein Kind haben zu wollen?

6. Was bedeuten für Sie Kinder?

7. Mit wem sprechen Sie über Ihre Behandlung? Wer unterstützt Sie während der Behandlungen? Wie reagieren diese Personen darauf?

8. Wie sehen und empfinden Sie die Rolle der Ärzteschaft vor, während und nach der Behandlung?

9. Was ist für Sie Zeugung?

10. Was ist für Sie „Leben“? Wann beginnt für Sie „Leben“?

11. Was bedeutet für Sie die Schwangerschaft?

12. Werden Sie Ihrem Kind erzählen, wie und wo es gezeugt wurde?

13. Was halten Sie von heterologer Insemination?

Würden Sie diese Möglichkeit, ein Kind zu bekommen, auch in Anspruch nehmen?

Würden Sie offen damit umgehen oder es ihrem sozialen Umfeld lieber verschweigen?

Aus welchen Gründen?

Paare, die durch heterologe Insemination schwanger wurden:

$\rightarrow$ Was hat Ihre Entscheidung für die het. Insemination beeinflußt?

$\rightarrow$ Wissen Ihnen nahe stehende Personen davon? Wenn ja, wie ist die Reaktion?

$\rightarrow$ Wollen Sie es Ihrem Kind später sagen?

$\rightarrow$ Haben Sie sich ausführlich mit dem behandelnden Arzt über diese Möglichkeit, schwanger zu werden, unterhalten?

$\rightarrow$ War der Ehemann bei Insemination dabei?]

14. Was halten Sie von Eizellenspende und Leihmutterschaft?

Warum ist Ihrer Ansicht nach Samenspende in Deutschland erlaubt und Eizellenspende nicht?

15. Was bedeutet für Sie die Ehe?

(Haben Sie beide den gleichen Nachnamen?

$\rightarrow$ Falls ja, welchen Nachnamen haben Sie bei der Hochzeit als Familiennamen ausgewählt? Gab es bestimmte Gründe für Ihre Entscheidung?

$\rightarrow$ Falls nein, warum nicht?

Welchen Nachnamen wird Ihr Kind erhalten?

16.Was bedeutet für Sie eine Familie?

17.Was bedeutet für Sie Mutterschaft?

18. Was bedeutet für Sie Vaterschaft?

19.Haben sich Ihre Vorstellungen im Verlauf der medizinischen Behandlung geändert? Inwiefern?

20.Haben Sie je an Alternativen zu einer medizinischen Behandlung der ungewollten Kinderlosigkeit gedacht wie bspw. Pflegeelternschaft oder Adoption?

Haben Sie darüber auch mit Ihrem Frauenarzt (überweisende Gynäkologen gesprochen? 
Haben Sie sich bei Beratungsstellen informiert?

Haben Sie darüber mit Ihnen nahe stehenden Personen geredet?

\section{Persönliche Daten}

- Geburtsjahr

- Geburtsort/wo aufgewachsen?

- Wohnort

- Schulbildung

- Beruf

- Seit wann verheiratet? 1. Ehe?

- Konfession

- ggfs. ehrenamtliche Tätigkeiten 


\section{Anhang 3: Leitfadeninterview Adoptiv- und Pflegeeltern}

\section{Komplex Familie und Verwandtschaft}

1. Mit welchen Personen fühlen sie sich besonders verbunden, zu welchen Personen fühlen sie sich zugehörig? Warum?

\section{Familie und Verwandtschaft?}

Definition/Unterschied/Wer wird dazu gezählt, wer nicht und warum?

Gibt es bei Ihnen einen Familienstammbaum?

Wer führt diesen bzw. vervollständigt ihn?

Familienwappen? -> der väterlichen oder der mütterlichen Seite?

Hat der Familienname eine besondere Bedeutung für Sie?

Familientage -> welche Seite der Verwandtschaft?

orale Familientraditionen?

Erbstücke? Wird etwas vererbt oder weitergegeben?

Namensgebung (von Großeltern, Eltern auf Kinder?)

physische Ähnlichkeiten?

Was bedeutet Blutsverwandtschaft und angeheiratete Verwandtschaft für Sie?

Wo liegt für Sie der Unterschied im Verwandtschaftsverhältnis?

Kennen Sie die Schwiegerverwandtschaft Ihrer Geschwister (Verwandtschaft der Ehepartner Ihrer Geschwister?

Welche der o.g. Personen werden wie bezeichnet (Onkel, Tante, Schwiegermutter..) und wie angeredet? Wie werden Sie angeredet?

Gibt es Personen, die mit Verwandtschaftstermini bezeichnet oder angeredet werden, die in Ihrer Genealogie nicht auftauchen (formal nicht zur Verwandtschaft gehören) wie bspw. Paten, Freunde der Eltern o.a.?

Gibt es Identifikationsfiguren als Kind oder auch jetzt noch? /Ferien früher wo? Zählen Sie auch die geschiedenen EhepartnerInnen zu Ihrer Verwandtschaft?

3. Mit welchen der aufgezählten (und/oder aufgezeichneten) verwandten Personen besteht eine emotionale Verbindung? Warum?

Gibt es besondere Erwartungen an Familie und Verwandtschaft?

$\mathrm{Zu}$ welchen nicht-verwandten Personen (bspw. FreundInnen) besteht eine emotionale Beziehung?

4.Mit welchen Personen haben Sie im Alltag zu tun?

\section{Wie sehen die Beziehungen mit diesen für Sie wichtigen Personen aus?}

Mit wem und wie oft? Von wem geht die Initiative aus?

Über was wird gesprochen? Wohnorte der Personen?

f) persönliche Treffen (-> Begrüßungsrituale)

g) telefonischer Kontakt

h) brieflicher Kontakt (Briefe, Karten......)

i) Festlichkeiten?

- Welche werden gefeiert und wer wird eingeladen bzw. nicht eingeladen? Wovon hängt das $a b$ ?

- Wer bekommt welche Geschenke, und welche Geschenke bekommen Sie?

- Verschicken Sie auch Pakete?

- Welche Personen laden Sie von Zeit zu Zeit zum Essen ein, und von welchen Personen werden Sie eingeladen?

j) gegenseitige Hilfeleistungen:

- An welche Personen würden Sie sich im Fall finanzieller Notlagen wenden? An wen auf keinen Fall?

- Wen würden Sie im Fall einer Krankheit versorgen und pflegen?

- Auf wessen Hilfe hoffen Sie im Alter, und für wen würden Sie im Alter sorgen? 
- Wen würden Sie um kleinere Gefälligkeiten bitten wie bspw. Babysitten, Blumen gießen während der Urlaubszeit o.a.?

- Gibt es eine Erwartungshaltung/eine Verpflichtung hinsichtlich bestimmter Personen?

6. Zu welchen Personen, die formal zu Familie/Verwandtschaft gezählt wurden, bestehen keine oder schlechte Beziehungen?

\section{Komplex Zeugung/Elternschaft}

„Mich interessiert Ihre persönliche Geschichte von dem Zeitpunkt an, als Sie sich gemeinsam oder jeder für sich entschlossen haben, dass Sie ein Kind/Kinder haben oder mit einem Kind zusammen leben möchten, bis zum heutigen Tag. Erzählen Sie mir doch bitte, welche Wege Sie gegangen Sie sind, welche Entscheidungen Sie getroffen haben und warum?"

\section{Persönliche Daten}

- Geburtsjahr

- Geburtsort/wo aufgewachsen?

- Wohnort

- Schulbildung

- Beruf

- Seit wann verheiratet? 1. Ehe?

- Konfession

- ggfs. ehrenamtliche Tätigkeiten 


\title{
Anhang 4: Soziobiografische Angaben zu den zitierten Personen
}

\author{
Kontext Reproduktionsmedizin
}

\section{Patienten}

Bachmann, Helga (39), Verkäuferin

Verheiratet seit 1986, „Kinderwunsch“ seit 1987. Bekam einen Sohn (3 Jahre) durch Inseminationen in einer Universitätsklinik. Befindet sich jetzt für ein zweites Kind zu Inseminationsbehandlungen in einer Praxis.

Baumann, Bernhard (42), Großhandelskaufmann,

Baumann, Marita (32), Eigenbezeichnung „Hausfrau“

Schwanger im vierten Monat durch eine Inseminationsbehandlung. Ein Kind von zwei Jahren.

Berger, Barbara (33), Marketing-Managerin,

Berger, Thomas (35), Bauingenieur

Herr Berger ist zeugungsunfähig aufgrund einer Erbkrankheit, Frau Berger hat keine Ovulation. Seit 1991 ist das Paar verheiratet und wünscht sich seitdem Kinder. Mittels Hormontherapien und der heterologen Insemination wurde Frau Berger schwanger und brachte 1995 einen Sohn auf die Welt. Zur Zeit des Interviews befindet sich das Ehepaar wieder in der Behandlung für ein zweites Kind.

Bergmann, Juliana (33), Rechtsanwaltsgehilfin,

Bergmann, Tim (38), Ingenieur

Verheiratet seit 1993. Frau Bergmann hat einen Eileiterverschluss. Das Paar befindet sich im zweiten IVF- Versuch (Kryozyklus).

Bilcher, Martin (37), Psychologe

Nicht verheiratet. Hat einen Sohn von vier Jahren durch „Spontanschwangerschaft" in einer anderen Partnerschaft. Jetzt homologe Insemination für zweites Kind.

\section{Budecker, Jörn (36), Sozialpädagoge}

Verheiratet seit 1990, seitdem „Kinderwunsch“. Das Ehepaar befindet sich im zweiten ICSI-Versuch und nimmt auch an einer Selbsthilfegruppe teil.

Dietrich, Anja (29), Speditionskauffrau

Verheiratet seit 1990, seit 1991 gibt es einen konkreten „Kinderwunsch“. Ihr Mann hat zu wenig befruchtungsfähige Spermien im Ejakulat, sie leidet unter ständig wiederkehrenden Zysten. Frau Dietrich hat schon zwei ICSI-Versuche in einer Universitätsklinik hinter sich und befindet sich nun nach einer Pause von einem Jahr beim insgesamt vierten ICSI-Versuch in einer niedergelassenen Praxis. 
Flirrau, Marie (35), Pädagogin

Verheiratet seit 1991. Seitdem möchte sie ein Kind, ist aber nicht schwanger geworden. Als klar war, dass ihr Mann ,steril“ ist, haben sie lange überlegt und sich jetzt für eine heterologe Inseminationsbehandlung entschieden.

Franz, Birgit (31), Grafik-Designerin,

Franz, Lothar (34), Architekt

Verheiratet seit 1993, „Kinderwunsch“ seit 1995. Erster ICSI- Versuch.

Glück, Marion (33), Architektin

Verheiratet seit 1994, „Kinderwunsch“ seit drei Jahren. Frau Glück war ohne ihren Mann zu einem Erstgespräch in einer Klinik, da sie sich erst mal informieren wollte. Vermutlich läuft es auf die heterologe Insemination hinaus. Sie sucht Unterstützung und Austausch in einer Selbsthilfegruppe.

Göbel, Sonja (37), Bürokauffrau

Verheiratet seit 1993, seitdem hat sie den konkreten „Wunsch“ nach einem Kind.

Frau Göbel befindet sich gerade - nach einem Kryozyklus - in der dritten IVF/ICSI-Behandlung.

Groß, Inge (38), Verkäuferin

Verheiratet seit 1991, seitdem gibt es einen konkreten „Kinderwunsch“. Ihr Mann ist zeugungsunfähig. Mit Hilfe der heterologen Insemination wurde sie nach 8 Versuchen schwanger und bekam einen Sohn. Sie befindet sich jetzt in der Behandlung für ein zweites Kind und hat schon 6 Versuche hinter sich, die nicht zu einer Schwangerschaft führten.

Hebler, Constanze (30), Selbständig

Kam 1997 zum Erstgespräch, worauf drei Inseminationen mit Hormonbehandlungen folgten. 1998 kommt sie zum Arztgespräch für IVF.

Heider, Maria (26), Kauffrau

Verheiratet seit 1996, „Kinderwunsch“ seit drei Jahren. Nach einmaliger homologer Insemination befindet sie sich nun in der zweiten ICSI-Behandlung.

Helming, Tatjana (36), Personalsachbearbeiterin,

Helming, Martin (38), Entwicklungsingenieur

Verheiratet seit 1991, seitdem haben sie auch einen konkreten „Kinderwunsch“. Nach vielen Arztbesuchen sowohl des Mannes als auch der Frau, bei denen sich herausstellte, dass er nur eingeschränkt zeugungsfähig ist und sie keinen regelmäBigen Zyklus hat, entschieden sie sich, erst einmal keine medizinische Hilfe in Anspruch zu nehmen. Zur Zeit des Interviews befindet sich das Ehepaar Helming in ihrer ersten IVF-Behandlung.

Hiller, Sabine (33), Sonderschullehrerin,

Hiller, Stephan (38), Finanzkaufmann

Seit 1992 ist das Paar verheiratet und wünscht sich seitdem auch ein Kind. Seit vier Jahren befindet sich die Hillers in Behandlung und haben schon verschiedene Behandlungsmethoden ausprobiert. Mit Hilfe der heterologen Insemination wurde Frau Hiller nicht schwanger, mit ICSI bekam sie 1995 einen Sohn. Befinden sich jetzt für ein zweites Kind wieder in Behandlung 
Jäger, Lea (36), Köchin

Verheiratet seit 1989, „Kinderwunsch“ besteht schon viel länger. Sie hat mit ihrem Mann eine Adoption überlegt, will aber lieber selber schwanger werden mit Hilfe der heterologen Insemination. Zur Zeit im sechsten Versuch.

Jansen, Lars (31), Großhandelskaufmann

Verheiratet seit 1995, seit mehreren Jahren nennt er einen „Kinderwunsch“. Das Ehepaar hat lange überlegt, ob sie medizinische Hilfe in Anspruch nehmen wollen und befinden sich zum gegenwärtigen Zeitpunkt zu einem Erstgespräch in der Klinik.

Kollo, Helga (29), Laborantin

Verheiratet seit 1993. Weiß schon lange, dass sie einen Eileiterverschluss hat. Zur Zeit in IVF-Behandlung, im dritten Versuch. Nimmt an einer Selbsthilfegruppe teil.

Kuschnick, Britta (31), Lehrerin,

Kuschnick, Hans (37), Anwalt

Verheiratet seit 1993, seitdem konkreten „Kinderwunsch“. Sie leidet ständig unter

Zysten. Das Paar befindet sich im zweiten Inseminationsversuch.

Lausitz, Karin (28), Medizinisch-Technische-Assistentin,

Lausitz, David (32), Apotheker

Verheiratet seit 1992. Zur Zeit der dritte IVF/ICSI-Versuch.

Leinemann, Elisabeth (34), Chemisch-Technische-Assistentin

Verheiratet seit 1988, seit ungefähr 1990 gibt es auch einen konkreten „Kinderwunsch“. Sie bekam zunächst Hormone, bis sie 1992 mit IVF begannen. Nach 13 Versuchen wurde sie schwanger und bekam einen Sohn. Nun ist sie nach zwei IVF/ICSI-Versuchen wieder schwanger.

Lenz, Christine (30), Rechtsanwältin,

Lenz, Karl (39), selbständiger Unternehmer Insemination (bisher ein homologer Versuch).

Ludwig, Henrike (32), Grundschullehrerin

Verheiratet seit 1995, seitdem hat sie auch einen konkreten „Kinderwunsch“. Sie ist zu einem Erstgespräch in der Klinik.

Mainer, Sybille (36), Ärztin

Verheiratet seit 1991, seitdem „Kinderwunsch“. Sie und ihr Mann haben zwei Jahre durch Geschlechtsverkehr versucht schwanger zu werden. Nach Untersuchungen wurde festgestellt, dass ihr Mann ,zeugungsunfähig“ ist. Jetzt erste heterologe Insemination.

Maywald, Henrike (35), Erzieherin

Verheiratet seit 1989, zur Zeit die dritte Insemination. 
Menge, Yvonne (36), Erzieherin

Verheiratet seit 1995, „Kinderwunsch“ seit 1994. Frau Menge verhütet seit zwei Jahren nicht mehr. Nach genauer Untersuchung wurden verklebte Eileiter festgestellt. Sie will nun eine IVF-Behandlung beginnen.

Meyer, Britta (33), Krankenschwester

Verheiratet seit 1990, einen Sohn durch ,Spontanschwangerschaft. Seit 1995 hat sie einen konkreten „Kinderwunsch“ nach einem zweiten Kind. Nach mehreren Inseminationsbehandlungen bekam sie durch die Inseminationsbehandlung einen Sohn.

Ottmers, Ilona (30), Verwaltungsfachangestellte,

Ottmers, Christoph (33), Lebensmitteltechniker

Verheiratet seit 1996, seitdem besteht auch ein „Kinderwunsch“. Herr Ottmers hat sich nach seinen zwei Kindern aus erster Ehe sterilisieren lassen. In der zweiten Ehe und dem erneuten Kinderwunsch gemeinsam mit Frau Ottmers ließ er sich Refertilisieren, was jedoch nicht zum Erfolg führte. Er unterzog sich einem MESA/TESE-Eingriff, wodurch Samenzellen gewonnen werden konnten, mit denen man die Eizellen von Frau Ottmers befruchtete. Das Paar befindet sich jetzt beim zweiten ICSI-Versuch.

Rösch, Lea (34), Beamtin

Verheiratet. Aufgrund diagnostizierter verschlossener Eileiter im dritter IVFVersuch.

Rudig, Katrina (33), Einzelhandelskauffrau

Nicht verheiratet, ein Kind aus einer vorangegangenen Partnerschaft. Die zweite Partnerschaft besteht seit fünf Jahren. Sie bezeichnet sich selber als seit vier Jahren ,ungewollt kinderlos“. Zur Zeit die vierte Insemination.

Rudlack, Johann (34), Vertreter

„Zeugungsunfähig“ aufgrund einer Mumps-Erkrankung in der Kindheit. Vierter heterologer Inseminationsversuch.

Schmidt, Maike (34), Verkäuferin

Verheiratet seit 1993, konkreter „Kinderwunsch“ besteht seit 1995. Frau Schmidt befindet sich in der zweiten Inseminationsbehandlung.

Schmitt, Martin (33), Lehrer

Verheiratet seit 1994, seit 1995 „Kinderwunsch“. Herr Schmitt hat keine befruchtungsfähigen Spermien im Ejakulat. Er ist mit seiner Frau zum Erstgespräch in der Klinik, um sich über die heterologe Insemination beraten zulassen.

Schulz, Bettina (30), Flugbegleiterin

Verheiratet seit 1995, seitdem in ICSI-Behandlung.

Schumann, Mathilde (37), Arzthelferin

Frau Schumann bekam 1996 mit Hilfe der IVF einen Sohn und befindet sich nun in Behandlung für ein zweites Kind (im zweiten IVF/ICSI-Versuch). 
Stephan, Jan (39), Bankangestellter

Verheiratet seit 1991. „Kinderwunsch“ seit acht Jahren. Das Ehepaar Stephan befindet sich seit vier Jahren mit Unterbrechungen in IVF-Behandlung.

Stiepel, Wilfried (38), Selbständig

Verheiratet seit 1994, ein Kind aus einer früheren Beziehung. Hat seit drei Jahren einen „Kinderwunsch“ mit seiner Ehefrau. Er ist mit seiner Frau zu einem Erstgespräch in der Klinik.

Töpfer, Sandra (36), Friseurin

Verheiratet seit 1990. Befindet sich im dritten heterologen Inseminationsversuch.

Toll, Robert (39), Optiker,

Toll, Melanie (32), Optikerin

Verheiratet seit 1987, seitdem „Kinderwunsch“. Drei IVF-Behandlungen in einer kassenärztlichen Praxis, jetzt befinden sie sich in einer Privatklinik.

Treis, Markus (30), Zimmermann

Nicht verheiratet, seit zwei Jahren gibt es einen konkreten „Kinderwunsch“. Herr Treis hat zu wenig befruchtungsfähige Spermien im Ejakulat, bei seiner Partnerin wurde ein Eileiterverschluss diagnostiziert. Beide befinden sich jetzt in ihrer ersten IVF-Behandlung. Wollen demnächst heiraten.

Wagner, Ira (33), Glasapparatebläserin

Verheiratet seit 1985, seit acht Jahren hat sie einen „Kinderwunsch“. Nach zweimaliger ICSI-Behandlung war sie schwanger, erlitt aber im sechsten Schwangerschaftsmonat eine Fehlgeburt. Frau Wagner befindet sich nun wieder in Behandlung.

Walter, Friedrich (41), Betriebswirt

Verheiratet seit 1988, seit 7 Jahren hat das Ehepaar einen konkreten „Kinderwunsch“. Bei dem Ehepaar Walter wurde die Diagnose ,idiopathische', d.h. ungeklärte „Sterilität“" gestellt. Sie versuchten zunächst mit Inseminationen, schwanger $\mathrm{zu}$ werden. Zeitgleich stellten sie auch einen Adoptionsantrag. Nach erfolglosen Versuchen machten sie einige Zeit Pause. Sie ließen sich nun bei einem Erstgespräch über die IVF-Methode informieren, da sie diese nun doch noch in Anspruch nehmen möchten.

Wieger, Marion (34), Journalistin

Verheiratet seit 1989, „Kinderwunsch“ besteht seit 1993. Frau Wiese bekam vor gut einem Jahr einen Sohn nach zwei homologen Inseminationsbehandlungen. Sie befindet sich jetzt in der Behandlung für ein zweites Kind.

Wille, Ute (36), Zahntechnikerin

Verheiratet seit 1991, seitdem hat sie einen konkreten „Kinderwunsch“. Ihr Mann gilt aufgrund einer Krebserkrankung als zeugungsunfähig. Sie haben zunächst mit Hilfe mehrerer heterologer Inseminationen versucht, ein Kind zu bekommen. Zweimal ist Frau Wille schwanger geworden, erlitt aber Fehlgeburten. Nach Einführung der ICSI-Methode in der reproduktionsmedizinischen Praxis konnten sie mittels MESA/TESE Samenzellen gewinnen und ihre Eizellen befruchten. Frau Wille ist nun in der 23. Woche schwanger. 
Zimmermann, Elisabeth (35), Verwaltungsfachangestellte Verheiratet seit 1996, seit fünf Jahren hat sie einen „Kinderwunsch“. Bei Frau Zimmermann wurde festgestellt, dass nur ein Eileiter durchgängig ist. Nach drei erfolglosen IVF-Versuchen hat sie eine Pause von einem Jahr gemacht, in dem sie und ihr Mann auch einen Adoptionsantrag gestellt haben. Sie möchte nun doch noch einmal versuchen, mit Hilfe von IVF schwanger werden.

\section{Ärzte und Ärztinnen}

Bircher, Eva Dr. (39), Gynäkologin und Psychotherapeutin, Reproduktionsmedizinerin, nicht verheiratet, kinderlos

Hagemann, Jan Dr., Biochemiker und Gynäkologe, Reproduktionsmediziner, verheiratet, ein Kind.

Heise, Peter Prof. Dr., Gynäkologe, Reproduktionsmediziner, verheiratet, drei Kinder.

König, Frank Dr. (43), Reproduktionsmediziner, verheiratet, drei Kinder.

Krüger, Norbert Dr. (43), Gynäkologe und Reproduktionsmediziner, verheiratet, zwei Kinder.

Wellmann, Rolf (45), Gynäkologe und Reproduktionsmediziner, verheiratet, zwei Kinder.

\section{Kontext Adoptionen und Pflegschaften}

Bechmann, Bianca (36), Lehrerin

Ein Adoptivkind im Alter von drei Jahren. Nach ersten Erfahrungen mit der Reproduktionsmedizin lehnte sie eine medizinische Behandlung ab und stellte einen Adoptionsantrag.

Behler, Evelyn (33), Ärztin

Adoptivmutter eines vierjährigen Kindes. Sie wusste schon mit 20 Jahren, dass sie keine leiblichen Kinder bekommen könnte (zur Ursache äußerte sie sich nicht näher).

Dammers, Ingeborg (44), Verkäuferin

Ein Adoptivkind im Alter von vierzehn Jahren, ein leibliches Kind im Alter von zehn Jahren und insgesamt sechs Pflegekindern in den letzten zehn Jahren

Gerlach, Hanna (42), Sozialpädagogin

Adoptivkind von 12 Jahren und zwei Pflegekinder im Alter von 10 und 13 Jahren.

Hansen, Margot (48), Eigenbezeichnung „Hausfrau und Mutter“

Zwei leibliche Kinder und vier Pflegekinder 
Hildmann, Anneliese (40), Altenpflegerin

Adoptivmutter eines sechsjährigen Kindes. Wurde mit ihrem ersten Mann nicht schwanger und stellte mit ihrem zweiten Mann gleich einen Adoptionsantrag.

Klein, Dorothea (38), Bäckereifachverkäuferin

Ein Adoptivkind im Alter von fünf Jahren, ein Pflegekind im Alter von vier Jahren. Als sie feststellte, dass sie durch Geschlechtsverkehr nicht schwanger werden konnte, stellten sie und ihr Mann einen Adoptionsantrag.

Kraus, Götz (45), Arzt

Ein Pflegekind und ein Adoptivkind. Äußerte sich nicht näher zu seinen Beweggründen, Kinder zu adoptieren und in Pflege zu nehmen.

Kruse, Astrid (37), Beamtin,

Kruse, Ingo (39), Polizist

Zwei Adoptivkinder im Alter von 7 und 1,5 Jahren. Herr Kruse ist „steril“. Für das Paar war von Anfang an klar, dass sie Kinder adoptieren möchten.

Küpke, Ulrich (73), Rentner

Leiblicher Vater von drei Kindern, Adoptivvater von einem Adoptivsohn und Pflegevater von 15 Pflegekindern in den letzten 25 Jahren

Schlote, Heike (38), Sozialarbeiterin

Adoptivmutter eines vierjährigen Kindes. Machte vier Inseminationsbehandlungen, bevor sie sich zu einer Adoption entschieden.

Wahren, Julia (38), Journalistin

Ein Adoptivkind von sieben und ein leibliches Kind von zwei Jahren.

Wellenreuther, Achim (43), Bankangestellter

Ein Adoptivkind im Alter von acht Jahren und zwei Pflegekinder im Alter von drei und fünf Jahren

Zinke, Ulla (38), Pferdepflegerin

Adoptivmutter eines achtjährigen Kindes. Sie und ihr Mann ließen sich ärztlich untersuchen, als sie nicht schwanger wurden. Es konnte aber keine medizinische Ursache festgestellt werden. So stellten sie einen Adoptionsantrag. 


\section{Anhang 5: Glossar medizinischer Fachausdrücke}

Anamnese
Compliance
Embryo
Endokrinologie
Endometriose
Follikel
GIFT

Hodenbiopsie

Hysterosalpingographie

\section{ICSI}

Infertilität

in vivo

in vitro

intrazervikal

intrauterin

IVF

Katheter

Keimbahnzellen

Keimzellen

Kryokonservierung

Laparoskopie
Krankengeschichte (Art und Verlauf)

aktive, selbstverantwortliche Mitarbeit eines Patienten im Therapieverlauf.

Frucht in der Gebärmutter während der ersten drei Schwangerschaftsmonate (bis zum Abschluß der Organentwicklung)

Lehre von der Funktion der Drüsen mit innerer Sekretion und der Hormone

Auftreten von Gebärmutterschleimhautgewebe außerhalb der Gebärmutter

Eibläschen, das die Eizelle enthält

Abkürzung: intratubarer Gametentransfer (reife Eizellen werden mit dem Sperma in die Eileiter eingespült

Gewebeentnahme aus dem Hoden zur Untersuchung

Röntgenologische Untersuchung der Gebärmutter und der Eileiter

Abkürzung: Intrazytoplasmatische Spermieninjektion, auch Mikroinjektion genannt (ein einziges Spermium wird direkt in die Eizelle injiziert)

Unfähigkeit, eine Schwangerschaft bis zur Geburt auszutragen. Beim Mann wird Infertilität gleichgesetzt mit Zeugungsunfähigkeit

im Körper

außerhalb des Körpers

im Gebärmutterhals

in der Gebärmutter

Abkürzung: In-vitro-Fertilisation (außerkörperliche oder Retortenbefruchtung genannt)

Schlauchsystem, über das Flüssigkeit zu- oder abfließt.

beinhalten die Erbanlagen, ohne Stoffwechselprozesse zu beeinflussen, und werden kontinuierlich von einer Generation auf die nächste übertragen.

Geschlechtszellen eines Organismus, z.B. Samenzellen und Eizellen

Einfrieren und Aufbewahren von Keimzellen bei $-196^{\circ} \mathrm{C}$

Bauchspiegelung 
MESA

Myome

Ovulation

Postcoitaltest

Prolaktin

Pronukleus

Sterilität

Superovulation

TESE

Tuben

Uterus

Varikozele

Vorkern

Zyste
Abkürzung: Micro Epididymal Sperm Aspiration (mikrochirurgische Gewinnung von Spermienvorstufen aus dem Nebenhoden)

gutartige Tumore der Gebärmuttermuskulatur

Eisprung

Untersuchung des Gebärmutterhalses unmittelbar nach dem Geschlechtsverkehr zur Beurteilung der Spermaqualität

Hormon, das besonders für die Milchbildung notwendig ist

\section{Vorkern}

Unfruchtbarkeit

hormonelle Stimulierung der Frau, um die Reifung mehrerer Eizellen in einem Zyklus zu bewirken

Abkürzung: testikuläre Spermienextraktion (mikrochirurgische Gewinnung von Samenzellen aus Teilen des Hodengewebes)

\section{Eileiter}

Gebärmutter

Krampfader am Hoden

Kern der weiblichen oder männlichen Keimzelle in der befruchteten Eizelle, vor deren Kernverschmelzung durch eine Kapsel abgeschlossene Geschwulst mit dünnoder dickflüssigem Inhalt 


\section{Anhang 6: Abkürzungsverzeichnis}

\begin{tabular}{ll} 
Abs. & Abschnitt (BGB) bzw. Absatz (GG) \\
a.F. & alte Fassung \\
Art. & Artikel \\
BGB & Bürgerliches Gesetzbuch \\
BGH & Bundesgerichtshof \\
BR & Bundesrat \\
DFG & Deutsche Forschungsgemeinschaft \\
DKF & Deutsche Klinik für Fortpflanzungsmedizin \\
EheG & Ehegesetz \\
EheRG & Ehereformgesetz \\
EschG & Embryonenschutzgesetz \\
EstG & Einkommensteuergesetz \\
FamR & Familienrecht \\
GG & Grundgesetz \\
GIFT & Intratubarer Gametentransfer \\
GRG & Gesundheitsreformgesetz \\
ICSI & Intrazytoplasmatische Spermieninjektion (Mikroinjektion) \\
IVF & In-vitro-Fertilisation \\
KindRG & Kindschaftsreformgesetz \\
KJHG & Kinder- und Jugendhilfegesetz \\
MESA & mikrochirurgische Spermatozoen-Aspiration \\
n.F. & neue Fassung \\
NJW & Neue Juristische Wochenzeitschrift \\
SGB & Sozialgesetzbuch \\
StGB & Strafgesetzbuch \\
TESE & testikuläre Spermienextraktion \\
\hline
\end{tabular}

Bei der Nennung von $\S$ und Abs. bezeichnen römische Zahlen einen Absatz, arabische einen Satz. 


\section{Literatur}

\section{Adolf, H.}

1937 Wortgeschichtliche Studien zum Leib/Seele-Problem. Sonderheft 5 zur Zeitschrift für Religionspsychologie. Wien.

\section{Atteslander, $\mathbf{P}$.}

1975 Methoden der empirischen Sozialforschung, 4. Aufl. Berlin/New York.

\section{Barbian, E./Berg, G.}

1997 Die Technisierung der Zeugung. Die Entwicklung der In-vitro-Fertilisation in Deutschland. Pfaffenweiler.

\section{Bargatzky, T.}

1997 Ethnologie. Eine Einführung in die Wissenschaft von den urproduktiven Gesellschaften. Hamburg.

\section{Barnes, J. A.}

1973 Genetrix: Genitor: Nature: Culture? In: Goody, J. (ed), The Character of Kinship: 61-73. London.

\section{Bayertz, K.}

1987 GenEthik. Probleme der Technisierung menschlicher Fortpflanzung. Reinbek bei Hamburg.

\section{Beck, U.}

1986 Risikogesellschaft. Auf dem Weg in eine andere Moderne. Frankfurt a.Mn.

Becker, A. E.

1995 Body, Self and Society: The View from Fiji. Philadelphia.

\section{Beck-Gernsheim, E.}

1988 Die Kinderfrage. Frauen zwischen Kinderwunsch und Unabhängigkeit. München.

\section{Beier, H. M.}

1997 Assistierte Reproduktion - Zum Stand der Therapieverfahren in der Bundesrepublik Deutschland. München.

\section{Bien, W./Marbach, J.H.}

1991 Haushalt - Verwandtschaft - Beziehungen: Familienleben als Netzwerk. In: Bertram, H. (Hg), Die Familie in Westdeutschland. Stabilität und Wandel in familialen Lebensformen: 3-44. Opladen.

Bundesverband der Pflege- und Adoptiveltern e.V. (Hg)

1994 Informationen für Adoptionsbewerber. Münster. (= InfoAdoption)

1996 Pflegekinder. Informationen für Pflegeeltern. Münster (= InfoPflege) 


\section{Carsten, J.}

1995 The Substance of Kinship and the Heat of the Hearth: Feeding, Personhood and Relatedness among Malays of Pulau Langkawi. American Anthropologist 22 (2): 223-241.

2000a Introduction: Cultures of Relatedness. In: Carsten, J. (ed): 1-37.

$2000 \mathrm{~b}$ (ed)., Cultures of Relatedness. New Approaches to the Study of Kinship. Cambridge.

\section{Coester, $M$.}

1983 Das Kindeswohl als Rechtsbegriff: die richterliche Entscheidung über die elterliche Sorge beim Zerfall der Familiengemeinschaft. Frankfurt.

1986 Das Kindeswohl als Rechtsbegriff. Sechster Deutscher Familiengerichtstag Brühl 1985: 35-51. Bielefeld.

\section{Coester-Waltjen, D.}

1986 Künstliche Befruchtung beim Menschen - Zulässigkeit und zivilrechtliche Folgen. Gutachten B für den 56. Deutschen Juristentag, Ziv. Abt.

\section{Collier, J./Rosaldo, M./Yanagisako, S.}

1982 Is there a Family? New Anthropological Views. In: Thorne, B./Yalon, M. (eds), Rethinking the Family: Some Feminist Questions: 25-39. New York.

\section{Csordas, T. J.}

1994 Self and Person. In: Bock, P. K. (ed), Psychological Anthropology: 331-350. London.

\section{Daniels, $\mathbf{K}$.}

1987 Semen Donors in New Zealand: Their Characteristics and Attitudes. Clinical Reproduction and Fertility 5 (4): 177-190.

1989 Semen Donors: Their Motivations and Attitudes Towards Their Offspring. Journal of Reproductive and Infant Psychology 7 (2): 121-127.

1998 The Semen Providers. In: Daniels, K./Haimes, E. (eds): 76-105.

Daniels, K./Haimes, E. (eds)

1998 Donor Insemination. International Social Science Perspectives. Cambridge.

Delaisi de Parseval, G./Janaud, A. 1986 [1983]

Ein Kind um jeden Preis. Ethik und Technik der künstlichen Zeugung. Weinheim.

\section{Delaney, C.}

1986 The Meaning of Paternity and the Virgin Birth Debate. Man 21: 494-513.

\section{Dolgin, J. L.}

1995 Family Law and the Facts of Family. In: Yanagisako, S./Delaney, C. (eds): 4767. 


\section{Duden, B.}

1987 Geschichte unter der Haut. Ein Eisenacher Arzt und seine Patientinnen um 1730. Stuttgart.

1991 Der Frauenleib als öffentlicher Ort. Vom Missbrauch des Begriffs Leben. München.

\section{Duden Herkunftswörterbuch}

1997 [1963]

Die Etymologie der deutschen Sprache. Bd. 7. Mannheim/Wien/Zürich.

\section{Durkheim, E.}

1921 La famille conjugale. Revue Philosophique de la France et l'Etranger 46 (XCI): 1-14.

Eckart, W. U.

1998 Geschichte der Medizin, 3. überarb. Aufl. Berlin/Heidelberg/New York.

\section{Edwards, J.}

2000 Born and Bred. Idioms of Kinship and New Reproductive Technologies in England. Oxford.

\section{Edwards, J. et al.}

1993 Technologies of Procreation. Kinship in the Age of Assisted Conception. Manchester/New York.

Edwards, J./Strathern, M.

2000 Including Our Own. In: Carsten, J. (ed), Cultures of Relatedness. New Approaches to the Study of Kinship: 149-167. Cambridge.

\section{Fehlmann-von der Mühll, M.}

1978 Verwandtschaft, Theorie und Alltag. Mit Beispielen aus einer Untersuchung unter jungen Züricher Familien. Dissertation. Zürich.

Feige, A. et al.

1997 Frauenheilkunde. München/Wien/Baltimore.

Fenner, B.

1984 Verwandtenbeziehungen in einem Dorf des rheinischen Braunkohlengebietes. Berlin.

Finkler, $\mathrm{K}$.

2001 The Kin in the Gene. The Medicalization of Family and Kinship in American Society. Current Anthropology 42 (2): 235-249.

Fischer, $\mathbf{H}$.

1990 Lehrbuch der genealogischen Methode. Berlin.

Foucault, M.

1974 Die Ordnung des Diskurses. München. 


\section{Franklin, S.}

1990 Deconstructing „Desperateness“: The Social Construction of Infertility in Popular Representations of New Reproductive Technologies. In: Mc Neil, M./Varcoe, I./Yearley, S. (eds): 200-230.

1991 Fetal Fascinations: New Dimensions to the Medical-Scientific Construction of Fetal Personhood. In: Franklin, S./Cury, C./Stacey, J. (eds), Off-Centre. Feminism and Cultural Studies: 190-205. London.

1992 Making Sense of Missed Conceptions: Anthropological Perspectives on Unexplained Infertility. In: Stacey, M. (ed): 75-91.

1993a Making Representations: The Parliamentary Debate on the Human Fertilisation and Embryology Act, In: Edwards, J. et al.: 96-132.

1993b Postmodern Procreation. Representing Reproductive Practice. Science as Culture 3 (4): 522-561.

1995 Postmodern Procreation: A Cultural Account of Assisted Reproduction. In: Ginsburg, F./Rapp. R. (eds): 323-345.

1997 Embodied Progress. A Cultural Account of Assisted Conception. London/New York.

1998 Making Miracles: Scientific Progress and the Facts of Life, In: Franklin, S./Ragoné, H. (eds): 102-118.

\section{Franklin, S./Ragoné, H. (eds)}

1998 Reproducing Reproduction. Kinship, Power, and Technological Innovation. Philadelphia.

\section{Gaul, H. F.}

1998 Die Neuregelung des Abstammungsrechts durch das Kindschaftsreformgesetz. In: Schwab, D. (Hg), Das neue Familienrecht. Systematische Darstellung zum Kindschaftsreformgesetz, Kindesunterhaltsgesetz, Eheschließungsrechtsgesetz und Erbgleichstellungsgesetz: 49-124. Bielefeld.

\section{Geertz, C.}

1983 Local Knowledge. New York.

\section{Giddens, A.}

1991 Modernity and Self-Identity: Self and Society in the Late Modern Age. Stanford.

1995 [1990]

Konsequenzen der Moderne. Frankfurt a. Mn.

Ginsburg, F. D./Rapp, R. (eds)

1995 Conceiving the New World Order. The Global Politics of Reproduction. Berkeley/L.A./London.

\section{Girtler, R.}

2001 Methoden der Feldforschung, 4. neu bearb. Aufl. Wien/Köln/Weimar. 


\section{Goody, J.}

1989 [1983]

Die Entwicklung von Ehe und Familie in Europa. Frankfurt a.Mn.

\section{Hagström, C.}

1996 Becoming a Father and Establishing Paternity. In: Lundin, S./Åkesson, L. (eds): 37-63.

\section{Haimes, E.}

1990 Recreating the Family? Policy Considerations Relating to the 'New' Reproductive Technologies, In: McNeil, M./Varcoe, I./Yearley, S. (eds): 154-173.

1992 Gamete Donation and the Social Managements of Genetic Origins. In: Stacey, M. (ed): 119-147.

\section{Haraway, D.}

1995 Die Neuerfindung der Natur. Primaten, Cyborgs und Frauen. Frankfurt/New York.

1997 Modest_Witness@Second Millennium.FemaleMan@C_Meets_OncoMouseÔ. Feminism and Technoscience. New York.

Harris, G.

1989 Concepts of Individual, Self, and Person in Description and Analysis. American Anthropologist 91 (3): 599-612.

\section{Harris, M.}

1989 Kulturanthropologie. Ein Lehrbuch. Frankfurt/New York.

\section{Hastrup, K.}

1978 The Semantics of Biology: Virginity. In: Ardener, S. (ed), Defining Females. The Nature of Women in Society: 49-65. London.

\section{Hauke, E.}

1937 Galen: Dass die Vermögen der Seele eine Folge der Vermischungen des Körpers sind. Dissertation. Berlin.

\section{Hauser-Schäublin, B.}

1989 The Fallacy of „Real“ and „Pseudo“ Procreation. Zeitschrift für Ethnologie 114: 179-194.

1991 „Verwandtschaft“ und „Reproduktion“. Vaterschaft, die Entleiblichung der Frau und die Entseelung des Menschen. In: Dies. (Hg), Ethnologische Frauenforschung: 306-331. Berlin.

1995 Das Ende der Verwandtschaft? Zeugung und Fortpflanzung zwischen Produktion und Reproduktion. In: Kaschuba, W. (Hg), Kulturen - Identitäten - Diskurse. Perspektiven Europäischer Ethnologie: 163-186. Berlin.

1997 Blick zurück im Zorn: Ethnologie als Kulturkritik. Zeitschrift für Ethnologie 122: 3-17.

1998 Humantechnologien und die Konstruktion von Verwandtschaft. KEA. Zeitschrift für Kulturwissenschaften 11: 55-75. 


\section{Hauser-Schäublin, B. et al.}

2001 Der geteilte Leib. Die kulturelle Dimension von Organtransplantation und Reproduktionsmedizin. Frankfurt/New York.

Hayden, C.

1995 Gender, Genetics, and Generation: Reformulating Biology in 'Lesbian Kinship'. Cultural Anthropology 10 (1): 41-63.

\section{Hiatt, L. R.}

1971 Secret Pseudo-Procreation Rites among the Australian Aborigines. In: Hiatt, L.R./Jayawardena, C. (eds), Anthropology in Oceania. Essays Presented to Ian Hogbin: 77-88. Sydney.

1990 Towards a Natural History of Fatherhood. The Australian Journal of Anthropology 1 (2-3): 110-130.

Hölzle, C.

1987 Kinderlosigkeit als Krise. Reproduktionsmedizin als Rettung? Psychische Probleme der Unfruchtbarkeit und ihrer medizinischen Behandlung. In: Zipfel, G. (Hg), Reproduktionsmedizin. Hamburg.

\section{Hoffmann-Riem, C.}

1989 Elternschaft ohne Verwandtschaft: Adoption, Stiefbeziehungen und heterologe Insemination. In: Nave-Herz, R./Markefka, M. (Hg): 389-411.

\section{Holy, L.}

1996 Anthropological Perspectives on Kinship. London/Chicago.

\section{Husserl, E.}

1993 Arbeiten an den Phänomenen. Ausgewählte Schriften. Herausgegeben und mit einem Nachwort versehen von B. Waldenfels. Frankfurt a. Mn.

\section{Illich, I.}

1975 Die Enteignung der Gesundheit. „Medical Nemesis“. Reinbek bei Hamburg.

Jensen, $\mathbf{J}$.

1995 Der Gegenstand der Ethnologie und die Befassung mit komplexen Gesellschaften. Zeitschrift für Ethnologie 120: 1-14.

\section{Jussen, B.}

1991 Patenschaft und Adoption im frühen Mittelalter. Künstliche Verwandtschaft als soziale Praxis. Göttingen.

\section{Kalitzkus, V.}

2001 Im Angesicht des Todes. Organtransplantation im Erleben von „Spendenden“ und Empfangenden in Deutschland. Dissertation an der Sozialwissenschaftlichen Fakultät der Universität Göttingen, noch unveröffentlicht. 


\section{Kettner, M.}

2001 Neue Formen gespaltener Elternschaft. Aus Politik und Zeitgeschichte 29. Juni 2001: 34-44.

\section{Knoll, E.-M.}

2001 Reproduktionsmedizinische Imaginationen. Überlegungen zum IVF-Diskurs aus ethnologisch-feministischer Perspektive. Diplom-Arbeit am Institut für Ethnologie, Kultur- und Sozialanthropologie, Wien, unveröffentlicht.

König, E.

1989 Körper - Wissen - Macht. Studien zur Anthropologie des Körpers. Berlin.

König, R.

1974 Die Familie der Gegenwart. München.

1976 Soziologie der Familie. In: Ders. (Hg), Handbuch der empirischen Sozialforschung, Bd. 7: Familie - Alter: 1-217. Stuttgart.

\section{Lang, $S$.}

1992 Wir Frauen ohne Kinder. Was Männer nie begründen müssen. Frankfurt a. Mn.

\section{Laqueur, $\mathbf{T}$.}

1986 Orgasm, Generation and the Politics of Reproductive Biology. Representations 14: $1-41$.

1990 Making Sex: Body and Gender from the Greeks to Freud. Cambridge, Mass.

\section{Laqueur, T./Gallagher, C. (eds)}

1987 The Making of the Modern Body. Sexuality and Society in the Nineteenth Century. Berkeley u.a.

\section{Lautermann, E. D.}

1974 Solidarität und Wohnen. Darmstadt.

\section{Leach, E.}

1967 Virgin Birth. Proceedings of the Royal Anthropological Institute for Great Britain and Ireland for 1966: 39-50.

\section{Le Gall, D./Martin, C.}

1997 Fashioning a New Family Tie: Step-Parents and Step-Grandparents. In: Gullestad, M./Segalen, M. (eds), Family and Kinship in Europe: 183-202. London/Washington.

Lesky, E.

1951 Die Zeugungs- und Vererbungslehren der Antike und ihr Nachwirken. Wiesbaden.

\section{Limbach, J.}

1989 Die rechtlichen Rahmenbedingungen von Ehe und Elternschaft. In: Nave-Herz, R./Markefka, M. (Hg): 225-241. 


\section{Lindemann, G.}

1993 Wider die Verdrängung des Leibes aus der Geschlechterkonstruktion. Feministische Studien 2: 44-54.

1994 Die Konstruktion der Wirklichkeit und die Wirklichkeit der Konstruktion. In: Dies./Wobbe, T. (Hg), Denkachsen. Zur theoretischen und institutionellen Rede von Geschlecht. Frankfurt a. Mn.

1996 Zeichentheoretische Überlegungen zum Verhältnis von Körper und Leib. In: Barkhaus, A. et al. (Hg), Identität, Leiblichkeit, Normativität: 146-175. Frankfurt a. Mn.

List, E.

1993 Die Präsenz des Anderen. Theorie und Geschlechterpolitik. Frankfurt a. Mn.

\section{Longino, $M$.}

1998 Die Pflegekindadoption. Berlin.

Lucke, D.

1998 Verwandtschaft im Recht - Rechtssoziologische Aspekte verwandtschaftlicher Beziehungen. In: Wagner, M./Schütze, Y. (Hg): 59-91.

\section{Lüderitz, A.}

1999 Familienrecht: ein Studienbuch. 27. Aufl. München.

\section{Lüschen, G.}

1970 Familie und Verwandtschaft. Interaktion und die Funktion von Ritualen. In: Ders./Lupri, E. (Hg), Soziologie der Familie: 270-284. Opladen.

1988 Familial-verwandtschaftliche Netzwerke. In: Nave-Herz, R. (Hg), Wandel und Kontinuität der Familie in der Bundesrepublik Deutschland: 145-172. Stuttgart.

1989 Verwandtschaft, Freundschaft, Nachbarschaft. In: Nave-Herz, R./Markefka, M. (Hg): 435-452.

\section{Luhmann, $\mathbf{N}$.}

1994 [1982]

Liebe als Passion. Zur Codierung von Intimität. Frankfurt am Main.

\section{Lundin, $\mathbf{S}$.}

1996 Power over the Body. In: Lundin, S./Åkesson, L. (eds): 13.36

\section{Lundin, S./Åkesson, L. (eds)}

1996 Bodytime. On the Interaction of Body, Identity, and Society. Lund.

Maine, $\mathbf{H}$.

1961 Ancient Law. London.

\section{Malinowski, B.}

1929 The Sexual Life of the Savages in North-Western Melanesia. London. 
1963 [1930]

Parenthood - The Basis of Social Structure. In: Ders., Sex, Culture, and Myth: 42-88. London.

Marbach, J. H.

1998 Verwandtschaftsbeziehungen und Abstammung - Eine Prüfung soziobiologischer und ethnologischer Thesen mit Hilfe familiensoziologischer Daten. In:

Wagner, M./Schütze, Y. (Hg): 91-127.

Martins, G./Breckwoldt, M./Pfleiderer, A. (Hg)

1994 Lehrbuch der Gynäkologie und Geburtshilfe. Stuttgart/NewYork.

Mauss, M.

1978 [1938]

Eine Kategorie des menschlichen Geistes: Der Begriff der Person und des „Ich“. In: Mauss, M., Soziologie und Anthropologie, Bd. II: Gabentausch, Soziologie und Psychologie, Todesvorstellung, Körpertechniken, Begriff der Person: 221-252. Frankfurt a. Mn.

1990 [1968]

Die Gabe. Form und Funktion des Austauschs in archaischen Gesellschaften. Stuttgart/New York.

\section{Mayring, $\mathbf{P}$.}

1993 Einführung in die qualitative Sozialforschung: eine Anleitung zum qualitativen Denken, 2. Aufl. Weinheim.

2000 Qualitative Inhaltsanalyse: Grundlagen und Techniken, 7.erw. Aufl. Weinheim.

McLennon, J.F.

1865 Primitive Marriage. Edinburgh.

McNeil, M./Varcoe, I./Yearley, S. (eds)

1990 The New Reproductive Technologies. New York.

Medick, H./Sabean, D. (Hg)

1984 Emotionen und materielle Interessen. Sozialanthropologische und historische Beiträge zur Familienforschung. Göttingen.

Meigs, A.

1989 The Cultural Construction of Reproduction and its Relationship to Kinship and Gender (New Guinea Highlands). In: Mac Marshall/Caughey, J.L. (eds), Culture, Kin and Cognition in Oceania: Essays in Honour of Ward H. Goodenough. Special Publication of the American Anthropological Association Nr. 25, Washington, DC: American Anthropological Association.

Merleau-Ponty, M.

1966 [1945]

Phänomenologie der Wahrnehmung. Berlin. 


\section{Mitterauer, M.}

1977 Der Mythos von der vorindustriellen Großfamilie. In: Mitterauer, M./Sieder, R., Vom Patriarchat zur Partnerschaft: 38-65. München.

Modell, J. S.

1994 Kinship with Strangers. Adoption and Interpretations of Kinship in American Culture. Berkeley/L.A./London.

\section{Moore, $\mathbf{H}$.}

1988 Feminism and Anthropology. Cambridge.

\section{Morgan, L. H.}

1868 A Conjectural Solution of the Origin of the Classificatory System of Relationship. Proceedings of the American Academy of Arts and Science 7: 436-477.

1910 [1877]

Ancient Society, or Researches in the Lines of Human Progress from Savagery through Barbarism to Civilisation. Chicago.

1970 [1871]

Systems of Consanguinity and Affinity of the Human Family. Smithsonian Contributions to Knowledge Vol. 17. Washington D.C.: Smithsonian Institution. Oosterhood N.B. - The Netherlands.

\section{Morris, B.}

1994 Anthropology of the Self. The Individual in Cultural Perspective. London.

Murdock, G. P.

1949 Social Structure. New York.

\section{Nave-Herz, R.}

1987 Bedeutungswandel von Ehe und Familie. In: Schulze, H. J./Meyer, T. (Hg), Familie: Zerfall oder neues Selbstverständnis?: 18-28. Würzburg.

\section{Nave-Herz, R./Markefka, M. (Hg)}

1989 Handbuch der Familien- und Jugendforschung, Bd. 1: Familienforschung. Neuwied/Frankfurt.

\section{Oberloskamp, $\mathbf{H}$.}

1993 Wie adoptiere ich ein Kind? Wie bekomme ich ein Pflegekind? Rechtliche Erfordernisse und Folgen, Kindesvermittlung, behördliches und gerichtliches Verfahren, 3. Aufl. München.

\section{Petersen, I.}

2000 Konzepte und Bedeutung von Verwandtschaft: Eine ethnologische Analyse der Parlamentsdebatten zum bundesdeutschen Embryonenschutzgesetz. Herbolzheim.

2002 Grenzkonflikte um Person und Leben. Kulturelle Wissensstrukturen in den Parlamentsdebatten zum Embryonenschutzgesetz und Transplantationsgesetz. Dissertation an der Sozialwissenschaftlichen Fakultät der Universität Göttingen, elektronische Veröffentlichung an der Universität Göttingen. 


\section{Pfeffer, $\mathbf{N}$.}

1987 Artificial Insemination, In vitro Fertilisation and the Stigma of Infertility. In: Stanworth, M. (ed): 81-97.

Pfeil, E./Ganzert, J.

1973 Die Bedeutung der Verwandten für die großstädtische Familie. Zeitschrift für Soziologie (2): 366-383.

\section{Pfleiderer, B.}

1995 Der Blick nach Drinnen: zur kulturellen Konstruktion medizinischen Tuns und Wissens. In: Dies./Greifeld, K./Bichmann, W., Ritual und Heilung: Eine Einführung in die Ethnomedizin: 163-198, 2. vollst. überarb. und erw. Neuaufl. Berlin.

\section{Pschyrembel, W.}

1993 Medizinisches Wörterbuch: Sonderausgabe Pschyrembel Klinisches Wörterbuch, 257. neu bearb. Aufl. Hamburg.

\section{Radcliffe-Brown, A. R.}

1950 Introduction. In: Ders./Forde, D. (eds), African Systems of Kinship and Marriage. London.

\section{Ragoné, H.}

1994 Surrogate Motherhood: Conception in the Heart. Boulder, CO.

1998 Incontestable Motivations, In: Franklin, S./Ragoné, H. (eds): 118-132.

\section{Raymond, J.}

1995 [1993]

Die Fortpflanzungsmafia. München.

\section{Reproduktionsmedizin}

$199915(84)$

\section{Richtlinien zur Durchführung der assistierten Reproduktion}

1998 Zweite Novellierung. Deutsches Arzteblatt 95 (49): A-3166-3171.

\section{Rosenbaum, $\mathbf{H}$.}

1982 Formen der Familie. Untersuchungen zum Zusammenhang von Familienverhältnissen, Sozialstruktur und sozialem Wandel in der deutschen Gesellschaft des 19. Jahrhunderts. Frankfurt a. Mn.

1998 Verwandtschaft in historischer Perspektive. In: Wagner, M./Schütze, Y. (Hg): 17-33.

Sault, N. (ed)

1994 Many Mirrors. Body Image and Social Relations. New Brunswick/New Jersey.

\section{Scheffler, H. W.}

1976 The „Meaning“ of Kinship in American Culture: Another View. In: Basso, K.H./Selby, H.A. (eds), Meaning in Anthropology: 57-91. Albuquerque. 


\section{Scheper-Hughes, N./Lock, M.}

1987 The Mindful Body: A Prolegomenon to Future Work in Medical Anthropology. Medical Anthropology Quarterly 1 (1): 6-41.

Schindele, E.

1990 Gläserne Gebär-Mütter. Frankfurt.

Schirren, C. et al.

1989 Unerfüllter Kinderwunsch. Leitfaden für Diagnostik, Beratung und Therapie in der Praxis. Köln.

\section{Schlieffen, A. Gräfin von}

1994 Offene Adoptionsformen - ein Grund zur Reform des Adoptionsrechts. Berlin.

\section{Schmidt, $\mathbf{H}$.}

1982 Philosophisches Wörterbuch. Neu bearbeitet von Georgi Schischkoff, 21. Aufl. Stuttgart.

Schmitt, A.

2000 Geteilte Mutterschaft. Eine ethnologische Studie über Herkunftsmütter in Deutschland. Magister-Arbeit am Institut für Ethnologie der Universität Göttingen, unveröffentlicht.

\section{Schneider, D. M.}

1980 [1968]

American Kinship: A Cultural Account, 2. Aufl. Chicago.

1984 A Critique of the Study of Kinship. Ann Arbor.

1992 Ethnocentrism and the Notion of Kinship. Man 27: 629-631.

Schütze, Y.

1991 Die gute Mutter. Zur Geschichte des normativen Musters „Mutterliebe“, 3. Aufl. Bielefeld.

\section{Segalen, M.}

1986 Historical Anthropology of the Family. Cambridge.

1997 Introduction, In: Gullestad, M./Segalen, M. (eds), Family and Kinship in Europe: 1-14. London/Washington.

\section{Snowden, R./Mitchell, G.D./Snowden, E.M.}

1983 Artificial Reproduction. London.

Spiro, M. E.

1968 Virgin Birth. Parthogenesis and Physiological Paternity. Man 3: 242-261.

\section{Stacey, M.}

1992a Social Dimensions of Assisted Reproduction, In: Stacey, M. (ed): 9-48.

1992b (ed), Changing Human Reproduction. Social Science Perspectives . London/Newbury Park/Delhi. 
Stanworth, M. (ed)

1987 Reproductive Technologies. Gender, Motherhood and Medicine. Minneapolis.

Stein, W./Sproll, E.

1995 Vom unwiderstehlichen Drang, Mutter zu werden. Mutterglück aus der Retorte?. Pfaffenweiler.

\section{Stein-Hilbers, M.}

1994 Wem gehört das Kind? Neue Familienstrukturen und veränderte Eltern-KindBeziehungen. Frankfurt/New York.

Strathern, A. J.

1996 Body Thoughts. Ann Arbor.

\section{Strathern, M.}

1992a After Nature: English Kinship in the Late Twentieth Century. Cambridge.

1992b Reproducing the Future: Anthropology, Kinship, and the New Reproductive Technologies. New York.

1992c The Meaning of Assisted Kinship. In: Stacey, M. (ed): 148-170.

1993a A Question of Context. In: Edwards, J. et al.:1-20.

1993b Regulation, Substitution and Possibility. In: Edwards, J. et al.:132-162.

1995 Displacing Knowledge: Technology and the Consequences for Kinship. In: Ginsburg, F./Rapp, R. (eds): 346-363.

1996 Enabling Identity? Biology, Choice and the New Reproductive Technologies. In: Hall, S./Gay, P. du (eds), Questions of Cultural Identity: 37-53. London/Thousand Oaks/New Delhi.

1997 Partners and Consumers: Making Relations Visible. In: Schrift, A.D. (ed), The Logic of the Gift. Toward an Ethic of Generosity: 292-311. New York.

1998 Divisions of Interest and the Language of Ownership. In: Hann, C. (ed), Property Relations. Renewing the Anthropological Tradition: 214-232. New York.

1999 Property, Substance \& Effect. Anthropological Essays on Persons and Things. London/New Brunswick, NJ.

2001 Gender - eine Frage des Vergleichs. In: Davis-Sulikowski, U. et al. (Hg), Körper, Religion und Macht. Sozialanthropologie der Geschlechterbeziehungen: 359-395, Frankfurt/New York.

\section{Strathern, M./Franklin, S.}

1993 Kinship and the New Genetics: An Assessment of Existing Anthropological Research. Manchester: University of Manchester, Department of Social Anthropology.

\section{Thorn, P.}

1999 Ach wie gut, dass niemand weiss...? Wie beeinflusst die Anonymität des biologischen Vaters die Beziehungen, die sich mit Hilfe der donogenen Insemination gebildet haben?. Diplomarbeit an der Fachhochschule Darmstadt, unveröffentlicht. 


\section{Tong, R.}

1995 Feminist Perspectives and Gestational Motherhood: The Search for a Unified Legal Focus. In: Callahan, J. (ed), Reproduction, Ethics, and the Law. Feminist Perspectives: 55-80. Bloomington/Indianapolis.

\section{Trautmann, T. R.}

1987 Lewis Henry Morgan and the Invention of Kinship. Berkeley/L.A./London.

\section{Ulrich, D./Strauß, B./Bohnet, H.-G.}

1988 Psychosomatische Aspekte von Fertilitätsstörungen. In: Appelt, H./Strauß, B. (Hg), Psychoendokrinologische Gynäkologie: 172-198. Stuttgart.

\section{Universal Lexikon}

1993 Lexikographisches Institut. Universal Lexikon in einem Band. Büchergilde Gutenberg. Frankfurt a. Mn.

\section{Vowinckel, G.}

1995 Verwandtschaft, Freundschaft und die Gesellschaft der Fremden - Grundlagen menschlichen Zusammenlebens. Darmstadt.

Wagner, M./Schütze, Y. (Hg)

1998 Verwandtschaft. Sozialwissenschaftliche Beiträge zu einem vernachlässigten Thema. Stuttgart.

\section{Waldenfels, B.}

2000 Das leibliche Selbst. Vorlesungen zur Phänomenologie des Leibes. Frankfurt a. $\mathrm{Mn}$.

\section{Weber-Kellermann, I.}

1974 Die deutsche Familie. Frankfurt a. Mn.

\section{Weston, $\mathbf{K}$.}

1991 Families We Choose: Lesbians, Gays, Kinship. New York.

\section{Wiemann, I.}

1998 Pflege- und Adoptivkinder. Familienbeispiele, Informationen, Konfliktlösungen. Reinbek bei Hamburg.

\section{Wiesenbart, $T$.}

1990 Großbritannien. In: Eser, A./Koch, H.-G./Wiesenbart, T. (Hg), Regelungen der Fortpflanzungsmedizin und Humangenetik 1. Eine internationale Dokumentation gesetzlicher und berufsständischer Rechtsquellen. Frankfurt a. Mn.

Wild, E.

1998 Adoption - Familienleben mit doppelter Elternschaft. In: Wagner, M./Schütze, Y. (Hg): 263-283.

\section{Winau, R.}

1982 Krankheitskonzept und Körperkonzept. In: Kamper, D./Wulf, C. (Hg), Die Wiederkehr des Körpers: 285-298. Frankfurt a. Mn. 
1983 Die Entdeckung des menschlichen Körpers in der neuzeitlichen Medizin. In: Imhof, A.E. (Hg), Der Mensch und sein Körper. Von der Antike bis heute: 209225, München.

Winkler, U.

1994 Der unerfüllte Kinderwunsch. Ein Ratgeber für kinderlose Paare. München.

Wittland-Mittag, A.

1992 Adoption und Adoptionsvermittlung - Selbstverständnis von Adoptionsvermittlern und-vermittlerinnen. Essen.

Yanagisako, S./Delaney, C. (eds)

1995 Naturalizing Power: Essays in Feminist Cultural Analysis. London.

Zonabend, F.

1996 An Anthropological Perspective on Kinship and the Family. In: Burguière, A. et al. (eds), A History of the Family Vol. 1: 8-71. Cambridge. 